Departamento de Automática, Ingeniería Eléctrica y Electrónica e Informática Industrial

Escuela Técnica Superior de Ingenieros Industriales

\title{
Strategies and Tools for Deployment and Optimization of Wireless Sensor Networks
}

Autor: Gabriel Noe Mujica Rojas

Máster en Electrónica Industrial por la Universidad Politécnica de Madrid

Director: Jorge Portilla Berrueco Doctor por la Universidad Politécnica de Madrid en Ingeniería Electrónica 



\section{Tribunal}

Tribunal nombrado por el Excmo. y Mgfco. Sr. Rector de la Universidad Politécnica de Madrid, el día de de 2017.

\section{Presidente:}

Vocales:

Secretario:

Suplentes:

Realizado el acto de lectura y defensa de la Tesis Doctoral el día de de 2017 en la Escuela Técnica Superior de Ingenieros Industriales de la Universidad Politécnica de Madrid.

Calificación:

EL PRESIDENTE

LOS VOCALES

EL SECRETARIO 



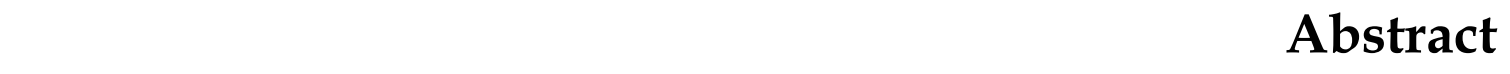

The progressive evolution and maturity process of the research field of Wireless Sensor networks (WSN) has encompassed new hardware and software approaches in order to face more demanding smart scenarios, where flexible and adaptable platforms play a fundamental role to properly address application requirements and service provision. Although the concepts of sensor networks and pervasive computing systems have been developed since the late nineties, the evolution of wireless communication strategies as well as the aim of a smart, sustainable and connected world came out with novel paradigms that are also defining the new horizon of WSN-based applications and related technologies. In particular, the progressive inclusion of the Internet of Things (IoT) as the main foundation for the "city of the future" implementation has defined a new way of approaching the development of embedded sensor systems.

This innovation process also implies new and more complex challenges due to the inherent integration of a wide range of different technologies, protocols and algorithms so that their performance and feasibility within final system implementations can be maximized as a whole. The complexity of converging these technologies into an efficient heterogeneous implementation makes the development cycle of a WSN not only a combination of different testing and optimization techniques at each stage of the development process (up until the final operability and maintenance of the system), but also a proper correlation among them, so as to guarantee the final success of the overall solution. In this way, there have been important contributions in the state of the art related to development and planning strategies of wireless embedded systems, including simulation techniques prior to the actual realization of the sensor network.

However, there is still a lack of well-defined frameworks, methodologies and tools to carry out the on-site deployment, commissioning, and validation activities as a support for developers and deployers to assure the cohesion and operability of the real WSN at the target scenario. Traditionally, the experimental phase of the development cycle beyond the simulation process has been accomplished with the assistance of testing infrastructures, and significant advances regarding the implementation of WSN testbed platforms have been discussed in the literature in recent years. However, fewer works attempt to provide a direct synergy between modelling and planning strategies with efficient deployment and on-site assessment techniques (particularly considering communication performance, wireless routing algorithms and networking mechanisms of the distributed sensor nodes).

In this work these challenges are addressed by proposing the design and integration of a comprehensive set of strategies and optimization tools in an in-field deployment platform, which allows performing a consistent on-site analysis, reconfiguration and 
performance evaluation of WSNs. For this purpose, the experimental and in-field commissioning phases are tackled in this thesis from a pre-deployment and on-site deployment perspective. On one hand, the former one is approached by proposing a new WSN testbed framework based on the modularity and flexibility of an embedded HW-SW node platform, from which the experimental analysis of WSN prototypes can be carried out considering:

- A WSN remote testing support scheme that allows seamless reconfiguration and execution of debugging actions on the target sensor nodes, so that more realistic experimentation of low-power resource-constrained sensor network prototypes and novel hardware and software developments can be performed upon the foundation of this modular architecture, and through a backchannelbased hybrid composition.

- Runtime partial reprogramming strategies by defining a memory-segmentsbased structure to modularize the functionalities under test as well as optimize the reconfiguration process of the embedded software, which is extensible to infield deployment and operational stages where energy-efficient functional block replacements or reprogramming procedures are one of the main keys to assure the long-term autonomy of the deployed system.

- A HW-SW integration platform to provide users with a complete support for an in-node embedded management of the architecture resources, low-level hardware controllers and system interfaces of the sensor devices.

On the other hand, the latter phase is accomplished by implementing a network deployment methodology along with the inclusion of runtime comparative analysis modules integrated in a unique on-site WSN deployment tool-chain (and within a mobile intelligent platform), in which a combination of simulation models with the actual system behaviour during the installation and commissioning process allows deployers to produce more adapted and accurate in-field results towards the operational release of the WSN-based system. This platform encompasses the integration of heterogeneous functionalities into a single element allowing for:

- Network/deployment models to properly define the main hardware and software elements that take part in the whole WSN architecture, so that a wellstructured modular input for and output from the on-site deployment subsystems can be provided. These models are associated with the design, synthesis and code generation process of the embedded platform, and based on the input requirements of the target system.

- In-field simulation engines for site estimation of network/node correlation based on the runtime parametrization and configuration actions carried out on 
the sensor devices, which can be interactively compared with the real performance evolution of the overall system, taking into account how such actions may affect the global behaviour of the WSN (beyond their local influence).

- Deployment optimization algorithms and on-site automatic guidance to efficiently perform the network correlation and configuration tasks, so that the cohesion of the whole WSN can be achieved taking into account a trade-off between energy/resource consumption and reliability among the involved interconnections (both the communication and operational side).

- Implementation of efficient multi-hop wireless mesh networking mechanisms into the modular HW-SW platform and connectivity evaluation strategies to analyse the performance of low-rate and resource-constrained communication protocols, particularly targeting dynamic and adaptable routing schemes.

- Integration of middleware-based support services and implementation of a debugging communication interface in the on-site deployment toolset in order to establish the runtime configuration and interaction with the sensor nodes in field, so that users are capable of providing a more comprehensive feedback to the system development cycle based on the comparative analysis of real plus simulated data along with the results of the on-site WSN evaluation process. Such abstraction layer is indeed in line with the network/deployment models, so that a consistent analysis and evaluation of the target system can be accomplished.

The design and implementation of the deployment toolset have been realized paying special attention to the provision of an intuitive and easy-to-use interface for conveniently assisting deployers during the in-field commissioning, evaluation and maintenance activities of the wireless system. In this way, the intelligent platform is mainly composed of a Hand-Held Device (HHD) in which the WSN technologies, infield simulation, optimization and performance assessment modules are effectively integrated so as to properly guide users throughout the whole process of deploying the target sensor network.

Real tests and experimental use cases have been carried out not only to validate the proposed strategies but also to put these capabilities into different application contexts, where the support and assistance of the designed pre-deployment and on-site deployment tools played an important role for the success of the aimed WSN-based systems.

In order to deeply address the work carried out in this thesis, the document has been organized in 6 chapters. In chapter 2, a comparative analysis of the state-of-the-art 
WSN testbed solutions is presented, together with the main motivations that outline the testbed framework proposed in this work. The HW-SW architecture along with the partial and remote reprogramming scheme are also presented in this chapter. Chapter 3 encompasses one of the major contributions of the present thesis, which is the design and development of the on-site WSN deployment and commissioning toolset, including the analysis of relevant issues related to the necessity of having an in-field HW-SW platform to carry out the configuration, analysis and performance evaluation of wireless sensor deployments. Based on this, chapter 4 summarizes the implementation of the deployment toolset and how the main capabilities of the proposed system have been integrated into a unique user interface. Chapter 5 is dedicated to describe several experimental test cases to validate not only the different strategies that integrate the on-site deployment toolset but also the overall system implementation in different application conditions, including further details regarding the challenges, design and development of a smart city demonstration scenario realized in the context of this thesis. Finally, conclusions, summary of scientific contributions as well as future research work are outlined in chapter 6 . 


\section{Resumen en Castellano}

El campo de investigación de las Redes de Sensores Inalámbricas (en inglés Wireless Sensor Networks, WSN) ha experimentado un importante y progresivo proceso de maduración que ha involucrado la definición de nuevas tecnologías a nivel hardware y software con el fin de abordar escenarios de aplicación mucho más demandantes, en los que plataformas flexibles y adaptables juegan un papel fundamental para la consecución de los requisitos de aplicación y provisión de servicios dentro de diversos entornos de implementación. Aunque el concepto de redes de sensores y computación ubicua se ha ido desarrollando desde la década de los noventa, la evolución de tecnologías de comunicaciones inalámbricas así como el objetivo de alcanzar sistemas globalizados sostenibles, inteligentes e interoperables han traído consigo nuevos paradigmas que de alguna manera también marcan el horizonte de las aplicaciones y tecnologías asociadas a las WSNs. En este sentido, es esencial destacar la inclusión del concepto del Internet de las Cosas (en inglés Internet of Things, IoT), que representa uno de los pilares en la implementación de las llamadas ciudades del futuro, y que marcan un nuevo enfoque en el desarrollo de sistemas embebidos inteligentes, de bajo consumo y distribuidos.

Dicho proceso de innovación implica a su vez nuevos y más complejos retos en el ámbito de las redes de sensores inalámbricas debido a la integración intrínseca de una amplia variedad de protocolos, algoritmos y tecnologías heterogéneas que permitan maximizar su viabilidad y rendimiento dentro de la implementación final de sistemas como un todo, más allá del funcionamiento de elementos individuales. La complejidad asociada a la convergencia de tales tecnologías hace que el ciclo de desarrollo de una WSN abarque no sólo una combinación de técnicas de optimización y experimentación en cada una de las etapas de dicho proceso de desarrollo (hasta la operación final y mantenimiento del sistema), sino también una adecuada correlación entre las mismas, para así garantizar el alcance de la solución completa objetivo. En este sentido, durante los últimos años ha habido importantes aportaciones en el estado del arte relacionados con estrategias de desarrollo y planificación de sistemas embebidos inalámbricos, incluyendo técnicas de simulación como preámbulo a la implementación real de la red de sensores.

Sin embargo, existe una carencia dentro de la definición de frameworks, metodologías y herramientas que proporcionen un completo soporte para el despliegue, puesta en marcha (que se enmarca dentro de las llamadas estrategias de commissioning) y validación en campo de WSN que permitan asegurar la cohesión y operación global del sistema dentro del escenario de aplicación objetivo. Normalmente, la fase experimental del ciclo de desarrollo más allá del proceso de simulación de la solución objetivo se lleva a cabo a través de infraestructuras de pruebas, en las que avances significativos 
en el área de plataformas testbeds de redes de sensores han sido propuestas en la última década, con el fin de dotar las actividades de experimentación con arquitecturas reales de depuración y evaluación. No obstante, una menor cantidad de trabajos de investigación se centran en analizar y proveer una sinergia directa entre estrategias de modelado y planificación con técnicas eficientes de despliegue y evaluación de redes de sensores in-situ (especialmente en términos mecanismos de interconexión y encaminamiento entre los nodos sensores distribuidos).

En este trabajo de investigación se abordan estos importantes retos mediante el diseño e integración de un completo set de estrategias y herramientas de optimización dentro de una novedosa plataforma de despliegue en campo, la cual permite llevar a cabo el análisis, reconfiguración y evaluación del rendimiento de la WSN de forma directa dentro del escenario de aplicación y de forma eficiente. Para conseguir este propósito, las fases de experimentación y puesta en marcha se plantean en esta tesis doctoral desde una perspectiva pre-despliegue y despliegue in-situ. Por un lado, la fase predespliegue se aborda a través de la propuesta de un nuevo framework para testbeds de redes de sensores inalámbricas basado en la modularidad y flexibilidad de una plataforma hardware-software embebida, sobre la cual el análisis experimental de prototipos para WSN se puede efectuar considerando los siguientes elementos:

- Un esquema de soporte para pruebas y experimentación remota que permita la reconfiguración y ejecución de acciones de depuración sobre los nodos sensores objetivo, de manera que un escenario experimental más realista basado en prototipos de redes de sensores de bajo consumo y recursos limitados pueda ser dispuesta, y de forma tal que nuevos prototipos hardwaresoftware se evalúen y optimicen dentro del marco de la arquitectura modular propuesta. Ello se plantea mediante la composición de un canal de depuración secundario híbrido.

- Estrategias de reprogramación parcial en tiempo real mediante la definición de una estructura basada en segmentos de memoria dentro de la plataforma modular de WSN, encaminado a modularizar las funcionalidades bajo test y evaluación y optimizar el proceso de reconfiguración del software embebido en los nodos. Dicha técnica de reprogramación es extensible a despliegues en campo y fases de operación estables de red, en el que el reemplazo y/o actualización eficiente de bloques funcionales en términos de consumo de recursos y energéticos representan uno de los principales elementos clave para obtener una mayor autonomía a largo plazo del sistema desplegado.

- Una plataforma de integración HW-SW que permita proporcionar a los desarrolladores de sistemas basados en nodos inalámbricos soporte para una efectiva gestión embebida en el nodo de los recursos disponibles en la 
arquitectura modular, además de controladores hardware e interfaces a nivel de sistema de los dispositivos sensores.

Por otro lado, la fase de despliegue se afronta en este trabajo a través del diseño e implementación de una completo set de herramientas interrelacionadas para llevar a cabo el despliegue, validación y ejecución de WSN en campo, en el que la definición de una metodología de despliegue junto con la inclusión de módulos de optimización y análisis comparativos en tiempo real in-situ son integradas en una dispositivo inteligente móvil, y que combina en una única plataforma soporte modelos de simulación con el funcionamiento real del sistema objetivo durante las tareas de instalación, configuración, puesta en marcha y evaluación del rendimiento de la red de sensores en todo su conjunto. Ello permite generar resultados más adaptados y precisos de cara a obtener una operación final eficiente de la WSN en el escenario de aplicación. Esta novedosa plataforma se basa en la integración de funcionalidades heterogéneas considerando los siguientes elementos de innovación:

- Modelos de despliegue y red con el fin de definir de forma adecuada los principales elementos hardware y software que formarán parte de la arquitectura global de la WSN, los cuales se encuentran estrechamente relacionados con el proceso de diseño, síntesis y generación de código del sistema embebido, y basado en los requisitos del sistema a desplegar. Ello permite proporcionar una estructura bien definida de entradas y salidas no sólo a los sub-sistemas que componen la herramienta de despliegue en campo sino también al ciclo de desarrollo de la red de sensores inalámbrica.

- Motores de simulación in-situ para así proporcionar al usuario deployer una estimación de la correlación nodo/red basado en acciones de reconfiguración y parametrización en tiempo de ejecución sobre los nodos sensores, cuyo resultado puede ser comparado de forma interactiva con la evolución del comportamiento real del sistema conjunto, y tomando en cuenta cómo dichas acciones pueden afectar de forma global el rendimiento de la WSN (mas allá de su influencia a nivel local).

- Algoritmos de optimización de despliegue y guía automática en campo que permitirán obtener la matriz real de correlación y configuración de red de modo eficiente, de forma tal que la cohesión de la WSN en todo su conjunto pueda ser alcanzada tomando en consideración un compromiso entre consumo de energía/recursos y la fiabilidad de las interconexiones involucradas entre los dispositivos inalámbricos (tanto a nivel de comunicaciones como a nivel de operación). 
- Implementación de técnicas eficientes de interconexión multi-salto malladas dentro de la plataforma modular HW-SW, combinadas con estrategias de evaluación de conectividad de los nodos a diferentes niveles de abstracción, con el fin de poder proveer soporte en el análisis del rendimiento de los protocolos de comunicaciones de baja tasa de transferencia de datos y recursos limitados en los dispositivos inalámbricos que forman parte de la WSN objetivo. En tal sentido, se toma en cuenta de forma particular la integración de esquemas de encaminamiento dinámico y adaptable en la arquitectura modular propuesta.

- Integración de una capa de abstracción de servicios soporte basada en una implementación middleware así como el diseño de una interfaz de depuración en campo dentro de la herramienta de despliegue, enfocado a establecer un canal de configuración e interacción en tiempo de ejecución con los nodos sensores de forma directa en el entorno de aplicación y en total sintonía con los modelos de despliegue/red definidos. Ello permite a los usuarios producir una realimentación mucho más completa al ciclo de desarrollo de la WSN en función del análisis comparativo de los datos reales y simulados de la red, en conjunción con los resultados obtenidos como salida del proceso de evaluación en campo del sistema global.

El diseño e implementación del set de herramientas de despliegue se ha realizado prestando especial atención a la generación de interfaces intuitivas y de fácil uso desde el punto de vista del deployer, para así poder proveer asistencia durante las tareas de puesta en marcha, evaluación y mantenimiento en campo de forma consistente. En este sentido, la plataforma inteligente se compone principalmente del denominado HandHeld Device (HHD) en el que las tecnologías de WSN, módulos de simulación in-situ, optimización y evaluación funcional son integrados de manera efectiva e interrelacionados entre sí, para así guiar al usuario durante todo el proceso de despliegue de la red de sensores inalámbrica en el escenario objetivo.

Se han llevado a cabo y analizado diversos casos de pruebas y experimentación dentro de este trabajo de investigación con el fin no sólo de validar las técnicas propuestas y su integración dentro de una plataforma unificada, sino también para contextualizar su aplicabilidad a través de distintos ámbitos reales prácticos, en los que el soporte y asistencia de las estrategias pre-despliegue y despliegue en campo han jugado un rol fundamental para la consecución de los objetivos y requisitos de tales sistemas basados en WSN.

Con el fin de abordar en detalle el trabajo realizado dentro del marco de la presente tesis doctoral, la organización del documento se ha desglosado de 6 capítulos. En el capítulo 2 se plantea un análisis comparativo del estado del arte de testbeds para WSN, además de exponer los principales motivos que engloban el diseño del framework de 
integración HW-SW para testbeds basados en la plataforma modular propuesto en este trabajo. La arquitectura hibrida desarrollada junto con las estrategias de reprogramación parcial y remota también se incluyen dentro de este capítulo. Por otro lado, el capítulo 3 abarca una de las principales contribuciones de esta tesis doctoral, que tiene que ver con el set de herramientas para el despliegue de WSNs en campo, incluyendo el análisis de aquellos aspectos que acentúan la necesidad de contar con una plataforma HW-SW bien definida y unificada para llevar a cabo la configuración, evaluación y mantenimiento en campo del despliegue de redes sensores inalámbricas. En base a ello, el capítulo 4 se ha dedicado a sintetizar la implementación a alto nivel del set de herramientas de despliegue y cómo las principales capacidades del sistema propuesto han sido integradas en una interfaz de usuario conjunta y heterogénea. En el capítulo 5 se describen algunos de los principales casos experimentales con el fin de validar no sólo las estrategias que integran la herramienta de despliegue en campo, sino también su inclusión dentro de diferentes condiciones de aplicación, incluyendo los retos, el diseño y desarrollo de un demostrador inteligente realizado dentro del contexto de la presente tesis y enfocado al ámbito de las Smart Cities. Finalmente, conclusiones, principales aportaciones científicas y resumen del trabajo de investigación futuro como fruto de esta tesis son presentados en el capítulo 6. 



\section{Contents}

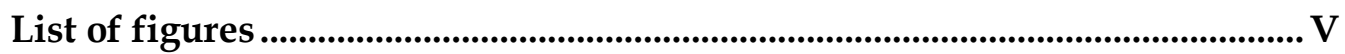

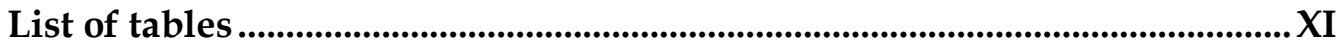

List of acronyms ............................................................................................

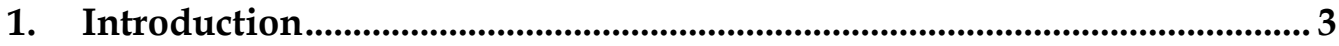

1.1. Wireless Sensor Networks: Evolution and challenges .......................... 3

1.2. The development of WSN-based applications .................................... 7

1.3. An in-field approach for experimenting in WSNs ................................ 8

1.4. Main objectives and scope of the proposed work .................................. 10

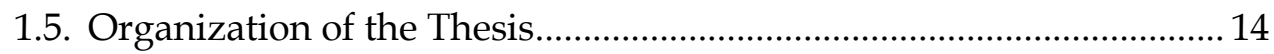

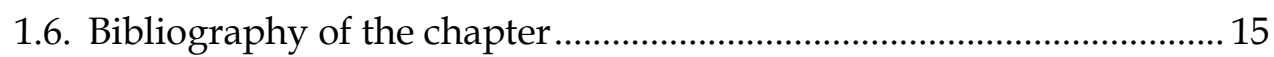

2. The pre-deployment stage of Wireless Sensor Networks ..........................19

2.1. Introduction....................................................................................... 19

2.2. WSN Testbed platforms in the state of the art..................................... 20

2.3. Proposed WSN testbed architecture and framework .......................... 33

2.3.1 The modular hardware platform ............................................................... 35

2.3.2 Backchannel interface approach ........................................................ 42

2.3.3 Functional blocks for supporting the experimental platform............50

2.3.4 HW-SW Integration platform: Software support libraries..................58

2.4. Remote and partial reprogramming architecture: A modular

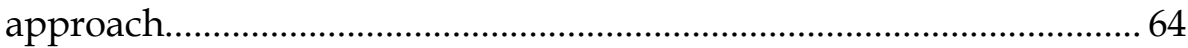

2.4.1 Challenges of reprogramming in WSNs and testbed

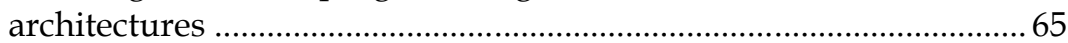

2.4.2 Remote Reprogramming techniques in the state of the art.................67

2.4.3 Proposed approach for a modular WSN partial reprograming .........71

2.4.4 Bootloader and recovery segment .......................................................... 79

2.4.5 Multi-application/experimentation support and dynamic

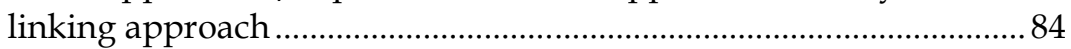

2.4.6 Application segment, User software prototyping ................................ 88

2.5. Applicability of the platform and test cases ...................................... 90

2.5.1 Scenario 1: Testbed characterization and remote reprogramming performance evaluation ............................................. 90

2.5.2 Scenario 2: Indoor deployment for planning tool analysis and

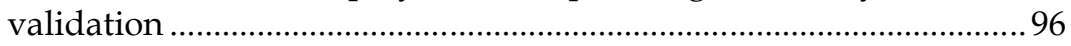

2.5.3 The WSN modular platform within IoT urban automation

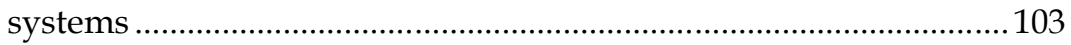

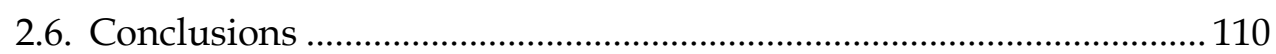

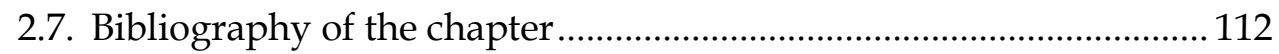


3. Deployment and on-site analysis of Wireless Sensor Networks ........... 121

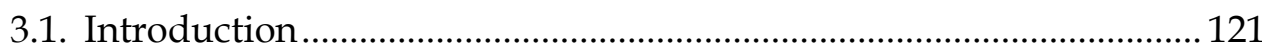

3.2. On-site deployment strategies in the state of the art: an open issue .

3.3. Adapting the modular platform to communication performance experimentation

3.4. A real perspective towards wireless mesh networking optimization based on the modular platform....................................... 133

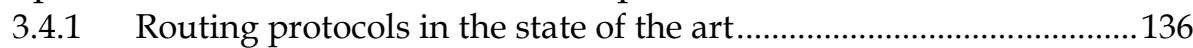

3.4.2 Cookie-Based-AODV implementation.................................................140

3.5. On-site deployment and commissioning process: requirements, considerations and proposed approach ................................................ 155

3.6. System architecture of the proposed approach ................................... 160

3.7. The Hand-Held Device in depth: on-site deployment methodology, analysis and performance evaluation strategies........ 163

3.7.1 Services and input model definition ....................................................163

3.7.2 Deployment connectivity analysis and in-field simulation engine …..........................................................................................177

3.7.3 Deployment methodology and on-site configuration strategies.....197

3.7.4 CB-RSim: Multi-hop mesh networking evaluation engine ...............222

3.7.5 Deployment dynamic guidance ...........................................................2. 239

3.7.6 HHD on-site interface to the WSN deployment .................................246

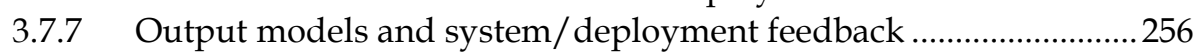

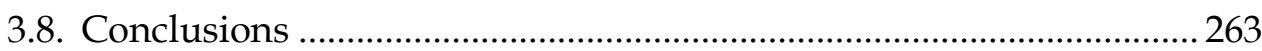

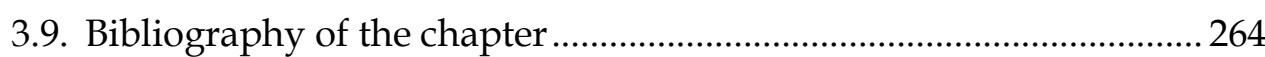

4. Deployment toolset implementation and interface................................ 273

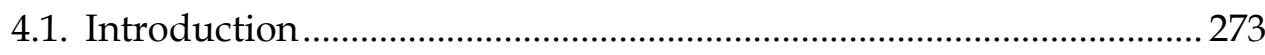

4.2. Application access and project selection ............................................. 274

4.3. Deployment and runtime optimization modules .............................. 275

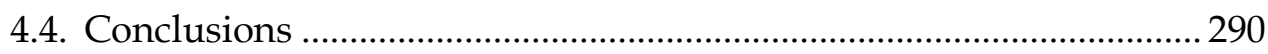

4.5. Bibliography of the chapter ................................................................ 291

5. Experimental tests and use cases .................................................................. 295

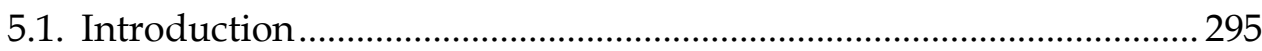

5.2. Test case 1: CB-AODV implementation and routing protocol

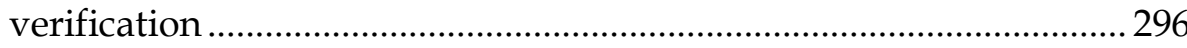

5.3. Metrics correlation and experimental characterization....................... 308

5.4. Test case 2: Outdoor Scenario A ............................................................. 309

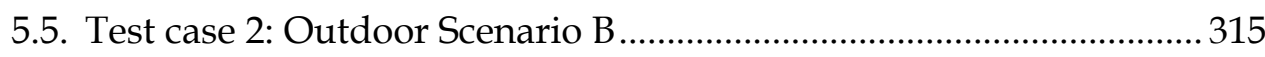


5.6. Smart parking demonstrator: WSN-DPCM European project

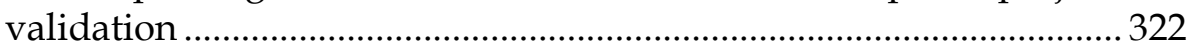

5.6.1 Demonstrator requirements and system architecture ........................325

5.6.2 Demonstrator implementation and on-site WSN deployment ........334

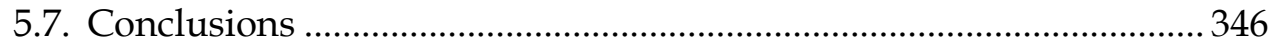

5.8. Bibliography of the chapter............................................................. 347

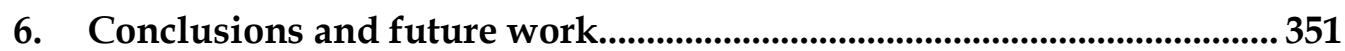

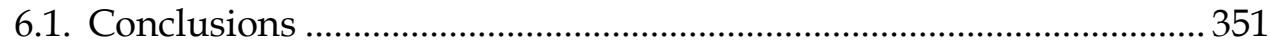

6.2. Research results and scientific references of the work ....................... 354

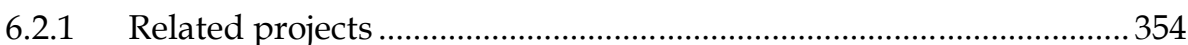

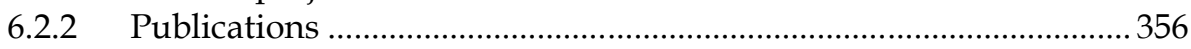

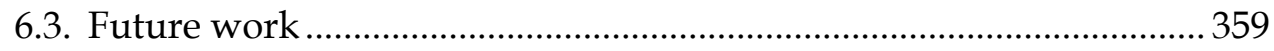

Bibliography of the Thesis........................................................................................... 363

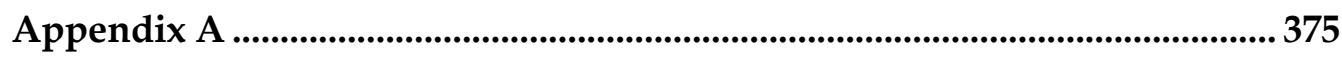

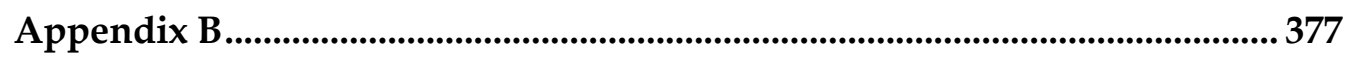





\section{List of figures}

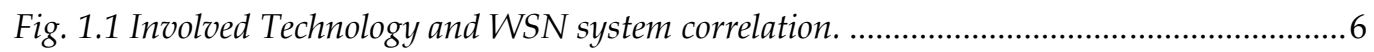

Fig. 1.2 System design cycle of a WSN-based application......................................................

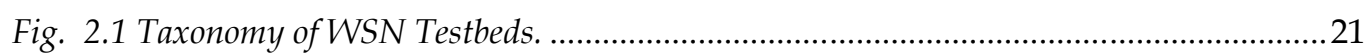

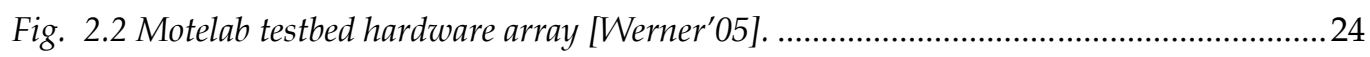

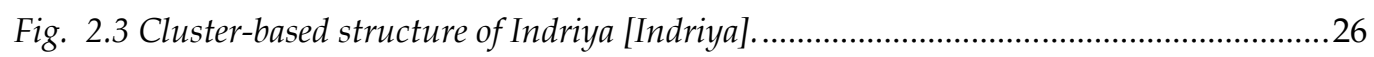

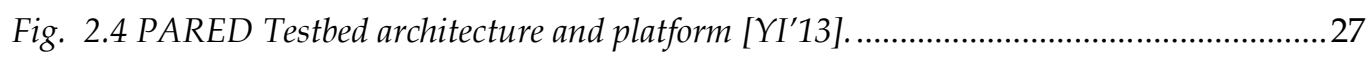

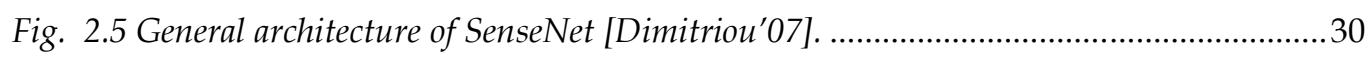

Fig. 2.6 General view of the control system of MOTEL [Förster'11] ........................................31

Fig. 2.7 Modular architecture of the Cookie hardware platform.................................................36

Fig. 2.8 Overall structure of the proposed processing layer design...........................................38

Fig. 2.9 Ultra-low power Cookie processing layer connected to the rest of the platform..............40

Fig. 2.10 Overall scheme of the proposed testbed infrastructure and involved elements. ............41

Fig. 2.11 Hardware implementation of the proposed Ethernet-based backchannel Cookie

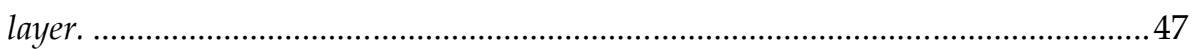

Fig. 2.12 Hardware implementation of the proposed wireless backchannel Cookie layer.............49

Fig. 2.13 General structure of the testbed framework implemented in the Cookie HW-SW

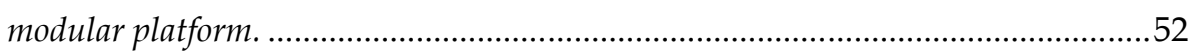

Fig. 2.14 Remote connection states and frame format for the testbed actions $\mathcal{E}$ blocks

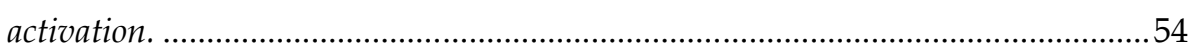

Fig. $2.15 \mathrm{HW}$-SW integration platform, main functional components and controllers...............59

Fig. 2.16 System architecture of the proposed partial reprogramming scheme ...........................74

Fig. 2.17 definition of classes and memory location for some of the component packages...........76

Fig. 2.18 Interaction schemes for the Bootloader/Recovery segment functionalities. ...................82

Fig. 2.19 Basic behaviour of the bootloader console and main interaction elements.....................84

Fig. 2.20 Multi-application/experiment independent projects for the on-the-fly partial

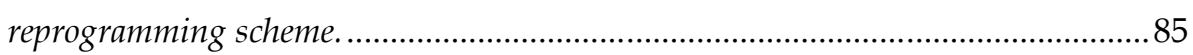

Fig. 2.21 Multi-application and partial reprogramming generation process. .............................8 89

Fig. 2.22 Basic structure of the WSN deployment for the testbed HW-SW platform

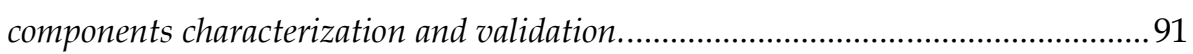

Fig. 2.23 Definition of the different memory areas for partial reprogramming structure. ..........92

Fig. 2.24 Characterization of the running application. Transitions of the sensor node operation (left), and observation setup for monitoring the platform interactions

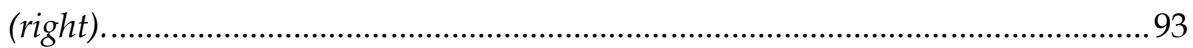

Fig. 2.25 Partial versus whole reprogramming runtime comparative analysis...........................94 
Fig. 2.26 Wi-Fi-based node configuration and application triggering, the console is then launched to execute or update a new application block.

Fig. 2.27 General view of the Java-based GUI for network and testbed support controlling.......98

Fig. 2.28 Comparative analysis of network topology considering simulation results (a) and experimental tests (b) .100

Fig. 2.29 Comparison of simulation and real results regarding accumulative RSSI values of node neighbourhoods.

Fig. 2.30 experimental results of the battery monitoring of the sensor node and network lifetime...

Fig. 2.31 Packet loss rate based on the overall network packet transmissions and receptions...102

Fig. 2.32 WSN data monitoring related to the different sensor measurements........................102

Fig. 2.33 Three different levels of User/developer interaction [OpenHAB]..............................105

Fig. 2.34 General Overview of the proposed system integration. ...........................................107

Fig. 2.35 Control frame and management interface for remote access and data aggregation of the top-level platform profile.

Fig. 2.36 Smart management system for urban/home automation based on the IoT Gateway

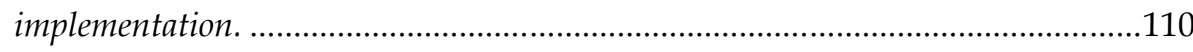

Fig. 3.1 Implementation of the IEEE 802.15.4-based Cookie communication layer..................130

Fig. 3.2 Data to be read from RXFIFO of the radio communication module [CC2420]. ...........132

Fig. 3.3 IEEE 802.15.4 Data frame depiction to be processed/generated [CC2420] [802.15.4] .132

Fig. 3.4 IEEE 802.15.4 module management integration overview in the Cookie HW-SW support platform.

Fig. 3.5 Routing protocols classification based on the various types of strategies....................137

Fig. 3.6 Exemplification of route discovery process, from node 1 to node 6. ............................142

Fig. 3.7 General structure of the new type of message DATA_ERR.......................................149

Fig. 3.8 General view of the CB-AODV implementation with the main functional blocks.......152

Fig. 3.9 General view of the involved phases and capabilities within a WSN application development. 158

Fig. 3.10 Functional components and system flow of the deployment and commissioning tool. .161

Fig. 3.11 Structural elements of the proposed Hand-Held Device system architecture. ...........163

Fig. 3.12 Structure of the deployment model definition as an input of the system. 169

Fig. 3.13 Basic representation of the first part of a deployment model considering the project and plan information as well as node and functional block instantiation...............170

Fig. 3.14 Node functional components composition based on blocks definition and instances. 173 
Fig. 3.15 Top-level JSON array that includes projects and plans identification of WSNs under development.

Fig. 3.16 Comparison of the in-field experimental results with respect to the model for TX power $=-3,-5$ and $-7 \mathrm{dBm}$. 184

Fig. 3.17 Experimental correlation between the RSSI and the LDR 188

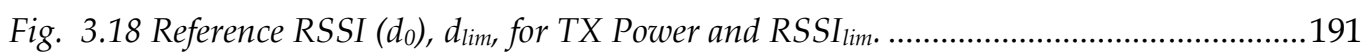

Fig. 3.19 basic definition of the first Tp uniform optimization strategy.................................192

Fig. 3.20 Representation of the cohesion-focused Tp optimization strategy..............................194

Fig. 3.21 Representation of the individual Tp optimization strategy......................................196

Fig. 3.22 In-field commissioning process and involved deployment/maintenance tasks based on network strategy-points. 202

Fig. 3.23 Genetic code representation of a candidate solution for the deployment methodology.

Fig. 3.24 Overview of the GA-based optimization algorithm and involved genetic operations. 214

Fig. 3.25 WSN deployment upon which the clustering-based optimization algorithm is exemplified. 216

Fig. 3.26 Summary of detection of branches and closed-groups for the target connectivity array.

Fig. 3.27 WSN for the evaluation of the deployment optimization methods. 220

Fig. 3.28 Comparison of the proposed deployment methodology and optimization strategies for different WSN scales. .222

Fig. 3.29 Results comparison of the deployment strategies considering GA and CA for 25, 28 and 52 nodes. 223

Fig. 3.30 Efficiency of the GA execution considering different configurations for the involved parameters.

Fig. 3.31 Evolution of the GA execution factor evaluation with respect to the variation of the population size, number of generations and convergence rate. 226

Fig. 3.32 Average results of the execution factor for the $G A$ and $C A$ strategies when varying the scale of the network.

Fig. 3.33 Route optimization mechanism based on $\mathrm{CH}$ dynamic evaluation. .234

Fig. 3.34 Local repair: a) failure on main path, b) failure on secondary path. 235

Fig. 3.35 WSN experimental test case and cluster formation result. 237

Fig. 3.36 Relationship of the number of control packets for every routing generation with respect to the overall amount of broadcasts. .239

Fig. 3.37 In-field relative position of the HHD when testing specific deployment areas. 241

Fig. 3.38 Structure of the defined packet framework to interface the HHD with the functional components and diagnosis blocks of the in-field sensors nodes. 247 


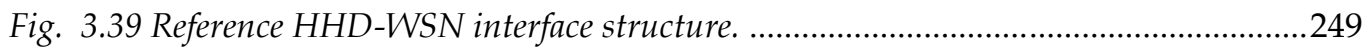

Fig. 3.40 HHD-MW integration structure and high-level services interface. ..........................254

Fig. 3.41 Common structure of the deployment output models..............................................257

Fig. 3.42 In-field report generation in accordance with the deployment functional flow

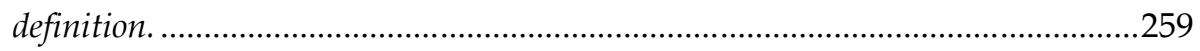

Fig. 4.1 Main log-in and project selection windows of the HHD ..........................................2275

Fig. 4.2 Deployment toolset main menu once the network model and input definitions are

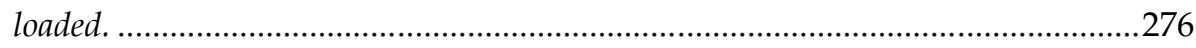

Fig. 4.3 Input models selection and visualization. ..........................................................276

Fig. 4.4 In-situ simulation module, where optimization engines are split into interactive

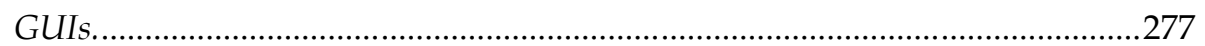

Fig. 4.5 Nodes correlation and transmission power optimization sub-system........................278

Fig. 4.6 Deployment optimization methods and algorithms - selection panel. ........................279

Fig. 4.7 Optimization evolution and result presentation views. .............................................279

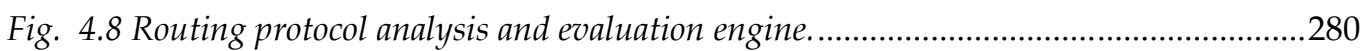

Fig. 4.9 Automatic Guide module. Activation and graphical inspection of various system

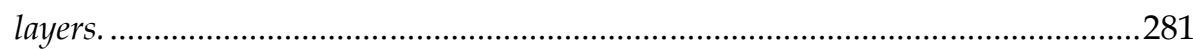

Fig. 4.10 Various options for users to interact with the toolset guide and the target WSN

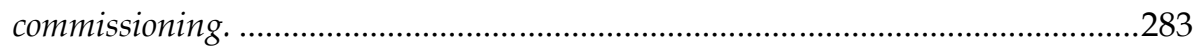

Fig. 4.11 Graphical representation of the test evolution process when selecting a node............283

Fig. 4.12 WSN deployment analysis and performance evaluation module distribution. ..........284

Fig. 4.13 Adaptable transducer management configuration and monitoring options based on

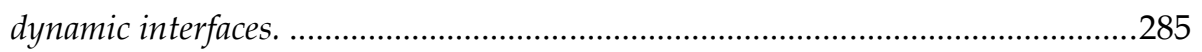

Fig. 4.14 Performance evaluation options and test results for WSN routing strategies. ..........286

Fig. 4.15 Interface to the functional blocks of the WSN node/middleware implementation. .....287

Fig. 4.16 Interactive map for the analysis of the network connectivity and real test results.....288

Fig. 4.17 Advanced-user configuration and monitoring modules........................................289

Fig. 4.18 Output reports generation. WSN deployment assessment and feedback. .................289

Fig. 4.19 General view of the HHD with the connection to HHNode.....................................290

Fig. 5.1 General view of the CB-AODV integration in the Cookie platform.............................2297

Fig. 5.2 Deployment of $3+1$ node to test the creation of the routing path. .............................299

Fig. 5.3 Route discovery process based on the information already available in the routing

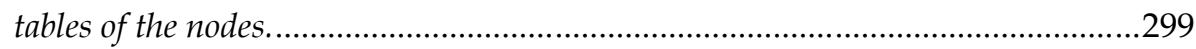

Fig. 5.4 Various data exchange configurations among the sensor nodes.................................300

Fig. 5.5 Evolution of the RSSI and LQI values among the WSN links...................................301

Fig. 5.6 RTT and RD delay measurement tests for the CB-AODV based multi-hop topology. 302

Fig. 5.7 2-Hop topology scenario for the routing delay analysis. ............................................302 
Fig. 5.8 Round-trip delay measurement samples for the 2-hop, double-branch topology scenario.

Fig. 5.9 Distribution of a 6-node sensor deployment to test the creation and data delivery by using the Cookies communication capabilities.

Fig. 5.10 Cookie-based mesh deployment array (left), and main results of the experimental scenario (right).

Fig. 5.11 Cookie-based aligned/string deployment array (left), and main results of the experimental scenario (right).

Fig. 5.12 Experimental correlation of RSSI and LQI parameters obtained from real tests and to be included within the estimation of quality metrics.

Fig. 5.13 WSN deployment scenario composed of 16 Cookie-based sensor nodes at ETSIIUPM.

Fig. 5.14 result comparison of the GA and CA methods for the WSN experimental scenario..311

Fig. 5.15 Comparative analysis of the in-field connectivity results with the simulation model outcome

Fig. 5.16 Graphical representation of the comparative analysis between the estimation and real on-site experimental data by using the HH interface.

Fig. 5.17 TX power optimization and configuration for the proposed experimental scenario...316

Fig. 5.18 Graphical representation of the comparative analysis between the estimation and real on-site experimental data of scenario B, by using the HH interface.

Fig. 5.19 in-field verification of node functionalities, a) sensor retrieving configuration and runtime measurement behaviour b) medium access assessment and analysis of connectivity metrics.

Fig. 5.20 analysis of the routing performance, a) creation of a route from node 9 to $1, b)$ packet delivery configuration and test results presentation.

Fig. 5.21 Evaluation of the routing rediscovery when detecting errors in route entries. 320

Fig. 5.22 Creation of an alternative route and results representation of the rediscovery mechanism by using the $\mathrm{HH}$ diagnosis tests visualization

Fig. 5.23 Smart parking demonstrator scenario for the DPCM toolset validation .327

Fig. 5.24 System architecture of the proposed smart parking demonstrator with the support

of the WSN-DPCM platform. .329

Fig. 5.25 Internal block diagram of the TelosB-based sensor node and external structure (Inetsis platform [DPCM-SD]).

Fig. 5.26 graphical representation of the infrared sensor and integration into the sensor node [DPCM-SD].

Fig. 5.27 Deployment model representation for the planned WSN demonstrator. .334

Fig. 5.28 DPCM toolset workflow for the design and implementation of the WSN. .335 
Fig. 5.29 Distribution of the car spaces in the target parking area at ETSII - UPM. .336

Fig. 5.30 HHD interface visualization in accordance with the defined deployment model for the ETSII smart parking demonstrator. 337

Fig. 5.31 nodes connectivity scheme results for the target WSN deployment..........................338

Fig. 5.32 HHD interface for the deployment methodology and WSN device connections. .......338

Fig. $5.33 \mathrm{HHD}$ interface capture related to the evaluation of the occupancy detection sensor..339

Fig. 5.34 App interface, log-in menu (left), and parking areas (right).

Fig. 5.35 App parking map and booking interface (left), slot status information (right). 343

Fig. 5.36 Booking reservation panel (left), parking place filtering for a specified time-slot (right).

Fig. 5.37 User reservation details and current status.

Fig. 5.38 Android notifications related to the user reservation status. 345 


\section{List of tables}

Table 2-1 Different voltage levels for the Cookie processing layers.

Table 2-2 Comparison of the reprogramming techniques by using different processing layers. ..94

Table 2-3 comparison of the reprogramming results for several configuration test cases. ............96

Table 3-1 comparison of different AODV implementations and proposals..............................154

Table 3-2 A reference block definition considering basic functional components within a WSN platform.

Table 3-3 Example of block instances and associated parameters within node objects, which uniquely identifies a particular attribute/property/configuration of the sensor platform.

Table 3-4 Generic path-loss factor values according to the application scenario.........................180

Table 3-5 Range of values for the experimental tests.........................................................181

Table 3-6 Relationship between TX power and reference RSSI(do). ........................................182

Table 3-7 Degradation of the LDR metric according to the RSSI. ...........................................188

Table 3-8 Distance limits with respect to the TX Power and its relationship with the RSSI

(reference and limit values).

Table 3-9 Parametrization of the deployment optimization strategies for the tests definition. ..221

Table 3-10 Comparative analysis of the routing strategies for the proposed experimental tests. 238

Table 3-11 summary of reference functionalities for runtime in-field connectivity evaluation

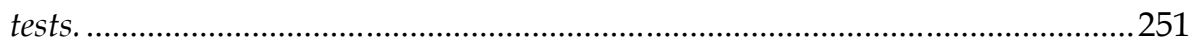

Table 3-12 Information reference to identify the report to be generated. ....................................258

Table 3-13 Summary of report identifiers and associated data objects. .....................................262

Table 5-1 Summary of configuration and diagnosis functionalities for the analysis of the CB-

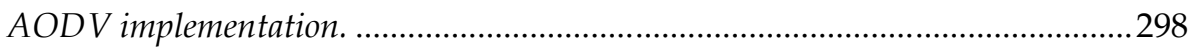

Table 5-2 Summary of the packet delivery results for the various data exchange configurations.

Table 5-3 Configuration parameters of the deployment optimization strategies for the target

application scenario

Table 5-4 Experimental results of the routing strategies by using ETX and E-aDM in the comparative analysis.

Table 5-5 General results of the comparative analysis of the estimation + real nodes correlation. 



\section{List of acronyms}

\begin{tabular}{|c|c|}
\hline $\mathrm{ADC}$ & Analogue to Digital Converter \\
\hline AODV & Ad-hoc On-demand Distance Vector \\
\hline ASCII & American Standard Code for Information Interchange \\
\hline CA & Clustering-based Algorithm \\
\hline CAN & Controller Area Network \\
\hline CB-AODV & Cookie-Based Ad-hoc On-demand Distance Vector \\
\hline CB-RSim & Cookie-Based Routing Simulation engine \\
\hline CMT & Commissioning and Maintenance Tool \\
\hline CSMA/CA & Carrier Sense Multiple Access with Collision Avoidance \\
\hline DHCP & Dynamic Host Configuration Protocol \\
\hline DPCM & Development, Planning, Commissioning and Maintenance tool \\
\hline E-aDM & Energy-efficient and adaptable Delivery Metric \\
\hline ETX & Expected Transmission Count \\
\hline FPGA & Field Programmable Gate Array \\
\hline GA & Genetic-based Algorithm \\
\hline GPIO & General Purpose Input/Output \\
\hline GPS & Global Positioning System \\
\hline GUI & Graphical User Interface \\
\hline HHD & Hand-Held Device \\
\hline HTTP & Hypertext Transfer Protocol \\
\hline $\mathrm{HW}$ & Hardware \\
\hline IDE & Integrated Development Environment \\
\hline IEEE & Institute of Electrical and Electronic Engineers \\
\hline
\end{tabular}




$\begin{array}{ll}\text { IP } & \text { Internet Protocol } \\ \text { IoT } & \text { Internet of Things } \\ \text { JSON } & \text { JavaScript Object Notation } \\ \text { JTAG } & \text { Joint Test Action Group } \\ \text { LDR } & \text { Link Delivery Ratio } \\ \text { LQI } & \text { Link Quality Indicator } \\ \text { MAC } & \text { Medium Access Control } \\ \text { MS-PRS } & \text { Memory-Segments-based Partial Reprogramming Scheme } \\ \text { MW } & \text { Middleware } \\ \text { NDoSq } & \text { Node Deployment optimization Sequence } \\ \text { OS } & \text { Operating System } \\ \text { PD } & \text { Powered Device } \\ \text { PLR } & \text { Packet Loss Rate } \\ \text { PoE } & \text { Power over Ethernet } \\ \text { PSE } & \text { Power Sourcing Equipment } \\ \text { PSS } & \text { Parking Support Server } \\ \text { PWM } & \text { Pulse Width Modulation } \\ \text { RAM } & \text { Random Access Memory } \\ \text { REST } & \text { Representational State Transfer } \\ \text { RFC } & \text { Request For Comments } \\ \text { RREQ } & \text { Route Request } \\ \text { RREP } & \text { Route Reply } \\ \text { RST } & \text { Roundrength Signal Indicator } \\ \text { RA delay Time }\end{array}$


SPI

Serial Peripheral Interface

SQL Structured Query Language

SW Software

TCP Transmission Control Protocol

UART Universal Asynchronous Receiver-Transmitter

UDP User Datagram Protocol

UI User Interface

URI Uniform Resource Identifier

USB Universal Serial Bus

VHDL Very-High Speed Integrated Circuit Hardware Description Language

WSN Wireless Sensor Network

uC Microcontroller 



\section{Chapter 1}

\section{INTRODUCTION}

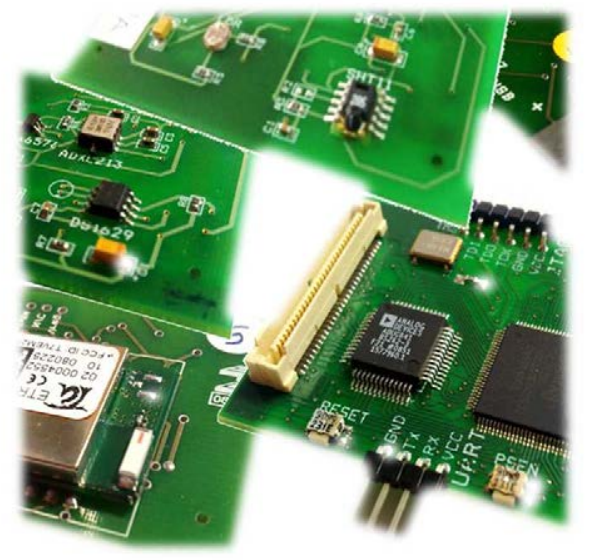

The research field of Wireless Sensor networks has experienced an important evolution during the last decade particularly considering the technological progress related to the paradigm of the Internet of Things, where the integration of novel hardware and software platforms involve more complex techniques to achieve an efficient implementation of interconnected sensor systems.

In this chapter an overall view of the main trends and challenges that comprise such a maturity process is approached, and questions related to the development and deployment of WSNs are addressed through the solutions proposed in this thesis. 



\section{Introduction}

\subsection{Wireless Sensor Networks: Evolution and challenges}

The research field of Wireless Sensor Network (WSN) has been growing and becoming more mature during the last decade in which novel hardware and software technologies have been intensively proposed by the research community, in order to increase its applicability and usability especially in this the new era of smart scenarios. The inherent nature of the WSNs, that is, the deployment of low-cost autonomous wireless sensor nodes to monitor and/or act on a target environment [Romer'04] makes this technology suitable for different application contexts, not only from a research perspective, but also in industrial use cases where the final/long-term operability and reliability of the distributed system is to be assured.

The essence of a WSN as a self-organized wireless distributed system has envisioned the creation of different solutions that encompass multidisciplinary areas related to embedded computing architectures, telecommunications, microelectronics and industrial technologies. In this way, most of the WSN use cases that can be traditionally found in the state of the art are enclosed within the following application scenarios:

- Environmental Sensing, where contributions regarding pollution monitoring have been proposed to provide industrial and government organizations with the ability to track pollution levels in factories and cities, including air quality and emission measurements. Likewise, the remote and spread nature of wireless sensor deployments together with stream-data gathering have broadened the opportunity to analyse and predict weather, seismic and volcano phenomena that are difficult to monitor otherwise [Ammari'12]. Habitat observation has also been explored by using WSNs in order to study the impact of climate and environment factors in animal existence as well as migration patterns [Othman'12]. However, the 
main issues when facing environmental monitoring are related to remote management and communication performance, scalability and adaptability, as well as energy efficiency and robustness of the system.

- Healthcare, including Body Area Sensor Networks for monitoring and studying vital parameters of athletes and patients, as well as detecting anomalies in chronic diseases so that assistance protocols can be activated. Diagnosis and treatment procedures can also be enhanced by applying wireless sensors for measuring specific changes in individuals' patterns related to particular ills [Ammari'12]. Most of the challenges that are faced in these types of applications are related to the creation of less/non-intrusive wearable or in-body solutions with well-defined capabilities of signal processing embedded in ultra-low power hardware elements, to assure the autonomy of the sensor nodes. Accuracy and privacy are also key aspects to be considered in this application context [Hanson'09].

- Agricultural Monitoring, comprising the observation and maintenance of crops by using, for instance, efficient irrigation systems based on humidity and volumetric water sensors, as well as moisture tracking and nutrient/fertilizer control. Moreover, the implementation of monitoring and control mechanisms in greenhouses based on temperature, humidity and light sensor nodes provides farmers with the possibility of achieving high quality crop growth in precision agriculture scenarios [Chaudhary'11].

- Industrial Applications, where different solutions have been proposed taking advantage of the flexibility and versatility of wireless sensor networks to enhance industrial production processes. A wide variety of application scenarios can be distinguished, such as process automation and service provision evaluation, in which different parameters (e.g. water and energy consumption, supply chain, waste monitoring, equipment condition and structural health) can be measured to analyse and even improve the performance of the 
production cycle [Erdelj'13]. Mining (including detection of toxic gases releases) and railway industries (mainly related to safety issues) are also key areas that benefit from WSNs [Ammari'12]. However, the main concerns when implementing WSN-based industrial applications are indeed related to the reliability, maintainability, heterogeneity and intrusiveness of the deployed system.

Although the concept of sensor networks and distributed computing systems has been developed since the late nineties, the evolution of wireless communication technologies as well as the aim of a smart, sustainable and connected world came out with novel paradigms that are also defining the new horizon of WSN-based applications and related technologies. In particular, the progressive inclusion of the Internet of Things (IoT) [Atzori'10] as the main foundation for the "city of the future" implementation has defined a new way of approaching the development of embedded sensor systems.

Furthermore, the WSN, IoT, and Smart City concepts are even more correlated not only because of their intrinsic nature (pervasive computing/sensing supported by flexible and dynamic communication capabilities) but also due to the increased number of convergence proposals from the research community trying to establish specific proprietary and non-proprietary technologies to cope with application requirements and service provision.

In this direction, novel trends in wireless sensor technologies are trying to face object-oriented heterogeneous frameworks from a high-abstraction/web-service level point of view, targeting applications such as smart metering, participatory sensing, traffic flow monitoring, smart home automation systems and energy efficiency in intelligent buildings management [Gubbi'12], thus moving forward to more demanding scenarios where flexible and adaptable platforms play a fundamental role.

However, this innovation process also implies new and more complex challenges due to the inherent integration of a wide range of different technologies, protocols, and algorithms so that their performance and feasibility in final implementations can be maximized. As shown in Fig. 1.1, the multidomain basis of the WSNs contributes to have continuous progresses in every 
field of expertise, such as novel hardware and software platforms, networking communication capabilities, and service-oriented management and monitoring strategies [Avilés'09]. However, this progressive evolution tendency also leads to considering the definition of efficient correlation and interconnection mechanisms among technologies as a must.

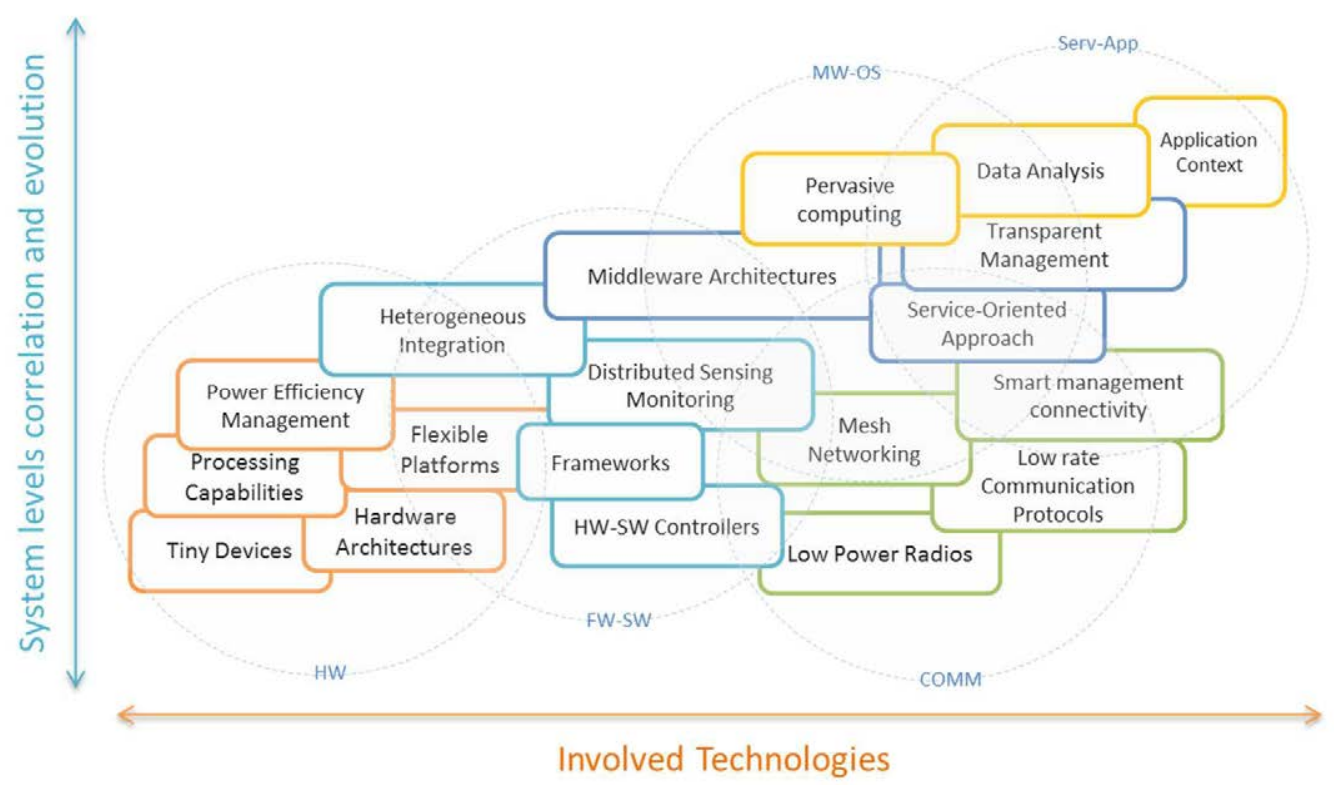

Fig. 1.1 Involved Technology and WSN system correlation.

This issue does not only refer to developing platforms and application-domain implementations as traditionally conceived in applied WSN works, but also how to debug, analyse, optimize and validate the wireless sensor deployments as a crucial verification process to ensure a proper release and reliability of the autonomous system.

Such perspective indeed reinforces the importance of consciously establishing the different phases of the system development flow so as to properly undertake the design and implementation of WSN-based applications. The complexity of converging these technologies into an efficient heterogeneous implementation makes the development cycle of a WSN a combination of different optimization techniques at every stage of the development process, up until the final operability and maintenance of the system. 


\subsection{The development of WSN-based applications}

As previously remarked, the development cycle of a WSN-based application goes from the requirement stage of the target implementation up until the final operation of the deployed system, but there are important interconnected processes in between that aim at enhancing the adaptability and final success of the solution. In Fig. 1.2, a general overview of what could be considered as a comprehensive design flow is shown, distinguishing different system domain interactions in order to progressively feed back the process of developing the target solution.

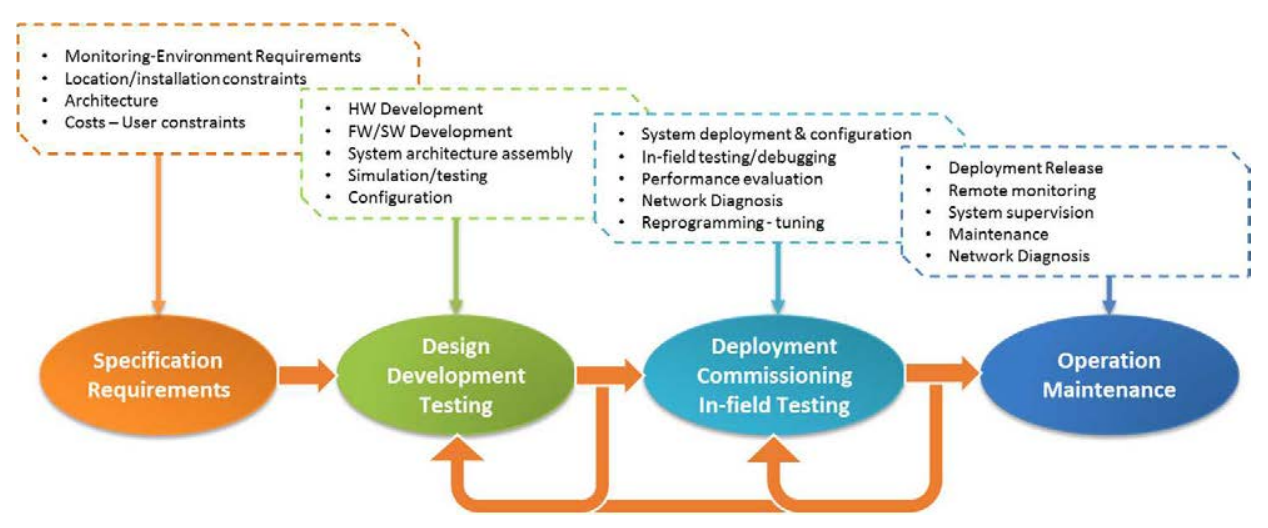

Fig. 1.2 System design cycle of a WSN-based application.

The initial specification stage will lay the groundwork of the subsequent design phases by defining the scope, limitations, variables and constraints of the target application, resulting in the functional and operational requirements of the system development.

During the design phase different technological approaches have to be considered specially related to hardware and software determination by taking into account the specifics of the objective scenario. Here novel trends regarding networking mechanisms, sensor platforms, drivers, libraries, and management frameworks are to be combined with planning tools [He'12] so that the integration and interconnection strategies can be defined accordingly.

Based on the outcomes of the design activity, the development of hardware and software prototypes includes the code generation process for every system 
implementation level (node-level, network-level, server+application level) as well as behavioural simulation strategies to analyse network/topological model definitions and theoretical parametrizations. In this case, synthesis and compilation tools can be combined with simulation mechanisms as part of planning tool chains so as to determine optimal deployment configurations.

It is important to highlight that there have been significant progresses in the literature in the area of planning tools to provide developers with the ability to analyse and optimize network and node models before testing and deploying the actual sensor network. Two types of simulation processes can be distinguished. On one hand, those that are focused on hardware, firmware and device model simulation [Du'14], and on the other hand tools that comprise connectivity and radio propagation engines to predict the communication performance of the nodes to be deployed [Castalia].

Moreover, some efforts have been focused on integrating middleware-like libraries into synthesis tools for automatic code generation based on existing functional blocks, so that the software prototyping can be sped up [Buckl'08]. However, when moving forward to the testing stage of the development process, a more realistic experimentation with the implemented technologies is to be carried out in such a way that an iterative optimization cycle can reflect considerable performance issues that might not be detected in previous simulation phases. Here the integration of hardware and software platforms into flexible experimental infrastructures plays and fundamental role in conjunction with the deployment and commissioning stages, where the on-site performance evaluation and runtime system configuration process will indeed determine not only the final operability of the implemented WSN, but also a comprehensive and necessary feedback to the design, modelling and development cycle.

\subsection{An in-field approach for experimenting in WSNs}

The idea of creating experimental mechanisms beyond WSN simulation capabilities has been indeed investigated during the last few years by researchers, trying to address testing strategies around what is called Testbeds for wireless sensor networks. The principle behind WSN testbeds is related to 
providing developers with a controlled HW-SW infrastructure composed of actual sensor nodes upon which experimental tests can be performed in realtime. This concept attempts to tackle the intrinsic limitations of the initial theoretical evaluations, which can be more noticeable in highly constrained wireless sensor deployments where energy, processing and communication restrictions may result on a solution far from the expected behaviour during the development phase [Imran'10].

In practice, in order for this approach to be a useful tool for WSN developers during the experimental and verification phase, a testbed architecture must be enclosed by flexible hardware and software platforms upon which different prototypes, protocols, algorithms and node management strategies can be tested, reconfigured and analysed at runtime. This will certainly lead to obtaining a better assessment of the applied models based on more realistic data.

Due to such a technological heterogeneity that characterises the WSN research field, there is a spread spectrum of different testbed solutions that certainly depend on the emphasis on one or other feature to be tested (e.g. scalability, heterogeneity, realistic environment, platform prototyping, protocol assessment, mobility)[Horneber'14]. However, most of the testbeds that propose a general experimental architecture are based on hardware and software platforms that are indeed well known but start being obsoletes, and much less flexible for experimenting with new technologies that come out with the evolution of the WSNs. This is a shortcoming in such platforms particularly in terms of runtime reprogramming and reconfiguration techniques, remote debugging and platform parametrization, modular prototyping, and resource handling in order to allow users to have a better understanding of what the behaviour of the WSN under test is, according to the running experiments.

On the other hand, unlike the WSN testbed research line there is still an important shortage when considering on-site deployment and commissioning strategies. This is certainly a critical stage since the operation of the final system will depend on the physical configuration and runtime testing capabilities to be applied for the performance evaluation of the developed WSN, in addition to the 
actual placement problems that come out with the on-site WSN establishment (mainly in terms of connectivity, processing, and sensing issues).

This phase is directly correlated with the planning/development stages so as to be able to carry out node readjustments and network communication optimizations from the basis of the simulated and implemented platform, prior to its final release. Therefore, the combination of modelling and planning techniques with novel deployment methodologies is set to be a key approach to guarantee the long-term operability, autonomy and efficiency of the deployed WSN-based solution.

Although that represents such a determining process in the overall development cycle, nowadays there is a lack of well-defined frameworks and methodologies not only to efficiently realize the on-site deployment tasks, but also to analyse and enhance the in-field communication performance, the wireless routing algorithms and the networking mechanisms of the distributed sensor nodes. Indeed, few works in the state of the art focus on deployment platforms to cope with these challenges and minimize the time, power and resource consumption during the installation, configuration and evaluation stages of the WSN.

\subsection{Main objectives and scope of the proposed work}

The aforementioned issues allow establishing the main guidelines of the present $\mathrm{PhD}$ thesis, in which a real approach for experimenting, debugging and evaluating real wireless sensor network deployments is undertaken in detail.

On one hand, the huge potential of the well-defined WSN modular platform proposed in [Portilla'10] that has been envisioned to provide an extensive support and adaptability to the evolution of WSN and IoT technologies defines the foundations for one of the overall objectives of the proposed work: the creation of a new testbed architecture and framework for experimenting with novel WSN hardware and software prototypes under the scope of such a promising technology. The underlying idea behind the creation of a flexible testbed platform is to provide developers with the capability of testing and analysing resource-constrained WSN implementations under a modular HW- 
SW platform, beyond the modelling and simulation strategies that can be applied in initial stages of the development cycle, as commented before.

Unlike state-of-the-art solutions, the proposed testbed framework targets to provide a modular and flexible support architecture that encloses three main aspects in a unique resource-constrained based scheme:

- A remote debugging infrastructure so that users can interact with the actual platform prototyping in a more transparent, comprehensive and reliable fashion.

- A memory-segmentation-based partial reprogramming strategy not only to optimize the reconfiguration process of the testbed experimentation, but also to modularize the type of functional blocks to be under test.

- A HW-SW integration platform to provide users with a complete support for an embedded management of the architecture resources, low-level hardware controllers and system interfaces of the nodes.

This testbed-based experimental approach has been conceived in such a flexible way that most of the proposed techniques can also be applied during the subsequent stages of the development process, that is, during the on-site deployment and parametrization capabilities of the modular platform.

On the other hand, in order to cope with the lack of well-defined frameworks designed to provide a comprehensive in-field performance evaluation and verification process of WSN-based systems, a novel on-site deployment and commissioning platform is fully proposed as one of the major contributions of this PhD thesis.

The basis of the proposed platform is the implementation of a network deployment methodology along with the inclusion of runtime comparative analysis modules integrated in a unique toolset, in which a combination of planning/simulation models with the actual system behaviour during the installation and commissioning process allows deployers to produce a feedback to the system development cycle, as a crucial step to assure its final operational release. The assessment and verification of the WSN to be distributed is based on 
the ability to trigger different diagnosis and reconfiguration techniques so that users can act on those parameters that might modify the overall performance of the sensor network, according to the observation of actual data.

This concept has been envisioned to provide a comprehensive evaluation of deployments based on mesh networks, where different levels of communication capabilities are to be considered, particularly regarding wireless routing mechanisms. In this direction, the implementation of efficient multi-hop techniques into the previously mentioned WSN modular platform is also proposed to be studied and assessed by using the deployment toolset approach.

Likewise, since the proposed tool targets the deployment configuration and onsite performance analysis of heterogeneous platforms, a middleware-based implementation for the system integration has been followed as an important contribution of the present work, so as to cover as different types of solutions as possible.

Based on the motivation and the overall objectives that define the scope of this $\mathrm{PhD}$ thesis, the main contributions and highlights of the proposed approach can be outlined as follows:

- A flexible and modular WSN testbed framework to provide users with a comprehensive experimental platform to test and debug lowpower resource-constrained sensor network prototypes and novel hardware and software developments upon the foundations of the work proposed in [Portilla'10].

- An efficient memory-segment-based partial and remote reprogramming strategy to support modular multi-experimentation into the testbed infrastructure, as well as provide users with a transparent way of managing the resources of the HW-SW platform. This method is indeed extensible to in-field deployment and operational stages where energy-efficient functional block replacements or reprogramming procedures are the key to assure the long-term autonomy of the deployed system. 
- A HW-SW integration platform based on the modular sensor node architecture so as to provide users with a support framework for the implementation and validation of novel prototypes under an efficient resource-usage scheme.

- A novel on-site deployment and commissioning toolset to provide developers and deployers with in-field strategies to configure, analyse and evaluate the designed WSN-based application. This goal encompasses different challenges that are tackled in the overall platform as described below.

- An intelligent deployment methodology and on-site automatic guidance based on the combination of simulation models with the integration of runtime optimization algorithms to efficiently carry out the configuration and node/network correlation tasks.

- Implementation of efficient multi-hop mesh networking mechanisms into the modular HW-SW platform and runtime connectivity evaluation strategies to analyse the performance of low-rate and resource-constrained communication protocols, particularly targeting dynamic and adaptable routing schemes.

- Integration of middleware-based support services and implementation of a debugging communication interface into the onsite deployment toolset in order to establish the runtime reconfiguration and interaction with the in-field sensor nodes. This is certainly intended to cover a wide spectrum of heterogeneous deployments, though the implementation has been focused on the proposed modular HW-SW platform. Based on the comparative analysis of the real plus simulation models together with the runtime reconfiguration process, users are capable of providing a more comprehensive feedback to the system development cycle, in correlation with the generation of online output models from the basis of the in-field commissioning and maintenance activities. 
Real in-field test cases as well as several application scenarios have been analysed in depth by using the proposed support strategies, so as to put the overall system implementation into different contexts and thus verify the flexibility, usability and effectiveness of the pre-deployment/on-sitedeployment approaches.

\subsection{Organization of the Thesis}

According to the aforementioned objectives, this $\mathrm{PhD}$ thesis is structured into two main blocks that clearly define the scope of the proposed pre-deployment and in-field-deployment strategies. In chapter 2, a comparative analysis of stateof-the-art WSN testbed solutions is presented, together with the main motivations that outline the testbed framework proposed in this work. The HWSW architecture along with the partial and remote reprogramming scheme are also presented in this chapter. Experimental results that include real test cases and testbed characterization are described to put the testbed framework into several application contexts. Final considerations are commented to summarize the contributions and relevant aspects of the work described in this first part of the $\mathrm{PhD}$ thesis.

Chapter 3 encompasses one of the major contributions of the present work, which is the design and development of the on-site WSN deployment and commissioning toolset. The relevant issue related to the necessity of having an in-field HW-SW platform to carry out the configuration, analysis and performance evaluation of wireless sensor deployments is firstly introduced. Then the analysis and optimization of multi-hop mesh networking techniques in the modular HW-SW platform are deeply described as one of the main target when accomplishing the deployment and performance assessment activities. Afterwards, the overall scheme of the proposed toolset is analysed along with the on-site deployment modelling and optimization strategies, before the description of the in-field automatic guidance and runtime system performance evaluation/reconfiguration capabilities. In this way, chapter 4 summarizes the implementation of the deployment toolset and how the main capabilities of the proposed system have been integrated into a unique user interface. 
Due to the relevance of the experimental test cases to validate not only the different strategies that integrate the on-site deployment toolset but also the overall system implementation in different application contexts, chapter 5 is entirely dedicated to describe such real scenarios. Moreover, since the proposed deployment platform has also been a fundamental part of the validation of a smart city demonstration scenario, further details regarding the design and development of this application use case are presented.

Finally, conclusions, summary of scientific contributions as well as future research works are outlined in chapter 6 , so that the main guidelines for the progression of the proposed technologies within the scope of the wireless sensor network research process are established.

\subsection{Bibliography of the chapter}

[Ammari'12] H. M. Ammari, N. Gomes, W. I. Grosky, et al., "Review of Applications of Wireless Sensor Networks", in Wireless Sensor Networks: Current Status and Future Trends, CRC Press, 1srt. Edition, 2012, DOI: 10.1201/b13092-3.

[Atzori'10] L. Atzori, A. Iera, Giacomo Morabito, "The Internet of Things: A survey", in Computer Networks, Vol. 54, Issue 15, October 2010, pp. 2787-2805, ISSN 13891286, http://dx.doi.org/10.1016/j.comnet.2010.05.010.

[Avilés'09] E. Avilés, J.A. García, “TinySOA: a service-oriented architecture for wireless sensor networks", in Service Oriented Computing and Applications, Vol. 3, Issue 2, pp. 99-108, June 2009.

[Buckl'08] C. Buckl, S. Sommer, A. Scholz, et al., "Generating a Tailored Middleware for Wireless Sensor Network Applications," in IEEE International Conference on Sensor Networks, Ubiquitous and Trustworthy Computing (SUTC'08), pp. 162-169, June 2008.

[Castalia] Castalia, a wireless sensor network simulator for low-power pervasive computing. https://castalia.forge.nicta.com.au.

[Chaudhary'11] D.D. Chaudhary, S.P. Nayse, L.M. Waghmare, “Application of Wireless Sensor Networks for Greenhouse Parameter Control in Precision Agriculture", in International Journal of Wireless \& Mobile Networks (IJWMN'11) Vol. 3, No. 1, February 2011.

[Du'14] W. Du, F. Mieyeville, D. Navarro , I. O'Connor, L. Carrel, "Modeling and simulation of networked low-power embedded systems: a taxonomy", in 
EURASIP Journal on Wireless Communications and Networking, Vol. 2014:106, doi:10.1186/1687-1499-2014-106.

[Erdelj'13] M. Erdelj, N. Mitton and E. Natalizio, "Applications of industrial wireless sensor networks", in Industrial Wireless Sensor Networks: Applications, Protocols, and Standards, CRC Press 2013, DOI: 10.1201/b14072-2.

[Gubbi'12] J. Gubbi, R. Buyya, S. Marusic, M. Palaniswami, "Internet of Things (IoT): A vision, architectural elements, and future directions", in Future Generation Computer Systems, Vol. 29, Issue 7, Sept. 2013, pp. 1645-1660, http://dx.doi.org/10.1016/j.future.2013.01.010.

[Hanson'09] M.A. Hanson, H.C. Powell, A.T. Barth, et Al., "Body Area Sensor Networks: Challenges and Opportunities," in IEEE Computer, Vol. 42, No. 1, pp. 58-65, Jan. 2009, doi: 10.1109/MC.2009.5.

[He'12] D. He, G. Mujica, J. Portilla, T. Riesgo, "Simulation tool and case study for planning wireless sensor network," in 38th Annual Conference on IEEE Industrial Electronics Society (IECON 2012), pp. 6024-6028, Oct. 2012.

[Horneber'14] J. Horneber, A. Hergenroder, "A Survey on Testbeds and Experimentation Environments for Wireless Sensor Networks," in IEEE Communications Surveys \& Tutorials, Vol. 16, no. 4, pp. 1820-1838, Fourth quarter 2014, doi: 10.1109/COMST.2014.2320051.

[Imran'10] M. Imran, A.M. Said, H. Hasbullah, "A survey of simulators, emulators and testbeds for wireless sensor networks," in International Symposium in Information Technology (ITSim 2010), vol. 2, pp. 897-902, June 2010, doi: 10.1109/ITSIM.2010.5561571.

[Othman'12] M. F. Othman, K. Shazali, “Wireless Sensor Network Applications: A Study in Environment Monitoring System", in Procedia Engineering, Vol. 41, 2012, pp. 1204-1210, ISSN 1877-7058, http:/ / dx.doi.org/10.1016/j.proeng.2012.07.302.

[Portilla'10] J. Portilla, "Plataforma Modular e Interfaces Genéricas de Transductores para Redes de Sensores Inalámbricas", PhD Thesis, Universidad Politécnica de Madrid, 2010.

[Romer'04] K. Romer, F. Mattern, "The design space of wireless sensor networks," in IEEE Wireless Communications, Vol. 11, no. 6, pp. 54-61, Dec. 2004, doi: 10.1109/MWC.2004.1368897. 


\section{Chapter 2}

\section{THE PRE-DEPLOYMENT STAGE OF WIRELESS SENSOR NETWORKS}

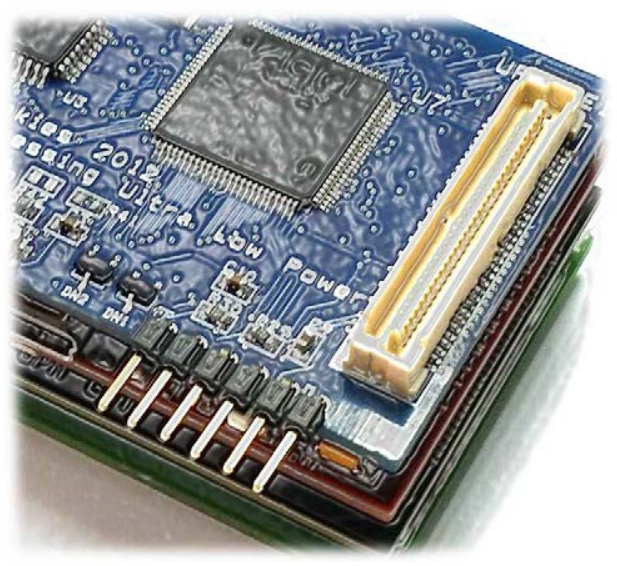

The development cycle of Wireless Sensor Networks shall encompass on one hand planning and simulation techniques prior to the final deployment of the target system, but on the other hand testing and evaluation mechanisms that can lead to analysing and optimizing the real hardwaresoftware prototypes under implementation. In this direction, the research field of WSN testbeds attempts to support the pre-deployment stage by means of providing real infrastructures under which developers can carry out experimental tests.

In this chapter some of the main state-of-the-art techniques for tackling the implementation WSN testbeds are analysed and a new flexible support platform based on a modular hardware architecture is proposed in detail. 



\section{The pre-deployment stage of Wireless Sensor Networks}

\subsection{Introduction}

The analysis and optimization of designed prototypes as part of the development process of wireless sensor networks have been traditionally performed during the planning and simulation stages prior to their integration into the final application, with a strong correlation with code generation activities. However, the nature of the WSNs as low-cost/highly-constrained adhoc networks made researchers further investigate in real mechanisms for experimenting, debugging and verifying the system implementation under more realistic conditions, not only in terms of the target environment itself, but also regarding the actual hardware-software platforms to be used as well as the effective interactions among the senor nodes.

The research field of WSN testbed platforms attempts to tackle the limitations of simulation tools by providing developers with a controlled HW-SW infrastructure composed of real sensor devices, upon which experimental tests can be performed at runtime. In this way, a more comprehensive evaluation of the design constraints, communication capabilities and node resource management can be carried out as a support to the theoretical models that are indeed part of the development cycle.

With the evolution of the WSNs, testbed platforms have also experimented a progressive growth though more in relation with the type of approach to be followed, that is, the particular focus the testbed is created for. While some researchers conceive their testbed architectures to support specific development areas such as power consumption optimization, network mobility or protocol stack implementation, there are works that target general infrastructures to serve as an overall support toolset for experimenting with diverse research or industrial HW-SW prototypes. 


\subsection{WSN Testbed platforms in the state of the art}

As previously mentioned, the research community attempts to provide developers of WSN-based technologies with a better understanding on how their final system implementation may perform based on fostering the use of testbed infrastructures for real evaluation and experimentation. The nature of the solutions that have been progressively proposed in the literature leads to establishing a classification of the main testbed research trends, which serves as a guideline to determine the scope and requirements when proposing a WSN experimental platform [Horneber'14].

In Fig. 2.1, a general overview of the main considerations when defining WSN testbed scenarios is depicted. The first (left-side) branch related to the system requirements outlines the focus of the research topic to be faced by the testbed platform, i.e., the aim the testbed is created for. This initial approach helps to distinguish a range of custom testbed implementations that are especially focused on one specific development target, rather than providing a testing environment support platform. For instance, in [Bal'09] an in-field tracking and localization testbed was developed in a manufacturing lab scenario in order to model environment effects that can be found in real deployments with similar conditions. They used TelosB [Polastre'05] connected to a central server which runs a java-based software application for controlling the experiments. However, this type of implementation seeks to characterize the specific WSN deployment configuration to verify their cooperative algorithms for localization tasks.

The necessity of allowing for general experimental evaluation schemes in WSN prototyping opened up the spectrum of testbed infrastructures that are primarily focused on providing an overall support for sensor node development, protocol analysis and runtime system debugging. Unlike the aforementioned solutions, the main idea behind this type of approach is related to trying to cover more than just one research use case, so as to create a more extensive solution. 


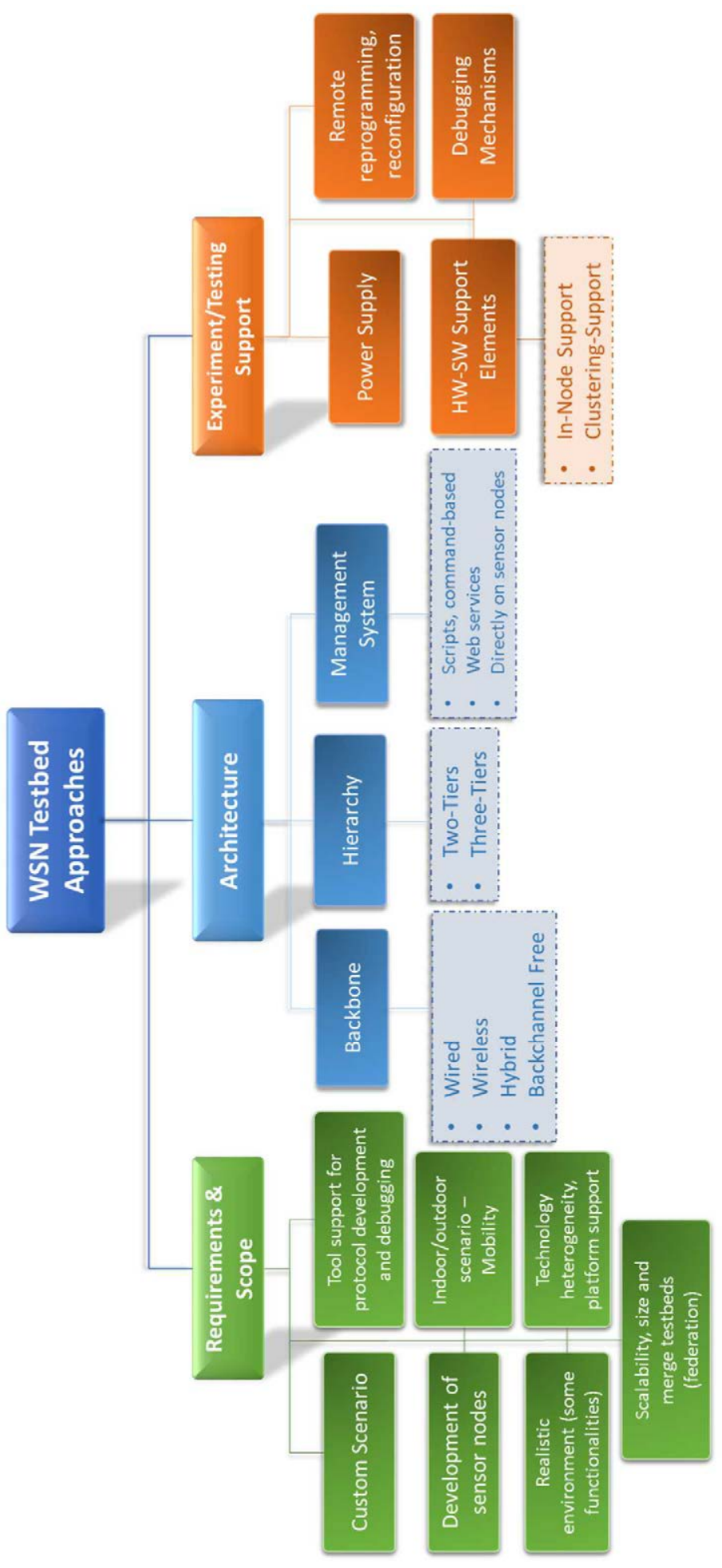

Fig. 2.1 Taxonomy of WSN Testbeds. 
As shown in the aforementioned scheme, there are important guidelines that encompass the definition of a WSN experimental platform. They can be mainly summarized in three specification criteria: System requirement/target, testbed architecture and experimental support capabilities.

From the point of view of system requirements, testbeds are built up trying to cover at least two development/evaluation support domains, particularly regarding: a) protocol implementation and evaluation, HW-SW prototype optimization and sensor platform development; b) realistic environment setup and final application emulation, together with network mobility and flexibility in indoor/outdoor scenarios; c) technology heterogeneity, platform accessibility, size and scalability, and experimental hybrid array.

In terms of the testbed architecture to be implemented, different configuration alternatives could be adopted. Such a decision certainly relies on the intended approach of the testbed platform to provide specific support for the abovementioned requirements. Scenically, since the underlying concept of a WSN testbed defines a support experimental platform, features such as transparency, reliability and testing accessibility have lead the determination of setting up backchannel-based testbeds. The discussion of a secondary communication channel centres the attention on the fact that debugging, reprogramming and experimental evaluation of the sensor network under test needs to be conducted in such a way that additional resources (e.g. network bandwidth) shall influence the normal operation of the system minimally. Here four main trends can be clearly distinguished according to the level of flexibility and cost-effective solution to be pursued, i.e., the adoption of either wired or wireless backchannel, a hybrid solution combining both approaches, or an alternative solution relying on a backchannel-free system array.

Two other important aspects that define the system architecture of testbed platforms are related to the type of hierarchy and the remote management mechanism to be employed. The former one focuses on the interaction level between the remote user and the installed sensor nodes. Two main configurations can be differentiated. On one hand, a two-tier array can be adopted, in which the sensor platforms are directly connected to the remote 
server via the backchannel communication (depending on the backchannel selection, intermediate elements take indirect part in the infrastructure, such as access points). In this type of setups nodes might be attached to auxiliary hardware elements that carry out and exchange debugging tasks with the server side. On the other hand, three-tier arrays are sometimes applied in order to perform the monitoring and evaluation activities with the aid of cluster devices, which control groups of sensor nodes within the testbed infrastructure. This is particularly useful in large-scale scenarios where the management of the sensor network is shared with an intermediate hierarchical layer.

Regarding aspects related to remote management mechanisms, some tendencies aim at employing web-service based techniques to remotely control and exchange information with the experimental platform, in addition to web interfaces targeting HTTP online access to the infrastructure. Other works tend to include the use of script-based control and command-like instruction sets to specify the actions to be triggered as well as the configuration of the data gathering, in combination with on-node management systems.

Based on this overall definition scheme, important contributions have been made in the state of the art that established the foundations of most of the proposed testbed infrastructures, particularly in terms of the type of architecture to be adopted as well as the support techniques for remote experimentation handling. Although there is a noticeable range of different works in the literature, most of them follow some patterns or tendencies regarding the applied technology when targeting the experimental platform to be implemented.

One of the first and most well-known testbeds is Motelab [Werner'05], which is an indoor open access platform for WSN testing deployed at Harvard University, which is based on MicaZ motes and TinyOS as the running operating system in the nodes. Nodes can be accessed by a PHP web interface that is the main user element for the system management, sensor monitoring and data logging, from which they can create different experiment tasks to be remotely executed. Another important software component that is part of its system 
structure is a MySQL database backend, which is responsible for storing collected data from experiments, and generating web content.

From the point of view of the hardware array setup, in Motelab Mica sensor nodes are connected to the central server through a backchannel approach for remote reprogramming and data logging capabilities. Such a connection to the testbed deployment is done via Ethernet communication, by using an Ad-hoc interface board for MicaZ nodes in its initial configuration, the MIB600 [MIB600], as shown in Fig. 2.2. The direct connection to the devices and the underlying functionalities rely on the TinyOS ServialForwarder capability. More than specific testing capabilities itself, what Motelab tries to provide users with is an overall deployment of sensor nodes for downloading and monitoring their running applications in a transparent fashion, as well as organizing server-side scheduling tasks for particular jobs execution.

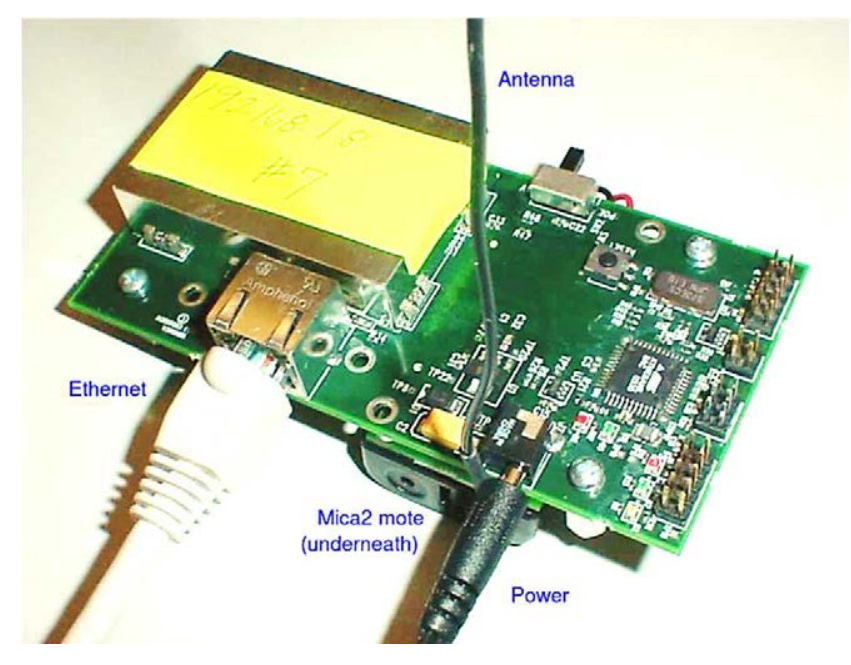

Fig. 2.2 Motelab testbed hardware array [Werner'05].

The main limitation of this approach is related to platform dependency, since the user experiments have to be coded under NesC for TinyOS in such Mica devices. Moreover, there is no software-based framework for developing the experimental applications under the testbed platform.

Unlike Motelab, Indriya [Indriya] uses an active-USB infrastructure as the testbed backchannel for reprogramming and power supplying the sensor nodes, which employs special USB connections called active cables in order to be able to 
reach up to 25 meters of connection. Indriya is a WSN testbed deployed at the School of Computing, National University of Singapore (NUS) and equipped with TelosB as node platforms, though they try to cover sensor heterogeneity by including different types of sensor modules, such as a WiEye Passive Infrared (PIR), magnetometer, accelerometer and ambient sensors for WSN application experimentation.

Since Indriya proposes a three-tier testbed configuration, the remote communication to the sensor nodes is performed indirectly via super nodes, commonly known as gateways that serve as cluster-heads (represented in Fig. 2.3). These types of devices are generally used for connectivity aggregation and some experiment support. In such a configuration, the control and accessibility of the deployed nodes is reached in two steps. In case of Indriya the clusterheads, that are MAC Mini PCs, are connected to the server by using an Ethernetbased communication, and from the nodes to the cluster-heads via the USB hubs by means of the aforementioned active cables. For instance, in subsequent versions of Motelab, they used a similar device array by including Stargate NetBridge single-board computers [NetBridge] as a bridge between the USB and Ethernet tiers, although in a one-to-one configuration basis (instead of a one-tomany setup, as Indriya does).

The main issue regarding Indriya testbed support lies on the fact that the experimental activities have to be carried out within the TinyOS and TelosB platform boundaries, and there is no specific action guidelines to perform prototyping evaluations and platform development under the testbed architecture, beyond the implementation of WSN applications via TinyOS coding. 


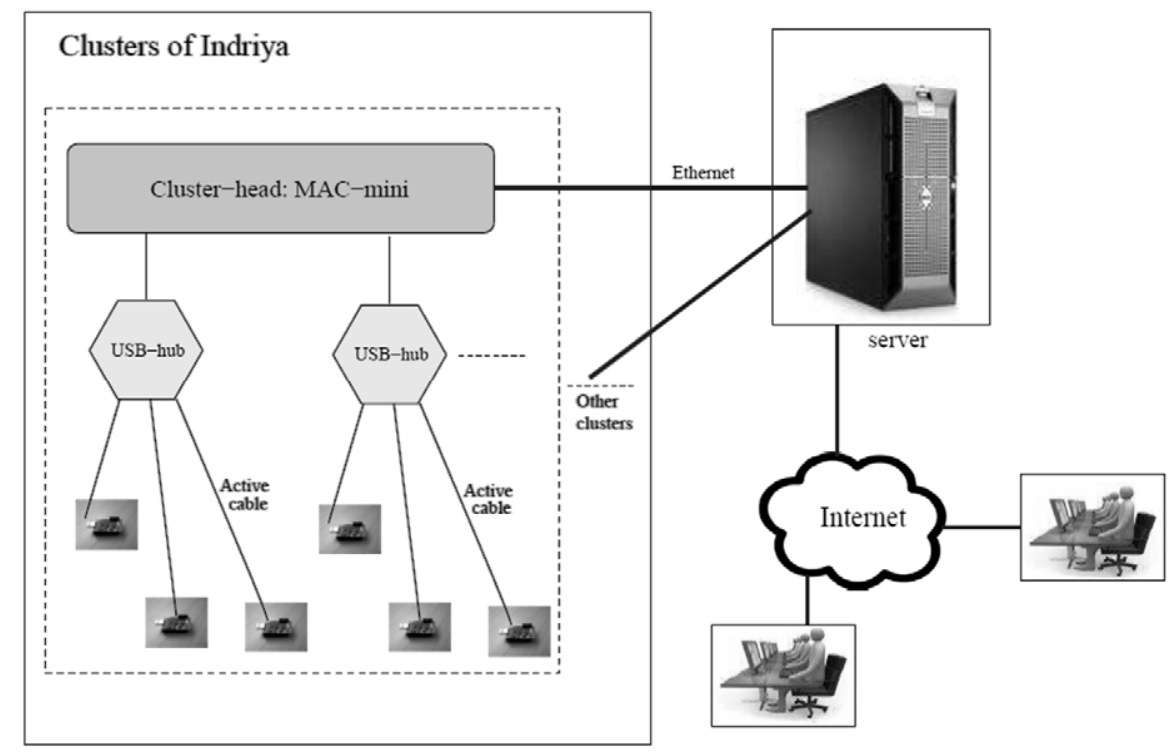

Fig. 2.3 Cluster-based structure of Indriya [Indriya].

Similar to Indriya, in Twist [Handziski'06] authors centre the support to the testbed architecture on the use of the USB interface. They include USB hubs between super nodes (NSLU2 [NSLU2], a discontinued resource-constrained Ethernet-to-USB device) and the sensor platform to cluster and power supply them. This testbed architecture was thought to be used with eyesIFX and TelosB nodes running TinyOS, and the main experimental support for debugging is done by using printf-based data logging.

In PARED [YI'13] authors propose a different approach from the previous testbeds infrastructures, in which they introduce various elements in a testbed structure in order to enhance the usability of the experimental array as a debugging, reprogramming and evaluation platform. First of all, their implementation relies on a Test Unit, which is composed of the sensor node platform in addition to the integration of a test board. The later allows introducing the concept of on-board testbed support devices, which encompasses node experimentation by means of attaching additional hardware elements to the available ports of the sensor platform. This could be done, for instance, to capture information related to analogue and digital signals, protocol lines and GPIO connections, as well as accessing specific ports for debugging purposes. 
In case of PARED platform, this approach is delimited to a 26-pins connector in the test board to which the control board (sensor node) is attached. This test interface includes data transfer port, BSL programming port, status monitoring port and power supply port, so as to provide a wider range of remote features to be monitored/performed. The design of the test board is based on an ARM 32bit Cortex-M3 MCU, whereas the sensor node contains a TinyOS compatible configuration based on the MSP430F5438A microcontroller [MSP430] and a CC2420 RF transceiver [CC2420], both from Texas Instruments.

Regarding the testbed backchannel configuration, unlike the previous works in which Ethernet and mainly USB setups are adopted, in PARED authors propose an alternative structure based on the combination of the CAN Bus plus $12 \mathrm{~V}$ power supply lines, as shown in Fig. 2.4. Their main contribution is related to the code dissemination process for reprogramming the nodes, in what they call parallel reprogramming. In such a technique, the binary code to be sent is divided into several parts and then broadcasted within a time slot, in which test devices can report failures during the loading process by using the CAN bus. The procedure is intended to be applied for whole reprogramming of the same image file throughout the network, as well as for detecting common errors among test units when performing the loading process.

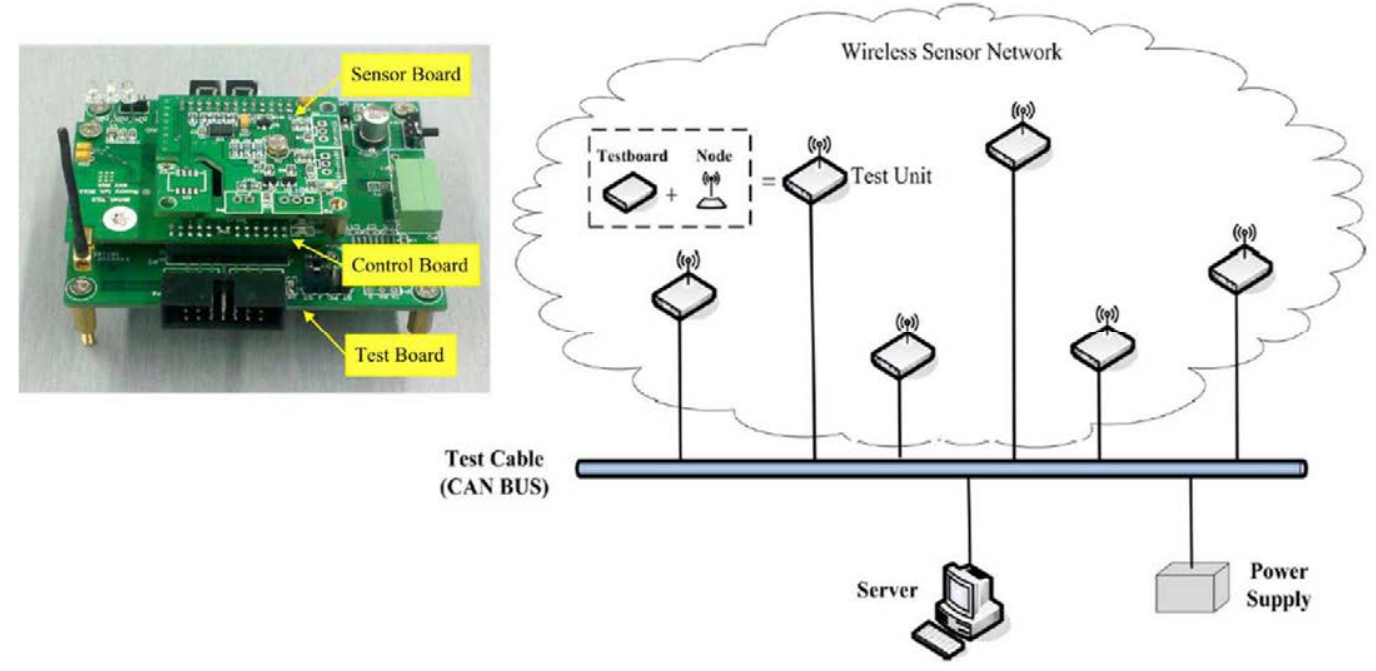

Fig. 2.4 PARED Testbed architecture and platform [YI'13]. 
Another interesting contribution is focused on adapting TOSSIM debugging statements (TinyOS code debugging at simulation level) to the PARED deployment. The main limitation of this technique is related to platform dependency, and the network development is certainly constrained to TinyOS code generation and execution.

Although most of the state-of-the-art testbeds are based on wired-backchannel infrastructures, there are also some works that propose the use of wireless platforms for debugging and reprogramming capabilities, but especially aiming to achieve greater flexibility, mobility and network adaptability within the experimental scenario. Sensei [Hermans'10] tries to address the problem of emulating sensor movement in real scenarios together with node reallocation and topology reconfiguration, features that are difficult to reproduce in fixed testbed infrastructures.

Their main focus is to provide sensor nodes with what they called sensor host, which are responsible for gathering the debugging and monitoring information from the sensor platform and transferring it to the side manager (the main bridge between the remote monitoring and the sensor hosts). Such an interconnection between the deployed sensor host and the side manager is performed by using IEEE 802.11 wireless communication as the support backchannel. Mobile sensor hosts are used to place the sensor nodes in accordance with the experiment to be carried out, and they are based on robots that can detect the surrounding environment to determine the next movement.

From the point of view of the testbed architecture, the main considered elements for building the sensor hosts are the Asus WL-500G wireless access points [WL500G] which run a modified distribution of Linux, and they include the sensor slots that are the main interconnection interfaces with the sensor nodes. This is generally done via USB (although a Bluetooth-based interface is also contemplated). Thus, platform debugging and evaluation capabilities are constrained to such a USB slot connection. What is interesting to highlight from these types of implementations is the fact that since there is no infrastructure rigidly established for the experimentation, the testbed platform can be applied in different target scenarios, hence increasing the flexibility and reusability of the 
system. The ability to move the experimental setups without extensive efforts refers to what is called nomadic testbeds [Horneber'14].

There are some testbeds that focus on evaluating sensing technologies within more realistic conditions so that a more precise characterization of the sensor measurements can be performed. In WINTeR [Slipp'08], a large scale deployment is proposed to evaluate and support industrial applications, based on programmable nodes controlled by a web interface. This testbed is based on two backchannels, the first one for controlling the data exchange to the nodes (Ethernet connection) and the second one for powering the nodes (USB connection). Their main target is to emulate harsh environments so as to analyse how conditions of final scenarios can impact the accuracy of the WSN development.

Although the use and development of testbed facilities based on the concept of backchannels for conducting the experimental capabilities is widely extended in the research community, it is also important to highlight that there are works that investigate the notion of backchannel-free strategies, on one hand trying to simplify the experimental architecture as well as reduce the cost of the hardware implementation; and on the other hand facilitating their applicability especially in two of the previous scenarios, i.e., targeting final application contexts as well as sensor network mobility and scalability. This is the case of SenseNet [Dimitriou'07], in which the primary objective of the testbed is to offer an experimental sensor network without a direct dependency on physical support infrastructures for node remote reprogramming, logging and runtime WSN interaction. In order to overcome the limitation of using the main wireless link as the testbed management channel, authors propose two alternatives to enhance such experimental array. Firstly, a custom routing protocol for collecting management packets from the sensor nodes to the central server, and secondly a flooding mechanism to transmit action messages from the server side to sensor nodes under test.

The testbed functionalities of SenseNet strongly rely on the packet structure of TinyOS, including additional fields related to the specification of users Ids as well as routing parameters. Moreover, nodes accessibility and remote 
reprogramming are subjected to TinyOS mechanisms such as the SerialForwarder and Deluge [Hui'04], which is a reprogramming strategy for code replacement under operating system (more details related to Deluge will be given in subsection 2.4 devoted to the proposed reprogramming architecture). A proof of concept was setup from the basis of $8 \mathrm{Mica} 2$ sensor nodes, so that performance of the wireless network when including the test packets is studied under different types of topologies. Their main concern is certainly related to verifying packet dropping depending on the deployment array, in addition to determining how the management of packet traffics for different users may affect the overall overhead of the wireless link. Another important issue is that since there is no hardware support platform for the testbed capabilities, the experimental actions and debugging activities are indeed limited to the software components of the running application. Fig. 2.5 shows the overall structure of their proposed system, in which the remote access to the WSN is performed via a Gateway element as a bridge component to the server side.

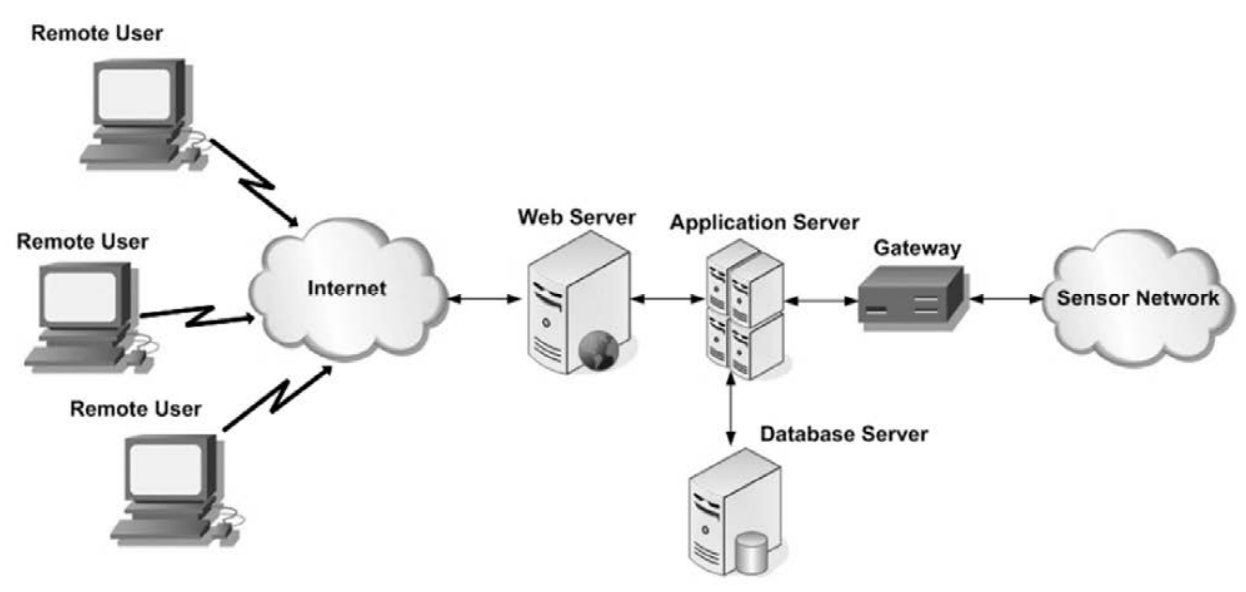

Fig. 2.5 General architecture of SenseNet [Dimitriou'07].

In this sense, authors of MOTEL [Förster'11] try to address network mobility and backchannel-free configuration in a flexible mobile WSN testbed. The main idea behind this proposal is the combination of mobile robots that carry the sensor nodes monitored by a camera attached to the ceiling (see Fig. 2.6), together with a software architecture intended to be used without any backchannel infrastructure. Such a software implementation, called FLEXOR, defines 
interfaces of software components and rules to interconnect them on demand, depending on runtime system specifications. The main aspect to be considered is the fact that all the functional modules are preloaded into the node memory, hence the reprogramming capability is rather a reconfiguration of the interconnections among modules.

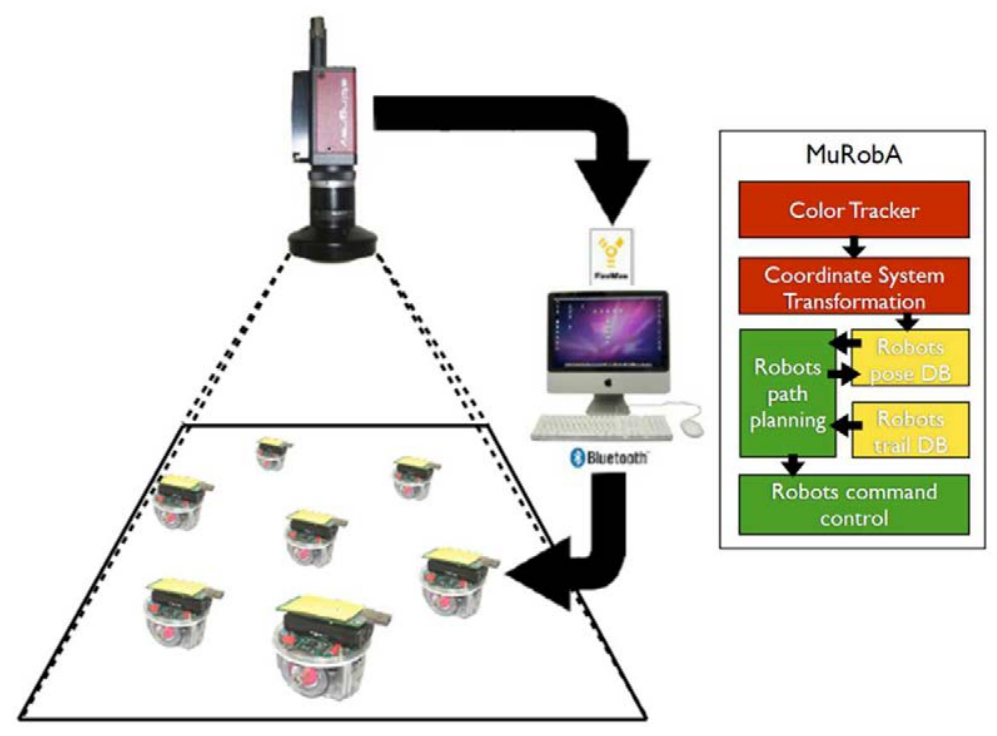

Fig. 2.6 General view of the control system of MOTEL [Förster'11].

Although minimal infrastructures for WSN experimental activities could seem a good choice in terms of cost-effective adaptive solution, this type of approach presents several drawbacks because of a lack of non-intrusiveness and transparency of the testbed capabilities, thus interfering in the normal operation of the wireless network and producing a less reliable solution for platform evaluation. Moreover, additional communication schemes have to be set in order to guarantee that problems regarding dissemination and access control can be avoided. This leads to producing inaccurate results in some of the experimental scenarios under test, such as network traffic analysis, power consumption characterization, routing protocol evaluation, connectivity map correlation, among others, particularly when network experiments get larger.

As previously remarked, there are different works in the state of the art proposing environment-controlled testing scenarios that are composed of a good amount of nodes. Most of such testbed platforms are certainly focused on 
providing users with different access services to make use of the deployed nodes. WSNTB [Sheu'08] is an example of service provision at the toplevel/server-side of the testbed architecture. They integrate different technologies to properly monitor and register data coming from the remote sensor nodes, such as user management for MySQL-based databases, event notifications and text editors. A web service approach is followed to handle the interaction of the user with the testbed.

However, fewer works try to provide well-defined rules to take advantage of the experimental architecture in the sense that developing, evaluating and properly debugging target prototypes can be sped up based on an established framework at sensor platform level. For instance, in [Weyer'09] authors propose a set of code templates intended to instrument the running application with specific logging codes, so that they can extract debugging information of the code execution process at runtime. Their implementation relies on adapting the code generation tool-chain of TinyOS to be able to add instrumentation code to the original file. In order to do so, specific pieces of code have to be included in the software modules and functions to be under test. This process will help users to perform data and message logging so as to trace the code execution in the sensor nodes. They use the Atmel NGW100 [NGW100] as a hardware adaptor attached to every node in order to transmit the control and logging packets via Ethernet connection. Power measurement capabilities are also provided in order to study power consumption patterns of the sensor platforms in relation with the code tracing process, considering that their proposed architecture has been mainly created to give support for developing energy-efficient applications.

Moreover, there is certainly a lack of works focused on testbed architectures that include debugging and evaluation capabilities at hardware or low-abstraction software levels. In Hint [Huangfu'10] authors attempts to tackle the limitation of signal observability in sensor nodes by proposing an experimental platform based on two main elements. First of all, a TelosB-based sensor platform design, call ZiNT, in which the expansion slot is extended with additional pins in order to be able to extract more information regarding the RF transceiver interface, microcontroller interfaces, analogue sensors and power supply lines. The signal 
information is extracted by using the second main element of the infrastructure, which is a development kit board that incorporates an FPGA Cyclone II EP2C8 from Altera [Cyclone] for signal acquisition, and an Atmel AT91SAM7X256 [AT91SAM7X] as the CPU of the test board, which includes the Ethernet MAC controller for the backchannel communication capabilities.

In order to make the sensor platform compliant with the test board, an additional adapter board has to be included in the hardware configuration array, so that physical signals from the sensor expansion connection can be correctly read. Hint theoretically supports reprogramming capabilities, although no strategies for runtime remote experimentation in terms of system reconfiguration and functional reprogramming techniques are proposed. Instead, the testbed platform is more intended to provide a comprehensive nonintrusive observation of node's transceivers at signal and packet parsing level.

As a final remark regarding state-of-the-art trends in WSN experimentation, there are some important contributions related to the integration of different remote testbeds into a larger experimental platform, targeting the use of heterogeneous technologies within what is known as testbed federations. In this context, several institutions try to unify the access, management, and interconnection of diverse testing infrastructures, thus sharing controlled deployment resources. WISEBED [Fischer'09] is a European initiative integrated by several research and academic centres whose main goal is the creation of a federation of distributed test laboratories for WSN technologies, interconnected via internet. A cross building among different platforms is carried out by providing users with $\mathrm{C}++$ based libraries and templates upon with software prototypes can be evaluated.

\subsection{Proposed WSN testbed architecture and framework}

In regard to the previous section, most of the state-of-the-art experimental platforms focus on providing remote access to a controlled deployment of sensor platforms based on well-known commercial nodes such as TelosB or MicaZ, so that they rely on the use of TinyOS-based software prototypes from the user perspective. Apart from the shortcomings already commented, particularly 
highlighting the lack of defined mechanisms for supporting in-node experimentation under such testbed schemes, there is a limitation on debugging and evaluation capabilities to be performed over those sensor architectures. First of all, although such hardware platforms have been widely used, they are becoming obsolete due to its low flexibility and their limited adaptation to hardware expansion and testing.

Secondly, dynamic and flexible experimentation based on efficient reprogramming mechanisms is not covered in most of the available testbed platforms, thus relying the testing and reconfiguration procedure rules entirely on the code developer. Third, there is a huge separation among rigid testbed platforms based on established backbone hierarchy, nomadic testbeds based on backchannel-free schemes, and environment-focus experimental platforms intended to address custom conditions of the target scenario. Hybrid approaches that try to take advantage of the benefit of such solutions, so as to provide a more comprehensive support for different experimental scenarios, are limited in the literature.

These main issues allow defining the scope of this chapter, where a new testbed architecture and framework for experimenting with WSN prototypes is presented. Its core functionalities have been created to address a hybrid solution, that is, most of them can be applied not only to the backchannel-based infrastructure that is proposed, but also to those application scenarios where observability, runtime reprogramming and debugging capabilities are particularly needed, so that the target implementation can be optimized. Moreover, the proposed solution is also considered as hybrid in the sense that the testbed support infrastructure has been conceived in such a way that a convergence between a nomadic strategy and a structured backbone scheme is achieved.

This approach is the result of a flexible and dynamic HW-SW support platform implementation to provide users with a defined testbed framework, which is primarily integrated to experiment with the modular node architecture proposed in [Portilla'10]. This sensor platform, called Cookies, relies on a modular system design in which the main capabilities that determine a WSN 
node (i.e. communication protocols, processing elements, power supply systems and sensor instrumentation/conditioning) are implemented in different hardware layers and interconnected by means of well-defined interface signals. Such a modularity is intended to provide a great degree of flexibility when integrating new technologies as well as adapting the platform to different application requirements. Moreover, reusability and interchangeability of designed layers define the main foundations of the Cookie platform, which means that whenever a new set of hardware components has to be included in a particular layer, the rest of the system can remain unaltered.

\subsubsection{The modular hardware platform}

In Fig. 2.7, a general overview of the Cookie platform is shown, where the main elements that compose the modular architecture are highlighted.

In the sensing layer, different technologies related to the physical observation of the target environment are integrated, in addition to the corresponding signal conditioning techniques as a pre-processing stage of the data acquisition system in the node. Due to the aforementioned modularity, this layer might be completely replaced by a new hardware design adapted to a particular scenario without modifying the rest of the involved layers. The only requirement is the compliance with the Cookie architecture in terms of the interconnection interface.

Depending on the type of application, in this layer actuation capabilities can also be included so as to trigger specific states of elements under control. Several versions of this sensing layer have been designed for the Cookie nodes within the scope of industrial application projects, including temperature, humidity and light sensors, accelerometers, $\mathrm{CO}$ and $\mathrm{CO} 2, \mathrm{pH}$ and water temperature, Global Positioning System (GPS) module, among others. Therefore, this layer is certainly application dependent and new prototypes can be designed according to the specific measurement requirements of the target scenario. 

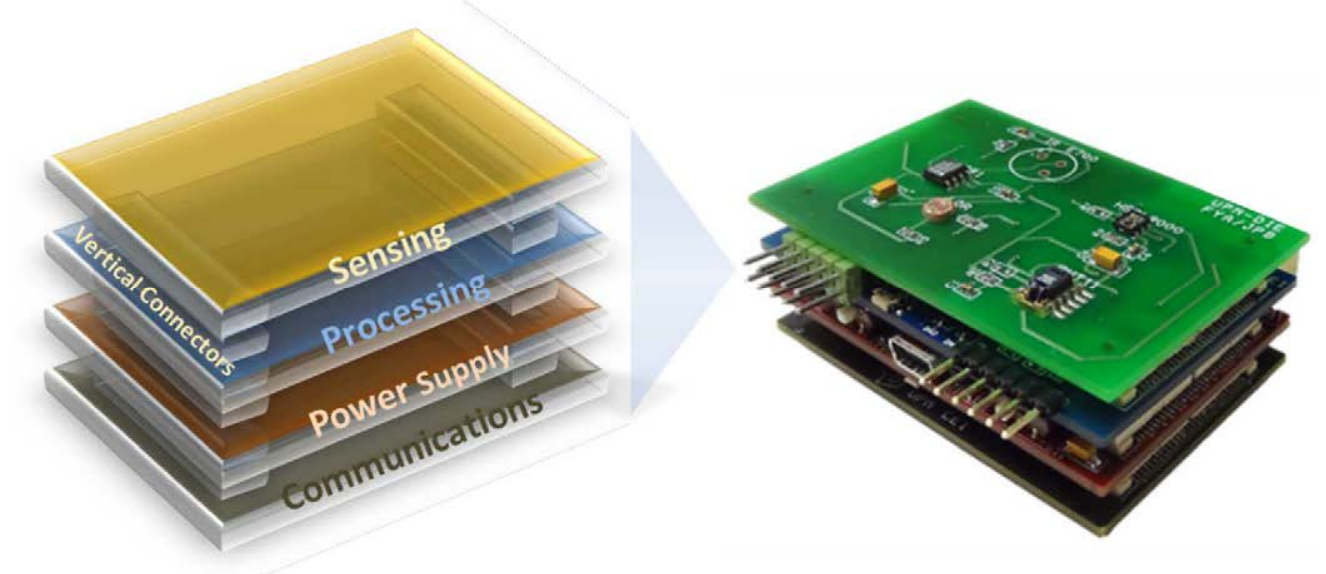

Fig. 2.7 Modular architecture of the Cookie hardware platform.

The communication layer is one of the most important elements in terms of the low-rate efficient wireless networking capabilities of the Cookie platform. Here different technologies related to medium access control and wireless connectivity techniques are integrated to establish the data communication and processing cooperation of the sensor nodes remotely. This modular layer has to be carefully designed since it is the one that usually contributes to increase the power consumption of the platform, hence specific interfaces for sleep modes and wake-up strategies are to be taken into account. A common practice within the Cookie platform development is to include hardware modules that already integrate the corresponding communication stack, such as Bluetooth and ZigBee. As described subsequently in this thesis, additional communication capabilities have been implemented to experiment with efficient mesh networking strategies for Cookie-based WSN applications.

Regarding the power supply stage, there have been different versions of this layer according to the type of power source to be used as well as the requirements of specific processing elements. The most common layer design relies on extracting and converting the input level from a rechargeable battery in order to provide the rest of the hardware platform with the corresponding voltage levels based on the Cookie-compliant characteristics. This layer also includes a USB connection port to take advantage of data and power supply lines of such a standard. There have also been some contributions intended to apply energy harvesting 
techniques to power-supply the node with, for instance, solar panels in those application scenarios where self-energy management of the sensor node without any human intervention is a critical concern.

The processing layer is the most important part and the core of the modular platform because it includes the hardware and software elements to provide the overall sensor node with an intelligent and efficient management scheme, as well as perform processing tasks based on the signals coming from and going to the rest of hardware layers. Several versions have been proposed targeting a trade-off between processing performance and low power consumption. The main feature that distinguishes this platform from state-of-the-art solutions is the combination of a microcontroller as the main processing element with a Field Programmable Gate Array (FPGA), whose versatility and flexibility allows including new interfaces and hardware coprocessing blocks in the system architecture. Furthermore, the FPGA plays an important role within the integration of debugging and remote management capabilities for the testbed framework.

In this work two heterogeneous processing layers have been used to implement the proposed WSN HW-SW technologies in the Cookie modular platform. The first one includes an ADuC841 microcontroller from Analog Devices (which integrates an 8051-based $64 \mathrm{kB}$ processing architecture for low-power embedded developments) and an FPGA Spartan 3 XC3S200 from Xilinx [Portilla'10]. The second one refers to the redesign of the processing layer as a contribution proposed in this work, in order to provide a higher degree of flexibility, observability and modularity combined with an ultra-low-power platform based implementation. It targets to provide a trade-off between power consumption efficiency in nomadic WSN testbed arrays and the adaptability and applicability of the HW-SW framework to different application/experimental scenarios.

In this way, three main aspects have been considered for the design of this new Cookie layer. The first one refers to the ability of the embedded system to adapt to different experimental configurations by allowing for several connectivity arrays of the processing elements to the rest of the platform. This means that, depending on the system requirements, the processing layer can be modified to 
offer different functional properties, as shown in Fig. 2.8, where the main modules and interconnections that define the structure of the layer are highlighted. The second one is related to the inclusion of an 8051-based processing architecture, which is in line with the defined HW-SW framework of the Cookies for resource-constrained sensor network applications. The third aspect lies on the compatibility with the aforementioned capabilities by integrating ultra-low-power hardware elements within the system architecture, seeking the balance between flexibility and power-awareness, as it has been remarked earlier.

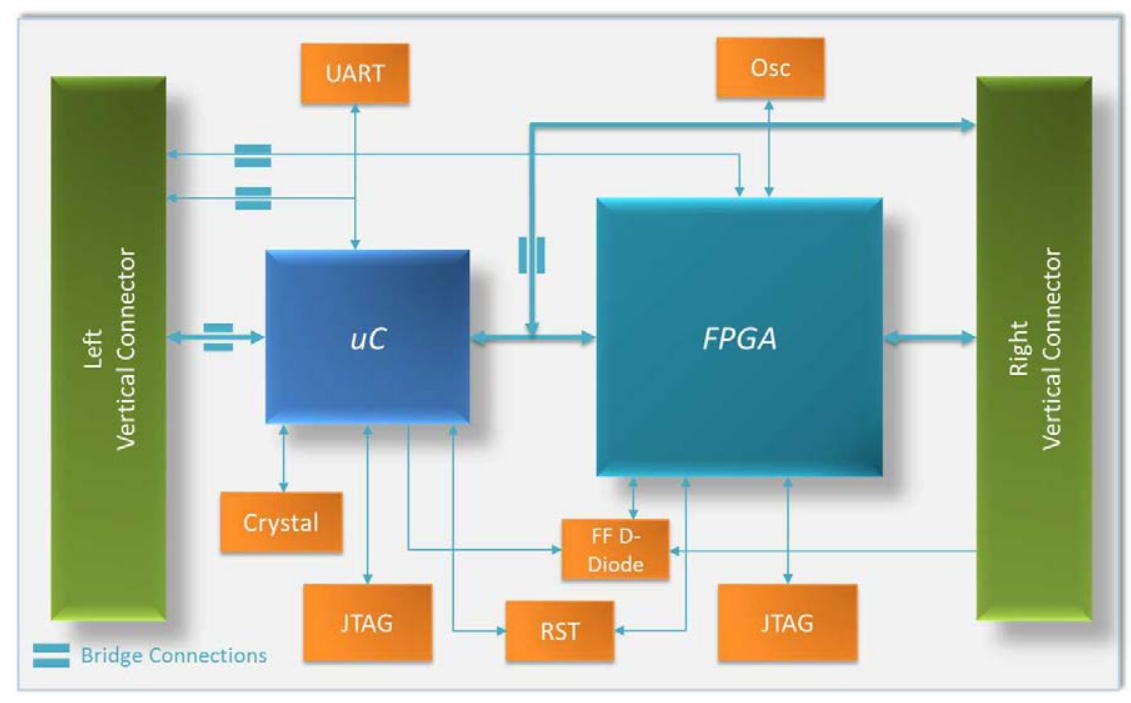

Fig. 2.8 Overall structure of the proposed processing layer design.

Based on the scheme proposed in Fig. 2.8, the designed processing layer provides five different operational modes, whose configurations can be made by using bridge connections among layer paths according to the WSN based system constraints definition for the target application/experiment, as follows:

- Microcontroller with ADC signal connections: this mode only includes the main processing element and it is thought to be used in those applications where the power consumption is the most critical aspect to be considered. This mode dedicates some of the port lines of the microcontroller for analogue input signal processing from the left-side of the Cookie interface. 
- Microcontroller without ADC signal connections: this is a variation of the former mode intended to provide the maximum number of digital input/output ports to the rest of the platform.

- FPGA as the core processor: in this case a trade-off between fast signal processing, digital connections availability and power consumption is sought. Thus, the I/O pins of the FPGA are spread between both vertical interfaces of the Cookie platform.

- Microcontroller with ADC signal connections and FPGA: this mode offers the widest possibility for platform experimentation in a single layer configuration array, so as to be able to combine the functional component capabilities and peripherals of the microcontroller with hardware block implementations for co-processing and debugging tasks in the FPGA. In this case, both analogue and digital sensor signals are contemplated.

- Microcontroller and FPGA: It offers a similar approach as the aforementioned mode though more ports of the microcontrollers are dedicated to digital interfaces of the Cookie node.

Silicon Laboratories provides ultra-low-power 8051-based microcontrollers families for long-term operability of embedded autonomous systems, including power-down/sleep modes that allow managing the active states of the device, which is intended to extend the battery lifetime of the platform. In this direction, the C8051F930 microcontroller [C8051F930] has been integrated into the new processing layer design, which offers a novel configuration architecture to assign different I/O ports to the internal peripherals, i.e., users can select analogue and digital peripherals to be located in any of the available physical ports of the microcontroller. This is particularly useful to enhance the adaptability and flexibility of the processing architecture when testing different layer prototypes and connection arrays. Such a cross-connectivity technology is used to promote the aforementioned operational modes of the designed Cookie layer.

The C8051F930 is composed of a $64 \mathrm{kB}$ flash program memory, internal and external RAM memories of 256 bytes and $4 \mathrm{kB}$ respectively, 10-bits ADC, four 16-bits timers and Smart Real Time Clock, UART and 2 SPI, and most 
particularly it supports a voltage supply range of $1.8 \mathrm{~V}$ to $3.6 \mathrm{~V}$, which can lead to obtaining $0.05 \mathrm{uA}$ of current consumption in sleep mode (besides idle and suspend modes that can also be configured).

Regarding the integration of an FPGA device, a trade-off between low-power consumption and capacity leads to considering the inclusion of an Actel (Microsemi) Igloo FPGA [IGLOO] since they provide several power-down configuration modes and particularly the defined Flash ${ }^{*}$ Freeze technologies, in which the power consumption can be dropped to $5 \mathrm{uW}$ and go back to normal operation within 1 us. The selected model has been the Igloo AGL030V5 that is a $1.2 \mathrm{~V}$ to $1.5 \mathrm{~V}$ low power flash technology programmable logic device composed of 30000 system gates, 768 D-flip-flops, 81 user I/O pins, and Flash*Freeze sleep mode control without needing to disconnect supply voltages.

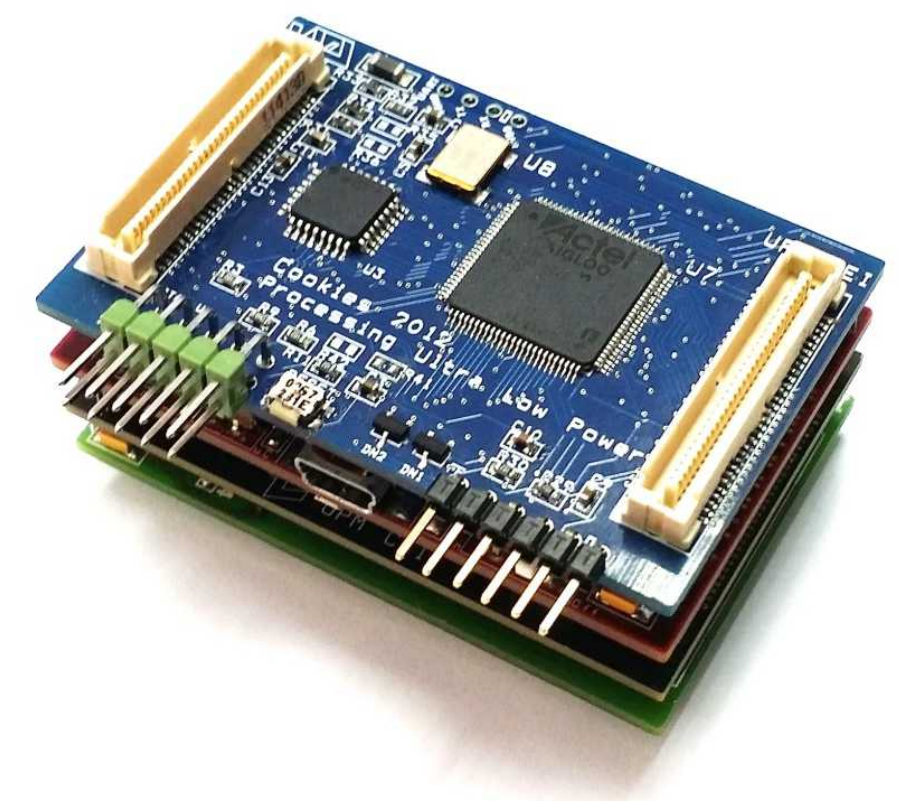

Fig. 2.9 Ultra-low power Cookie processing layer connected to the rest of the platform.

As a result, the implementation of the proposed processing layer is shown in detail in Fig. 2.9, where the involved elements are integrated into a Cookiecompliant hardware design.

It can be seen that the key to achieve such a level of flexibility in this modular platform are the vertical connectors that are placed at both side of every layer 
(and top/bottom sides), playing not only a physical role of adaptation but also sharing the analogue and digital interconnection signals among the different designed boards. There are 60 pins per connector, thus a total amount of 120 signals can be used to interface and exchange data among the hardware layers. The physical connection on the left-side of the layer is considered as the analogue bus because analogue signals are commonly interconnected to it, whereas on the right-side digital interfaces are gathered likewise.

The testbed infrastructure proposed in this work takes advantage of this degree of signals accessibility to provide a comprehensive remote interaction with the platform interfaces, so as to obtain a better understanding of the sensor behaviour at runtime.

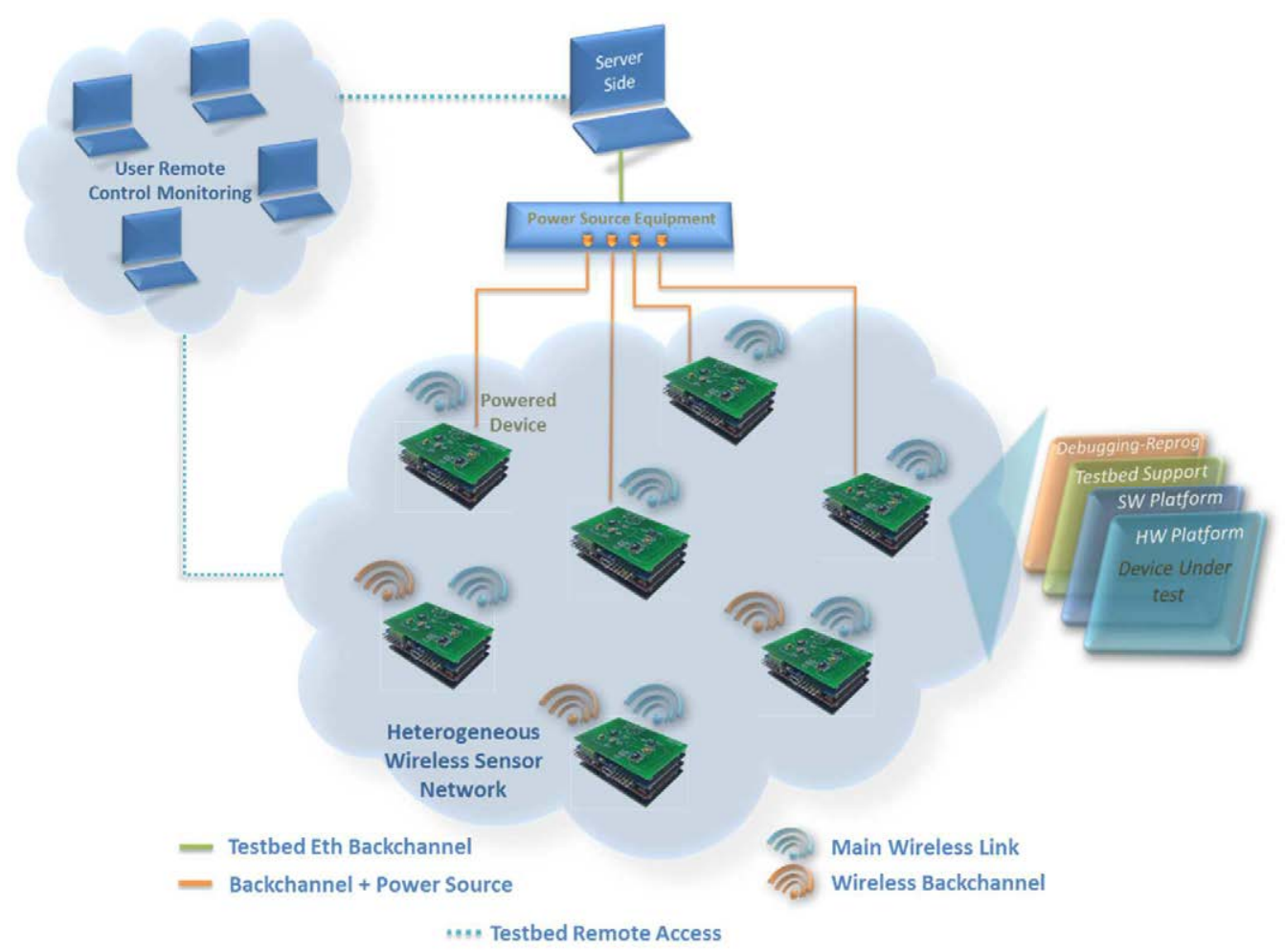

Fig. 2.10 Overall scheme of the proposed testbed infrastructure and involved elements.

In Fig. 2.10 a general overview of the proposed testbed infrastructure is depicted, where the integration of hardware and software elements constitutes the baseline of the framework. In order to cope with the main challenges related to remote access to the device under test, debugging capabilities, and multi- 
experimentation based on efficient reprogramming strategies, the combination of a flexible backchannel infrastructure with a memory-segments-based partial and remote reprogramming scheme is presented.

\subsubsection{Backchannel interface approach}

As mentioned before a hybrid approach for the backchannel-based testbed infrastructure is followed, in such a way that a unification of a structured backbone built on a wired interconnection along with a flexible highperformance wireless communication interfaces is proposed. Therefore, the advantage of both strategies are considered to implement a solution that covers a broader spectrum of experimental conditions.

In this way, the first aspect to be taken into account is the integration of the testbed communication interface into the Cookie modular platform. As shown in Fig. 2.10, the main requirement of the backchannel structure to be tackled is the ability for users to access the experimental platform remotely in a reliable and non-intrusive way with respect to the main communication link of the WSN. One of the decisions that have been allowed for is the use of an Ethernet-based connectivity interface so that mechanisms for accessing and controlling the testbed platform from intra/internet sides can be established. Secondly, two important analysis of a WSN are associated with the energy consumption characterization of the sensor node and dynamic topology reconfigurability, which can be studied by using strategies for turning on/off the remote platforms accordingly. Therefore, the ability to provide and control the power supply of the devices under test shall also be included in conjunction with the backchannel communication capabilities.

Since robustness, reliability, high performance accessibility and bandwidth availability determine the usefulness of the backchannel interface, Ethernet fits such requirements allowing for a trade-off between feasibility of implementation in a laboratory environment and the integration of available technologies upon the IEEE 802.3 stack [802.3], such as the well-known TCP/IP protocols as well as upper abstraction layers for system services and application interfaces. 
Moreover, in order to address the aforementioned requirement regarding power supply capabilities, the idea of integrating the Power over Ethernet technique (PoE) is addressed, so that power and data can be obtained from the same cable (instead of using two different sources of connection for each one), hence reducing the complexity of the interconnections and the installation setup. Although the original IEEE 802.3 includes 4 twisted pairs of cables but only two of them are used, new evolutions of the standard try to take advantage of the available pairs so that the inclusion of some additional features can enhance its performance (for instance, allowing Fast Ethernet at $100 \mathrm{Mbps}$ ). Particularly, the substandard 802.3af [802.3] introduced the concept of transmitting up to $15.4 \mathrm{~W}$ of power supply from an emitter point to a receiver over the available twisted pairs.

However, hardware elements are not commonly prepared to support such a configuration, thus a particular set of components are considered in order to be compliant with the PoE mechanism. There are two main involved devices: the Power Sourcing Equipment (PSE) and the Powered Device (PD). The latter is the target device which will be powered and connected to the backchannel data interface, whereas the PSE is the one in charge of power supplying the end device as well as providing the remote data connection. In case of the PSE, there are two possible configurations to be adopted. On one hand, a Midspan device can be used, which is an external power injector between a normal Ethernet switch and the PD. On the other hand, an Endspan device that works exactly as a network switch but integrates de PoE by injecting both the power and data to the end devices. When the power is supplied over the free twisted pairs, i.e. pins 4-5 and 7-8, such a configuration is also known as Mode B within the standard. Besides, in case the power is provided over the same twisted pairs, i.e., pins 1-2 and 3-6, such a configuration is also known as Mode A within the standard.

It can be noticed that the second option presents a reduced configuration array since the switching and power supplying functionalities are integrated in the same device. Therefore, seeking as less complexity of installation and interconnections for the backchannel infrastructure as possible, an Endspanbased approach has been adopted as the PSE for the PoE technique. Several 
commercial solutions were evaluated trying to have a trade-off between cost/performance and number of devices to be connected, and the selected device was the Netgear GS110TP PoE switch [GS110TP], which has 8 ports that support PoE and 10/100 Mbps Ethernet data communication. This switch provides up to $59.3 \mathrm{~W}$ dynamically distributed over the available Ethernet ports, which means that depending on the number of connected devices the maximum supplied power might varied, never exceeding the $15.4 \mathrm{~W}$ of the standard. As commented later on in the PD description, this supplied values are more than enough for the power requirements of the Cookie platform. Another important feature that has been taken into account in this device was the capability of managing and monitoring the power usage of the PD, particularly to be able to schedule turning on/off actions in specific periods of time. This will help to study network topology changes as well as how experimental prototypes may affect the overall power consumption performance of the sensor platforms.

Regarding the PDs, the modular nodes are the end-devices that receive both power and data lines over the Ethernet port. Therefore, in order to establish the proposed backchannel interconnection with the sensor platforms, a new Cookiecompliant hardware design for the testbed interface has been taken into account.

Focusing the attention firstly on the power supply issue, a PoE compliant implementation has been carried out so that nodes that are part of the testbed infrastructure can be powered accordingly. The voltage supply levels that the architecture commonly handles are shown in Table 2-1 (which refers to the standard levels VDD, V1 and V2 of the Cookie platform, and considering their correspondence with the processing layers in this case). As it can be seen, since every modular layer has different designed versions depending on the technology that was particularly integrated, special attention in the compatibility among different supplied voltage levels has to be paid, by means of creating a flexible design in such a way that a wider range of Cookie layers can be perfectly connected to the platform. In this direction, a new PoE-based modular layer that serves as the main element to support the backbone infrastructure for the Cookie nodes was created. 


\begin{tabular}{|c|c|c|c|}
\hline Processing Layer & VDD & V1 & V2 \\
\hline C8051F930-based & $3,3 \mathrm{~V}$ & $\begin{array}{c}1,5 \mathrm{~V} \\
\text { (Actel } \\
\text { AGL030V5 FPGA) }\end{array}$ & $\begin{array}{c}2,5 \mathrm{~V} \\
\text { (C8051F930 uC) }\end{array}$ \\
\hline ADuC841-Based & $\begin{array}{c}3,3 \mathrm{~V}[>2,8 \mathrm{~V}] \\
\text { (ADuC841 uC, Xilinx } \\
\text { Spartan 3 FPGA IOs) }\end{array}$ & $\begin{array}{c}1,2 \mathrm{~V} \\
\text { (Xilinx Spartan 3 FPGA } \\
\text { core) }\end{array}$ & $\begin{array}{c}2,5 \mathrm{~V} \\
\text { (Vcc Aux Xilinx } \\
\text { Spartan3 FPGA) }\end{array}$ \\
\hline
\end{tabular}

Table 2-1 Different voltage levels for the Cookie processing layers.

First of all, in order for a PD to be compliant with the PoE standard regardless of the adopted mode, the female socket RJ-45 that receives the signals from the Ethernet cable has to be configured so that specific output power pins are physically available. For instance, common sockets have the unused pairs connected to ground, while other specific ports do extract the corresponding signals and then make them available externally. That is the case of the SI-52008F from Bel Fuse [SI-52008-F], which indeed provides the output signals for both PoE operational modes.

Since the voltage at the PD ranges from $37 \mathrm{~V}$ up to $57 \mathrm{~V}$ according to the standard, and the maximum voltage supply level of the Cookie platform is $3.3 \mathrm{~V}$, a DC/DC converter has to be included to adapt the incoming signals in the designed layer. Therefore, an IEEE 802.3af PoE-compliant PD module was integrated to adapt such values, particularly from the Ag9400 family manufactured by Silvertel [Ag9400], which has been designed to also provide the power classification and PoE signature required during the negotiation between the PSE and PD, before supplying the corresponding power. In Ag9400 series nominal output voltages can be found, such as $24 \mathrm{~V}, 12 \mathrm{~V}, 5 \mathrm{~V}$ and $3.3 \mathrm{~V}$. For this particular case, the Ag9403 provides $3.3 \mathrm{~V}$ and $6.6 \mathrm{~W}$ as maximum output power that is enough for the requirements of the Cookie platform as PD.

As mentioned before, it is important to highlight that this testbed support layer is designed to provide power levels to different modular layers of the Cookie platform, and it is particularly noticeable in case of the processing layers where several voltage values are handled. Therefore, apart from the resulting $3.3 \mathrm{~V}$ output of the PoE module, ultra-low-power linear regulators are included to provide $2.5 \mathrm{~V}, 1.5 \mathrm{~V}$ and $1.2 \mathrm{~V}$ to the rest of the layers in accordance with the 
hardware configuration of the node. These voltage values are then distributed to the rest of the platform through the Cookie vertical connectors.

With the PoE standard completely covered, the main issue to be faced is related to the question of establishing a communication link between the user's remote access and the sensor platform under test, so that runtime experimental reprogramming, parametrization and debugging capabilities can be performed. Since the aforementioned capabilities are carried out with the support of the processing elements included in the Cookie nodes, a data interface between the designed and the processing layers shall be defined so that testbed frames can be remotely exchanged through the Ethernet backchannel. In order to do that, a hardware module that implements an embedded Ethernet protocol stack controlled via Serial Peripheral Interface bus (SPI) has been incorporated into the designed layer, targeting an efficient straightforward integration and portability of the solution into the different Cookie processing components that are part of the architecture.

In this way, the selected module was the WIZnet W5200 [W5200], which enables a high-speed SPI access to the TCP/IP and Ethernet stack layers from a host device in a more transparent fashion. Apart from the main features that can be distinguished from its embedded implementation (such as hardwired TCP/IP Protocols support, 10BaseT/100BaseTX Ethernet MAC/PHY, up to 8 independent sockets working simultaneously) an important aspect that is considered for the support of the backchannel performance is its internal $32 \mathrm{kBs}$ of memory for buffering TX and RX data.

In Fig. 2.11 a general view of the designed Cookie-compliant Ethernet layer with the integration of the described elements is depicted, together with the physical implementation of the hardware layer. As seen in the figure, due to the dimensions of the PoE module as well as the RJ-45 port, the physical design of the board has been created in such a way that the standard dimensions of the Cookie nodes are kept (40x60 millimetres) plus an additional extension in width to include these elements without disturbing the connection to other modular layers at both sides. The final implementation of the board fits in $80 \times 60 \mathrm{~mm}$. Another important feature to be highlighted is the fact that since a combination 
of power supplying and data communication elements are combined in the designed board, two intermediate layers in the layout have been used as voltage and ground regions respectively, in addition to the top and bottom layers for routing signals and placing components' footprints. Therefore, the designed board is composed of a total of 4 layers on its hardware implementation.
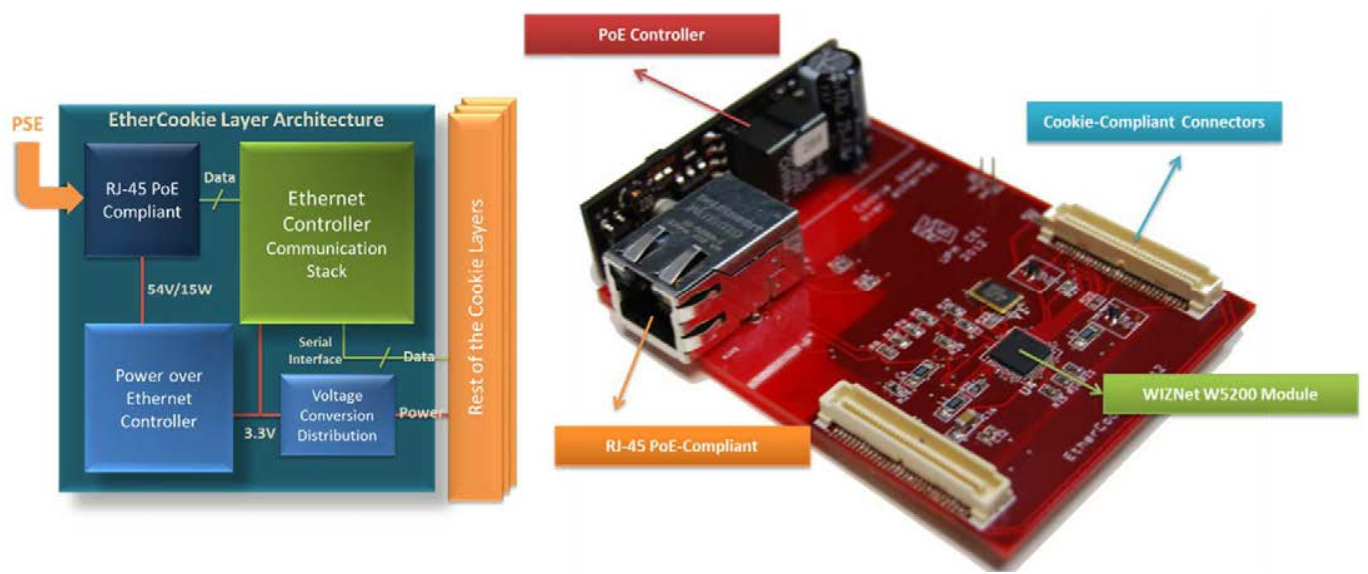

Fig. 2.11 Hardware implementation of the proposed Ethernet-based backchannel Cookie layer.

As a result of this approach in which the integration of several technologies allows establishing a reprogramming and debugging channel, the proposed innode WSN testbed framework has the support of a dedicated data communication interface to remotely carry out the tasks of the experiments.

In addition to this, the proposed architecture focuses on addressing a flexible and adaptable testing platform so that a wider spectrum of experimental activities can be covered by using the provided framework. In order to accomplish that, a hybrid solution in which the already explained backbone structure is combined with the inclusion of a high performance wireless backchannel is considered in this work. The main idea is to face experimental requirements regarding mobility, testbed deployment reconfiguration and nomadic functionality, where a fast reorganization of the sensor nodes can be easily performed. Moreover, since the IoT paradigm tends to gather heterogeneous platforms based on ad-hoc interconnection technologies upon $\mathrm{Wi}-\mathrm{Fi}$, the integration of a novel communication layer into the Cookie modular architecture indeed expands the interoperability and heterogeneity of the proposed solution to different application contexts. 
Therefore, the design and development of an IEEE 802.11 based hardware layer pursues on one hand the support and scalability of the testbed architecture in different experimental scenarios as a backchannel interface approach, and on the order hand the creation of gateway nodes where low-rate communication technologies and high performance wireless infrastructures converge in an embedded solution. As it can be realized, such an approach fosters the possible usability of the testbed architecture also in mixed indoor/outdoor scenarios.

One of the main concerns regarding such an implementation refers to the power consumption modes of the technology to be used, as well as how the integration of two different wireless communication protocols that work at the same frequency band may affect their normal operation. There are several works in the state of the art focused on experimental analysis of this aspect, such as the one proposed in [Sakamuri'08] in which authors studied the interoperability between IEEE 802.11 and IEEE 802.15.4, and they conclude that a proper configuration of different operational channels will result on an adequate operability of both protocols under the same platform and scenario. Regarding the power consumption issue, typical Wi-Fi modules can consume up to $250 \mathrm{uA}$ in sleep modes, which is not a viable solution within the scope of the WSNs. However, new approaches based on the so-called Low-power Wi-Fi open a new range of possible solutions where the power consumption can be reduced down to $4 \mathrm{uA}$ in sleep mode, whereas $30 \mathrm{~mA}$ and $200 \mathrm{~mA}$ in reception and transmission modes can be achieved respectively, according the configuration of the devices [Tozlu'12].

A similar approach with respect to the design of the Ethernet-based backchannel layer has been followed to implement the Cookie-based Wi-Fi communication board, in the sense of integrating a hardware module that provides access to the corresponding protocol stack by using a standard interface. Particular requirements are also taken into account regarding network configuration, module parametrization and power supply modes, especially related to external interruptions and timers so that the management of such states can be carried out from a host device. Another important aspect to be allowed for is the 
capability of including different modes of operation such as ad-hoc and accesspoint configurations.

Based on this, the selected module was the RN-131C from Roving Networks (now part of Microchip) [RN131] that offers a $2.4 \mathrm{GHz} 802.11 \mathrm{~b} / \mathrm{g}$ ultra-low power embedded solution, which includes the TCP/IP stack on-board, secure Wi-Fi authentication modes such as WEP-128 and WPA2-PSK, low-power and HW-SW wake-up mechanisms, and built-in networking solutions such as DHCP, UDP and HTTP client. Moreover, the module integrates a $1 \mathrm{MB}$ flash memory and $128 \mathrm{kB}$ of RAM, allowing several firmware versions to be simultaneously saved, particularly to include ad-hoc and access point connection modes. An ASCII UART-based interface provides host devices with the capability of configuring and controlling the module, besides the possibility of a remote configuration over Wi-Fi via Telnet.

Likewise, a modular design has been followed keeping the dimensions of the Cookie standard layers, and the result of the implementation is shown in Fig. 2.12.

Both hardware layer solutions represent the main elements of the proposed hybrid testbed infrastructure to provide a robust, flexible and reliable backchannel interface to remotely access, interact and experiment with the modular architecture of the WSN sensor platform.

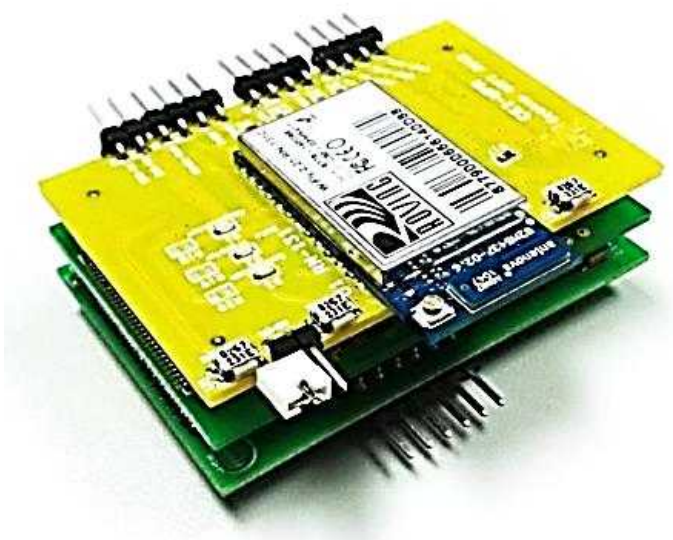

Fig. 2.12 Hardware implementation of the proposed wireless backchannel Cookie layer. 


\subsubsection{Functional blocks for supporting the experimental platform}

As explained in previous sections, the proposed framework targets to provide in-node support capabilities for experimenting with the modular platform, aiming to apply such functional blocks not only during the pre-deployment stage but also in those scenarios where runtime testing and debugging actions are required. Therefore, these hardware and software support components are implemented by using the processing architecture of the Cookie nodes, i.e., a system convergence between functionalities that are integrated both in the microcontroller and the FPGA.

Considering this, one of the goal of the testbed platform is the capability of debugging and analysing the behaviour of the wireless sensor platform beyond the typical prinft-based procedure at the top level application of the microcontroller. Hence, the FPGA takes an important role to perform lowabstraction level functionalities as the support co-processing element of the proposed framework. In this way, five main interaction levels are defined to categorise the type of experimental actions to be performed, as follows:

- Signal/GPIO level: the control and observability of signals allows performing actions at hardware level, such as reset and wake-up modes of the working elements in the sensor platform, hardware activation/deactivation based on GPIO event detection, reprogramming/reconfiguration modes triggering, and interconnection switching among modules.

- Interface/communication level: this is related to the use of welldefined interfaces such as SPI and UART to execute debugging routines with the involved peripherals and modules that are working in the sensor platform at runtime. As described in following paragraphs, this is particularly important for the establishment of a remote debugging connection to the microcontroller, in order to extract internal information at peripheral and code execution level.

- Component/peripheral level: in correlation with the aforementioned level, the analysis of the configuration and behaviour of the internal 
peripherals of the microcontroller allows obtaining information related to analogue and digital sensor processing, timing performance, memory usage and resource accessing, interface and interruption handling. This level combines functional blocks of the FPGA with debugging routines of the processor to carry out the aforementioned tasks.

- Implementation and application levels: these are related to the software implementation and the verification of functional components as part of the application prototyping. The remote and partial reprogramming techniques proposed in subsequent sections are the main elements to support these interaction levels.

Focusing the attention on the first two interaction levels, an embedded block structure to remotely trigger specific control actions under the experimental platform is implemented into the FPGA of the Cookie node. In order to command the functional blocks that compose such structure, the backchannelbased data communication approach has to be integrated by means of including configuration, decoding/encoding and frame processing engines, so that the data coming from and going to the testbed infrastructure can be effectively used to manage the experimental platform.

In Fig. 2.13, an overall scheme of the functional blocks that comprise the control structure at signal and interface level is represented in detail, where the backchannel configuration as well as the correlation among the different involved components are highlighted. 


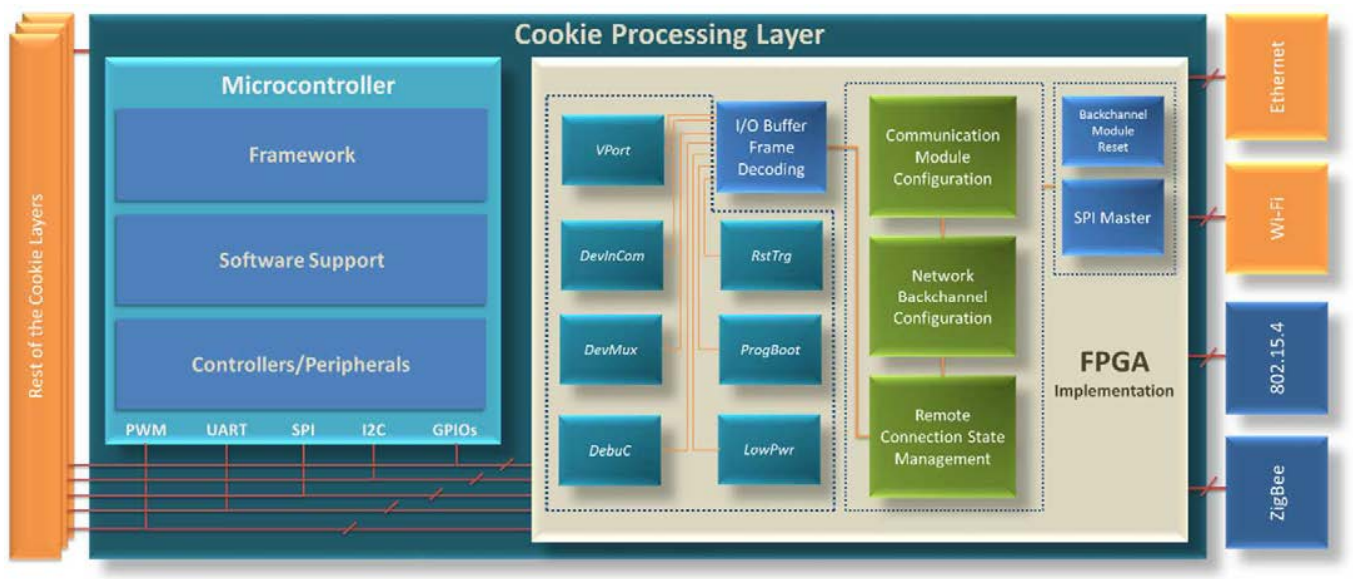

Fig. 2.13 General structure of the testbed framework implemented in the Cookie HW-SW modular platform.

The basic element of the low-level integration with the backchannel infrastructure is the implementation of the SPI communication interface, so that the interaction with the WIZnet module can be established. Two important aspects have to be considered in this context. First, the SPI block has to be created as a master device, because the different SPI modules that can be connected to the modular platform act in slave mode, not only in case of the WIZnet, but also in other hardware layers, including memory and communication elements. Secondly, different SPI operational modes are supported, particularly mode 0 and 3 which are the ones implemented in the WIZnet module. The operational modes of an SPI interface are related to the configuration of a specific clock polarity and phase in order to synchronize the transmission to and reception from the MOSI (Master Output, Slave Input) and MISO (Master Input, Slave Output) signals. The other two involved signals are the interface clock (output from the master, which can be configured up to 11 $\mathrm{MHz}$ in this architecture), and the slave select (output from the master) to activate the slave module to which the write/read process will be performed.

Together with the SPI block, the WIZNet reset sequence generation is also included at this level, in order to be able to properly set the operational state of the module as well as activate the rest of the control blocks. Based on these signal-level control blocks, the defined mechanism to access the internal registers of the WIZnet is fully implemented as a second stage of the backchannel 
interaction, so that the subsequent configuration of the Ethernet module can effectively be carried out. Three main parameters are to be taken into account in the memory access process, as follows:

- Addressing bytes: Specification (2 bytes) of the memory address of the register/memory sector to be accessed.

- Write/read operation: action to be performed and specified in the MSB of the third byte of configuration.

- Number of bytes to be read/written: the number of bytes is specified in 15 bits, according to the type of action (e.g. module register, socket register or TX/RX buffer accessing).

In this way, an important implemented feature is the capability of generating what is called burst processing, in which a sequence of consecutive data bytes is read/written from a reference pointer of the memory map structure. This is also particularly useful when injecting a sequence of remote testing data to a selected hardware module, as well as for high-rate reprogramming and reconfiguration purposes.

The backchannel configuration block makes use of the aforementioned functionality to set up the network parameters of the module, so that the testbed platform can establish the data connection by means of the communication infrastructure. Configurations regarding the protocol to be used for the remote data exchange process is realized in this operational stage, particularly regarding IP parameters (IP Address, subnetwork masks, gateway address).

Moreover, the socket communication over a TCP connection is included as a predefined mechanism in this configuration, though UDP, for instance, can also be used. Depending on the number of channels to be configured (up to 8 simultaneous sockets) a memory reservation scheme is performed such that each one of them can manage a particular relative sector within the memory map for data buffering and socket registers. Moreover, an individual TX/RX buffer size can be set up considering that, in case of only using one debugging channel, up to $16 \mathrm{kB}$ can be reserved each for data transmission and reception. Therefore, 
within this size, the memory space might be dynamically assigned for every configured channel (see Appendix A for additional details).

As a result of the backchannel interface configuration process, the network connection engine is in charge of monitoring and processing the testbed communication states as well as retrieving available action frames from the assigned memory. The socket state of the channel $n$ is supervised by means of the register Sn_SR (as defined in [W5200]), which can adopt the values represented in Fig. 2.14.

Besides the states that perform the testbed communication configuration, socket initialization, and integrity verification tasks, the CHn_ESTABLISHED state includes the abstraction block to decode and process the testbed action frames that are remotely transferred, so that proper functionalities can be triggered accordingly. Before entering into the analysis of the functional blocks that encompass such triggering actions, a breakdown of the defined testbed action frame is explained following the format proposed in Fig. 2.14, in which the different fields that compose its overall definition are highlighted. It is intended to provide a scalable specification body regarding the type of functional blocks and debugging connections to be made, as well as their underlying configuration.

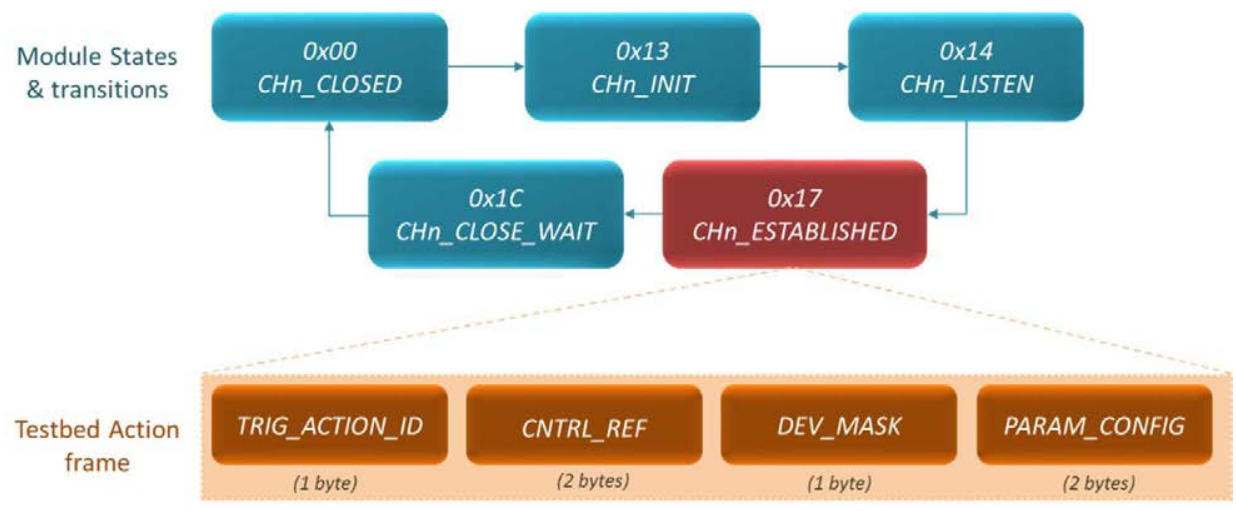

Fig. 2.14 Remote connection states and frame format for the testbed actions \& blocks activation.

As shown in the figure, the basic entity of the frame format is a lightweight header that includes the following configuration: 
- TRIG_ACTION_ID (1 byte): defines the type of functional block to be triggered, so that further remote debugging tests can be performed. Based on the set of defined blocks, the activation of the experimental connections as well as the modification of the operational modes of the hardware modules are carried out according to the specification of this frame field.

- CNTRL_REF (2 bytes): provides a reference code for the generated testbed packet. In combination with TRIG_ACTION_ID, it allows uniquely identifying the received action for subsequent tracking of the debugging state and related data.

- DEV_MASK (1 byte): refers to the designation of the hardware module that will be involved in the action or debugging connection to be executed. Depending on the type of functional block, more than one device module might be set in this field. Every hardware module that can be connected to the processing layer is masked into one of the bits of the defined field.

- PARAM_CONFIG (2 bytes): this field includes specific configurations of the action to be executed, if the functionality to be triggered requires an initial configuration (e.g., interface operational mode selection).

Based on this approach, specific functional blocks for providing debugging actions and test interconnection schemes have been defined to contribute to the overall architecture of the proposed testbed framework, which are described as follows.

Reset triggering (RstTrg - [0x01]): this functional block lies on the generation of the signal sequence for restarting the specified hardware module. Since the signal evolution for the restarting task depends on the hardware module upon which the action is triggered, several sub-blocks constitute the basis of this action. The main implemented reset sequence targets the hardware restart of the microcontroller device, whereas the second elements to be considered are the wireless communication modules such as ZigBee, IEEE 802.15.4 and Wi-Fi. More 
than one device can be masked, so that a reset triggering action on several modules is performed in order to restart a specific experimental operation of the sensor node.

Programming mode (ProgBoot - [0x02]): like the aforementioned block, this functionality controls the signals that allow triggering the programming mode of the processor, so that remote reprogramming capabilities can be performed by using the on-chip downloader kernel. Moreover, in case of the wireless communication layers, the programming mode can be activated in order to update the firmware of the modules remotely.

Sleep/power-down modes (LowPwr - [0x03]): processors and radio communication modules generally provide several levels of sleep configurations according to the powering states of internal peripherals. Two of the most common mechanisms to switch from low power and ultra-low power modes to active mode are implemented in this functional block, that is, either by using a communication interface such as UART or by generating an external interruption in the corresponding GPIO or reset input of the device, respectively. This operation is especially applicable in case of studying power consumption strategies and transitory wake-up states of the hardware nodes, in addition to analysing topology changes when specific nodes enter into power-save modes.

Interface virtual port (VPort - [0x04]): this capability is intended to establish a direct link with the devices included in the modular platform by using one of the implemented debugging interfaces, i.e., a remote connection with, for instance, an attached communication module through the backchannel as if a local serial port (via UART) is opened with such devices, so that testing and emulation data can be burst, including packet injection. This functionality particularly allows experimenting with protocol control frame generation and communication stack development, in which simulation and emulation mechanisms require the inclusion of hardware in the loop.

Devices intercommunication gathering (DevInCom - [0x05]): this block allows monitoring internal interconnections between the microcontroller and other hardware modules, such as communication or memory devices, so as to analyse and evaluate how packet generation at the software side of the system 
implementation is realized. It serves as a transparent packet gathering according to how the hardware modules are interconnected among them. This is especially important for tracing data processing bugs and application execution states.

Module interface switching (DevMux - [0x06]): it provides interconnection options to dynamically commute the hardware association among elements that share the same communication interface, so as to facilitate the experimental array of different devices in the same sensor platform under test. This is especially interesting when the microcontroller is to be connected to different external peripherals via UART serial interface, such as the integration of two different network communication and/or memory modules.

Microcontroller debugging Interface (DebuC - [0x07]): This functionality is a combination of three main interaction elements in order to provide users with the ability to use the embedded debugging kernel of the microcontroller so that standard tools for code debugging can be performed at runtime. To this extent, a serial VPort and the on-chip debugging component of the microcontroller (described later on in the software support platform) can be combined with a remote IDE (Integrated Development Environment) in order to execute integrated debugging tools for program testing and analysis of the internal elements of the microcontroller (for instance, Keil Development tools from ARM [Keil] have been used in this case for testing and debugging the processing elements included in the Cookie nodes within the context of the testbed remote experimentation platform). Therefore, a direct access to the program execution process as well as internal peripheral configuration/modification capabilities can be accomplished in order to obtain a more comprehensive understanding of the implementation of software components and functional libraries. This flexible mechanism that relies on the support of the backchannel infrastructure contributes in the provision of a very dynamic and adaptable debugging strategy so that developers can benefit from code testing features in order to speed up the development of the embedded software. 


\subsubsection{HW-SW Integration platform: Software support libraries}

Unlike most of the state-of-the-art works in which the testbed platforms rely on embedded software implementations focused on TinyOS-based developments, in this thesis a well-defined software support platform is also proposed as part of the testbed framework in order to provide users with not only the described experimental infrastructure but also a complete set of software components so that prototypes can be easily made, by using this HW-SW integration platform following the flexibility and modularity of the Cookie development.

The main idea behind this concept is the creation of a software support framework upon which developers can build up and test novel WSN designs by using the resources of the hardware architecture in an easy yet efficient fashion. Such a support is primarily accomplished by means of a set of embedded software libraries that are correlated from the basis of a system-abstraction-level structure. The modular concept of the Cookie platform is indeed followed in the software support platform as well, particularly in the sense that functional components can be easily included or replaced according to the type of hardware array that needs to be developed and tested.

Furthermore, the aforementioned system structure also intends to provide a rapid prototyping approach so as to avoid entering in low-level details in those situations where users are particularly interested in experimenting with software prototypes and applications at a higher abstraction level of the implementation. As mentioned before, these functional components are certainly designed as a hybrid approach for both the testbed architecture as well as during the deployment stage of the WSN.

In Fig. 2.15 a general view of the HW-SW integration platform is shown, where the inclusion of the functional components that encompass the testbed framework is highlighted (It is important to remark that such an embedded software implementation has been tested and validated in both processing layers of the Cookie modular platform). 


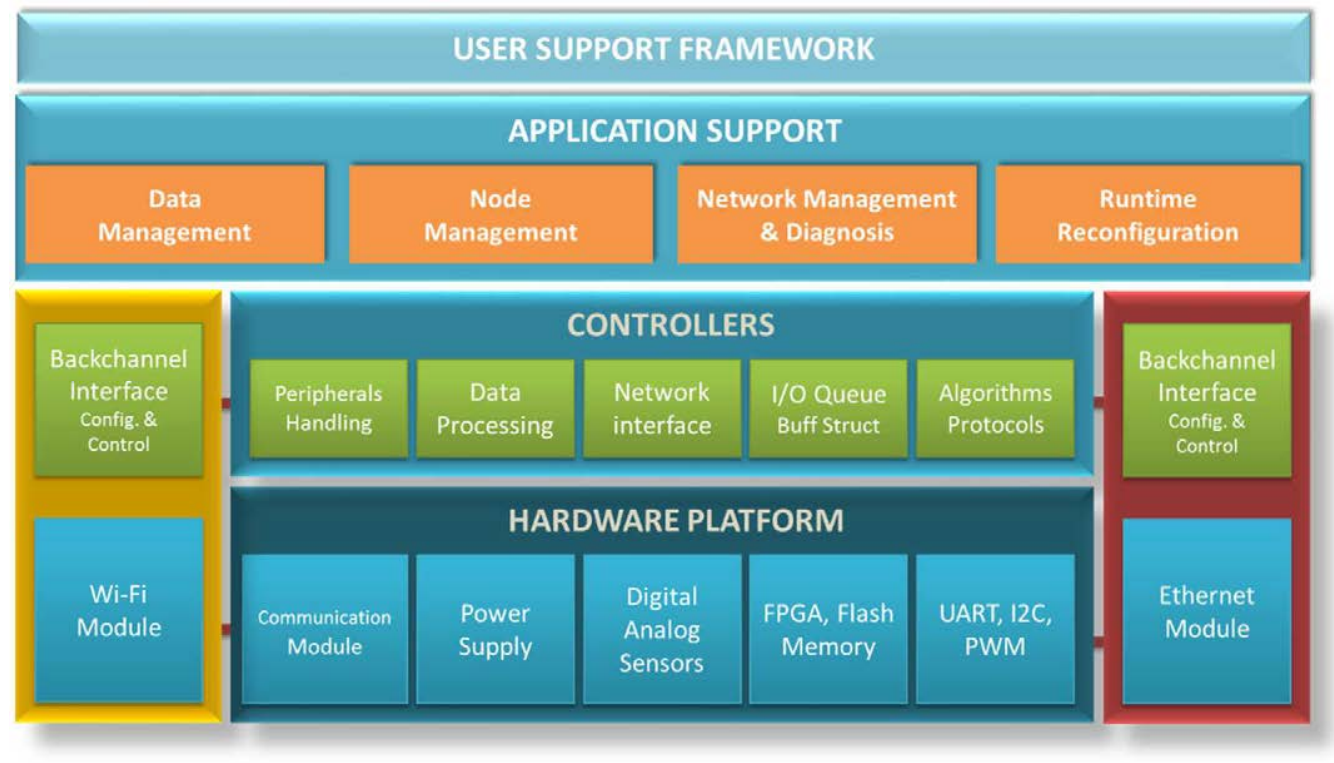

Fig. 2.15 HW-SW integration platform, main functional components and controllers.

The foundations of the HW-SW integration platform rely on the definition of low-level controllers that correspond to the management of the hardware layers and functional blocks of the modular architecture. This management capability is carried out by the main processing element of the platform, the microcontroller, which means that a resource-constrained approach has to be considered. Moreover, the software support components are implemented in such a way that users can make use of the libraries as low-abstraction-level resources upon which different software and service implementation approaches can be integrated. The implementation of the software support platform has been developed under the scope of $C$ language, which significantly speeds up the learning and development curve of new hardware and software prototypes.

The basic composition of the software controllers can be split into four major groups. First of all, drivers and interfaces for wireless communication devices, so that a proper configuration and control of these elements can be effectively done. Secondly, analogue and digital sensing acquisition handling, so that the different hardware components that compose the sensor layers of the Cookie node can be used accordingly. Third, controllers related to the internal peripherals of the microcontroller as well as those included in the FPGA for co-processing tasks 
and the memory layer. And finally the management of the functional components that are directly related to the testbed infrastructure, such as debugging capabilities, configuration and reprograming components.

Based on this, the low-level functional components for the control of the hardware elements are mainly implemented in the following repository of software libraries:

CEI_wComm: This library refers to the management of the radio communication protocol to be used in the sensor node, including the following features:

- Radio and network parametrization, comprising radio channel and transmission power configuration, PAN identification, among others.

- $\quad$ Node role determination, based on network topology definition and multi-hop strategies.

- Sleep modes configuration, in relation with the type of external triggering for wake-up actions.

- Packet management, referring to the type of control and data packets to be received from the communication module, as well as the message prompting setup.

This library is at the same time divided into several sub-libraries that refer to the specific device configuration (in reference to the communication module to be used), as well as wireless connectivity configuration and network establishment. Therefore, three main libraries can be distinguished, that is, two versions of the CEI_zb library (supporting the ETRX2 from Telegesis for $2.4 \mathrm{GHz}$ band [ETRX2], and the ZigBit from Atmel that works at $868 \mathrm{MHz}$ [ZigBit], both of them ZigBee modules) and the CEI_802.15.4 library that supports the CC2420 IEEE 802.15.4 module from Texas Instruments [CC2420] (which has been included in a new Cookie communication layer as described subsequently in the next chapter).

CEI_queue: this set of libraries is directly related to the one described before, because it is essential to define a dynamic buffering mechanism in order to be able to register and handle the data coming from and going to the communication modules and thus the wireless sensor network. Therefore, two 
circular buffers per module are configured and controlled by the library, in addition to the underlying memory management for optimizing the data access. CEI_peripherals: Here the low-level communication buses and interfaces for connecting to the external devices are driven, so the peripherals of the microcontroller are properly configured and handled. Moreover, the protocol interface for communicating with digital transducers that are managed by the FPGA is also included in this library. The most relevant components can be summarized as follows:

- ADC (Analogue to Digital Converter) management, for initializing and configuring the ADC controller, so that measurements from the analogue sensors can be properly performed and provided to upper software layers.

- TIC (Time Interval Counter) operation modes, for handling timing and task scheduling strategies, as well as the management of the external events, based on interruption supervision.

- Serial interface management, in which communication buses such as UART and SPI used to interface network and memory devices are set up, in order to initialize the corresponding serial port for data transmission and appropriate interrupt handlers for message reception routines.

- Error management, based on internal watch-dog-driven components for handling possible failures that may be produced during data gathering and processing tasks, especially when bugs related to wireless data losing and communication errors between devices may affect the normal behaviour of the system.

- FPGA blocks interaction, in which the generic interface proposed in [Portilla'07] for digital sensing acquisition is implemented, so that components such as the temperature and humidity sensor SHT11 from Sensirion [SHT11] and the 2-axis accelerometer ADXL210 from Analog Devices [ADXL210] included in Cookie sensor layers can be employed. 
CEI_DataProcess: The functional components implemented in this library rely on the sensing and data gathering provided by CEI_peripherals as the input for performing measurement conversion, adjustment and correction actions. Temperature and humidity, $\mathrm{pH}$ and water temperature, $\mathrm{CO}$ and $\mathrm{CO}$, acceleration, strain gauge or light intensity sensors can be processed, allowing the generation of information in standard and adapted terms. Besides, this library includes functionalities for monitoring and processing the power consumption of the node in runtime, based on a current monitoring circuit that is implemented on the power supply layer of the modular platform.

CEI_tbChannel: the set of libraries that composes this software package refers to the additional support of the backchannel interface approach, in particular regarding the implementation of complementary components for controlling the Wi-Fi communication approach in those situations where clustering techniques are applied, so that the integration of several wireless connectivity schemes have to be dynamically handled.

In addition to the above-described libraries that represent the HW-SW integration of the modular platform, the provision of embedded management capabilities on top of the software controllers are allowed for. They are intended to provide a system development and testing framework at software level, upon which users can manage the resources of the Cookie platform. These functionalities are defined within the following software repositories:

CEI_dataManagement: it includes configuration and monitoring directives for smart data acquisition, processing and filtering, such as allowing for the determination of threshold rules for transmitting data to the root node, transducer sampling and measurement report scheduling, and on-demand data generation based on remote retrieving requests.

CEI_nodeReconfigManagement: it provides capabilities for supporting experimentation and testing activities under the modular architecture, particularly targeting the performance of remote reprogramming and debugging tasks. In this way three main elements can be distinguished. First, CEI_reconfig and CEI_bootloader libraries are implemented to provide an efficient remote and partial reprogramming mechanism as well as system recovering (as explained in 
the following section) for multi-application experiments, and embedded resource execution options for selecting and modifying the type of experiment to be launched, respectively. Secondly, a debugging library for 8051-based architectures [ISD51] is integrated so as to allow code execution testing and runtime peripheral configuration and evaluation. And third, a power manager that works in conjunction with the already mentioned hardware blocks in order to monitor the energy consumption of the sensor platform, in order to detect specific threshold values in relation with the running application. Sleep mode configuration properties are also handled in this functional component.

CEI_networkManagement: it refers to the libraries related to the wireless network testing and how the interaction of the sensor node with its surrounding environment is in terms of communication and networking capabilities, including the following features:

- Node identification, covering type of node and included sensing capacity, functionalities and role within the network, as well as MAC level identification.

- Link assessment, where values related to quality of the interconnections with the neighbourhood can be analysed, in addition to network diagnosis regarding Radio Strength Signal Indicator (RSSI) and Link Quality Indicator (LQI), Packet Loss Rate (PLR) and multi-hop path evaluation, so as to have a better understanding about the dynamics of the network topology.

- Node reconnections monitoring, produced by factors such as platform reset, network connection error, and lack of radio coverage.

CEI_tb_wf: this includes the embedded software implementation of Wi-Fi communication module drivers in order to perform advanced control and management tasks, particularly in those WSN scenarios where a heterogeneous Cookie gateway array is configured. Three main elements can be distinguished in this set of libraries. First, the module configuration process to set up internal parameters of the hardware such as connection interface definition, firmware update and download options. Secondly, network connection capabilities for 
further communication settings regarding the creation of or connectivity establishment to ad-hoc networks or access point (AP) - infrastructure mode, and HTTP data transmission. And third the module/network status handling related to scan, reconnect and leave the active connection mode, as well as sleep configuration accessing and triggering.

CEI_framework: this packet represents the entry point for the creation of new application prototypes by using the HW-SW support architecture, from which users can apply the previously described resources for executing, testing and debugging their embedded implementation under the modular platform. As explained in the subsequent sections, this framework is particularly important from the user point of view for the production of experiments within the memory-segmentation-based partial reprogramming architecture of the WSN testbed.

\subsection{Remote and partial reprogramming architecture: $A$ modular approach}

Remote reprogramming strategies are one of the key aspects to be allowed for when performing experimentation and debugging capabilities in WSN testbed architectures. The concept of testing new embedded software prototypes under the sensor node platform such that a dynamic execution of different implementation models is carried out plays a fundamental role for the analysis and optimization of the system development process. Based on the accomplishment of testbed debugging activities, an inherent modification and refinement of the functional components under design has to be effectively and interactively performed. Moreover, efficient reprogramming mechanisms represent a must during the deployment and operational release of WSNs, especially for the maintenance and further long-term updates of the running application. The reliability and flexibility of the reprogramming technique to be applied may lead to obtaining an overall performance improvement of the cost, time and energy consumption of the maintenance process.

While in case of the testbed infrastructure the remote reprogramming tasks are realized by using the high-performance backchannel interface approach, during 
the deployment and operational phases of the WSN-based application the update and code replacement possibilities are to rely on the applicability of highly efficient reprogramming and dissemination techniques, due to the lowrate and energy-constrained nature of the system.

\subsubsection{Challenges of reprogramming in WSNs and testbed architectures}

The wireless sensor node reprogramming issue can be analysed from several angles according to the type of modification or update that the embedded implementation under experimentation/operation needs. On one hand, a common practice when modifying the behaviour of the sensor node is related to the definition of a set of code rules for node parametrization (also known as functional reconfiguration) so that lightweight triggering frames can be disseminated throughout the wireless network to execute a particular update of the running application. In this way, three main update levels can be distinguished for this type of reprogramming, i.e., application parametrization, functional properties adjustment, and peripheral/module configuration. The advantage of this kind of approach is the ability to resolve specific bugs or perform corrective actions at runtime without a significant amount of data to be transferred remotely, whose impact is thus reflected in energy, time and resource consumption during the reprogramming process.

However, such a perspective rather refers to a behavioural modification of the running implementation, instead of the accomplishment of an embedded code reprogramming process, which is conceived for the replacement of functional components, experimental software prototypes or even algorithms to be evaluated under the distributed sensor network. On the other hand, in order to address the aforementioned capability, the code replacement procedure and core reprogramming methodologies are to be performed such that a trade-off between energy efficiency, flexibility and reliability of the mechanism can be properly addressed. From the on-site deployment perspective, energy and reliability represents a more critical aspect, whereas in terms of the testbed support platform flexibility and experimental adaptability is a major concern. Furthermore, dynamic reprogramming support techniques are to enhance the testability of different network and application technologies, by means of 
allowing for multi-experimental schemes where several software test units can be allocated and scheduled into the hardware platform for their subsequent execution.

The main issue regarding remote reprogramming capabilities in WSN testbeds is that most of the support techniques rely on whole code replacement strategies, in which a new configuration image is downloaded into the program memory of processor element so as to run a new experiment. The consequence of this action will produce three main interconnected effects on the system performance: first, the transient power consumption associated with the reprogramming process in terms of both the intrinsic consumption of the processor while reprogramming and the data communication consumption while transmitting/receiving the configuration file in the sensor node (particularly in on-site operational mode); secondly, the time spent during the reprogramming task that is primarily determined by the size of the program image, which will affect not only the energy/resource usage of the sensor platform and the network itself, but also the dynamic transition window of the programming mode that may influence the overall runtime operability of the sensor nodes under test (particularly if a whole configuration file has to be downloaded every time an update/replace action has to be performed); and third the feasibility and reliability associated with the code dissemination process of the programming file, because as the implementation image gets larger, the probability of losing packets increases accordingly, especially in the actual operation of the network, where the remote reprogramming is carried out by using the low-rate wireless link that is not certainly prepared to handle a great amount of data. The later issue has a direct impact on the power consumption of the sensor nodes as well, because of the number of retransmissions and code verification actions needed to guarantee the integrity of the configuration file remotely transferred to the platform memory.

An alternative to reduce such effects while trying to perform an efficient mechanism for node remote reprogramming is related to introducing the concept of partial reprogramming. The idea behind such an approach lies on changing the running embedded implementation partially depending on the type of functional component to be tested or updated. However, this concept 
poses important challenges in relation with the management of the processing and memory elements of the involved microcontroller in order to efficiently support such partial updating/replacement capability, especially considering that the underlying programming optimization scheme should be as transparent as possible from the user/code-developer point of view. Furthermore, the dynamics of the testbed architecture has to be also taken into account when integrating reprogramming techniques due to the debugging and testing nature of the system, unlike general code generation and programming procedures. This means that the software support platform for in-node experimentation and component prototyping makes the structure of a partial reprogramming scheme a combination of static, dynamic and management/linking functionalities, so that an efficient testability of embedded software developments under the testbed platform can be provided taking into account the framework composition.

In this way, some contributions in the literature focus on module replacement mechanisms based on the support of operating systems, in addition to component interconnection reprogramming in which various functional blocks are downloaded into the sensor node and then the reprogramming procedure refers to how such components are correlated among them to generate a specific desired behaviour of the application [Weyer'09].

\subsubsection{Remote Reprogramming techniques in the state of the art}

Different approaches regarding node reprogramming capabilities have been addressed in recent years trying to provide WSNs with the ability to remotely change/update the embedded software while coping with the intrinsic limitations related to radio communication performance and power consumption, computational resource usage, and operational degradation associated with the reprogramming costs. In this way, several types of reprogramming strategies can be classified distinguishing whole code replacement, virtual-machine based application abstraction support, loadable and relocatable modules including dynamic linking, differential-based and incremental reprogramming [Liu'11]. 
Full code replacement refers to the reprogramming of the whole software implementation running on the sensor node by fully compiling a completely new image and then overwriting the program memory of the processor element. This is one of the most common ways of performing the reprogramming task since no additional capabilities for binary matching or special functionalities related to dynamic linking and relocation optimization techniques are needed. Its main drawback is the associated overhead in terms of data delivery, time and power consumption during the updating stages of the remote platform, apart from its poor performance concerning flexibility, modularity, and fast generation of in-node test units.

Deluge [Hui'04] tries to overcome some of these limitations by integrating a dissemination protocol for fast delivery of the program image in a reliable fashion. It has been one of the main reprogramming techniques for wireless sensor nodes under TinyOS operating system, which makes use of the Trickle algorithm [Levis'04] for code propagation but considering large data objects to be delivered. A key aspect that is considered in Deluge is binary partitioning for object dissemination and software update comparison. However, a strong dependency on an external flash memory to download the whole image into the sensor node is contemplated, so that a subsequent comparison of what parts of the code are to be replaced can be carried out prior to the final memory writing process. Moreover, the Deluge component has to be included in the new application to be transferred so that the reprogramming process can be evaluated at the node side. Therefore, Deluge's best efforts are focused on data delivery but not on the size of the object, and only support monolithic TinyOS image reprogramming. Stream [Panta'07] tries to mitigate the shortcoming associated with transferring the reprogramming protocol code along with the full image by pre-installing the Stream component into the nodes before the deployment.

Virtual Machine (VM) based platform support is thought to promote both high level abstraction for code development and application code updating without being necessary to reprogram the whole embedded software scheme (particularly the part that refers to the common system code), although the performance 
overhead is penalized due to its native code translation support, therefore generating a less efficient resource consumption and code execution. Moreover, the sensor node reprogramming is usually performed at application level, thus low-abstraction modifications are not contemplated in such cases. Some contributions have been focused on adapting the concept of general VMs to resource-constrained sensor platforms, such as Maté [Levis'02] which was one of the first implementations that inspired subsequent tiny VMs for WSNs. Mate was built on top of TinyOS components as a bytecode interpreter, based on a set of generic instructions included in capsules that hide the details of the OS. Its reprogramming essence relies on installation of capsule versions. Darjeeling [Brouwers'09] is a VM created to execute a subset of Java language in microcontrollers with limited memory resources. It addresses the issue of dynamic reprogramming by rather considering the coexistence of several applications into the same node and then triggering their execution accordingly.

Loadable modules is another alternative to perform partial reprogramming based on the support of operating systems such as ContikiOS and SOS [Dunkels'05] [Han'05]. In [Dunkels'06] authors introduce the concept of linkable modules for ContikiOS, in which the module to be loaded into the embedded platform contains information regarding functional components and variables of the kernel, whose references have to be resolved by using either pre-defined linking or dynamic linking. Also, depending on the location of the loadable module into the memory scheme, the references to its included functions are also considered, which thus refers to the process of relocation. Pre-linked module schemes refer to absolute addressing of system components, whose information have to be available at compilation time. This procedure reduces the complexity of the linking management in those applications where small modifications are contemplated, keeping the rest of the system unaltered, and also avoids possible overhead related to the transmission of relocation information. In ContikiOS the dynamic linking for the object files is performed by using a compact version of the Executable Linking Format and the Contiki file system [Dunkels'06]. Although TinyOS does not support loadable modules per se, recent contributions aim at transforming it into a more dynamic OS but trying to keep the same programming model [Munawar'10], also adding modularity by 
dividing the applications into static and exchangeable parts [Gauger'09], though in that case the system components cannot be reprogrammed.

Incremental reprogramming considering a differential-based approach is another technique for updating the running software implementation of the sensor node, which relies on calculating the differences between the old and new version of the binary code, so that a delta (a reduced image that represents such differences between program versions) can be transferred. This is a desirable option for performing small modifications on the operational code related to fixing bugs or minor updates in the same embedded software. One of the first concerns when allowing for this type of mechanism is that the differential approach is performed at byte level, without considering the structure of the application implementation. Although this can reduce the overhead associated with code transferring, it may actually lead to generating an important overhead regarding memory reprogramming itself [Koshy'05]. Based on this, if the size of the delta is considerable big, the updating task could be as if a whole reprogramming is performed.

The concept of incremental linking has been addressed in [Koshy'05] by taking into account possible updates in functional components of the running software. Instead of shifting binary codes in the program memory when part of a functionality grows or shrinks, updating regions are defined to support such changes avoiding overlapping other functions. The operations are realized by using copy and insert instructions of the diff-script that is transmitted and executed into the sensor node. However, the relocation mechanism could lead to having very intensive memory management as well as fragmentation of the defined regions, thus an overall rewriting is to be done to guarantee a more efficient memory usage. Zephyr [Panta'11] addresses the issue of byte-level comparison for delta generation by first performing application-level modifications based on structuring the software implementation, which can intrinsically focus the binary mismatches. However, in order to avoid dynamic linking as well as mitigate functional components shifting, Zephyr makes extensive use of an external memory in which the structure and operations of the reprogramming tasks are performed. It includes five main elements within such 
an external structure before writing the program memory of the sensor node: the reprogramming builder, the old and new application images, the delta script and the indirection table for the new built image. After rebuilding the new image version in the external memory, the whole program memory is then rewritten with the updated application. The work proposed in [Jeong'09] follows a similar approach in the sense of using an external memory where the image decoding process is carried out, and then the bootloader is called to fully write the program memory with the new application update. However, authors focus the code update task on the generation of the delta at byte level, without considering application structure and software component shifting.

Most of the proposed mechanisms are focused on code replacement once the sensor network is in its operational stage, in order to modify specific functionalities or fixing errors that may subsequently appear on the released distributed system. Fewer works attempt to support testbed capabilities for innode flexible experimentation, especially for hybrid and nomadic architectures where a trade-off between efficiency on the reprogramming mechanisms and modularity of the experimental scheme is needed. Instead, most of the testbed platforms rely their support for reprogramming tasks on only providing the backchannel communication structure though further composition of modular and dynamic techniques to perform such reprogramming is not usually specified.

\subsubsection{Proposed approach for a modular WSN partial reprograming}

In order to provide a flexible reprogramming mechanism not only to experiment with new embedded software prototypes under the modular testbed architecture but also to guarantee a high degree of functional updating in final application scenarios when using the proposed hardware-software platform, a new partial reprogramming technique is addressed in this thesis. The idea behind the proposed strategy is to support updates and modifications in both the hybrid testbed infrastructure and WSN deployments, allowing for a trade-off between efficiency and modularity of the reprogramming system. In order to accomplish this, three primary aspects are pursued: energy and resource 
consumption enhancement, reprogramming time optimization, and modular location and replacement capabilities.

The first one is related to how the structural implementation of the reprogramming architecture is designed such that the code reconfiguration can be resource and energy efficient. This is also correlated with the partial reprogramming of modular components that allows the optimization of the configuration time, particularly compared to a whole reprogramming mechanism. Then, such a well-defined structural scheme of the embedded software allows the inclusion of different experiment and application blocks into the sensor node, which can be independently programmed, executed, updated and replaced. This level of modularity is to be supported by the software components of the testbed architecture, so that the user application blocks can make use of the platform libraries without the need of including/reprogramming them every time a new application is updated. Moreover, an efficient interconnection of the software support components of the Cookies and the application blocks shall be assured such that a dynamic control of application execution is performed, which is also to consider transparency/decoupling when reprogramming functional elements of the support platform itself.

Due to the nature of the proposed technique as a support to platform experimentation and prototype testing within the testbed architecture, such a partial reprogramming scheme is mainly focused on software component modularity for code loading and replacement. As described in the following paragraphs, this approach does not only reduce the amount of data to be transferred to the remote sensor nodes, but also the process and cost of reprogramming itself, since only specific areas of the memory structure are updated. In this way, the reprogramming scheme will be firstly addressed from the application perspective and thereafter details related to modifications on the software support platform are provided.

The first aspect that has been taken into account refers to the design of the system structure as the baseline for the partial reprogramming capabilities. In this direction, a memory-segments-based framework for the Cookies platform 
is proposed in this work as a contribution to enhance the system flexibility and modularity at both hardware and software levels. Two main areas can be distinguished within the overall perspective of the architecture. The first one contains the software support platform for the provision of hardware controllers as well as prototype experimentation and debugging functions, as shown in previous sections. The second one corresponds to the different modular blocks that contain the user application/experiment implementations, which make use of the former one in a transparent fashion. One of the differences between this approach and, for instance, loadable-module techniques, is their dependency on OS-based implementations. Moreover, as described subsequently, modifications on the kernel of the system (support area) are fully addressed.

However, in order to efficiently handle a seamless correlation between these areas as well as control the execution and interconnection of software components, additional optimization features are to be contemplated within the reprogramming scheme, which also involves a modification of the compilation, code relocation and generation processes. At this point it is again important to highlight that the Cookies modular platform relies on low-power embedded processors based on the 8051 architecture. This means that the created memorysegments-based partial reprogramming scheme (MS-PRS) is focused on the structure of this architecture, although the underlying concept can be extensible to other types of processing elements. The system implementation has been validated in two different processing layers of the Cookie modular platform, i.e., the one based on the ADuC841 [ADuC841] microcontroller from Analog Devices, and the ultra-low-power layer previously proposed, which includes the C8051F930 [C8051F930] from Silicon Labs.

An overall view of the system architecture is shown in Fig. 2.16, where the main elements that compose the reprogramming scheme are highlighted. 


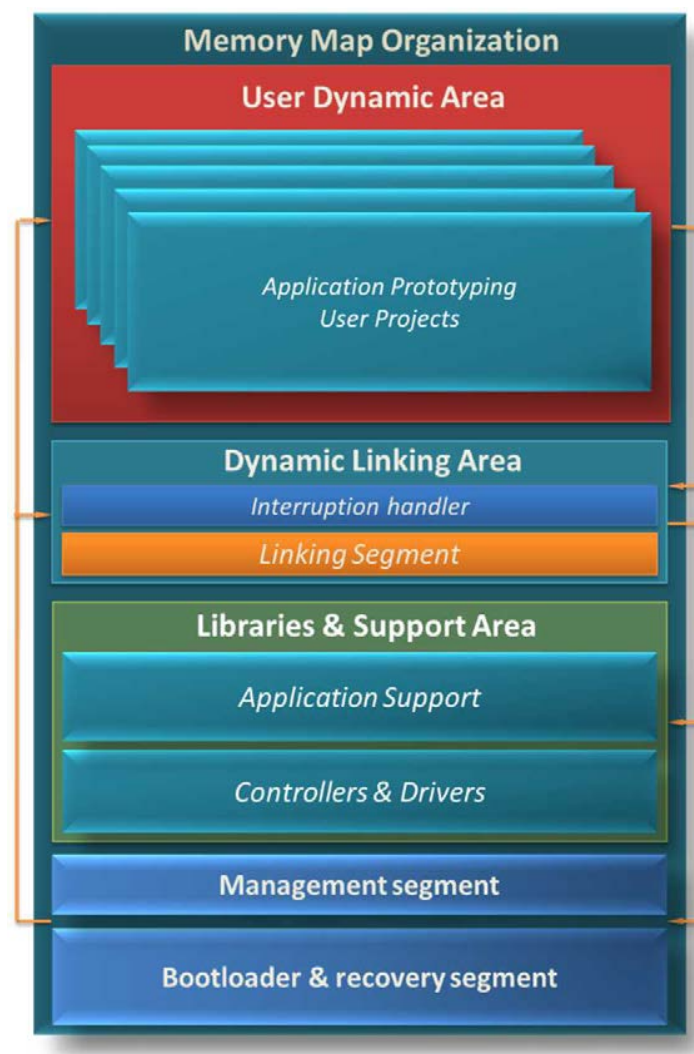

Fig. 2.16 System architecture of the proposed partial reprogramming scheme.

Apart from the aforementioned areas, the reprogramming engine is supported by a bootloader and recovery segment as well as a dynamic linker and application execution area. The basic concept to be tackled is the memory relocation capability within the processing architecture. The program memory distribution in both cases comprises $64 \mathrm{kB}$ of flash memory, plus $4 \mathrm{kB}$ of data memory. Depending on the implementation (e.g. in case of the ADuC841) $2 \mathrm{kB}$ of the program memory are dedicated to on-chip downloading/debugging kernels. In order to carry out the modification of the compilation process as well as the relocation, segment definition and linking options, the C51 compiler and some of the Keil Development tools from ARM has been used [Keil], which include a wide range of 8051-based microcontrollers from different manufacturers.

Within the definition of the support area, different segments related to the software support elements are to be included in order to provide the rest of the 
system with the controllers and management libraries, in accordance with the defined hardware-software integration platform. Such functional components and drivers are considered as an integral part of the system, especially for the testbed infrastructure, which means that in case the application area has to be replaced or updated, the support segments will remain unaltered, thus reducing the amount of data to be reprogrammed.

In this way, the location of the software libraries is assigned by a memory slot scheme definition, in which segments for the functional components included in them are specified. Depending on the type of reprogramming strategy to be adopted for the support area (package prototyping or operational component release), a memory slot reservation process is performed for every functional component. Otherwise, the allocation is realized by placing the software elements from the basis of a specified memory slot address that determines the distribution point of the library. As shown in the .MAP file of the compilation process illustrated in Fig. 2.17 segments can be assigned to software components by specifying the symbol of the function and the associated memory (the first three columns correspond to the started/ended memory address and length respectively, while the last two columns refer to the target memory and the symbols of the segments).

Since special symbols such as constant-type segments have to be explicitly located with this procedure, and due to compiler constraints related to segment ordering with respect to the program execution vector, a modular alternative based on the definition of new memory classes for every support package is adopted. Every library is associated with both data and program memory classes with a compilation directive that identify the symbol of the defined class, and then they are included in memory slots so as to be properly located in the support area, as represented in the map file of Fig. 2.17, where a new type of memory class appears (CODE_PERIPHERAL) along with the segments that are included in it (starting from $0 \times \mathrm{xB} 00$ in the figure). 


\begin{tabular}{|c|c|c|c|c|c|c|c|}
\hline $002 \mathrm{~B} 1 \mathrm{CH}$ & $002 \mathrm{~B} 3 \mathrm{CH}$ & $000021 \mathrm{H}$ & BYTE & UNIT & CODE & ?PR?_CEI_DP_COSENSOR?_CEI_DATAPROCESS & 7 \\
\hline О02B3DH & $002 \mathrm{~B} 5 \mathrm{CH}$ & $000020 \mathrm{H}$ & BYTE & UNIT & CODE & ?PR?_CEI_DP_ADCDATA2INTT?_CEI_DATAPROCESS & \\
\hline 002BSDH & $002 \mathrm{~B} 7 \mathrm{CH}$ & $000020 \mathrm{H}$ & BYTE & UNIT & CODE & ?PR? CEI-UTİLS ISDIGIT? C̄EI ŪTILS & \\
\hline 002B7DH & 002В93В & $000017 \mathrm{H}$ & BYTE & UNIT & CODE & ?CO? $\overline{\mathrm{PR} U E B A} \overline{-}$ & \\
\hline $002 \mathrm{~B} 94 \mathrm{H}$ & ОО2ВАВН & $000015 \mathrm{H}$ & BYTE & UNIT & CODE & ?PR?_CEI_DP_STRING2NUM?_CEI_DATAPROCESS & \\
\hline ОО2ВA9H & ОО2BВAH & $000012 \mathrm{H}$ & BYTE & UNIT & CODE & ?PR? -CEI_UTILS_ABS?_CEI_UTIT'S & \\
\hline О02BВBH & 002BC7H & 00000DH & BYTE & UNIT & CODE & ?PR?_CEI_DP_2BȲTESJÖIN?_CEI_DATAPROCESS & \\
\hline OOBBOOH & OOBC78H & $000179 \mathrm{H}$ & BYTE & UNIT & $\begin{array}{l}\text { CODE_PERIPHER } \\
\text {-ALS }\end{array}$ & 2PR?_CEI_SERIAL_INTERRUPT?_CEI_PERIPHERALS & \\
\hline ООВС79H & ООВСВВВ & $000043 \mathrm{H}$ & BYTE & UNIT & $\begin{array}{l}\text { CODE_PERIPHER } \\
\text {-ALS }\end{array}$ & ?PR?_CEI_WATCHDOG_INTERRUPT?_CEI_PERIPHERALS & \\
\hline ООВСВСН & ООВСЕAH & $00002 \mathrm{FH}$ & BYTE & UNIT & $\begin{array}{l}\text { CODE_PERIPHER } \\
\text {-ALS }\end{array}$ & PPR?_CEI_PER_GET_FPGAVALUE?_CEI_PERIPHERALS & \\
\hline ООВСЕВH & OOBD18H & $00002 \mathrm{EH}$ & BYTE & UNIT & $\begin{array}{l}\text { CODE_PERIPHER } \\
- \text { ALS }\end{array}$ & ?PR?_CEI_PER_INITIC?_CEI_PERIPHERALS & \\
\hline ООВD19H & ООВD44H & $00002 \mathrm{CH}$ & BYTE & UNIT & $\begin{array}{l}\text { CODE_PERIPHER } \\
\text {-ALS }\end{array}$ & 2PR?_CEI_PER_INISERIAL?_CEI_PERIPHERALS & \\
\hline ООВD45H & ООВD5AH & $000016 \mathrm{H}$ & BYTE & UNIT & $\begin{array}{l}\text { CODE_PERIPHER } \\
\text {-ALS }\end{array}$ & ?PR?_CEI_PER_MODETIC?_CEI_PERIPHERALS & \\
\hline ООВD5BH & OOBD6DH & $000013 \mathrm{H}$ & BYTE & UNIT & $\begin{array}{l}\text { CODE_PERIPHER } \\
-A L S\end{array}$ & ?PR?_CEI_PER_WRITE2SERIAL?_CEI_PERIPHERALS & \\
\hline ООВD6ЕH & ООВD7EH & $000011 \mathrm{H}$ & BYTE & UNIT & $\begin{array}{l}\text { CODE_PERIPHER } \\
-A L S^{-}\end{array}$ & 2PR?_CEI_PER_INIWD?_CEI_PERIPHERALS & \\
\hline OOBDTFH & OOBDEEH & $000010 \mathrm{H}$ & BYTE & UNIT & $\begin{array}{l}\text { CODE_PERIPHER } \\
- \text { ALS }\end{array}$ & PPR?_CEI_PER_GETADCVALUE?_CEI_PERIPHERALS & \\
\hline OOBDSFH & ООВD9AH & $00000 \mathrm{CH}$ & BYTE & UNIT & $\begin{array}{l}\text { CODE_PERIPHER } \\
- \text { ALS }\end{array}$ & ?PR?_CEI_PER_ADCCONVERSION?_CEI_PERIPHERALS & \\
\hline ООВD9BH & OOBDASH & 00000BH & BYTE & UNIT & $\begin{array}{l}\text { CODE_PERIPHER } \\
\text {-ALS }\end{array}$ & ?PR?_CEI_PER_INIFPGA?_CEI_PERIPHERALS & \\
\hline ООВDA6H & ООВDABH & $000006 \mathrm{H}$ & BYTE & UNIT & $\begin{array}{l}\text { CODE_PERIPHER } \\
- \text { ALS }^{-}\end{array}$ & PPR?_CEI_PER_INIADC?_CEI_PERIPHERALS & \\
\hline \multicolumn{8}{|c|}{ Class symbols for_CEI_Perihperals components in code memory } \\
\hline$\ddot{* * * *}$ & 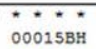 & $\begin{array}{c}X \mathrm{X} \mathrm{R} \\
00015 \mathrm{CH}\end{array}$ & $\begin{array}{l}\text { I A } \\
\text { BYTE }\end{array}$ & $\begin{array}{l}\text { EMC } \\
\text { UNIT }\end{array}$ & $\begin{array}{llll}\mathrm{Y} * * \\
\text { XDATA }\end{array}$ & PXD?PRUEBA & \multirow[t]{17}{*}{ \pm} \\
\hline $00015 \mathrm{CH}$ & $000177 \mathrm{H}$ & $00001 \mathrm{CH}$ & BYTE & UNIT & XDATA & XDATA_GROUP_ & \\
\hline $000178 \mathrm{H}$ & 0010FFH & $000 \mathrm{~F} 8 \mathrm{H}$ & $\ldots$ & $\cdots$ & $* * \mathrm{GAP} * *$ & & \\
\hline $001100 \mathrm{H}$ & 00110B日 & $00000 \mathrm{CH}$ & BYTE & UNIT & XDATA_UTILS & ?XD?_CEI_UTILS_STRCPY_PART?_CEI_UTILS & \\
\hline $00110 \mathrm{CH}$ & $001117 \mathrm{H}$ & $00000 \mathrm{CH}$ & BYTE & UNIT & XDATA_UTILS & ?XD?_CEI_UTILS_STRSTR? _CEI_UTTILs̄ & \\
\hline $001118 \mathrm{H}$ & $001122 \mathrm{H}$ & $00000 \mathrm{BH}$ & BYTE & UNIT & XDATA_UTILS & ?XD?_CEI_UTILS_STRNCPY?_CEI_UTILS & \\
\hline $001123 \mathrm{H}$ & 00112BH & $000009 \mathrm{H}$ & BYTE & UNIT & XDATA_UTILS & ?XD?_CEI_UTILS_STRCPY?_CEI_UTILS & \\
\hline $00112 \mathrm{CH}$ & 001133н & $000008 \mathrm{~B}$ & BYTE & UNIT & XDATA_UTILS & ?XD?_CEI_UTILS_STRNCMP?_CE $\bar{I}$ _UTILS & \\
\hline $001134 \mathrm{H}$ & $001139 \mathrm{H}$ & $000006 \mathrm{H}$ & BYTE & UNIT & XDATA_UTILS & ?XD?_CEI_UTILS_STRLEN?_CEI_UTILS & \\
\hline О0113АH & 00113FH & $000006 \mathrm{~B}$ & BYTE & UNIT & XDATA_UTILS & ?XD? - $\mathrm{CEI}_{-}^{-} \mathrm{UTILS}_{-}^{-}$STRCMP?_CEI_UTILS & \\
\hline $001140 \mathrm{H}$ & $001143 \mathrm{H}$ & $000004 \mathrm{H}$ & BYTE & UNIT & XDATA_UTILS & ?XD?_CEI_UTILS_FABS?_CĒIUT̃ILS & \\
\hline $001200 \mathrm{H}$ & $001211 \mathrm{H}$ & $000012 \mathrm{H}$ & BYTE & UNIT & $\begin{array}{l}\text { XDATA_DATAPRO } \\
\text {-CESS }\end{array}$ & ?XD? _CEI_DP_PHSENSOR?_CEI_DATAPROCESS & \\
\hline $001212 \mathrm{H}$ & $00121 \mathrm{FH}$ & 00000EH & BYTE & UNIT & $\begin{array}{l}\text { XDATA_DATAPRO } \\
\text {-CESS }\end{array}$ & ?XD?_CEI_DATAPROCESS & \\
\hline $001220 \mathrm{H}$ & $001224 \mathrm{H}$ & $000005 \mathrm{H}$ & BYTE & UNIT & $\begin{array}{l}\text { XDATA_DATAPRO } \\
\text {-CESS }\end{array}$ & 2XD?_CEI_DP_INT2STRING?_CEI_DATAPROCESS & \\
\hline 001225H & 001228: & $000004 \mathrm{H}$ & BYTE & UNIT & $\begin{array}{l}\text { XDATA_DATAPRO } \\
\text {-CESS }\end{array}$ & ?XD?_CEI_DP_VOLTAGEMONITORING?_CEI_DATAPROCESS & \\
\hline $001229 \mathrm{H}$ & $00122 \mathrm{CH}$ & $000004 \mathrm{H}$ & BYTE & UNIT & $\begin{array}{l}\text { XDATA_DATAPRO } \\
\text {-CESS }\end{array}$ & ?XD? _CEI_DP_CURRENTMONITORING?_CEI_DATAPROCESS & \\
\hline $00122 \mathrm{DH}$ & $001230 \mathrm{H}$ & $000004 \mathrm{H}$ & BYTE & UNIT & $\begin{array}{l}\text { XDATA_DATAPRO } \\
\text {-CESS }\end{array}$ & PXD?_CEI_DP_SHT11_CONVERSION?_CEI_DATAPROCESS & \\
\hline
\end{tabular}

Class symbols for_CEI_DataProcess and_CEI_Utils components in data memory

Fig. 2.17 definition of classes and memory location for some of the component packages.

This process is also performed for data memory slots, in which segments are defined within the external RAM of the 8051 architecture. As a result, a welldefined memory structure is configured for the support area to relocate both code and data segments. As mentioned before, the distribution scheme for data and functions of the support segments considers two main approaches: the first one in which functional components and hardware controllers that are in their final release version are compacted into a memory slot whose maximum size corresponds to the aggregation of the associated libraries; and the second one where a memory reservation strategy is to be adopted so that future 
modifications of functional components or the inclusion of new software elements are contemplated.

In order to understand such capabilities, consider the possibility of modifying the organization of this area in order to include new libraries for the management and control of different hardware developments. In this way, the area of interest is calculated as follows:

$$
A_{l}=\sum_{n=1}^{N s}\left(L_{s n}+O_{s n}\right)
$$

$A_{1}$ is the location area for the software support platform within the MS-PRS, which contains the libraries and components to be used. $\mathrm{L}_{\mathrm{Sn}}$ is the size of the slot $\mathrm{n}$ in bytes, which depends on the total sub-area of every library in its current version. $\mathrm{N}_{\mathrm{S}}$ is the amount of libraries to be included in this support area, whereas the $\mathrm{O}_{\mathrm{Sn}}$ represents the memory reservation as an offset that can be assigned to every slot in order to include replacements, modifications or changes in the current version of the libraries.

The determination of the offset value for every slot can be carried out by using several memory reservation optimization criteria. One of them is based on the definition of component update weights either in accordance with the size of the library or considering their release/development level (e.g. for those preliminary software support prototypes a progressive updating process may be allowed for). Another alternative is to assign a generic offset among the memory slots, in which case equation 2.1 remains as follows:

$$
A_{l}=\left(O_{s} * N_{s}\right)+\sum_{n=1}^{N s} L_{s n}
$$

When considering the release versions of the support libraries to be used in final implementations where the size of the segments is to be optimized, no $\mathrm{O}_{\mathrm{sn}}$ is assigned to any sub-area, thus the first element of the right side of the equation 2.2 is null, resulting on a smaller size of the area. In this case, the main target of the testbed experiments is related to the usage of the dynamic area for testing and analysing algorithms, software developments, and application prototypes 
on top of the support area, which hence stays fixed. Instead, in case of contemplating platform experiments where modifications of the support libraries are carried out, every available sub-area for library updates can be calculated as follows:

$$
A_{s n}=L_{s n}+O_{s n}+O^{\prime}{ }_{s n-1}+O^{\prime}{ }_{s n+1}
$$

$\mathrm{A}_{\mathrm{sn}}$ is the maximum size per slot that can be updated. Based on an adjacentoffset sharing principle, the size of the support area is optimized to minimize the isolated memory space, without being necessary to reprogram the whole memory sector, which thus maximizes the efficiency of the partial reprogramming mechanism for the support segments of the Cookies software platform.

The aforementioned expressions allow changing the location parameters of the memory classes and function symbols, from which the new compilation configuration files are subsequently generated according to this memory slot calculation process. For instance, when considering a release version of CEI_Utils, CEI_DataProcess, CEI_queue, and CEI_peripherals, the code distribution based on the memory class assignation can be performed as CODE_UTILS (C:0xB000-C:0xB439), CODE_DATAPROCESS(C:0xB440-C:0xB8BF), CODE_QUEUE (C:0xB8C0 - C:0xBAFF), CODE_PERIPHERALS(C:0xBB00 - C:0xBDFF), where the starting point of distribution is $0 \times B 000$ in this case.

In general terms, it is also necessary to specify the start-up point of the program to be executed after system hard-reset, the memory slot from which the software block will be written when performing the reprogramming process, and the location of the interruption vectors associated with the relocated software. In this way, .A51 and .OPT files are modified to include on one hand the definition of the start-up execution point to be located at the bootloader/recovery segment from which the active application program is triggered, and on the other hand a common interruption linker that will redirect the execution routine to the interruption handler of that active application program. The data and code memory slot addresses from which the code distribution is to be performed can also be included in the .OPT definition. With this, the compilation process will optimize the amount of memory space to be used for the defined slot relocation, 
thus minimizing gaps in between software components unless specified in the memory slot reservation process.

Regardless of the type of relocation method to be applied, functional components and their associated data resources are placed in the new reserved support area (program + data memory) to which application blocks will dynamically and transparently access. This means that modifications on the memory classes and/or symbols relocation will be performed by the partial reprogramming engine without affecting the top-level application access to the support area, which is addressed by the dynamic linking definition.

\subsubsection{Bootloader and recovery segment}

The remote and partial reprogramming mechanism embraces the capability of the sensor nodes to receive the configuration file and then reprogram the corresponding memory segments according to the update of a functional component or the replacement/inclusion of application prototypes. In works such as [Panta'11] [Jeong'09] the reprogramming technique is carried out by the support of an external memory. Therefore, the reprogramming process is composed of two main stages: image composition into the external flash memory, and whole program memory writing. Moreover, in [Hui'04] the reprogramming engine is remotely transferred along with the image to be updated. Unlike such approaches, in this work the reprogramming strategy is performed on-the-fly, which means that no additional hardware and software elements for the reconfiguration process are needed but the implementation of a recovery segment in the support area of the proposed architecture. This allows reducing the complexity of the reprogramming as well as the power consumption associated with additional external memories and whole reprogramming tasks into the internal processor memory. Moreover, in order to reduce the size of the reprogramming image, the recovery segment includes the bootloader engine as a fundamental part of the system, which means that it is preconfigured into the architecture as a memory-protected area. Therefore, it will not be transferred every time a remote reprogramming task is triggered. 
Although the programming image to be remotely transferred to the sensor nodes is considerably smaller thanks to the MS-PRS, and since in case of the WSN testbed architecture a reliable backchannel infrastructure supports the reprogramming process, additional mechanisms have been included to enhance the reliability of the on-the-fly configuration technique, particularly targeting hybrid deployments where the integrity of the binary code might be affected by the communication performance.

As mentioned before, the 8051-based microcontrollers included in the Cookie processing layers are composed of $64 \mathrm{kB}$ of program memory and $4 \mathrm{kB}$ of data memory. While the data memory can be directly written by accessing the corresponding address, the code memory is by default write-protected. Therefore, a download mode configuration has to be set (either by introducing a key sequence for write-enabling or by modifying the configuration of a dedicated special function register) so that the content of the program memory can be modified by using defined writing instructions but pointing at the code memory addresses.

Since the program memory is structured in pages, depending on the memory address that is intended to be written (specified in two separated bytes), the first step is to erase the page that contents such a code position, and thereby one or more bytes can be written in the specified memory addresses. Finally, the download mode is closed so as to reestablish the normal operation of the data memory addressing. The same procedure is carried out when using variables that are saved into the flash memory for non-volatile management purposes.

The bootloader segment integrates the program memory accessing, erasing, writing and verification routines, although further mechanisms have to be implemented in order to process and execute the commands associated with the received reprogramming image. After compilation process, the binary code transferred to the sensor node is encoded on the basis of the HEX format [HEX], in which the programing information is divided into several coding records that are included in the memory writing file. Every record is firstly composed of a start symbol, then the length of the data contained in the current record, followed by the 16-bit memory address and the record type code. Thereafter, the 
n-bytes data sequence precedes the checksum value that serves as one of the mechanisms to verify that the record has been properly received without errors.

The binary code is transferred by opening a VPort of the testbed and then sending the sequence of records that correspond to the compiled source code of the memory segments to be reprogrammed. Apart from the checksum calculation during the programming process to prevent possible packet losing effects related to the on-the-fly remote transaction, three additional elements are combined to guarantee the integrity of the reprogramming scheme. The first one is the Cookie-defined Watchdog component, which detects connectivity problems of the testbed/network interface and packet reception latencies. A specific timeout can be configured as a threshold for triggering the watchdog functionalities in order to reestablish the availability of the affected segment, so that it can be replaced or updated. This feature is supported by memory block masks that determine the current state of the programming area. The second one is a predefined recovery application that is executed once a failure is detected during the configuration process. This minimalistic code is included within the memory-protected area (thus it cannot be corrupted during reprogramming tasks) in order to restore the reprograming engine options. The third one is the remote use of the RstTrg block to externally reestablish the microcontroller and thus reboot the recovery segment.

In Fig. 2.18 an overall view of the bootloader/recovery segment structure is illustrated, in which the different sources that can execute the reprogramming engine are highlighted: it can be triggered from the dynamic area (application block); when the processor is reset or the Cookie-Watchdog component is activated; or from the management segment that includes internal interruptions related to scheduled/synchronous tasks or external interruptions from the VPorts to remotely launch a new partial reprogramming process. In case of malfunctions or failures when the application/experiment is running, the Cookie-Watchdog or specific debugging tasks can launch the recovery segment in order to update or restore the corresponding component or segment remotely.

After performing one of the defined reprogramming actions by using an embedded command-based console interface (clearing, replacement, update, 
recovery, reprogramming, verification of the specified memory slot), the bootloader will call the management segment to execute the corresponding memory segment accordingly.

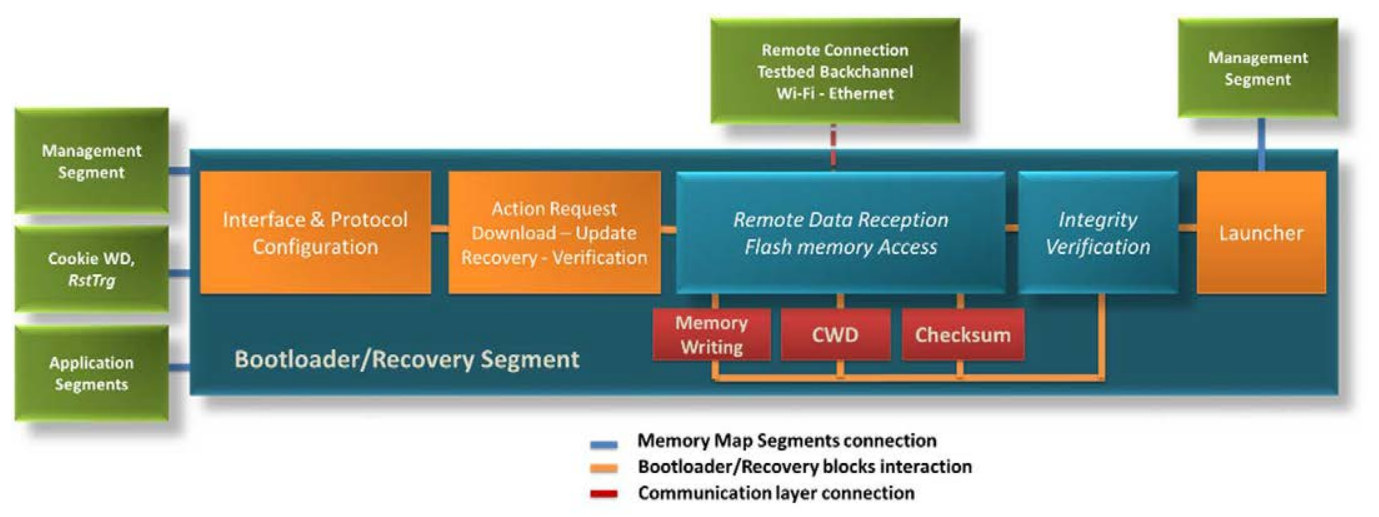

Fig. 2.18 Interaction schemes for the Bootloader/Recovery segment functionalities.

The support to the aforementioned capabilities for the reprogramming engine is realized by means of the _CEI_Bootloader library, whose core functionalities are mainly composed of the following components:

- _CEI_bl_bDecoder: performs the decoding tasks that allow interpreting the incoming partial reprogramming image, so as to identify the type of received data and the memory area to be written, based on the binary format already mentioned before.

- _CEI_bl_eraseSlot: this functionality erases the associated memory pages of the specified memory slot, so that it can be available for downloading a new application/experiment programming file. The Slot_State_n mask that controls the current status of the target slot $n$ (programmed, empty) will also be erased to indicate its availability for new experiments. Furthermore, in case of erasing the active application, the recovery segment will update the Active_Prog parameter according to the information of the management segment, i.e., either by internally triggering a new memory slot or by receiving a new configuration from the console interface to be executed.

- _CEI_bl_updateCode: this function makes use of the aforementioned components to write the specified segment, by first verifying that the 
associated memory pages are properly erased, and then checking the type of data and address information to be handled during the record writing operation into the code memory. Since the process is carried out on-the-fly, the VPort interface is established and then the previously mentioned reprogramming integrity mechanisms are activated, including the checksum evaluation.

- _CEI_bl_console: integrates the in-system user options provider to remotely handle the different memory segments of the MS-PRS, so that the state of the software components and experiments included in the node can be verified, updated or replaced. Users can access the state of the Slot_State masks as well as the Active_Prog, and perform the following options: 1) a new code downloading procedure 2) update the content of one of the memory segments 3) restart or execute a different already-stored experiment 4) provide the state of available application blocks 5) show errors and information related to the reprogramming and recovery process. The console can also be activated by triggering an external interruption sequence from the debugging blocks engine to dynamically access and evaluate the state of the multi-experiment/application architecture of the testbed framework. As depicted in Fig. 2.19, this component makes use of the proposed functionalities to generate the actions related to the on-thefly reprogramming and memory management process implemented into the provided bootloader. The interactions among elements are also highlighted so as to illustrate the behaviour of the system when performing the reprogramming tasks. 


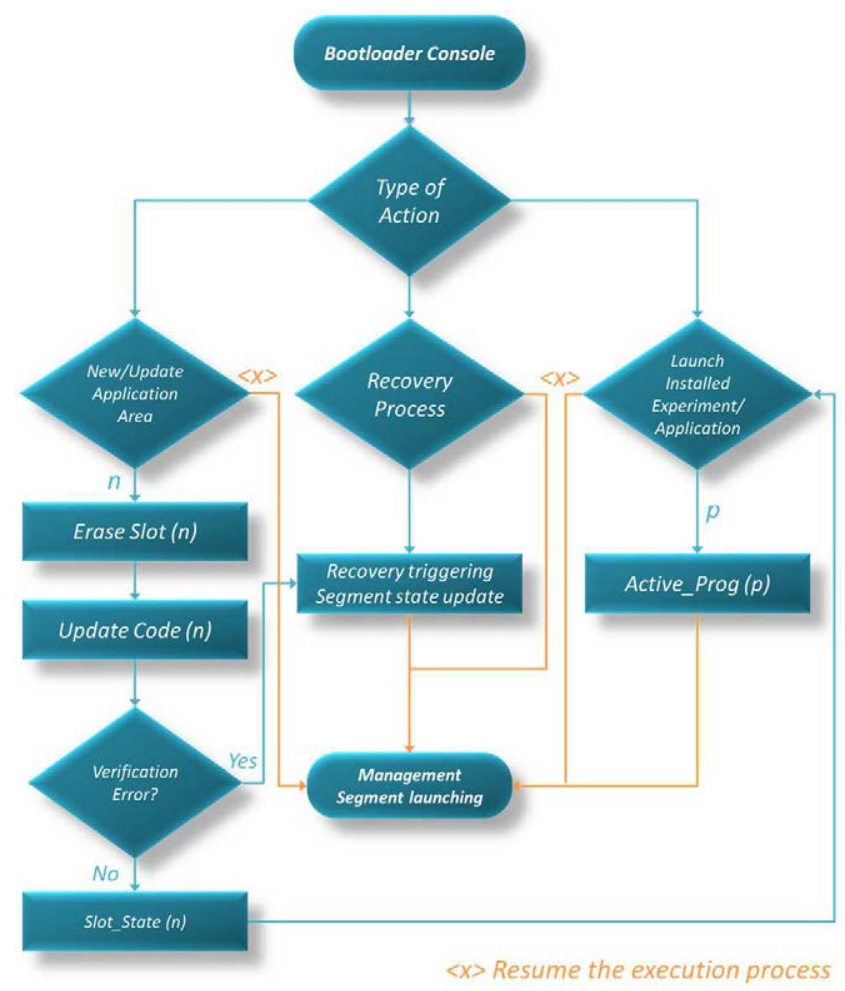

Fig. 2.19 Basic behaviour of the bootloader console and main interaction elements.

\subsubsection{Multi-application/experimentation support and dynamic linking approach}

As previously remarked, the proposed modular approach encompasses the inclusion of different independent experiments and application prototypes within the MS-PRS, so that they can be reprogrammed and triggered without modifying the rest of the platform. In order to support such a flexibility, the software relocation is combined with a dynamic linking strategy, so that the application blocks can be seamless correlated with the software support area. This also means that in case of modifying functional components of the libraries, no additional changes have to be performed at the application side either. Moreover, in other to manage the application triggering task, a segment execution handler helps the testbed architecture control the experiments that are programmed and active in the memory map. 
The basic idea behind this concept is exemplified in Fig. 2.20, in which an independently-developed user application can be linked with the software support platform. The user application project makes use of the functional components and drivers of the defined framework but they do not have to be included into the final program image, hence reducing the size of the reprogramming file to be transferred. The main element to establish the interconnection between the support project and the user experiment/application projects is defined by two independent interface objects that determine the functionalities to be connected to the dynamic area (they are internally defined in the form of ASM directive blocks within the independent projects). As shown in Fig. 2.20 the components of the support area are defined as external resources, whereas the application blocks specify the type of public resources to be used, considering that they are independently compiled and programmed in different memory segments of the reprogramming scheme.

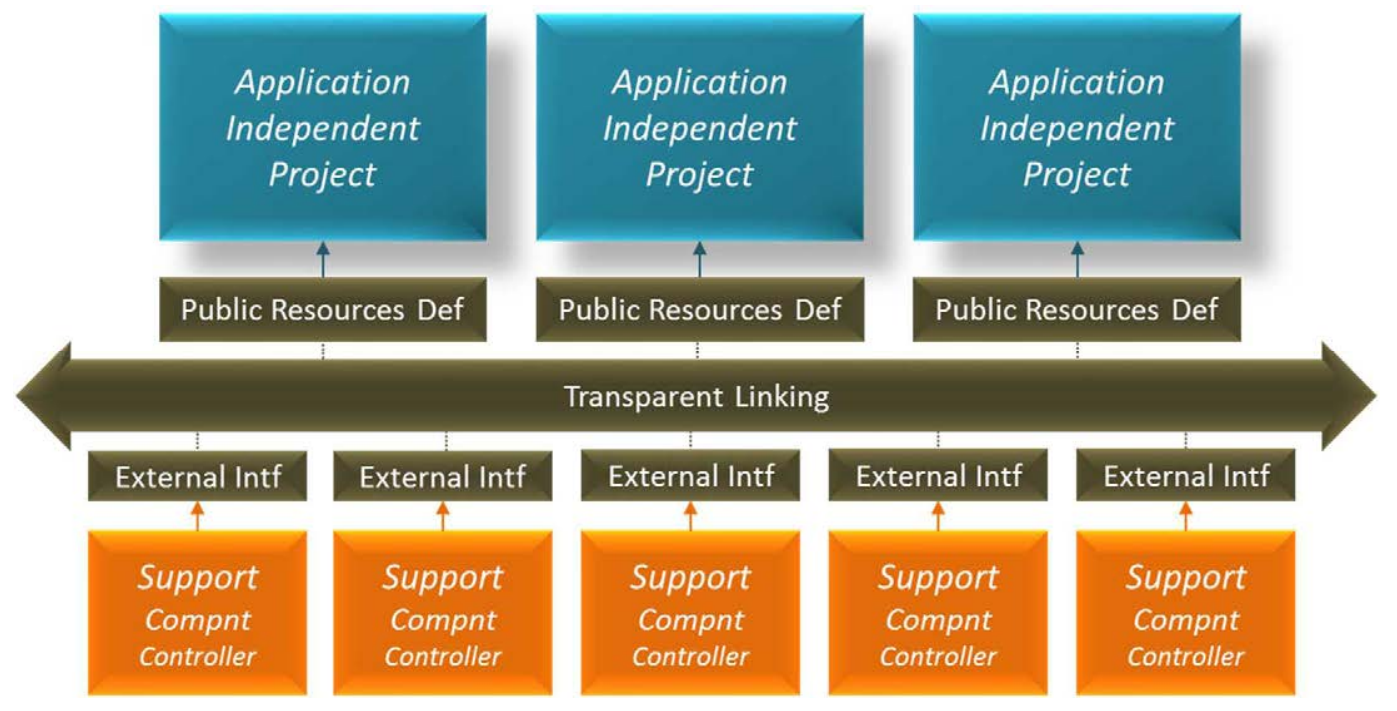

Fig. 2.20 Multi-application/experiment independent projects for the on-the-fly partial reprogramming scheme.

However, although a transparent function sharing can be performed between support and dynamic areas, the minimum parameter to be specified at low-level within the public resource interface definition is the memory location of the components. This means that the application segments need to be aware of the addressing information of the support elements to be used. This could produce 
inconsistencies when partially reprogramming software components of the platform or even memory addressing overlapping when externally referring to a resource, unless the linking information of the different segments is modified as well. This could significantly reduce the modularity and transparency of the partial reprogramming architecture and thus penalize the independence among software development projects within the proposed framework. Therefore, a dynamic linking approach is implemented to assure that these shortcomings can be diminished as much as possible. This is achieved by defining a common linking region in which a relative addressing mechanism is established to abstract the software component usage from the application level.

At the compilation side of the application segment projects, the public resource interface includes the definition of the functions used in the code that are to be dynamically linked to (whose addresses refer to the linking region instead of the absolute location), while in the support segment projects the functional components to be shared are specified within their interface definition as externals, though unlike the previous case the dynamic redirection instruction to the actual resource location is then included. As a result, the interfaces converge at the linking region and from there the redistribution to one or other segment is dynamically performed. Therefore, the cross-interaction among the software resources of different memory areas is transparently realized without low-level addressing and location dependencies.

Regarding the parameters of the functional components, their transfer is optimized by using the $R n$ registers of the processing architecture, which provide up to 8 bytes that are distributed depending on the type of parameter to be passed to (for instance, in case of generic pointers, 3 bytes are used to specify the type of memory together with the 16-bit address). They are also combined with dedicated memory positions for those particular situations where more data need to be passed, whose addresses are included in the function symbols that require additional memory for such parameter transferring (specifying the memory identifier and the location of the parameter).

Two important aspects are also tackled to enhance the flexibility of the multiapplication structure, which are related to independent interruption handling as 
well as segments execution and state control. On one hand, the proposed system provides user projects with the possibility of implementing their own interruption management functions in order to make use of some of the internal microcontroller resources such as real time clock and interval counter modules or GPIO-based events in an independent fashion. For this, the dynamic linking segment performs a runtime redistribution of the interruption vectors to the active application/experiment segments when the corresponding action happens.

By default, those application interruption vectors will be placed at the beginning of every block definition following the same order of interruption offsets defined by the processing architecture. Then, the dynamic linking calculates the position of such a vector as Active_Prog + IntVtOffst (active application block parameter and vector offset according to the interruption identification). In this case, the behaviour of the application once the interruption vector is triggered will be explicitly defined by the user. The addressing information of the functions that determines this behaviour is internally specified by using the previously mentioned interface abstraction definition (.ASM objects of the application/experiment projects).

On the other hand, in order to manage the segment execution process as well as control and update the state of the different application blocks, the Active_Prog parameter is combined with the Slot_State_n masks that contain information of the different application segments included in the dynamic area, which are handled by the management segment in combination with the bootloader/recovery segment. These are key elements to provide multiapplication management support, taking into account that there are two main ways of selecting the application to run. The first one is the asynchronous assignment, in which a selection request can be performed remotely in order to switch from one application to another. This request, as an asynchronous interruption, will execute the console and then execute the selected experiment segment or download a new partial reprogramming file. The second possibility is a synchronous or pre-programmed assignment, which is intended to be used for assigning application time slots so that predefined experiments could run 
during a specific and reserved period of time. This feature allows users, for instance, to download tree different application prototypes and schedule their execution in order to conform a multi-experimental scenario for testing several in-node prototypes. In order to accomplish this, an internal time interval counter is used within the management segment to assign and monitor activation periods for the selected application/experiment blocks.

This feature is also important from the point of view of node restarting, because the management segment can trigger application blocks without being necessary to perform a hard reset or system reboot of the microcontroller, which is certainly time and energy consuming particularly considering its applicability at the operational stage of the WSN.

\subsubsection{Application segment, User software prototyping}

As remarked previously, the partial reprogramming scheme has been designed and implemented in such a way that from the user perspective the underlying mechanisms for supporting segment correlations and system modularity are transparent, and several applications can be developed and downloaded independently among each other. As shown in Fig. 2.21 users are provided with a project framework that primarily contains the structure of the platform under which their code implementation is to be carried out. The main elements to be distinguished are as follows:

- Interface definition object (in the form of .ASM) for the dynamic linking information to the support area.

- $\quad$ The STARTUP-51 file to create the platform configuration of the 8051based target device and custom configurations.

- The project option file for memory addressing and compilation directive settings.

- Header files of the Cookies software support libraries to be used for the application prototyping. 
- The top-level software templates in the form of a $C$ file that defines the framework for developing a new embedded software under the Cookies HW-SW platform.

- The project file that comprises target selection, further compilation options, symbols and system configurations that are already incorporated for the proper generation of the partial reprogramming scheme.

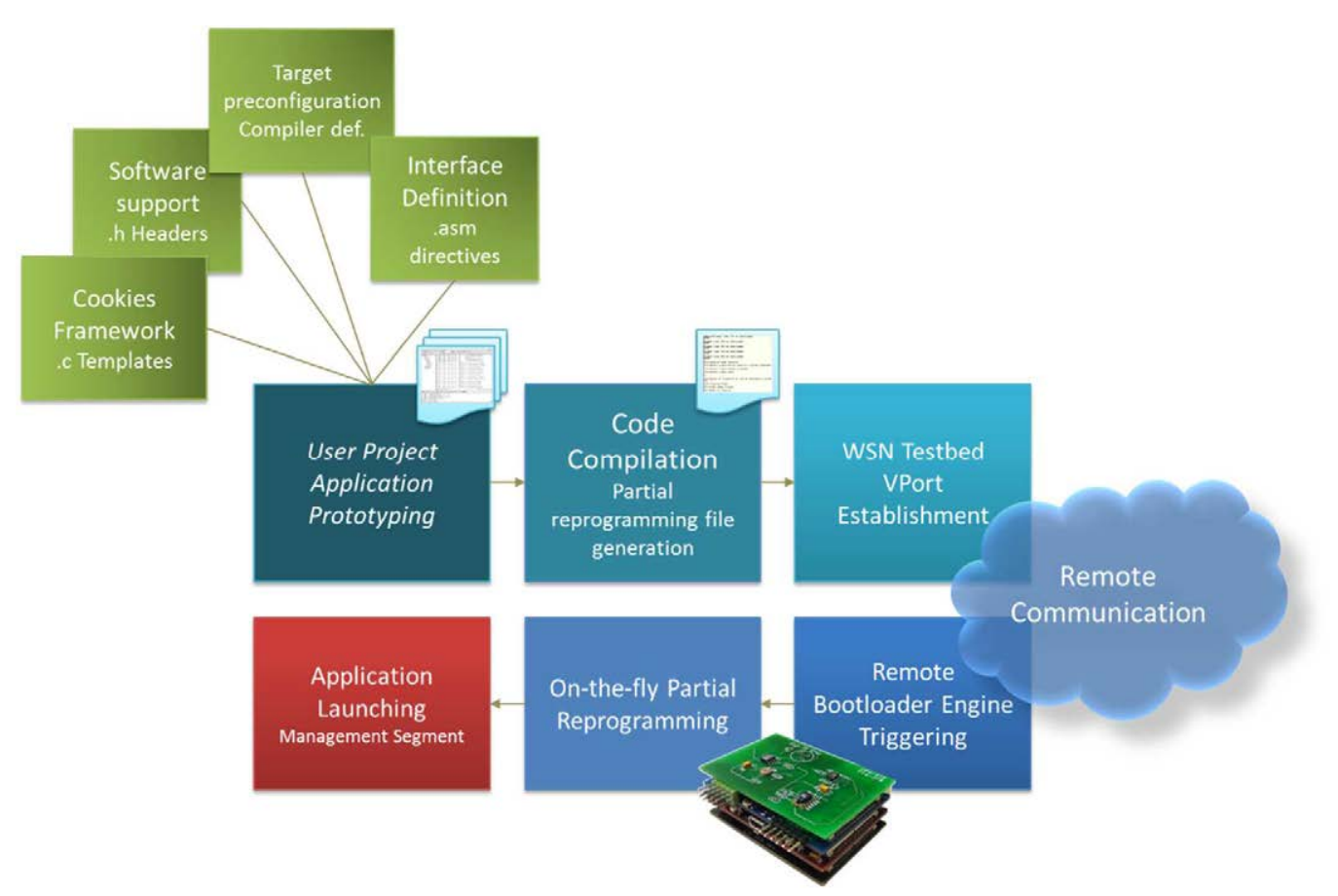

Fig. 2.21 Multi-application and partial reprogramming generation process.

It is important to highlight that the proposed MS-PRS has been fully implemented in both processing layers of the Cookies platform, and hence the process of creating a new application based on the project development framework is the same from the user point of view. After the software implementation is realized and the programming file is generated, a VPort can be opened to establish a new remote connection with the target platform, and then start the partial reprogramming process with the support of the bootloader/recovery segment. 


\subsection{Applicability of the platform and test cases}

In order to analyse the potentials and applicability of the proposed HW-SW testbed framework in different experimental scenarios taking into account its hybrid nature, and also trying to put the designed capabilities into various WSN contexts, different sets of test cases are proposed under which the overall structure of the testbed architecture as well as the benefits of the partial reprogramming scheme can be applied. The main target is to verify the observability, controllability, and adaptability of the proposed system implementation within different testing scenarios, as well as address the remote usability and effectiveness of the designed HW-SW support components. In this way, the action and configuration command execution process to control the behaviour of the WSN system is combined with the integration of a lightweight server application to provide users with a basic set of functionalities seeking the ease of usage of the testbed platform under such use cases.

\subsubsection{Scenario 1: Testbed characterization and remote reprogramming performance evaluation}

In order to verify the hardware and software elements of the testbed backchannel infrastructure, an indoor hybrid WSN testbed deployment has been carried out in the Centre of Industrial Electronics, in which a combination of the Ethernet-based and Wi-Fi-based layers was set up. In Fig. 2.22 the deployed sensor platforms with the backchannel-based and nomadic structure is shown, considering a heterogeneous configuration of the network not only regarding the testbed support layers, but also the use of both C8051F930-based and ADuC841-based processing layers so as to compare the performance of the remote reprogramming capabilities as well as the software support components.

Regarding the rest of the Cookie layers for the main WSN application, the sensor node is composed of a ZigBee-based communication layer that includes the ETRX2 module from Telegesis, and the sensor layer that integrates an SHT11 device for temperature and humidity measurements, together with a light dependent resistor sensor. 


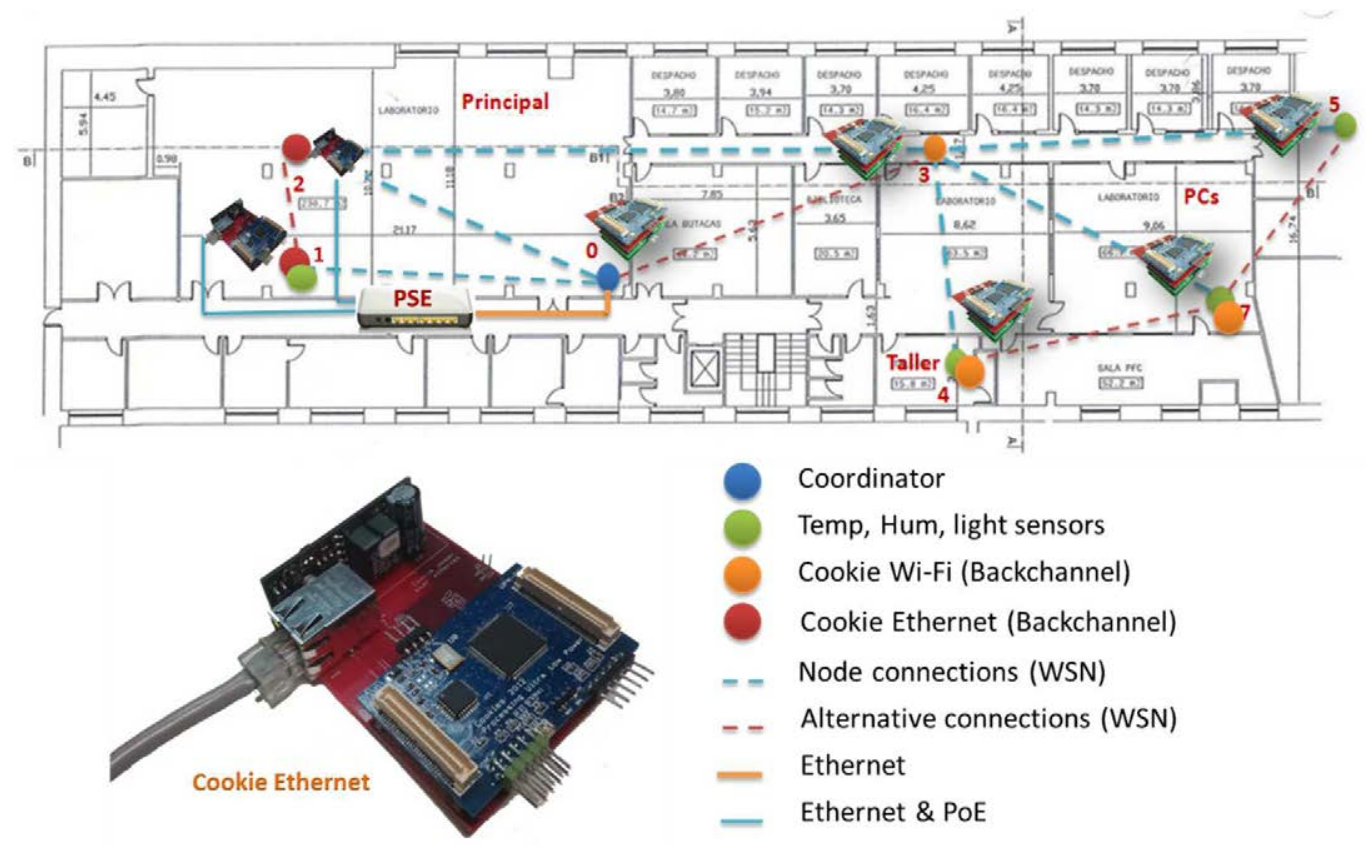

Fig. 2.22 Basic structure of the WSN deployment for the testbed HW-SW platform components characterization and validation.

In this particular test case, the memory segmentation scheme has been distributed as shown in Fig. 2.23, where the user dynamic area for experimental purposes can be divided into $4 \mathrm{kB}$ per block (which can be reconfigured depending on the user requirements). Moreover, as also highlighted in Fig. 2.23, the support area was also included from the position 0xB000 to 0xDFFF, which contains the software libraries for providing users which Cookie HW-SW support components, as well as the management and recovery segment from 0xE000 for the remote reprogramming and back-up features.

The basic functionality of the WSN application prototype to be downloaded is configured such that the sensor measurement information is transmitted from the sensor nodes to the coordinator every 2 seconds and, after 5 consecutive data transmissions, the nodes are to be configured to enter in sleep mode. For that, ZigBee communication configuration and management components have to be included into the node, in addition to analogue and digital signal processing functions to handle the measurement data from the sensor layer of the Cookie platform. 


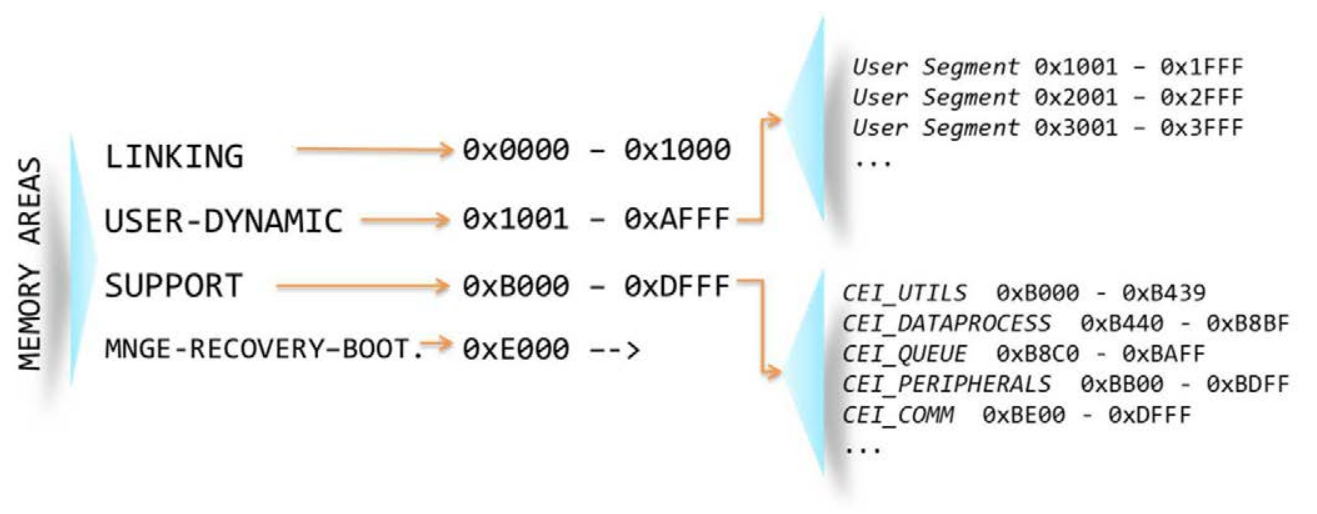

Fig. 2.23 Definition of the different memory areas for partial reprogramming structure.

In this way, several sets of functionalities of the proposed platform are used. First, the backchannel communication interface is configured to be able to trigger the VPort for establishing the remote reprogramming process, the LowPwr block to set the processing and communication modules in sleep modes, RstTrg to remotely reset the platform once the testbed and WSN application configurations were performed, and the DevInCom functionality to remotely and transparently monitor the status of the internal interconnection and data exchange between the ZigBee stack and the embedded software implementation in the microcontroller.

Second, the bootloader and recovery segment evaluation is carried out by performing the remote partial reprogramming and launching process for the described WSN application. As shown in Fig. 2.24 (left), the runtime execution of the application block written allows verifying the functional behaviour of the memory segmentation and dynamic linking capability of the MS-PRS, where the different stages of the configured modes are highlighted. Then, the controllers for managing the different internal and external peripherals involved in the running implementation are tested to effectively provide the user application with the transparent support architecture.

In order also to further analyse such functionalities of the platform with respect to the remote observation of the gathered testbed data, an experimental instrumentation for signal monitoring has been set up as shown on Fig. 2.24 (right). Based on this, it can be seen the aforementioned transitions of the sensor node operation in Fig. 2.24 (left), where the first part corresponds to the 
configuration of the platform. Thereafter the sensor processing and transmission process is accomplished (highlighting the transferring consumption picks associated with the wireless communication module) before entering the powerdown mode.

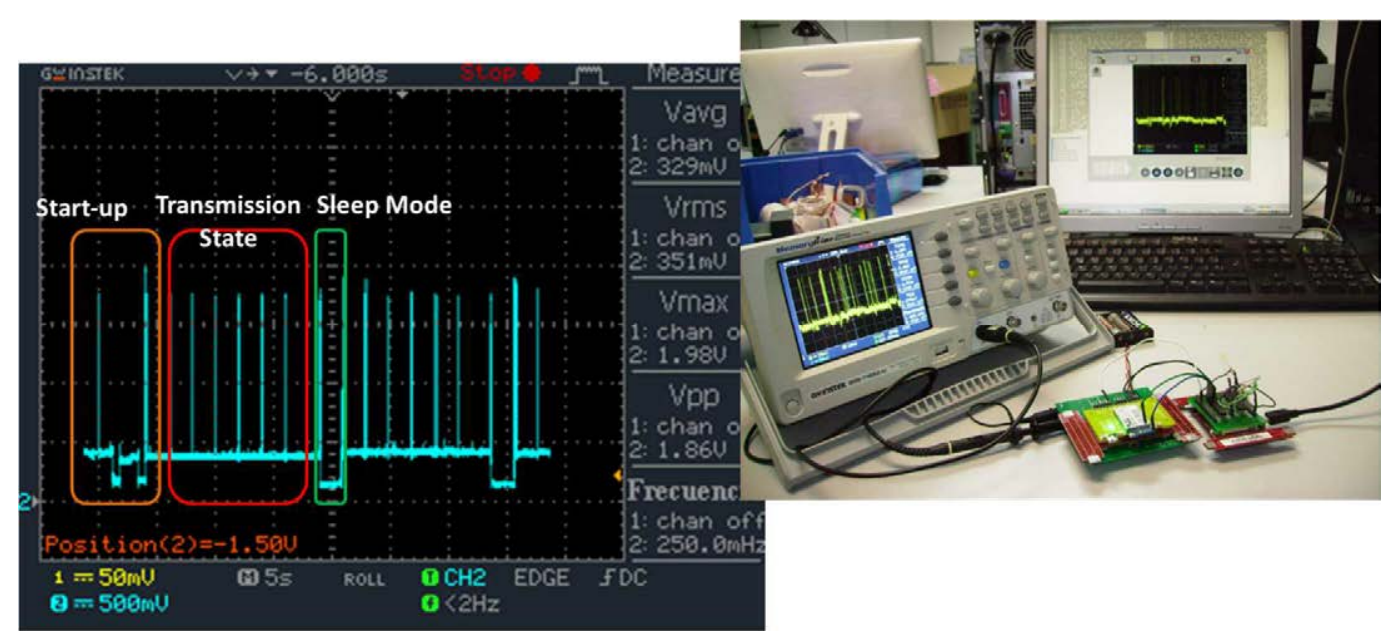

Fig. 2.24 Characterization of the running application. Transitions of the sensor node operation (left), and observation setup for monitoring the platform interactions (right).

The remote programming mechanism has been evaluated by comparing the performance of whole versus partial reprogramming in both processing layers. As shown in Table 2-2, there is a clear benefit on applying the proposed reprogramming architecture, since the size of the configuration file is up to 30 times smaller than the normal complete flash writing, thus reducing considerably the amount of data to be transferred as well as the time spent during the downloading and on-the-fly reprogramming tasks (95\% of time and data-transfer reduction in this case). This analysis is supported by the signal capturing of Fig. 2.25, where a noticeable difference between both techniques can be highlighted. It is also interesting to remark the comparison of the average consumptions in active mode between nodes using one or other processing layer, obtaining $32.26 \mathrm{~mA}$ in case of the ADuC841-based node, whereas a value to $11.94 \mathrm{~mA}$ (three times less in normal operational mode) is achieved in the C8051F930-based node. 


\begin{tabular}{|c|c|c|c|c|}
\hline \multirow{2}{*}{$\begin{array}{c}\text { Remote Reprogramming / } \\
\text { Processing Layer }\end{array}$} & \multicolumn{2}{|c|}{ ADuC841 } & \multicolumn{2}{c|}{ C8051F930 } \\
\cline { 2 - 5 } & Time & $\begin{array}{c}\text { Programming } \\
\text { File (KB) }\end{array}$ & Time & $\begin{array}{c}\text { Programming } \\
\text { File (KB) }\end{array}$ \\
\hline Partial Reprogramming & $664 \mathrm{~ms}$ & 1,14 & $952 \mathrm{~ms}$ & 1,63 \\
\hline Whole Reprogramming & $19,20 \mathrm{~s}$ & 32,5 & $21,70 \mathrm{~s}$ & 36,8 \\
\hline
\end{tabular}

Table 2-2 Comparison of the reprogramming techniques by using different processing layers.
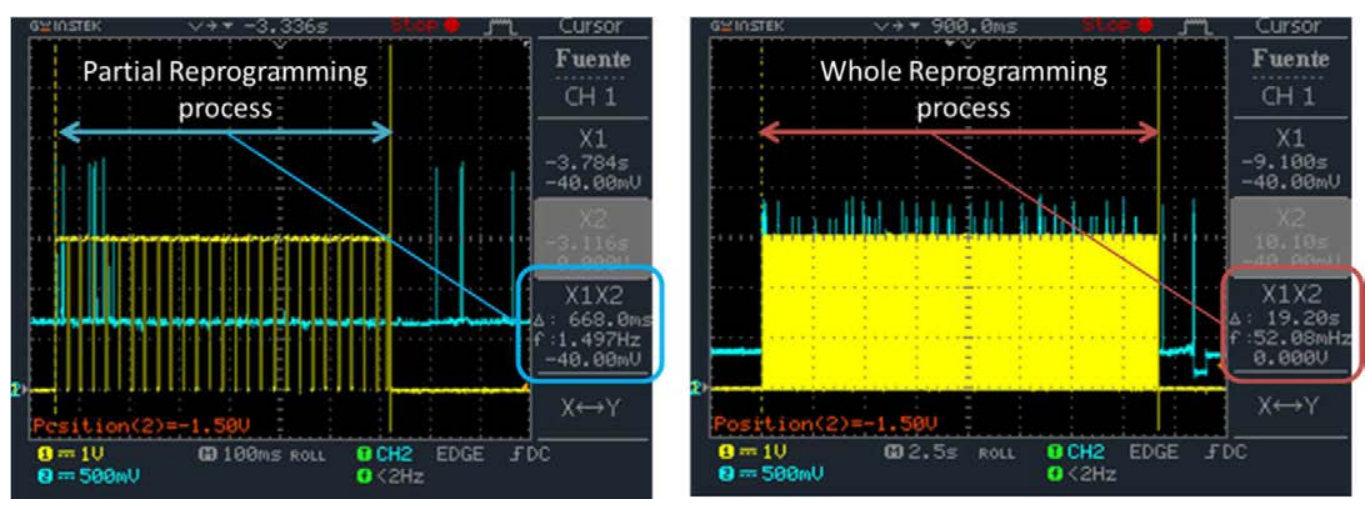

Fig. 2.25 Partial versus whole reprogramming runtime comparative analysis.

This also allowed verifying the operation of the backchannel-based node connection by transferring both partial and whole reprogramming file through the proposed testbed infrastructure. Moreover, the normal operation flow of the running application and also the transitions among segments (including the recovery segment when triggering the remote reprogramming process) were validated.

The second set of test cases focused on including several application blocks within the reprogramming architecture for the management of the hybrid wireless sensor node array, which allows modifying not only the configuration of the Wi-Fi-based testbed interface but also to test several connectivity options when using it as a gateway platform. Four main application segments are considered as remarked in Fig. 2.26: the first one for configuring the sensor node to be connected to an access point of the CEI, from which the remote data is collected and the reprogramming process is triggered (After the node is already connected to the local network, a TCP communication is established from a 
remote point to the sensor devices by using a VPort); the second one allows for the creation of an independent ad-hoc secondary network for the testbed capabilities; the third block aims at transferring the gathered information from the hybrid nodes to a remote HTTP web-server for data collection; and finally the fourth test targets the inclusion of a gateway-node configuration in one of the points of the network (in this case the coordinator node) to establish a dynamic data exchange between the low-rate sensor network and the highperformance wireless communication interface.

As seen in Fig. 2.26, the initial configuration of the Wi-Fi module is realized and then the corresponding applications blocks are reprogrammed and subsequently executed, obtaining the results represented in Table 2-3. While for a whole reprogramming of one of this application use case the process takes up to $23.94 \mathrm{~s}$ and $42 \mathrm{kB}$ of programming file, the partial reprogramming structure allows obtaining less than 3 seconds (2.85 s for $5 \mathrm{kB}$ ) which represents an important amount of time and data-transfer reduction, in addition to enhancing the capability of including several application segments within the same HW-SW platform. This also leads to producing a more energy-efficient reprogramming process not only from the point of view of the hybrid and nomadic testbed architecture, but also for those final application contexts in which the HW-SW support framework is used, particularly where energy-aware mechanisms are a must. 


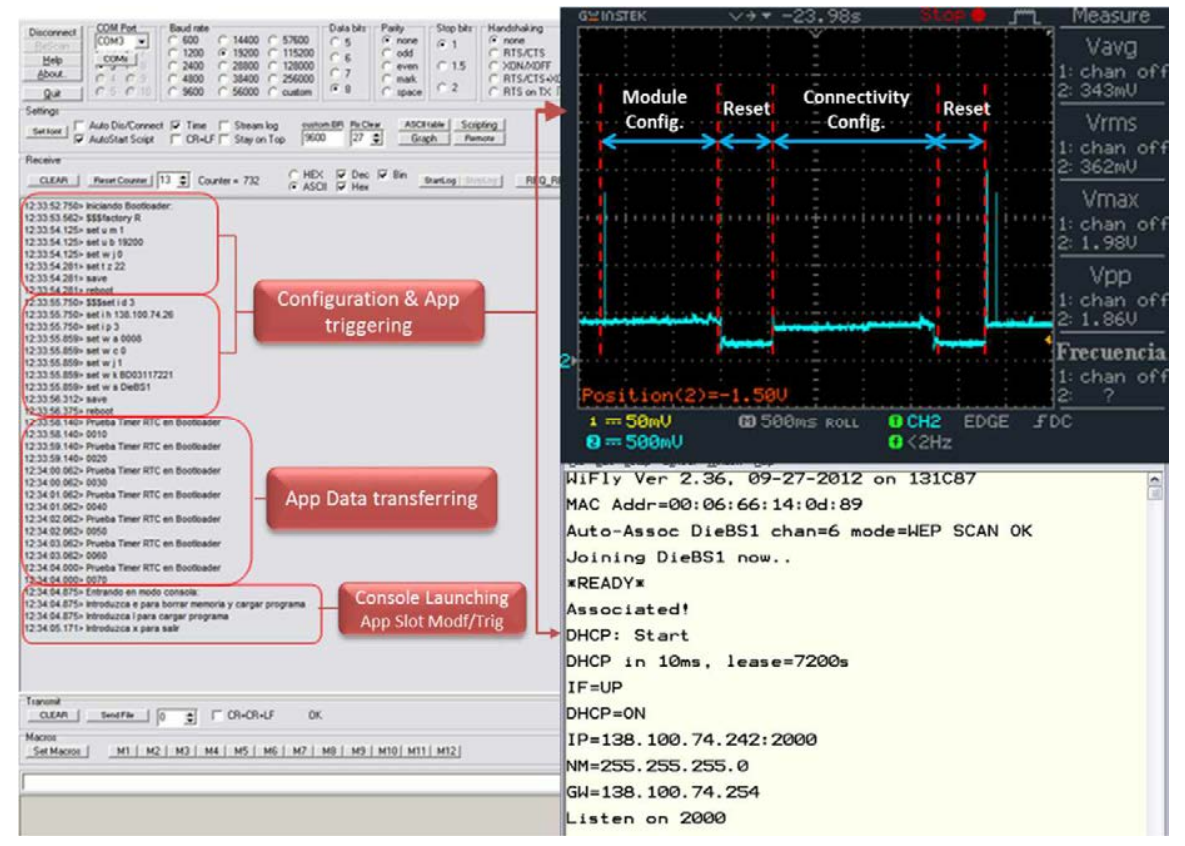

Fig. 2.26 Wi-Fi-based node configuration and application triggering, the console is then launched to execute or update a new application block.

\begin{tabular}{|c|c|c|}
\hline \multirow{2}{*}{ Experimental test cases } & \multicolumn{2}{|c|}{ Over-the-air data transferred } \\
\cline { 2 - 3 } & Whole reprogramming & Partial reprogramming \\
\hline Ad-Hoc network & $37 \mathrm{~KB}$ & $2.21 \mathrm{~KB}$ \\
\hline Access Point Config./Connection & $36 \mathrm{~KB}$ & $2.65 \mathrm{~KB}$ \\
\hline HTTP configuration & $34 \mathrm{~KB}$ & $1.81 \mathrm{~KB}$ \\
\hline ZigBee-Wi-FiGW Network & $42 \mathrm{~KB}$ & $5 \mathrm{~KB}$ \\
\hline
\end{tabular}

Table 2-3 comparison of the reprogramming results for several configuration test cases.

\subsubsection{Scenario 2: Indoor deployment for planning tool analysis and validation}

In this second use case scenario the main target is to analyse and validate the performance of a planning tool development [He'14] by using the proposed WSN testbed platform, so that the real behaviour and test results of the sensor network can be compared with what is expected from the theoretical node placement optimization process. The proposed HW-SW platform served as a support tool to perform the real test cases targeting the validation of the 
planning capabilities for WSN. Thus, the framework was used to develop the involved embedded applications, validate the use of the support area to manage the operation of the nodes, and perform the reprogramming and parametrization actions of the test scenario to dynamically modify the composition of the desired experiment. In this case, since flexibility on node location changes is needed to set up different types of indoor WSN deployment arrays at CEI to support the iterative simulation process with actual data, a wireless testbed structure was adopted.

The primary objective relates to controlling the following parameters within a ZigBee WSN in order to study system optimization models considering node sensing coverage, radio propagation and connectivity, lifetime of the network and data delivery metrics, so that a comprehensive result space for the performance evaluation of planning simulations can be generated by using the testbed capabilities:

- Neighbours information, link quality and radio signal strength indicators, which are periodically retrieved from the sensor nodes in order to study the evolution and variability of the network connections during the deployment lifetime.

- Routing map of the deployment that is obtained by registering the subsequent hops of the generated routes from the sensor nodes to the destination of the network.

- Battery level of the wireless nodes, which is computed and transferred periodically by every node in order to estimate the overall lifetime of the network, as well as study the energy balance among the different areas of the deployment. It will be evaluated in correlation with the configuration of the data processing and transmission rate of the nodes (as shown in the experimental results, an intensive network traffic in terms of sensor measurement sending ratio is generated in order to see the evolution of the battery budget).

- Packet Loss Rate, by means of using the data frames that are sent between two remote multi-hop points. They are observed by 
generating and analysing node packet sequences, in addition to processing the TX and RX times for the transferred packet.

- Sensing measurements that are gathered at the coordinator/server side of the network by defining a data delivery configuration in the sensor nodes, so that they can periodically transmit the processed values of the integrated sensors.

A lightweight graphical interface has been created in order to control and monitor the sensor network not only from the point of view of the running application itself, but also the underlying testbed parameters to evaluate the performance of the wireless deployment. As seen in Fig. 2.27, apart from the observation area where the real-time gathered information is presented, several remote runtime reconfiguration and action commands can be triggered, including neighbour and routing tables composition as well as timing configuration and parameter request/publication operations.

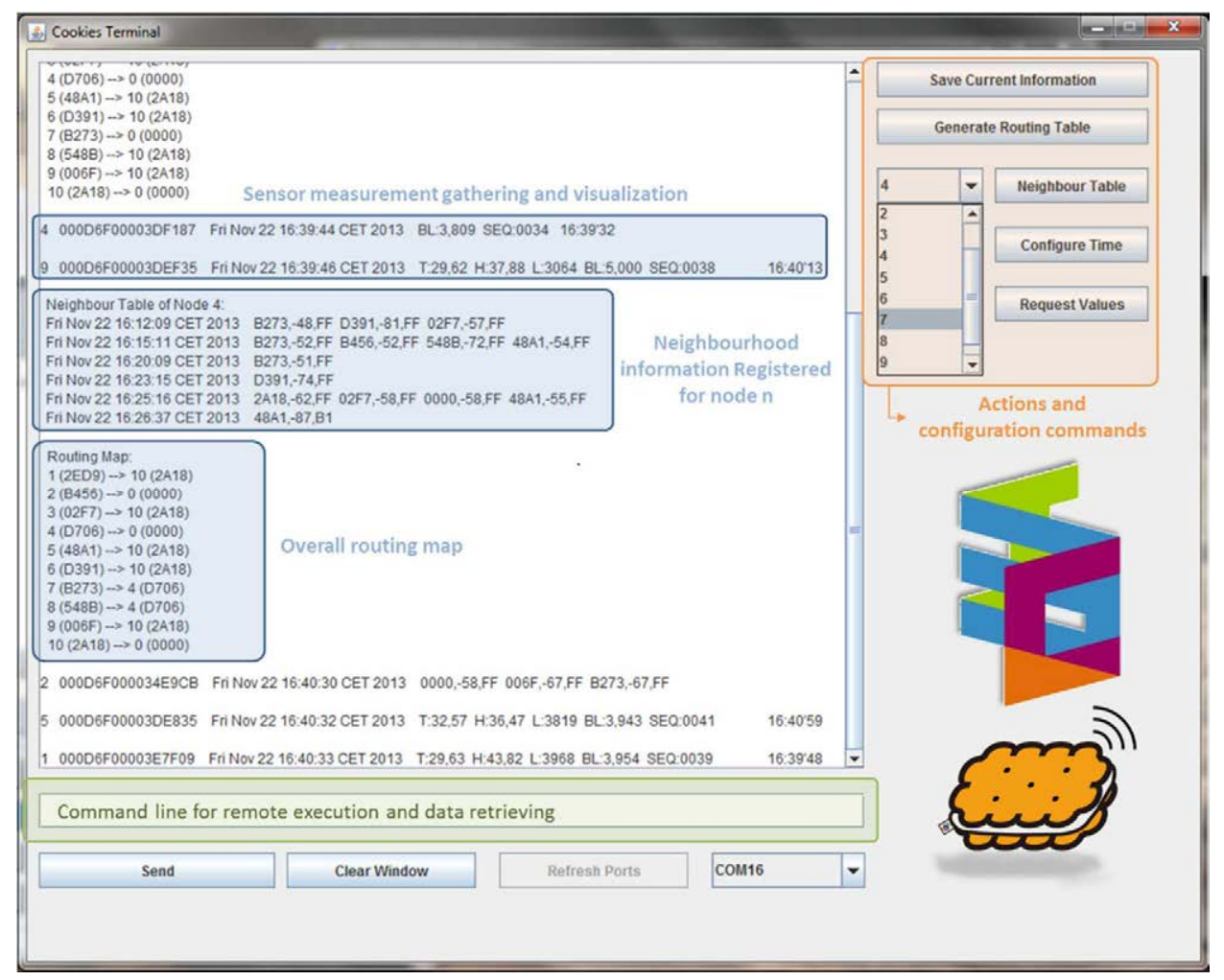

Fig. 2.27 General view of the Java-based GUI for network and testbed support controlling. 
Moreover, the aggregated data is prompted with the identification of the sensor nodes under tests and the sequence numbers so as to keep track of the data acquisition packets. Besides the user interface to control the testing and observability tasks, an underlying data log system registers all the WSN testbed activities in order to be able to carry out further off-line analysis related to the sensor network execution and system performance.

In Fig. 2.28, an analysis of network topology generation based on simulation plus real system behaviour is presented, where the actual performance of the deployed sensor nodes is obtained by using the testbed support platform and compared with one of the candidate solutions from the planning tool. The predictability of neighbourhood discovery has been assessed by comparing the neighbour tables of the nodes (N1 to N17) with two different parametrization methods of the simulation engine. As a result, the accuracy of the neighbour detection reaches $88 \%$ with respect to the real testbed data; obtaining a mean error for the RSSI value of $4.29 \mathrm{~dB}$ and a standard deviation error of $5.06 \mathrm{~dB}$ for the first parametrization, while $3.80 \mathrm{~dB}$ and $3.61 \mathrm{~dB}$ respectively for the second method. Fig. 2.29 shows such a comparative analysis by overlapping the simulation and real accumulative RSSI values of every node and their neighbours, in which the encountered differences between predicted and detected nodes are highlighted with maximum negative picks. 


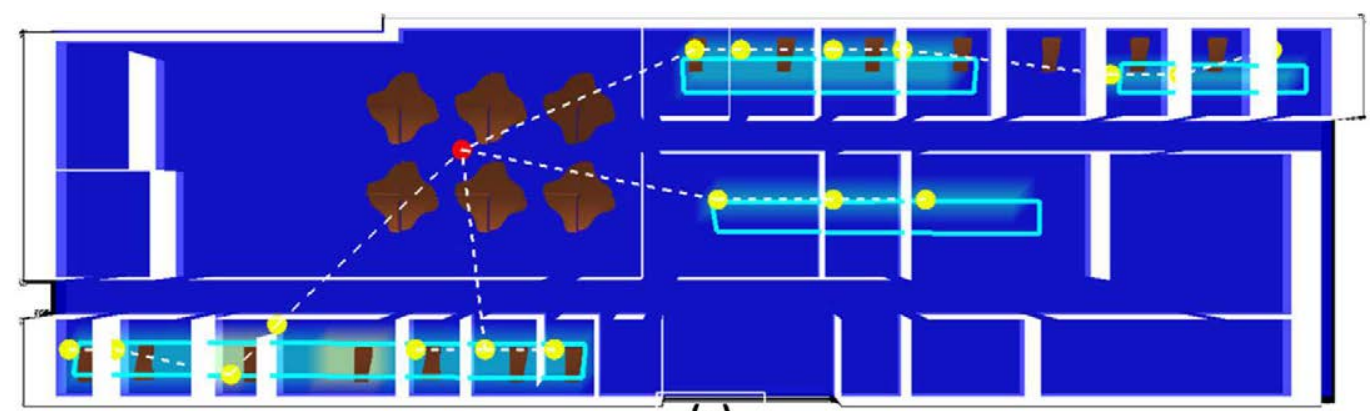

(a)

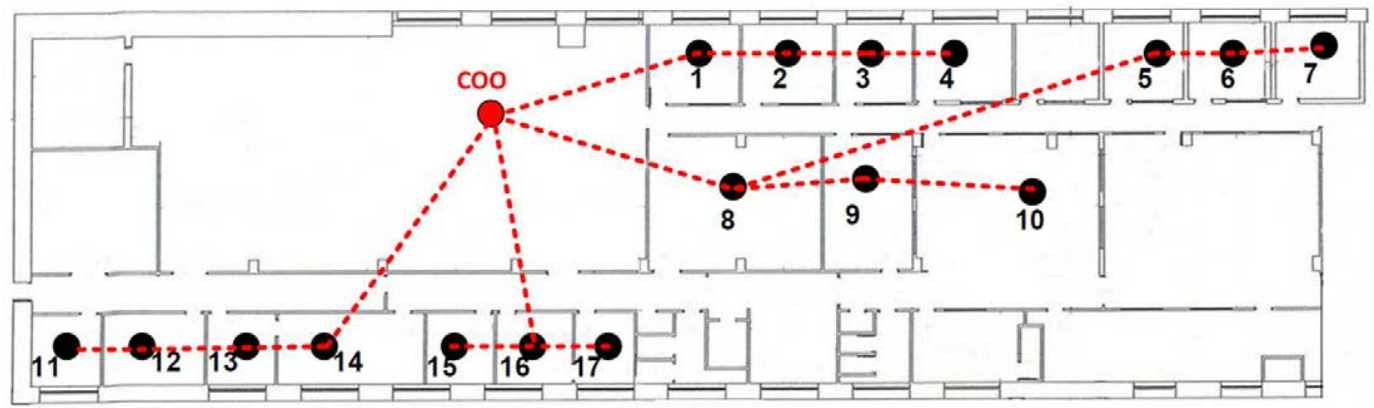

(b)

Fig. 2.28 Comparative analysis of network topology considering simulation results (a) and experimental tests (b).

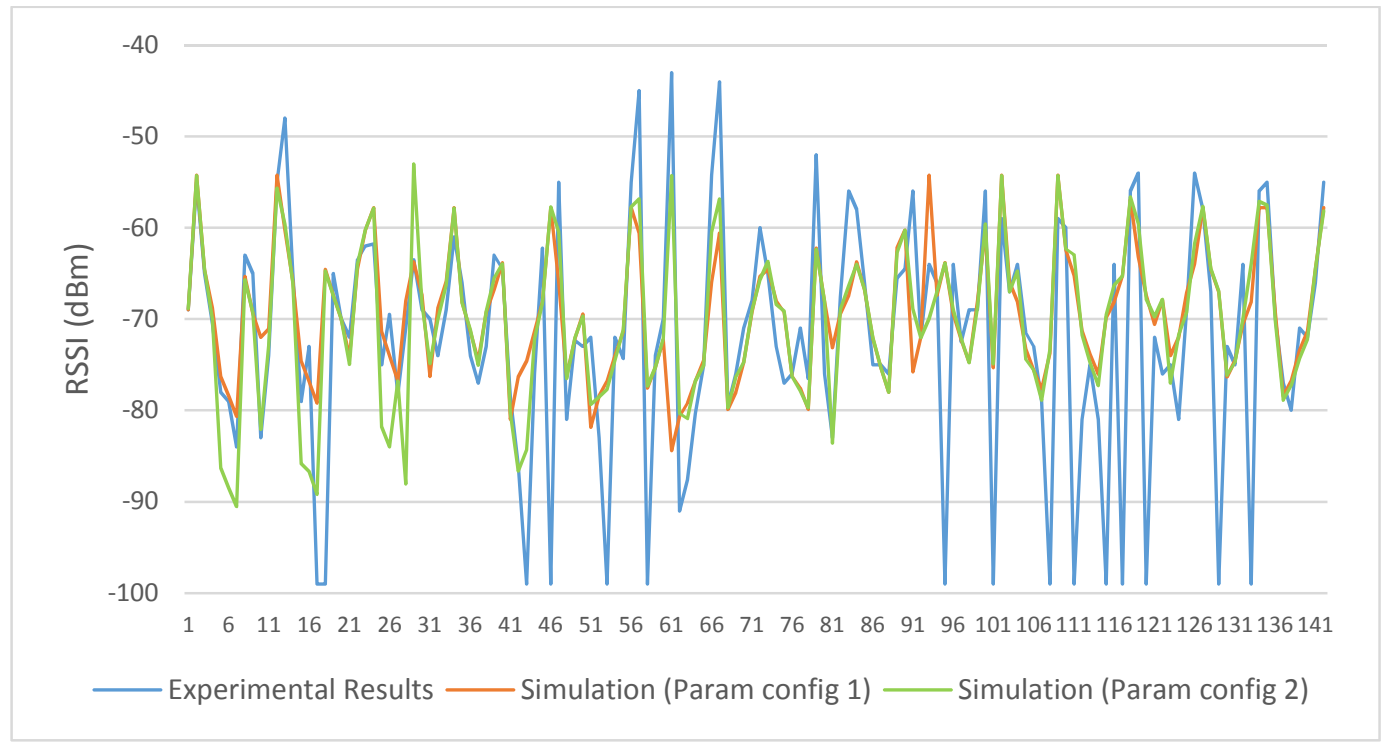

Fig. 2.29 Comparison of simulation and real results regarding accumulative RSSI values of node neighbourhoods.

A traffic load mechanism based on transmitting the measurement information from the sensor nodes with a periodicity of 4 samples per minute has been 
configured in order to analyse the battery consumption degradation in those critical nodes that are penalized by the packets distribution and forwarding actions. In Fig. 2.30, the experimental results related to battery level measurement evolution in nodes 1, 8, and 14 are shown in detail. According to the real tests, Node 1 represents the lowest lifetime of the critical nodes with a total of 8.3 hours of intensive traffic activity before running out of battery. The packet loss rate performance was also computed for every sensor node and the results are illustrated in Fig. 2.31, which allows calculating the overall packet drop rate of the deployment as percentage of data lost with respect to the total amount of transmitted data throughout the network, obtaining $90 \%$ of effective delivery, which thus represents a $9 \%$ of difference with respect to the estimated value by the simulation results $(99 \%)$.

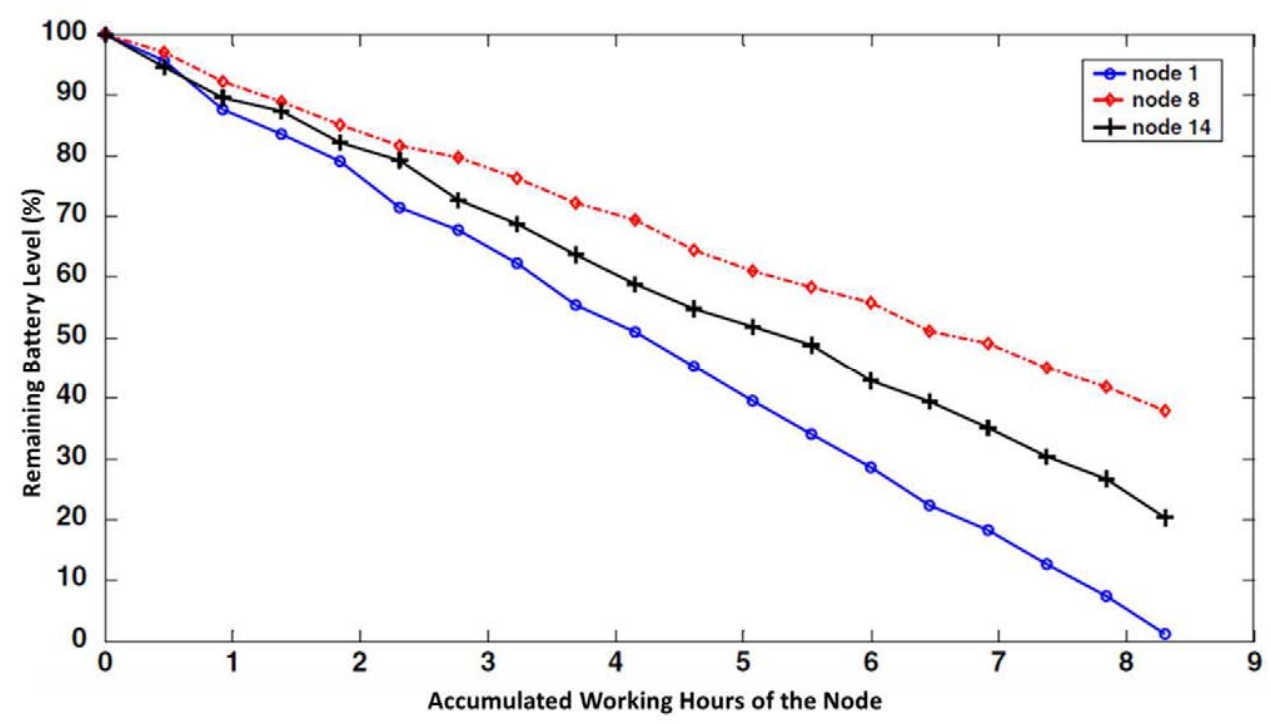

Fig. 2.30 experimental results of the battery monitoring of the sensor node and network lifetime.

Finally, real data related to environment monitoring of the indoor laboratory by using the deployed WSN was collected and process to provide an overall perspective of the sensor performance. As shown in Fig. 2.32, the average of the temperature and humidity taken from the recorded data related to each sensor node measurement produce stable values without significant changes during the observation time $\left(29^{\circ} \mathrm{C}\right.$ and $47 \%$ respectively, from $16: 00$ to $21: 00$ in the figure). 
In case of the light level of the lab, it can be seen that since the light system is turned off after a certain hour the value suddenly drops (from $90 \%$ to $0 \%$ ).

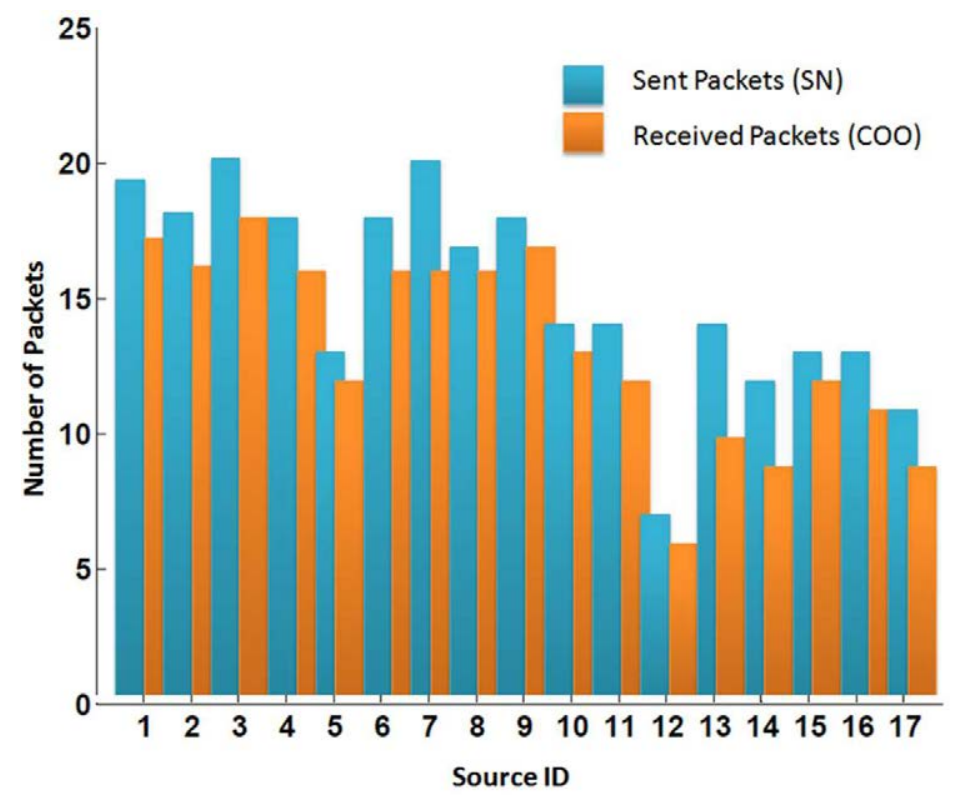

Fig. 2.31 Packet loss rate based on the overall network packet transmissions and receptions.

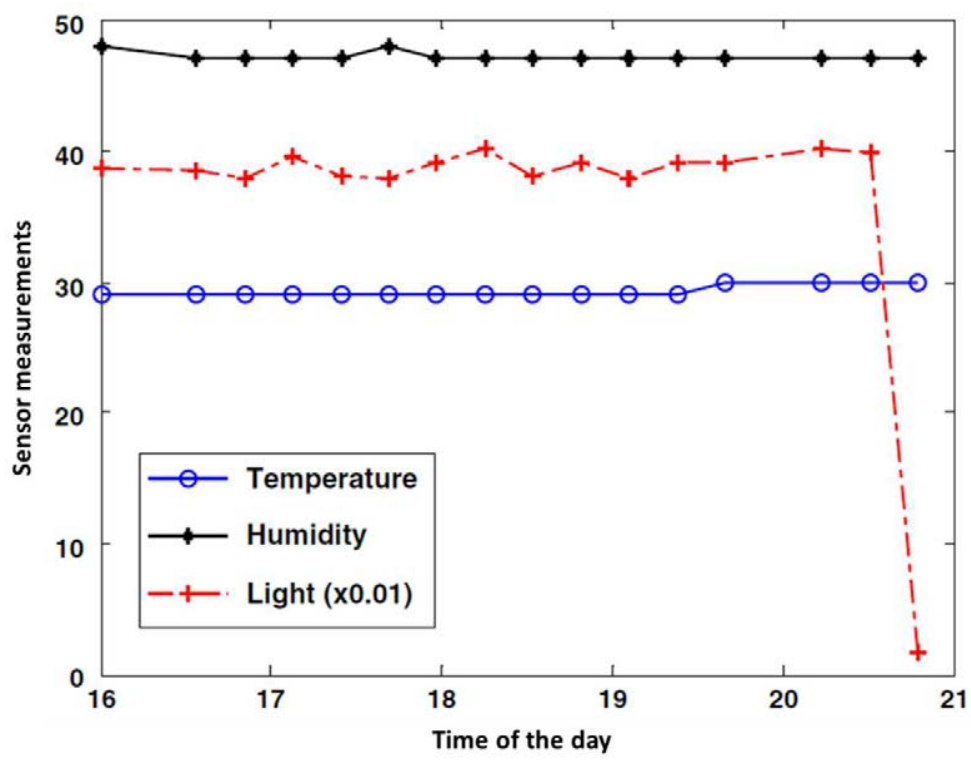

Fig. 2.32 WSN data monitoring related to the different sensor measurements. 


\subsubsection{The WSN modular platform within IoT urban automation systems}

The flexibility and modularity of the HW-SW integration platform together with the partial reprogramming strategies proposed in this chapter can leverage the integration of Cookie-based WSNs with a variety of technological approaches that are coming out with the evolution of the IoT paradigm. In this way, the experimental experience upon a new approach for urban automation is used to propose the integration of the HW-SW platform with a powerful solution for IoT. The basis of this system implementation lies on the use of an open-source gateway-level platform for gathering the diversity of different vendor solutions and platform heterogeneity into an IoT middleware-driven architecture.

This work-in-progress integration has emerged as a result of the collaboration with the Distributed Systems Group at Trinity College Dublin, in Ireland, where the implementation of an IoT platform for smart urban and home automation system was carried out to empower the applicability of self-learning techniques for autonomous demand-side energy management, with the support of WSNs.

OpenHAB [OpenHAB] has been conceived to meet requirements related to the interoperability and technology synergies within a hardware/protocol transparent support solution upon which different agents can be involved to create a comprehensive automation environment. Although specially focused on home automation systems, OpenHAB features can be extrapolated to a variety of urban networking cases where novel smart objects are to be seamless integrated. As in middleware architectural conceptions, OpenHAB does not focus on replacing manufacturer software capabilities, but instead offers a top-level integration environment for the correlation of heterogeneous technologies in such a way that an efficient management of the overall system operation is better achieved. This is not only important considering the fast growing of technologies that come out with the IoT evolution process, but also particularly from the user point of view, where the access and consumption of system services is to be enhanced.

OpenHAB defines as a basic principle the notion of Items, which represents the elemental abstraction for functionalities and capabilities. This produces a totally transparent use of an Item without being aware of implementation 
dependencies, i.e., no references to the underlying technology (protocol access, hardware interface, etc.) that implements the control of an attribute/parameter (for instance, temperature sensor) will appear at the Item-level management. This indeed fosters a straightforward reusability and replacement of low-level components without changes at the application-user abstraction layer. Likewise, in the other side of the system architecture the concept of Bindings is applied to implement platform, services and communication interfaces with the smart objects and things. OpenHAB leverages the integration of OSGi as the core runtime architecture of the middleware platform, which produces a pure Javabased solution.

The OSGi Alliance [OSGi] has defined a comprehensive Java-based dynamic software component model system that allows a modular creation and execution of functional components, called Bundles, as well as the interaction rules that determine how bundles will be connected. Apart from the Bundles (that are the software components to be developed by users/developers) OSGi specification defines the Service layer which determines how bundles are dynamically connected, and the Modules layer which establishes the rules for a bundle to export/import code. Besides, the Life-Cycle layer provides the API to dynamically install, start, stop, update and uninstall bundles [OSGi].

OpenHAB creates bindings from the basis of OSGi bundles and provides the OpenHAB Event Bus, which allows items to access the services provided by the component implementation, in addition to performing a decoupled communication among bindings. When events occur or actions are performed, bundles shall notify other involved bundles via the common communication bus, so that a proper cross-connectivity is achieved. Although this can be successfully applied to stateless services, OpenHAB also implements an Item Repository in order to keep track of the state of the Items. In this way, two main events can be distinguished within the Event Bus operation. On one hand, commands allow triggering particular actions or state changes in a binding and thus in a device, and on the other hand state updates allow properly notifying such a modification to the involved actors. These interactions are supported by a 
$\mathrm{pub} / \mathrm{sub}$ mechanism from the basis of the OSGi EventAdmin service, and associated event delivery strategies.

Three main levels of user/developer interactions with the middleware can be identified, as depicted in Fig. 2.33.

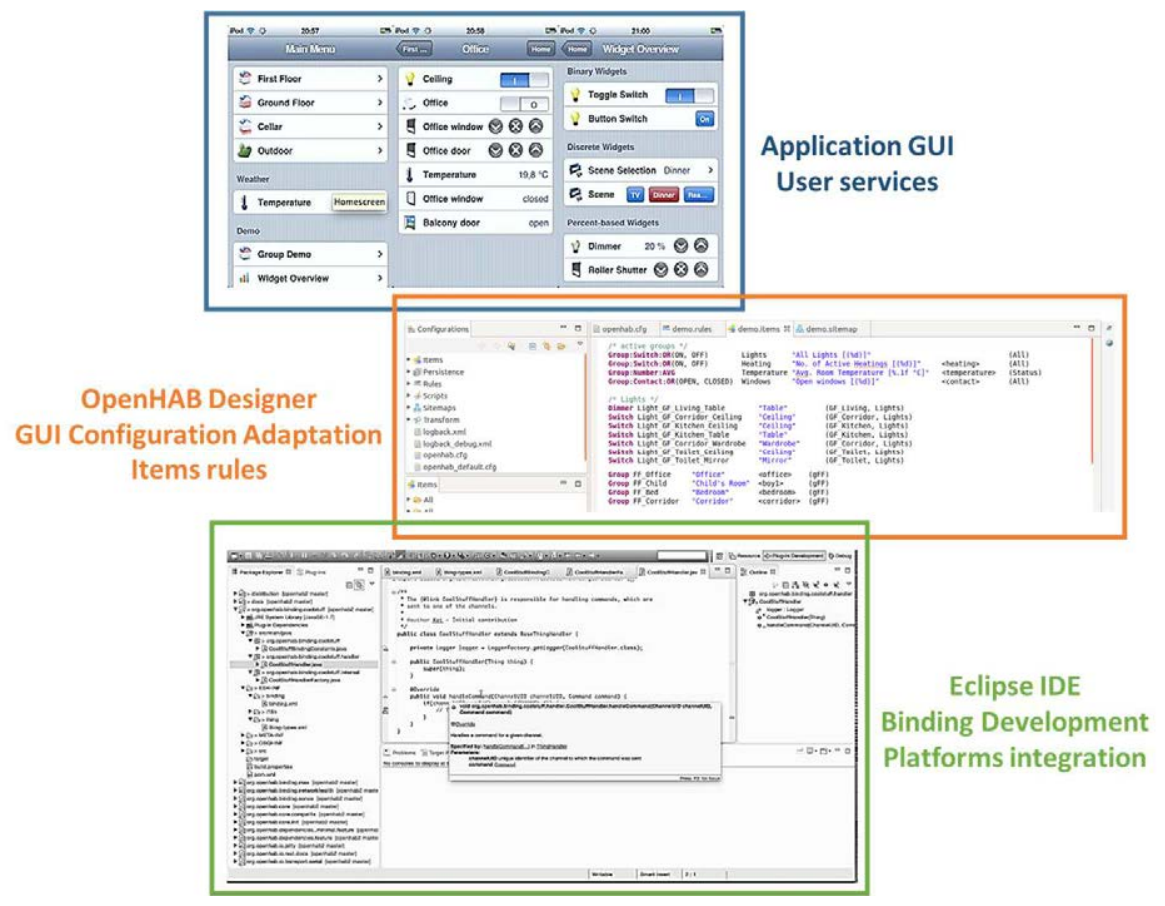

Fig. 2.33 Three different levels of User/developer interaction [OpenHAB].

First, user services, which corresponds to the top-level application, and relies on the GUI from which users can interact with the services and the deployed systems, by receiving information about the subscribed items and perform actions on them. The second level refers to the application development, in which users can create and customize their own automation system, by making use of available bindings to create and configure Items as well as OpenHAB runtime environment. For this, OpenHAB designer allows an easy edition of configuration files such as .items, .sitemap, .cfg and .rules, so that the high-level implementation of the target application can be customized, including the GUI that will be visualized at the user side. The third interaction level relies on the development and integration of platforms proper, technologies and services by using the OpenHAB binding structure, i.e., developers can create their modular 
components and integrate them in the system architecture. This is carried out through the OpenHAB development functionalities under Eclipse IDE, by using interfaces for Event bus communication, command and actions management, and bundle configuration. After validation, bindings are exported and encapsulated as add-ons in the runtime environment, which then are dynamically initialized and started.

The aim of integrating this approach with the modular platform focuses on merging already existing technologies that are present in OpenHAB in the form of available Bindings as well as the gateway-side runtime environment with the development of new modular components, so as to create an embedded heterogeneous management system for urban automation networks. In order to accomplish this, a combination of low-rate and low-power wireless sensor devices and IP-based elements is realized, as shown in Fig. 2.34, where the overall view of the proposed system is highlighted. The kernel of the system is an embedded Gateway platform upon which the OpenHAB runtime core is included. For this, a Raspberry Pi 2 B combined with the Raspbian Operating System [Raspberry] is considered as the central management component while the correlation with the sensor/actuator objects is realized by using three main communication approaches, as follows:

- Wi-Fi based wireless communication: this encompasses binding external interfaces with smart devices in order to control, for instance, the state of home/urban powered-elements.

- Wi-Fi/Serial virtual interface to the WSN: the connection of the management elements and bundle components to the wireless distributed sensor systems based on Cookie nodes is performed following two main concepts. On one hand, a WSN Network Interface can be included from the basis of a serial connection between the embedded platform and a Cookie node (taking advantage of the VPort approach proposed early in this chapter). The later provides direct data access to the sensor network by encapsulating communication control dependencies and hardware details. On the other hand, a low-power-Wi-Fi-to-Low-rate Cookie 
Node Adapter allows a seamless integration of the WSN data gathering with the embedded platform, based on the Wi-Fi-based communication layer also proposed in this work.

- AP connection for internal-external data accessibility: while the internal communication among participant elements can be carried out from the basis of Ad-Hoc networking scheme, an AP-based infrastructure suites well for external remote access to the smart management system, so that users have both options to monitor the behaviour of the overall application and related services.

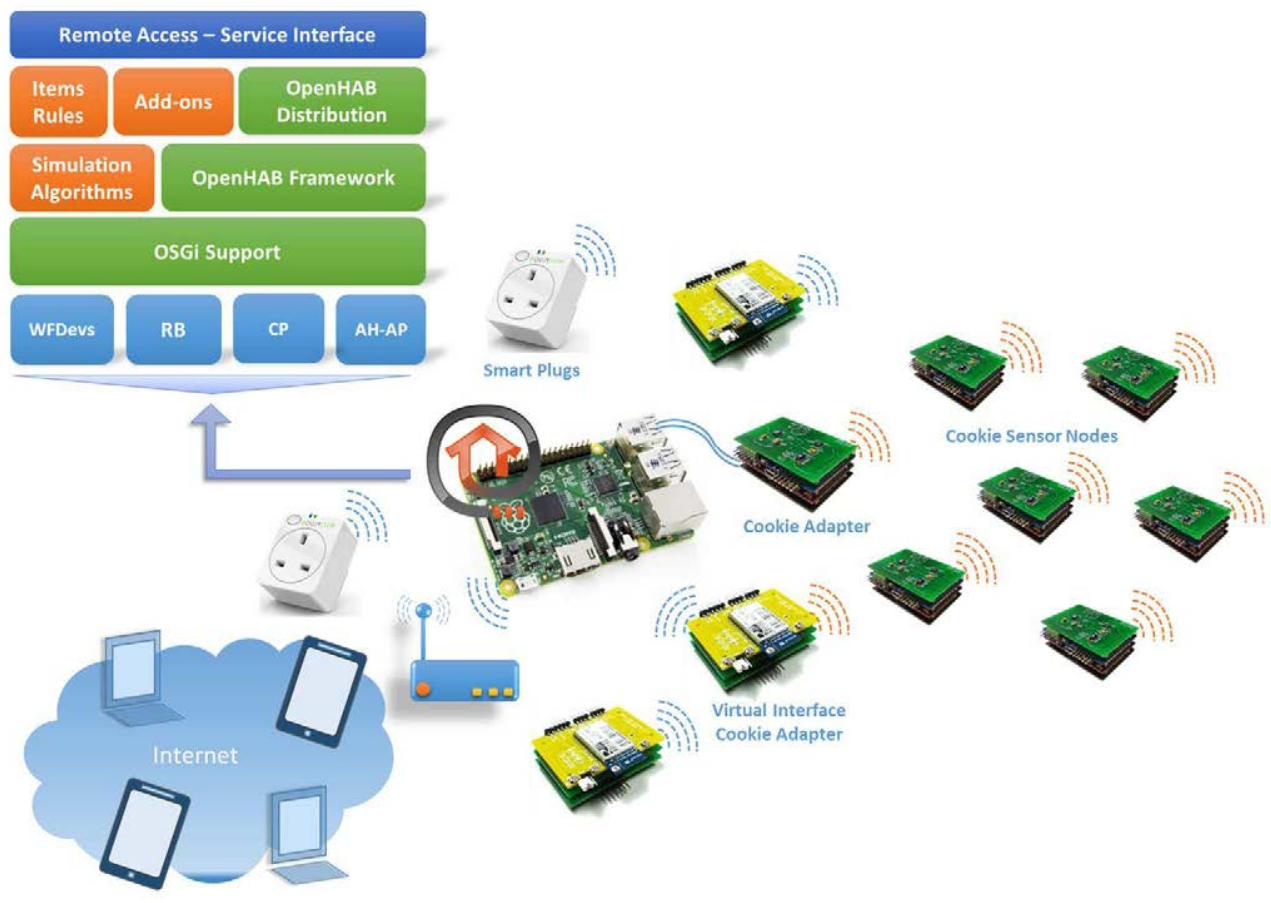

Fig. 2.34 General Overview of the proposed system integration.

In this direction, the system definition is composed of four main add-ons in line with the aforementioned interface definitions and encompassing the integration of various types of smart objects:

- _SmartPlug_Binding: includes the integration of vendor interfaces to access the services of Smart Plugs such as WifiPlug [WifiPlug]. Main capabilities to control this smart devices lies on switching Items to manage the state of the plug in relation with events on light and 
temperature sensor measurements, as well as timing windows to configure the operational duty cycles.

- _PubSub_Binding: encompasses the reusability of binding functionalities already included in OpenHAB such as an MQTT client [Hunkeler'08] to configure and establish publish-subscribe mechanisms with a distributed broker. This is useful when employing technologies related to smart metering applications such as Flucksometer [Flukso], which has been used for implementing the demand-side management system commented in the following paragraphs.

- _CookieServiceInface_Binding: implements the Cookie service management, considering the configuration of platform and sensing measurement attributes as well as topics subscription, including network event handling and WSN data aggregation.

- _CookieVirtualInface_Binding: establishes the virtual interface with the sensor network through the configuration of the Cookie node adapter connection.

The service provision of the Cookie-based sensor network can be realized by considering a top-level platform profile combined with a control frame handling, which allow the configuration of sensor data publication, attributes monitoring and configuration. Fig. 2.35 highlights common parameters that are considered within the implementation of this Cookie profile, including reference identifiers for the type of parameters and the sensor classification profile.

As mentioned before, this proposal came out as a result of the integration of the intelligent IoT Gateway in an autonomous demand-side management system for residential/community energy consumption awareness. The main idea of this system relies on estimating the energy consumption of users (based on consumer profile) in order to predict those loads that can be deferred to low demand periods instead of triggering then in critical consumption peaks. 


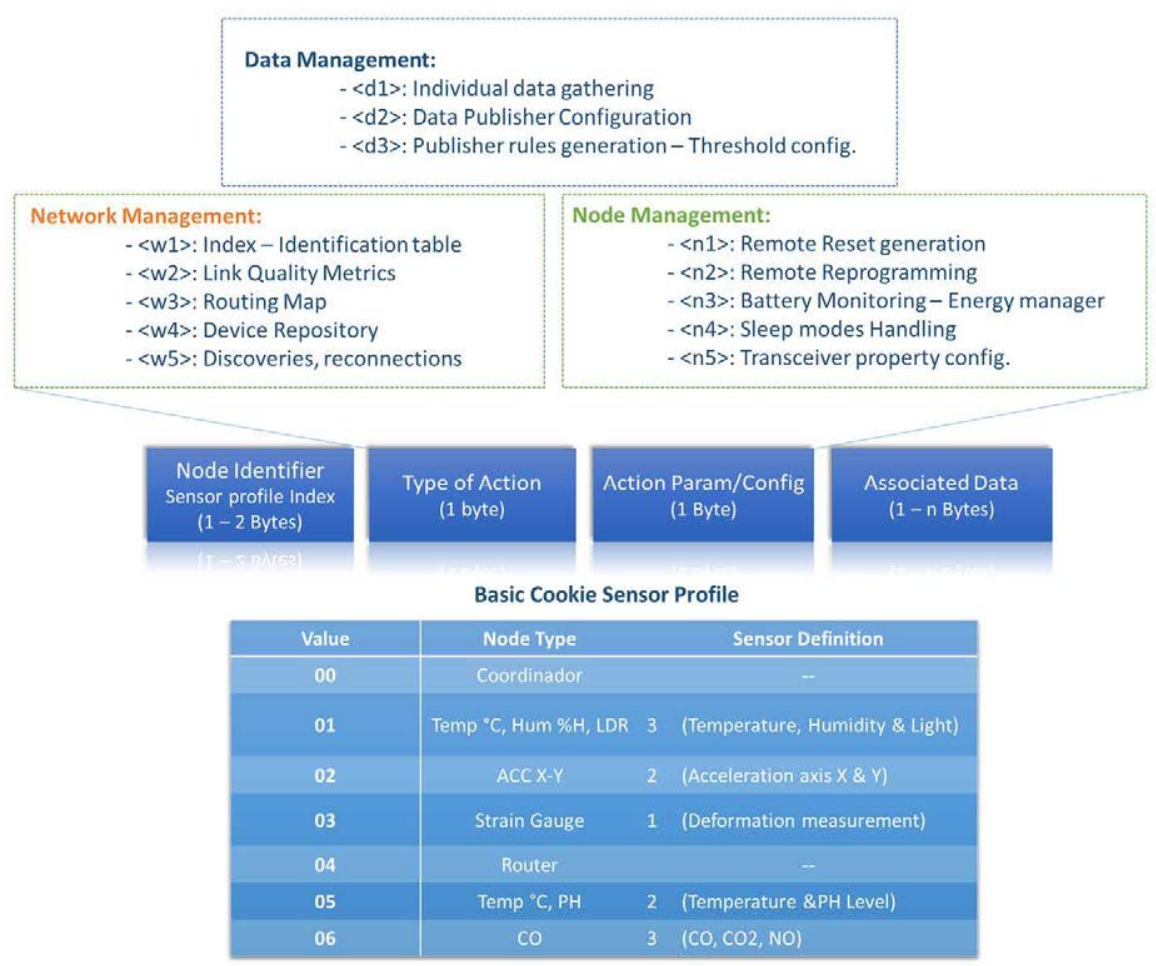

Fig. 2.35 Control frame and management interface for remote access and data aggregation of the top-level platform profile.

The smart management system implemented during the research activities at DSG addresses the integration of a multi-agent system by using a reinforcement learning approach, where the interface to the optimization engines are implemented as modular bindings to be controlled through OpenHAB. Based on the information provided by the IoT Gateway to the learning engine (related to the real behaviour of the sensor/actuator network according to the user + agent activity), the intelligent algorithms will automatically execute actions on the connected elements to reduce the energy consumption and self-adapt to the demand profile.

Fig. 2.36 shows the implemented architecture where the gateway-side encompasses the Multi-Agent System Interface, the integration of virtual elements in the form of simulation bindings for modelling the behaviour of particular elements, and bindings for sensor and actuator interfaces as well as the smart metering system, from which the runtime energy consumption is 
obtained and fed back to the estimation engine. This system has been successfully created and integrated with both the IoT sensor/actuator elements and the learning engine interface, whose prove of concept was exhibited by conforming an interactive platform demonstrator within the scope of the Future Cities research workshop at TCD.

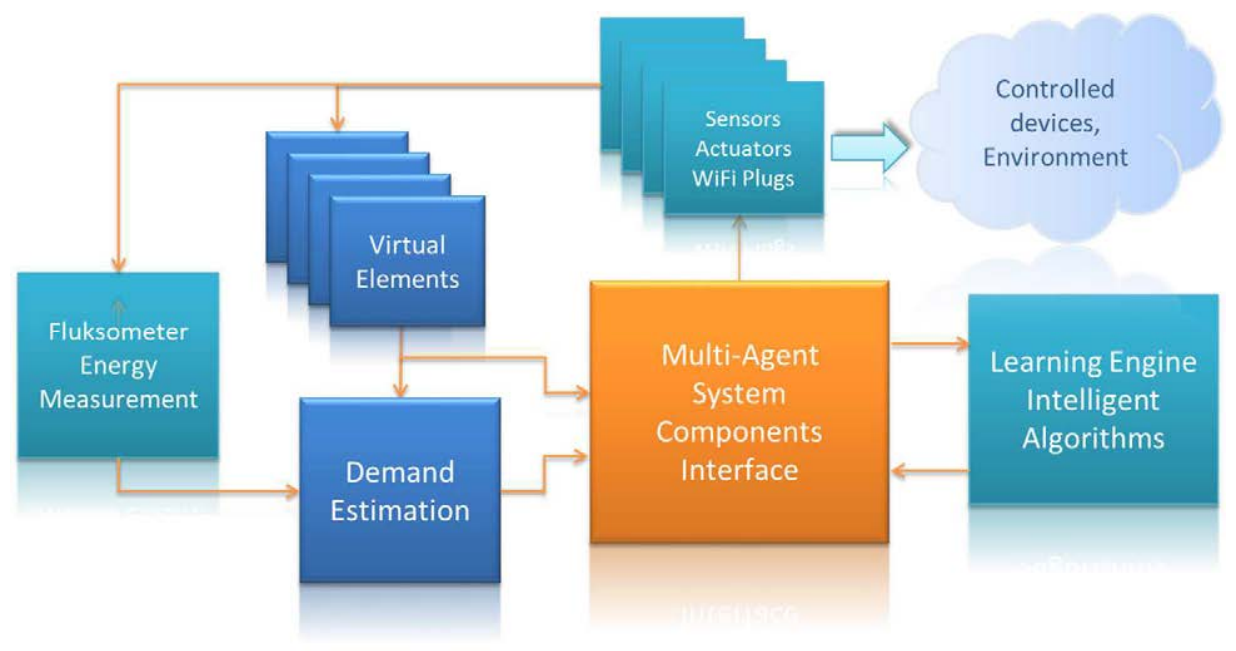

Fig. 2.36 Smart management system for urban/home automation based on the IoT Gateway implementation.

\subsection{Conclusions}

The real experimentation during the pre-deployment stage of the WSN development process is to be supported by flexible prototyping mechanisms as well as testing capabilities for further evaluating the sensor network under implementation. In this chapter, a hybrid approach towards the experimental analysis of WSNs has been proposed focusing the testbed infrastructure on a combination of a well-defined backchannel-based architecture with an adaptable testing support platform, which can accommodate readjustments in accordance with the specifics of the target network under test. Such a hybrid nature refers not only to the support channel for the remote experimentation alongside the actual behaviour of the system (structural + "nomadic-based" composition), but also to the applicability of the designed support functionalities in the operational 
stage of the deployment network, thanks to the embedded focus of part of the testing functionalities.

While most of the testbeds proposed in the state of the art concentrate on the general structure of the laboratory deployment itself, in this work special attention is paid to supporting the analysis and verification process from the basis of a well-defined experimentation framework, under which users can test different HW-SW prototypes at runtime, particularly considering the partial reprogramming scheme as well as the in-node functional bocks for remote debugging at different implementation levels. The proposed support platform based on the Cookies modular architecture focuses on including further analysis mechanisms beyond the high-level application-wise testing perspective encountered in traditional testbed arrays, and addresses three of the pillars for a testbed support platform: a) reprogramming, b) monitoring and management, c) debugging and interaction.

The MS-PRS allows not only the optimization of the reprogramming tasks both within the testbed infrastructure and the WSN operation itself, but also the modularity of the embedded software implementation of the sensor node (in line with the flexibility of the hardware platform), which enriches the way functional components can be effectively and independently allocated, updated and replaced without affecting the overall structure of the system. Such approach has been tested within various real use cases, where the suitability and workability of the proposed architecture was verified throughout the performance of remote monitoring, debugging and reprogramming tasks of the sensor networks under experiment, contributing to obtaining a complete operation of the target WSNbased applications.

Besides the test cases presented in this chapter, the proposed HW-SW integration platform has supported the implementation of Cookie-based WSN industrial applications, including sensor network deployments and experimental use cases within the scope of research projects. This encompassed not only the development and testing stages of the system prior to its final release, but also the embedded management of the modular platform when the operational stage of the network took place. 


\subsection{Bibliography of the chapter}

[802.3]

[ADuC841]

[ADXL210]

[Ag9400]

[AT91SAM7X]

[Bal'09]

[Brouwers'09]

[C8051F930]

[CC2420]

[Cyclone]

[Dimitriou'07]

[Dunkels'05]

[Dunkels'06]
IEEE 802.3 Working Group, standards for Ethernet network, http://www.ieee802.org/3/

ADuC841 Microcontroller, Analog Devices, http://www.analog.com/

ADXL210 Low-Cost $\pm 10 \mathrm{~g}$ Dual-Axis Accelerometer http://www.analog.com/en/products/obsolete/adxl210.html

IEEE 802.3af PoE PD, Ag9400 Series Sivertel, http:/ / www.silvertel.com/ products/ poe-power-over-ethernet/26-poemodules/165-ag9400.html

32-bit ARM-based microcontroller, Atmel SAM7X series http://www.atmel.com/devices/SAM7X256.aspx

M. Bal, H. Xue, W. Shen, and H. Ghenniwa, "A testbed for localization and tracking in wireless sensor networks" in IEEE Systems, Man and Cybernetics, SMC 2009. pp. $3581-3586$.

N. Brouwers, K. Langendoen, P. Corke, “Darjeeling, a feature-rich VM for the resource poor", In 7th ACM Conference on Embedded Networked Sensor Systems (SenSys'09), pp. 169-182, New York, NY, USA, 2009.

C8051F92x-F93x Ultra Low-Power MCUs, Silicon Labs, http://www.silabs.com/

Single-Chip 2.4 GHz IEEE 802.15.4 Compliant and ZigBee Ready RF Transceiver, http://www.ti.com/product/CC2420

FPGA Cyclone II Family, Altera http://wl.altera.com/devices/fpga/cyclone2/overview/cy2-overview.html

T. Dimitriou, J. Kolokouris, and N. Zarokostas, “Sensenet: a wireless sensor network testbed", In Symposium on Modeling, analysis, and simulation of wireless and mobile systems (MSWiM '07). ACM, pp. 143-150, New York, USA, 2007.

A. Dunkels, B. Grönvall, T. Voigt, "Contiki - a lightweight and flexible operating system for tiny networked sensors", in 29th IEEE International Conference on Local Computer Networks, pp. 455-462, 2004.

A. Dunkels, N. Finne, J. Eriksson, T. Voigt, "Run-time dynamic linking for reprogramming wireless sensor networks" In 4th international conference on Embedded networked sensor systems (SenSys'06), pp. 15-28, New York, NY, USA, 2006. 
[ETRX2] ETRX2 2.4GHz ISM band transceiver based on the Ember EM250 single chip ZigBee/IEEE802.15.4 solution, Telegesis http://www.telegesis.com/support/legacy-products/etrx2-zigbee-module/

[Fischer'09] I. Chatzigiannakis, S. Fischer, C. Koninis, et al., “WISEBED: An Open LargeScale Wireless Sensor Network Testbed", In International conference on Sensor Applications, Experimentation, and Logistics (SENSAPPEAL'09), Athens, 2009.

[Flukso] Flukso, an open-source web-based community metering application, https://www.flukso.net/

[Förster'11] A. Förster et al., "Poster abstract: MOTEL: Towards flexible mobile wireless sensor network testbeds," in European Conference on Wireless Sensor Networks (EWSN'11), Poster Session, pp. 1-2, Germany, Feb. 2011.

[Gauger'09] M. Gauger, P. Marrón, C. Niedermeier, “TinyModules: code module exchange in TinyOS", in 6th international conference on Networked sensing systems (INSS'09), pp. 175-182, Piscataway, NJ, USA, 2009.

[GS110TP] Netgear GS110TP, 8-port Gigabit PoE Smart Managed Switch http://support.netgear.com/product/GS110TP

[Han'05] C. Han, R. Kumar, R. Shea, E. Kohler, M. Srivastava, “A dynamic operating system for sensor nodes", In 3rd international conference on Mobile systems, applications, and services (MobiSys'05), pp. 163-176, New York, USA, 2005.

[Handziski'06] V. Handziski, A. Köpke, A. Willig, and Adam Wolisz, “TWIST: a scalable and reconfigurable testbed for wireless indoor experiments with sensor networks" In international workshop on Multi-hop ad hoc networks: from theory to reality (REALMAN '06). ACM, pp. 63-70, New York, USA, 2006.

[He'14] D. He, “A novel methodology for planning reliable wireless sensor networks", PhD Thesis, Universidad Politécnica de Madrid, 2014.

[Hermans'10] F. Hermans, O. Rensfelt, P. Gunningberg, L.A. Larzon, E. Ngai, “Sensei-UU - A Relocatable WSN Testbed Supporting Repeatable Node Mobility", in international workshop on Wireless network testbeds, experimental evaluation and characterization (WiNTECH '10), pp. 63-70, New York, USA, 2010.

[HEX] ARM Technical Support, "Intel Hex File Format". [Online]. Available: http://www.keil.com/support/docs/1584.

[Horneber'14] J. Horneber and A. Hergenröder, “A Survey on Testbeds and Experimentation Environments for Wireless Sensor Networks", in IEEE Communication Surveys \& Tutorials, Vol. 16, No. 4, pp. 1820 - 1838, November 2014. 
[Huangfu'10]

[Hui'04]

[Hunkeler'08]

[IGLOO]

[Indriya]

[ISD51]

[Jeong'09]

[Keil]

[Koshy'05]

[Levis'02]

[Levis'04]

[Liu'11]
W. Huangfu, L. Sun, and J. Liu, “A High-Accuracy Nonintrusive Networking Testbed for Wireless Sensor Networks", in EURASIP Journal on Wireless Communications and Networking, Vol. 2010, 15 pages, doi:10.1155/2010/642531.

J. W. Hui and D. Culler, "The Dynamic Behavior of a Data Dissemination Protocol for Network Programming at Scale Categories and Subject Descriptors", In International Conference on Embedded Networked Sensor Systems (SenSys '04), pages 81-94, Baltimore, USA, 2004.

U. Hunkeler, H. L. Truong and A. Stanford-Clark, "MQTT-S - A publish/subscribe protocol for Wireless Sensor Networks," in Conference on Communication Systems Software and Middleware and Workshops, pp. 791-798, Bangalore, 2008.

IGLOO Low-Power Flash FPGAs family, Microsemi, http://www.microsemi.com/products/fpga-soc/fpga/igloo-overview

M. Doddavenkatappa, M. C. Chan, and A. L. Ananda, "Indriya: A lowcost, 3D wireless sensor network testbed," in TridentCom, pp. 302-316, Apr. 2011.

In-System Debugger, ARMKEIL microcontroller tools, http://www.keil.com/c51/isd51.asp

J. Jeong, D. Culler, "Incremental Network Programming for Wireless Sensors", in International Journal of Communications, Network and System Sciences (IJCNS), Vol. 2 No. 5, pp. 433-452, 2009.

Keil development tools for the 8051 Microcontroller Architecture, http://www.keil.com/c51/

J. Koshy and R. Pandey, "Remote incremental linking for energy-efficient reprogramming of sensor networks," in Second European Workshop on Wireless Sensor Networks (EWSN'05), pp. 354-365, Istanbul, Turkey, 2005.

P. Levis, D. Culler, "Maté: a tiny virtual machine for sensor networks", in 10th international conference on Architectural support for programming languages and operating systems (ASPLOS'02), pp. 85-95, New York, NY, USA, 2002.

P. Levis, N. Patel, S. Shenker, D. Culler, "Trickle: A self-regulating algorithm for code propagation and maintenance in wireless sensor networks", Technical report, University of California at Berkeley, 2004.

X. Liu, K. M. Hou, H. Shi, C. Guo, H. Zhou, "Efficient and portable reprogramming method for high resource-constraint wireless sensor nodes," in IEEE 7th International Conference on Wireless and Mobile Computing, Networking and Communications (WiMob'11), Wuhan, pp. 455-460, 2011. 
[MIB600] Memsic MIB600 Gateway for $\quad$ MICA motes http://www.memsic.com/wireless-sensor-networks/MIB600.

[MSP430] 16-bits Ultra-low-power microcontroller produced by Texas Instruments, http://www.ti.com/product/MSP430F5438A

[Munawar'10] W. Munawar, M. H. Alizai, O. Landsiedel, K. Wehrle, "Dynamic TinyOS: Modular and Transparent Incremental Code-Updates for Sensor Networks", in IEEE International Conference on Communications (ICC'10), pp. 1-6, Cape Town, South Africa, 2010.

[NetBridge] Stargate NetBridge embedded sensor network gateway, Crossbow Technology, http://www-xbow-com/

[NGW100] NGW100 Network Gateway Kit, Atmel Corporation http://www.atmel.com/tools/NGW100MKIINETWORKGATEWAYKIT.as $\mathrm{px}$

[NSLU2] Network Storage Link for USB 2.0 Disk Drives from Linksys, http://www.linksys.com

[OpenHAB] OpenHAB, a framework for home automation systems and IoT technology integration, available online: http:/ / www.openhab.org/

[OSGi] OSGi, Dynamic module framework and specifications for Java programming, OSGi Alliance, https://www.osgi.org/

[Panta'07] R. K. Panta, I. Khalil, S. Bagchi, "Stream: Low Overhead Wireless Reprogramming for Sensor Networks", in 26th IEEE International Conference on Computer Communications (INFOCOM'07), pp. 928-936, Anchorage, AK, 2007.

[Panta'11] R. K. Panta, S. Bagchi, S. P. Midkiff, "Efficient incremental code update for sensor networks," ACM Transactions on Sensor Networks, vol. 7, no. 4, pp. 132, Feb. 2011.

[Polastre'05] J. Polastre, R. Szewczyk, D. Culler, “Telos: enabling ultra-low power wireless research", in International Symposium on Information Processing in Sensor Networks (IPSN'05), pp. 364-369, April 2005.

[Portilla'07] J. Portilla, T. Riesgo, A. Abril, A. de Castro, "Rapid prototyping for multiapplication sensor networking", 12 November 2007, SPIE Newsroom. DOI: $10.1117 / 2.1200711 .0851$.

[Portilla'10] J. Portilla, "Plataforma Modular e Interfaces Genéricas de Transductores para Redes de Sensores Inalámbricas", PhD Thesis, Universidad Politécnica de Madrid, 2010. 
[Raspberry] Raspberry Pi 2 Model B, the second generation Raspberry Pi, and Raspbian Debian-based Operating System, https://www.raspberrypi.org

[RN131] RN131 ultra-low power embedded 802.11 b/g Wi-Fi module http:/ /www.microchip.com/wwwproducts/Devices.aspx?product=RN131

[Sakamuri'08] D. Sakamuri, "NetEye: A wireless sensor network testbed", Master's thesis, School of Wayne State University, Detroit, MI, USA, 2008.

[Sheu'08] J. Sheu, C. Chang, C. Sun, W. Hu, “WSNTB: A Testbed for Heterogeneous Wireless Sensor Networks", in International Conference on Ubi-Media Computing (U-Media'12), 338 - 343, China, 2008.

[SHT11] SHT1x Digital Humidity \& Temperature Sensor, Sensirion https://www.sensirion.com/products/digital-humidity-sensors-for-reliablemeasurements/digital-humidity-sensors-for-accurate-measurements/

[SI-52008-F] Bel Fuse components, http://www.belfuse.com/

[Slipp'08] J. Slipp, C. Ma, N. Polu, J. Nicholson, M. Murillo, S. Hussain, “Winter: Architecture and applications of a wireless industrial sensor network testbed for radio-harsh environments", in IEEE conference of Communication Networks and Services Research Conference (CNSR'08), pp. 422 -431, 2008.

[Tozlu'12] S. Tozlu, M. Senel, M. Wei, A. Keshavarzian, “Wi-Fi Enabled Sensors for Internet of Things: A Practical Approach", in IEEE Communications Magazine, Vol. 50, No. 6, pp. 134 - 143, 2012.

[uVision]

[W5200]

ARMKeil uVision IDE and uVision Debugger, http://www2.keil.com/mdk5/uvision/

W5200 Hardwired TCP/IP embedded Ethernet controller, WIZnet http://www.wiznet.co.kr/product-item/w5200/

[Werner'05] G. Werner-Allen, P. Swieskowski, M. Welsh, “Motelab: a wireless sensor network testbed" in International Symposium on Information Processing in Sensor Networks (IPSN'05), pp. 483 - 488, USA, April 2005.

[Weyer'09] C. Weyer, C. Renner, V. Turau, and H. Frey, "A roadmap for hardware and software support for developing energy-efficient sensor networks", in Proc. 8th GI/ITG KuVS FGSN, pp. 67-70, Aug. 2009.

[WifiPlug] Wi-Fi Plug for remote wireless control of powered devices, http://www.wifiplug.co.uk/

[WL-500G] Asus WL-500G, OpenWrt distribution https://wiki.openwrt.org/toh/asus/wl500gp 
[YI'13] K. Yi, R. Feng, N. Yu, P. Chen, "PARED: A testbed with parallel reprogramming and multi-channel debugging for WSNs," in IEEE Wireless Communications and Networking Conference (WCNC'13), pp.4630-4635, China, April 2013.

[ZigBit] ZigBit 900MHz Module with Balanced RF Output, 784/868/915MHz 802.15.4/ZigBee OEM,

Atmel http://www.atmel.com/tools/ZIGBIT900MHZMODULEWITHBALANCED RFOUTPUT.aspx 



\section{Chapter 3}

\section{DEPLOYMENT AND ON-SITE}

ANALYSIS OF WIRELESS SENSOR NETWORKS

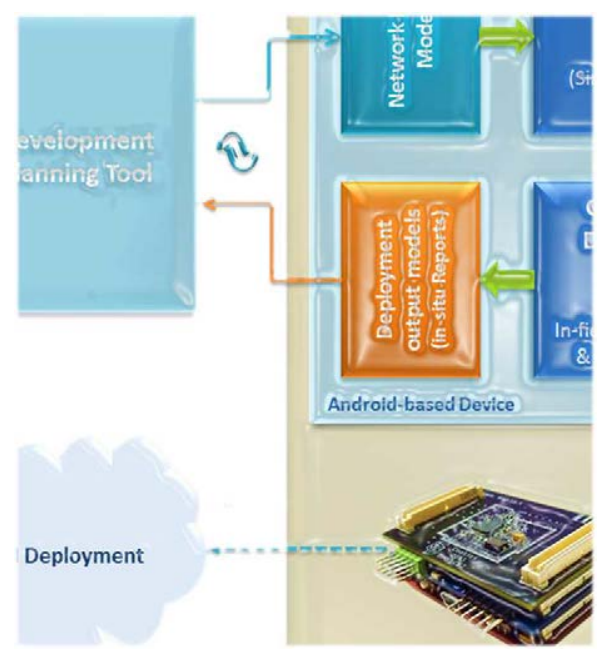

In this chapter the main elements that take part in the analysis, configuration and optimization of WSN deployments are addressed together with the description of a new in-field toolset that is proposed in this thesis to assist the site installation, parametrization, performance evaluation and maintenance of the sensor nodes at the target environment, particularly considering the complexity of such tasks in medium/large-scale wireless mesh networks.

The importance of creating a proper synergy between the development and simulation stages with the real deployment of WSNs represents a key aspect to guarantee not only the final cohesion of the sensor network but also the operability and maintainability of the overall wireless platform in field. To this extent, although there have been significant contributions in the state of the art related to planning and modelling sensor systems, the provision of well-defined tools and methodologies to carry out the on-site deployment of WSNs is still an open issue to be further explored. 



\section{Deployment and on-site analysis of Wireless Sensor Networks}

\subsection{Introduction}

One of the most critical stages during the development cycle of WSN-based applications is related to the intrinsic deployment of the sensor nodes and its onsite verification/optimization as a fundamental step for the final operational release of the system. As described in previous chapters, the process of designing and implementing a distributed sensor network based on the integration of hardware and software technologies can be supported by different tools that aim at providing a way of planning, developing, simulating and testing the conceived prototypes according to the initial requirements of the system.

Planning and simulation strategies attempt to approach the deployment of sensor nodes in such a way that the efficiency of the platform in terms of cost, coverage, network topology and connectivity is maximized with a minimum number of elements to be installed. Hardware design, embedded software development, system modelling and behavioural simulation techniques aim at addressing the input operational requirements of the target application while coping with the challenges associated with the development of the autonomous multi-hop WSN, and particularly considering resources and energy constraints. The testbed support architecture proposed in the previous chapter serves as a platform synergy to experiment and validate implementation prototypes as well as novel technologies within the aforementioned strategies.

However, when tackling the real on-site deployment of wireless sensor networks important challenges regarding the actual specifics and conditions of the target scenario are to be taken into account, and they may certainly influence the operability, efficiency and performance of the released system. Moreover, the process of deploying and commissioning the theoretically planned WSN should 
lead to promoting further in-field optimizations and performance analysis, since the installation and debugging actions may significantly affect the overall interrelation of the wireless distributed platforms, not only considering the individual elements that compose the sensor network but also the system as a whole.

In this work the concept of WSN commissioning refers to those actions that involve the in-field installation, configuration, deployment performance evaluation and diagnosis in order to properly start up the target sensor network. Such process shall result not only on the operation of the overall system but also on the ability to remotely monitor the deployed nodes accordingly.

In this direction, one of the main aspects to be allowed for when carrying out the deployment and verification activities relates to the performance assessment and enhancement of network connectivity issues, not only regarding wireless communication links but also the multi-hop mechanisms to be used, especially when targeting wireless mesh networking scenarios. Moreover, an efficient deployment methodology should contemplate the transient power and resource consumption during the installation, parametrization and reprogramming steps, the time spent during the commissioning stage depending on the evaluation strategy to be adopted, and the actual placement problems that come out with the in-field WSN establishment, mainly in terms of coverage, processing and sensing performance.

However, there is an important lack of well-defined frameworks, methodologies and tools to carry out the on-site deployment, commissioning, validation and maintenance activities as a support to assuring the final long-term operability of the WSN-based system. The effective combination of modelling and planning strategies with efficient deployment and on-site performance assessment techniques are to be taken into account to guarantee the final reliability and autonomy the WSN, but such a synergy is still an open issue that has not been properly addressed in the literature yet. 


\subsection{On-site deployment strategies in the state of the art: an open issue}

Unlike the research field of WSN testbeds in which there have been different types of solutions in the literature to approach the application prototyping and experimentation at the pre-deployment stage, there is a shortage of established works to tackle the problem of deploying, commissioning and maintaining WSNs such that the cohesion and runtime operation of the real network is efficiently achieved during the on-site configuration/parametrization, performance evaluation and release activities. Traditionally, the deployment of sensor networks has been addressed from a planning/simulating perspective, though the actual behaviour of the implemented platform might be far from the expected operability and theoretical results of the designed system.

Some contributions have centred their attention on embedded initialization mechanisms such as the work described in [Meier'09], in which authors propose the adoption of a flooding-based synchronization strategy to configure discovery and expiration time windows within which nodes can perform neighbour search and link assessment. Operational modes are triggered by dedicated points of the network, such as sink and root nodes, from which the mode changing process is disseminated. When a network call is triggered and propagated throughout the network deployment, nodes configure a polling interval that serves as a reference period for neighbour discovery. Since a process of network flooding during the discovery phase is performed over the whole network, both collisions avoidance and starting-expiration time shifting among nodes are to be considered as major concerns to assure the accuracy of the technique. More than a maintenance strategy, it main focus relies on providing network layer with a more consistent discovery mechanism prior to running routing and dissemination tasks, particularly in case of protocols such as CTP [Gnawali'13].

A maintenance system to be embedded into the sensor nodes has also been proposed in [Cao'08], which intends to provide maintenance services during the operational stage of the WSN by defining system coherency state policies. Such maintenance policies mainly rely on triggering conditions when encountering 
sensing failures and communication coverage failures, especially considering protocol coherent states, which are organized as Global view of coherent states (system-wise, such as time synchronization requirements), and Local view of coherent states (node-wise, such as neighbourhood awareness). The maintenance engine collects the coherent requirements and states, and then floods the list of protocols for the maintenance service throughout the network. The global state monitoring is realized by the base station according to the policy specification, which collects and processes the performance information from individual nodes based on their local monitor. This local monitoring system running on the sensor nodes is configured with the list of protocol policies propagated from the base-station controller so that the maintenance services are set to gather and transmit specified states, such as battery level remaining, number of neighbours and link quality relationship with the node's neighbourhood. Based on the processing of the collected states, maintenance actions are flooded from the base station, for instance resynchronization of the overall network or link rediscovery for connectivity enhancement.

Other works, such as the one proposed in [Ringwald'07], make use of Deployment Support Networks (DSN), which are wireless networks temporarily installed during the deployment process alongside the actual WSN. The main target of this infrastructure is to provide two different radio communication capabilities, one for passive listening of network traffic and another for debugging channel connections through DSN nodes in order to distribute the gathered information to a nearby sink point (from which the deployment analysis will be performed). Therefore, such a debugging implementation relies on a passive monitoring system based on the deployment of additional network platforms in field. Such a side network is to be removed once the expected performance of the WSN system is achieved. In order to forward the overheard packets from the sensor network up to the sink of the tree, the DSN nodes use Bluetooth as the support communication. The system then relies on a message decoder which is in charge of parsing the stream of packets and pass it to the data stream processor, which allows the analysis of the incoming packets by applying operators such as filtering, state detection, and aggregation windows. The system is certainly focused on providing developers and deployers with a 
way of passively observing the field operation of the network in order to detect possible failures such as communication malfunction or non-working nodes.

In [Yu'10] a general diagnosis framework has been proposed following a similar approach as the aforementioned work in the sense of using an out-of-band channel for overhearing the WSN. Sniffing nodes are introduced into the network in order to gather raw radio data which then undergo a series of processing phases based on the structure of the framework, including packet merging and decoding, in accordance with the configuration of the message structure, packet and event filtering (the former one for detecting individual messages and the later for a sequence pattern identification), and classification and visualization. Rules for grouping filtered packets can also be established so as to subsequently produce a proper representation to the user. Therefore, the ultimate objective of such approaches is rather the provision of a packet analysis regarding data exchange among the deployed sensor nodes, though further commissioning, maintenance and on-site reconfiguration capabilities are not certainly contemplated.

Seeking a lightweight portable tool for in-field monitoring and visualization, in [Selavo'06] authors propose the implementation of a mobile user interface module for WSN nodes, particularly targeting their integration into the MICAz platform. The main objective is provide deployers with a compact and portable device for performing in-field data logging, graphical visualization and power consumption measurement features, so that further analysis regarding the operation of the sensor network can be done at the deployment scenario. For this, the proposed hardware design aims at including an attachable module into the MICAz that comprises an LCD screen and five way navigation buttons, a memory storage interface compliant with SD and MMC, and a shunt-based power consumption meter that provides a connection to the test node in order to measure and calculate its consumed energy. TinyOS drivers for controlling the LCD are provided together with functions to manage graphical features of the screen, considering the possible inclusion of modules for creating GUI menus. The main advantage of this prototype lies on its lightweight nature that allows accessing the in-field sensor nodes by using a small device. However, an 
important shortage of processing resources and capabilities for on-site debugging, diagnosis and maintenance tasks indeed produces a strong limitation of the actions to be carried out by the deployer, thus reducing the WSN deployment and commissioning support. In fact, it can be seen that the device is an extension of a resource-constrained sensor node by adding more data memory capacity and a display hardware for graphical interface purposes, but no further functionalities for enhancing the deployment, maintenance and performance assessment strategies are addressed.

On the other hand, some research trends regarding deployment techniques for WSNs centre the attention in random strategies [Wang'13] in which nonuniform, uniform and grid distributions are combined with coverage optimization analysis [Chatterjee'2014] to maximize/balance the connectivity of the sensor network to be deployed. Node placement strategies targeting network lifetime and network coverage enhancement have also been addressed in the state of the art, in which optimization methods for energy consumption improvements can be achieved by using constrained and relay-node-based deployment schemes, such as in [Kenan'10] and [Misra'10]. In this direction, even though several optimization approaches can be distinguished for performing more efficient network distributions, actually most of the current solutions certainly face the problem of deploying WSNs from the point of view of planning/simulation techniques, but not as the result of a proper on-site real deployment, configuration and performance evaluation/enhancement methodology.

Moreover, the evaluation of multi-hop mesh networking strategies have been usually addressed from a simulation perspective, by using frameworks such as the works presented in [Sobeih'06] and [Varga'08], for simulating protocols and communication networks. However, fewer proposals try to address practical assessment of network connectivity and in-field analysis of routing mechanisms, such as the work presented in [Wei'13], where authors proposed a toolkit for evaluating collection tree protocols from a simulation point of view as well as translating their TinyOS implementation to a controlled testbed, where nodes are connected to PCs to gather data and statistics, which is more focused on a 
laboratory approach for pre-deployment analysis. Similarly, in the approach proposed by Dongliang and Guoying [Dongliang'11], the network testing information is collected by the sink node through the main wireless link, which then retransmits the data to a remote performance analysis and evaluation system, by means of using a wired connection or Wi-Fi.

\subsection{Adapting the modular platform to communication performance experimentation}

Apart from the debugging and diagnosis strategies on the actual hardwaresoftware implementation of the platform under deployment, the on-site performance evaluation of the sensor nodes to be installed and maintained strongly depends on the analysis and enhancement of the wireless communication capabilities, particularly when considering resource-constrained multi-hop wireless mesh networks. The optimization of WSN-based systems shall consider the distribution, integration and cooperative processing of the spread devices as a critical aspect to assure the final operability and reliability of the planned application. The communication performance is a key aspect to establish an overall system correlation as a whole network, more than the individual capability of deployed sensor nodes.

In this direction, the low-power profile nature of WSN-based applications leads to studying different communication technologies that cover physical and medium access control mechanisms, the integration of efficient routing protocol optimization algorithms, going up to the application-domain abstraction level. In this way, the de facto standard for low-rate wireless personal area networks IEEE 802.15.4 [802.15.4] integrates the basic elements of the two lower layers of the OSI model upon with novel technologies for efficient interconnection and data dissemination strategies among the wireless sensor platforms can be implemented. In fact, the ZigBee specification [ZigBee] is built from the basis of this standard, in addition to including network layer and different application profiles. 
To this extent, the main approach of the Cookie modular platform as a support framework for experimenting with novel hardware and software prototypes, together with the analysis and real implementation of optimized mesh multihop networking mechanisms within the proposed WSN technologies, has promoted the design and integration of a new wireless communication layer for the modular platform in this work, based on the IEEE 802.15.4 standard. Such a layer implementation serves as one of the main interface elements to establish the on-site debugging, dynamic routing and dissemination strategies, so as to guarantee the interaction with and among the sensor nodes under deployment. Furthermore, this Cookie layer allows the ability to expand its interoperability among several WSN platforms that integrates this protocol as the underlying connectivity mechanism for underpinning upper communication technologies, which thus increases the flexibility and heterogeneity of the Cookie-based sensor nodes.

As the rest of the designed communication layers, the main target is to integrate a low-power radio module that is used as a peripheral element by the host side (i.e. the processing layer by means of the corresponding microcontroller) in order to manage the wireless connectivity of the platform and its interaction with the sensor network. The module has been selected considering a wellknown RF transceiver family for embedded wireless sensor systems, the CC2420 [CC2420], which is a 2.4 GHz IEEE 802.15.4 compliant module developed by ChipCon (now Texas Instruments) for low-power and resource-constrained wireless platforms. It is a widely spread transceiver that is included in different WSN platforms such as TelosB and other clone sensor nodes, so the wireless communication interoperability with these types of hardware can be performed.

This module provides support for packet handling and data buffering, clear channel assessment, radio signal strength/link quality indicators and $250 \mathrm{kbps}$ of effective data rate, among other functions that are accessed by using a SPI interface with the processing host. Depending on the output transmission power to be configured, the TX current consumption can vary from $8.5 \mathrm{~mA}(-25 \mathrm{dBm})$ up to $17.4 \mathrm{~mA}(0 \mathrm{dBm})$, also taking into account that there are two different types of low-power modes available. During the idle mode the current consumption 
reaches $426 \mathrm{uA}$ while in power-down mode this value is reduced to $20 \mathrm{uA}$. Moreover, the transceiver integrates an on-chip voltage regulator to supply $1.8 \mathrm{~V}$ for internal elements of the module, based on an input voltage range from $2.1 \mathrm{~V}$ to $3.6 \mathrm{~V}$. According to the regular values handled by the Cookie modular platform, this input voltage is set to $3.3 \mathrm{~V}$. The activation/deactivation of this internal regulator is controlled externally by using a VREG_EN signal from the processing layer, thus allowing a third power-down mode (1 uA maximum).

Regarding the interface signals for managing the communication module as well as packet transmission/reception operations, the following inputs/outputs have been considered when integrating the transceiver with the rest of the platform:

- SO (Slave Out), SI (Slave In), CLK (Clock), CSn (Chip Select): these are the SPI signals to control the slave module from the master processor.

- RESETn: it serves as a hardware restart signal for performing a chip reset to the module.

- SFD: this pin allows detecting the beginning of a new frame by means of the Start of Frame Delimiter that corresponds to a PHY packet.

- FIFO and FIFOP: these pins are used to control the state of the RXFIFO so as to detect the availability of data bytes in the input buffer of the module, as well as verify that an incoming packet has been received if the address recognition mechanism is activated.

- CCA: refers to the Clear Channel Assessment control signal for performing the CSMA-CA mechanism of the standard in order to properly access the medium.

The hardware design was conceived to include 4 layers within the proposed layout of the board, two of them for power supply and ground planes respectively. The result of the implemented Cookie-compliant IEEE 802.15.4based wireless communication layer is shown in Fig. 3.1, where its integration with the rest of the modular node by using the vertical connection interfaces is highlighted, following the corresponding standard dimensions of the platform. 

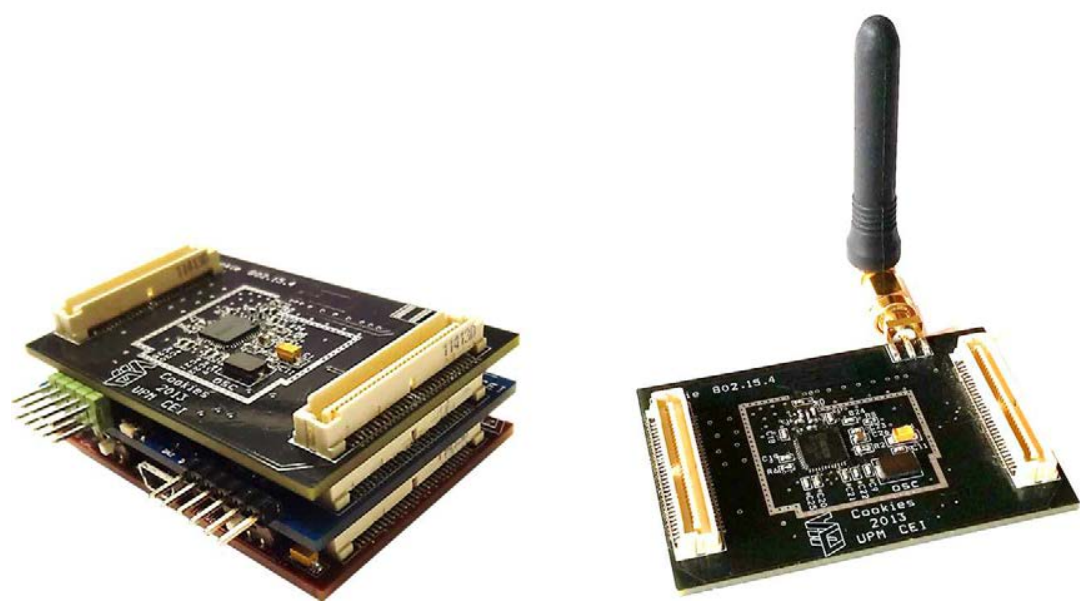

Fig. 3.1 Implementation of the IEEE 802.15.4-based Cookie communication layer.

The integration of this standard with the modular architecture is certainly twofold, since a set of functional components has also been implemented within the software support platform so that the communication module can be controlled by using the defined HW-SW framework. Considering the same modular structure of the proposed libraries for managing the Cookie platform, a new library is included into the framework with the following key main components that provide access and control to physical and MAC functionalities:

- CEI_Comm15-4_moduleConfig: it includes functions to configure the SPI connection to the radio module as well as the actions for properly initializing the peripheral prior to releasing the normal operation mode, such as voltage regulator activation, addressing and channel configurations, DSN frame generation, and automatic acknowledge execution activation.

- CEI_Comm15-4_regControl: allows modifying the 16-bit internal registers in order to control and set parameters related to the behaviour of the module and the communication performance, such as power transmission and automatic generation of acknowledge packets, as well as the execution of strobe commands. These are actions 
mapped in register addresses to automatically perform a particular task, distinguishing turning on the crystal oscillator, RX enabling, and clearing the input/output buffers.

- CEI_Comm15-4_memoryManag: integrates functions to manage the RAM memory of the device that is used to allocate the input/output data buffers as well as save information related to PAN identification and module address.

- CEI_Comm15-4_bufferHandler: it makes use of the aforementioned component to control the RX/TX FIFOs of the module, so that the incoming and outgoing data packets can be processed accordingly. From the reception point of view, when the address recognition is activated, the FIFOP signal is used to process the data reception in RXFIFO based on the packet frame shown in Fig. 3.2, taking into account the length of the packet, the MPDU (MAC Protocol Data Unit), the RSSI and average correlation value (that is employed for the LQI metric calculation), and the CRC (Cyclic Redundancy Check) flag which indicates the integrity status of the received data. Regarding the data transmission process, together with the payload to be sent via the TXFIFO, the IEEE 802.15.4 header is included in the buffer particularly considering the MAC frame and the FCF (Frame Control) as shown in Fig. 3.3. Then, the medium access control mechanism provided in the following component is triggered, in order to proceed with the data transmission correctly.

- CEI_Comm15-4_macGen: this set of functionalities implements the medium access control specified in the IEEE standard [802.15.4], which corresponds to the CSMA/CA algorithm (Carrier Sense Multiple Access with Collision Avoidance), whose basic idea is to check the availability of the channel after a random period of time specified as backoff periods, where each period corresponds to aUnitBackoffPeriod symbols [802.15.4]. The number of backoff periods that a node is to wait before performing the medium access 
assessment is increased every time the attempting process is carried out.

- CEI_Comm15-4_PwrMode: it allows modifying the state of the radio module by configuring one of the defined power modes of the transceiver (Off, Power-down, Idle, Normal operation), as well as changing the transmission power parameter according the specifics of the wireless communication and consumption performance needs.

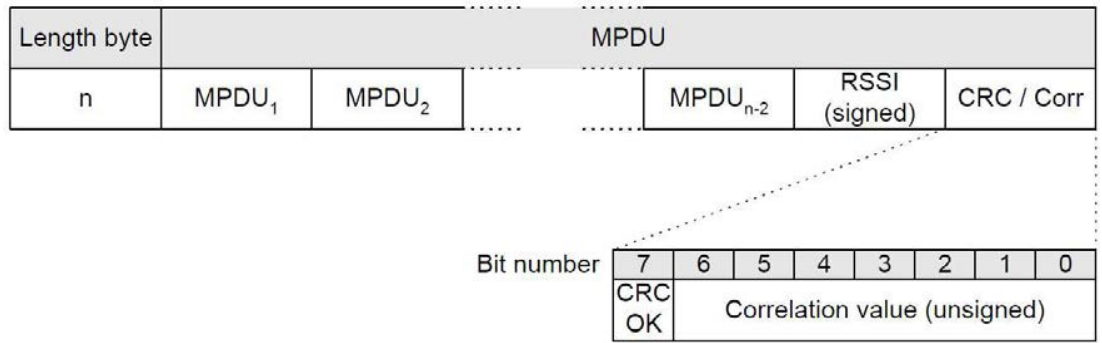

Fig. 3.2 Data to be read from RXFIFO of the radio communication module [CC2420].

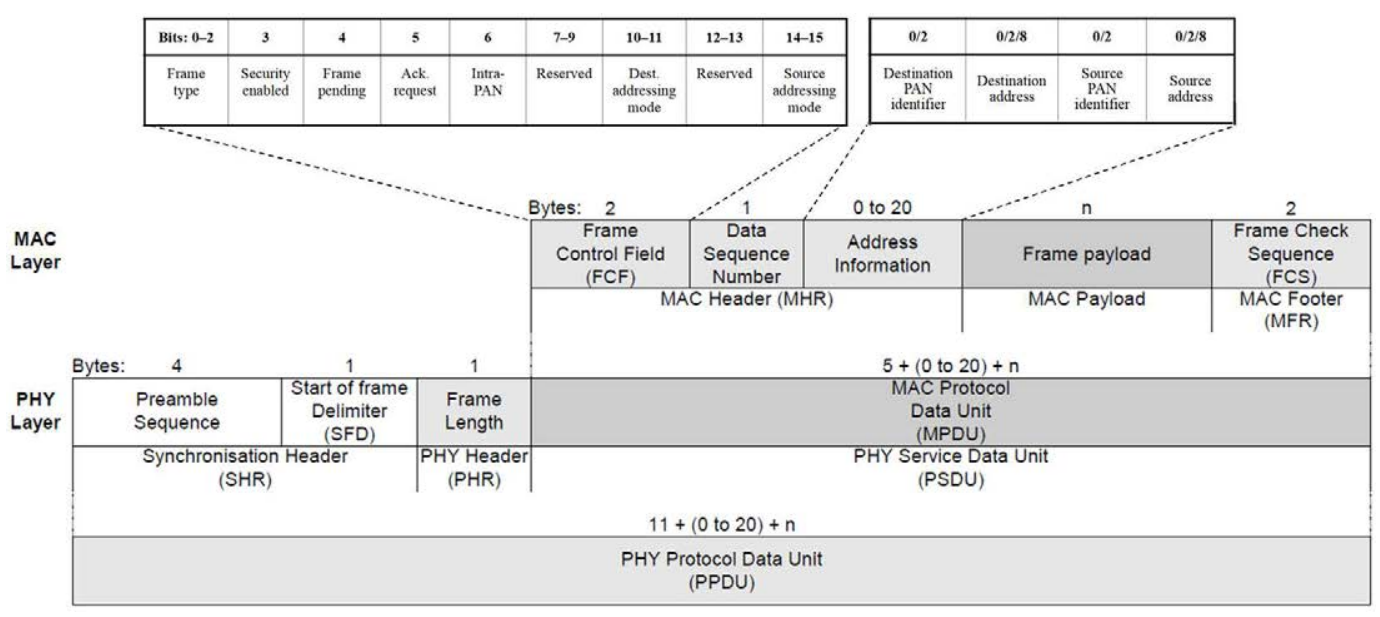

Fig. 3.3 IEEE 802.15.4 Data frame depiction to be processed/generated [CC2420] [802.15.4].

The integration of the proposed software libraries with respect to the overall HW-SW support framework is shown in Fig. 3.4. It can be seen that the controllers provide a well-defined interface that is subsequently used by the upper implementation layers, which make use of the proposed components to 
integrate different communication technologies above the IEEE 802.15.4 standard, as addressed in the following sections with the implementation of a mesh multi-hop networking approach under the Cookie modular platform, based on a dynamic routing protocol optimization process.
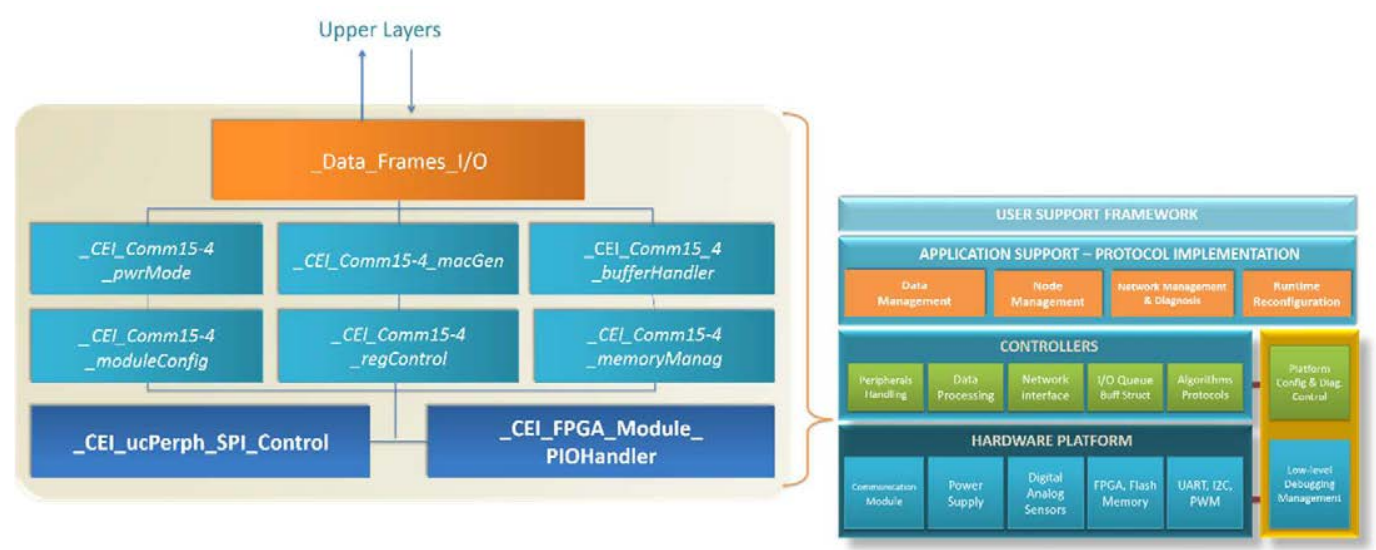

Fig. 3.4 IEEE 802.15.4 module management integration overview in the Cookie HW-SW support platform.

\subsection{A real perspective towards wireless mesh networking optimization based on the modular platform}

The communication performance evaluation and optimization during the deployment phase relies on analysing not only the in-field connectivity problems that come out when carrying out the commissioning process but also the actual adaptability and suitability of the implemented data dissemination strategies to the target operational requirements, so that the efficiency and final reliability of the wireless distributed system can be properly reached. A key element to assure such a level of effectiveness and robustness when developing the resource-constrained wireless system is certainly related to the implementation of dynamic and adaptable routing algorithms, especially in multi-hop mesh networks. In this context, routing problems in WSNs have been studied from different research angles, depending on the target application, topologies needed for specific scenarios and environments, network mobility and scalability, and data aggregation [Goyal'12]. 
On one hand, from the point of view of the network structure to be adopted, three major groups of routing algorithms can be distinguished. The first one is the flat routing protocols [Pang'06], in which nodes have the same role in order to collaborate in achieving the goal of the wireless deployment in a reliable way. The creation process of remote communication paths can be started and commanded by any device of the network, and connections are not restricted to data packet retransmissions to centralized points or collecting nodes. Flexibility is a key aspect covered in these types of protocols due to the possibility of having different alternatives to disseminate the sensor information, which is important in unstable environments where the quality of the links are prone to be affected. The second group is the hierarchical-based routing protocols, in which two main types of roles are defined. Sensor nodes are used to gather the target measurements whereas cluster nodes retransmit these data to the different destination points. Such a configuration aims to reduce the power consumption of the sensor nodes, since they do not need to perform intensive routing tasks and then special sleep modes can be adopted for the former type of device [Pantazis'13]. Moreover, depending on the type of large-scale deployment distribution it might be more suitable to split sensor nodes into several subnetworks or groups trying to avoid overall network control packet density, by creating a structural topology based on node functional role assignment. However, these routing protocols are mainly intended to be used in stable environments where the probability of communication failures is lower, and the redundancy criteria are not a critical network requirement. The third group is focused on location-based protocols, in which nodes find the routing alternatives by knowing their location (using signal-strength-based algorithms or adding GPS-based hardware to the sensor implementation) with respect to the surrounding neighbour nodes [Chen'09].

On the other hand, considering how routes are discovered and created, routing algorithms can also be classified in proactive, in which the mechanism periodically or continuously attempts to determine the routes even if they are not used, so that the corresponding path is already available whenever a node needs to send a packet to a destination point [Mohseni'10]. Although this schema reduces the time delay when a packet has to be transmitted, it has poor 
performance in unstable environments where the routes are prone to be changed or in network mobility, in which the frequency of the connectivity reconfiguration is much greater, in addition to its high energy and resource consumption to maintain the routes. In order to cope with these limitations, a second classification is included, the reactive protocols, in which the route determination is performed on demand [Mohseni'10]. This means that the first time a source node needs to send a packet to a destination node the route mechanism will attempt to discover a connection path and then keep such route for subsequent transmissions. Although this schema may increase the delay of the transmission process due to the route request procedure, it is more flexible and less resource consuming in those environments where the connectivity can be more unstable.

Most of the aforementioned mechanisms and state-of-the-art routing protocols have been evaluated by using simulation tools and modelling process, which give to some extent an idea of what the functionality of the mechanism will be. However, fewer works focus on implementing and integrating routing protocols in real hardware platforms and tested in actual scenarios in order to analyse the performance and the efficiency of those implementations under real and operational conditions of the deployment, as well as dealing with the resource limitations of sensor nodes. In this context, it is essential to evaluate and validate the implementation of routing algorithms and network connectivity in real wireless platform and deployments, as a support to theoretical simulation models that cannot predict certain constraints and limitations in the behaviour of the system.

Furthermore, the behaviour of routing protocols running in actual hardware platforms might be far from the expected simulation results with respect to the final operability of the system, especially in terms of communication performance, due to limitations concerning the core and memory architecture on which routing algorithms are to be implemented, as well as computational and processing limitations that are not taken into account during the modelling process. A real implementation of flexible and optimized routing protocols under the modular HW-SW support framework, their integration with the IEEE 
802.15.4 based communication layer, and their performance evaluation within actual deployment scenarios by using the on-site commissioning toolset (proposed later on in this chapter) are addressed in this thesis work, so that a real perspective to mesh networking analysis, debugging and optimization can be tackled.

In this context, two main approaches are followed. On one hand, the implementation of a flexible AODV-based (Ad-hoc On-Demand Distance Vector) optimized flat routing algorithm is proposed for the modular platform to be also included within the routing performance evaluation engine of the onsite commissioning toolset. On the other hand, a hierarchical routing optimization technique that relies on the combination of a Cluster-based protocol and a flat-based strategy for mesh networks is also included within the evaluation engine, so that a runtime comparative analysis of different multihop dissemination strategies can be performed. The underlying goal is to provide a set of routing optimization mechanisms in order to study and assess their suitability to WSN applications.

\subsubsection{Routing protocols in the state of the art}

As remarked previously, there are various routing protocol strategies that can be adopted depending on the requirements of the WSN deployment as well as the application/scenario constraints to be tackled. Due to the inherent instability nature of the WSN communications, a dynamic mechanism to discover the multi-hop data path between two remote points is a prior decision when implementing a particular routing technique. Moreover, there are design requirements that might determine how nodes shall disseminate data throughout the deployment, such as centralized traffic models, where one or more coordinators serve as sink elements of the data flow; differences in processing, memory and energy constraints according to the sensor features; cooperative models where distributed processing and path redundancy are key aspects to be considered; and mobility or volatile interconnections in which the routing paths are variants during different operational stages. 
In Fig. 3.5, based on the analysis of the main types of categories of routing techniques, different proposals can be distinguished, such as GAF (Geographic Adaptive Fidelity) [ $\mathrm{Xu}^{\prime} 01$ ], which uses location information to establish coherent association with surrounding nodes, so that they can determine which of them can sleep while the others route packets. The main idea behind this technique relies on creating virtual grids within the covered communication area of the distributed network, where nodes are associated according to their GPS-based location. These virtual grids are determined such that all nodes from adjacent micro areas can fully communicate among each other, and they are considered as equivalent points in terms of routing cost. This means that any pair of points of adjacent grids can be selected to establish a communication path while the rest of the nodes stay in sleep mode. In order to produce a balanced energy conservation scheme, a cooperative coordination among the nodes that belong to the same grid has to be performed such that they determine which nodes go to sleep modes.

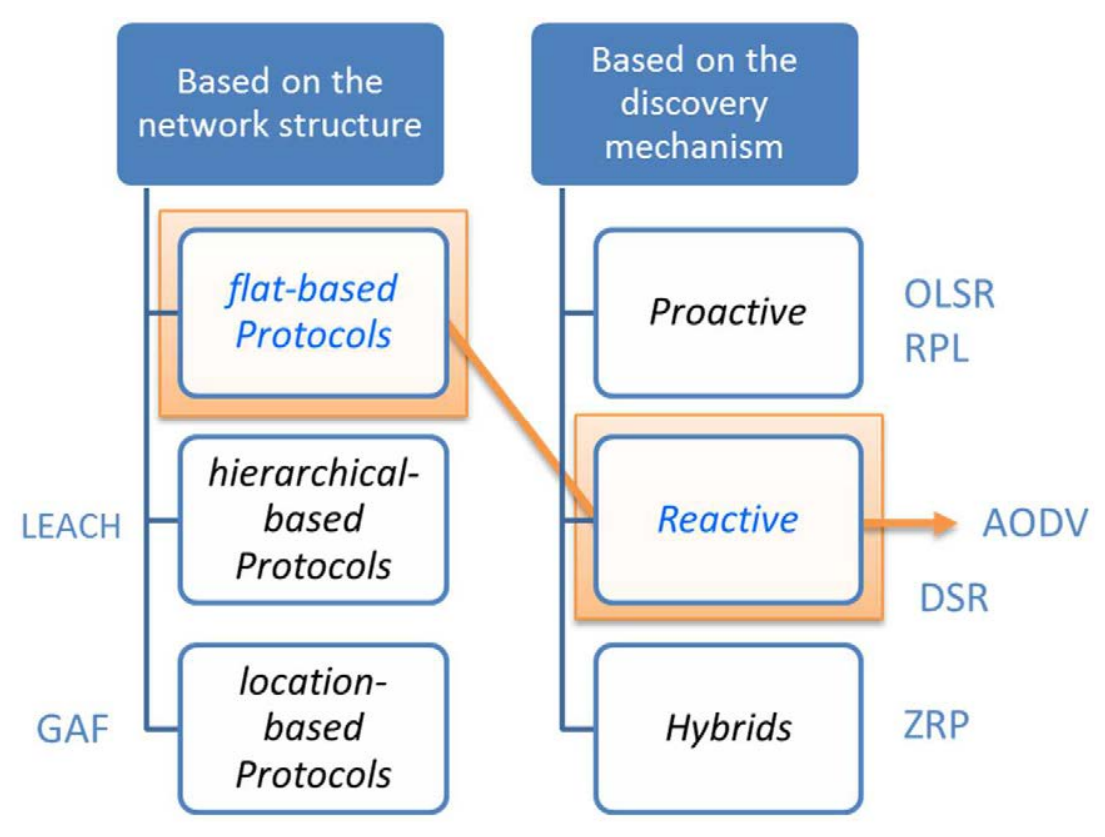

Fig. 3.5 Routing protocols classification based on the various types of strategies.

In LEACH (Low Energy Adaptive Clustering Hierarchy) [Aslam'12] sensor nodes transmit information to cluster heads that then distribute the data in a 
compressed fashion to the base stations, so the energy consumption is balanced trying to extend the network lifetime. This strategy randomly selects and rotates a number of cluster nodes that gather the data from their corresponding group and then transmit an aggregated packet to the sink point, thus they serve as routers of the network information to the base stations. Although the initial approach considered a single-hop scheme between sensors and cluster nodes, and from those clusters to the sink point (which assumes that the former one will have full direct communication will the latest), further optimizations allowed for the creation of multi-hop communication among the cluster nodes in order to reach the base station.

OLSR (Optimized Link State Routing) [Jacquet'03] is a proactive protocol that tries to minimize rerouting latencies by maintaining and rediscovering paths so that they can be available even if they have not been demanded, which increases the overhead of the network. In order to minimize such an inherent overhead associated with topology information exchanging, this technique defines MultiPoints Relay (MPR) neighbours that are in charge of retransmitting such control messages to the rest of the network. The protocol uses Hello and Topology Control messages to maintain the routes towards the destination points of the networks as well as identify and organize the MPR nodes within the neighbourhood.

RPL (Routing Protocol for Low power and Lossy Networks) [Winter'12] is a collection-based protocol that forms a tree topology to define routes to the collecting points of the network. Each node has its corresponding parent through which data packets are transmitted to a root node, although they can also be associated with multiple parents. Such a hierarchy is determined by the definition of what is called ranks, which represents a relative position of a node with respect to the root of the tree structure. Ranks are computed according to the translation of one or more metrics in the form of objective functions that eventually define the optimized routes to the target root, though the protocol specification does not set a particular metric.

ZRP (Zone Routing Protocol) [Haas'02], that combines the advantages of a reactive mechanism among different defined network zones while applying a 
proactive technique among the nodes of a neighbourhood. In order to accomplish this hybrid approach, routing zones are defined in each node as a radius that is expressed in the form of number of hops. The maximum distance between the reference node and the peripheral/border nodes is defined by the zone radius. Hence, nodes that are within the boundaries are called interior nodes. A proactive mechanism is then performed in order to discover and maintain the routes from a sensor node to its interior and peripheral points. When a node decides to send a packet within its zone radius, it checks the routing information that has been generated proactively. Instead, if the destination point is outside the zone, a reactive discovery process is triggered from the peripheral nodes to the objective destination.

The well-known CTP (Collection Tree Protocol) [Gnawali'13] implemented under TinyOS can be classified as a data-centre-based protocol, where the directionality of the data flow is defined from sensor devices to coordinator nodes by using a tree schema based on the quality of the interconnections. This collection protocol relies on the generation of a routing gradient based on the link quality estimation among the sensor nodes, starting from the root point of the deployment. The route discovery and selection is based on an announcement procedure that stars from the root and is then disseminated throughout the network by using beaconing messages, whose rate is controlled by adaptable transmission intervals.

Another popular mechanism is the Dynamic Source Routing (DSR) [Johnson'09], which is a reactive protocol where the entire path between two nodes is stored in the information/control headers of the packets. The behaviour of the protocol is similar to an on-demand mechanism in that it generates a discovery process only when a new route is requested. This mechanism does not use periodic messages to maintain the routing information, and is well-suited for stable scenarios where communication and mobility changes are not frequent. During the discovery phases nodes append their own addressing information to the received request packet and then forward it to the surrounding nodes, which increases the traffic overhead in those situation where the variability of the interconnections is to be taken into account. 


\subsubsection{Cookie-Based-AODV implementation}

Based on the inherent flexibility of the Cookie nodes to be adapted to different WSN application contexts, and also according to the state-of-the-art analysis of the previous section, the AODV-based routing protocol [Perkins'03] has been selected as one of the most suitable algorithm to be integrated into the HW-SW platform, due to its adaptability to different network environments as well as its dynamic reconfigurability in ad-hoc network topologies.

Taking advantage of the benefits of this flat-based reactive routing protocol, a modified version is proposed to be adapted and implemented into the real HWSW node platform, especially targeting dynamic and unstable environments, where a versatile routing mechanism has to be adopted. The proposed implementation, called CB-AODV (Cookie-Based AODV), aims to help developers to analyse different aspects, constraints and requirements in the use of reactive routing protocols under real ad-hoc mesh WSN application scenarios.

\subsubsection{Basic operation of the protocol}

The AODV protocol uses 4 different types of control messages to create and maintain the routes of the network. These messages are named RREQ (route request message), RREP (route reply message), RERR (route error message) and HELLO. Moreover, every node of the network keeps on one hand a routing table with different fields that include information for the maintenance of the routes, and on the other hand a local sequence number that allows the system to avoid the formation of possible loops within the network, as shown subsequently in this section. Likewise, every entry in the routing table contains the last available sequence number of the destination node of such route. Hence, the control messages include information related to the source and destination sequence numbers, so that a runtime comparative analysis of route states and their update can be performed.

When a node decides to send an application packet to another remote node of the network, the first step is to check if it has the route to the destination point in its own routing table. If so, the message is then sent to the next node of the route. This is repeated along the whole path until the packet reaches the final 
destination. In case that the node does not have information of the route, it will store the packet and start the route request process by broadcasting a RREQ message. The devices that receive this message will rebroadcast it until one of the nodes has information of the route, or the destination itself is the one that receives such request message. In both cases the node will send a RREP to the previous node until it reaches the source node that requested the route, and then the application packet previously stored is finally sent through the discovered path.

In this way, with the propagation of the RREQ, the nodes update their corresponding routing table with the information of the source node, thus creating what is called the inverse route. In addition to this, nodes also create a route table entry with the information of the node from which they received the RREQ, hence creating routes to their neighbour nodes. With the propagation of the RREP, a direct route is created, which implies that the different nodes where the RREP hops create a route to the destination point.

In Fig. 3.6, the process of discovering a route from node 1 to node 6 is shown, as well as the different messages that are sent to carry out the aforementioned procedure. As it can be seen, the distance vector approach relies on the fact that the nodes are not aware of the entire information of the network to create the multi-hop path to the target destination. Instead, they do register their surrounding neighbourhood from which the orientation to the destination point can be obtained (next hop of the route), and the distance that is certainly related to the cost metric (e.g. number of hops) to get to the target node.

The computed routes are only valid during a limited period of time, and once they are not used within this timeout they are marked as invalid, and therefore they are no longer available for use. The original routing protocol defines the HELLO messages that are broadcasted periodically by all the nodes of the network in order to maintain the routes. However, as presented in the following subsections, these types of messages are not included in the modified implementation, aiming to have a more efficient performance in terms of power and resource consumption by reducing the network overhead. Whenever a route changes its state from active to inactive due to not being used in a period of time 
or due to a link failure in the propagation of a data packet (no acknowledge received in any of the attempts to send a packet to the next hop), the node that detects the failure sends a RERR to all of its precursors nodes, i.e., those nodes that use the former node as their next hop. The precursors will then repeat the same process until the nodes that used the fail route eliminate it from their routing table.

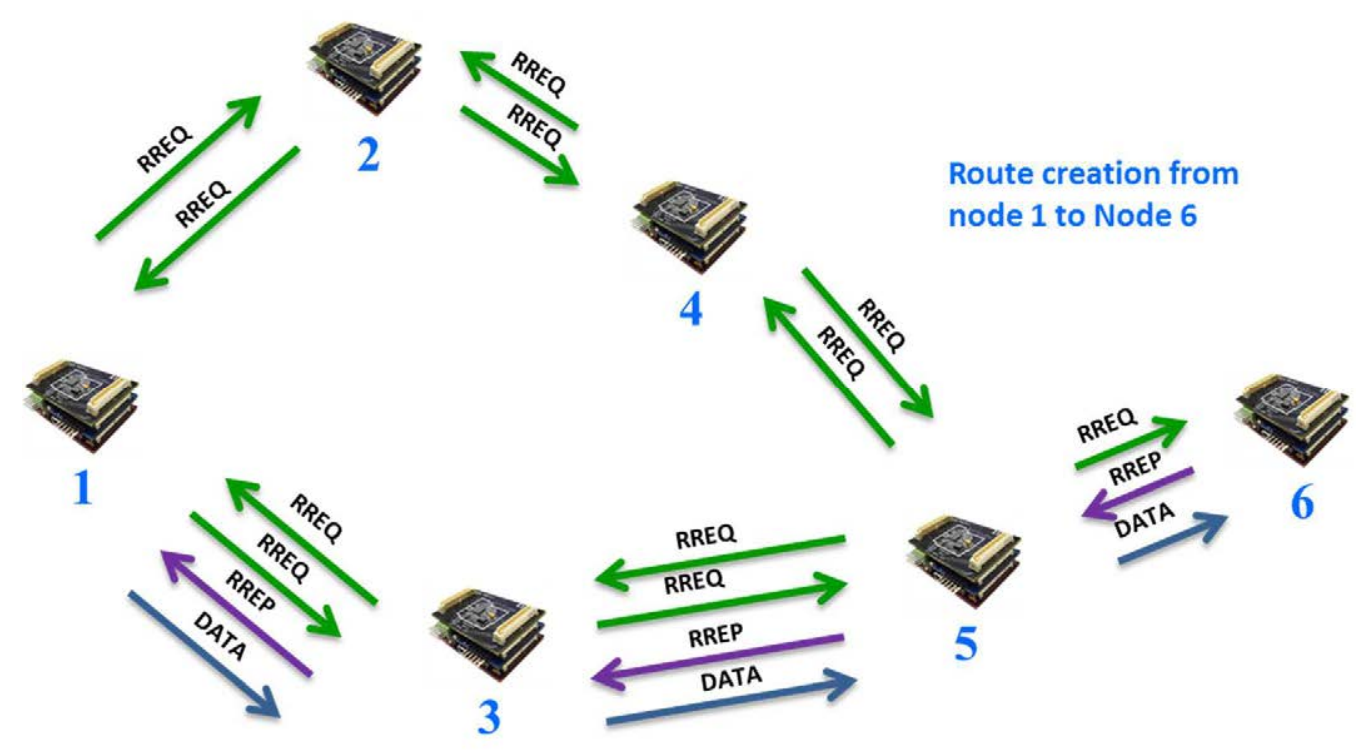

Fig. 3.6 Exemplification of route discovery process, from node 1 to node 6.

In this overall behaviour, in order to effectively perform the routing mechanism, the main information that has to be kept and processed by every node is as follows:

- Destination address.

- Sequence number of the destination node.

- Sequence number state.

- Route state.

- Number of hops \& link cost metric.

- Next hop.

- Precursor lists. 
- Lifetime of the route.

\subsubsection{CB-AODV: Adaptation to the WSN modular platform}

The AODV routing protocol was initially implemented to be used in MANET networks (Mobile Ad-hoc Networks), where a dynamic interaction and selforganized communications among the different devices that participate in the mobile wireless network array take place, particularly considering the decentralized nature of the ad-hoc configuration. Despite all the similarities with respect to the WSNs, wireless nodes in sensor networks are distinguished by having much less processing capabilities and the battery autonomy has to be taken into account in order to enhance the overall lifetime of the deployment. Because of that, it is necessary to adapt the routing strategy to the WSN characteristics and especially targeting the architecture of the Cookie HW-SW platform and its usage in real ad-hoc wireless sensor application scenarios, though taking advantage of the dynamic operation and flexibility of the mechanism.

In this way, the proposed adaptation is focused on adding new functionalities to the original protocol to make the behaviour of the mechanism more efficient to resource-constrained WSNs, and not including some others aiming to improve and optimize the overall performance of the algorithm for the purpose of the Cookie node connectivity, and thus reducing the resource consumption in this system architecture. The CB-AODV implementation has been fully developed in line with the specific resources of the Cookie hardware platform, so that the memory scheme is highlighted to take full advantage of the architecture.

First of all, there are basic adaptations that have been adopted to guarantee an efficient implementation in terms of power consumption and resource handling, particularly because there are features in the routing specification that are not needed within the context of WSNs:

Elimination of the time to live of the routes and suppression of HELLO messages: With this optimization it is possible to reduce overhead of the network (messages are not sent periodically for route maintenance). Moreover, since the dynamics of a WSN is based on having duty cycles in which sensors remain in stand-by, there 
is no need of expending additional resources to check if the routes have been used for a period of time, thus reducing the implementation size. In any case, even if the deployment requires higher data traffic, routes are maintained by combining three elements: ACK packets during data transmissions (MAC layer), RERR messages, and a new type of dissemination integrity process that is introduced later on in this section. Therefore, the maintenance of the routing paths can be checked/performed in a reactive fashion as well (only when needed), thus avoiding wasting energy and simplifying the implementation.

Expanding Ring Search algorithm removed: This algorithm is based on searching the routes by using RREQ messages with a low TTL (time to live, i.e., maximum number of hops that the message can do during the discovery process), so that the area of searching is limited. If no RREP is received, then the RREQ is rebroadcasted and the TTL is increased until the route is discovered or the TTL has reached its maximum value. This algorithm is used to avoid flooding the network in crowded topologies, at the expense of increasing the latency.

In addition to the aforementioned modifications, new features and additional properties have also been included in CB-AODV in order to increase its performance without penalizing the memory and processing resources of the architecture. These important characteristics are classified as follows.

Implementation of different types of route cost metrics: The discovery metric is a fundamental parameter used to compare the different route alternatives that can be obtained during a route discovery process. In terms of processing and implementation, the easiest way to make a decision is just register the first path found during the discovery mechanism (no buffers of control messages have to be used to handle different alternatives). However, within the context of dynamic communications in WSN deployments, this approach is not enough to assure the effectiveness of the remote interconnections and the quality of the data transactions. On the other hand, the metric applied in the AODV protocol is the number of hops, also known as minimum Hop Count (i.e., as less number of hops as possible to determine what route is the best encountered), which may have poor performance in unstable and "crowded" WSN application environments. This approach could reduce the latency during the packet 
delivery, but actually, if the different links along the path are unstable in terms of the quality of the connection, transmissions attempts could considerably increase, thus penalizing the time and energy consumption of the involved nodes during the communication transactions.

Therefore, it is fundamental to allow for other types of metrics that can discover the best routes in terms of the quality of the interconnections among the wireless nodes. For this purpose, metrics that use a parameter called LDR (Link Delivery Ratio) for measuring the percentage of messages that will be successfully sent between 2 nodes are considered. The main idea is to study the reliability of two remote points in terms of data delivery by calculating the quality of the transaction between every pair of nodes along the route path, which can be directly calculated by triggering a determined amount of packet transmissions between them. Of course, this method cannot be afforded in a real WSN because it is energy and bandwidth consuming, so there are studies in the state of the art in which the main efforts are focused on how to correlate the LDR with the most common data quality indicators, which are provided by the IEEE 802.15.4 layer: LQI (Link Quality Indicator) and RSSI (Radio Signal Strength Indicator).

In the Cookies implementation, a correlation between the LQI and the LDR for the CC2420 radio transceiver has been studied based on the work proposed in [Gomez'10], but also adding the RSSI parameter to the estimation so that a more comprehensive model can be used. The main target is to obtain a real correlation of both parameters for the Cookie platform by applying an experimental setup with the designed communication layer, so that the prediction model of the data delivery is more adapted to the hardware platform.

Based on this approach, the experiments were configured in an outdoor space scenario at the ETSII-UPM, considering two Cookie nodes with the same position at the same height ( $1 \mathrm{~m}$ from the ground) and distance samples starting from $1 \mathrm{~m}$ up to $35 \mathrm{~m}$. Moreover, the range of the TX power configuration of the radio modules is swept from $0 \mathrm{dBm}$ to $-25 \mathrm{dBm}$, so two levels of interactions are fully considered to guarantee a higher amount of experimental data to be correlated, obtaining up to 4236 test/measurement cases during the testing process in the field testbed scenario. As a result, the comparisons and main 
contributions for the in-field modelling of the communication and quality metrics are presented in the experimental analysis chapter.

Based on those results and considering the analysis proposed in [Gomez'10], the inclusion of different types of quality-based route cost metrics is taken into account, so that the CB-AODV can be evaluated under several routing discovery decisions, as follows:

- PATH-DR (Path Delivery Ratio) metric: this metric calculates the percentage of messages that theoretically will be successfully sent in a route. It is obtained by computing the LDR parameter in every pairof-points of the route, i.e., the link quality estimation per hop [Chen'06] [Gomez'10].

- ETX (Expected Transmission Count) metric: represents the estimation of the expected number of transmission required to successfully deliver a packet over a link between two nodes [Couto'03]. The total ETX route cost metric is the sum of the computed values throughout the involved links of the path. Hence, this metric tries to minimize the number of hops by taking into account the possibility of link failures, considering for its calculation the quality of the LDR parameter [Gomez'10].

- Hop Count: as explained before, it is the original metric used in the protocol, which consumes less memory resources due to its ease of calculation and implementation.

It is important to highlight that the modularity of the implementation allows changing the corresponding metric depending on the target application and the conditions/requirements of the scenario, by using reconfigurable parameters within the implementation properties.

On the other hand, in this work a new type of routing metric is also introduced: E-aDM (Energy-efficient and adaptable Delivery Metric) targeting the enhancement of the network lifetime, by considering energy and resource factors in every computed cost calculation. The main idea behind this approach is to bring the possibility of reconfiguring the influence of every particular node 
along the path depending not only on the estimation of the LDR, but also on other factors that could affect the performance of specific nodes or neighbourhoods during their operational behaviour within the WSN.

The calculation of the metric is obtained on the basis of the path delivery indicator evolution throughout the route under evaluation, in which every node computes and incorporates its link delivery estimation in the control packet during the discovery process. However, before indexing such indicator, the node first balances its value by applying the following expression as a multiplier (3.1), which includes the elements that could influence its performance in long-term operation:

$$
\delta=1-\gamma
$$

where $\gamma$ is (3.2):

$$
\gamma=\left(C_{p l} \cdot w_{p l}+C_{b c} \cdot w_{b c}+C_{i b} \cdot w_{i b}\right) / w_{l}
$$

$\mathrm{C}_{\mathrm{pl}}$ is a neighbourhood factor representing the number of precursors that use the node as their next hop, so it can indicate the degree of saturation with respect to other nodes along the path or in the distribution of the routing tasks. The $\mathrm{C}_{\mathrm{bc}}$ is the energy consumption factor, which expresses the amount of battery level that has been used from the beginning of the node's operation. It is computed as the maximum voltage value of the connected battery minus its runtime measured value. This node's consumption is also correlated with the TX power configuration of the radio communication module to be used, as well as the estimation of the long-term autonomy based on its operational/computational duty cycle. $C_{i b}$ is a firmware implementation indicator which considers the number of effective functional and processing blocks that have been integrated in addition to the general controllers of the SW support platform to perform application-dependent tasks, so it measures the processing load that a node has in order to carry out its functions. $\mathrm{w}_{\mathrm{pl}}, \mathrm{w}_{\mathrm{bc}}$ and $\mathrm{w}_{\mathrm{ib}}$ represent the weights of every condition according to how critical they are considered for a particular node or deployment. They can go from 1 to 5 ( 1 for those topologies where rely nodes are more critical along the routing path, thus less redundancy; and 5 for those nodes which have higher computational load in terms of processing capabilities 
within the wireless deployment) and can be reconfigured depending on the target requirements/constraints and the type of deployment plan, while $w_{l}$ represents the general weight of the involved link, which is the quality estimation with the preceded hop in this case.

It can be seen that, the greater this factor is (the conditional elements are higher, i.e., the number of precursor nodes, the number of implement blocks and the consumed power), the worse the measured metric would be for a particular node. With this schema, a trade-off between performance and energy efficiency is taken into account, so that energy and routing balance throughout the deployment can be achieved. Moreover, regarding network diagnosis capabilities, the balance of these factors speeds up the detection and debugging of possible non-functional nodes in terms of, for instance, power consumption anomalies by means of correlating the number of implemented blocks, the lists of precursor nodes and the power mode configuration with the actual in-field energy spent per node and their operational behaviour.

New type of message: in addition to the included features, in this work a new type of message has been implemented for the CB-AODV protocol, called DATA_ERR. The aim of this new control message is to enhance the dissemination capabilities of fragmented information by supporting integrity verification of the data flow, in addition to the local repair and rediscovery processes. Whenever an intermediate node discovers a link failure when transmitting a data packet, after sending the RERR (to its precursor list) it will also send the originate message to the source node indicating that it is a DATA_ERR message. When the originator of the message receives the DATA_ERR, it will store the data packet and then try to discover an alternative path to send the message, so that an information checking process is performed in order to avoid losing critical data in unstable WSN application environments.

This is especially useful when splitting an information stream that targets the reconfiguration of specific functional blocks of the HW-SW platform, where several data packets have to be efficiently sent to assure the correct configuration mechanism, or in cooperative networks where the efficiency in data distribution 
is enhanced. The structure of the control packet is similar to those already defined, as depicted in Fig. 3.7.

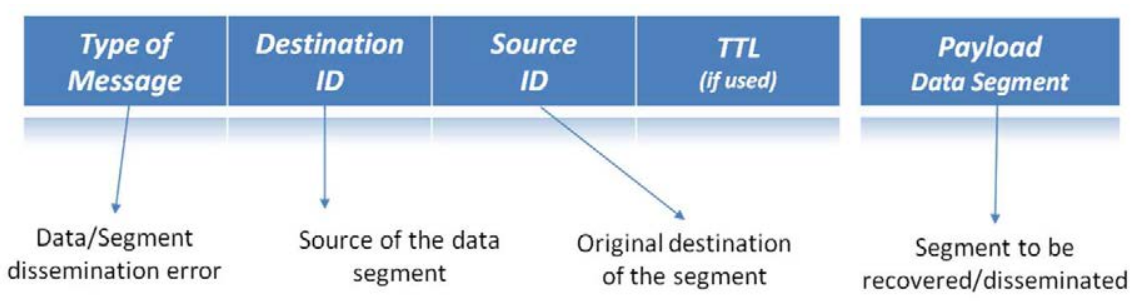

Fig. 3.7 General structure of the new type of message DATA_ERR.

There are also other types of protocol adaptations proposed in the state of the art such as [TinyAODV] to reduce the need of memory and processing capabilities as well as the complexity of the protocol by not incorporating some of the functionalities of the technique in such implementations. However, those modifications are not included in CB-AODV (although the modularity of the implementation allows "plug-in/out" several functionalities to be included in the target node, aiming to create a more compressed integration when needed), because they also reduce the performance of the protocol implementation and were not necessary reductions within the Cookie platform context, in which a trade-off between performance efficiency and resource constraints is adopted to assure its flexibility in different application scenarios. Those features are described as follows:

Suppression of the Local Repair mechanism: The Local Repair is a functionality in which an intermediate node discovers a link failure when sending a message and, instead of generating a RERR message, it will try to find an alternative route without the need of notifying such event to the packet source. If the Local Repair mechanism is deleted it is possible to reduce the consumed memory because an output queue is no longer needed when trying to rediscover a new route in order to keep the pending data. However, it decreases the protocol performance, since the dynamic capabilities of fixing localized problems is restricted to the originator of the messages, so that more discovery actions have to be disseminated and wasted. Hence, the Local Repair Mechanism is indeed included in CB-AODV. 
Precursor list: As explained before, when a node detects that a route is no longer valid because a failure and no alternatives were found during the local repair mechanism, the RERR messages are sent to the precursor nodes of the route that has failed. This means that, from the implementation point of view, it is necessary to include a record of precursors for every routing table entry, which increases the memory usage. However, this is an important aspect to be considered for a better performance of the protocol, since if precursor lists are not implemented, the propagation of the RERR messages is then carried out as the propagation of RREQ messages is, which may flood the network and they might cause the suppression of routes that did not need to be eliminated. Therefore, in CB-AODV, this feature is fully supported to obtain a better dynamic behaviour of the protocol.

Route cost metric implementation: As previously explained, there is a possibility of omitting the inclusion of metrics for selecting more efficient routes when discovering them. In this way, the node only generates a RREP for the first received RREQ and not for the rest that it might receive during the discovery process (even if they are better routes). This option allows reduced memory in the routing tables and the size of the control message frames, but decreases the effectiveness and robustness of the protocol. Moreover, by appropriately applying metrics, a correct balance of the energy consumption of the nodes with respect to communication tasks can be achieved. As described before, in the CBAODV implementation it is possible to select and customize this metric capability by changing the configuration of the discovery mechanism according to the application constraints.

Only the final destination is capable of generating RREP messages: This has the advantage that no loops (circular paths in which the message continuously hops never reaching the destination) are made because the intermediate nodes cannot generate RREP. The sequence number is a field in the routing table and in the control messages created to avoid the generation of loops, as well as serves as a comparative parameter to analyse how updated routes are. Every node in the wireless network has to keep updated its own sequence number, whereas every routing table entry contains the latest available sequence number of the 
destination. In this way, if no loops can be created, then sequence numbers are no longer needed. However, this type of mechanism aims to reduce the amount to retransmission packets that can be avoided without wasting energy and processing resources on them. Therefore, the sequence numbers are fully supported in CB-AODV to allow intermediate nodes to generate RREP messages during the discovery procedure.

Elimination of RERR messages: In a very compact implementation, the inclusion of RERR messages can be omitted to decrease the complexity of the protocol, but it can lead to having a quite poor handling of errors especially in unstable scenarios where links are frequently prone to fail.

\subsubsection{Integration of $C B-A O D V$ in the HW-SW platform}

The design and integration of CB-AODV has been carried out based on the resources and the modular HW-SW architecture of the Cookie nodes, and on the basis of the developed communication layer. In this way, the libraries that handle the proposed features are included on top of the IEEE 802.15.4 controller that was created to manage the hardware implementation. This set of lightweight libraries, which has been coded in $C$ language on the basis of the proposed framework under the 8051-based architecture, is defined to support the Cookie communication capabilities in an efficient and resource-optimized fashion, considering that other implementations have been defined to be used over operating systems such as TinyOS.

In summary, the implementation is mainly composed of 3 blocks of libraries, as shown in Fig. 3.8, where CB-AODV is integrated within the context of the Cookie HW-SW support platform and data processing. The main block $\left(C B \_A O D V\right)$ is in charge of performing the main functionalities and the dynamics of the routing mechanism regarding control message coding/decoding and protocol information management, based on the packet frames coming up from the MAC layer handler. $C B_{-} A O D V \_$Table is in charge of handling the routing tables as well as the reserved memory for this purpose, making use of low-level memory controllers for optimizing the access and management of the routing information entries. On the other hand, $C B \_A O D V \_Q u e u e$ controls the input/output buffers 
as well as the RREQ registers to handle the protocol information in terms of discovery and dissemination processes. The type of implementation to be downloaded is controlled by using configurable parameters definition that allows making a more compact mechanism (based on the basic behaviour of the protocol) or a full/high performance implementation with all the designed capabilities.

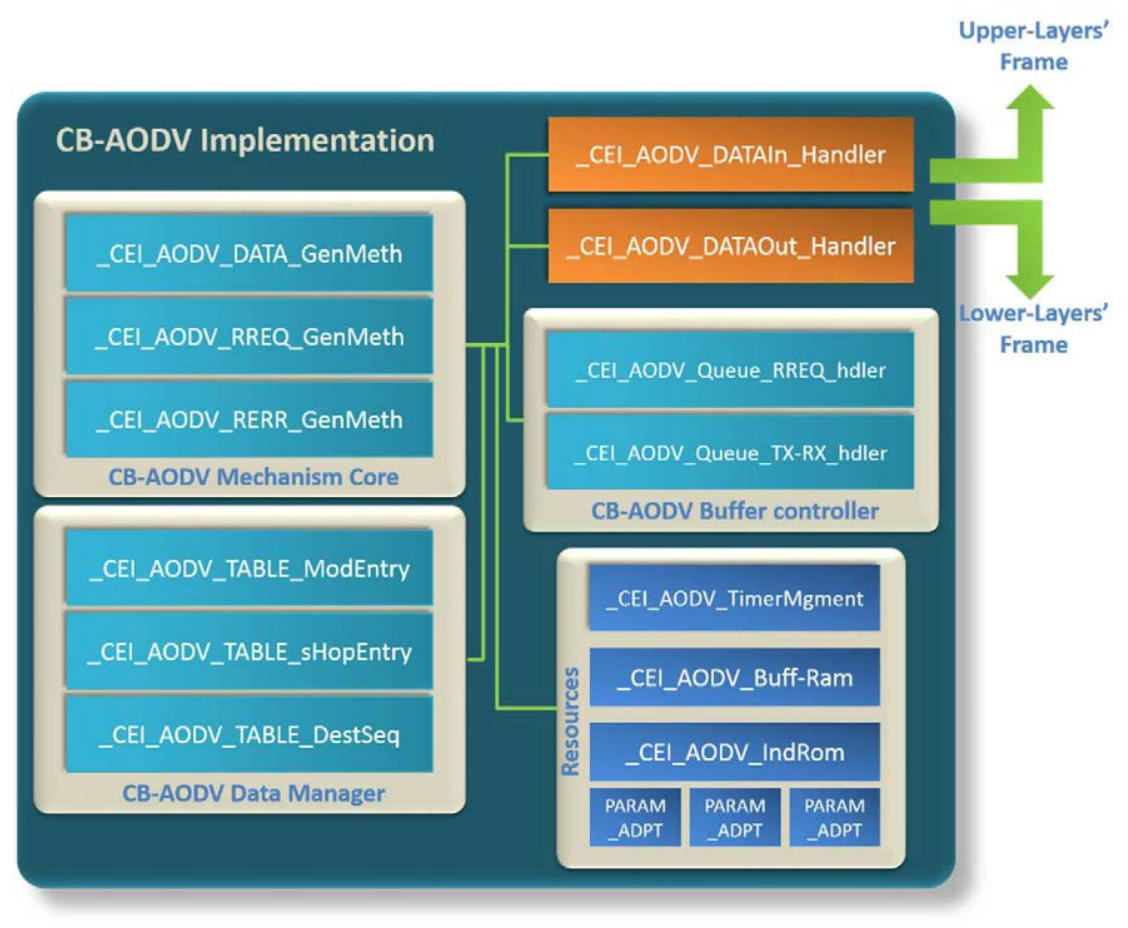

Fig. 3.8 General view of the CB-AODV implementation with the main functional blocks.

It can also be seen that the implementation makes use of different peripheral controllers of the architecture to modularize the data handling, so that the debugging process and the internal usability of the system is highlighted. Besides, as expected, the protocol implementation has been carried out in such a modular way that, from the point of view of the platform users, the application abstraction layer is provided with simple functions for sending packets to and receiving from remote destination points in a transparent way. 


\subsubsection{Comparative analysis of AODV implementations and proposals}

In Table 3-1, a comparison among different AODV implementations and proposals is shown in detail (based on the comparative analysis proposed in [Gomez'06]), in which CB-AODV is included. It is important to highlight that there are many proposals in the state of the art that have been analysed and implemented in simulation, unlike the one presented in this work which has been completely integrated in a new WSN HW-SW platform to be used in mesh network applications and thus evaluated in real WSN deployments.

The CB-AODV implementation has similar features compared to the NSTAODV, which is one of the most popular AODV implementations for TinyOS and is based on the TinyAODV implementation, being in this comparison the ones with more characteristics from the original AODV protocol as defined in the RFC and, therefore, the ones that will theoretically have better performance. The main difference between CB-AODV and NST-AODV is that the former one incorporates the precursor lists allowing the protocol to have better efficiency in the propagation of the RERR messages as well as the implicit detection of critical points along the routing path; the inclusion of the DATA_ERR message that targets a more efficient data dissemination process in critical and unstable scenarios; and the proposal of energy-efficient based cost metrics to enhance the dynamic routing mechanism taking into account the real conditions of every node in the target scenario, thus increasing the performance of the implementation.

As it can be seen in the table, the inclusion of the precursor list has not been added in some of the implementations or proposals. This is because of the additional memory resources that are needed for its inclusion. In CB-AODV, this feature has been added because there is an available internal EEPROM in the core architecture of the Cookie platform, so this free memory space was efficiently used to keep the routing tables. Moreover, another important optimization is also proposed in terms of memory usage. For those applications in which the size of the network is partitioned in less than 255 nodes, the control message frames can be reduced by means of using a 1-byte-based address field. 


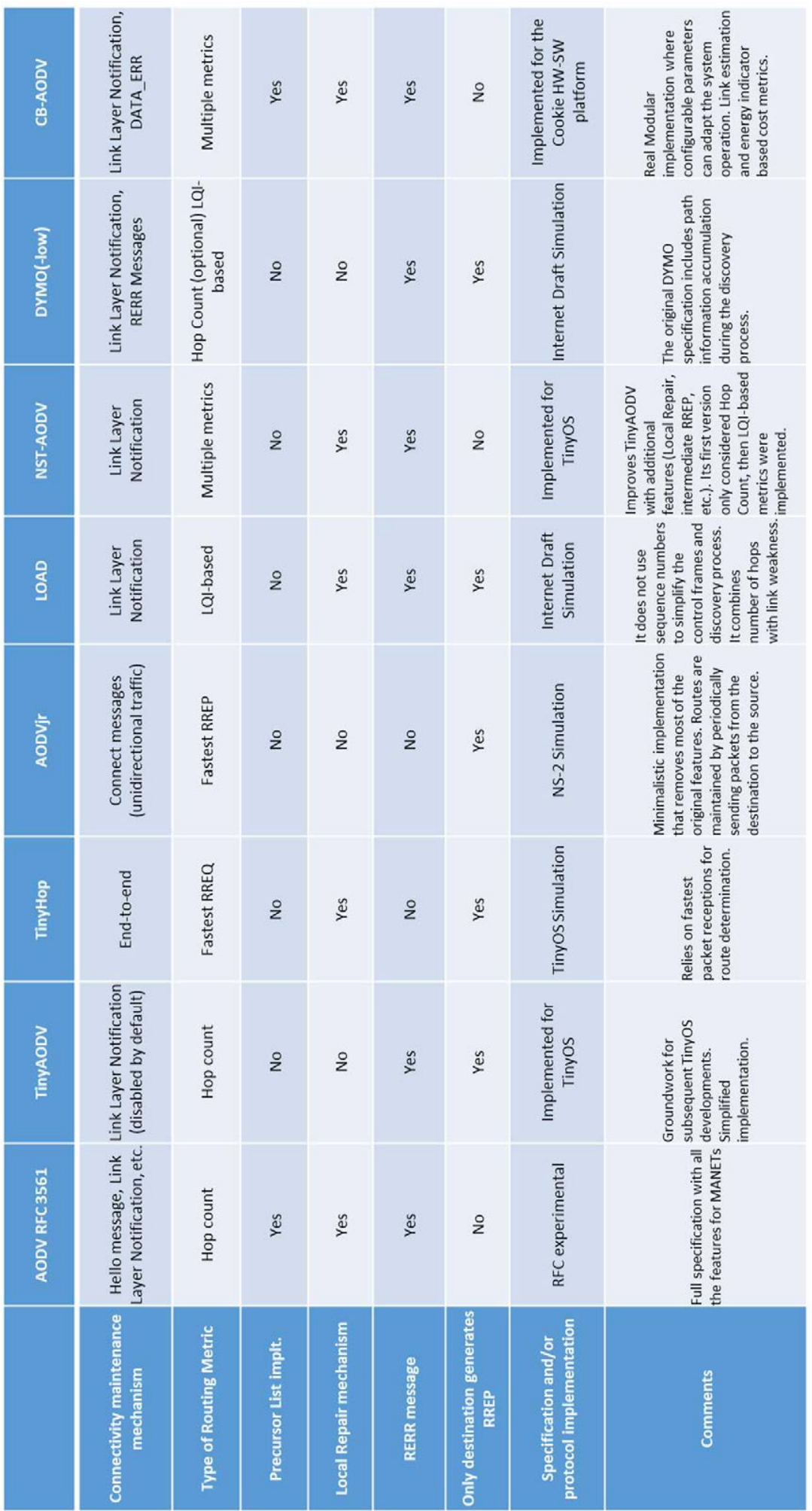

Table 3-1 comparison of different AODV implementations and proposals. 
In this case, the available memory space of the CB-AODV architecture for the routing tables can also be reduced and fixed. This option aims to increase the performance of the protocol since the latency due to routing table entry searching is reduced (memory access) by assigning a predefined position in the table entries for every node, hence speeding up the internal transaction of data processing elements.

As mentioned earlier in this section, the analysis and integration of different dynamic multi-hop optimization strategies for wireless mesh networking has also been addressed by including on one hand the AODV mechanism within the routing protocol optimization and performance analysis engine of the proposed toolset, and on the other hand an optimized hierarchical routing strategy so as to provide users with a better understanding on how the performance of the multihop communication techniques in mesh networks could be. For this, a section devoted to the CB-RSim engine is presented latter on in this chapter.

\subsection{On-site deployment and commissioning process: requirements, considerations and proposed approach}

The development cycle of a WSN-based system shall comprise the application of heterogeneous tools in order to efficiently implement, test and optimize the network design objective. This iterative system development flow involves the interrelation of development, planning and simulation strategies in order to generate the output information that serves as the starting point to carry out the deployment and on-site performance evaluation of the implemented sensor network application.

Let's first consider a system development cycle in which a proper integration of different technologies to pursue the goal of implementing a WSN application is faced. The first stage of the ideal flow is related to the formulation of the specification of the system to be developed and deployed, considering the main requirements and constraints associated with the functional and operational demands of the target application. The outcome of this phase will lead to outline the reference system architecture that better fits with the requested attributes 
and related components. The design phase comprises on one hand the conceptual planning and system/location survey that define the groundwork for network topology structure as well as node placement directives, in order to produce the initial network models information accordingly; and on the other hand the application logic description that defines the system components for the accomplishment of the desired functionality. This includes the selection of suitable hardware and software platforms as well as communication protocols under with the system operation is built, encompassing libraries and drivers for the node platform, processing elements for data aggregation, block interconnection definitions and sensor acquisition and monitoring techniques.

The planning and development phases shall enclose the integration of multidomain tools in order to perform the code generation and network component implementation activities in accordance with the system definitions of previous stages. The planning tool is responsible for providing virtual field node placement capabilities as well as radio propagation models in order to evaluate the suitability of connectivity links among the sensor nodes via simulation modules. Node positioning and radio communication configuration options are to be available in order to study network topologies and coverage issues, so that a theoretical network array can be generated to maximize the overall WSN connectivity with a minimum number of relay nodes. The development tool shall provide functionalities for the creation and management of new or existing libraries of components in order to perform code generation, compilation and application synthesis. The application can be built both for network behavioural simulation and for actual device programming and configuration, based on firmware production and according to the different types of WSN elements that take part in the system implementation.

It is important to notice that there shall be a direct relationship between the planning and development stages since they provide relevant information regarding channel configuration, field positioning and node/network platform properties that serve as input parameters to enhance not only the simulation models themselves but also the analysis of the real behaviour of the wireless sensor system under deployment/maintenance. 
During the testing phase, lab infrastructures are considered for prototype testing in combination with an iterative simulation process, where the result of the development and planning phases can be analysed, allowing then the readjustment of configuration parameters within the system development and code generation process. This leads to producing network and configuration models that will define the entry point for the deployment and commissioning phases. The deployment stage comprises the in-field installation, node positioning adjustment and initial configuration tasks in accordance with the output information generated from the previous steps of the development cycle. Then, the on-site performance evaluation of the WSN can be accomplished such that an efficient optimization process allows a proper refinement of the distributed system functionalities and overall network behaviour, considering actual connectivity parameters, environment conditions, sensor acquisition and processing, and runtime data dissemination mechanisms. Thereafter, the in-field testing stage encompasses the execution of debugging and diagnosis capabilities in order to analyse and compare the performance of the real operation of the physical WSN with what is expected from the modelling and simulation process. As a result, a more comprehensive feedback to the system development process can be provided so as to guarantee a more appropriate completion of the application requirements. The commissioning phase covers the final installation and initialization of the network functionalities as the outcome of the deployment phase, in which the application data processing and monitoring services are released, thus obtaining the operational state of the developed WSNbased system. Subsequent maintenance tasks are to be also considered in order to apply repair and troubleshooting actions upon the running application, so that the long-term operability and overall network life-time can be properly enhanced.

In Fig. 3.9 the overall correlation of the different described phases along with the involved heterogeneous tools is highlighted. Three important aspects have to be taken into account within the integration of the aforementioned systems. First of all, the application description entry which allows the interaction of the developer/deployer with the platform. This corresponds to the definition of UIs that support the actions to be executed. Secondly, component interrelation is to 
be done in such a way that the different tools can exchange the associated input/output generated data so as to contribute with the overall system implementation and optimization. Such a correlation can be achieved by creating well-defined interfaces among the implicated subsystems. One of the most suitable approach in this type of multi-domain platforms is the design of a model-based architecture, in which the different components are fed with structured models that are built and shared by using a common platform bus or system repository. This communication interface works as a project support component to store platform/network definitions as well as retrieve/update the results generated by the various participant tools. The third element that shall take part in the underlying integration is a middleware-oriented hardwaresoftware abstraction at different levels of the WSN system implementation (device-side and tool-side), so that technology encapsulation and low-level platform dependencies are handled by providing on one hand a set of library elements for a common development process, and on the other hand a middleware service interface for accessing and interconnecting platform attributes/components not only from the point of view of synthesis and compilation but also at real-time operation.

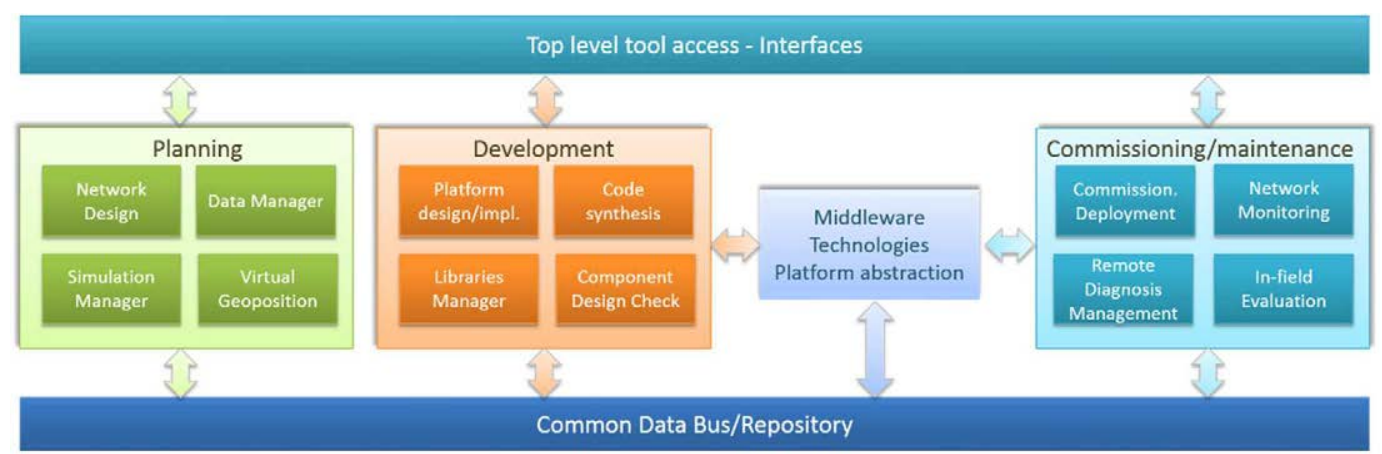

Fig. 3.9 General view of the involved phases and capabilities within a WSN application development.

As described previously, although there are different contributions in the literature regarding individual tools to accomplish the different steps of the predeployment phases, the integration of planning, simulation and modelling capabilities with a well-defined on-site deployment and commissioning toolset is a pending issue. This is particularly important considering that there is a 
shortage of frameworks, methodologies and tools to execute the in-field installation and performance assessment of the developed WSN, as fundamental phase to validate and subsequently maintain the released wireless system, especially allowing for the described system development cycle and the associated integration process.

In this thesis the aforementioned concept is addressed by proposing a new deployment and on-site evaluation tool, which integrates in a unique system architecture a complete set of functionalities to efficiently perform the installation, configuration and runtime in-field analysis of the target WSN, in accordance with the platform implementation, network simulation models, and code development process generated during the previous stages of the overall design and development cycle. The effective implementation of the WSN in-situ, i.e., the real deployment and commissioning of the sensor networks in the final planned scenario presents important issues to be faced so that the final desired operation of the system can be assured.

These key aspects to be considered include the transient power and resource handling during the installation, setup, tuning and reconfiguration steps, the effectiveness of the deployment and maintenance stages depending on the evaluation strategy to be adopted, and the actual placement problems that come out with the in-field WSN establishment, mainly in terms of connectivity, processing and sensing performance. Therefore, the effective combination of modelling and planning strategies with efficient deployment, commissioning and assessment techniques are to be taken into account to guarantee the final autonomy of the WSN system.

Moreover, the performance of the communication techniques and particularly routing strategies under the real operational conditions is essential to assure the effectiveness and the reliability of the final application where wireless nodes are to be used. The analysis and evaluation of data dissemination mechanisms, network topologies and routing protocols has been commonly approached from a simulation perspective, although realistic environments include unpredictable situations that cannot be covered in simulation models. Therefore, it is also crucial to establish an in-field based support mechanism to test the WSN 
connectivity under the real deployment scenario, thus allowing developers to analyse and compare functionalities from different abstraction levels, so that an on-site enhancement of the deployment performance and its operational lifetime can be achieved.

Based on this approach, in this work an evaluation and diagnosis support tool for the in-field activities is implemented within the proposed deployment and commissioning framework (which also supports the real testing of the implemented CB-AODV routing protocol within final application contexts). A synergy between simulation techniques and runtime evaluation of the physical WSN deployment is one of the main targets pursued in this work.

\subsection{System architecture of the proposed approach}

According to the aforementioned requirements and considering the on-site nature of the proposed deployment toolset, the system architecture approach is based on the integration of heterogeneous hardware and software technologies, whose implementation core relies on the concept of a mobile smart device with which deployers can accomplish the in-field configuration and performance evaluation activities. Such an element shall combine on one hand WSN capabilities to directly interact with the sensor nodes under deployment, and on the other hand advanced processing, computational and communication attributes to perform runtime on-site system analysis and optimization tasks. Deployers are to be capable of executing configuration, testing and assessment actions based on the provision of a dynamic user interface that will provide a comprehensive deployment guidance according to the WSN application to be commissioned.

Based on this approach, the proposed platform implementation is based on the integration of a mobile device with a node platform that includes the WSN technologies to establish a communication interface with the sensor nodes. That results on the development of a Hand-Held Device (HHD), where the WSN node platform to be integrated is referred to as the Hand-Held Node (HHNode). Thus, the overall deployment platform will encompass the definition of a Deployment, Commissioning and Maintenance Tool - Hand-Held Device. 
Before addressing the design and implementation of the internal technologies that build the overall system architecture, this platform can be firstly represented in accordance with the functional flow that takes part during the described development and deployment cycle, as depicted in Fig. 3.10. The basic element that serves as the entry point of the platform is the input network model definition, which represents the outcomes of the planning, development and simulation stages. Such models constitute the basic starting information to build the on-site deployment scheme, and shall include references regarding the hardware and software implementation of the embedded sensor platforms to be installed. That comprises the number and type of nodes that compose the WSN, considering positioning information, functional components and their configuration parameters, and block properties that determine the node/network adaptation to the target application. Along with this definition, input simulation information is also contemplated for the subsequent comparison of theoretical models with the actual behaviour of the WSN. Such a simulation data is represented in the form of a radio connectivity scheme among the nodes that outline the initial definition of the deployment.

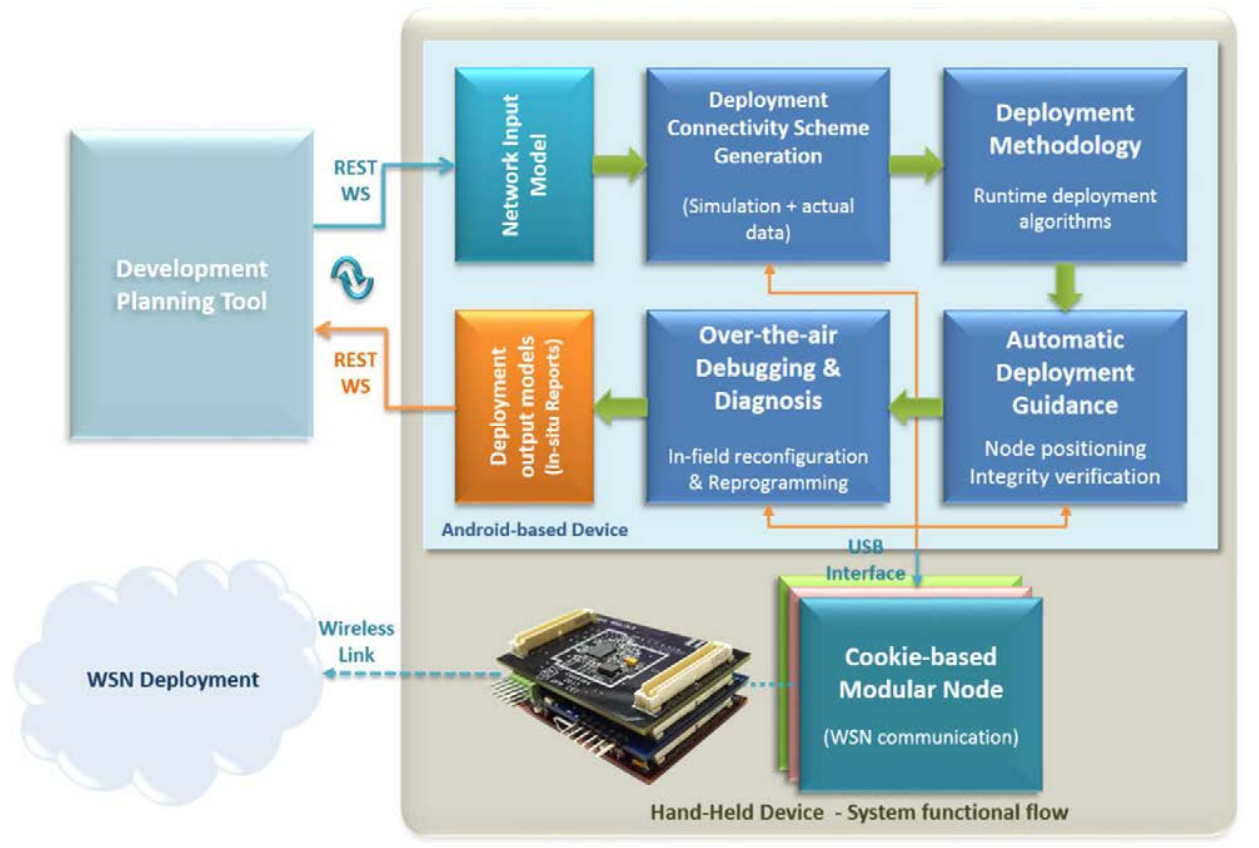

Fig. 3.10 Functional components and system flow of the deployment and commissioning tool. 
The outcome of the aforementioned input information retrieval is the generation of the internal deployment model that allows the tool to dynamically prepare the data management subsystem as well as the radio connectivity correlation engine of the HHD. From then on, the network optimization process, the deployment methodology and automatic guidance can be triggered with a direct implication of the real behaviour of the sensor nodes by using the HHNode communication interface, from which data regarding configuration, monitoring and diagnosis actions can be exchanged in-situ. This certainly represents a second interaction stage of the deployment tool that takes into account the actual functional components implemented and programmed in the nodes, as well as the middleware-based technologies for controlling and modifying internal properties of the embedded platform.

The on-site performance analysis, evaluation and optimization activities based on the deployment toolset capabilities will automatically generate output WSN models for subsequent refinement of the development tasks, so that a twofold enhancement process can be performed, i.e., during the deployment and maintenance stages as well as in system update phases if needed. That corresponds to another level of interaction within the system functional flow with respect to external elements that shall compose the overall development process, as shown in Fig. 3.10.

Based on the outlined functional flow of the deployment toolset and considering key requirements in the architecture definition such as: portability and autonomy, storage and memory handling, auto-localization, visualization, processing and runtime on-site computation, aggregation, and wireless communication capabilities, the system design and implementation can also be depicted as shown in Fig. 3.11, where the kernel of the toolset is the inclusion of an AndroidOS-based smart device on which the proposed architecture and the on-site WSN optimization engine is developed, in combination with its integration with the modular HW-SW platform also provided in this work. Such an integration array allows encompassing different WSN communication strategies depending on the application requirements, and especially targeting IEEE 802.15.4-based technologies. 


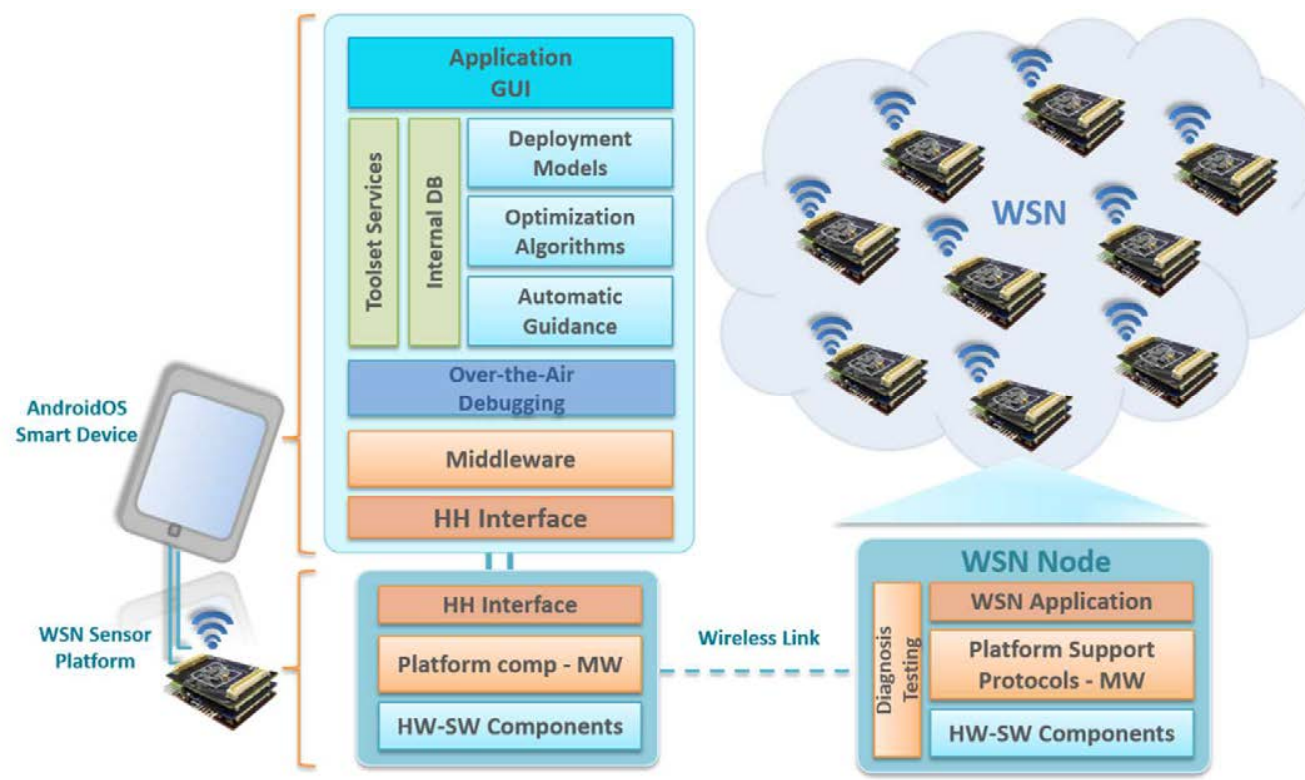

Fig. 3.11 Structural elements of the proposed Hand-Held Device system architecture.

\subsection{The Hand-Held Device in depth: on-site deployment methodology, analysis and performance evaluation strategies}

The Hand-Held Device has been conceived such that the requirements and challenges associated with the proposed system architecture of the deployment and commissioning toolset can be properly fulfilled by combining a set of functional elements and technologies in a unique mobile platform, encompassing the implementation of optimization algorithms within the deployment engine. Based on this, the starting point to be addressed focuses on the network model analysis and definition, as described in the following paragraphs.

\subsubsection{Services and input model definition}

The initial specifications and node/network attributes of the WSN to be deployed are materialized in the form of an application project definition, in which models that contribute in the system implementation are built. In this work the creation and update of the project definitions is realized by using a 
system repository that serves as an information storage for the network models to be generated. Such a repository is considered both as an online service for the HHD access and as an internal information provider within the self-storage of the HHD. While for the second case the created and downloaded models are stored by using the internal memory management system of the HHD, the online repository is handled by using service-oriented technologies.

The Web Services paradigm was conceived to establish an interoperable interaction between applications of different nature that are running under heterogeneous platforms, machines, language programming and frameworks, without the need of knowledge about the internal structure or implementation among them. It is therefore a standard definition on how to communicate different multi-domain systems by only considering a unified data transfer format, as the main way for information exchanging. Such a data exchange is commonly performed via Web technologies, particularly using the HTTP protocol upon which the data representation is transferred, usually considering an object-oriented approach. The Web Service transaction is performed between a service consumer that requests information from another application and a service provider, which then generates a response in the corresponding encoded format.

There are two main technology approaches when addressing the implementation of Web Services. On one hand, the description of the data can be done by using an XML specification (Extensible Markup Language) and transferred by means of SOAP (Simple Object Access Protocol), which defines the rules for data exchange based on XML [SoAP]. The body of the SOAP envelope is usually associated with (but not restricted to) a WSDL description (Web Services Description Language), which defines an XML-based public interface with a collection of bindings and endpoints that determine the services to be provided/consumed. SOAP commonly works on top of HTTP to carry out the data transfer process, although other protocols may also be used.

One the other hand, REST (Representational State Transfer) has become a clear alternative to SOAP due to its simplicity, flexibility and ease of implementation. The theoretical definition of REST refers to a set of architectural principles to 
establish a web-service-based interaction of components within a distributed system [Belqasmi'11]. In practice, REST describes interfaces that use HTTP as a standard way for managing system resources, which means that applications/platforms access and exchange the representation of such resources by using the defined HTTP-operations, without further abstraction level considerations. The resources that are available in a service provider are addressed by using a Uniform Resource Identifier (URI), which allows the univocal determination of a resource. The consumer application uses the URI to get the information of a resource, whose representation is returned back in the corresponding format. Hence, the application shall be able to interpret such a data representation, which is commonly in the form of a standard encoding format, such as HTML, XML, or JSON (JavaScript Object Notation). Apart from the resource identifier and the type of action to be performed, applications do not need to have further information about intermediary elements to access the services, beyond the request itself. Moreover, there is no session association between service providers (server) and consumers (client), thus considering the communication mechanism as stateless, where every transaction is treated independently (additional information is included in the resource itself, if needed).

Therefore, the main way of using a REST Web Service is by applying the explicit methods defined in HTTP, which corresponds to:

- POST, for the creation of a resource in the server.

- GET, for reading a resource.

- PUT, for updating/modifying a resource.

- DELETE, for removing a resource.

The type of representation to be expected in a resource request can be specified in the HTTP header by using MIME-types, such as application/json in the Content-Type for JSON, or application/xml for XML format. REST does not apply any restriction on the type of resource representation to be used, although the most common ones are XML and JSON, as mentioned before. However, the simplicity, easy to interpret and generate, and lightweight feature of JSON 
makes it suitable for its applicability in web-service-based data exchange, particularly because it is very efficient in terms of the data to be transferred, since the notation is more focused on the content than in the format and structure [Nurseitov'09].

The primary element of a JSON representation is the collection of key-value pairs that compose a JSON object. While the key is specified as a string, the value of a key can be expressed as follows:

- String (a sequence of zero or more Unicode characters).

- Number (used for any numeric type, including integers and floating point numbers).

- Boolean (in the form of true or false).

- A JSON object.

- A JSON array (it refers to a list of ordered values, which can take the form of any of the aforementioned formats).

Since the content of a key can be an object itself, more detailed structures can be created based on linking objects and arrays, so that the information can be comprehensively depicted. Such a notation certainly makes JSON representation easy to handle and analyse, both from the point of view of human and machine interpretation and searching. Another interesting feature related to JSON is the generation of JSON schemas, which declare the structure that the JSON object to be exchanged shall have (similar to XSDL for XML), including the expected fields that are required and the type of values associated with every key and arrays. It is therefore a formal description of the JSON content structure and constraints to be used during the service transaction, whose information is particularly useful for documenting and validating the generation of the involved JSON objects on a project.

The intrinsic modularity and flexibility of JSON combined with the REST Web Service capabilities to interface multi-domain platforms have been applied in this work to represent the network model definition and data exchange transactions between the system repository and the deployment toolset. In this 
direction, the approach that has been considered regarding the system repository technology from which the Hand-Held Device will extract and locate the simulation/deployment models is based on an Apache HTTP server [Apache], upon which the services for downloading and registering the involved models are contained.

More specifically, the Eclipse environment and tools for Java EE and Web application development [JavaEE] have been combined with Jersey Framework [Jersey] for implementing RESTful Web Services in Java, and Apache Tomcat Server [Tomcat] for deploying the implemented services.

The input network model is the primary element for the definition of the initial deployment guidelines to carry out the installation, configuration and onsite performance analysis of the target application, which is loaded according to the established Web Service capabilities, or by using the internal repository of the HHD. The JSON object is the representation mean to describe the planning and development information to be used as the input deployment specification of the system. Based on that, a comprehensive WSN model structure has been designed to specify both the overall sensor network attributes and the nodes implementation definition, in accordance with the HW-SW embedded platform to be deployed and considering the MW component interfaces. The system information description defines the minimum input properties to construct the deployment and data management system in order to carry out the in-field activities.

As shown in Fig. 3.12, the network model hierarchy is primarily composed of two major objects in line with the aforementioned attributes classification. The network definition object contains general information mainly regarding the following items:

- Network size: includes the number of nodes that compose the WSN deployment and thus the scale of the node object instances that are included in the network model. This is a result of the planning stage where the type of sensors and route nodes have been preliminary decided, in order to cover the application specification along with a minimum network coverage. 
- Node type instance: refers to the type of node platform instances that are needed within the WSN structure, including ROOTN_DEF, SENSORN_DEF, and NWKINTF_DEF that corresponds respectively to the number of root, sensor and network interface definitions contemplated in the model.

- Communication protocol attributes: encompass channel communication identification, radio interface configuration and wireless protocol properties, which will allow the HHD to tune not only the WSN nodes to be configured, but also the deployment tool interface component.

- Project and Plan references: each deployment model is associated with a plan entity that also belongs to a WSN project definition. The management of the models is organized by defining a structure split into application projects as overall system containers, and plan entities whose scope is delimited by the related project. While the project encompasses a target system implementation and requirements that will be addressed by the planning and development activities, plan entities determine different possible outcomes that can be analysed and evaluated. Models are referenced based on unique project and plan identification items, which also allows handling different versions of the input model generations. As explained later on in the URI description for the implemented Web Services, a top-level application project model provides the basic information of the projects and plans available in the system repository, including details of the applications under development.

- Network simulation sub-model reference: this parameter points at the input simulation instance in which a network correlation scheme among pairs of points (referred to as 2-Ns) of sensor nodes is provided alongside the model, if available. This simulation representation shall mainly include the attributes of the radio propagation results in the form of an array instance that contains a collection of 2-Ns communication objects, including transmitter and 
receiver configuration and quality evaluation metrics. As described subsequently, this simulation model representation can be compared and switched to an on-site simulation+real network model, which is dynamically generated in-situ based on modelling and experimental results integrated into the deployment methodology engine.

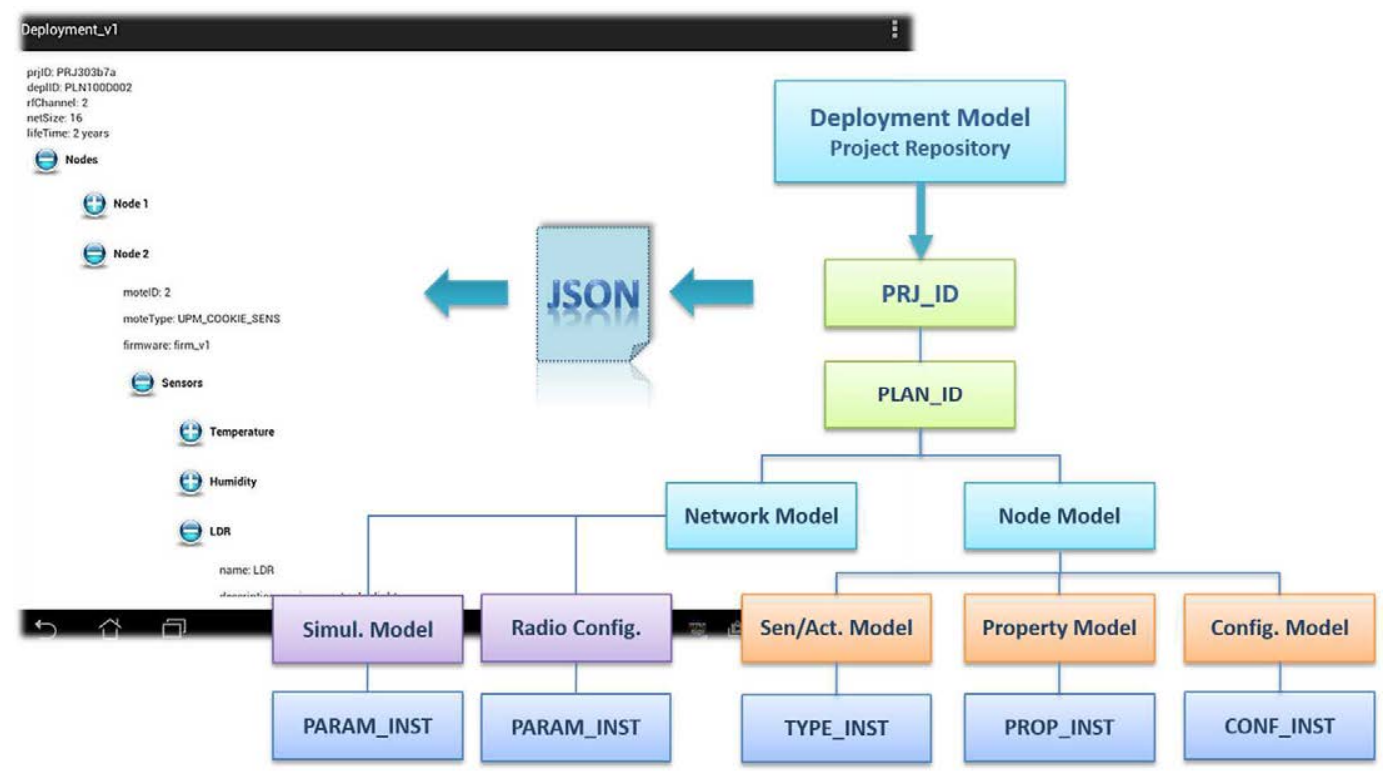

Fig. 3.12 Structure of the deployment model definition as an input of the system.

The node definition that encompasses the WSN network model implementation has been designed under the notion of a modular object representation, which considers on one hand four major levels of platform/component description, as follows:

- Sensor/Actuator entities: correspond to elements and attributes that define the HW-SW implementation of the sensors or actuators to be included within the application development.

- Property entities: include the characteristics of a platform component (for instance, sensors, radio communications, core processing), which define and interface the implementation.

- Configuration entities: provides the model abstraction to carry out modifications in changeable parameters as well as runtime configuration within particular entities (sensor, actuators or other platform/property components). 
Fig. 3.13 shows a basic JSON representation of a deployment model considering the baseline elements to be included within the WSN definition. Depending on the network dimension that forms the initial definition of the target deployment, a number of node objects will be instantiated within the network model accordingly. Every object is built by several component instances from the basis of the aforementioned definition of entities, which assemble the implemented platform to be installed.

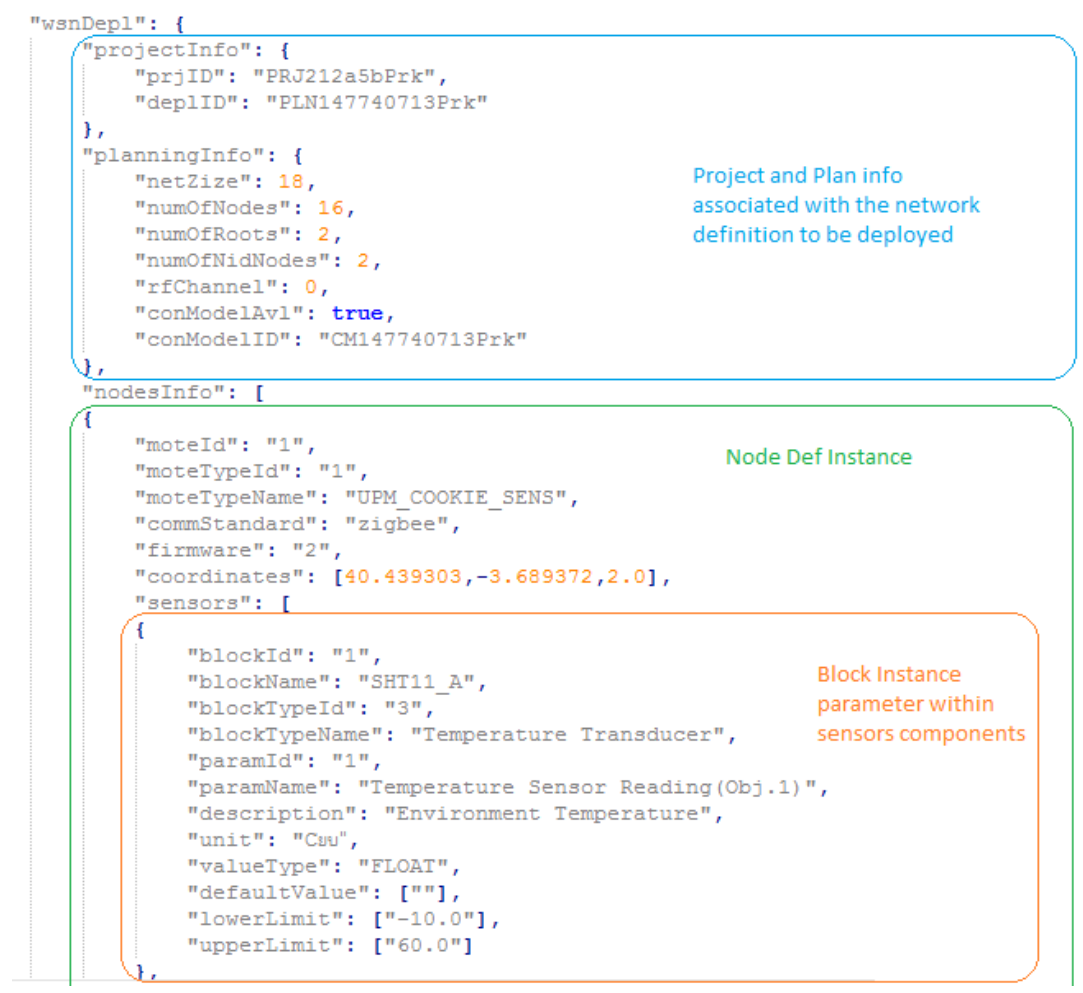

Fig. 3.13 Basic representation of the first part of a deployment model considering the project and plan information as well as node and functional block instantiation.

The node object is depicted considering firstly general node attributes and different component instances. Every node object is well determined based on three identification parameters, whose range scope is the deployment model (overall), as follows:

- MoteID: is the unique reference for the particular node object instantiation. It corresponds to an integer value (16-bits).

- MoteTypeID: refers to the node type definitions explained before, which can allow determining the main features of the object model. 
- MoteTypeName: provides a basic description label of the type of platform associated with the node object instantiation, for instance, for the Cookie modular platform.

Another important node attribute refers to the provision of the physical wireless node position within the target scenario. Such information is materialized in the form of three-dimension coordinate specification of the sensor location $\{\langle x\rangle,\langle y\rangle,\langle z\rangle\}$, which is a direct outcome of the planning stage. This feature will be subsequently used to perform the deployment methodology and in-field automatic guidance.

The components abstraction that defines the node representation has been conceived taking into account an object-oriented approach, in which three hierarchical elements depict the basic structure of a functional component: first, a generic bock type definition that contains the basic abstraction of the component (it corresponds to a component class); second, a block instance that particularizes the generic type definition with the specific attributes and configuration capabilities related to the real implementation of the component in the platform; and third, component parameters definition, which determines the inputs/outputs of the block instance and serves as abstraction interfaces to configure and monitor component attributes within the real implementation. Such a component description is illustrated in Fig. 3.14, where the expression of a particular component based on the aforementioned object-oriented definition is exemplified.

As in the node object representation, every component is uniquely determined by using three identification pairs, each one linked to the type, instance and parameter definition respectively, as follows:

- BLOCK_TYPE: identifies the functional component class from which the block instance is realized, in accordance with a generic block type repository. Such a repository is built from the basis of general HW-SW components that can take part within the platform implementation process. A common component definition regarding the reference modular platform is shown in Table 3-2, which considers functional abstractions and interfaces for sensors, radio modules, platform control 
blocks, publish/monitoring attributes, among others. It can be realized that such a component repository can be seen as a set of libraries and categories that determine the scope, properties and interface of a block implementation. This approach allows reusability and extensibility of the provided framework for different application developments. Its identification is global and unique for the component library definition to be used.

- BLOCKDEF_INST: represents a specific instance of a block type in relation with the general block definitions. It is the particularization of a component class integrated into the node platform, so that it can be properly interfaced and treated. For instance, considering a block type definition of temperature transducers that is instantiated to particularize the implementation of a SHT11 or a LM35 sensor. While the top-level hierarchy refers in both cases to the transducer definition, two different and unique instances are done for temperature sensors in case they are integrated in the node object, for example. This also applies in other types of fields, such as radio communication component definitions, platform properties and data processing configurations. In fact, the different instances that define the functional components of the node object representation are also classified according to the aforementioned platform description level (transducers, communications, properties, configurations). Regarding the scope of the instance reference, the model implementation has been approached by using two alternative ways. On one hand, every block can be associated with the sequence number of instances of a particular block type, i.e., the boundaries of the block identification is the number of instances of a block type definition. On the other hand, blocks can be associated with the sequence number of instances of a particular node, i.e., in this case the boundaries of the block identification is the number of component implementations that belong to a particular node.

- BLOCKDEF_PARAM: corresponds to the parameters that constitute the input and output references of a functional component, in the form of readable and/or writable values definition for a particular block 
instance. It is therefore the actual data representation of the associated block's attributes, which can be a sensor magnitude or measurement correction factor determination, a radio communication configuration, or the description of a runtime readable property. Thus, it allows a proper interface and management abstraction of the HW-SW functionality included in the node platform implementation. The sequence number of the parameters is local for every block instance, which means that the identification field refers to the number of parameters of each particular block.

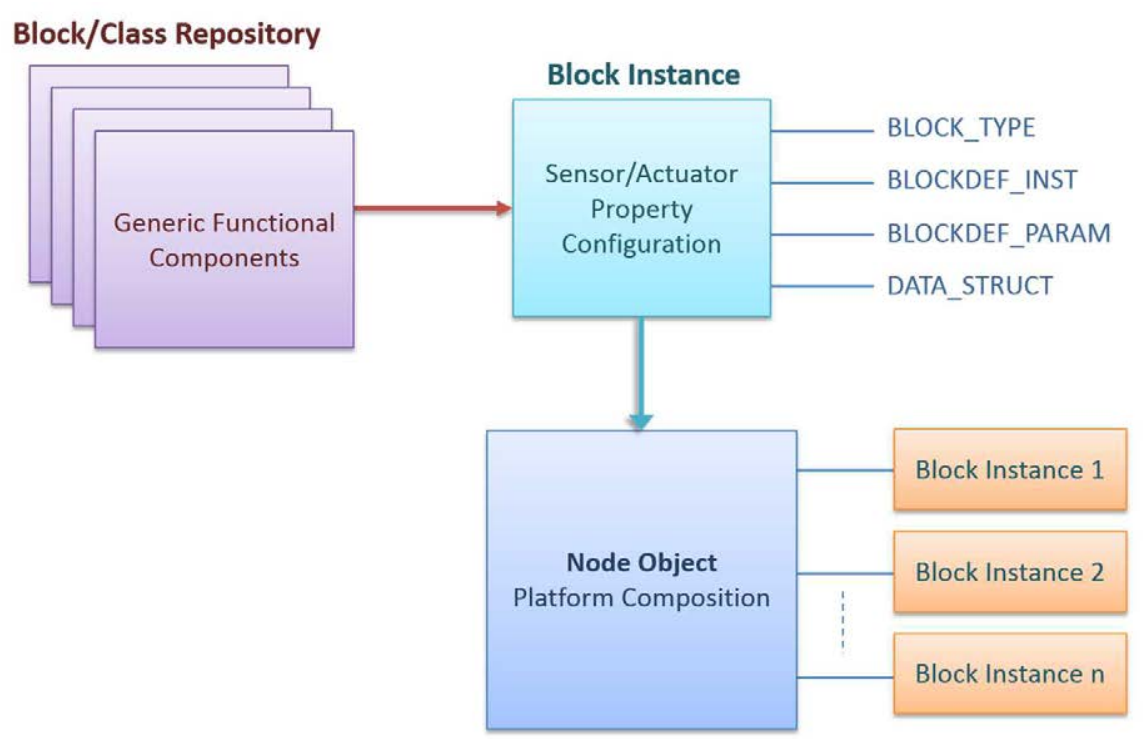

Fig. 3.14 Node functional components composition based on blocks definition and instances.

The identifier and descriptor of every hierarchy level along with the node object identification allow an unambiguous reference to blocks and parameters within the overall system model, as shown in Table 3-3 where a representation of different functional components is highlighted by using the aforementioned structure. Hence, such a sequence reference will certainly provide a well-defined entry to any value, parameter or state of a particular implemented block of the node objects and the overall network implementation. 


\begin{tabular}{|c|c|}
\hline $\begin{array}{c}\text { Block Definition } \\
\text { Identifier }\end{array}$ & Block Type Definition \\
\hline 1 & Radio_Transceiver \\
\hline 2 & Battery_Transducer \\
\hline 3 & Temperature_Transducer \\
\hline 4 & Humidity_Transducer \\
\hline 5 & Led_Perph \\
\hline 6 & ACC_Transducer \\
\hline 7 & Device_Control \\
\hline 8 & Param_Publisher \\
\hline 9 & Timer_Monitoring \\
\hline 10 & Protl_Metric \\
\hline 11 & Net_Inface \\
\hline 12 & Mem_Manager \\
\hline 13 & Light_Transducer \\
\hline 14 & Distance_Transducer \\
\hline 15 & Telemetry_Data \\
\hline
\end{tabular}

Table 3-2 A reference block definition considering basic functional components within a WSN platform.

Moreover, as shown in Appendix B, where a schematic representation of functional components based on various block type definitions is illustrated, instances can include parameters associated with any of the described platform levels, i.e., a transducer component can encompass not only parameters regarding the sensor entity, but also properties that can be accessed as well as configuration capabilities that can be modified (such as measurement factor correction or publishing interval). A block instance of Radio_Transceiver block type (e.g. whose instance represents the component implementation of a IEEE 802.15.4 module) can provide on one hand readable properties regarding link quality metrics, packet transmission rate, reception count and drop rate, and on the other hand configuration parameters related to TX power, power-down modes, and medium access settings. 


\begin{tabular}{|c|c|c|c|}
\hline Node Object & Block Definition & Block Instance & Instance Parameter \\
\hline 1 & $\begin{array}{c}\text { Temperature_Transducer } \\
\text { (defID:3) }\end{array}$ & $\begin{array}{l}\text { SHT11_A } \\
\text { (instID:1) }\end{array}$ & $\begin{array}{c}\text { Sens_Reading } \\
\text { (parmID:1) }\end{array}$ \\
\hline 1 & $\begin{array}{l}\text { Humidity_Transducer } \\
\text { (defID:4) }\end{array}$ & $\begin{array}{c}\text { Si7006_B } \\
\text { (instID:2) }\end{array}$ & $\begin{array}{l}\text { Sens_Sampling } \\
\text { (parmID:2) }\end{array}$ \\
\hline 2 & $\begin{array}{c}\text { Led_Perph } \\
\text { (defID:6) }\end{array}$ & $\begin{array}{c}\text { RGB } \\
\text { (instID:2) }\end{array}$ & $\begin{array}{c}\text { Green_State } \\
\text { (parmID:3) }\end{array}$ \\
\hline 2 & $\begin{array}{c}\text { Radio_Transceiver } \\
\text { (defID:1) }\end{array}$ & $\begin{array}{c}\text { IEEE_802.15.4 } \\
\text { (inst/D:1) }\end{array}$ & $\begin{array}{l}\text { RSSI_Value } \\
\text { (parmID:1) }\end{array}$ \\
\hline 2 & $\begin{array}{c}\text { Radio_Transceiver } \\
\text { (defID:1) }\end{array}$ & $\begin{array}{l}\text { IEEE_802.15.4 } \\
\text { (inst/D:1) }\end{array}$ & $\begin{array}{l}\text { TX_Power } \\
\text { (parmID:3) }\end{array}$ \\
\hline
\end{tabular}

Table 3-3 Example of block instances and associated parameters within node objects, which uniquely identifies a particular attribute/property/configuration of the sensor platform.

This deployment model definition has been conceived such that a high degree of modularity, scalability and flexibility of the network representation is achieved as the entry point for the HHD not only to build the internal data management system that serves as the support for the deployment, commissioning and maintenance activities, but also to establish a well-defined interconnection scheme to the functional components that integrate the WSN HW-SW implementation. Therefore, it allows determining the interface, data types and parameter structures of platform blocks and related properties/configuration elements.

Its reusability to apply available components and create new ones is an important attribute that distinguishes its design and implementation within the proposed platform. Furthermore, this abstraction model will support and ease in-field runtime execution tasks between the HHD and the WSN, especially when considering a middleware integration process as shown in latter sections.

Based on this network model representation, a JSON object can be created and then downloaded by the HHD from the system repository, via Web Services. In this way, four main URIs are defined as the interface to retrieve the input models as well as upload the subsequent output models according to the on-site deployment activities. The basic definition of the URI is expressed as 
http://<domain-server-ip $>:<$ port $>/<$ server-name $>/$, upon which different services to get/post resources are provided. As commented previously, the generation of the models is correlated with the creation of application projects and plans.

In order for the deployment tool to obtain information related to available projects, a top-level JSON that contains an array of project information objects is also considered, which includes their descriptions as well as the associated plans identification. This service is provided in the form of a GET method and the URI extension /projects/. In Fig. 3.15 a basic representation of this JSON object is illustrated, in which projects and plans IDs are introduced, in addition to including the active plan reference that corresponds to the current network planning of the associated project. Moreover, plans of a particular project can be filtered by specifying the project reference as /projects/<projectid $>/$ plans/, which thus allows retrieving the information object of a particular target project. From any of these methods, by specifying project and plan ID two main models can be downloaded from the system repository. The first one is the deployment model itself, by using /projects/<projectid>/plans/<planid $>/$ deplmodel, whereas the associated network connectivity correlation scheme can be retrieved (if available) from $/$ projects/<projectid $>$ /plans/<planid $>$ /netsconnt.

Therefore, the web services for downloading the project and plans based model from which the HHD deployment engine is initialized is summarized as follows:

- General information of projects and plans: base URI + /project/. Response: JSON object with array of project objects.

- Plans associated with a particular project reference: base URI + /projects/<projectid>/plans/. Response: JSON object with plan information objects.

- Deployment model downloading for a particular project and plan reference: base URI + /projects/<projectid>/plans/<planid>/deplmodel. Response: JSON representation of the network model definition including the array of nodes objects and their corresponding bock instances. 
- Network connectivity correlation downloading for a particular project and plan reference: base URI + /projects/<projectid>/plans/<planid>/netsconnt. Response: JSON representation of the connectivity scheme in the form of an object array with the radio communication correlation among the involved nodes.

Besides these retrieving options, the output deployment models can be uploaded by using the HTTP POST method, as shown in the output report generation section.

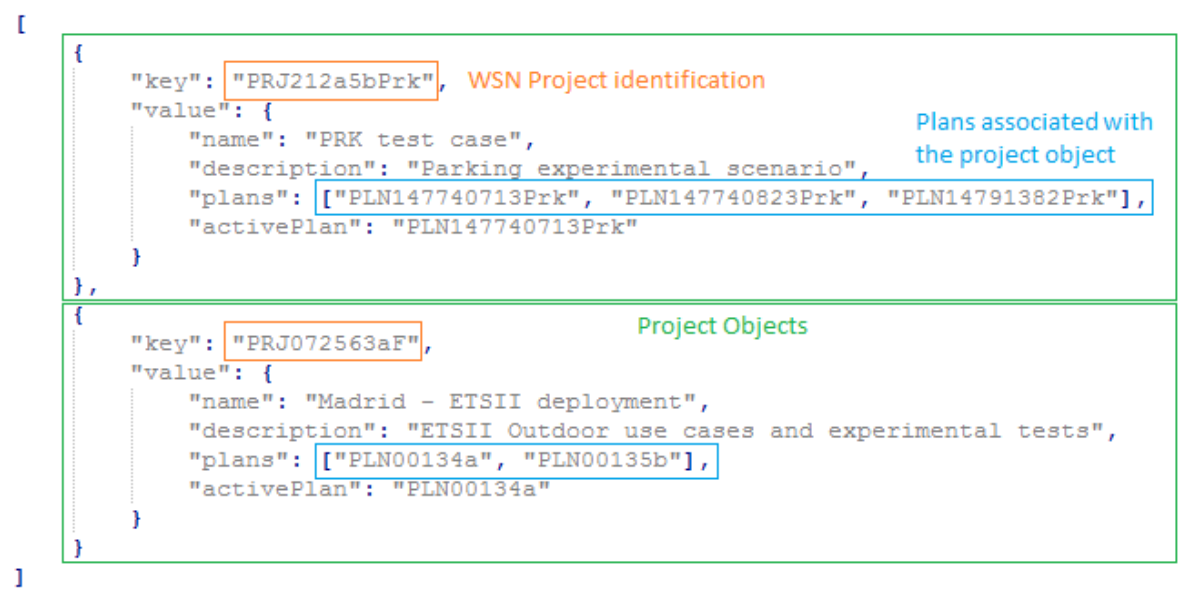

Fig. 3.15 Top-level JSON array that includes projects and plans identification of WSNs under development.

\subsubsection{Deployment connectivity analysis and in-field simulation engine}

Based on the definition and initialization of the deployment tool capabilities in accordance with the input models, the node correlation scheme can be generated in order to start the analysis and subsequent optimization of the network connectivity and topology. In combination with the theoretical node coordinates generated from the input definition, an on-site deployment connectivity engine is proposed to dynamically calculate the node correlation scheme, so that infield modifications in terms of node installation, positioning and parameter configuration can be directly evaluated online. This can lead to analysing insitu how particular changes in the network deployment might modify the overall system correlation and communication performance. This is especially 
important in this stage of the development and validation flow, taking into account that possible unexpected conditions may be encountered when carrying out the installation, configuration and performance assessment activities. Such a node connectivity correlation engine is conceived from the basis of including experimental-based radio connectivity patterns, by using real measured data for the production of adapted in-field simulation models.

Moreover, this connectivity analysis and optimization stage will provide a twofold support for the overall deployment toolset. First, it will supply the deployment methodology sub-system with an experimental estimation of the node connectivity in order to consider what nodes are correlated before executing the deployment mechanism; second, it will dynamically optimize the radio transmission power configuration of the nodes depending on real connectivity models generated from the actual hardware to be used in-field, which is materialized in a direct optimization of the network topology. The main target in this case is to have a trade-off between reliability of the connections, network communication requirements and power consumption efficiency, by only expending the necessary energy and resources on connecting the complete WSN system.

Together with the deployment methodology engine and the on-site network performance evaluation, this is an important optimization approach within the deployment process since the sensor network is addressed as a whole entity composed of collaborative elements, whose efficient correlation optimization will guide users to generate a more adapted system configuration array for the target scenario. As described subsequently, once the deployment stage is executed, a real connectivity scheme is generated and compared to the former estimation, so that the development phase can be fed back to enhance the simulation models and implementation strategies but considering the gathered data from the specific conditions of the target scenario.

In order to generate the node correlation scheme among the 2-Ns of the network, the main target is to integrate a connectivity model such that it can be dynamically executed without extensive computation during the on-site evaluation process. It will serve as a reference scheme for the provision of a 
general overview on how specific changes in the network installation or configurations can affect the overall network correlation. Although in the state of the art there is a numerous amount of different techniques to calculate the radio propagation between two different wireless nodes, one of the most well-known and general solutions that could fit effectively in outdoor scenarios is the Logdistance path loss model [Botta'13], which is an experimental model that computes the path loss for a signal between a transmitter and a receiver, taking into account the distance between both points as well as the path loss at a defined reference distance. It is expressed as shown in equation 3.3.

$$
\frac{P_{r}\left(d_{0}\right)}{P_{r}(d)}=\left(\frac{d}{d_{0}}\right)^{n}
$$

Applying logarithms and considering the power variables in $\mathrm{dBm}$, the expression remains as represented in equation 3.4.

$$
P_{r}(d)=P_{r}\left(d_{0}\right)-10 \cdot n \cdot \log _{10}\left(\frac{d}{d_{0}}\right)
$$

$P_{r}\left(d_{0}\right)$ represents the received signal power at a reference distance $d_{0}$ from the transmitter, $P_{r}(d)$ the received signal power at the receiver, and $n$ the path-loss exponent factor which implies the dependency regarding the specific propagation environment where the wireless communications are performed. The expression is finally represented in accordance with the radio signal strength values as reflected in equation 3.5.

$$
\operatorname{RSSI}(d)=\operatorname{RSSI}\left(d_{0}\right)-10 \cdot n \cdot \log _{10}\left(\frac{d}{d_{0}}\right)
$$

It can be realized that the propagation model can be tuned according to particular conditions by using experimental values for $d_{0}, P_{r}\left(d_{0}\right)$, and $n$, which allows producing more adapted estimations of the radio connectivity correlation scheme. In fact, there are typical path-loss exponent values depending on the application environment, as proposed in works such as [Kang'07], and summarized in Table 3-4. However, the HHD provides deployers with not only a set of experimental parameters to select and generate the connectivity simulation based on real registered factors, but also functions to carry out real in-field 
measurements so that scenario-adapted and $\mathrm{HW}$-adapted values can be produced.

Therefore, an estimation of the connectivity scheme according to a more accurate experimental path-loss custom factor is created both for the type of hardware platform to be used and the propagation environment. Such configuration capabilities and the lightweight connectivity estimation make the integration of these types of propagation models suitable for the in-field network correlation analysis as an additional support element for online readjustments and optimization tasks.

\begin{tabular}{|c|c|c|}
\hline \multirow{2}{*}{ Scenario } & n factor \\
\hline \multirow{2}{*}{ Outdoor } & Free space (general) & 2 \\
\cline { 2 - 3 } & Urban (more-dense) area & $2.7-4.3$ \\
\hline \multirow{2}{*}{ Indoor } & Line-of-sight & $1.6-1.8$ \\
\cline { 2 - 3 } & Obstacles & $4-6$ \\
\hline
\end{tabular}

Table 3-4 Generic path-loss factor values according to the application scenario.

In this direction, real tests have been performed in an outdoor scenario at the ETSII by using the HHD in order to obtain an adapted path-loss factor for the implemented IEEE 802.15.4-based communication layer proposed in this work within the modular platform, in addition to finding the reference RSSI at distance $d_{0}$ for the whole range of TX power of the aforementioned layer.

The experimental setup is composed of two wireless nodes that work as transmitter and receiver elements, with which packets will be exchanged to measure the RSSI parameter considering two main variables within the test cases: a range of different distances between the two points, and the TX transmission configuration values for the communication module. Moreover, LQI and LDR metrics are also gathered in order to study parametric correlation among the various quality indicators, so that further estimation models can be generated based on the experimental performance analysis.

Table 3-5 shows the range of distances and TX powers for the experimental scenario, from which more than 900 different tests have been performed 
considering a combination of these parameters, then triggering and gathering up to 7208 samples, which were registered and processed by using the HHD accordingly. Based on this, in Table 3-6 the main results regarding the correlation between the TX power configuration range and the RSSI $\left(\mathrm{d}_{0}\right)$ values gathered at a reference distance $d_{0}=3 \mathrm{~m}$ are shown, which were computed as the average of aggregated distance-power reference data.

\begin{tabular}{|c|c|c|c|c|c|c|c|c|c|}
\hline Variable & \multicolumn{10}{|c|}{ Range of Values } \\
\hline \multirow{2}{*}{ Distance $(d)$} & 1 & 2 & 3 & 4 & 5 & 6 & 7 & 8 & 10 \\
\cline { 2 - 12 } & 12 & 14 & 16 & 19 & 22 & 25 & 30 & 32.5 & 35 \\
\hline TX Power $(\mathrm{dBm})$ & 0 & -1 & -3 & -5 & -7 & -10 & -15 & -25 \\
\hline
\end{tabular}

Table 3-5 Range of values for the experimental tests.

By using the expression represented in equation 3.6, where the $n$ factor is found from equation 3.5, the path-loss value is obtained for every RSSI sample at distance $d$ between transmitter and receiver and with respect to the aforementioned reference $\operatorname{RSSI}\left(\mathrm{d}_{0}\right)$. Then, the average experimental factor is computed according to the overall results of $n$ for the whole range of distances considered. As a result, a reference path-loss parameter for outdoor scenarios based on the modular WSN platform and for the proposed wireless communication layer is provided in (3.7).

$$
\begin{gathered}
n_{e}=\frac{\operatorname{RSSI}\left(d_{0}\right)-\operatorname{RSSI}(d)}{10 \cdot \log _{10}\left(d / d_{0}\right)} \\
n_{e}=1,77
\end{gathered}
$$

Such experimental outcomes allows comparing the integrated connectivity model with the actual gathered data, so as to analyse whether the radio signal and packet degradation curves tendency match well in both cases. As shown in Fig. 3.16, three different curves in relation with the TX power configuration have been selected as samples to illustrate the similarities on the model tendency with respect to the real experimental data, verifying the suitability of such a lightweight in-field simulation capability for outdoor scenarios, particularly to provide deployers with a fast general overview of possible connections and 
quality indicators among the sensor nodes to be installed, in addition to generating quick previews of such a correlation scheme if modifications in configuration and positioning are realized.

This in-field modelling capability serves as a groundwork to analyse and incorporate different impact and attenuation parameters based on experimental data, as well as including factors such as the log-normal shadowing effect that is expressed as a zero-mean Gaussian random variable and standard deviation $\sigma$ (whose term includes variations in the signal strength due to fluctuations in the propagation environment), represented as $X_{\sigma} \sim N(0, \sigma)$ in $\mathrm{dBm}$.

\begin{tabular}{|c|c|}
\hline TX Power (dBm) & RSSI(d0) (dBm) \\
\hline 0 & $-58,6$ \\
\hline-1 & $-60,5$ \\
\hline-3 & $-61,6$ \\
\hline-5 & $-64,0$ \\
\hline-7 & $-66,0$ \\
\hline-10 & $-67,3$ \\
\hline-15 & $-75,8$ \\
\hline-25 & $-85,1$ \\
\hline
\end{tabular}

Table 3-6 Relationship between TX power and reference RSSI(do).

In order for deployers to include connectivity restrictions in combination with the network correlation scheme, three main model configurations can be triggered during the in-field simulation generation, particularly regarding the path-loss factor depending on environment conditions in particular areas of interest. This will allow setting topology constraints that are also used as an input information to the node transmission optimization algorithms. Three main levels of connectivity configurations are then provided, as follows:

- Outdoor mode: Allows for a general outdoor scenario without major interference and attenuation elements that affect the overall performance 
of the 2-Ns connections other than the tendency of the obtained propagation parameters (particularly considering $\mathrm{n}=1.77$ ).

- Intermediary attenuation mode: this includes those scenarios where the connection between transmitter and receiver are quite influenced by attenuation elements and obstacles that might penalize the strength of the signal. Such a condition is configured by mainly tuning the path-loss factor to be applied to the propagation model. In this case, the path-loss factor will be set in the range of $n=2.8$ as an intermediary point to model the aforementioned state.

- Connectivity restriction mode: allows setting connection constraints in those situations where the correlation between two points is limited or certainly unstable, hence considering and marking such a connection as unreached. As mentioned before, this option also provides the possibility of performing topology changes and connectivity correlation scheme reconfiguration based on on-site requirements and restriction agents, so that subsequent optimization algorithms can take into account such factors to find a more adapted solution.

The network connectivity evaluation allows further analysis of the real state of the WSN deployment especially considering the balance of node interconnections throughout the network. For this, three main conditions are considered to evaluate the optimization of the network communication, especially in mesh networking scenarios where link distributions play an important role to guarantee the cohesion of the deployment, as well as the effective communication among remote points:

- Isolated nodes: refers to those points that do not establish a direct link with its surrounding area due to coverage limitations (location constraints or transmission configuration).

- Critical points: in which traffic congestion is focused on nodes that have a bigger load in packet retransmissions and routing functions. As in the other cases, this is associated with factors related to network distribution and node coverage capabilities. 
- Redundant connections: considered as an ideal condition for multi-path communication and packet delivery reliability, since communication between remote points is supported by several interconnection routes. A trade-off between feasibility, platform cost (in terms of number of redundant points) and power consumption/lifetime are to be taken into account for the configuration strategy to be adopted, although such state also depends on the actual specifics of the deployment, including placement and coverage problems that are encountered when performing the field installation and evaluation process.
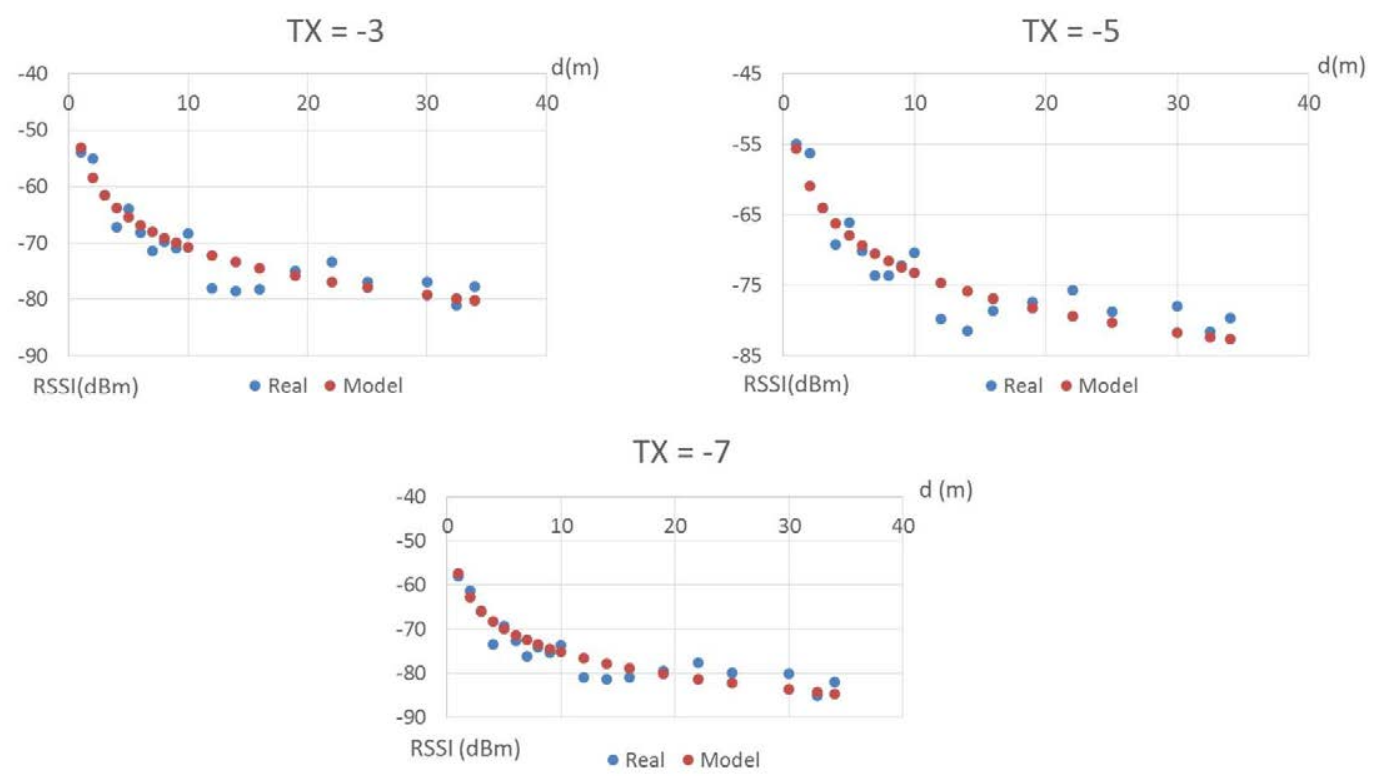

Fig. 3.16 Comparison of the in-field experimental results with respect to the model for TX power $=-3,-5$ and $-7 \mathrm{dBm}$.

It is important to highlight that the aforementioned states and the network connectivity scheme allow to remark the effects of modifying the deployment properties of a particular node with respect to the rest of the WSN, i.e., the optimization process has to be performed considering that changes in nodes or areas may affect the global network correlation. Therefore, the system optimization has to be carried out in such an efficient way that a trade-off solution between communication support balance and network cohesion is addressed. 
Based on this approach, the network connectivity engine generates the output correlation scheme that serves both for the connectivity optimization mechanisms as well as the deployment methodology engine, as described in the following sections.

\subsubsection{Topology optimization mechanism}

Taking advantage of the benefits of the generated node/network correlation scheme, a topology optimization capability is built within the connectivity analysis engine in order to provide more balanced network interconnections, in terms of reliability of the communication links among the nodes and the energy consumption optimization associated with data transmission of the sensor nodes. Generally in WSN deployments wireless sensor nodes are usually configured with the maximum transmission power in order to theoretically assure a greatest level of reachability and reliability of the interconnection links among them as possible. However, power consumption associated with radio communications represents one of the most relevant components in the overall consumption of the sensor nodes and thus in the lifetime of the network. This issue has been addressed in the literature by considering topology control mechanisms in WSNs, especially targeting transmission power optimization approaches [Khemapech'07].

The transmission power control and optimization concern is certainly related to an energy reduction during communication between pairs of points of the network, but it can also have effects in reducing high density of wireless medium access (and hence the number of possible retransmissions), as well as computational efforts when encountering a high degree of multi-path alternatives to reach remote points. Therefore, reliability and efficiency are not always related to configuring the transmission power parameter with the highest possible value of the radio communication module range.

In this way, transmission power optimization has been approached from different perspectives in the state of the art, including techniques such as the work proposed in [Lin'06], where authors target the adjustment of the transmission power configuration of each node with respect to their 
neighbourhood, by means of registering the best possible value for every neighbour that guarantees a minimum quality of the link. This can lead to produce an important overhead particularly in dense and large-scale deployments to initialize, execute and keep such a scheme. A relationship between the transmission power and the link quality parameters is studied to establish a predictive function that allows configuring an adapted transmission power for every neighbour node along time, based on the monitoring of link changes.

In [Liu'03] authors focus on covering heterogeneous deployments where the range of transmission configuration values may be different in every node, i.e., those situations where the maximum possible power of one node is higher than that of one or more neighbours. For this, nodes compare their transmission power $P_{x}$ with their vicinity nodes, and if one of them cannot properly reply to the origin $\left(P_{y}\right.$ max $\left.<P_{x}\right)$ then a re-broadcast procedure is performed to register a multi-hop path to such an origin. Other works in the literature centre the transmission power optimization on finding a predefined (threshold) number of neighbours per node with the lowest power value. If such a threshold level is not achieved, the transmission parameter is reconfigured to restart the neighbour verification process with a better power value.

Most of the works focus their attention either in keeping the list of neighbours and then try to readjust the transmission power, or in defining minimum neighbour links correspondences, which can produce matching areas where optimization requirements are covered, but with lack of cohesion among them.

In this work a different approach is followed considering an overall network topology optimization from the basis of the generated connectivity scheme. Instead of keeping the neighbour parameter as an initial reference to optimize the transmission power of every node, the optimization is realized by targeting the cohesion of the whole network with a) a reduced power consumption with respect to the maximum transmission configuration, b) reliability and quality of the generated topology, c) a trade-off between redundancy and medium access efficiency. 
The basis of the connectivity optimization relies on the evaluation of the link quality among the 2-Ns of the network, which can be done either by triggering and retrieving packets between two nodes and then computing the packet reception ratio [Son'04], or by using estimation models such as in [Lin' 06]. In this work, RSSI, LQI and LDR based experimentations are intensively analysed and assessed focused on the proposed modular HW-SW platform, so that a direct correlation between the transmission power configuration, the delivery reliability of encountered links, and the quality parameters is allowed for.

In this way, the experimental results from previous sections for obtaining a more accurate propagation model have been combined with additional tests in order to study the impact of the quality of the interconnections in the adjustment of the transmission power value. The first aspect to be taken into account is the evaluation of the reliability of a connection between two nodes based on the quality of such a link, regardless of the transmission power configuration. The analysis of the connection at physical and medium access level for the modular communication layer is performed from the basis of the RSSI and LQI values provided by the integrated IEEE 802.15.4-based radio transceiver. Independently of the transmission power, the reliability margin is associated with the packet delivery/reception ratio of a direct link between two points, which can be correlated with the RSSI parameter from the real gathered data.

As shown in Fig. 3.17 based on the real experimental results by using the modular sensor nodes, a correlation between RSSI and LDR is established to analyse the progression of the delivery ratio with respect to the measured radio signal strength at the receiver point. It can be seen that up until a certain range of RSSI values, the LDR presents a stable behaviour (100\% of delivery), while from these bounds the link exhibits more unstable result, which hence could affect the suitability of such a link to be reliably used for the communication between the involved pairs of points. A summary of these observations are also collected in Table 3-7, where an important degradation of the LDR for RSSIs between -85 and $-89 \mathrm{dBm}$ is visible. This represents a critical connection, and thus it can be marked as a very weak link that therefore does not guarantee a minimum data transfer consistency between the nodes. 


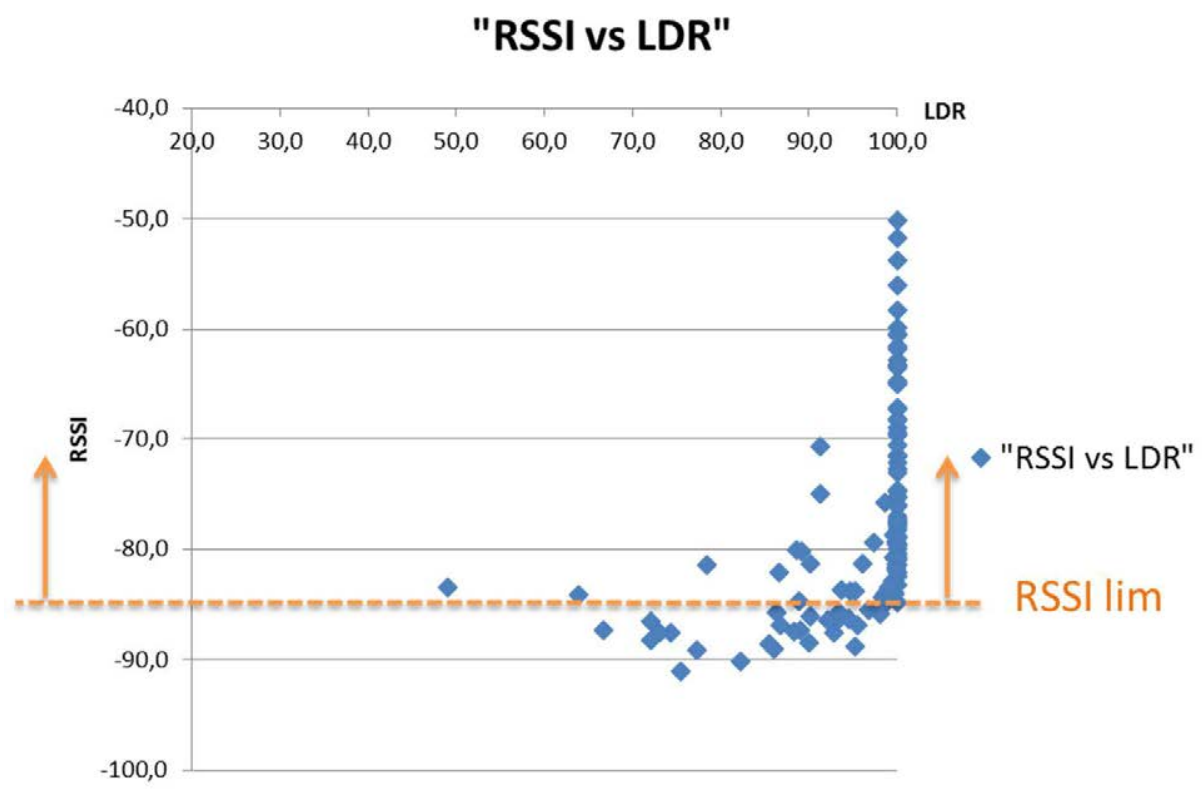

Fig. 3.17 Experimental correlation between the RSSI and the LDR.

In order to consider a link reliable enough to mark two nodes as connected, a conservative RSSI value is taken into account from the basis of the experimental results found. Hence, the threshold value upon which the LDR metric will be higher than $95 \%$ is $-85 \mathrm{dBm}$, i.e., LDR $>95 \%=>$ RSSI $>-85 \mathrm{dBm}$, so the RSSI limit to be considered for the connection condition is defined as $\mathrm{RSSI}_{\mathrm{lim}}=-85 \mathrm{dBm}$.

\begin{tabular}{|c|c|}
\hline LDR (\%) & RSSI (dBm) \\
\hline 100 & $>-84$ \\
\hline$[99,95]$ & {$[-84,-85]$} \\
\hline$[95,75]$ & {$[-85,-87]$} \\
\hline$[75,60]$ & {$[-87,-90]$} \\
\hline$<60$ & $<-90$ \\
\hline
\end{tabular}

Table 3-7 Degradation of the LDR metric according to the RSSI.

Then, based on the radio propagation model and considering the RSSI values at distance reference (which also imply the transmission power range), as well as the aforementioned RSSI lim, another parameter that can also be provided to evaluate the connectivity between 2-Ns is a distance limit, which is expressed as shown in equation 3.8. This parameter is $d_{l i m}$. 


$$
d_{\text {lim }}=d_{0} \cdot 10^{\left(\frac{R S S I\left(d_{0}\right)-R S S I_{\text {lim }}}{10 \cdot n}\right)}
$$

As shown in Table 3-8 and also represented in Fig. 3.18, a relationship between the transmission power configuration, the RSSI at the distance reference and the obtained $d_{\text {lim }}$ is highlighted, which allows the provision of a reference parameter overview to decide whether the connectivity between the sensor nodes may be suitable or, instead, changes shall be performed to enhance the performance of the links. According to these results, the following considerations can be highlighted:

- An overall network connectivity perspective can be provided to deployers so that runtime optimizations may be performed, not only in terms of the transmission power configuration but also an experimental overview of suitable distances between 2-Ns. By setting one of this parameter, the other one can be adjusted so as to optimize the corresponding connection within the proposed limit parameters. This means that, for instance, if restrictions in node positions imposed by deployment constraints are to be taken into account, the transmission power value may be readjusted to enhance the power consumption of the node.

- The RSSI lim found is of great importance since regardless of the transmission power configuration, the reference RSSI/distances or the radio propagation factor, this parameter will set the boundaries from which a connection is considered as unstable and thus avoidable. Within these limits, the power configuration is to be carry out such that a balance between network cohesion, energy awareness and communication robustness is achieved. This metric will allow to define the notion of node's direct links, with which the topology optimization is performed by assuring a minimum consistency of the connectivity distribution throughout the deployment. 


\begin{tabular}{|c|c|c|c|}
\hline $\begin{array}{c}\text { TW Power } \\
(\mathbf{d B m})\end{array}$ & RSSIr (do) & $d \lim (\mathbf{m})$ & $\begin{array}{c}\text { RSSI lim } \\
(\mathbf{d B m})\end{array}$ \\
\hline 0 & $-58,60$ & 93 & \\
\hline-1 & $-60,50$ & 73 & \multirow{2}{*}{-85} \\
\hline-3 & $-61,58$ & 63 & \\
\hline-5 & $-64,00$ & 46 & \\
\hline-7 & $-66,00$ & 36 & \\
\hline-10 & $-67,33$ & 30 \\
\hline-15 & $-75,75$ & 10 \\
\hline-25 & $-85,08$ & 3 \\
\hline
\end{tabular}

Table 3-8 Distance limits with respect to the TX Power and its relationship with the RSSI (reference and limit values).

Based on these outcomes, the main target is to provide deployers with a set of topology optimization mechanisms from which a complete array of transmission powers for the whole network deployment is provided by using the Hand Held Device, so that in-field reconfiguration capabilities can be dynamically realized accordingly. So, based on the RSSI estimation for every link of the mesh network and considering the distribution of the deployment, the proposed algorithms aim to find firstly a general optimized transmission power with respect to the maximum communication range, and then based on such an enhanced scheme the topology is readjusted in a second optimization phase to guarantee a reduced power consumption with a proper link quality map.

So the first phase of the topology optimization process within the proposed connectivity engine is the uniform power algorithm, in which the target is to homogeneously reduce the transmission power configuration of the overall network such that the cohesion of the network can be kept with a better power consumption profile, i.e., a transmission power that assures the necessary robustness and consistency of the nodes interconnections with an improvement of the energy spent for transmission purposes. 


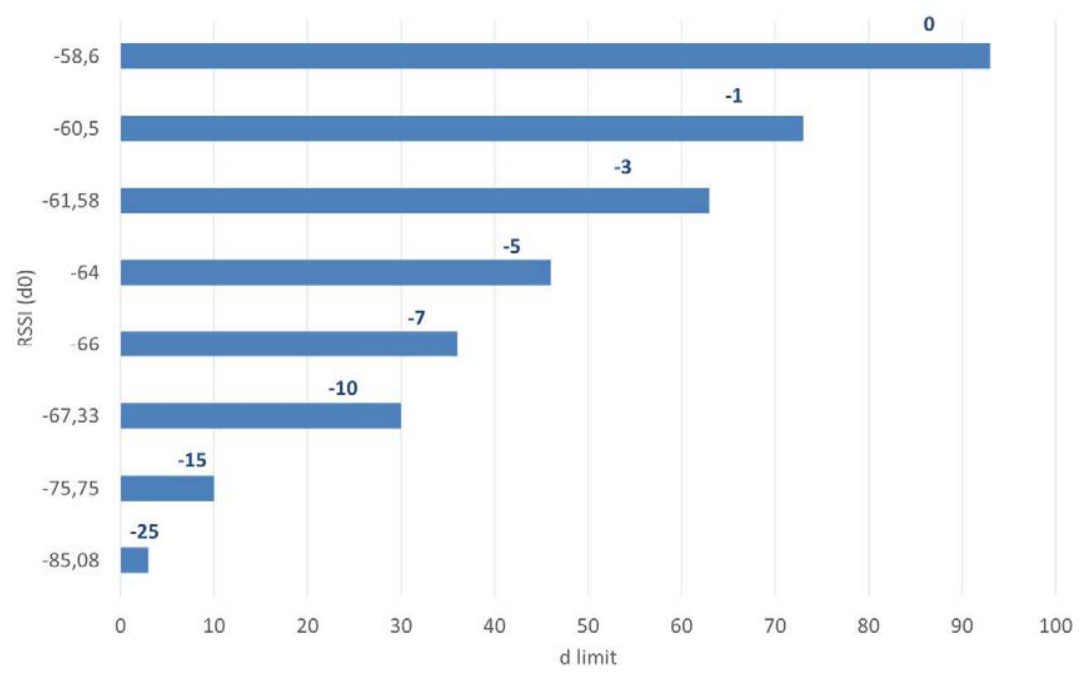

Fig. 3.18 Reference RSSI $\left(d_{0}\right)$, $d_{\text {lim, }}$ for TX Power and RSSI $I_{l i m}$.

Two main alternatives are considered within the uniform power generation scheme. The first one seeks the avoidance of nodes in isolated state, so that a minimum connection with at least one surrounding neighbour is assured. This means that every node shall be interconnected with another neighbour point whose link quality is within the reliable boundaries (i.e., based on the RSSI lim parameter). The algorithm starts with the minimum transmission power configuration as the triggering parameter to begin the evaluation of the sensor node connections, as follows.

- Consider such a first configuration as $t \mathrm{p}_{\min } \in \mathrm{TP}_{\text {range, }}$ from which node $n_{i}$ $\in N$ evaluates the discovery of $S_{i}$ neighbours, where $S_{i} \geq Q$, and $N$ is the size of the network. $\mathrm{Q}$ is the desirable neighbour discoveries, which is 1 in this case for the maximum topology optimization.

- For $n_{i}$ the RSSI parameter is calculated with respect to the rest of $\mathrm{N}$ so as to encounter those nodes above $\mathrm{RSSI}_{\text {lim }}$ that are considered as neighbours. The process is then repeated for all the nodes that are part of the WSN deployment.

- If $n_{i}$ does not find any suitable surrounding neighbour $\left(S_{i}=0\right)$, then $t p_{\text {min }}$ is increased to compute a new generation of possible reliable links for each sensor node of the network. The idea is therefore to verify those 
sensor nodes that are marked as isolated points to improve such status with a new discovery round.

- In case each node matches the condition of at least $Q$ encountered reliable link, current $t p_{\min }$ is then set to be configured on the wireless nodes.

The algorithm is also illustrated in Fig. 3.19 to show the different stages of this first level of optimization. This uniform power optimization scheme is certainly a suitable mechanism to be implemented in those networks where a homogeneous physical distribution is expected, so that a balanced connectivity map in terms of link quality is combined with an overall topology reduction.

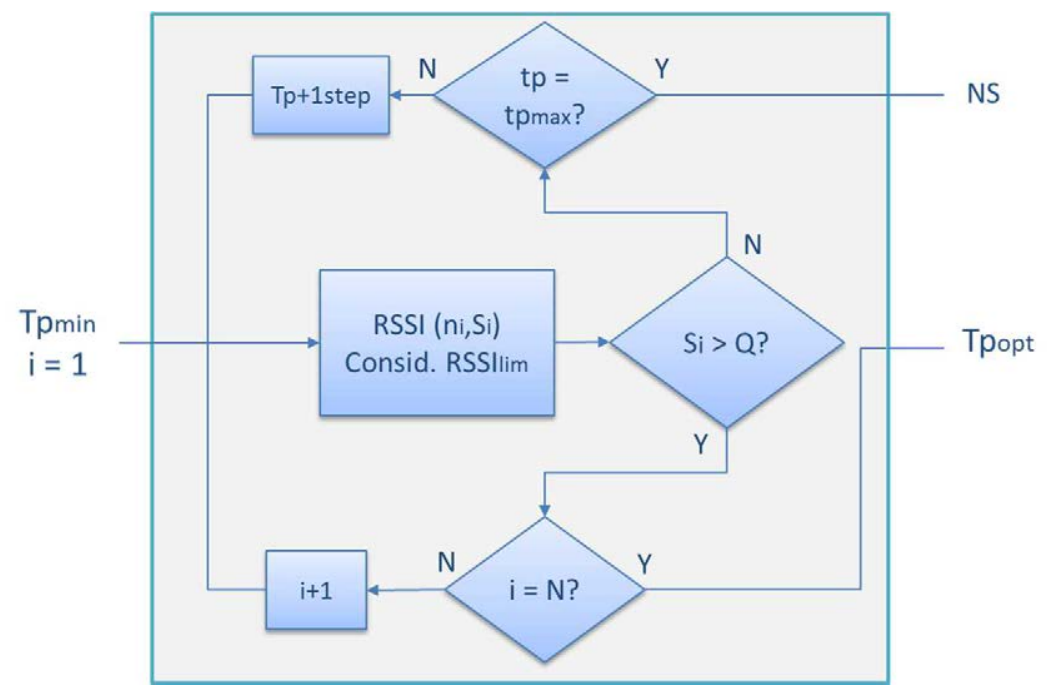

Fig. 3.19 basic definition of the first Tp uniform optimization strategy.

An important remark to be highlighted from the aforementioned procedure is that even though more optimized connections are generated, situations where groups of 2-Ns are not interconnected among them will lead to creating isolated groups of nodes throughout the deployment. The capability of the HHD as an on-site deployment optimization and performance evaluation tool allows adjusting these types of circumstances by means of applying the following criteria:

a) Dynamic recalculation of relay nodes in specific areas of the deployment scenario in order to enhance the node correlation 
between isolated groups. Such a solution shall also be evaluated with the support of the real connectivity correlation scheme generated during the deployment activities. In this direction, a trade-off between energy cost and platform cost is to be assessed, in order to make a decision on whether to include additional enhancement points according to the in-field conditions. Since deployment limitations and restrictions may be encountered during the installation phase, deployers can set particular link constraints as previously described, so as to tune the topology generation scheme. Based on this, the HHD will subsequently guide users to perform the relay node positioning and configuration process.

b) When possible (depending on the placement constraints and location requirements for the sensors), node repositioning can also be adjusted in-situ to modify the connectivity performance with respect to their surrounding neighbourhood. Based on such a refinement process, a new topology optimization can be produced at runtime to analyse the impact of the on-site changes in the overall connectivity scheme. The HHD will then provide an automatic comparison of the generated calculations and the real quality links throughout the deployment.

Regardless of the applicability of these enhancement actions, a second alternative within this optimization stage is proposed to guarantee the cohesion of the network with respect to the previously produced connectivity scheme. The main idea relies on coping with possible isolated group generation by creating at least one communication path along the network, so that all the nodes will be totally reachable from any point of the deployment by at least one common interconnection array, as follows:

- The algorithm starts with $t p_{\min } \in T P_{\text {range }}$ and $n_{1} \in I_{\text {array }}$ that defines an identification array to trace which nodes of $\mathrm{N}$ have been discovered and marked as a valid interconnection.

- $\quad n_{1}$ evaluates its surrounding neighbourhood to detect those links that are within the quality boundaries set, and then the encountered nodes $\left(S_{1}\right)$ are included within the evaluation series to subsequently analyse 
whether they discover nodes that are not part of the already identified and validated progression.

- Then, if the process ends without the validation of $N$ nodes in $I_{a r r a y}$ it means that at least one breaking point of consistency is found, so $t p_{\min }$ is increased to re-evaluate the discovery progression.

- Instead, if $I_{\text {array }}$ complexion fits the overall interconnection of $N$ with the configured transmission power, then the algorithm releases the new topology optimization scheme, so that on-site refinements and assessments can be performed in accordance with the provided connectivity map.

This optimization stage is illustrated in Fig. 3.20, where the discovery and validation progression of the proposed algorithm allows the avoidance of possible disjoints among network connectivity areas. Thus, minimum transmission power configuration with reliable links among sensor nodes and overall network cohesion is assured, by applying these two topology optimization phases.

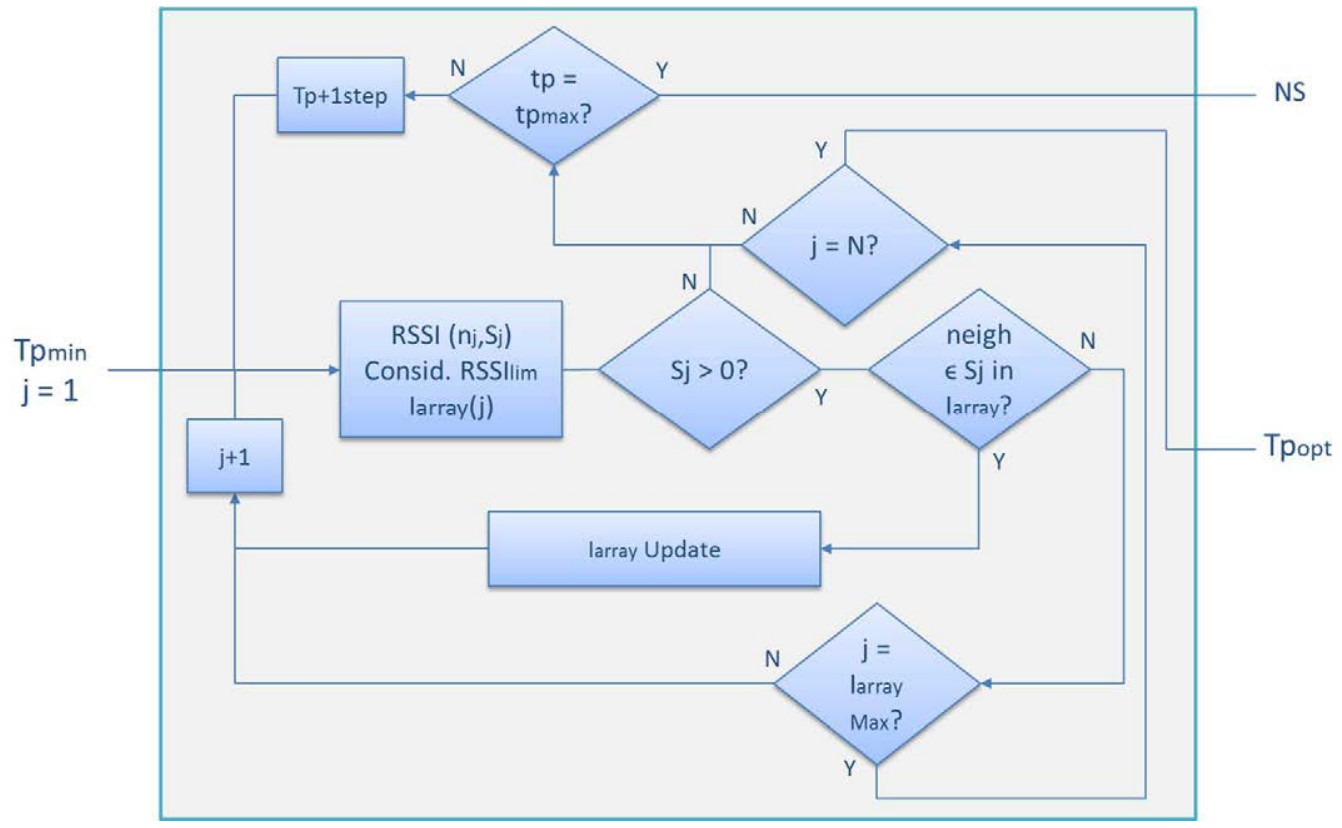

Fig. 3.20 Representation of the cohesion-focused Tp optimization strategy. 
As mentioned before, this first stage of connectivity enhancement mainly targets more homogeneous distributions of mesh networks where a trade-off between power consumption and robustness can be obtained with a uniform configuration scheme.

A second stage is allowed for to optimize configuration of every sensor node in the form of individual transmission powers, which means that the adaptation will be performed based on observing the particular conditions of each node, so that specific unbalancing points do not strongly penalize the rest of the configurations. The proposed individual power algorithm then follows a nodebased approach as a further enhancement step within the optimization process. It attempts to address those situations where the deployment connectivity may present more irregularities that can be solved with the configuration adjustment of particular points of the network.

Based on the result of the previous stage, the evaluation is done in this case from the point of view of $n_{i}$, i.e., each node individually reduces its transmission power and then evaluates the impact of such an action on the detection of $S_{i}$, obtaining a new discovery $S_{i}{ }^{\prime} . t p_{\text {min }}{ }^{\prime}$ is validated whenever $S_{i}^{\prime}$ is at least equal to $S_{i}$, otherwise $n_{i}$ assumes $t p_{\min }$ as its optimized transmission power. Therefore, this stage focuses on keeping the same level of consistency by assuring the overall connection of the whole network though links that can be reliably preserved with a more reduced transmission power are then optimized, by adapting the configuration of the involved nodes accordingly. Fig. 3.21 illustrates the progression of the algorithm starting from the outcome of the uniform power method. Moreover, a degree of redundancy is achieved to guarantee the avoidance of isolated points in case of node failures, also considering in this respect the ability to dynamically analyse whether a level of $t p_{\min }$ is to be increased whenever an isolation state is locally detected. 


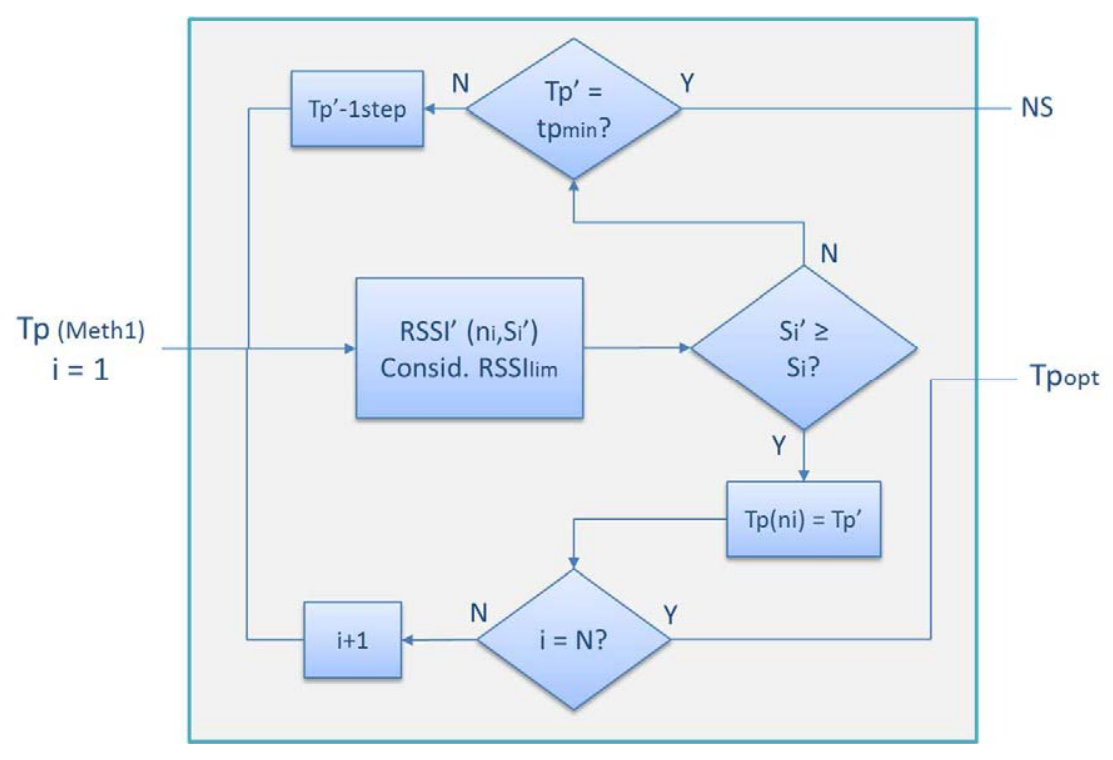

Fig. 3.21 Representation of the individual Tp optimization strategy.

\subsubsection{Output connectivity optimization scheme}

Based on the described techniques to generate the network connectivity and topology optimization process for the target WSN deployment, the outcomes of this subsystem are provided in the form of an output node correlation scheme, in which a connectivity matrix is produced with information that contains input parameters and properties for the following components of the functional flow of the deployment tool. This matrix highlights the interrelation between every pair of points of the network considering four main elements:

- The transmission power configuration for every node of $\mathrm{N}$, in accordance with the result of the optimization algorithms.

- The radio propagation factor configuration for specific links of the network, based on either the available options or custom factors generated and tuned by using the HHD in-field experimental procedure.

- The link quality values as a result of the connectivity models and simulation engine, considering the RSSI, LQI and LDR parameters to evaluate the connectivity between the involved nodes.

- Overall topology representation in the form of the network connectivity map view, in order to analyse and evaluate the optimization models with 
the overlapping of the real behaviour of the sensor nodes (whose layer is generated by using the runtime platform configuration and data gathering process).

As mentioned before, this correlation scheme will support the functionalities of the subsequent components that take part in the deployment toolset, particularly considering a) the deployment methodology engine that will be supplied with the neighbour matrix based on the nodes connectivity results b) the routing protocol evaluation subsystem to analyse the dissemination strategies upon the wireless connections at layer $2 \mathrm{c}$ ) the on-site deployment guidance procedure to configure, evaluate and verify the behaviour of sensor nodes with respect to the expected modelling results, so that on-site optimization and enhancement iterations can be accomplished accordingly.

Then, a real connectivity scheme will be generated in-situ and represented in combination with the modelling results, so that deployers are capable of analysing whether modifications of parameters can certainly readjust and optimize the correlation of the sensor nodes. Thus, a more adapted solution can be dynamically reconfigured to obtain an efficient solution for the final operation of the target application.

\subsubsection{Deployment methodology and on-site configuration strategies}

The combination of the deployment models, the output network connectivity scheme and the node correlation matrix will serve as the input information to carry out the on-site deployment and commissioning activities, whose associated strategies will determine the cohesion of the network (particularly considering the map of reliable connections throughout the network), its operability and the groundwork for a proper maintainability of the wireless sensor system. The importance of this stage within the WSN development cycle relies on the fact that the way the installation, configuration, evaluation, system release and maintenance are performed represents a critical point for the assessment and improvement of previous stages and its real effectiveness with the actual HWSW platform under deployment. 
This means that the issue of deploying a WSN in such a way that both the cohesion and the maintainability of the network can be assured poses important challenges to be faced, especially when adding energy efficiency as another variable to the problem analysis.

The setup of the sensor nodes not only in terms of the mesh networking communication mechanisms but also in relation with the platform parametrization (hardware/software properties and application tuning) might be unified by means of triggering a collaborative configuration procedure from the mobile on-site deployment tool, in which the target node executes a frame dissemination task in order to generate two main actions: network connectivity correlation with its surrounding environment (and thus establishing links information for routing and topology purposes), as well as platform setup where specific parameters regarding application support services (data transmission mask, service provision, and power mode configurations) and node properties (such as node weight determination, which refers to the generation of routing metrics and cluster-based configuration entries) are distributed among its enclosed area.

In both cases, the performance verification, reconfigurability and maintainability processes could seem to be a subsequent generation of configuration dissemination actions among the sensor nodes that compose the wireless mesh networking array. However, this scenario becomes a non-trivial execution task and even a less stable scheme when the WSN-based system is a conjunction of points in a large-scale deployment, where problems related to distributed processing capabilities, network flooding and power dropping situations may affect the overall performance and long-term autonomy of the application.

Therefore, the deployment phase shall provide an optimized way of performing the in-field network configuration and maintenance activities, and a comprehensive feedback to the system development flow based on the on-site evaluation capabilities (considering, for instance, real limitations of node coverage and communication establishment, or placement problems that come out during the installation process), so that a refinement of the planning and implementation process can be realized accordingly. However, although 
contributions in the literature have been focused more on the provision of planning and simulation techniques, well-defined deployment and maintenance strategies based on the implementation of in-field optimization methodologies are still a pending issue for WSNs, especially targeting the assurance of an efficient consistency of the wireless network with a resource and energy awareness scheme.

In this work this issue is addressed by proposing a new perspective of analysing and tacking the on-site deployment of WSNs, by means of designing and developing a deployment methodology engine within the HHD, in order to provide deployers with the optimization of the configuration and maintenance tasks considering a) the resource and power consumption during such phases and their impact in the overall network functional release, $b$ ) the cohesion of the network taking into account the system as a whole interrelation of the sensor nodes rather than analysing them only as individual elements c) the integrity, long-term operability and reliability of the wireless distributed system based on the generation of the real enhanced connectivity scheme.

The problem of deploying a WSN relates to performing the placement and initial setup of the sensor nodes (by following the location information as well as the setting parameters from the generated network models) in such a way that the sequence of configuration tasks to be carried out complies with the abovementioned requirements, and particularly allowing for the time and energy factors to guarantee the desired network scheme. Although this process may be feasible to realize and a straightforward task in small-scale deployments by iteratively adjusting each node, as mentioned before when facing medium or large scale mesh networking scenarios the problem of efficiently deploying the sensor platforms becomes an important issue to be optimized, which is also extrapolated to the system debugging and maintenance tasks. This leads to addressing the study and definition of a deployment methodology to be accomplished by using the Hand Held Device in field. For that, an optimization process shall be executed in the mobile platform at runtime (within an affordable 
computational time considering the on-site nature of the tool), from which different deployment configuration and evaluation sequences are obtained.

The basis of the installation and configuration process relies on an in-field positioning, local parametrization and global configuration. These three phases are certainly correlated since the positioning and local configuration of a node may condition how its global configuration and establishment is accomplished. After site location and the initial parametrization, node $n i \in N$ shall trigger a surrounding configuration process that allows on one hand verify the communication properties and the propagation/connectivity performance in the target area, and on the other hand set a correlation setup with its neighbourhood especially considering topology strategies. This procedure involves the execution of a control and acknowledgement packet exchange scheme among the implied nodes under configuration/assessment.

Then, after the deployer carries out such tasks on the target node by using the $\mathrm{HHD}$, the next position in the configuration sequence is executed and the communication performance evaluation is then generated. The way such a deployment/maintenance sequence is accomplished may condition how efficient the configuration process and the final cohesion of the network is produced, not only regarding the associated resources spent but also the sensor nodes correlation scheme and topology correctness.

In this direction, the deployment procedure of the wireless mesh network can be realized considering a node-to-node configuration array, in which each node is installed, evaluated and checked with respected to the previous points already tested in the sequence. Once the sensor node is validated, it can remain in standby state while the rest of the points are involved in the deployment execution process, unless it takes part in the correlation scheme generation of another node under evaluation, where an active state is allowed for to carry out the packet control exchange mechanism (for instance, the second point in the sequence establishes a configuration process with the first node that had previously been installed). The method is then performed with the subsequent points of the configuration array up until the overall topology and network platform is obtained. 
Such a procedure can be suitable for small-scale networks where the number of nodes to be deployed allows an easy step-by-step evaluation of each sensor platform even if the surrounding area is not yet deployed. However, in medium/large-scale mesh deployments where the complexity of the network configuration and node correlation scheme increases, a second alternative is also taken into account to improve not only the deployment evaluation but also the maintainability and operability of the global WSN system. This method aims at finding strategic points of the network upon which the seeking topology and configuration scheme can be produced with a minimum time and resource cost.

Through such key elements of the deployment, the configuration, performance analysis and optimization can be performed with the necessary control execution actions (without spending additional resources and processing when they can be avoided, and reducing extensive flooding mechanisms), and they provide a better assessment on how specific configurations or modifications of the network condition may affect the behaviour of the overall wireless system. Hence, such points can lead to producing a more optimized way of efficiently performing the on-site deployment, debugging and maintenance tasks on the distributed system, and with the most energy/resource effective cost.

After an initial placement phase, the mobile paltform triggers the control actions in those key elements that compose the configuration dissemination scheme, from which both the local and neighbourhood collaborative parametrization is performed. Since the surrounding nodes are already available to establish the connectivity scheme, only those points that have not been configured and evaluated yet will activate the configuration process, while the rest stay in standby state. Moreover, as shown in Fig. 3.22, key points allow a better management of the control packet dissemination by reducing the data exchange process in those 2-Ns correlations that have already been accomplished, thus no unnecessary transmissions are realized. Therefore, the discovery and dissemination control mechanism supports an enhanced generation of the desired network correlation scheme. 


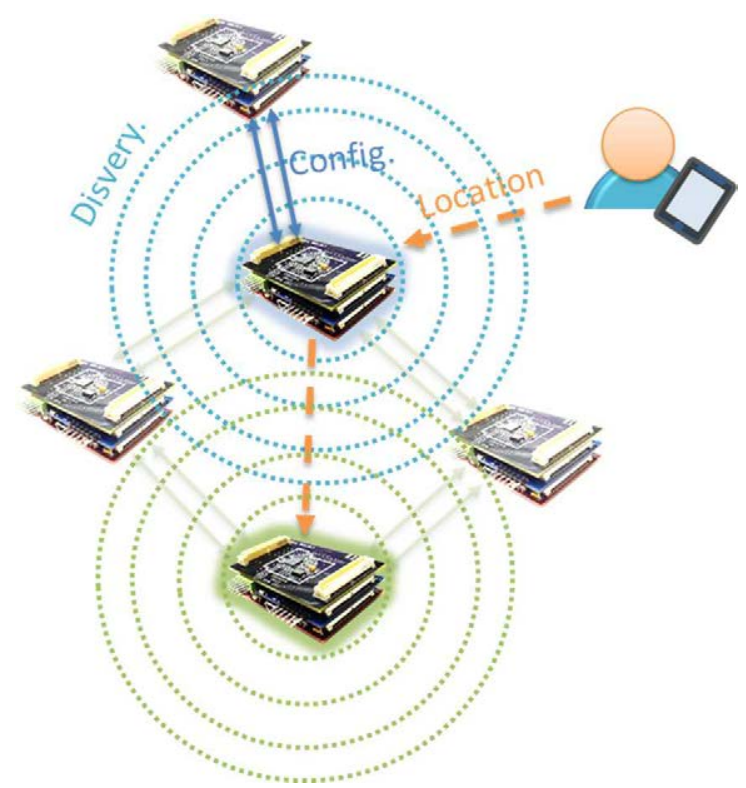

Fig. 3.22 In-field commissioning process and involved deployment/maintenance tasks based on network strategy-points.

Based on that, the proposed optimization process considered in this methodology relies on the dynamic calculation of the key strategy-points of the mesh networks (from here on Kps), upon which the configuration dissemination scheme is realized with an effective resource+energy cost. However, the actual efficiency of this execution process does not only depend on the number of encountered points, but also on how the sequence of dissemination tasks is performed along the Kps scheme. Thus, the optimization of the type and number of Kps also relies on the definition of the execution sequence to be carried out at the deployment and maintenance stage, so as to minimize the level of redundancy when applying the configuration tasks to obtain the same overall dissemination and network correlation scheme.

\subsubsection{Modelling of the deployment and maintenance methodology}

In order to be able to evaluate the aforementioned deployment methods and propose an optimized sequence to effectively accomplish the explained process, a proper modelling of the deployment methodologies is to be created so that dynamic and runtime optimization algorithms can be employed accordingly. An important aspect to be highlighted is the inclusion of actual WSN hardware 
parameters as part of the modelling process, especially in terms of power/resource consumption and radio communication configurations. This is achieved by determining the actions that compose the deployment and configuration dissemination strategy in the form of a problem cost function, which will serve as the main component to compare different possible solutions. The main target is to minimize this cost function within an affordable computational time (considering the nature of the tool as an on-site mobile mechanism).

To produce such a modelling process, the inclusion of the sensor node resource consumption (related to every stage of the discovery, configuration and dissemination mechanism) is considered into the cost function, which is materialized as the transient energy associated with the corresponding actions to be triggered. As explained previously, the deployment methodology relies on executing the configuration actions from the HHD based on the discovery and dissemination control mechanisms, whose process is performed in relation with the configuration sequence, the number of involved nodes and their location, and the node correlation scheme. In this way, three main resource consumption factors are taken into account to express the cost functions associated with the deployment methodology process, as follows.

Node deployment sequence, transient cost. The first factor to be considered is the transient cost associated with the configuration and commissioning transitions among the sensor nodes under deployment while the execution sequence is carried out by the mobile platform. This means that this cost representation corresponds to the process of deploying or maintaining the WSN in field, which refers also to the influence of activating a network setup mode while the deployer/maintainer triggers the deployment configuration sequence. As shown in equation 3.9, the cost is expressed as the average energy weight of the network right before starting the deployment/maintenance sequence (placement/installation stage), plus the transitions among the points of analysis, that is represented as a resource consumption and time cost factor for every 2-Ns of the sequence. 


$$
f_{t r}=\alpha \cdot N+\sum_{i=2}^{p} P_{s b} \cdot y_{d} \cdot \delta_{(i-1, i)}
$$

Where $a$ corresponds to the cost factor of the initial placement/installation phase per node (associated with the Kps-based technique), $P_{s b}$ refers to the power/resource consumption in stand-by state while the transition among sensor node evaluation occurs, and $y_{d}$ is the number of points that are in such state. In case of the first method, this factor will correspond to the number of nodes being deployed when node $i$ is the next configuration point target, i.e., $i-1$, while for the second method it will refer to the $\mathrm{N}$ nodes involved in the deployment/maintenance scenario. Likewise, $p$ can be in the form of the $\mathrm{N}$ target nodes for the former case, or the number of Kps of the deployment/maintenance sequence for the second one, considering Kps $<N$. $\delta$ factor represents the transition weight between the i-enth node within the evaluation process and the previous target point $i-1$.

An efficient generation of the node deployment sequence (NDoSq) (based on the optimization algorithms explained subsequently), in addition to the discovery and configuration dissemination mechanisms, will assure the cohesion of the network according to the provided network correlation scheme, but with energy/resource cost minimization effectiveness.

Discovery and link correlation scheme generation costs. After identifying the target node from the configuration sequence, the on-site deployment support tool triggers a controlled discovery mechanism in which the surrounding area is evaluated, so as to update the link correlation scheme with those 2-Ns that have not been analysed/configured yet. $n_{i}$ of $N D o S q$ emits a localization control packet with information related to the discovery process, and the surrounding neighbours that are under the boundaries of reliable connections will answer the request considering a medium access control condition, in which the communication channel is assessed by the discovered neighbours $n_{r} \in m_{i}$. Then, each encountered node will emit a discovery feedback packet to $n_{i}$ in order to establish the 2-Ns link correlation state. 
In this binding phase the mesh network information is updated (particularly regarding neighbours indexing) both in the involved nodes and in the deployment tool, so that a dynamic recalculation and refinement of the models can be performed in situ. During this procedure, the action execution among the different comprised nodes are computed as a) the relationship between the radio transmission power configuration and the energy weight of the sequence point under commissioning; b) its associated nodes during the discovery and acknowledgement process. Based on the aforementioned routine, Clear-To-Send mechanisms (CTS) for Collision Avoidance (CA) are also allowed for within the cost evaluation process, so as to properly express the operation slots among the involved nodes, as seen in eq. 3.10.

$$
\begin{gathered}
f_{s r(i)}=\left(E_{T(i)}+\sum_{r=1}^{m} E_{R(r)}\right)+\left(\sum_{r=1}^{m} E_{a(i, r)}\right)+ \\
\left(\sum_{r=1}^{m}\left[(m-r) \cdot E_{c k(r)} \cdot \xi+(r-1) \cdot E_{s}\right]\right)+\left((p-m-1) \cdot E_{s n}\right)
\end{gathered}
$$

Four main elements can be distinguished within the cost expression shown in eq. 3.10 .

- First, the target node that triggers the action involving the discovery mechanism with the surrounding neighbours. It is represented as the resources spent at the source and destination points during the performed function.

- Then, the association stage is expressed as redundancy filtering and 2-Ns establishment, which includes the cost representation of the recognition execution between the target and discovered points (the number of encountered points to be configured is expressed as $m$ ). This action execution also allows a bidirectional neighbour tables indexing for subsequent routing and dissemination optimization purposes.

- Medium access influence is defined as the average power during standby and active transitory modes of the surrounded-nodes of the target points multiplied by a CA slot factor $\xi$. It includes those nodes that change their state once the binding process between them and the target node is finished. 
- Finally, the cost weight of passive elements that are not directly involved in the discovery dissemination process at that point of the deployment sequence is also included as an overall stand-by offset of the rest of the sensor nodes.

The main difference between point-to-point and key-point based strategies in the cost function representation is the $p$ factor, which will be equal to the number of deployed nodes $i$ in the former case and $N$ as the overall number of nodes contained in deployment/maintenance scheme for the second case, where the number of target nodes included in NDoSq will be Kps $<N$, as mentioned before.

Reconfiguration and parametrization cost based on the node correlation scheme: once the node association is properly performed from the corresponding node of the deployment sequence under action, the configuration transaction is then triggered between the target point and the correlated node, so as to establish a runtime parametrization in relation with application level services as well as network settings for communication and multi-hop purposes, but particularly focusing on link metrics and node weights for the mesh routing optimization strategies as well as cluster-based dissemination mechanisms. In this way, the HHD activates a configuration control packet to be emitted from the target node of NDoSq to $n_{r}$, which then receives the setting parameters and answers back with additional information regarding node properties and link quality metrics, so that the evaluation process is done for the 2-Ns under the correlation scheme generation.

As shown in eq. 3.11, the function of the configuration execution is expressed as the cost weight of the target node and the setting-point during the cross configuration exchange process, which includes the transmission power of both nodes as well as the active mode consumption when the emission and reception of the configuration actions are realized (the two points involved), in addition to the stand-by offset of the nodes that are not directly involved in the performed correlation action (the $p$ points of the deployment method).

$$
f_{\text {conf }(i, r)}=E_{t r(i, r)}+(p-2) \cdot E_{s n}
$$


The application of this phase within the deployment process represents a fundamental part for the dynamic updating of the network simulation models based on the on-site gathered data, so that a refinement of the optimization technique can be accomplished according to a comprehensive feedback from the deployment tool. Moreover, the result of this strategy indeed enhances the reduction of flooding schemas that significantly affect the overall power and resource consumption of the network platform.

The described cost function representations allow not only expressing the different factors that are included into the different operations for obtaining the network correlation scheme, but also analysing how the configuration and interconnections of nodes can be performed such that an efficient cohesion and maintenance of the network is assured without penalizing the deployment in terms of communication, processing and power consumption. The general representation of the cost functions for the proposed methodologies is shown in eq. 3.12 and 3.13, in which the explained underlying actions are represented for both deployment/maintenance methods:

$$
f_{\cos t^{1}}=f_{t r}+\sum_{i=2}^{N}\left\lfloor f_{s r(i)}+\sum_{r=1}^{m^{\prime}(i)} f_{\text {conf }(i, r)}\right\rfloor
$$

Where $m^{\prime}(i)$ in this case represents the available-deployed points that can be configured when deploying $i$, and $N$ is the number of nodes of the target deployment.

$$
f_{\cos t^{2}}=f_{t r}+\sum_{i=1}^{p}\left\lfloor f_{s r(i)}+\sum_{r=1}^{m_{(i)}} f_{\operatorname{conf}(i, r)}\right\rfloor
$$

$m_{(i)}$ represents the encountered nodes that have not been configured/parameterized yet, whereas $p$ refers to the Kps in the deployment configuration sequence to be carried out.

\subsubsection{Deployment optimization algorithms}

Based on the definition of the deployment and maintenance methodology scheme as well as the cost functions that allow the evaluation of different strategies to be performed, the next step relies on the ability to dynamically 
optimize the result of these functions such that the commissioning task can be carried out efficiently with a minimum cost of operation. The main target is the applicability of optimization mechanisms to obtain an enhanced trade-off between the cohesion of the network, by efficiently generating the real connectivity correlation scheme, and a minimum resource utilization during the overall on-site configuration and evaluation tasks.

This process is to be accomplished considering the nature of the on-site deployment tool in terms of time and processing capabilities. Since it is a dynamic optimization method based on both modelling and real data from the actual behaviour of the WSN under deployment/maintenance, efficient though affordable runtime optimization techniques are to be adopted. The first consideration that could seem to be a solution for the optimization problem is the application of what is named here as swap-based algorithm, which refers to the assessment of each solution as a combination of every possible NDoSq, which will lead to comparing and obtaining the most efficient result for a given deployment scenario. It relies on the permutation of the array of nodes that composes the WSN such that all the possible combinations (considering the network correlation scheme) are obtained. Then, each result is evaluated in order to calculate and compare their associated costs, so that the most optimized solution (with the least cost outcome) and the corresponding deployment array can be provided accordingly.

Consider a network with a number of nodes equal to $3(\{1,2,3\})$, the swap-based algorithm will generate 6 possible solutions for NDoSq, which means that the spectrum of possible combinations (3!) are covered and evaluated. The main problem of this solution is its performance and scalability in medium/largescale WSNs, since the algorithm makes the problem of optimizing a network composed of $N$ nodes rely on the evaluation of $N$ ! possible solutions to generate an optimized configuration sequence, i.e., in order to add a node into the evaluation of a WSN of N nodes, the number of possibilities is $\mathrm{N}+1$ times larger, which is not time and computationally feasible for the on-site support tool.

In order to guarantee a trade-off between solution efficiency and time/processing effort of the mechanism, the concept of bio-inspired 
optimization algorithms has been postulated as a clear alternative in the literature to solve optimization problems with computational feasibility. These types of strategies refer to evolutionary computation to generate a solution space based on optimal or suboptimal results, by applying natural evolution and selection principles. In this direction, the so-called genetic algorithms are included within these types of techniques, whose iterative process mimics the natural selection from Darwin's theory, in which the aptitude of the individuals will condition their survival and reproduction within the evolution process of the species. Likewise, their application in optimization problems will generate solutions whose aptitude allows assuring an optimal and suitable result.

In the proposed toolset, a genetic-based algorithm has been implemented as part of the runtime optimization process, in which several modifications were integrated in order to adapt the dynamics of this mechanism to the problem of deploying a WSN by using the proposed methodologies. One of the main advantages of this algorithm is that the scale of the network does not strongly condition its performance to produce the subsequent candidate solutions, so it can be applied to different types of mesh network arrays. Instead, the parametrization of the algorithm could influence the evolution of the results and the computational time effort to obtain optimal outcomes.

There are two main requirements that serve as the starting point to apply the genetic algorithm to the optimization problem. The first step is the generation of candidate solutions that are expressed as a population composed of individuals. Traditionally in genetic algorithms individuals are represented as strings of binary codes, which might simplify the subsequent operations and evaluations that the candidate solution has to undergo. However, in case of the deployment methodology, the genotype of the individual is created as an array of nodes that corresponds to the NDoSq. For instance, consider the sequence identification of Fig. 3.23, which defines the genetic code of the individual and thus allows encoding the candidate solution as an array equal to: $\{2,5,3,1,6,4\}$.

The creation of the genotype is independent on the type of methodology to be applied, which means that during the aptitude assessment process the candidate solution can suffer an additional optimization in case of the Kps-based methods 
(in accordance with the proposed 2-Ns redundancy detection) that will check the active key nodes of the configuration sequence (it can be seemed as an implicit simplification of the encoding size), though the genetic code remains as it is for the previous operators. This means that in case of the aforementioned example the final optimization solution for the deployment problem based on the second method could produce a NDoSq $=\{2,3,6\}$.

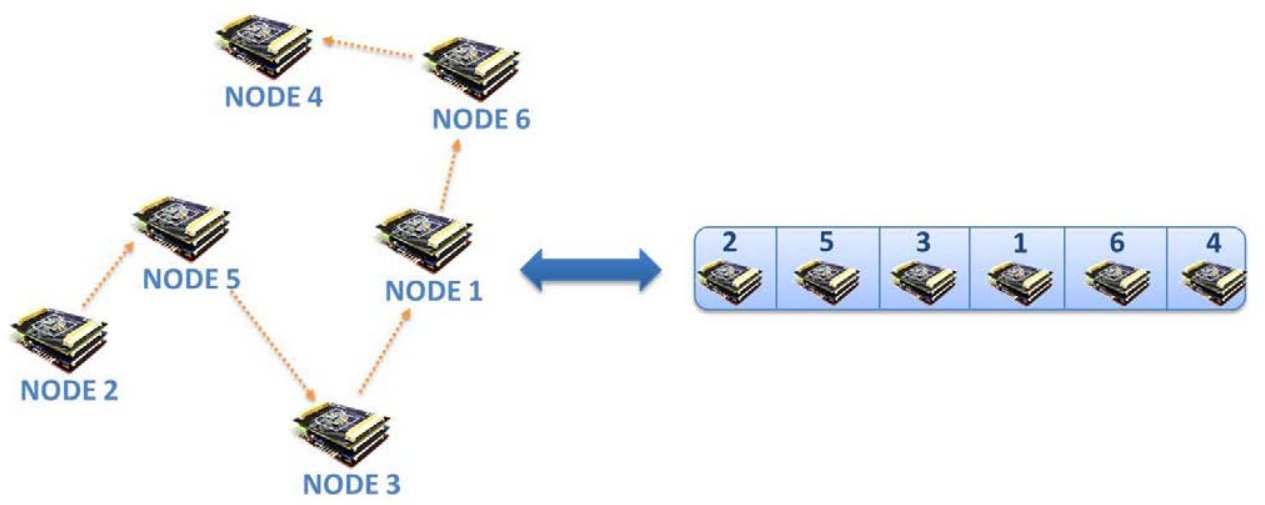

Fig. 3.23 Genetic code representation of a candidate solution for the deployment methodology.

The second consideration relies on the definition of an aptitude function that allows the evaluation and comparison of different candidate solutions, which serves as the main element for the optimization algorithm to analyse the degree of feasibility of survival of an individual to the next generation of the evolution process. The problem cost functions proposed in the modelling process are used in such a way that the higher the result is, the less the aptitude of the deployment optimization sequence will be, i.e., the fitness expressions are defined as (3.14), where $f_{\text {cost }}$ can be (3.12) or (3.13) depending on the type of method to be used:

$$
f_{f}=\frac{1}{f_{\text {cost }}}
$$

Therefore, based on the genotype of a candidate solution, it can be evaluated by applying the corresponding functions of the selected method and then verifying the fitness result for the associated NDoSq. While in case of the point-to-point strategy such assessment is performed based on the verification of the whole genetic code of the individual, in case of the Kps-based method only the key 
strategy-points that form the optimized sequence will be checked and validated during the analysis and evaluation process of the candidate's genetic code (i.e., in the aforementioned case the fitness is evaluated based on the second NDoSq). In order to define a sensor node as a key strategy-point in the sequence, a dynamic record of 2-Ns evaluation is tracked so that those following points of the configuration array in which the redundancy detection action comes out with new 2-Ns that had not previously been assessed are marked as Kps and added to the register function, otherwise the sensor node under analysis is considered as checked. Hence, the resulting sequence is reduced to those encountered strategy-points of the configuration array.

Along with the genotype encoding and the evaluation strategy to be adopted based on the definition of the fitness functions, the individuals that compose a population shall undergo a series of operations that take part within the geneticbased optimization process. In order to produce an enhanced population in every generation of the genetic evolution (the very first population is randomly generated with a number of possible NDoSq according to the input population parameter), a selection process is performed, in which candidates of the population are chosen for their evaluation and application of the evolution cycle. Those individuals that have more aptitude to evolve undergo the crossover operation, in accordance with the fitness function outcome. The selection is accomplished by randomly getting a reduced number of candidates and thereafter the best solution of such a group is registered to be used with a candidate of another group, which results from applying the procedure once again. Both individuals then go through the crossover process.

Once two candidates are selected from the current population, they are recombined to generate new children individuals, whose genetic code contains features from their parents' genotypes. The application of the crossover operation in this case has to allow for the nature of the solution encoding, which is a sequence of sensor nodes that are uniquely identified and then not repeated within the array. This means that the crossover technique to be used shall take into account such a condition when extracting a segment of one parent and combining it with the corresponding segment of the other parent. For the 
proposed optimization implementation a modified two-point-based crossover is applied, in which a randomly selected segment both in terms of position of the array and in its length is transferred to the child's genotype, which is placed in the same position of the parent. Then, the rest of the genetic code is completed with the information of the genotype of the second parent, by transferring those nodes that have not been included yet following their position in the sequence, i.e., the code of the other parent is evaluated in order to incorporate those elements that are still missing in the available positions of the child, as illustrated in Fig. 3.24.

This procedure is repeated until a new population is created with new valid individuals that have characteristics of the best candidate solutions from the current population, though they first have to undergo the mutation evaluation process before their inclusion into the subsequent evolution cycle. The mutation operation refers to the possibility of an individual to suffer an alteration of its genetic code, so that a new genetic material is included into the population. The importance of this stage of the evolution process relates to avoiding similarities among genotypes of a population that could lead to producing a premature convergence into local optimal solutions, thus stopping the evolution cycle. When performing the mutation, each individual is evaluated with a modification probability factor that will determine whether one or more genes are going to be altered. In case of binary encoding, the simplest way of performing such a change is by just flipping the position of the individuals' genetic code under mutation (from 0 to 1 and vice versa). However, due to the nature of the deployment optimization problem, the mutation is realized by following a swapping mechanism in order to assure a coherent gene alteration, as illustrated in Fig. 3.24. In this way, if a gene has to mutate, a random position of the genotype is selected in order to be exchanged with it. The probability that a gene mutates is usually small, so that a trade-off between the evolution of the following population and the convergence criteria is obtained. Therefore, in case of the proposed algorithm the mutation rate is set to $1.5 \%$.

The process of selection, crossover, and mutation are repeated up until a new population is generated with the result of the described genetic operations. In 
the proposed deployment optimization algorithm the replacement phase is also enhanced by implementing an additional operation, which is known as elitism. The elitism refers to the incorporation of individuals with the highest aptitude to the new population without any alteration, which guarantees that the next generation will have at least one candidate solution with the same aptitude with respect to the previous generation. The main goal of this safety function is the avoidance of possible degradation of the aptitude from one population to another, which might happen when the recombination and mutation of suitable candidates create a solution with less quality results.

An overall representation of the described operations and functionalities is shown in Fig. 3.24, where every stage of the optimization process is also illustrated with the corresponding genetic operator.

Once a new population is successfully generated, termination conditions are evaluated to analyse whether a final candidate is selected from the solution space obtained at that point of the evolution process, in accordance with the fitness result. Two main conditions are considered within the implementation of the optimization algorithm, as follows:

- Maximum number of generations achieved. Users can configure this parameter before executing the optimization algorithm, taking into account that the higher this value is, the longer the system takes to find the final solution, since more number of evolved populations are assessed. As shown in the analysis and parametrization of the algorithm, a trade-off between computational time and optimal results is studied so as to provide deployers with default configurations seeking an enhanced runtime behaviour of the optimization process.

- Convergence threshold overtaken. This parameter can also be configured by users beforehand in order to set a convergence rate that allows stopping the evolution process, and thereafter an optimized candidate solution is proposed. In case of the proposed deployment methodology, the convergence criteria is determined by the percentage of individuals that have the same aptitude within a population (provided that the fitness value is at least as high as the best solution encountered so far). 


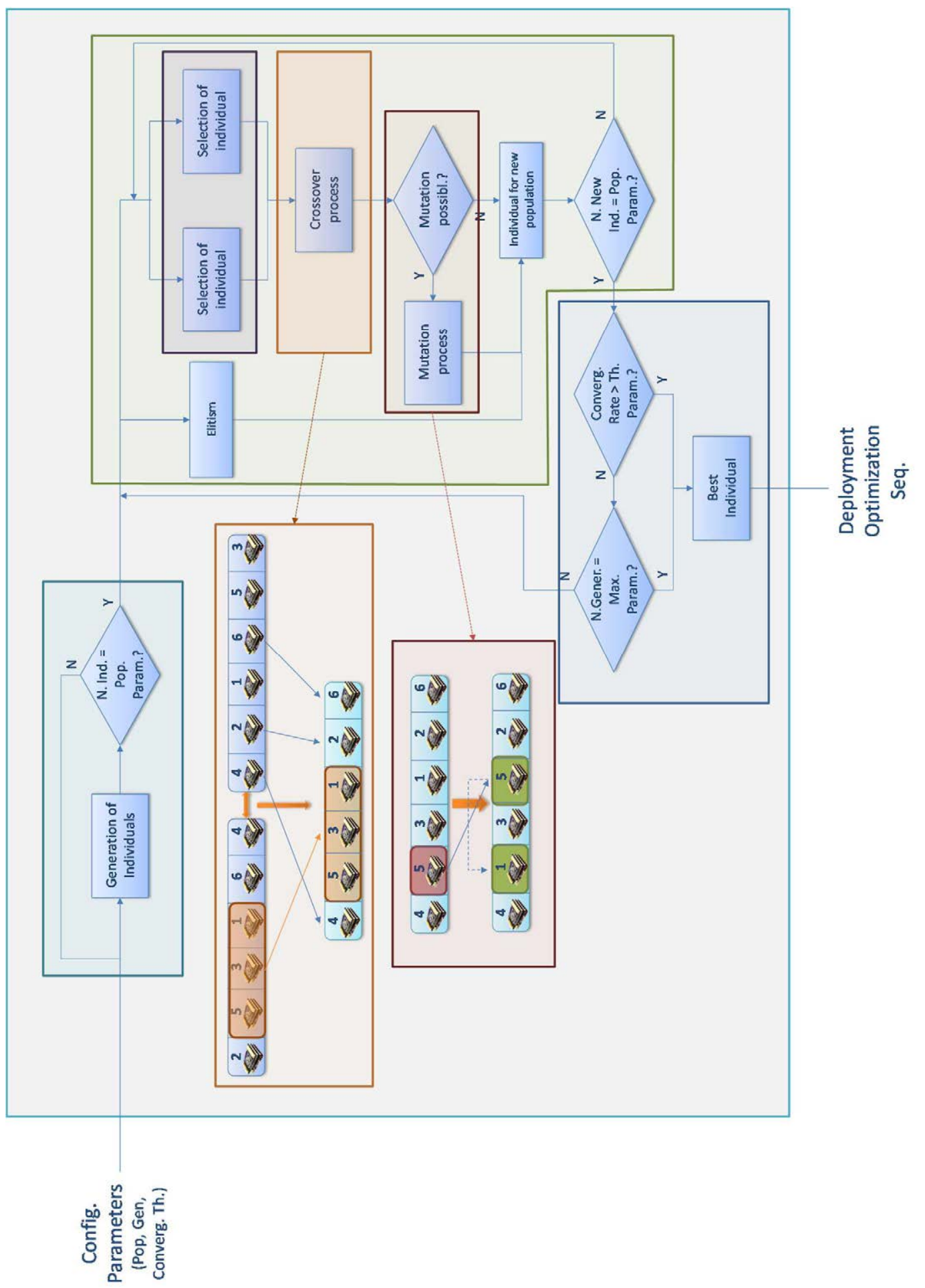

Fig. 3.24 Overview of the GA-based optimization algorithm and involved genetic operations. 
This means that various deployment sequences with the same cost result can reduce the generation of long unnecessary iterations before terminating the genetic evolution.

Moreover, in order to avoid a premature convergence of the algorithm, the threshold value is recommended to be set between 90 and $95 \%$.

Therefore, three main parameters will determine the underlying performance and solution space generation of the optimization process: the size of the population, the number of generations and the convergence rate. How these parameters are configured will constrain the computational time and effort to produce an optimal deployment configuration array, considering the size of the network as an element that increases the complexity of the genetic operators.

A third optimization technique has been designed and implemented within the deployment methodology engine, which focuses more on the modularity of the deployment and maintenance tasks as well as the ease of debugging and verification process. The optimization problem is divided into several groups in which the configuration of sub-deployments can be performed transparently with minimum dependencies between them in terms of parametrization and local node correlation. The main advantage of this mechanism is the ability to validate different parts of the target deployment in such a modular way that the analysis and evaluation of possible errors or connection mismatches are more focalized in specific areas of the network. Thus, from the deployer point of view, it allows an enhanced local testability process.

This clustering algorithm relies on a spreading method for distribution detection, in which the seeking configuration sequence seed starts from a reference node and then, based on the network correlation scheme, it is coherently spread throughout those subsequent neighbour points that match the dynamically generated diffusion branch. Unlike the swap-based method, since the possible diffusion branches are constrained by the network connectivity map, the total possible solutions will be certainly much less than $n !$. Two main phases can be considered within this strategy, firstly the clustering process according to the distribution detection, and secondly the optimization of the deployment sequence and groups interrelation allowing for the created clustering scheme. 
Consider the example of the network shown in Fig. 3.25, where node $n_{1}$ is the reference node, and the detection seed is spread by using the adjacent nodes $n_{2}$ and $n_{11}$. Then, they produce a separate dynamic sequence identification starting from the former one that can detect two different branches: 2-3-5 $\left(b_{1}\right)$ and 2-4-5 $\left(b_{2}\right)$. Considering $b_{1}, n_{5}$ will encounter three different possible branches trough $n_{4}$, $n_{6}$ and $n_{7}$, in which $n_{4}$ will allow setting a close group as 2-3-5-4-2 $\left(c_{1}\right)$, while $n_{6}$ and $n_{7}$ produce $5-6-7-5\left(c_{2}\right)$.

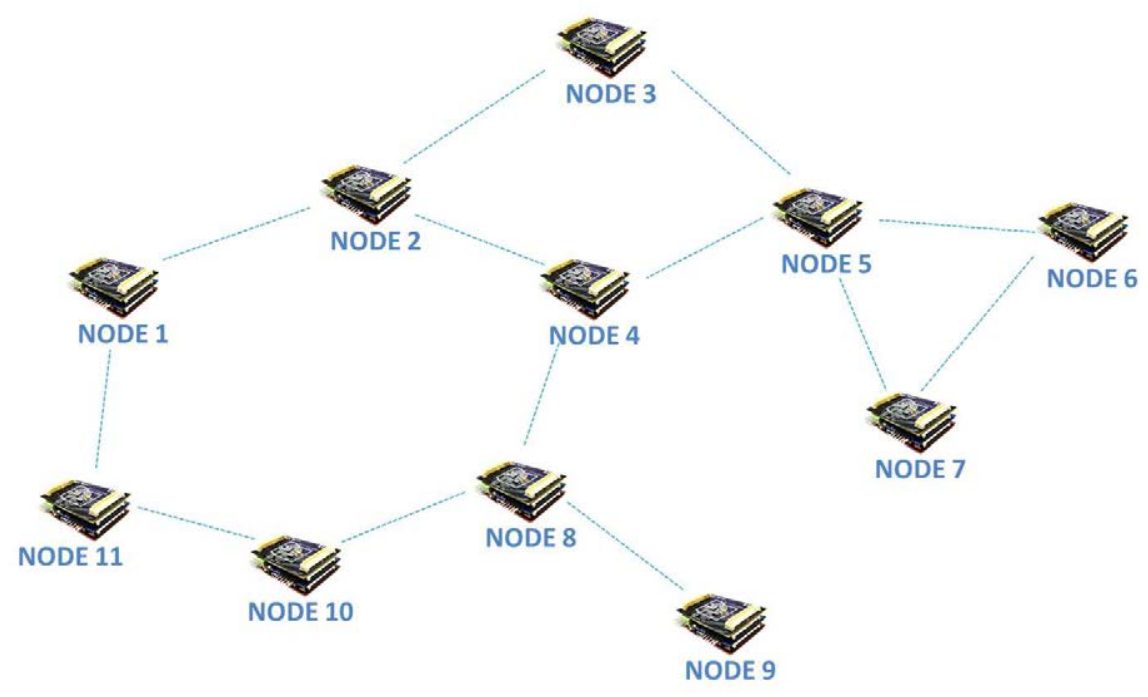

Fig. 3.25 WSN deployment upon which the clustering-based optimization algorithm is exemplified.

Thereafter, branch $b_{1}$ is extended by means of the detection of $n_{4}$ to finally produce a close group as 1-2-3-5-4-8-10-11-1 $\left(c_{3}\right)$, while branch $b_{2}$ will produces 1 2-4-8-10-11-1 $\left(c_{4}\right)$ through the identification of a branch by $n_{4}$. In this two cases an open branch is also identified considering the connection of node $n_{8}$ and $n_{9}$, which is marked as an end-point for subsequent evaluations. Finally, when attempting to perform the spreading identification through $n 11$, the process identifies that possible branches have already been produced and analysed in previous diffusions, so the system ends with the provision of the encountered groups to the processing and filtering functions. A summary of the aforementioned mechanism is illustrated in Fig. 3.26, in which the generation of the different branches and closed groups are highlighted by means of the distribution detection arrows. 
Groups are filtered in order to discard those that do not match with the following criteria:

- Maximum number of nodes per group: the scale of the group can be configured either by setting a proportional parameter with respect to the overall size of the network, or by means of a limit value entered by the deployer. For instance, in case this parameter is configured as $N c_{\text {lim }}=8, c_{3}$ will be discarded, whereas the rest of the groups are kept as valid solutions.

- Groups contained in other groups: in case the groups that contain smaller groups encountered into them fulfil the set boundaries, by default the biggest ones will have a higher priority. In the explained example, if $\mathrm{Nc}_{\text {lim }}$ is set to $8 c_{1}$ and $c_{4}$ will remain as valid groups. Instead, if the limit value is increased, $c_{3}$ absorbs them to unify such deployment area.

- Duplicity detection: in those situations where groups composed of interception points of two or more groups, i.e., a group which has nodes that are really part of others (not necessarily the same), they can be discarded as unnecessary groups.

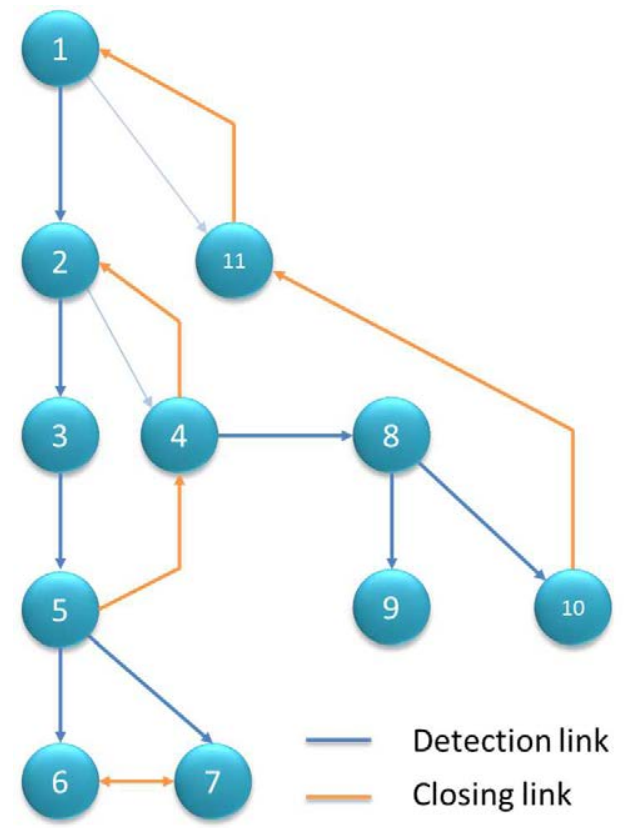

Fig. 3.26 Summary of detection of branches and closed-groups for the target connectivity array. 
Once the deployment groups are generated and processed based on the described clustering mechanism, users can perform the installation and configuration process by modularly verifying the different groups and then analyse the intersection points that allows the interconnection among them. Nevertheless, a deployment methodology can also be applied to this modular scenario from the basis of the already proposed optimization techniques. So, as mentioned before the second stage relies on the optimization of the deployment configuration sequence though by considering the generated groups along with an efficient interconnection process to guarantee the final cohesion of the network.

In this way, the swap-based algorithm (for instance, for those networks in which $N c_{\text {lim }}$ is set to be $<10$ ) and most particularly a modified version of the geneticbased algorithm are used to find an enhanced deployment sequence, although keeping the integrity of the encountered clustering identification within the optimization problem. When the genetic algorithm evaluates an individual, the genetic code is dynamically reconfigured once a node is detected as part of a deployment group, such that the genes that corresponds to nodes of such a group can be evaluated before continuing with the following gene of the genotype.

For instance, if a genotype of an individual to be evaluated is composed of the following sequence $s_{1}=\left\{n_{1}, n_{4}, n_{7}, n_{2}, n_{3}, n_{6}, n_{5}, n_{8}\right\}$ and there is an encountered group $c_{1}$ composed of $\left\{n_{3}, n_{6}, n_{7}, n_{8}\right\}$, when analysing the genetic code it might be restructured as $s_{1}^{\prime}=\left\{n_{1}, n_{4}, n_{7}, n_{3}, n_{6}, n_{8}, n_{2}, n_{5}\right\}$ to comply with the clustering result. This means that in the moment of evaluating $n_{7}$, since it belongs to $c_{1}$, those elements that are also part of it are included as the next points of the evaluation process (considering also an internal optimization of the sequence of such a group based on its entry point, in this case $n_{7}$ ). Obviously, to keep the coherence of the genotype, the subsequent nodes are ordered without including the already reconfigured segments $\left(n_{2}\right.$ and $n_{5}$ in the aforementioned example). Once the process is performed on the groups that are to be taken into account in the configuration sequence, the genetic operations are applied as in previous cases. 
The question is now related to how groups are included as a sub-array of the individual's genotype, which refers to the way of carrying out the deployment methodology within the clustering areas. For this, another modified optimization mechanism is employed to find an enhanced sequence, although allowing for the input and output points of the groups based on the configuration sequence to be evaluated. Consider the aforementioned example where $c_{1}$ is included in the reconfiguration of $s_{1}$. The group $c_{1}$ undergoes the operations of the optimization algorithms in order to calculate an optimal solution considering $n_{4}$ and $n_{2}$ as previous and following points of the configuration sequence, respectively. As a result, a combination of the overall sequence optimization with local group sequence optimizations is realized, so that a trade-off between optimal candidate solutions and deployment/maintenance modularity is provided to users by using the HHD.

As shown latter on in the optimization parametrization and experimental results, even though the clustering mechanism may offer higher costs (which is related to the aforementioned constraints that are set in the optimization algorithm), its advantages can ease the deployment and maintenance tasks from the user point of view, especially regarding the determination, isolation and repairing of possible failures as well as connectivity problems when producing the real node correlation scheme, thanks to the modularity of system. Moreover, the combination of this technique with the Kps-based method will provide enhanced results to the optimization problem.

\subsubsection{Parametrization and performance analysis of the optimization strategies}

In order to evaluate how different parametrizations can influence the performance of the proposed optimization methods as well as compare their efficiency and applicability to the network deployment problem, several experimental test cases have been set and analysed. The main idea is to combine various configuration cases to study how the algorithms perform to produce an optimal solution space for the distributed sensor networks under deployment. For that, apart from the variation of the parameters related to every optimization 
mechanism, the scale of the deployment and its distribution are also taken into account within the evaluation process.

In Fig. 3.27 the deployment baseline to carry out the different test cases is shown, which presents a heterogeneous mesh distribution and from which several network scales are taken to perform experiments with different number of nodes, as one of the variables to be considered. Moreover, Table 3-9 summarizes the parameters that are also considered for the generation of a comprehensive set of tests so as to evaluate and compare the outcomes of the proposed strategies. Besides the number of sensor nodes, it can also be highlighted the parameters related to the genetic-based algorithm, in which a range of values for the number of generations, the convergence rate and the population size is included.

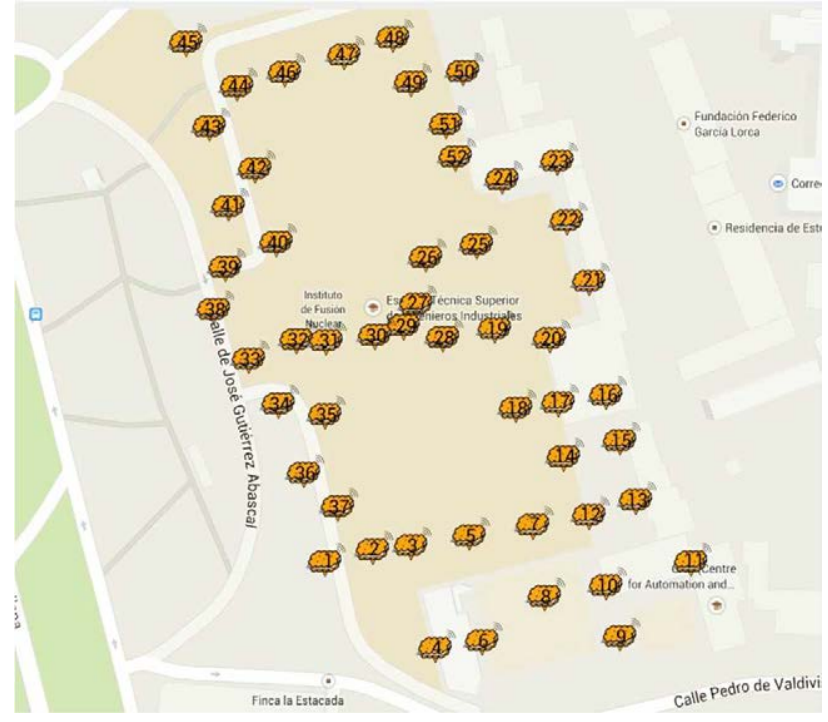

Fig. 3.27 WSN for the evaluation of the deployment optimization methods.

As mentioned earlier in the clustering algorithm, the size of the groups can also be adjusted, so in these experiments such a parameter is set as a proportional value of the size of the network. Moreover, the number of generations, population and convergence rate are set to be 100, 100 and $85-90 \%$ respectively in case of applying the modified genetic-based optimization mechanism for the clustering algorithm. As a result, more than 700 tests were produced by using the Hand Held Device and the results automatically registered to analyse the 
impact of such parametrization, so that users can be provided with a set of balanced reference values and deployment guidelines for triggering the optimization methods accordingly.

Fig. 3.28 and Fig. 3.29 capture some of the main results gathered from the test cases, considering the scale of the network and the outcomes of the cost functions for the point-to-point method and the Kps-based method in every optimization strategy. In case of the genetic-based algorithm the results correspond to the average cost for every scale of the deployment, while in case of the clustering algorithm the results refer to the group parameter configuration equal to $30 \%$.

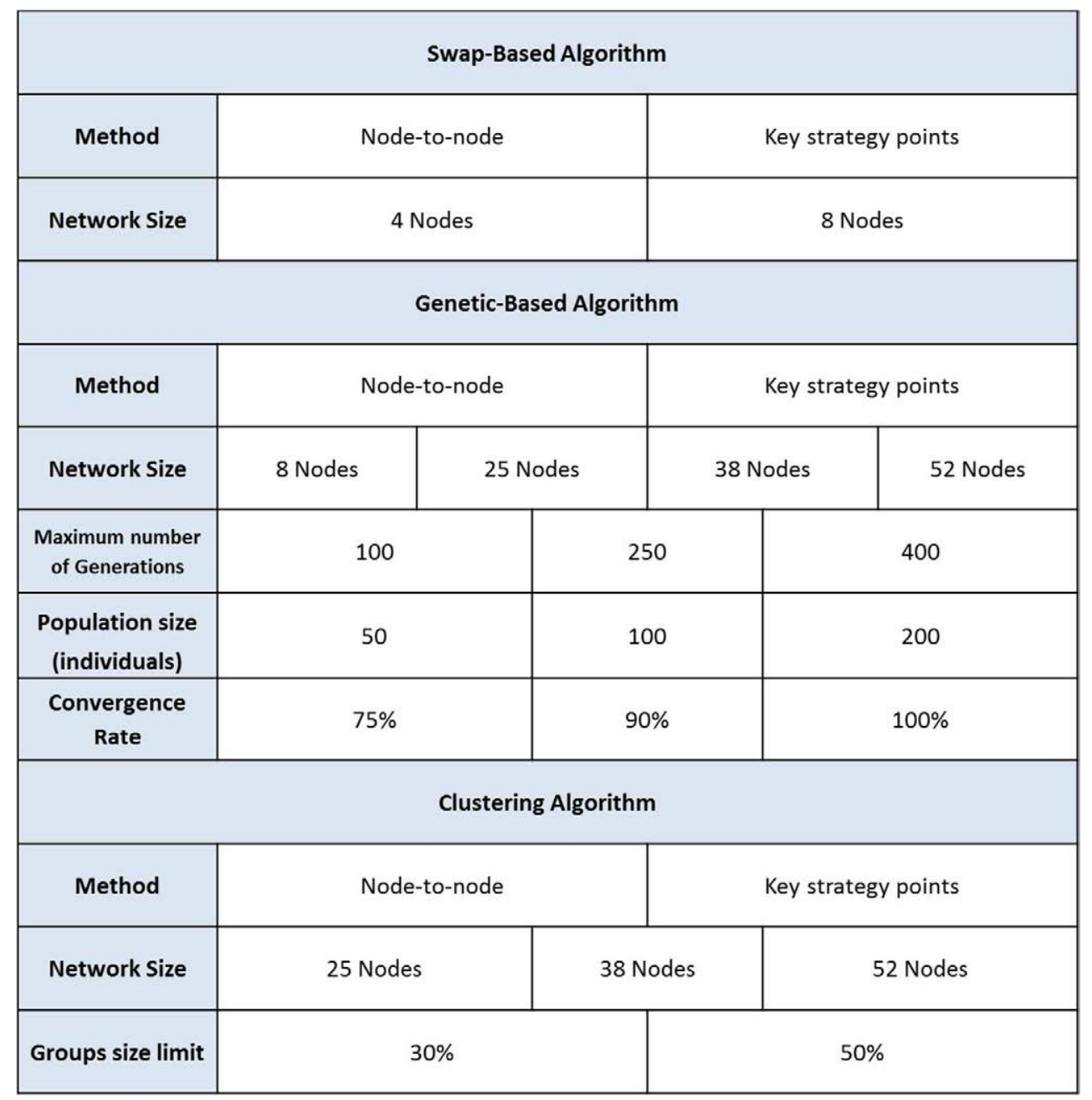

Table 3-9 Parametrization of the deployment optimization strategies for the tests definition. 
It can be seen that in each method the cost results increase (and then the fitness decrease) as the size of the network grows, since the configurations and deployment operations raise, as expected. However, in case of the clustering algorithm, such increase is more accentuated when applying the point-to-point method, where a clear difference with respect to the Kps-based method appears. This is certainly originated by the fact that restrictions in the genetic-algorithm to consider the generated groups into the genotypes produce that optimal solutions provide higher costs than the unconstrained mechanism. As remarked before, the clustering strategy has been conceived to have a trade-off between modularity of the deployment and maintenance tasks and cost optimization, while it can be realized that the genetic-based algorithm offers better performance in terms of optimal cost-result provision, particularly for the pointto-point strategy. This situation occurs because of the stand-by factor that has a higher impact in the Kps-based method than in the previous one, though the key strategy-point also offers important advantages when performing the maintenance and debugging capabilities once the network is released.

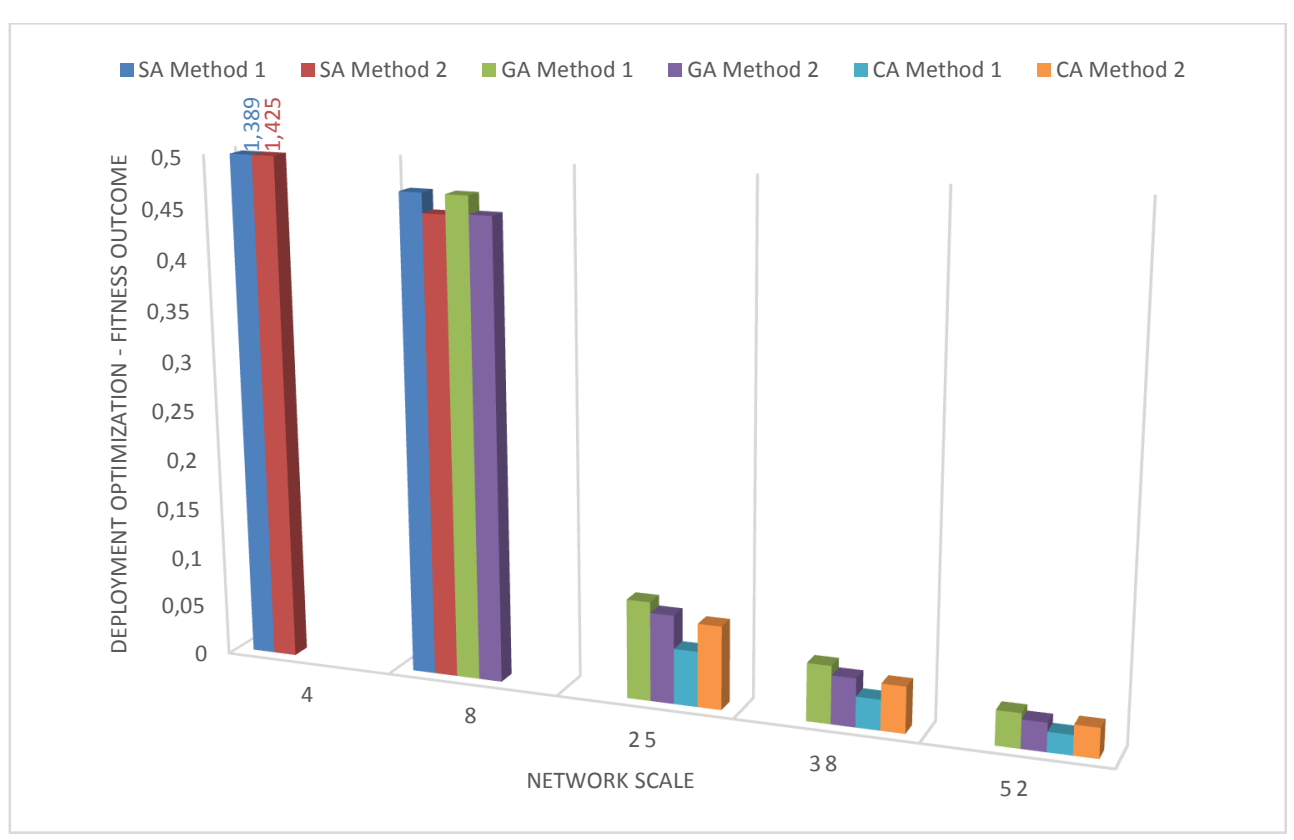

Fig. 3.28 Comparison of the proposed deployment methodology and optimization strategies for different WSN scales. 
On the other hand, as the network gets larger and spread, the redundancy optimization strategy plays an important role since the cost associated with the dissemination execution actions does not shoot up when applying the second method, which thus allows an enhanced configuration process.

It is also important to notice that in case of applying the clustering algorithm with the second method, the cost results are almost the same as in case of the genetic-based algorithm, which highlights the suitability of combining both techniques in medium-large scale deployments from the user perspective, so as to have modularity together with strategy-points for platform evaluation and validation. Such differences in the clustering algorithm are related to the fact that the Kps-based strategy reduces the mentioned restrictions, because the configuration sequence may not include the whole segment of a group into the individual's genotype.

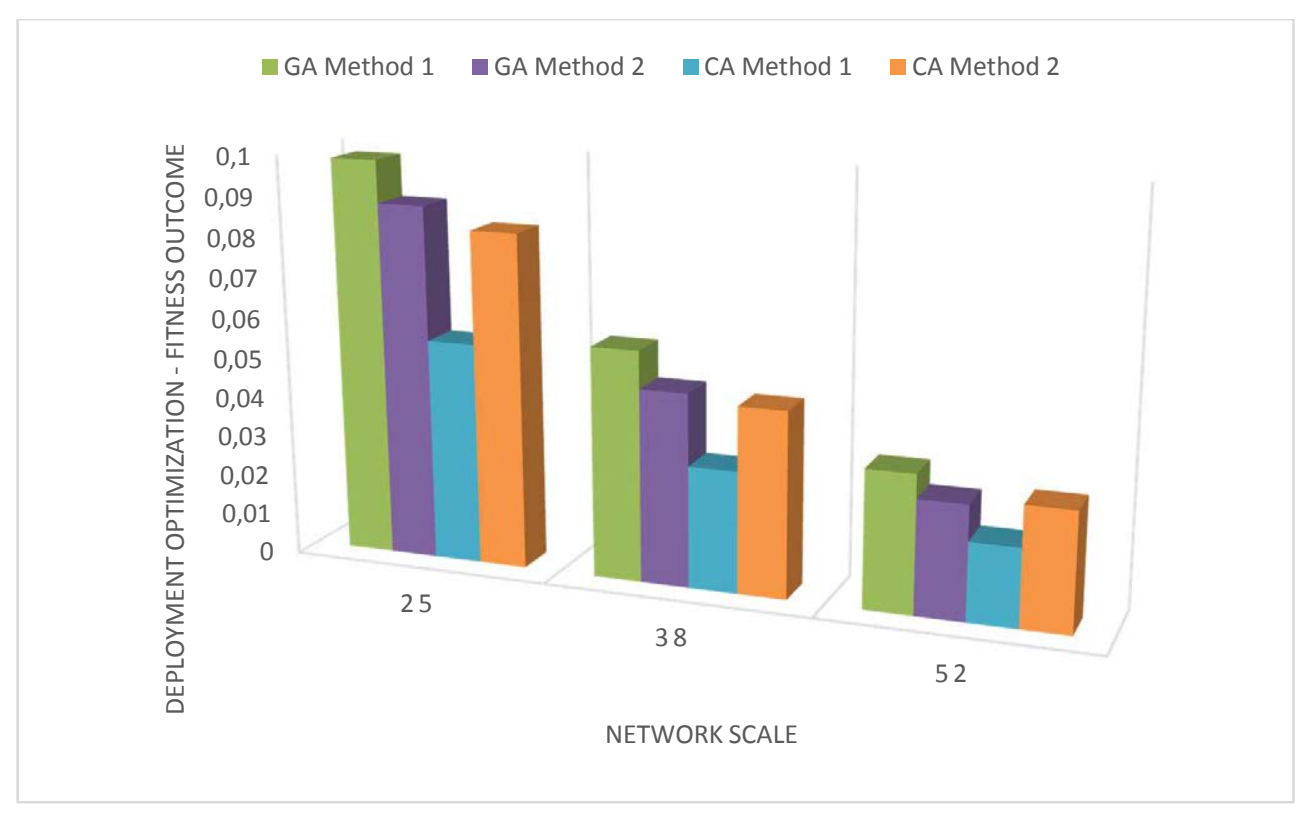

Fig. 3.29 Results comparison of the deployment strategies considering GA and CA for 25, 28 and 52 nodes.

Regarding the computational effort to calculate the solution space in each optimization method, results showed that the cost of the solution improves as the maximum number of generations and the populations get larger, although the computational time also grows. Since this is also a fundamental part to be 
considered due to the nature of the HHD as an on-site deployment and commissioning tool, an execution factor has been created to evaluate the balance between optimal result, time spent to generate it and the number of involved nodes, which is expressed as follows (3.15).

$$
f_{\text {ex }}=\frac{N}{f_{\text {cost }} \cdot t_{c}}
$$

Based on this, in order to analyse the effect of the setting parameters in the performance of the genetic-based algorithm, Fig. 3.30 illustrates the comparison of the population size and the number of generations for a convergence rate of $100 \%$. As it can be extracted from the figure, the execution cost factor is maximized when using lower values for the configuration parameters, whereas it is penalized as the population and number of generations are risen, where the computational time increases rapidly with respect to the cost optimization improvement. The main question focuses on which parameter is more preferable to be modified considering such a behaviour. It can be highlighted that the gradient is more accentuated in those regions of the representation where the number of generations increases than in case of the population size. Thus, the convergence rate plays a fundamental role to assure that in those situations where the number of generations is oversized, the execution time will not be penalized. Therefore, a good option to be followed is the increase of the population size (i.e. 100) and the number of generations configured either as a medium value (i.e. 100 or higher), or increasing it together with a variation of the convergence. Moreover, according to the analysis of the obtained results, a convergence limit of $75 \%$ was too low for several cases, so a default value can be set around $85 \%$. 


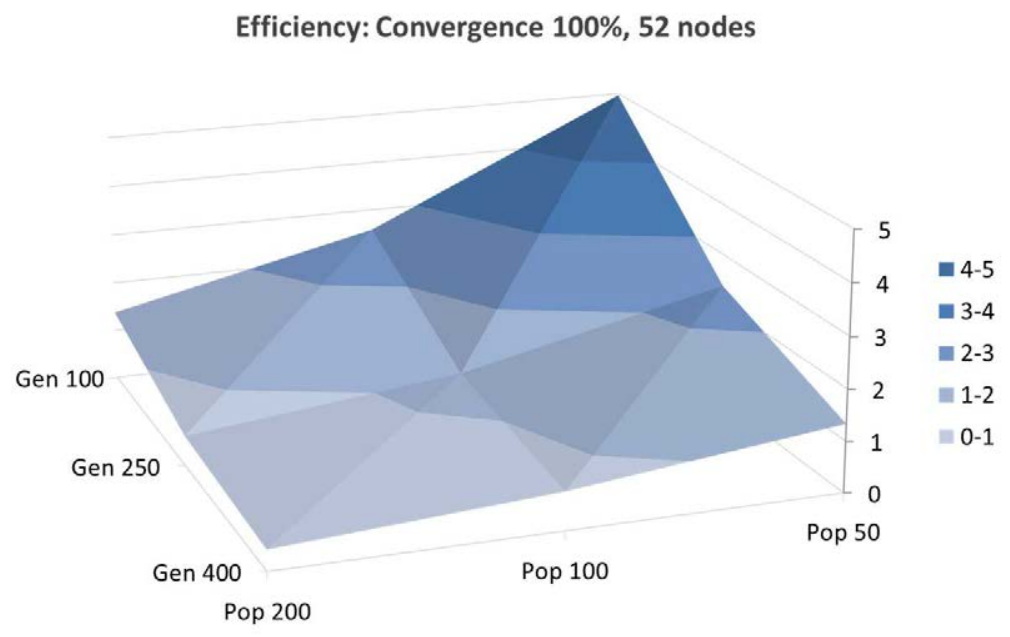

Fig. 3.30 Efficiency of the GA execution considering different configurations for the involved parameters.

Regarding the comparison of the execution factor among the different optimization strategies, as shown in Fig. 3.31 and Fig. 3.32 the application of the genetic-based algorithm for the point-to-point method provides better performance with respect to the other solutions. Such results are associated with a higher level of computational effort that involves the Kps-based method particularly in combination with the clustering algorithm, which thus has an impact in the time spent to find an optimal solution space.

The HHD provides users with the capability of automatically calculating and analysing different optimization strategies for the target scenario, so that a more adapted solution can be achieved. Nevertheless, based on these results and the different observations from the behaviour of the optimization engine, some guidelines can be followed when undertaking one or other deployment strategy. For small scale deployments the swap-based algorithm can certainly suite well with the optimization requirements, whereas in case of larger scales the geneticbased algorithm provides much better results both in terms of cost and computational time. 


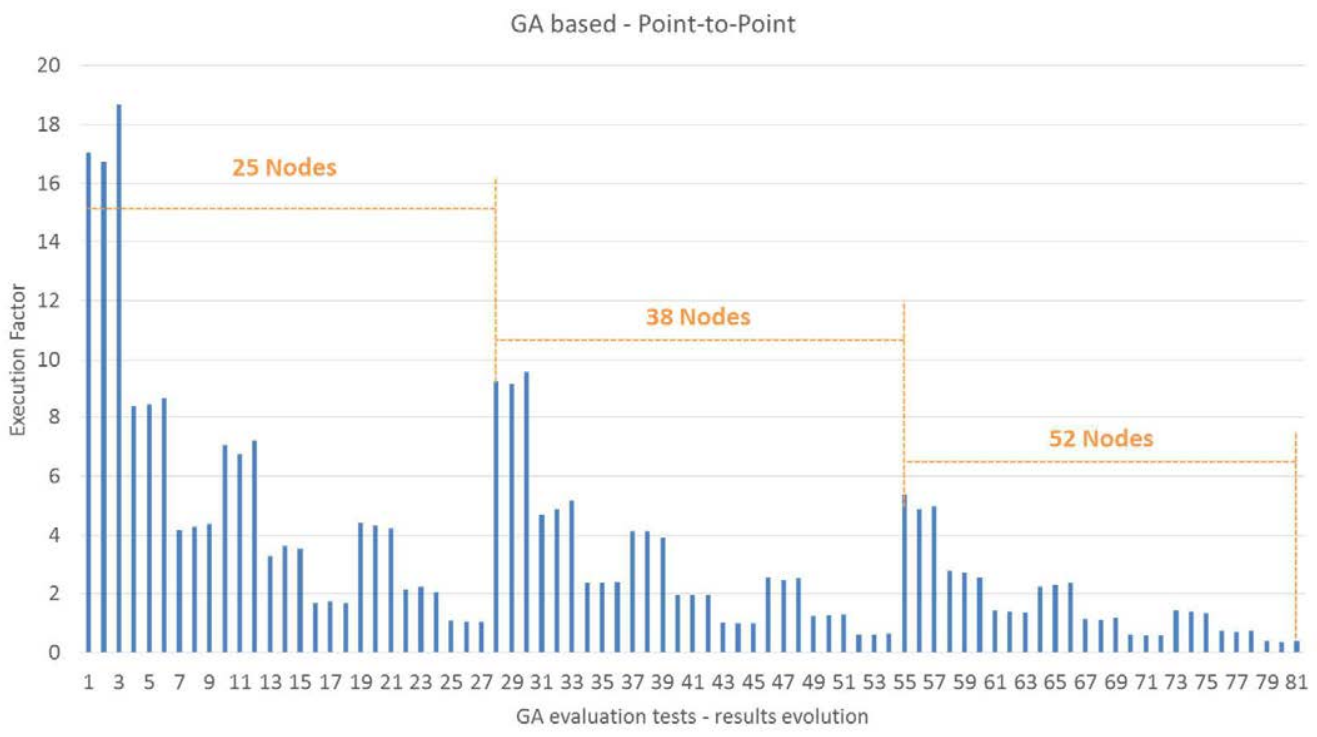

Fig. 3.31 Evolution of the GA execution factor evaluation with respect to the variation of the population size, number of generations and convergence rate.

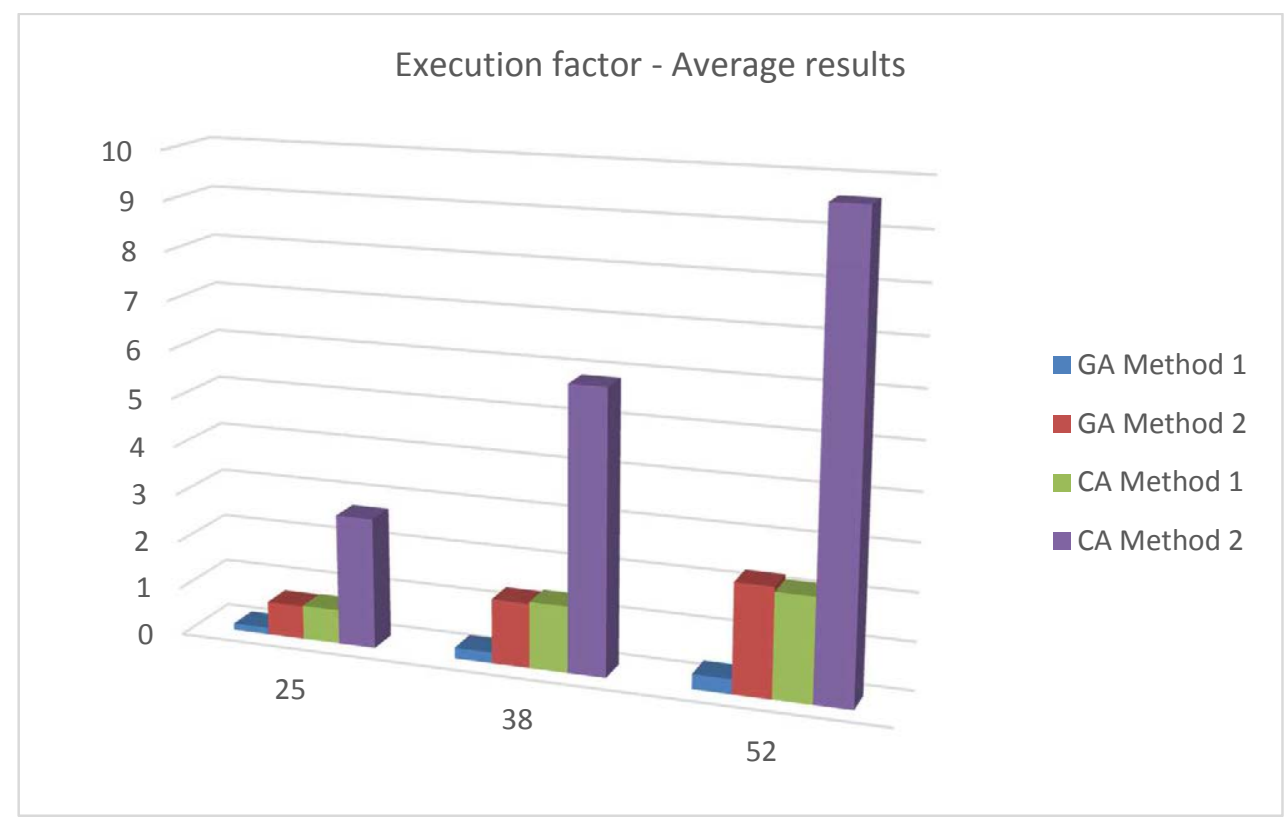

Fig. 3.32 Average results of the execution factor for the GA and CA strategies when varying the scale of the network.

Depending on which of these factors is more critical from the point of view of the deployer requirements, the parameters shall be tuned accordingly, 
particularly considering the results regarding the population size and the number of generations. In this way, the combination of the genetic-based algorithm with the point-to-point strategy offers appealing solutions for a stepby-step/progressive methodology more focused on the sensor nodes themselves, whereas joining the genetic-based or the clustering algorithm with the Kps-based method is certainly suitable for carrying out an overall analysis of the network correlation scheme especially in maintenance phases, as well as provide key points not only for triggering debugging actions from the HHD but also targeting the enhancement of control dissemination packets in line with the routing strategies to be adopted.

\subsubsection{CB-RSim: Multi-hop mesh networking evaluation engine}

As commented previously, one of the main objectives of the on-site deployment and commissioning toolset proposed in this chapter is to provide the ability to analyse and evaluate the performance of real implementations of WSN strategies under the actual HW-SW embedded platform and within the target application scenario, particularly regarding multi-hop communication optimization mechanisms (as the one presented in previous sections), so that a better understanding of the mesh wireless sensor network operation can be obtained from different angles and abstraction levels.

In this way, the Cookie-based Routing protocol Simulation and performance analysis engine of the proposed toolset (CB-RSim) combines the real runtime behaviour of the sensor network deployment with the inclusion of different multi-hop communication models in order to assess their suitability to the WSN application objective, serving as a decision-making element to execute in-field refinements and reconfiguration tasks under the real sensor nodes operation. The idea behind this concept relies on providing deployers with the ability to compare the data that is obtained from the network under deployment/maintenance with on-site routing simulation models, so that a further analysis of route discovery processes, remote connectivity and path metric evaluation can be better accomplished. 
Based on this approach and considering the evaluation of mesh networking strategies, the proposed system pursues the integration of different types of routing optimization mechanisms, allowing for two major communication schemes in this work. On one hand, flat communication strategies according to AODV-based protocols such as the one proposed in previous sections; and on the other hand a hierarchical communication scheme based on a clustering approach that is also addressed to be integrated within the simulation and evaluation engine. In the following paragraphs the main aspects and design objectives of the cluster-based communication strategy is tackled, while the implementation of the routing protocol simulation and evaluation engine is addressed in subsequent sections.

\subsubsection{Hierarchical routing mechanisms in mesh networks}

As described in previous sections, flat-based routing protocols such as the AODV mechanism provide a very flexible and adaptable alternative to wireless mesh networking and particularly to those application scenarios where the behaviour of the deployment as well as the instability of the communications make the use of dynamic routing strategies a must. In addition to this, in largescale deployment distributions it may also be suitable to split it into several subnetworks or groups trying to avoid flooding the overall network with control packets, so that the performance of the dissemination process can be more focused in such situations.

This is one of the main reasons why hierarchical routing protocols appear as an alternative to create a structural topology based on assigning different functional roles to the sensor nodes according to specific requirements. One of the wellknown mechanisms within this approach are the clustering protocols [Sharma'11], where routing capabilities rely on the creation of cluster nodes that are in charge of conducting the data retransmissions among different deployment areas or groups. In this way, cluster-based routing approaches divide geographically the network into groups and set a corresponding status to each node in the protocol mechanism. The information of each region is focused on some nodes, called cluster-heads, which are responsible for ensuring communications within their domain. Therefore, the overall deployment 
information is distributed among backbone-like nodes that collaborate in disseminating the data between remote points in a multi-hop network scheme.

Based on this approach, many of the cluster-based routing protocols split the network into clusters according to the planning stage results prior to the deployment phase, although the real instability of communications and thus the need of hierarchy changes has to be also taken into account. Other protocols establish cluster-heads in a flat-based fashion by using a stochastic algorithm [Heinzelman'00] or by comparing nodes 'height' with the corresponding neighbours according to their identification number [Gavalas'06]. Generally, in any of these protocols, in case of adding new nodes to the network deployment, they would usually try to find a cluster-head in their neighbourhood and then get attached to it. If failed, they would become cluster-heads themselves.

\subsubsection{Hybrid routing approach for the evaluation engine}

Right after running the deployment optimization algorithms, a new model of the mesh network that includes the result of the nodes parametrization is created, which then serves as the entry point for CB-RSim to generate the routing optimization scheme to be evaluated from the basis of the deployment objective.

Targeting the applicability to medium and large WSNs, besides the evaluation of the AODV routing technique a combination of flat and cluster-based strategies are taken into account as an additional feature for users to analyse various scalable routing mechanisms for mesh networking scenarios. The underlying idea behind this functionality is to provide users with the ability to a) experiment with different multi-hop communication approaches, b) analyse and evaluate the suitability of such strategies for the target application scenario, c) based on the feedback from the real behaviour of the system perform reconfiguration and parametrization tasks if needed.

In this direction, the default strategy within the routing modelling and evaluation engine in addition to the AODV mechanism is a hierarchical structure from the basis of the work proposed in [Rezaee'09], which has been implemented into CB-RSim with some further additional features. The routing 
strategy is composed of four possible types of node states or functional roles, as follows:

- Undefined status, when the node is not included in the hierarchy, being the default initial status for the nodes of the network before executing the deployment optimization engine.

- Participant status, when the node is part of a cluster defined by a Clusterhead node, from which it requests information when needed.

- Cluster-linker status, when the node may be both part of a cluster or part of two different clusters, thus being responsible for the communication between cluster-heads and serves as a highway/backbone for information exchanging.

- Cluster-head status, when the node is in charge of its corresponding cluster. It generally has a series of participant nodes under its domain, and manages advanced procedures within the cluster regarding the multi-hop communication mechanism.

CB-RSim provides users with the generation of the clustering formation analysis (using the network connectivity schemes as the inputs of the evaluation engine, as commented before), whose process follows the basis of the aforementioned work though including additional modifications to enhance the capabilities of the cluster-based routing mechanism. According to such an algorithm, in a first stage every node in the network launches a limited number of broadcast messages that are usually called hello or live messages and will be received by nearby nodes, which will use the incoming data to create new entries in their Neighbour Tables.

The determination of the different types of states will depend on a metric-based calculation from the basis of the previous stages of the optimization process, which will then generate an overall scheme of suitable clustering roles for the sensor nodes. Based on this, when a node changes its status to cluster-head, it will notify its surrounding neighbours of such a modification, which will act according to the following rules: 
- If the neighbour node has an undefined status, this will become a participant node and set the source neighbour as its cluster-head. Information about its neighbourhood will be sent to its new cluster-head, which will save it in the corresponding Cluster Table.

- If the neighbour node is another cluster-head, this will use data received to create a new entry in its Cluster-head Neighbour Table. Then, the source cluster-head node will set itself as cluster-linker in the table.

- If the neighbour node is a participant that has a different cluster-head (therefore, it belongs to another cluster), this node will send a clusterlinker request to its own cluster-head.

Although in the first two situations the status is directly updated, the latest case produces a cluster-linker validation process, in which the cluster-head that receives the request will verify whether the source cluster-head has been previously discovered. If not, the destination cluster-head will register the route to the new cluster-head and reply positively to its participant node, which then becomes cluster-linker. This node will include the route between both clusterheads into its Linker Table and notify the source cluster-head with the positive cluster-linker creation, which then saves the information of the destination cluster-head into its own cluster table. A similar process is carried out when a cluster-linker node receives a live message from a cluster-head that has not been registered in the linker table. In that case, a linker request is sent to every registered cluster-head, whose positive replies are then forwarded to the new cluster-head.

In order to keep information of the node status updated to assure the integrity of the multi-hop communication, as soon as a node changes its functional role, it must broadcast a live message to notify its neighbours and then wait for an acknowledgement response from them, so that the information entries are properly changed. In such a scenario, a participant node could contact to another participant of a different cluster-head. The behaviour is the same as if it had received a live message from the cluster-head of its neighbour; however, a new parameter has been added within the update operation, both into the clusterlinker request and the positive reply message, which is the linker-coupling, with 
the id of the other participant node. Both participants will then become clusterlinkers and they will mutually add each other and their cluster-heads to each corresponding linker table. This will also happen between two cluster-linkers or between a participant node and a cluster-linker. In such a case, cluster-linkers will check in their linker tables for the cluster-head of the other node rather than checking its own cluster-head.

If an undefined node contacts a participant or cluster-linker node, it does not change its status. Therefore, after a certain amount of time, if a node remains undefined, it checks its neighbour table and sends a cluster-head request to its best neighbour. When the neighbour node receives the message, it automatically becomes a cluster-head and notifies its neighbourhood. Then, the undefined node becomes a participant node and sets its neighbour as its cluster-head. Once there are no undefined nodes left, the cluster forming process ends.

As seen in the cluster formation process, in order to manage and maintain the routing mechanism, every node has to handle two routing information tables regardless of its type of role: a Neighbour Table that contains information related to every node within its radio communication range, and a Routing Table. In addition to this general structure, cluster-heads have to include two more information entries: the Cluster-head Neighbour table in which data related to cluster-head neighbours and the associated cluster-linker in between are registered, and the Cluster Table that comprises the participant nodes that belong to the cluster as well as their associated neighbours, such that clusterheads can be completely aware of nodes 2-hops ahead from them. There are cluster-based routing mechanisms proposed in the state of the art that include this 2-hop information in every node of the wireless network [Y $\mathrm{u}^{\prime} 08$ ], whereas the one implemented here manages this capability through the cluster-linker as a result of the optimization process, which thus leads to reducing the network overhead and resource cost of the routing strategy.

Finally, the cluster-linker nodes also include an additional table (the Linker Table) for registering the information about pairs of cluster-heads that use the cluster-linker as the main interconnection point between two network areas. 
Based on the cluster formation process, CB-RSim also provides the routing discovery and maintenance mechanism to establish and analyse multi-hop connections between remote-2-Ns of the mesh network. In this way, the implemented routing discovery follows a hybrid approach, in which communication between nodes is made on-demand. As AODV does, each node keeps information of the next-hop in the route to a destination node. While in flat routing strategies such as AODV the source node generates a broadcast with the RREQ control message to initiate the discovery procedure, in the clusterbased approach the RREQ is sent to the associated cluster-head, which then checks both its neighbourhood and its routing table for the destination node. If the source node is a cluster-linker node, it will send the request to every clusterhead registered in its linker table. A cluster-head that receives a RREQ message checks both its neighbourhood and its Routing Table for the destination node. If it does not find the destination point, the RREQ message is forwarded to its neighbour cluster-heads through the corresponding cluster-linker, which also checks its neighbour table and routing table before forwarding the message to the corresponding cluster-head. When a node discovers the destination node in its neighbourhood or in its routing table (or the destination node itself receives the RREQ message), it sends back a RREP control message to the previous cluster-head.

A dynamic optimization might be considered when the RREP is travelling back to the source route by using the established route during the RREQ process, because the cluster-heads can execute a runtime comparative analysis in order to dynamically design an alternative adjacent route based on their cluster tables. As shown in Fig. 3.33 the involved cluster-head makes a local evaluation based on the already available routing management information, and if succeed it will notify the nodes involved, add the alternative adjacent route to the RREP message and let the next cluster-head continue developing the optimization process. When the last cluster-head receives the RREP message, it checks whether the alternative path is more suitable or not in terms of the multi-hop communication cost metric and then sends the adjacent solution to the source node. The main advantage is that such strategy relies on already available 
information from the cluster-heads, so there is no impact in terms of network flooding and packets overhead.
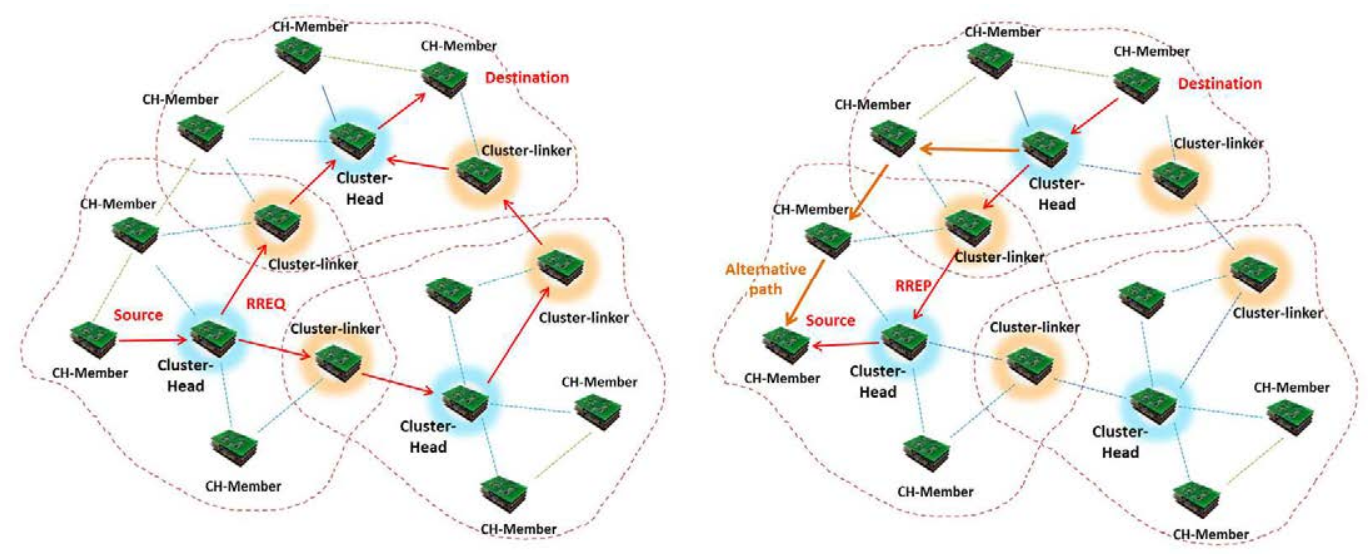

Fig. 3.33 Route optimization mechanism based on $\mathrm{CH}$ dynamic evaluation.

Regarding the route maintenance mechanism, in order to verify the integrity of the routing performance between remote points and assure the multi-hop communication in the proposed protocol, two different types of failures can be defined:

- A failure in a main path: the error is detected in the path that goes from a cluster-head to another cluster-head or from a cluster-head to any of its participant nodes.

- A failure in a secondary path: the error is detected between two participant nodes or between a cluster-linker and a participant node. In a particular case, it might also be found between two cluster-linkers from different paths.

When the main failure is found in the path between cluster-heads, an alternative path can be found just as the route discovering strategy: forwarding a message from cluster-head to cluster-head. In addition, a cluster-head can use its information about 2-hops-away nodes and try to locate an alternative clusterlinker route. If a new route is found, the old path between cluster-heads is dismissed, and the cluster-linkers involved remove the path in their linker tables, and they become normal nodes if necessary. However, if the new route is not suitable according to certain criteria (such as cost overrun), both cluster- 
heads involved may require all of their participants to send a live messages in order to find a new cluster-linker if possible. Only if no alternative way is found to reach the cluster-head, the solution will be to alert the source node and then reorganize the network structure, launching again the cluster formation procedure.

In case the failure takes place between a cluster-head and its participants, the cluster-head would look for the lost node in its cluster table at a distance of 2 hops. If it is found, messages addressed to the lost node will be forwarded to the most suitable intermediate node in the cluster.

If not found, it is likely that the node is isolated from the network. Secondary failures are much easier to repair. If an error in communications is located between two participant nodes, the participant will request its cluster-head an alternative path. The cluster-head will try to find an alternative path looking on its cluster table, and if it failed to find one, the cluster-head will forward a message to its neighbour cluster-heads. If the failure is detected by a clusterlinker, a request to several cluster-heads can be done at a time. Both types of failures and the described local repair procedures for the hybrid routing strategy are shown in Fig. 3.34.

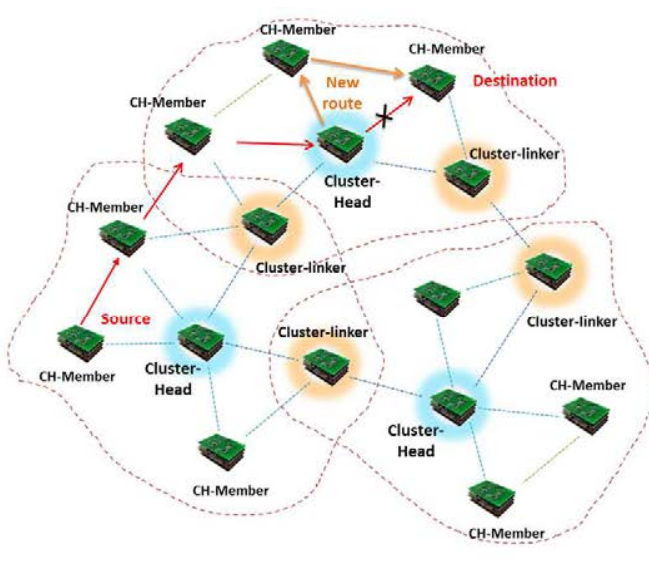

a)

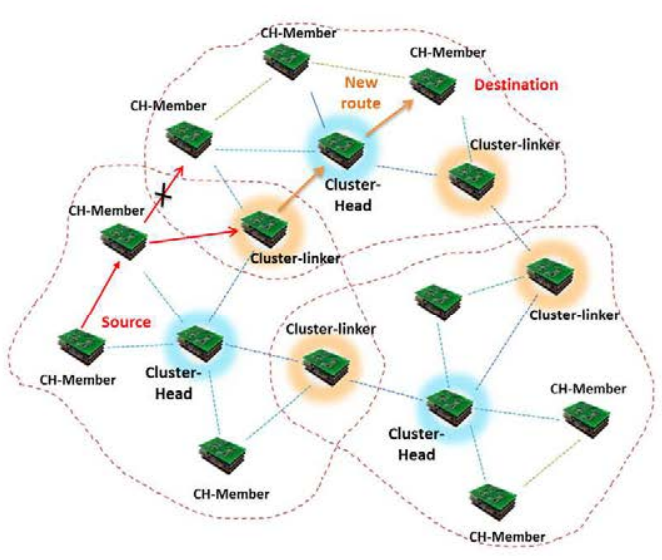

b)

Fig. 3.34 Local repair: a) failure on main path, b) failure on secondary path.

As shown subsequently in the implementation of the HHD functional subsystems, the simulation and evaluation engine provides users with a set of 
integrated functions to easily analyse and compare the behaviour of the included routing optimization mechanisms in situ, by accessing the list of available protocols and select one of them to evaluate its performance in accordance with the input network correlation and optimization models, and by configuring triggering actions as well as monitoring events on the system. This means that the proposed evaluation tool is focused on its on-site usability, that is, provide users with a dynamic tool to analyse the operation of the sensor network deployment in-field and in runtime, following the nature of the HHD. From this, the routing discovery, maintenance and update techniques can be studied from a real point of view to verify their suitability for the network scale conditions, deployment distribution and connectivity/platform constraint issues.

The implementation of these strategies has been conceived as a support framework to include further routing optimization mechanisms within the CBRSim engine for, their in-field evaluation and validation. The details of the user interface and interactions with the deployment toolset are described latter on in the next overall system implementation chapter.

Consider the experimental test case depicted in Fig. 3.35, in which a mesh wireless sensor network is set to be analysed by using the routing strategies in CB-RSim. The cluster-based optimization process determines the cluster-head and the cluster-linker points (in purple and blue respectively) that correspond to the hierarchy parametrization, where blue lines refer to the main multi-hop paths while green lines involves secondary routes (the flat scheme is expressed by all the interconnection lines). Based on the protocol implementations and the node modelling framework included into the routing evaluation engine, cluster formation and discovery procedures have been evaluated. For instance, a communication request such as $X>Y$ implies that node $X$ sends a RREQ message to subsequent points in order to find node $Y$. The route from node $X$ to node $Y$ that passes through node $k$ is represented as ' $X-\ldots-k-\ldots-Y$ '. If the RREP response is generated by node $f$, which has found node $Y$ in its Neighbour Table or in its Routing Table, the route is represented as ' $X-\ldots-k-\ldots-[f]-\ldots-Y$ '.

The main parameters that are taken into account in this experimental comparison are the energy consumption of a routing path, the cost of 
discovering and maintaining the communication between two remote points and the dissemination execution actions among the network connections, which are respectively computed as the involved broadcasts of the selected mechanism, the total amount of broadcasts spent and the number of hops associated with the discovery paths. Moreover, routes that are displayed in brackets correspond to the alternative routing optimization of the hybrid solution.

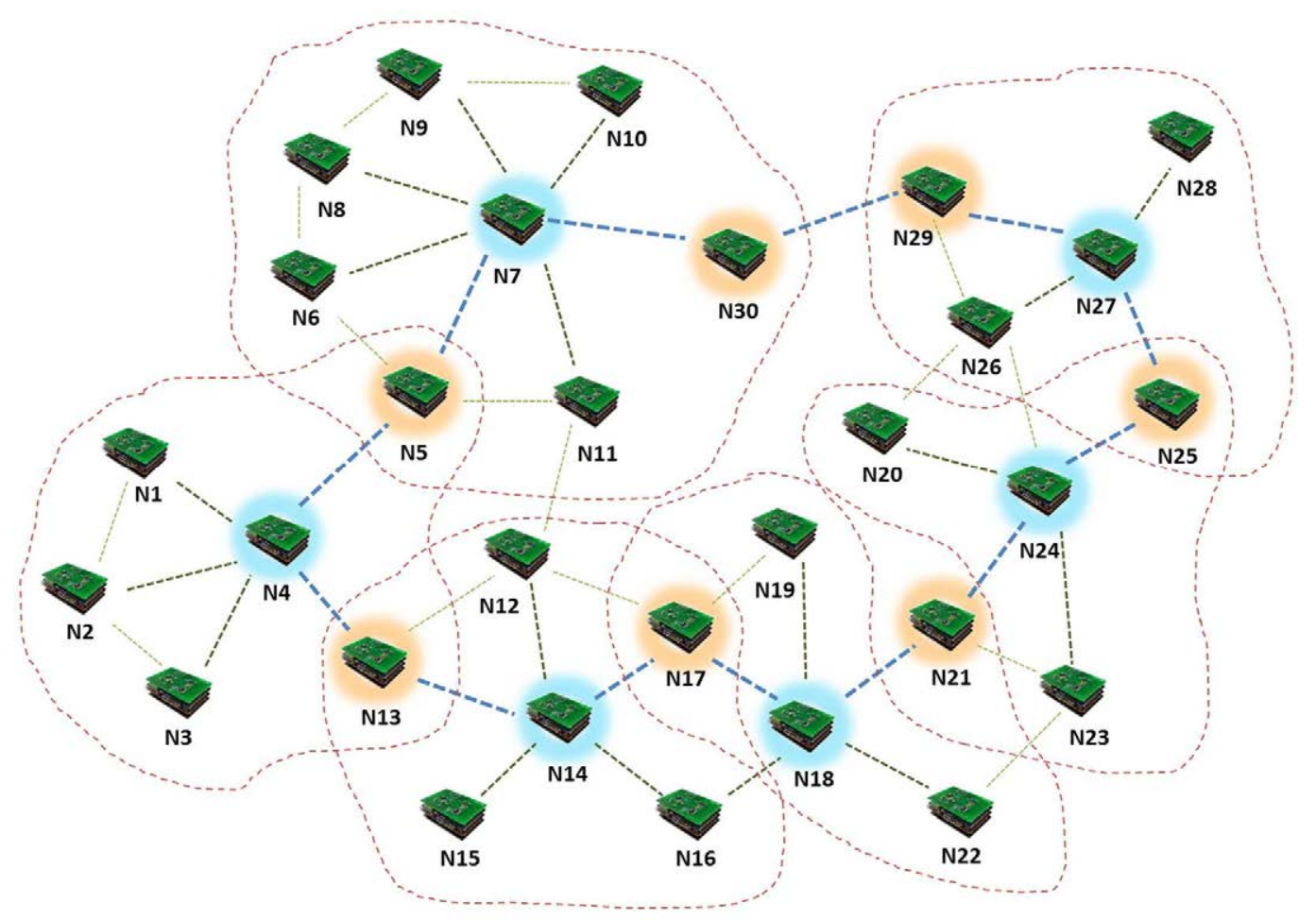

Fig. 3.35 WSN experimental test case and cluster formation result.

Table 3-10 summarizes several route discovery test cases from different remote points in order to realize a comparative analysis between a flat-based approach and the hybrid technique, by using the data processed by the deployment support tool. A first quick analysis can be highlighted related to the evolution of the dissemination control packets. While in case of the AODV-based routing approach the main focus is related to highly dynamic environments in which the on-demand technique allows a better adaptation to unstable application scenarios, the hybrid technique provides suitable results when the cluster-based formation can keep a medium or high degree of unaltered states for a 
determined period of time. This means that in case of the former strategy the control dissemination efforts rely on the discovery process itself, so that it tends to increase as the scale of the network and the number of demanded communication between different remote points gets larger, whereas in the cluster-based strategy the initial cluster formation requires an intensive packet exchange process that increments almost proportional to the scale of the network, although the subsequent routing discovery ideally provides a quit availability of the different paths without further broadcasting actions.

\begin{tabular}{|c|c|c|c|c|c|c|c|c|c|c|}
\hline \multirow{2}{*}{$\frac{\text { Request }}{\text { Cluster formation }}$} & \multicolumn{2}{|c|}{ Route found } & \multicolumn{2}{|c|}{ Number of hops } & \multicolumn{3}{|c|}{ Broadcasts involved } & \multicolumn{3}{|c|}{ Total Broadcasts } \\
\hline & - & & - & & 160 & 90 & & 160 & 90 & \\
\hline $3>28$ & $\begin{array}{l}3-4-5-7-30-29-[27]-28 \\
(3-4-5-7-30-29-27-28)\end{array}$ & $3-4-5-7-30-29-27-[28]$ & $\begin{array}{l}7 \\
(7)\end{array}$ & 7 & 0 & 0 & 29 & 160 & 90 & 29 \\
\hline $8>22$ & $\begin{array}{c}8-7-5-4-13-14-17-[18]-22 \\
(8-7-11-12-17-18-22)\end{array}$ & $8-7-11-12-17-[18]-22$ & $\begin{array}{c}8 \\
(6)\end{array}$ & 6 & 0 & 0 & 25 & 160 & 90 & 54 \\
\hline $25>15$ & $\begin{array}{l}25-24-21-18-17-[14]-15 \\
(25-24-21-18-16-14-15)\end{array}$ & $\begin{array}{c}25-24-21-18-17-[14]- \\
15\end{array}$ & $\begin{array}{c}6 \\
(6)\end{array}$ & 6 & 0 & 0 & 27 & 160 & 90 & 81 \\
\hline $24>3$ & $\begin{array}{l}24-21-18-17-14-13-[4]-3 \\
(24-20-19-17-12-13-4-3)\end{array}$ & $24-26-[29]-30-7-5-4-3$ & $\begin{array}{l}7 \\
(7)\end{array}$ & 7 & 0 & 0 & 15 & 160 & 90 & 96 \\
\hline $11>20$ & $\begin{array}{c}11-7-30-29-27-25-[24]-20 \\
(11-7-30-29-26-20)\end{array}$ & $11-12-17-19-20$ & $\begin{array}{c}7 \\
(5)\end{array}$ & 4 & 0 & 0 & 24 & 160 & 90 & 120 \\
\hline $14>7$ & $\begin{array}{l}14-13-4-5-[7] \\
(14-12-11-7)\end{array}$ & $14-12-[11]-7$ & $\begin{array}{c}4 \\
(3)\end{array}$ & 3 & 0 & 0 & 20 & 160 & 90 & 140 \\
\hline $11>3$ & $\begin{array}{c}11-[7]-5-4-3 \\
(11-5-4-3)\end{array}$ & $11-[5]-4-3$ & $\begin{array}{c}4 \\
\text { (3) }\end{array}$ & 3 & 0 & 0 & 15 & 160 & 90 & 155 \\
\hline $20>22$ & $\begin{array}{c}20-24-21-[18]-22 \\
(20-24-23-22)\end{array}$ & $20-24-[23]-22$ & $\begin{array}{c}4 \\
(3)\end{array}$ & 3 & 0 & 0 & 9 & 160 & 90 & 164 \\
\hline $16>29$ & $\begin{array}{c}16-14-13-4-5-7-30-[29] \\
(16-18-21-24-26-29)\end{array}$ & $16-18-19-20-[26]-29$ & $\begin{array}{c}7 \\
(5)\end{array}$ & 5 & 0 & 0 & 24 & 160 & 90 & 188 \\
\hline $26>10$ & $\begin{array}{c}26-27-29-30-[7]-10 \\
(26-29-30-7-10)\end{array}$ & $26-29-30-[7]-10$ & $\begin{array}{c}5 \\
(4)\end{array}$ & 4 & 0 & 0 & 25 & 160 & 90 & 213 \\
\hline $6>24$ & $\begin{array}{c}6-7-30-29-27-25-[24] \\
(6-7-30-29-26-24)\end{array}$ & $6-[7]-30-29-26-24$ & $\begin{array}{l}6 \\
(5)\end{array}$ & 5 & 0 & 0 & 4 & 160 & 90 & 217 \\
\hline $\begin{array}{l}\text { Local Repair: } \\
\qquad 12>17\end{array}$ & $12-14-17$ & $12-14-17$ & 2 & 2 & 0 & 0 & 24 & 160 & 90 & 241 \\
\hline
\end{tabular}

Table 3-10 Comparative analysis of the routing strategies for the proposed experimental tests.

The control frame dissemination and flooding costs of both techniques intersect when the number of remote-2-Ns raises 7-8 different totally new discoveries, which allows defining a reference value upon which one or other solution may suit better for the target network communication scenario. Obviously, this will indeed depend on the network distribution and topology scheme of the WSN. For instance, in dense and spread networks, although the AODV-based technique may generate additional control dissemination actions to find a 
suitable route, in case of the cluster-based strategy the encountered paths might be penalized in terms of number of hops to reach the destination point because routes are produced from the basis of the cluster-head interconnections. The hybrid solution enhances this by applying the dynamic alternative route generation process proposed previously. Moreover, as remarked in Table 3-10, by taking advantage of the application of the deployment methodology actions (particularly by using the NDoSq based on the Kps-based parametrization), the number of required broadcast messages can be reduced thanks to the optimization of the control packets to be used for producing the network correlation scheme, which serves as the starting point to generate the cluster structure accordingly. In Fig. 3.36, the control dissemination effort tendencies to obtain the different required multi-hop paths are compared, where the boundaries to start considering one or other solution for the target mesh network allows users to have an overall view of the impact of the route discovery tasks.

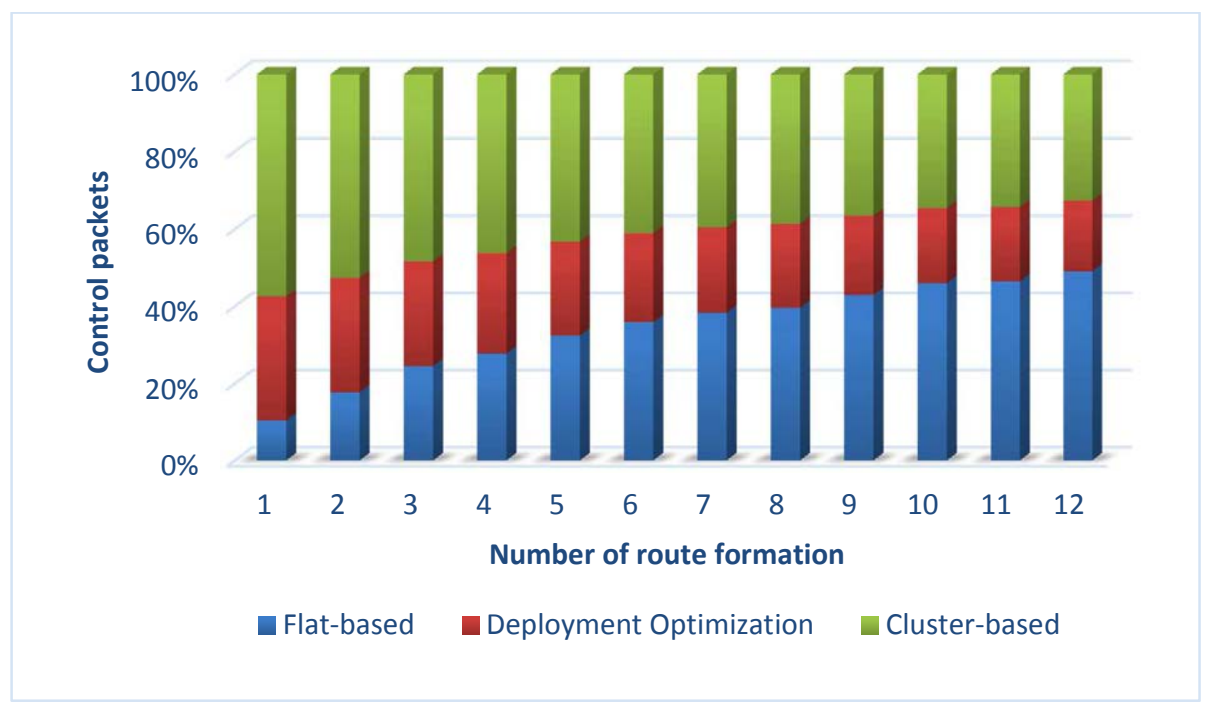

Fig. 3.36 Relationship of the number of control packets for every routing generation with respect to the overall amount of broadcasts.

\subsubsection{Deployment dynamic guidance}

Once the network connectivity generation and optimization engines produce the overall deployment schemes based on the data and functional flow described in 
previous sections, the deployment and maintenance toolset provides a dynamic deployment guidance which makes use of the input network models in order to adapt the configuration and performance evaluation activities to the target WSN under commissioning. The underlying idea relates to an easy and dynamic interaction of the deployer with the sensor nodes to be deployed, by means of using the in-field configuration capabilities provided by the HHD, and supported by a well-defined over-the-air interface approach. Thus, the mobile platform will simplify the testing tasks with the assistance of the toolset and GUI functionalities.

In order to accomplish this, the deployment guidance is composed of five main interaction stages that provide different levels of testing and evaluation actions, summarized as follows:

- Platform positioning, installation process.

- Node configuration, platform-level interaction.

- Low-abstraction evaluation, medium access level analysis and configuration. Network correlation scheme generation/updating.

- Routing/network performance evaluation, deployment level interaction.

- Overall system assessment and validation, comparative analysis. Reconfiguration and parametrization refinement.

As described in the implementation details of the HHD, the mobile positioning and overall network visualization is provided by using a layer-based management interface, in which the basic element upon which the different deployment options are overlapped is a Google-map-based interface [Maps]. This map visualization serves as a reference indication for users to carry out the positioning and installation process, in accordance with the geo-location information that is gathered from the input network models, which contain the physical coordinates of the sensor nodes to be deployed. The positioning of the mobile platform can be seen also from the WSN point of view beyond the real location of the HHD, i.e., two different types of locations can be highlighted. One of them is the in-field location based on the GPS capabilities of the smart device, and the other one is its positioning with respect to the wireless network itself, 
which refers to the coverage of the sensor nodes under deployment/maintenance. The later can be seen as a relative positioning in relation with the network, which is provided with the support of the HHNode.

As shown in Fig. 3.37, the positioning of the HHD allows a direct communication with the embedded HW-SW platforms to perform the configuration and analysis/diagnosis services, considering the different levels of interaction mentioned before. This is a key factor to take into account, because such a mobile attribute of the HHD provides a more transparent way of accomplishing the in-field testing and debugging tasks, without further structural implementation. The over-the-air actions are supported by a debugging component included in a small footprint within the embedded software of the sensor nodes, in combination with the commissioning and diagnosis components integrated into the HHD.

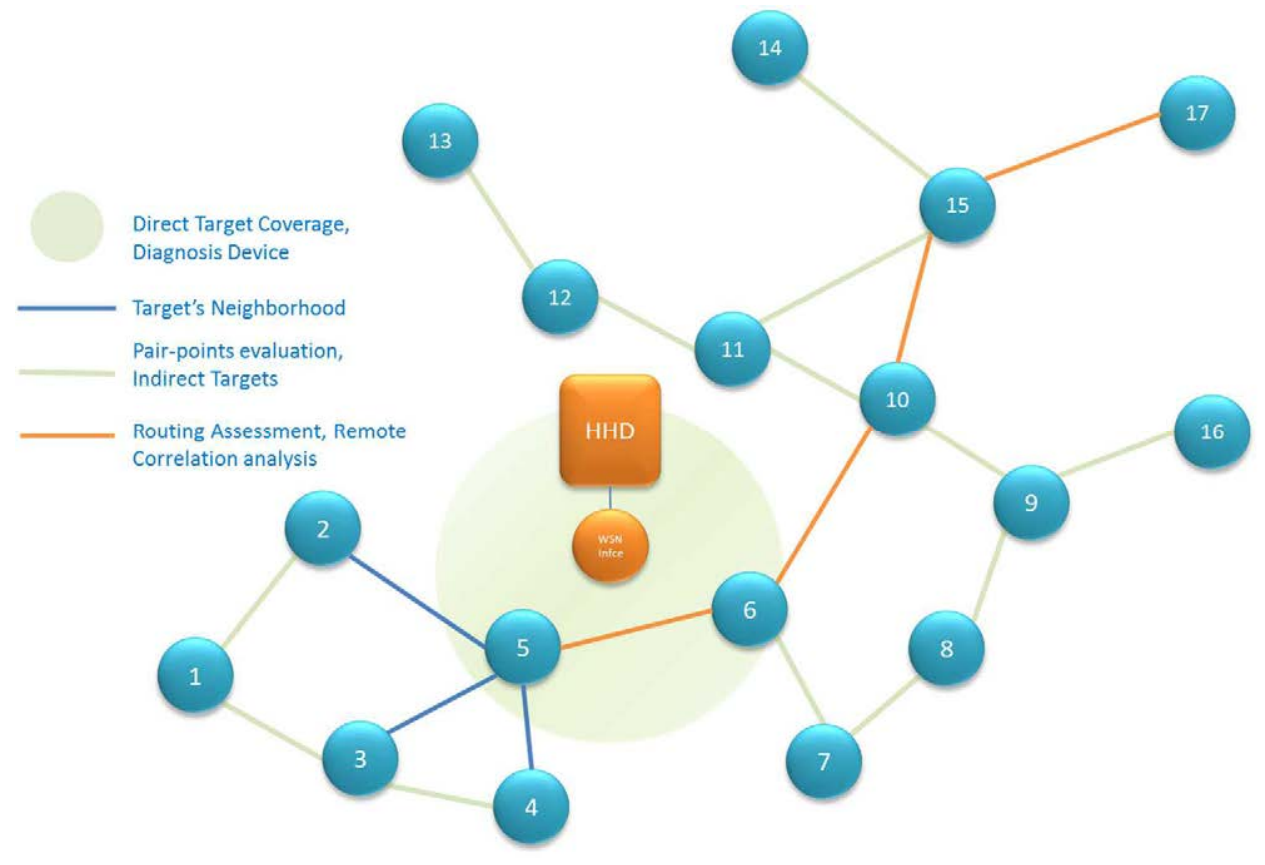

Fig. 3.37 In-field relative position of the HHD when testing specific deployment areas.

Upon the map visualization and navigation layer, the different functionalities related to deployment of the WSN can be activated, particularly considering the network connectivity and optimization schemes previously generated, so that a runtime comparative analysis with the actual data can be performed by 
executing the in-field tests from the dynamic evaluation layer. For instance, a reconfiguration of node's properties, the modification of their location or the inclusion of a new node to enhance the communication coverage of a certain area allows the automatic recalculation of the connectivity links and the execution of the associated tests, in order to evaluate how such an action can impact the overall network correlation scheme and the performance of the involved deployment area.

Once the physical installation of the target node is performed by using the HHD, initial configurations can be carried out to start its correlation with the WSN, considering the configurable blocks that are included in the input model definition for such a node. The basic elements to be considered regardless of the HW platform are the communication module parametrization (initial TX power and power-down modes, node type profile if available), node metric and weight factor configuration. Together with this parametrization, the node interaction with the environment is also evaluated, which corresponds to the configuration and performance analysis of the sensor/actuators that are implemented into the WSN node.

Based on loading the block definition, the HHD can configure the publishing period of the sensor device by using the associated block type, block instance and parameter identification, and then trigger the over-the-air measurement gathering service from the target node, so that runtime sensing values can be stored and processed to evaluate its correctness with respect to the expected behaviour of the transducer. This will allow users to tune the data acquisition and conversion function to properly calibrate the sensing magnitude for the specifics of the environment under monitoring. Moreover, a runtime automatic processing of the gathered data is realized to provide several metrics for users to assess of the behaviour of the sensor acquisition: average and medium measurement magnitudes, maximum and minimum sensing window, measurement slope and sensor tendency characterization.

The next stage refers to the evaluation of the sensor nodes from the point of view of the quality of the interconnections and platform communication coverage (local/surrounding level), by following the defined deployment methodology 
tasks. This will lead to generating the real connectivity scheme at MAC level, which is dynamically updated in the form of a layer overlapped into the deployment map interface. In this way, the network correlation scheme is produced based on an iterative process where every node gathers bidirectional information related to their corresponding neighbours/pairs-of-points in terms of quality of the link, radio signal strength, and packet delivery metrics. This information can be compared with the connectivity models at runtime by activating the simulation layers within the map interface, in order to detect possible mismatches between actual and modelled links/paths.

For this, the selection of a sensor node to be evaluated provides the possibility of triggering several in-field tests, distinguishing a) medium access protocol assessment and packet analysis execution, b) surrounding detection for local correlation and node entry association, c) neighbour configuration and bidirectional quality evaluation, including metric calculation. A MAC frame decoder is also included by gathering protocol frames regarding exchanged information among the nodes, containing a format configuration as well as a network packet classification. By using the packet analyser capabilities, low-level frames can be monitored in order to evaluate the generation/reception of packets from a lower abstraction level, so that problems regarding medium access and frame configuration can be detected.

Such abstraction level serves as the baseline to carry out the performance evaluation of the routing optimization strategies and the analysis on how their associated control dissemination mechanisms are correlated with the generated network connectivity scheme. The HHD allows on one hand obtaining information related to the routing protocol information management, such as routing table entries considering the following items:

- Number of hops from the source node to the destination point. It allows the comparison of the metric-based routing decision with respect to the actual connectivity map.

- Next hop used to reach the destination node, which indicates the vector information of the created route. 
- Value of the associated metric for the route discovery process, considering the proposed set of quality metrics within the routing protocol implementation.

- Number of precursor nodes and local-repairs for the table entry, which allows further stability analysis of the communication links to establish the multi-hop connection between the target node and the destination point.

On the other hand, real tests regarding the creation and evaluation of routes between pairs of remote points can be triggered from the HHD, so that runtime outcomes considering the following metrics can be provided, apart from the aforementioned items:

- Sent/received packet ratio, Packet Loss Rate (PLR). A packet delivery test is proposed to evaluate the performance of the routing capabilities as well as parameters regarding path discovery/rediscovery. The evaluation tool can launch this test by configuring the pairs of points to be assessed (having coverage with at least one of them), the number of packets to be transmitted and the data/parameter setups according to the specific test to be carried out. The diagnosis component executes the packet delivery tasks from the target node and then calculates the PLR that is presented and stored in the corresponding database entry of the tool.

- Route Rediscovery Rate (RDR) - link failure. The number of rediscoveries are computed by storing the information of the next hop with which a failure comes out (note that each node stores coverage information on their neighbour table). This process can help users to detect possible link and instability problems and critical points in specific deployment zones, as well as analyse the impact of the selected metric configuration to avoid the creation of overhead areas.

The routing map can be compared with the connectivity/MAC level in order to evaluate the performance of the routing protocol in terms of efficiency on the metric usage, asymmetry on the remote-2-Ns, node isolation, congestion, and 
loops avoidance. This runtime automatic correlation process leads deployers to deciding whether specific routing and network configuration criteria shall be adopted in accordance with the dynamics of the system behaviour.

Based on the network models and connectivity schemes, the deployment methodology tasks and the real experimental tests carried out in-situ, a comparative analysis of the real behaviour of the distributed sensors is accomplished in order to refine and reconfigure specific parameters seeking a runtime performance enhancement of the overall WSN. For this, the HHD offers a dynamic system assessment matrix in which the stored information of the deployment activities can be on one hand contrasted with the simulation results and on the other hand verified from a global/system-wise perspective, where internal, local and overall network correlation levels shall be evaluated for further tuning actions. This will lead to analysing possible mismatches of the experimental outcomes with respect to the estimation models, in order to detect those network points that may be source of possible failures, particularly in terms of connectivity costs, critical nodes and unbalanced deployment areas, as well as bottlenecks and redundancy levels.

For instance, considering the comparative relation of the node connectivity scheme with the performance of the routing strategies, the data distribution throughout the wireless mesh network might be affected by a lack of reliable connections that leads to producing worst results in terms of the control and data dissemination indicator metrics. In order to overcome such a situation, users can execute a series of quick runtime operations attempting to enhance the performance of the influenced area, based on the inclusion of additional deployment constraints or requirements into the optimization options, so as to update the deployment models accordingly:

- Transmission power recalculation and on-site reconfiguration, taking into account how current and updated changes may affect the involved local node correlation scheme.

- Site relocation and dynamic computation of the connectivity map to analyse the influence of such modifications in the overall deployment area. This can be supported by the use of rely nodes, which allow the 
enhancement of weak zones that present important unexpected limitations and thus affect the normal operation of the nodes.

- Incorporation of connectivity constraints (e.g., reconfiguring the experimental model factors based on the registered data from the on-site deployment activities) to avoid the correlation of 2-Ns that present higher fluctuations in the quality metric boundaries with respect to the rest of the correlation scheme, so that an enhancement of the connectivity map can also be recomputed and improved consequently.

In order to interface the debugging and diagnosis components included both in the HHD (through the HHNode) and the embedded software implementation of the sensor nodes, a control packet framework has been designed for the overthe-air execution of the testing capabilities from the proposed deployment and maintenance functionalities. As shown in Fig. 3.38, the baseline format of the packet headers follow the network deployment model description in which the functional block definition, block instance and parameter configuration interface identifiers are to be included so as to specify the type of action to be carried out.

The block repository is complemented with the inclusion of additional component definitions to provide block interfaces for the diagnosis tasks, considering the functionalities in Table 3-11. Thanks to the modularity of the functional blocks definition, different components for debugging, configuration and testing actions can be included and scaled within the embedded software implementation of the wireless nodes and the HHD, which can be updated and reprogrammed in accordance with the type of evaluation to be performed (in line with the deployment model generation for the target WSN application).

\subsubsection{HHD on-site interface to the WSN deployment}

As mentioned before, in order to establish the in-field communication with the sensor nodes under deployment/maintenance for the evaluation and diagnosis control packet exchange capabilities, the HHD makes use of the HHNode through which the low-level WSN protocol is implemented. However, the effective runtime interaction of the deployment toolset with the wireless sensors is carried out by means of the design and integration of a well-defined platform 
interface into the smart device (considering its implementation within the Android OS based development).

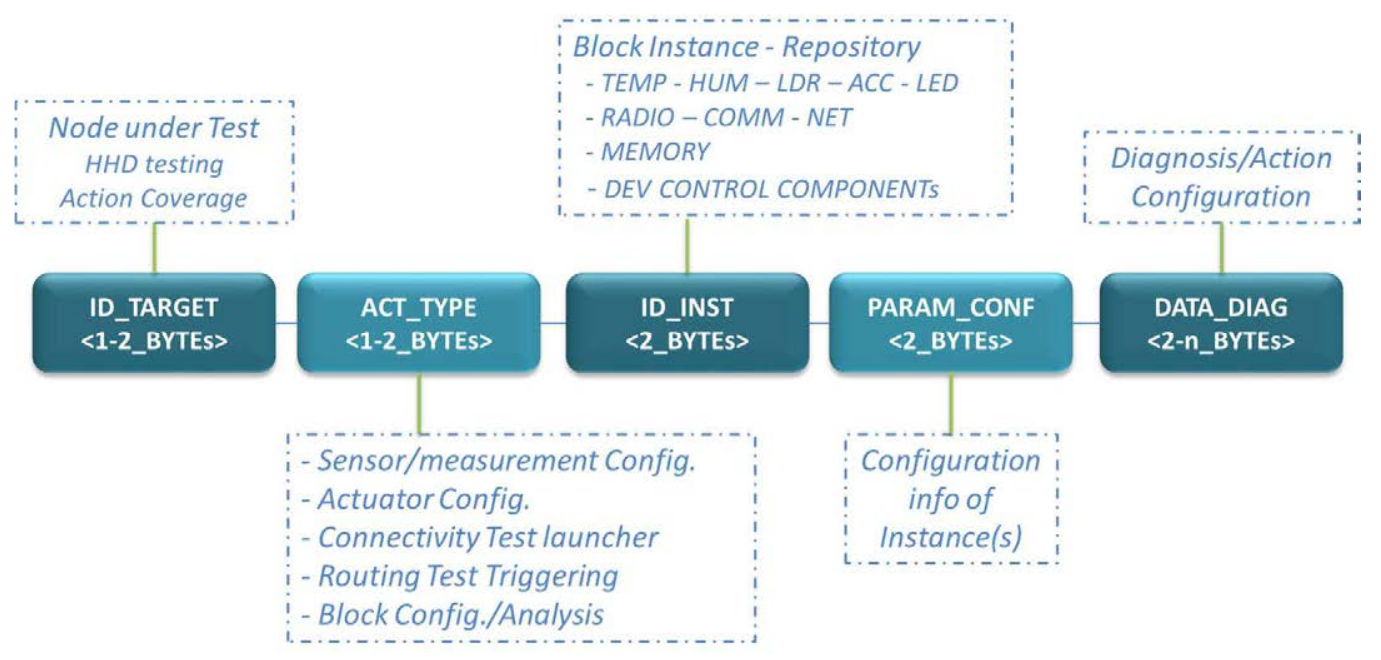

Fig. 3.38 Structure of the defined packet framework to interface the HHD with the functional components and diagnosis blocks of the in-field sensors nodes.

The basic element to outline such interface approach relies on an interrelation of the low-abstraction-controller of the smart device with the HHNode through the physical connection between both platforms. For this, a USB-Host scheme has been adopted as the underlying connectivity structure of this heterogeneous integration, where the mobile device provides both power and data to the HHNode. At this point, the first requirement to be tackled is an efficient management of the node connection identification and interface loading, so as to establish a proper communication of the hardware controller with the corresponding attached embedded platform, being a seamless transaction for the upper implementation layers of the deployment toolset. The designed controller has been conceived to manage the connection and data aggregation from the USB port considering the following items:

- Hardware automatic detection and node identification registration.

- Packet interruption handling for data gathering/dispatching and input/output frame processing threading.

- Bundle composition to encapsulate the network deployment data structure for layer information interchanging within the tool. 
This controller definition serves as the foundation to create and integrate three main interface approaches within the HHD implementation:

- Reference HHD-WSN interface and Cookies HW-SW platform integration.

- Middleware technology integration for heterogeneous developments.

- TinyOS-based platform interactions.

The Reference HHD interface determines the abstraction layer for managing the WSN debugging and diagnosis services by using the underlying controller definition, which is described as follows.

\subsubsection{Reference HHD-WSN interface approach}

Fig. 3.39 depicts the basic elements that compose the HHD-WSN interface abstraction layer within the deployment tool, in which the low level controller hhUsbDevice that handles the HW port and HHNode data interface represents the base of the management for the upper layers. It includes on one hand the USB host port configuration and thread definition to establish the runtime communication with the in-field WSN by using the plugged HHNode, and on the other hand the encapsulation of the low data structure of the gathered/dispatching data for information provisioning to side layers.

Thereon, hhUsbDeviceInterface and hhNodeManager include the decoding/encoding mechanisms by using the bundle encapsulation and control interface methods provided by the aforementioned layer in order to execute/process the HHD-WSN configuration and evaluation tasks, following the specification of the component instances to be acted on, i.e., considering block type, block instance and block parameter definitions in line with the network deployment model.

In this way, by loading node object definition properties, in-field data requests and execution of commands can be triggered by specifying node ID and type of object (Sensor/actuator, property, configuration, network parameter), as well as the associated data of the action to be performed. For instance, if a threshold detection for a sensor measurement is to be configured, the window boundary parameters are specified in the form of a data structure in dataObjectDef. 


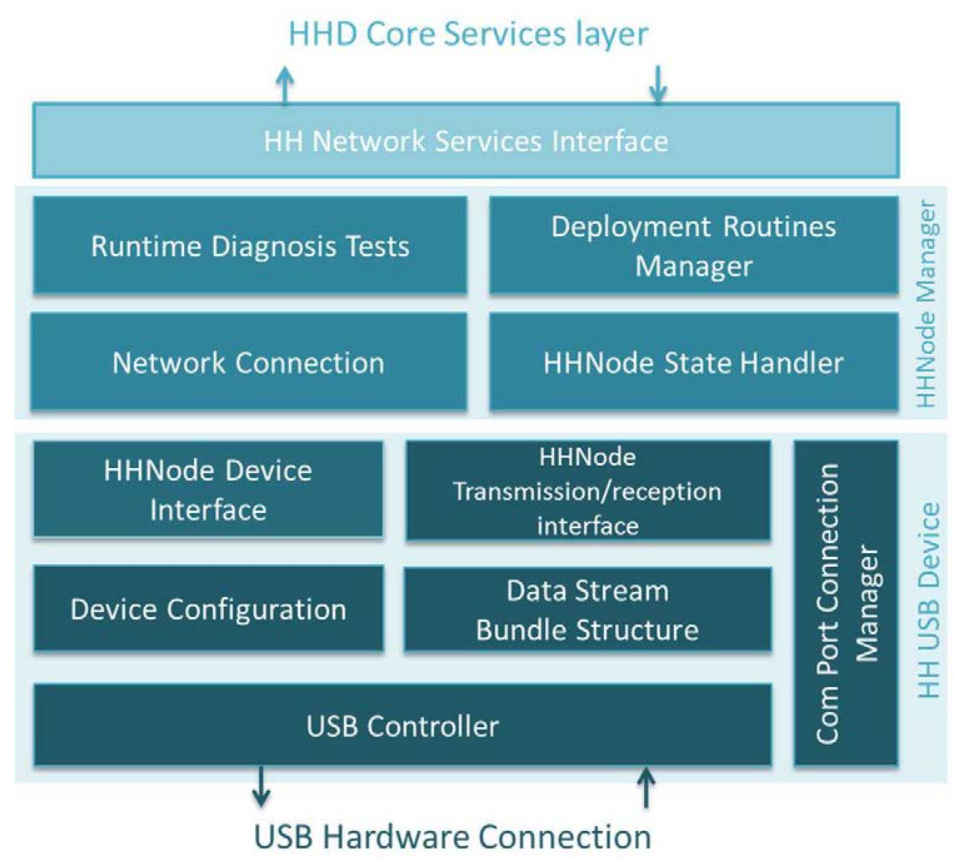

Fig. 3.39 Reference HHD-WSN interface structure.

Based on this, the hhNodeServices component provides upper layers with basic methods for triggering in-field deployment tests regarding the deployment methodology and routing evaluation tasks, allowing for the following functionalities, (summarized in Table 3-11):

- hhDisvTest: allows the execution of the HHD relative positioning in terms of surrounding neighbours detection. The execution call received as an output a dataObjectRet containing the $\mathrm{HH}$ neighbour information including the medium access communication metrics with the encountered nodes (RSSI, LQI and LDR).

- hhNodeTest: provides node configuration and neighbourhood runtime evaluation execution to establish local correlation among the target node and its enclosing communication area. The input of the execution call is the targetId of the node under configuration, the communication block type definition identifier and the radio module instance block according to the deployment model information. As a result, neighbour table entries are updated for subsequent 2-Ns correlation and connectivity evaluation, whose information is received in a dataObjectRet containing 
neighbouring list information and associated connectivity parameters for metric computation and quality analysis.

- hhNodeNeighTest: triggers controlled neighbour correlation and bidirectional connectivity evaluation between the targetIdX and the neighIdY, including the computation of the real link quality metrics between the 2-Ns in the callback, as well as node property states as configured in dataConfigDef.

- hhNodeRouteTest: allows the execution of routing performance evaluation tests by analysing path discovery, packet loss rate and routing metric generation between selected remote-2-Ns within the mesh multi-hop network. The execution call retrieves as input the configuration $\operatorname{targetId}(X, Y)$ related to the nodes under evaluation and the packet delivery configuration in case of executing a runtime transmission test (including number of packets, transaction frequency, and metric configuration for testing various types of route discovery and maintenance mechanisms).

- hhNodeRouteEval: along with the aforementioned test, this option allows the evaluation of the routing performance and maintenance of the endto-end communication path by retrieving parameters related to the information management of the routing protocol, considering a) number of table entries of targetId, b) items regarding the registered route (destination point, next hop and cluster information if applicable, metric parameter and number of hops), and c) routing role performance including precursor nodes, number of rediscoveries and packet delivery assessment.

Moreover, methods for configuration and interface state service monitoring are also provided in this component, so that a high level management of the HHNode connection and site coverage assessment can be performed. Moreover, as shown in the implementation chapter, a packet analyser capability is also built as an additional deployment toolset component to allow deployers to gather low-level communication frames transparently from the in-field data 
exchange of the WSN nodes, so that further behavioural patterns can be extracted and studied.

\begin{tabular}{|c|c|c|}
\hline Functionality & Parameters & Outcome \\
\hline hhDisvTest & $\begin{array}{l}\text { - Communication block } \\
\text { definition } \\
\text { - Radio module block instance }\end{array}$ & $\begin{array}{l}\text { - HH neighbour discovery } \\
\text { - Identification information } \\
\text { - } \text { Quality metrics (RSSI, LQI, } \\
\text { LDR) }\end{array}$ \\
\hline hhNodeTest & $\begin{array}{l}\text { - } \quad \text { targetld to be under } \\
\text { evaluation/test }\end{array}$ & $\begin{array}{l}\text { - Target neighbour discovery } \\
\text { - Identification and entry } \\
\text { generation/update }\end{array}$ \\
\hline hhNodeNeighTest & $\begin{array}{l}\text { target } I d X \text {, neighld } Y \text {, according } \\
\text { to the neighbour discovery } \\
\text { entries. } \\
\text { - Properties configuration and } \\
\text { functional block identifiers. }\end{array}$ & $\begin{array}{l}\text { - 2-Ns correlation, node } \\
\text { configuration. } \\
\text { - Bidirectional evaluation } \\
\text { - Quality metrics (RSSI, LQI, } \\
\text { LDR) }\end{array}$ \\
\hline hhNodeRouteTest & $\begin{array}{l}\text { - targetld }(X, Y) \text {, remote 2-Ns } \\
\text { - Packet delivery configuration: } \\
-\quad \text { Number of packets } \\
-\quad \text { Transmission } \\
\text { frequency/burst } \\
\text { mode } \\
-\quad \text { Routing Metric }\end{array}$ & $\begin{array}{l}\text { - Path discovery/rediscoveries } \\
\text { - Packet Loss rate } \\
\text { - } \text { Metric value }\end{array}$ \\
\hline hhNodeRouteEval & $\begin{array}{ll}\text { - } & \text { targetldX } \\
\text { - } & \text { Network block definition } \\
\text { - } & \text { Routing block instance }\end{array}$ & $\begin{array}{l}\text { - Table entries } \\
\text { - Routing items: } \\
\text { - } \quad \text { Destination point } \\
\text { - } \quad \text { Next hop/cluster node } \\
\text { - } \quad \text { Maximum number of } \\
\\
\text { - } \text { hops } \\
\text { - } \text { Rotric parameter } \\
\text { - Precursor points } \\
\text { - } \quad \text { Rediscovery metric } \\
\text { - } \text { Packet delivery } \\
\\
\quad \text { assessment }\end{array}$ \\
\hline
\end{tabular}

Table 3-11 summary of reference functionalities for runtime in-field connectivity evaluation tests. 


\subsubsection{Middleware technology integration}

Seeking the interoperability of the HHD through different HW-SW platforms and heterogeneous technologies, and considering also its integration into a complete development tool chain [DPCM] (as commented in the experimental results), another important feature that has been addressed within the system implementation of the proposed deployment, commissioning and maintenance tool is its integration with a Middleware-based technology development. The primary objective of the middleware structure proposed in [DPCM-MW] is the provision of a common abstraction for various existing WSN-based technologies and vendor dependent resources, which fosters an efficient encapsulation for application logic connection between the server-side, gateway functionalities, and in-node hardware and embedded software implementation. As such, the middleware baseline technology provides the following attributes to the WSN development flow:

- Abstraction framework for WSN node platforms, both hardware and hardware-OS-based, and system level functions.

- Application development decoupling from low-level component implementation.

- Cross-connectivity between heterogeneous systems at application level.

- High level composition and portability over heterogeneous embedded programming platforms, including the reusability of standards and existing specifications.

On one hand, the basic element to be distinguished within the definition of this middleware architecture is the modelling and interconnection of component units, which are specified by means of using of SysML block description [SysML], and library of components that are interfaced with the development tool by using configurable parameters. On the other hand, in order to create interoperable common abstraction entities, this middleware technology encompasses the adoption of standards related to sensing, monitoring and controlling tasks that are usually considered in WSN-based systems. In this direction, a combination of IEEE 1451-based family of standards and OGC SWE 
related specifications [SWE] has been combined in [DPCM-MW] to represent the abstraction model for common platform entities that have to be handled by the middleware, highlighting the following attributes:

- Transducer, storage and communication functionalities, which allow network and application level abstractions.

- Processing functional blocks, which represent the application logic abstraction and algorithmic component of the system.

- Parameter representation, which outlines the data model definition for the abstraction entities.

- Actions, Events and Time functionalities, which define the execution and interconnection models among functional components.

The middleware incorporates the high level implementation of component abstraction libraries in order to directly access and configure the integrated functional blocks within the embedded software platform by using a defined Java-based interface approach. Two main packages can be distinguished within the implementation of the middleware abstraction layer: Java-based libraries for the main core components and functional blocks integration (in stdlib), and middleware base libraries for high abstraction implementation of MW services (in runtime). On top of it, the middleware provides access to functionalities by using set/get calls, in which block instances and port selection can be specified for data configuration, retrieving and observation triggering, considering the following inputs (see Fig. 3.40):

- DispatchAddress: specifies the targetId of the node from which the information will be gathered/configured. MW allows SR-based addressing scheme as the input object to specify the nodes to be involved in the execution process, i.e., considering a $\{\langle$ origin $\rangle,\langle$ inter $\rangle,\langle$ dest $\rangle\}$ object (where the destination point can also be referred to a broadcastmode addressing for acting on the neighbour nodes of the intermediate points).

- Port selection: corresponds to the specification of the functional component and parameter to act on, i.e., it allows the identification of the 
ports upon which the execution action is performed, which is associated with the functional components included in the embedded implementation of the sensor node, and shall be in line with the network model definitions.

- pValue: refers to the object that defines the decoding/encoding body of the data structure for the parameter to be written/retrieved. Depending on the nature of the property/configuration definition related to the specified block, the value type will be of different representation instantiations.

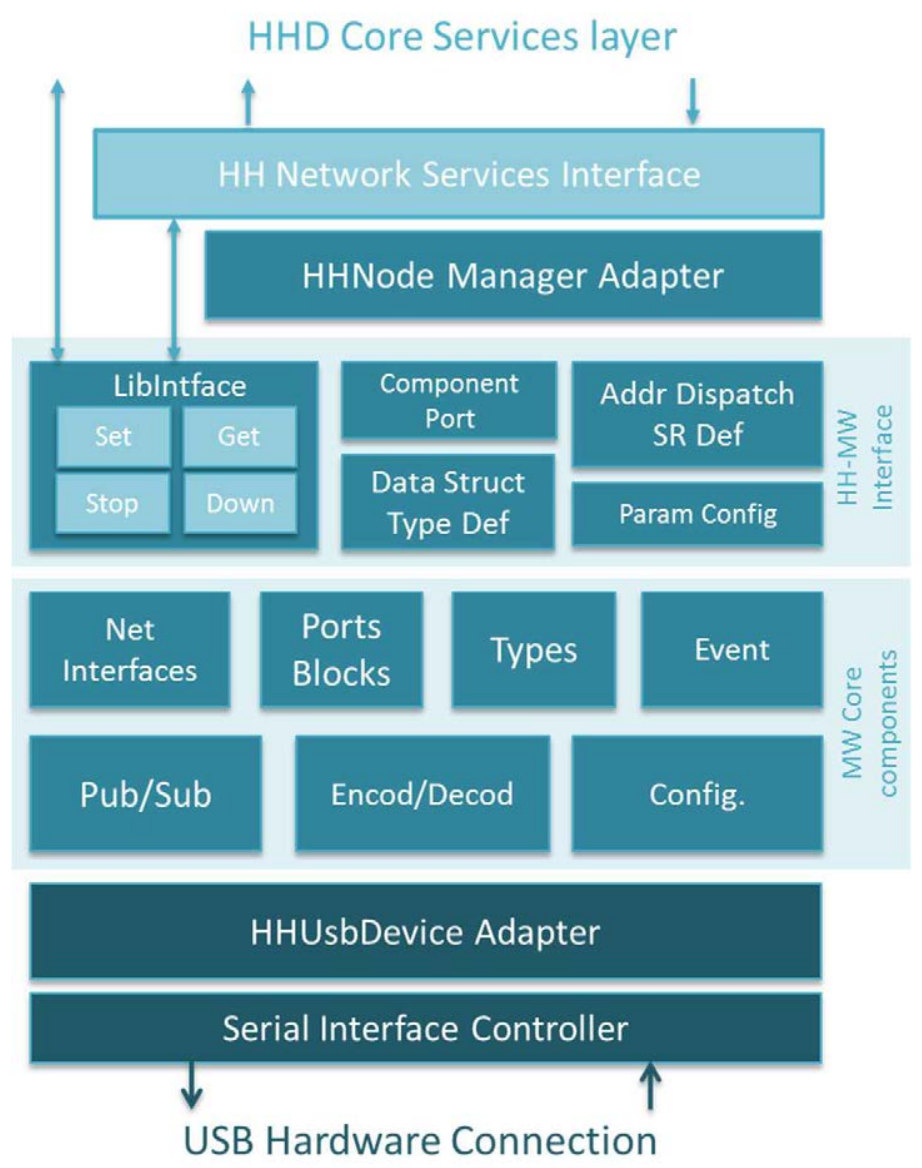

Fig. 3.40 HHD-MW integration structure and high-level services interface.

The integration of such a technology within the HHD has been realized by designing and implementing a wrapper-like component interconnection in 
which the abstraction layer definition of the HHD is interfaced with the middleware libraries as depicted in Fig. 3.40. This means that a two-side correlation with the middleware components is encompassed. On one hand, hhUsbDeviceAdapter manages the low-level HW control of the USB-host-port connection and provides the source of packets that streams the real-time data from the WSN to the MW core components and vice versa. On the other hand, the hhMwServicesInface creates the high-level abstraction from the described MW entry-point functions to the HHD service interface, so that the structure and execution of the designed evaluation routines remain transparent, thus promoting reusability and interoperability of the core functionalities.

As shown in Fig. 3.40, the middleware services interface adapter makes use of the hhMwLibIntface that includes the init, get, set, stop, sdown methods to access the middleware components and then produces a seamless integration of hhNodeServices including the associated evaluation tests execution. This includes the specification of the block type and block instance definition, as well as the block parameter to identify the embedded component property upon which the reading/configuration action is performed. This indeed allows a proper interaction of the HHD with the WSN and the real implemented functional blocks within the sensor platforms, based on the network deployment models and node object instances.

\subsubsection{TinyOS-based platform interactions}

A third level of platform interoperability has been taken into account targeting the interaction with TinyOS-based sensor node implementations, considering the integration of some of the tools provided by such operating system for data aggregation and platform monitoring [Levis'05][Levis'09]. TinyOS provides utilities and Java-based code for retrieving data from the sensor nodes into a base station, which allows decoding TinyOS data frames. It uses a bridge node as the sink point of the network that gathers packets from the radio communication module and forwards them to the serial port, and vice versa. In this work these Java-based libraries are integrated into the Android-OS implementation by adapting the PacketSource abstraction of TinyOS. Commonly, the basic communication medium between the base station-node and the 
gateway/server-machine is a serial port configuration, and in operating systems such as Ubuntu or Windows the serial packet source is specified as serial@/tev/ttySx and serial@COMx, which then make use of native low-level controllers of TinyOS for managing the serial communication capabilities.

The serial packet source has been adapted in the HHD to integrate the hhUsbDevice management capabilities with the TinyOS PacketSource and underlying interfaces, so that tools such as net.tinyos.tools.listen can be transparently used. For this, Packetizer and ByteSource implementations are used with a NativeSerial adaptation so as to provide upper layers with a seamless integration of TinyOS-focus data frame analysis and processing. Moreover, by modifying the bridge node to act as an in-field transparent traffic forwarder integrated in the HHNode and triggering the Listen utility in the smart device, HHD can make use of the received packets to decode data exchange transactions among sensor nodes for traffic pattern analysis in-situ (working as a mobile packet analyser) without interfering in the normal packet dissemination operation of the network.

\subsubsection{Output models and system/deployment feedback}

Based on the on-site commissioning, configuration and performance evaluation capabilities that the HHD provides deployers with, and according to the described functional flow of the in-field optimization actions by using the deployment toolset engines, the HHD automatically generates output models which contain relevant information of the network performance as well as the result of the comparative analysis of the simulations with the real behaviour of the wireless nodes and the overall deployed system assessment. This report generation process allows deployers to create a comprehensive automatic feedback to the planning and development cycle based on the composition of on-site real processed information, so that further refinement of the simulation models and configuration properties can be carried out, in accordance with the outcomes of the distributed sensor network.

Such a system feedback is realized by means of a web-service based interface approach in the same way as in case of the input models retrieving, but using a 
POST method to upload the deployment report to the remote server. These output reports are produced based on the processing of the data related to every stage of the on-site deployment activities and configuration/performanceevaluation routines, from which different summarized result schemes are generated as well-defined groups of models. In this way, such models have been designed following the same modular structure as the input model definition approach, considering their implementation in the form of JSON objects. The subsequent analysis of the outcomes of this reporting process will allow an offline study and enhancement of the network model generation for better adaptation of the pre-deployment stages to the real behaviour and constraints of the actual on-site WSN application.

The general structure of the common output model definition is shown in Fig. 3.41, where four main objects contain the following information for the determination of the type of report to be generated:

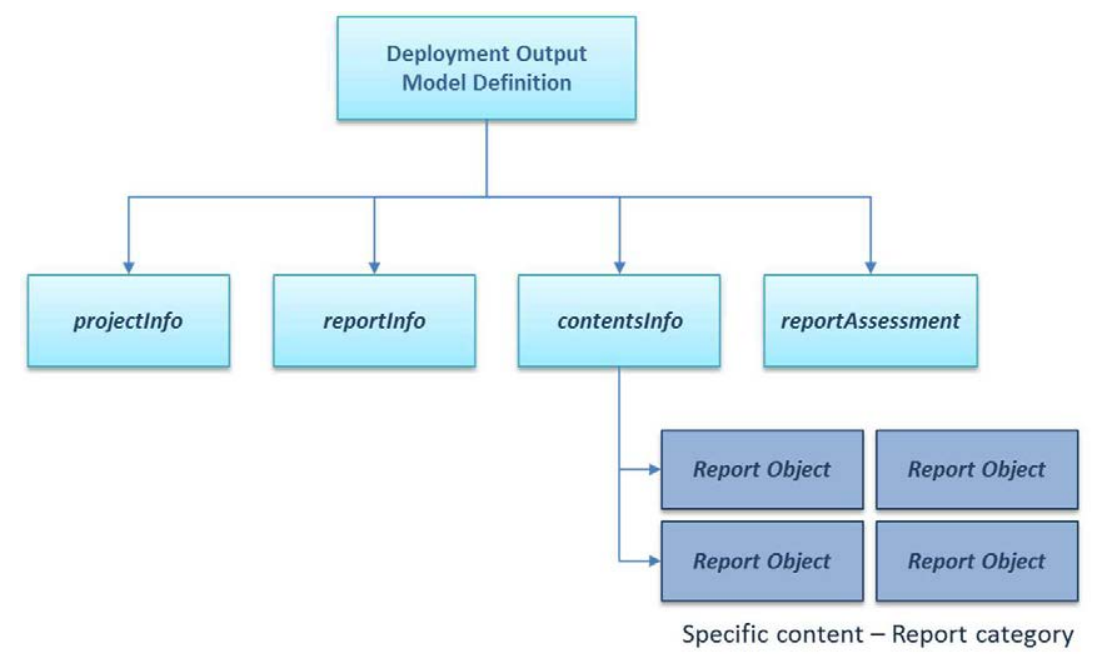

Fig. 3.41 Common structure of the deployment output models.

- projectInfo: contains the basic information of the project and plan identification the report is associated with. This data is correlated with the input network model and common to the output models to be generated within such a project and plan.

- reportInfo: includes general information related to the identification and reference description of the type of output report to be produced. Table 
3-12 summarizes the main fields that are contained in this object, which uniquely identify each output report generation.

- contentsInfo: provides the body of the output report information with the specific results of the associated deployment data and performance evaluation process outcomes, with respect to the report category.

- reportAssessment: allows incorporating specific details regarding the results that are reported in the output contents, so that further descriptions of the related data can be provided for better analysis.

\begin{tabular}{|l|c|}
\hline \multicolumn{1}{|c|}{$\begin{array}{c}\text { Information } \\
\text { Item }\end{array}$} & Description \\
\hline reportld & Identifier of the report. Unique reference \\
\hline reportType & Type of report to be generated/uploaded \\
\hline creationDate & Date-Hour of creation \\
\hline reportName & User report name based on the type of model \\
\hline description & General description of the report \\
\hline groupld & Identifier of the group the report refers to \\
\hline
\end{tabular}

Table 3-12 Information reference to identify the report to be generated.

Based on this common model structure, eight different types of output model categories have been implemented in order to cover various configurations, optimizations and performance assessment phases of the deployment and maintenance process. In Fig. 3.42, a defined output model classification is summarized according to the on-site functional flow, whose domainrelated/specific information are described as follows (considering the report identification represented in Table 3-13). 


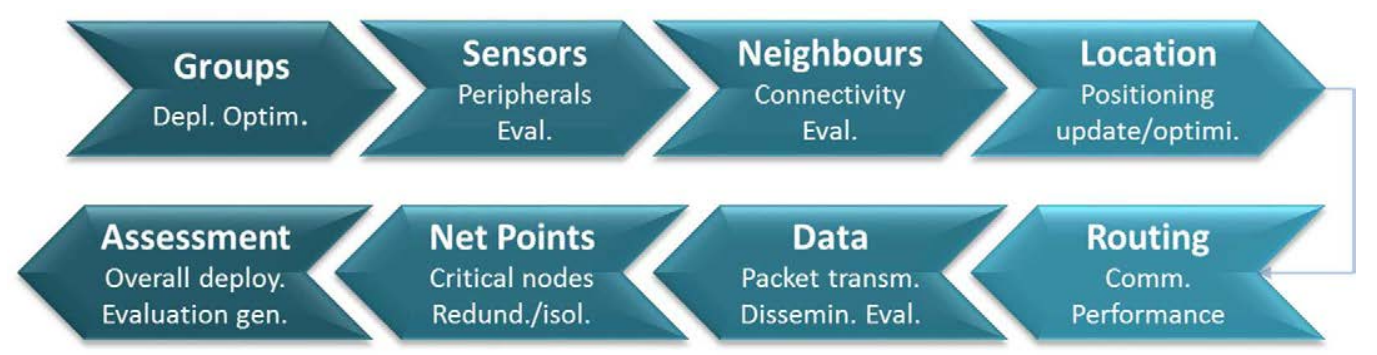

Fig. 3.42 In-field report generation in accordance with the deployment functional flow definition.

GROUPS_DEF [DR01n]: contains a JSONArray whose elements are defined as JSONObjects with information of network groups, in accordance with the deployment methodology generation. This will serve as a reference for subsequent output reports identification. It includes the reference ID of the generated group, the number of nodes that belong to such a group and their associated nodeId.

SENSORS_EVAL [DR02n]: provides in-field information regarding configuration and sensor/actuator performance evaluation with respect to the measured values, so that the interaction of the nodes with the target environment can be analysed and validated. The JSONArray is created with the number of nodes that have been analysed, which are represented as JSONObjects with the following information:

- moteId (value): reference identification of the node in accordance with the deployment model definition.

- Sensor-Actuators (Array): array of objects with sensors/actuators of moteId that have been evaluated and processed.

- Sensor-actuator info (object): in case of both sensors and actuator objects, the reference identification of the associated block instances is provided. For sensor data, the number of sampled values is included together with baseline metrics for further analysis of the measurement performance, such as maximum and minimum sensed bounds, as well as average and medium values of the gathered data. In case of the actuator evaluation, states registry and actions triggering are provided to verify the proper operation of the integrated component. 
NEIGH_EVAL [DR03n]: this output model encompasses the generation of the real node connectivity correlation scheme based on the data obtained from the deployment methodology tasks. It provides information related to the quality of the interconnections among the sensor nodes with respect to relevant information regarding platform positioning, transmission power configuration and surrounding neighbouring correlation. Hence, the JSONArray contains $N$ objects with the following parameters:

- moteId (value): reference identification of the node in accordance with the deployment model definition.

- Coordinates (array): geo-positioning coordinates of the sensor node in the target scenario.

- txPower (value): transmission power configuration of the sensor node associated with the registered data of the correlation test.

- neighboursInfo (object): contains similarly the aforementioned information for the encountered node in addition to bidirectional evaluation of the link quality metrics, primarily considering RSSI, LQI and LDR parameters though further metric implementations can be included within the array of metric objects, with a description of the type and nature of them.

LOCATION_UPTE [DR04n]: provides information of in-field node positioning updates for connectivity and node correlation enhancement, as well as possible restrictions in the installation of the sensor nodes with respect to the theoretical location, or constraints in the application scenario that had not been taken into account during the planning phase. In this way, the JSONArray provides the number of modified locations in the form of objects that contain the information of both the initial and updated geo-positioning coordinates of the sensor nodes under evaluation, in addition to a description of the motivations for such changes in line with the mentioned situations.

ROUTING_REG [DR05n]: offers the analysis results of the routing management information generation and routing discovery entries within the embedded implementation of the multi-hop communication protocol, such as for CB- 
AODV. The JSONArray contains the number of evaluated node objects which include the following items:

- moteId (value): reference identification of the node in accordance with the deployment model definition.

- entryInfo (array): provides the array of objects with the information of the discovered routes, considering the destination id of the node entry, next point of the path and end-to-end hop-count, as well as the composition of evaluation objects that contain the type description, units and values of the available implemented quality metrics, such as PDR and E-aDM.

DATA_DISTR [DR06n]: contains the outcomes of the packet dissemination tests in order to evaluate the routing discovery and maintenance mechanisms implemented within the routing protocols engine. This model follows a similar scheme as ROUTING_REG in addition to including the ratio of transmitted and received packets from the source node moteld to the remote destination point included in destinationInfo object. Moreover, as in previous cases, evaluation parameters are included in the form of metric object definition including type description, units and values associated with the test, such as PLR and RDR.

NTPOINTS_EVAL [DR07n]: includes the detection of critical-points and bottlenecks of the network or specific deployment areas, in addition to highlighting possible isolation points at routing level with respect to the path discovery generation, which are computed by means of using processing route entries, precursor node lists and the neighbour correlation scheme. The JSONArray is built with node objects that contains the moteId of the node to be remarked and pointEvalInfo objects for describing the type of encountered points. GLB_ASSESSMENT [DR08n]: this output model serves as a general report generation to highlight overall evaluation results and in-field optimization tasks carried out during the deployment/maintenance activities, so that a better understanding of the real behaviour of the system can be obtained. The JSONArray includes objects with the reference assessment type and the description of network optimization, errors or scenario constraints that may condition the performance and final operation of the deployed system. 
It is also important to highlight that although the described output reports can be created and updated during the on-site deployment, commissioning and maintenance activities by using the HHD, the internal data management system of the deployment toolset registers and saves the whole databases as well as related test data and models in order to export more comprehensive results, targeting subsequent offline analysis and processing of the real in-field behaviour of the WSN.

\begin{tabular}{|c|c|c|}
\hline Type of Report & Identifier & Objects Parameters \\
\hline GROUPS_DEF & DR010xx & $\begin{array}{l}\text { - } \quad \text { Groups ID generation } \\
\text { - } \\
\text { Associated nodes of the group } \\
\quad \text { Node ID array }\end{array}$ \\
\hline SENSORS_EVAL & DR020xx & $\begin{array}{ll}\text { - } & \text { Node references } \\
\text { - } & \text { Sensor-Actuator objects (block reference) } \\
\text { - } & \text { Evaluation metrics }\end{array}$ \\
\hline NEIGH_EVAL & DR030xx & $\begin{array}{ll}\text { - } & \text { Node references } \\
\text { - } & \text { Coordinates } \\
\text { - } & \text { TX Power } \\
\text { - } & \text { Neighbour objects } \\
\text { - } & \text { bidirectional evaluation metrics }\end{array}$ \\
\hline LOCATION_UPTE & DR040xx & $\begin{array}{ll}\text { - } & \text { Node references } \\
\text { - } & \text { Reference coordinates array } \\
\text { - } & \text { Updated location objects } \\
\text { - } & \text { Modification description }\end{array}$ \\
\hline ROUTING_REG & DR050xx & $\begin{array}{l}\text { - } \quad \text { Node references } \\
\text { - } \quad \text { Routing entry objects } \\
\text { - }\end{array}$ \\
\hline DATA_DISTR & DR060xx & $\begin{array}{ll}\text { - } & \text { Node references } \\
\text { - } & \text { Destination points objects } \\
\text { - } & \text { Packet delivery information } \\
\text { - } & \text { Metrics evaluation }\end{array}$ \\
\hline NTPOINTS_EVAL & DR070xx & $\begin{array}{ll}\text { - } & \text { Node references } \\
\text { - } & \text { Type of network point detection } \\
\text { - } & \text { Metrics evaluation, neighbour correlation }\end{array}$ \\
\hline GLB_ASSESSMENT & DR080xx & $\begin{array}{ll}\text { - } & \text { Assessment object } \\
\text { - } & \text { Evaluation type reference } \\
\text { - } & \text { Description, overall assessment }\end{array}$ \\
\hline
\end{tabular}

Table 3-13 Summary of report identifiers and associated data objects. 


\subsection{Conclusions}

In this chapter a new deployment and maintenance toolset for in-field wireless sensor networks has been proposed in order to cope with the lack of welldefined frameworks and support platforms for performing the activities associated with the deployment, configuration and optimization of the WSNbased system. The design, implementation and integration of the deployment strategies proposed in this work are materialized in the form of an intelligent mobile system, a Hand-Held Device (HHD), which provides users with an onsite simulation, evaluation and optimization methodology to efficiently achieve the cohesion of the target multi-hop mesh network. This portable device assists deployers during the in-field operations by interactively triggering interconnected functional modules of the integrated deployment guidance, so as to properly acquire a better understanding of the behaviour or the system with respect to what is expected from the estimation results.

The practical focus of the proposed platform aims at balancing the development and planning stages of WSNs with a real perspective of the implemented network in field, combining simulations and optimization techniques with the actual data obtained from the deployed sensor nodes, so that a more comprehensive feedback to the rest of the development cycle can be achieved. To this extent, the deployment platform has been specially conceived to have a proper alignment with both the outcomes of the planning/development process and the HW-SW implementation of the WSN-based system. For that, a two-side integration has been realized, i.e., by means of the input/output network deployment models definition, and the middleware abstraction layer for seamless runtime access and configuration of the embedded software of nodes.

In this context, the mesh networking strategies proposed for the Cookie nodes have been implemented from the basis of the software support platform presented in chapter 2 , in combination with the design of a new modular communication layer in order to enhance the suitability of the modular platform to be adapted to various types of connectivity patterns, as well as communication protocols based on the de-facto standard for WSNs, IEEE 
802.15.4. The real implementation of CB-AODV contributes to such an adaptability to dynamic multi-hop communication scenarios. The parametrization capability of the routing technique along with its real performance evaluation by using the deployment tool indeed allows a more efficient generation of the end-to-end communication paths throughout the wireless network.

Furthermore, the analysis of the deployment methods towards an efficient configuration and cohesion of WSNs showed that, by automatically identifying an optimal $N D o S q$, the real network correlation scheme can be generated with a minimum operation cost, based on applying one of the optimization mechanisms proposed in this work. Depending on the type of network to be deployed as well as the seeking trade-off between computational effort, energy awareness and long-term maintainability of the WSN, users may either select a suitable method automatically provided by the tool, or tune the CA/GA-based mechanisms to obtain more specific results for the target scenario.

\subsection{Bibliography of the chapter}

[802.15.4] IEEE Standard Part 15.4: Wireless Medium Access Control (MAC) and Physical Layer (PHY) Specifications for Low-Rate Wireless Personal Area Networks (LRWPANs) 1 October 2003.

[Apache] Apache HTTP server, an open-source web server, Apache Software Foundation, https://httpd.apache.org/

[Aslam'12] M. Aslam, N. Javaid, A. Rahim, U. Nazir, A. Bibi and Z.A. Khan, "Survey of extended LEACH-based clustering routing protocols for wireless sensor networks", in proceedings of IEEE 9th International Conference on High Performance Computing and Communication and IEEE 14th International Conference on Embedded Software and Systems (HPCC-ICESS), pp. 1232-1238, June 2012.

[Belqasmi'11] F. Belqasmi, R. Glitho and C. Fu, "RESTful web services for service provisioning in next-generation networks: a survey," in IEEE Communications Magazine, vol. 49, no. 12, pp. 66-73, December 2011.

[Botta'13] M. Botta, M. Simek, "Adaptive Distance Estimation Based on RSSI in 802.15.4 Network", in Journal of Radioingenieering, vol.2, no.4, 2013.

[Cao'08] Q. Cao and J. A. Stankovic, "An In-Field-Maintenance Framework for Wireless Sensor Networks", in Proceedings of the 4th IEEE international conference on 
Distributed Computing in Sensor Systems (DCOSS '08), Springer-Verlag, Berlin, Heidelberg, 457-468, 2008.

[CC2420] Single-Chip 2.4 GHz IEEE 802.15.4 Compliant and ZigBee Ready RF Transceiver, http://www.ti.com/product/CC2420

[Chatterjee'2014] P. Chatterjee, N. Das, "Coverage constrained non-uniform node deployment in wireless sensor networks for load balancing," in Applications and Innovations in Mobile Computing (AIMoC'14), pp.126-132, Kolkata, 2014.

[Chen'06] B. Chen, K. Muniswamy-Reddy, and M. Welsh, "Ad-hoc multicast routing on resource-limited sensor nodes", in Proceedings of the 2nd international workshop on Multi-hop ad hoc networks: from theory to reality (REALMAN'06), New York, NY, USA, pp. 87-94, 2006.

[Chen'09] M. Chen, V. Leung, S. Mao, Y. Xiao and I. Chlamtac, "Hybrid geographical routing for flexible energy-delay trade-offs", in IEEE Transactions on Vehicular Technology, vol. 58, no. 9, pp. 4976-4988, Nov. 2009.

[Couto'03] D. De Couto, D. Aguayo, J. Bicket, and R. Morris, “A high-throughput path metric for multi-hop wireless routing", in Proceedings of the 9th annual international conference on Mobile computing and networking (MobiCom'03), New York, NY, USA, pp. 134-146, 2003.

[Dongliang'11] X. Dongliang and W. Guoying, "SPAE: a dynamic wireless sensor network performance analysis and evaluation platform", in Proceedings of 4th IEEE International Conference on Broadband Network and Multimedia Technology (IC$\left.B N M T^{\prime} 11\right)$, October 2011, pp. 545-548.

[DPCM] Development, Planning, Commissioning and Maintenance Toolset for Wireless Sensor Networks (WSN-DPCM), ARTEMIS-JU European project, http://www.wsn-dpcm.eu/

[DPCM-MW] WSN-DPCM European Project, Platform heterogeneity \& Middleware, http://www.wsn-dpcm.eu/

[Gavalas'06] D. Gavalas, G. Pantziou, C. Konstantopoulos, B. Mamalis, "Lowest-ID with Adaptive ID Reassignment: A Novel Mobile Ad-Hoc Networks Clustering Algorithm", in Proceedings the International Symposium of Wireless Pervasive Computing, January 2006.

[Gnawali'13] O. Gnawali, R. Fonseca, K. Jamieson, M. Kazandjieva, D. Moss and P. Levis, "CTP: an efficient, robust, and reliable collection tree protocol for wireless sensor networks", in ACM Transaction on Sensor Networks (TOSN), vol. 10, no. 3, 2013.

[Gomez'06] C. Gomez, P. Salvatella, O. Alonso and J. Paradells, “Adapting AODV for IEEE 802.15.4 Mesh Sensor Networks: theoretical discussion and performance 
evaluation in a real environment", in proceedings of the International Symposium on a World of Wireless, Mobile and Multimedia Networks (WoWMoM'06), Niagara-Falls, Buffalo, NY, June 2006.

[Gomez'10] C. Gomez, A. Boix and J. Paradells, "Impact of LQI-based routing metrics on the performance of a one-to-one routing protocol for IEEE 802.15.4 multihop networks", in EURASIP Journal of Wireless Communication and Networking. 2010, pp 1-20.

[Goyal'12] D. Goyal and M. Tripathy, "Routing protocols in wireless sensor networks: a survey", in Proceedings of Second International Conference on Advanced Computing Communication Technologies (ACCT'12), pp. 474-480, January 2012.

[Haas'02] Z.J. Haas, M.R. Pearlman, P. Samar, The Zone Routing Protocol (ZRP) for Ad Hoc Networks, draft-ietf, July 2002.

[Heinzelman'00] W. R. Heinzelman, A. Chandrakasan, H. Balakrishnan, “Energy efficient communication protocol or wireless microsensor networks", in Annual Hawaii International Conference on System Sciences, Maui, 2000.

[Jacquet'03] P. Jacquet and T. Clausen, Optimized Link State Routing Protocol (OLSR) 2003, RFC 3626.

[JavaEE] Java Enterprise Edition and Web application development, Oracle Technology Network, http://www.oracle.com/technetwork/java/javaee

[Jersey] Jersey RESTful Web Services framework, open-source support for developing RESTful in Java, https://jersey.java.net/

[Johnson'09] D. Johnson, Y. Hu and D. Maltz, The Dynamic Source Routing Protocol (DSR) for Mobile Ad Hoc Networks for IPv4 2009, IETF RFC 4728.

[Kang'07] J. Kang, D. Kim and Y. Kim, "RSS Self-calibration Protocol for WSN Localization", in 2nd International Symposium on Wireless Pervasive Computing, San Juan, 2007.

[Kenan'10] Xu Kenan, H. Hassanein, G. Takahara, W. Quanhong, "Relay Node Deployment Strategies in Heterogeneous Wireless Sensor Networks," in IEEE Transactions on in Mobile Computing, vol.9, no.2, pp.145-159, Feb. 2010.

[Khemapech'07] I. Khemapech, A. Miller, I. Duncan, “A survey of transmission power control in wireless sensor networks", in Proceedings of the 8th Annual Postgraduate Symposium on the Convergence of Telecommunications, Networking and Broadcasting, pp. 15-20, June 2007.

[Levis'05] P. Levis, S. Madden, J. Polastre, R. Szewczyk et. al., “TinyOS: An operating system for sensor networks", In Ambient intelligence, pp. 115-148, Springer Berlin Heidelberg, 2005. 
[Levis'09] P. Levis, D. Gay, "TinyOS Programming”, Cambridge University Press: Cambridge, 2009.

[Lin'06] S. Lin, J. Zhang, G. Zhou, L. Gu, J. A. Stankovic, and T. He, “ATPC: adaptive transmission power control for wireless sensor networks", in Proceedings of the 4th international conference on Embedded networked sensor systems (SenSys'06). ACM, pp.223-236, New York, NY, USA, 2006.

[Liu'03] J. Liu and B. Li, "Distributed topology control in wireless sensor networks with asymmetric links," in IEEE Global Telecommunications Conference (GLOBECOM '03), 2003, pp. 1257-1262 vol.3.

[Maps] Google Maps Android API, available on: https://developers.google.com/maps/documentation/android-api/

[Meier'09] A. Meier, M. Woehrle, M. Weise, J. Beutel and L. Thiele, "NoSE: Efficient Maintenance and Initialization of Wireless Sensor Networks," in 6th Annual IEEE Communications Society Conference on Sensor, Mesh and Ad Hoc Communications and Networks, pp. 1-9, Rome, Italy, 2009.

[Misra'10] S. Misra, D. H. Seung, X. Guoliang, T. Jian, "Constrained Relay Node Place-ment in Wireless Sensor Networks: Formulation and Approximations," in IEEE/ACM Transactions on Networking, vol.18, no.2, pp.434-447, April 2010.

[Mohseni'10] S. Mohseni, R. Hassan, A. Patel and R. Razali, “Comparative review study of reactive and proactive routing protocols in MANETs", in proceedings of 4 th IEEE International Conference on Digital Ecosystems and Technologies (DEST'10), pp. 304309, Dubai, April 2010.

[Nurseitov'09] N. Nurseitov, M. Paulson, R. Reynolds, C. Izurieta, “Comparison of JSON and XML Data Interchange Formats: A Case Study", Caine, 2009, 157-162.

[Pang'06] K.L. Pang and Y. Qin, "The comparison study of flat routing and hierarchical routing in ad hoc wireless networks", in proceedings of 14th IEEE International Conference on Networks (ICON'06), 1, pp. 1-6, Singapore, September 2006.

[Pantazis'13] N.A. Pantazis, S.A. Nikolidakis and D.D. Vergados, "Energy-efficient routing protocols in wireless sensor networks: a survey", in IEEE Communications. Survey and Tutorials, vol. 15, no. 2, 2013, pp. 551-591.

[Perkins'03] C. Perkins, E. Belding-Royer and S. Das, Ad hoc On-Demand Distance Vector (AODV) Routing, 2003, RFC 3561.

[Rezaee'09] M. Rezaee, M. Yaghmaee, "Cluster based Routing Protocol for Mobile Ad Hoc Networks", INFOCOMP, Journal of Computer Science, Vol.8, no. 1, pp.30-36, Feb.2009. 
[Ringwald'07] M. Ringwald, K. Römer, and A. Vitaletti, "Passive inspection of sensor networks", in Proceedings of the 3rd IEEE international conference on Distributed computing in sensor systems (DCOSS'07), Springer-Verlag, Berlin, Heidelberg, 205222, 2007.

[Selavo'06] L. Selavo, G. Zhou and J. A. Stankovic, "SeeMote: In-Situ Visualization and Logging Device for Wireless Sensor Networks," in 3rd International Conference on Broadband Communications, Networks and Systems, San Jose, CA, 2006, pp. 1-9.

[Sharma'11] S. Sharma and S. K. Jena, "A survey on secure hierarchical routing protocols in wireless sensor networks", in Proceedings of the International Conference on Communication, Computing \& Security (ICCCS '11), ACM, New York, NY, USA, 146-151, 2011.

[SoAP] Simple Object Access Protocol, specifications and technical recommendations, https://www.w3.org/TR/soap/

[SysML] UML-based System Modelling Language, Object Management Group (OMG), http://www.omgsysml.org/

[Sobeih'06] A. Sobeih, J.C. Hou, L.-C. Kung, N. Li, H. Zhang, W.-P. Chen, H.-Y. Tyan and H. Lim, "J-Sim: a simulation and emulation environment for wireless sensor networks", in IEEE Wireless Communications, vol. 13, no. 4, pp. 104-119, Aug. 2006.

[Son'04] D. Son, B. Krishnamachari and J. Heidemann, "Experimental study of the effects of transmission power control and blacklisting in wireless sensor networks", in First Annual IEEE Communications Society Conference on Sensor and Ad Hoc Communications and Networks (SECON'04), pp. 289-298, 2004.

[SWE] Sensor Web Enablement (SWE) framework and standards, Open Geospatial Consortium (OGC).

[TinyAODV] TinyAODV, AODV implementation for TinyOS, http:/ / tinyos.stanford.edu/tinyos-wiki

[Tomcat] Apache Tomcat Server, open-source webserver implementation of JavaEE specifications, http://tomcat.apache.org/

[Varga'08] A. Varga and R. Hornig. "An overview of the OMNeT++ simulation environment", in Proceedings of the 1st international conference on Simulation tools and techniques for communications, networks and systems $\mathcal{E}$ workshops (Simutools '08). ICST, Brussels, Belgium, Article 60, 10 pages.

[Wang'13] Y. Wang, B. M. Kelly and X. Li, "On the network connectivity of wireless sensor net-works following a random and non-uniform distribution," in IEEE 9th 
Conference on Wireless and Mobile Computing, Networking and Communications (WiMob'13), pp.69-74, 7-9 Oct. 2013.

[Wei'13] S. Wei, M. Hashemi, I. Warsawski, M. Laifenfeld, D. Starobinski and A. Trachtenberg, "TeaCP: a toolkit for evaluation and analysis of collection protocols in wireless sensor networks", in Proceedings of IEEE International Conference on Microwaves, Communications, Antennas and Electronics Systems (COMCAS 2013), pp. 1-5, Tel Aviv, October 2013.

[Winter'12] T. Winter, P. Thubert, A. Brandt, J. Hui, R. Kelsey, P. Levis, K. Pister, R. Struik and J. Vasseur, RPL: IPv6 Routing Protocol for Low Power and Lossy Networks March 2012, IETF RFC 6550.

[Xu'01] Y. Xu, J. Heidemann and D. Estrin, "Geography-informed energy conservation for ad-hoc routing", in proceedings of ACM/IEEE International Conference on Mobile Computing and Networking (MobiCom'01), pp. 70-84, Rome, Italy, July 2001.

[Yu'08] J. Y. Yu, P. H. J. Chong and M. Zhang, "Performance of Efficient CBRP in Mobile Ad Hoc Networks (MANETS)", in IEEE 68th Vehicular Technology Conference (VTC'08), pp. 1-7, Calgary, BC, 2008.

[Yu'10] D. Yu, "DiF: A Diagnosis Framework for Wireless Sensor Networks," in INFOCOM IEEE Conference on Computer Communications Workshops, San Diego, CA, 2010, pp. 1-5.

[ZigBee] IEEE 802.5.4-based specification for low-rate low-power wireless personal area networks, ZigBee Alliance. 



\section{Chapter 4}

\section{DEPLOYMENT TOOLSET}

\section{IMPLEMENTATION AND INTERFACE}

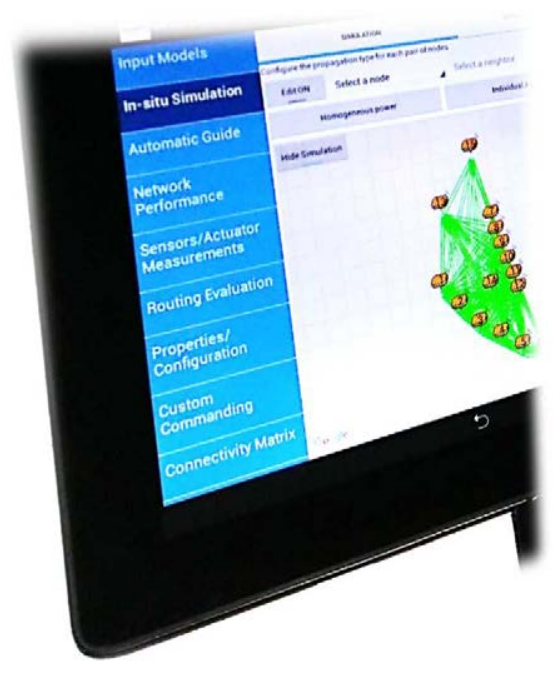

The implementation of the on-site deployment toolset has been approached in such a way that users can be guided during the in-field activities in an intuitive and interactive fashion, combining in a unique mobile device the simulation, performance evaluation and optimization strategies with the real behaviour of the wireless nodes.

In this chapter an overall view of the main functional modules and components that integrate the HHD interface are presented, which are in line with the capabilities proposed in the previous chapter. 



\section{Deployment toolset implementation and interface}

\subsection{Introduction}

The design and implementation of the deployment toolset has been conceived in accordance with the main nature and requirements of the proposed platform, i.e., an in-field easy-to-handle support element that allows deployers to carry out the configuration and optimization of the WSN in-situ. For that, a smart mobile device represents the core of the HHD, upon which the proposed system architecture and related capabilities are implemented and properly integrated as a whole deployment platform. The Android operating system has been used as the main baseline to develop the various software components that compose the deployment tool, considering the $\mathrm{v} 4.03$ (or higher) as a reference version to be included in a tablet device for an optimum experience with the top-level application (an Asus Transformer 10" was employed for the system implementation, which includes an NVIDIA Tegra3 Quad Core processor at 1 $\mathrm{GHz}$, and 1 GB of RAM memory). Thus, a Java-based modular implementation that allows an easy integration of functionalities and library of components was accomplished from the basis of the Android development framework.

Focusing on a proper unification of the deployment capabilities into the HHD as well as an adequate access and interaction with the top-level interfaces from the user perspective, the system implementation of the toolset has been realized considering the use of Android Fragments, which favours modular and dynamic user interfaces particularly intended to larger screens, such as in tablets. With this technique the layout of an Activity in Android (the basic entity of an application, which is created in accordance with a .xml for the graphical definition and java for the behavioural and logic implementation) is divided into different fragments and views, which gives the ability to dynamically modify user interface representations and concurrent distributions at runtime, in 
addition to encapsulating interface and behavioural components into the definition of modules, hence obtaining an adaptable composition of the elements that comprise the target application. This is especially important for the proposed toolset since the integration of heterogeneous techniques and optimization engines, in-field simulations and performance analysis, are to be properly and modularly combined within a unified platform (which shall also be presented in an easy-to-access manner to the WSN deployer). Moreover, Fragments allows the management of their own lifecycle as well as input events, so that it can be treated as a subset of the activity container.

To this extent, the Android developing framework provides various types of project templates to create the desired application model and UI structure, although the proposed toolset has been implemented from the basis of the Master Detail Flow pattern [AndroidDev]. Subsequent figures show the model of the toolset interface, which is mainly composed of a) a fixed fragment that gives access to the various modular components/sub-systems implemented within the HHD (left-side of the tool), b) dynamic fragments that will be changed and combined based on the selection of the available modules (centre and right-side of the tool), c) application activities, containing on one hand the fragments for the toolset main menu, and on the other hand the top-level entry point of the deployment capabilities through the WSN project access and network models selection (see Fig. 4.1 and Fig. 4.2). Such a modularity allows an easier extension of the toolset functionalities by adding new options at the leftside fixed fragment, in combination with its corresponding implementation and fragment interface presentation.

\subsection{Application access and project selection}

The toolset relies on the provision of the project definition and associated network models as the input to prepare the different modules of the system, in accordance with the WSN to be deployed. In this way, as shown in Fig. 4.1, after the user logs in/registers, the selection of the project and related WSN plan can be carried out so that the corresponding deployment definition is downloaded from the project repository, which then allows feeding the functional 
components of the platform with the specifics of the target network. Several plans can be associated with a particular project, so once the latter is selected from the left-side option, the list of the available plans is loaded into the rightside option list. The underlying WS interfaces support the access and retrieving of the network models based on the described JSON objects definition. Nevertheless, users are also able to select available projects that had previously been downloaded and stored into the HHD, so that off-line actions can be further realized from the basis of already defined network models. In any of these cases, after the project references are selected and the start button is pressed, the HHD loads the toolset main menu with the fragment-based structure, so that the different deployment functionalities and optimization engines can be explored/employed.

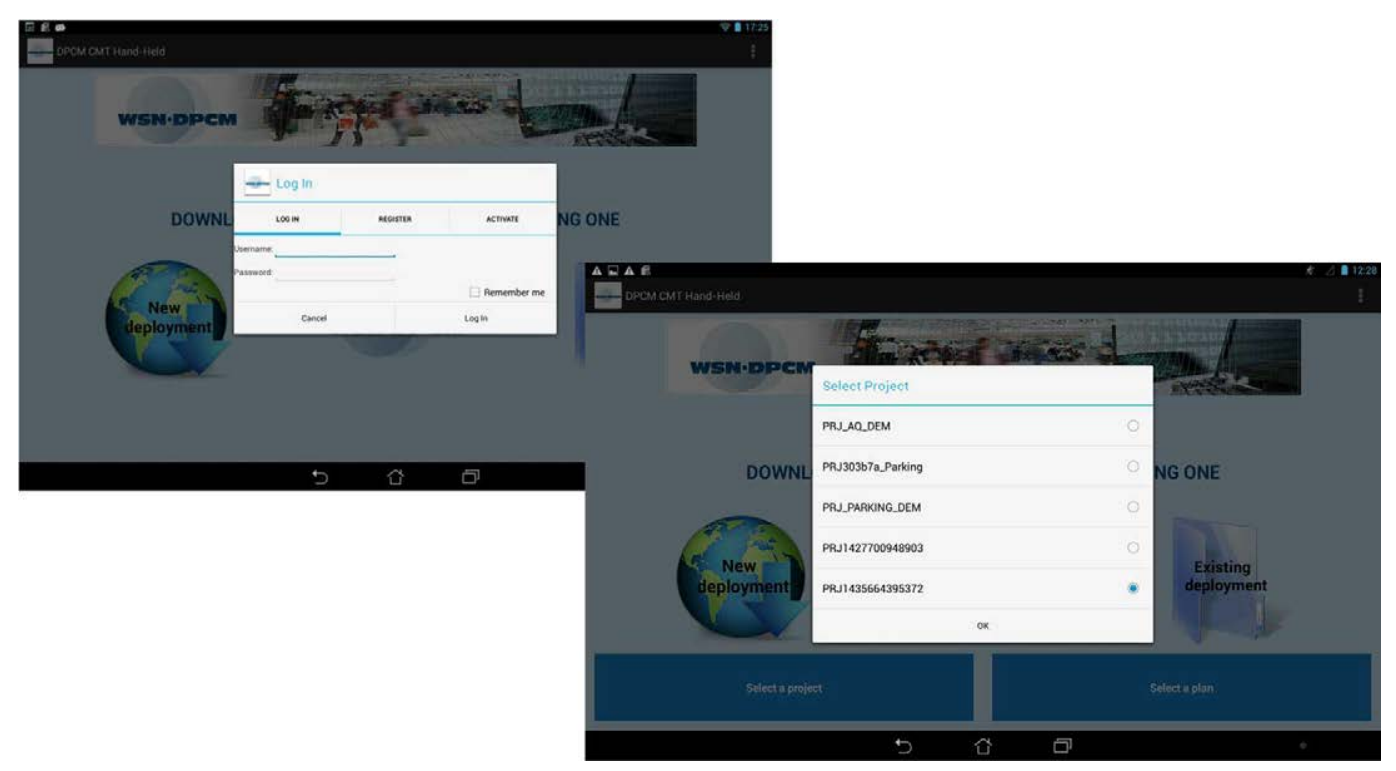

Fig. 4.1 Main log-in and project selection windows of the HHD.

\subsection{Deployment and runtime optimization modules}

Fig. 4.2 presents the general view of the main menu for the set of deployment capabilities, where the left-side panel corresponds to the different on-site optimization, evaluation, and configuration modules to be used during the WSN installation activities. Such a list of options refers not only to the subsystems that 
compose the overall toolset, but also to the distribution of the different stages of the deployment over which users will be guided.

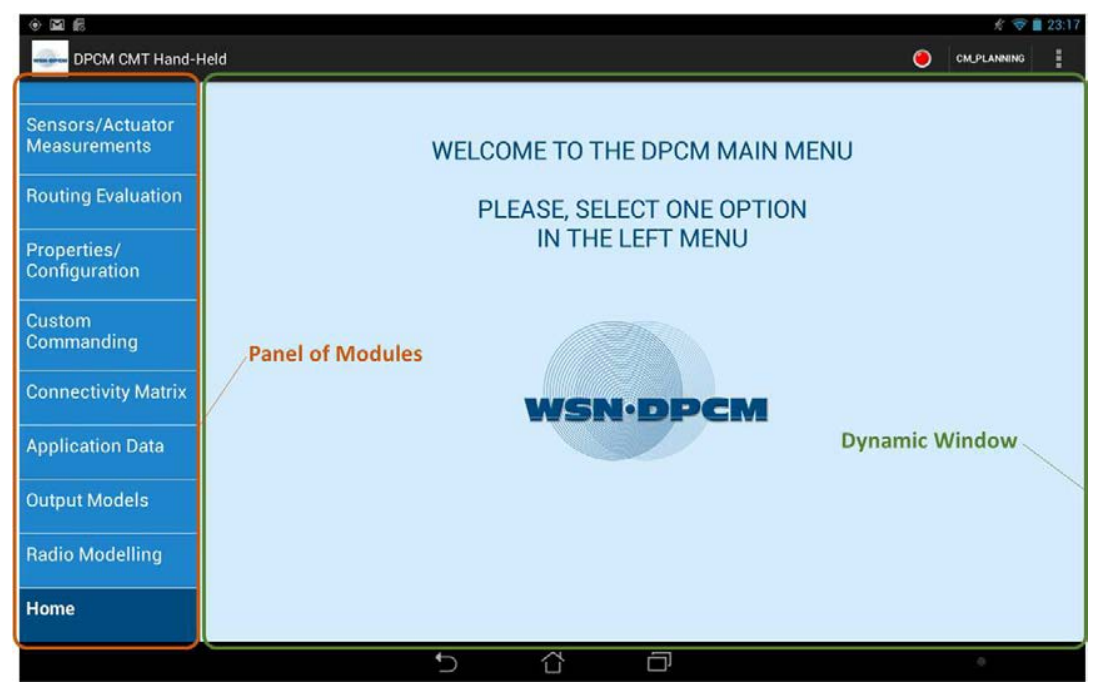

Fig. 4.2 Deployment toolset main menu once the network model and input definitions are loaded.

The first module corresponds to the input models representation, where both the deployment model definition as well as the external connectivity scheme (if available) can be explored as shown in Fig. 4.3, so that details regarding the number and types of sensor nodes as well as the functional components that integrate the HW-SW definition of the devices may be preliminary analysed by the deployer.

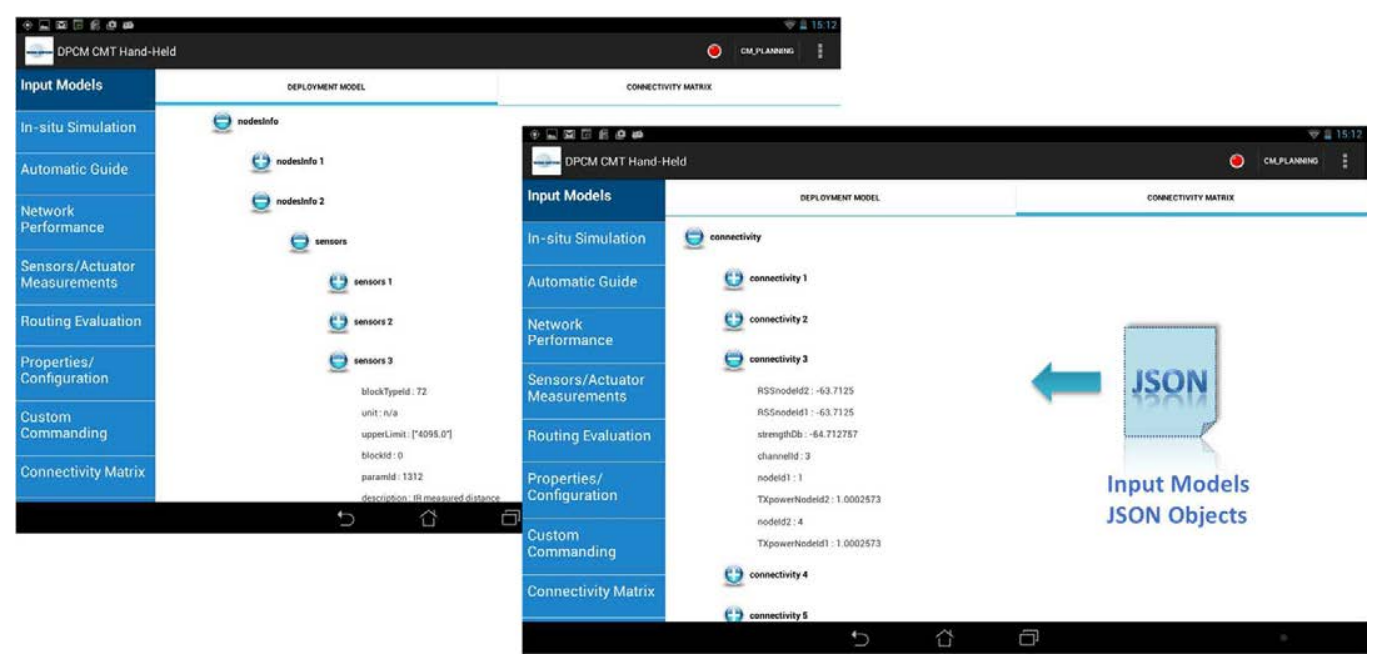

Fig. 4.3 Input models selection and visualization. 
Tag-based interfaces are also exploited to present more intuitive views and dispositions of the module's options/windows. Moreover, on the upper-right corner a set of functions allows a) the selection of different options that are dynamically adapted in line with the current fragment, b) the configuration of the type of connectivity scheme to be used, c) and the status of the HHNode connection.

The In-situ Simulation module contains three different optimization and evaluation engines that can be switched through the selection of the corresponding tag, as displayed in Fig. 4.4. Such subsystems are internally correlated in order to dynamically feed them with the results generated by each other, so an interactive optimization can be performed accordingly. The GoogleMaps API capabilities [Maps] are used to show the target application environment and related map functionalities. In the connectivity evaluation and performance analysis tag, if activating the simulation engine toggle button, a set of configuration options that allows specifying particular connectivity and propagation constraints are viewed, apart from the TX power optimization algorithms that can be triggered by using one of the available activation buttons (in green and yellow, see Fig. 4.5).

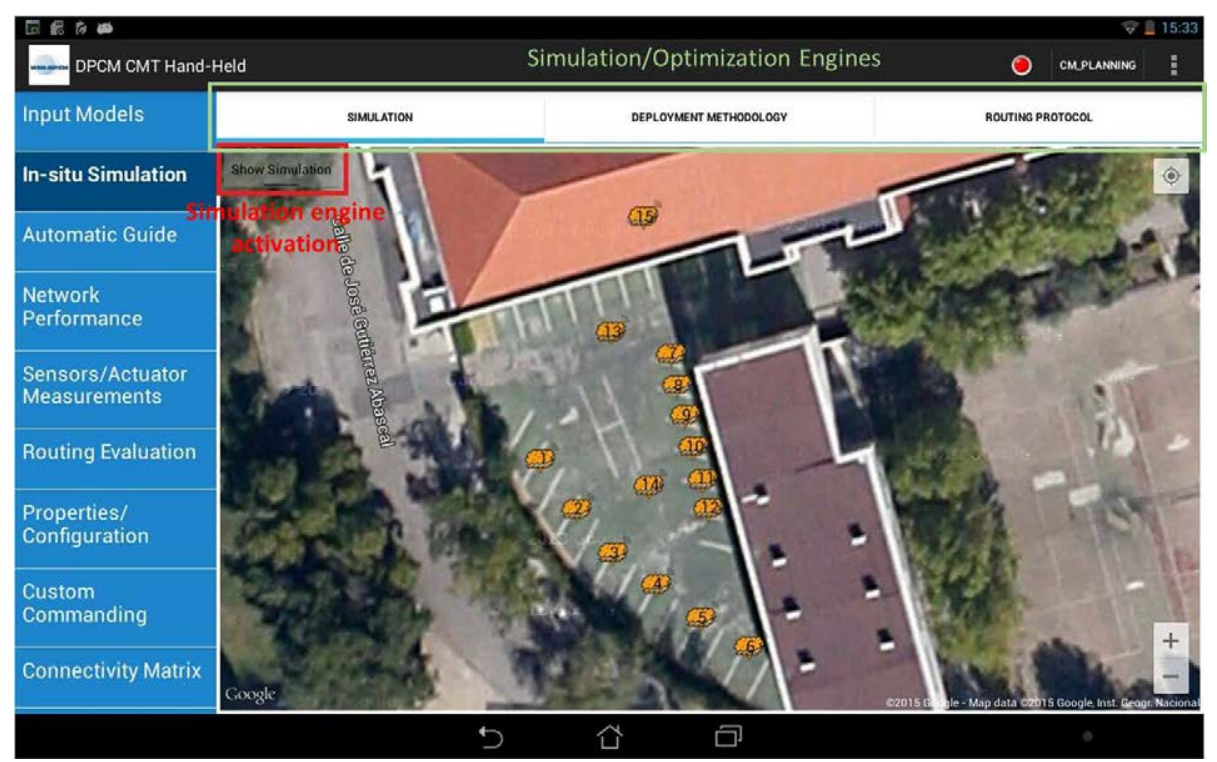

Fig. 4.4 In-situ simulation module, where optimization engines are split into interactive GUIs. 
While the map of the scenario shows a graphical view of the sensor nodes and how the connectivity among them is simulated, the panel at the centre-right position of the tool provides runtime information related to the optimization evolution as well as the results of the connectivity evaluation. Since the deployment tool implementation and interfaces are thought to include additional types of optimization strategies, if a method is integrated the option shall appear on the list of available connectivity correlation capabilities and updated in the top-right corner of the segment interface.

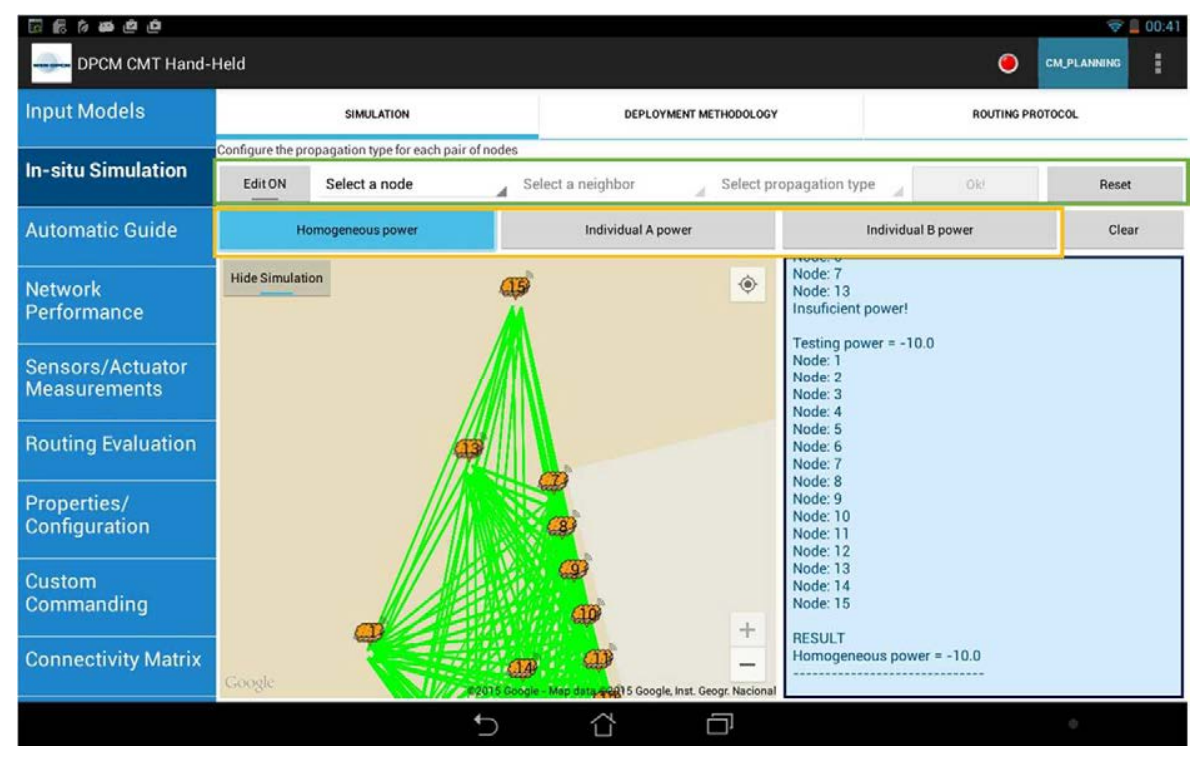

Fig. 4.5 Nodes correlation and transmission power optimization sub-system.

The Deployment Methodology tag provides the optimization engine based on the proposed strategies and algorithms for the in-field deployment, configuration, and real connectivity correlation tasks. As highlighted in Fig. 4.6, users shall select one of the methods to be applied (or an additional option to let the system automatically provide the best solution for the target scenario) as well as the type of optimization mechanism to be executed (in blue and red, respectively), considering that the SA and the CA will be enable for selection depending on the scale of the network (the former for small networks and the latter for larger sizes).

When selecting the GA-based optimization, the configuration parameters are automatically pre-set in accordance with the previously described tuning 
process. As shown at the status view in Fig. 4.7, once the algorithm is triggered the runtime evolution process can be monitored/analysed and stopped an an time by the user, providing the best result obtained so far. Regarding the CAbased optimization, the entry configuration corresponds to the maximum number of nodes per group.

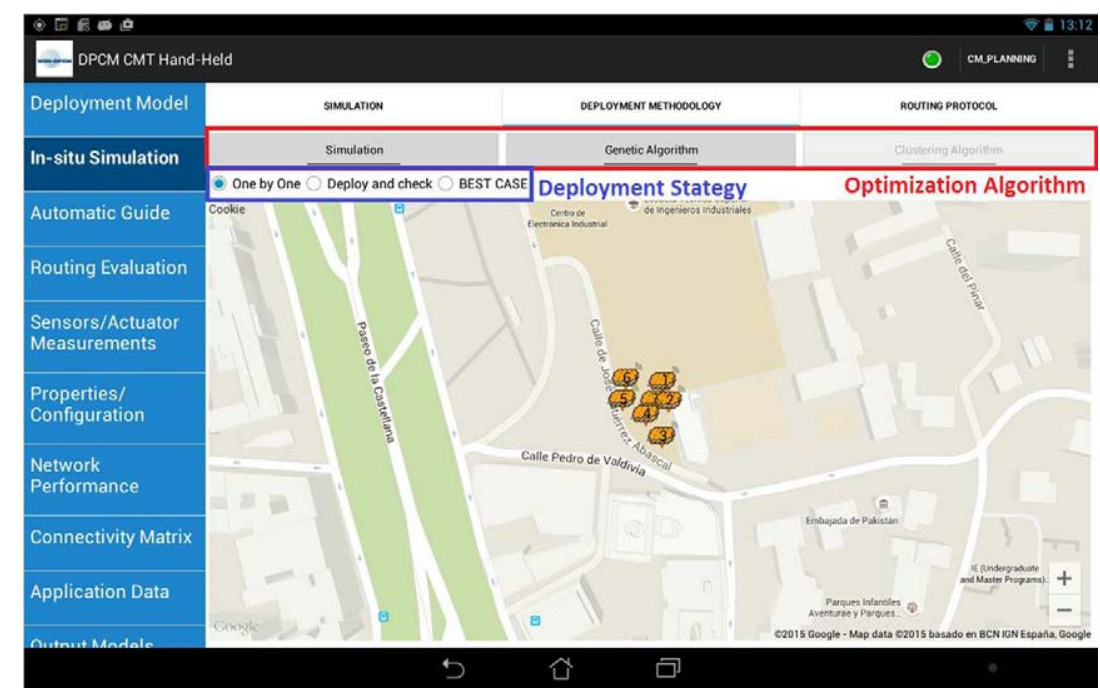

Fig. 4.6 Deployment optimization methods and algorithms - selection panel.

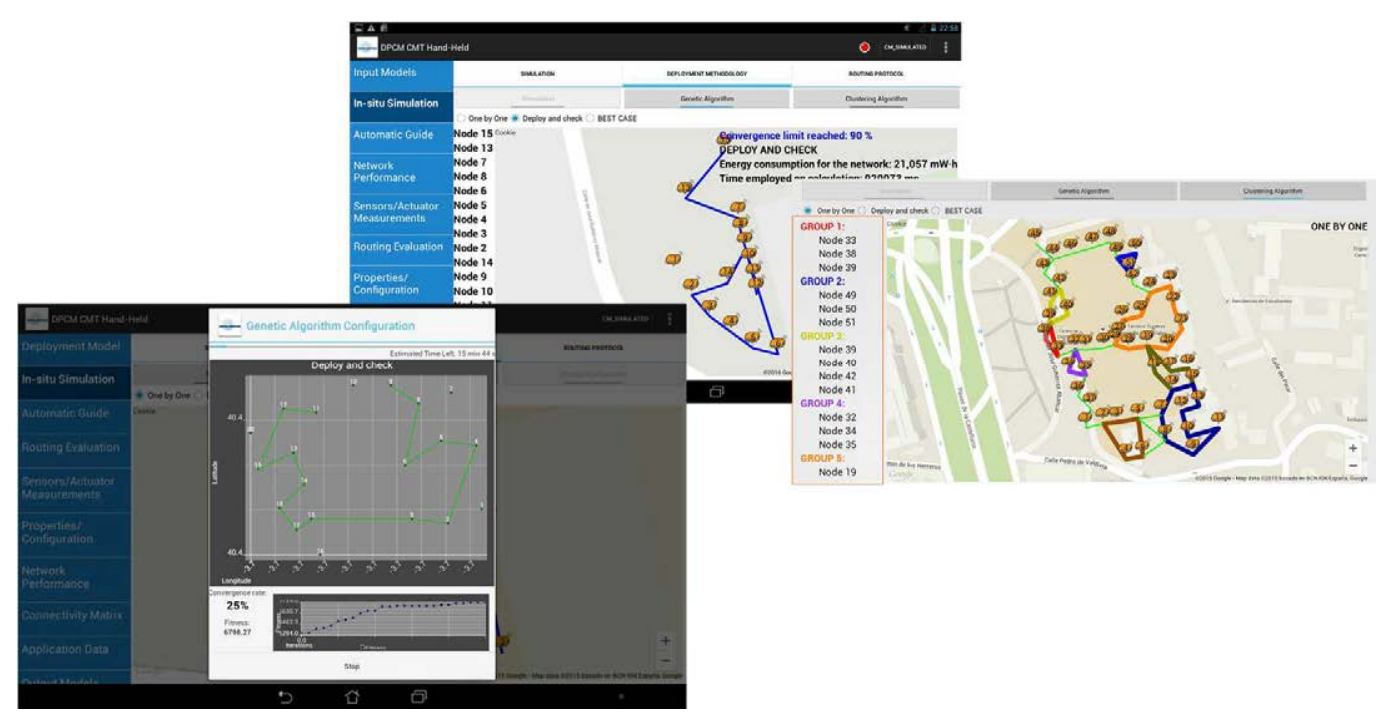

Fig. 4.7 Optimization evolution and result presentation views.

After the execution process of the selected mechanism ends, the result panel shows on one hand the optimal deployment sequence in the form of the array of 
nodes to be configured/analysed and its correspondence in the network map (in case of the Kps-based method by double-pressing on the sensor nodes a list of the correlated points is automatically displayed), and on the other hand quantitative values regarding the computational time as well as the overall cost optimization in accordance with the parameters under objective, such as energy consumption or number of execution tasks (Fig. 4.7).

The Routing Protocol tag supports the analysis and comparison of the different multi-hop strategies of the sensor network in situ. First, based on the input models and the connectivity correlation scheme, nodes' neighbourhood can be highlighted and parameters related to link metrics and hierarchy status in case of the cluster-based routing strategy are provided to the users, in addition to the information that a node handles when selecting it. Users can access a list of available protocol implementations and select one of them for the analysis.

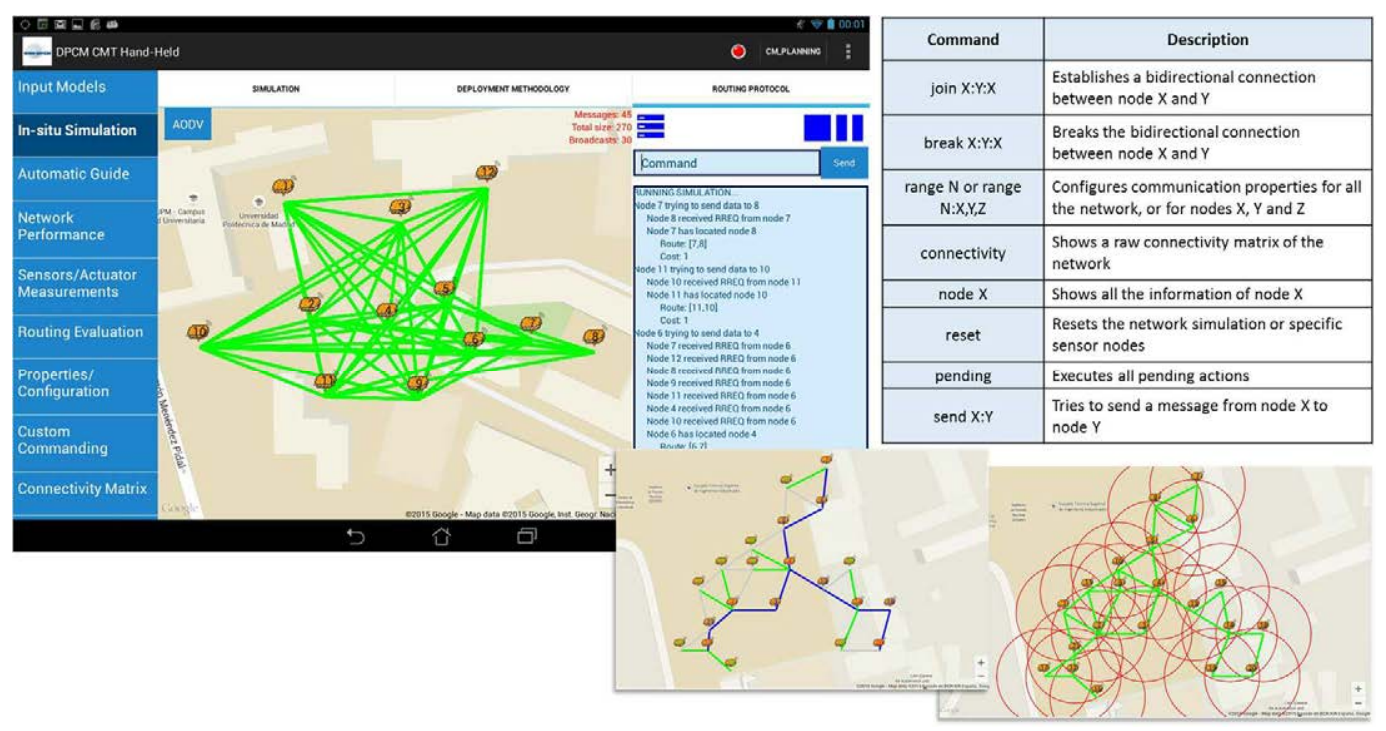

Fig. 4.8 Routing protocol analysis and evaluation engine.

Based on this, the routing simulation and evaluation mechanism can be triggered within two different types of executions: A single simulation, which lies on the communication between two specific remote points of the deployment according to the selected protocol; and a progressive simulation, in which two random nodes are periodically selected to establish a communication and data dissemination process based on the routing strategy to be adopted, 
generating in both cases all involved protocol routines to find the destination node and transmit the data packet. As shown in Fig. 4.8, different actions and events can be triggered by means of using a set of defined commands on the protocol evaluation console, which also logs the activities related to the multihop communication among the sensor nodes. During the runtime evaluation and when the execution process ends, users can check statistics on the right upper corner of the map and access the updated information gathered by the nodes.

The Automatic Guide module offers a graphical interactive supervision for users to perform the on-site deployment installation, configuration and evaluation tasks, allowing for a runtime comparison of the estimation models with the real evolution of the WSN behaviour at the target area. As shown in Fig. 4.9, the group of toggles at the upper side of the fragment allows activating the different optimization schemes obtained in previous stages, considering the type of simulation model selected at the action bar (the colour of the connectivity lines will change in relation with such a selection). If one of the options has not been executed jet, a message warning the action that shall be performed will be displayed accordingly.

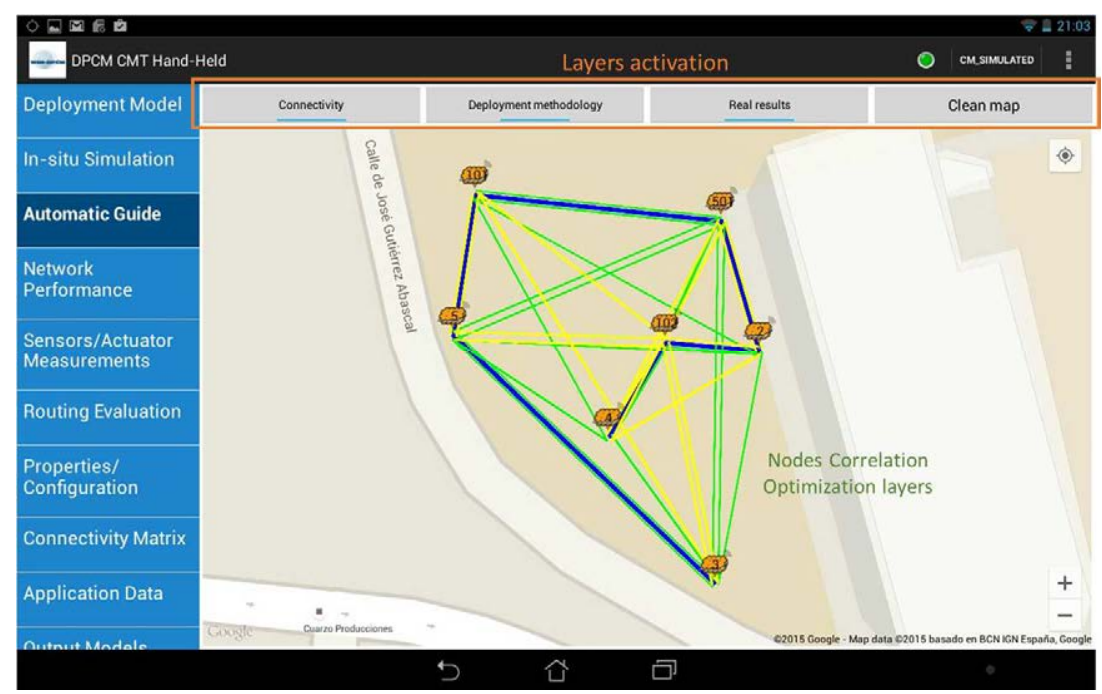

Fig. 4.9 Automatic Guide module. Activation and graphical inspection of various system layers.

Fig. 4.9 also shows how layers can be overlapped to graphically analyse the network/node correlation (in green expected connections among the nodes, blue 
the optimization sequence currently followed, and yellow connections and 2-Ns correlations already established). In case users update the position of a node during the testing process (long-press action on the map), a dialogue will automatically appear to provide deployers with the option of specifying the type of modification and further information related to such an update action. Moreover, a single-press action on any of the nodes will display the menu with the different available functionalities to be used, mainly allowing for the following items (as shown in Fig. 4.10):

- Node information: provides basic information related to the sensor node implementation and the current status of various parameters, such as firmware, coordinates, protocols, among others (see Fig. 4.10).

- Drive me here: allows launching the predefined navigator so that users can be guided to the position where the node is to be deployed.

- Check/Uncheck node: provides options to track and update the deployment status of the sensor node according to the in-field configuration and evaluation process.

- Update node with current location: changes the node coordinates based on the current position of the user with the HHD, whose modification also produces the corresponding update in the deployment models and network definitions.

- In-field node/network tests: allows triggering connectivity tests and performance evaluation analysis from the selected node to the surrounding neighbourhood as well as to remote points of the network. As shown in Fig. 4.11, the UI interactively guides users throughout this process highlighting real encountered nodes and communication links on the map, apart from updating the status of the nodes correlations at runtime.

It is important to highlight that the different actions performed during the deployment and evaluation activities also generate the underlying modifications in the different models and the data management system of the toolset, which 
will be automatically reflected in the output reports to be uploaded to the project repository as well.

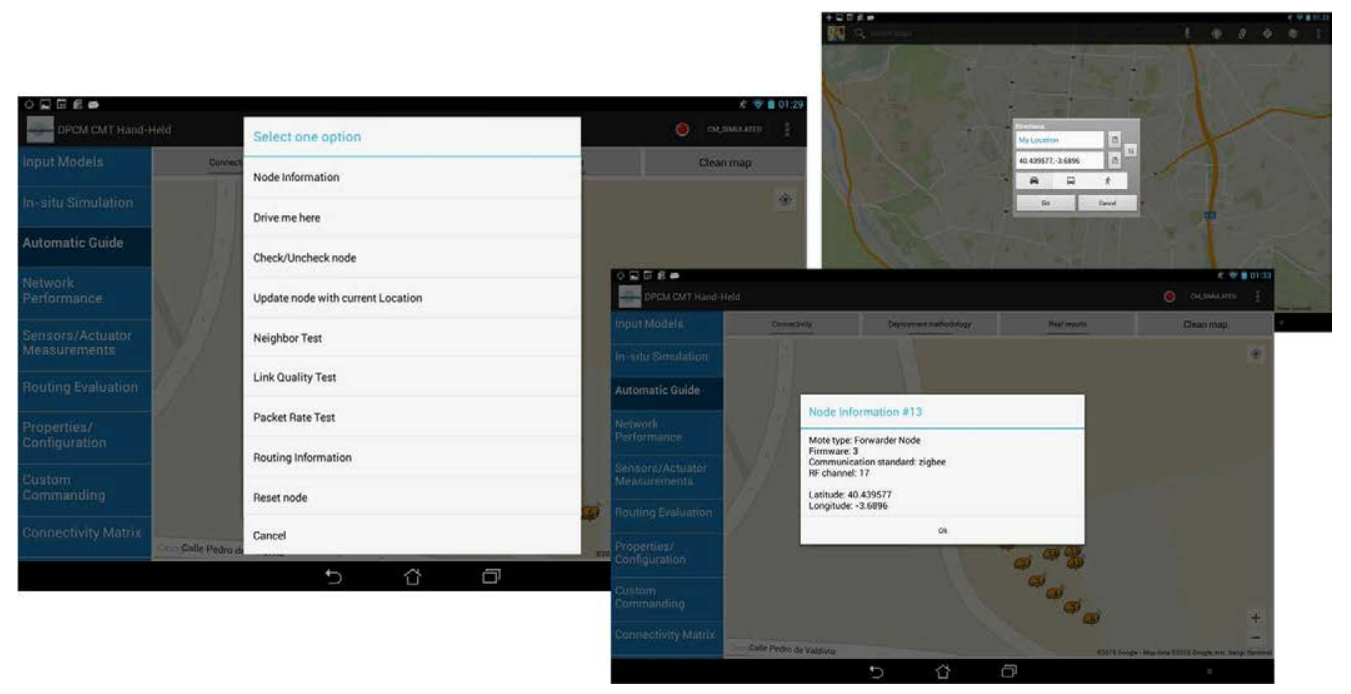

Fig. 4.10 Various options for users to interact with the toolset guide and the target WSN commissioning.

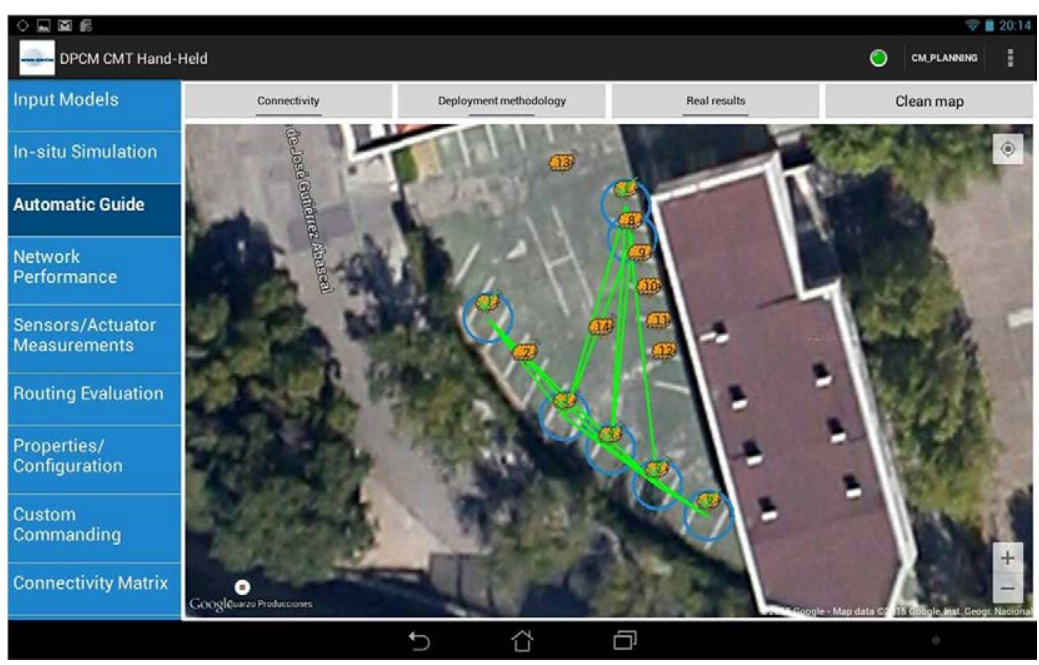

Fig. 4.11 Graphical representation of the test evolution process when selecting a node.

The Network Performance module provides users with a set of functionalities to carry out on-site network performance evaluation and 2-Ns correlation tests. This fragment is composed of various dynamic interfaces that are reconfigured in accordance with the network model (as the node identification panel on the right-side of the window in Fig. 4.12, and the configuration button that allows 
selecting the functional parameters of the network model associated with radio communication properties to be used for the performance analysis, such as connectivity metrics) and the results of the test execution process (for instance, the selection panel of correlated/ destination points for a particular node Id).

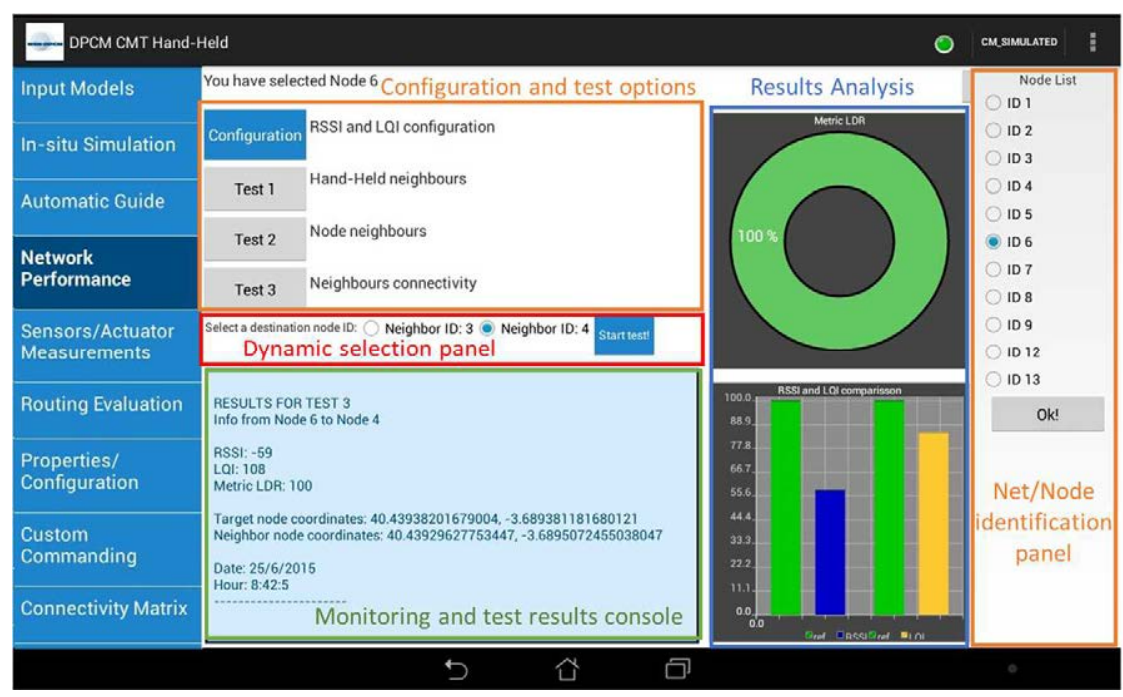

Fig. 4.12 WSN deployment analysis and performance evaluation module distribution.

When selecting a node from the identification panel, users can set up the TX power configuration value and then trigger any of the available tests in the target deployment area. The results of the execution process will be provided both in the information console (in green) and in the graphical representation of the various data outcomes (in blue).

The Sensor/Actuator Measurements module provides users with functionalities to configure sensing acquisition and monitoring services, so that the evaluation and calibration of the implemented sensors/actuators can be performed in-situ. As shown in Fig. 4.13 the transducer selection array automatically adapts to the node checked in the identification panel, and thereafter users can set up a data publishing period in order to get runtime information from the selected sensor. Every sensor/actuator can be seen as a measurement channel that can be configured to analyse the behaviour of the transducer, with the support of the dynamic plot views and consoles that will be automatically inserted whenever a data acquisition has been triggered. When selecting a transducer to be analysed, 
plots are added to the monitoring view so that the comparison of different measurements can be performed concurrently.

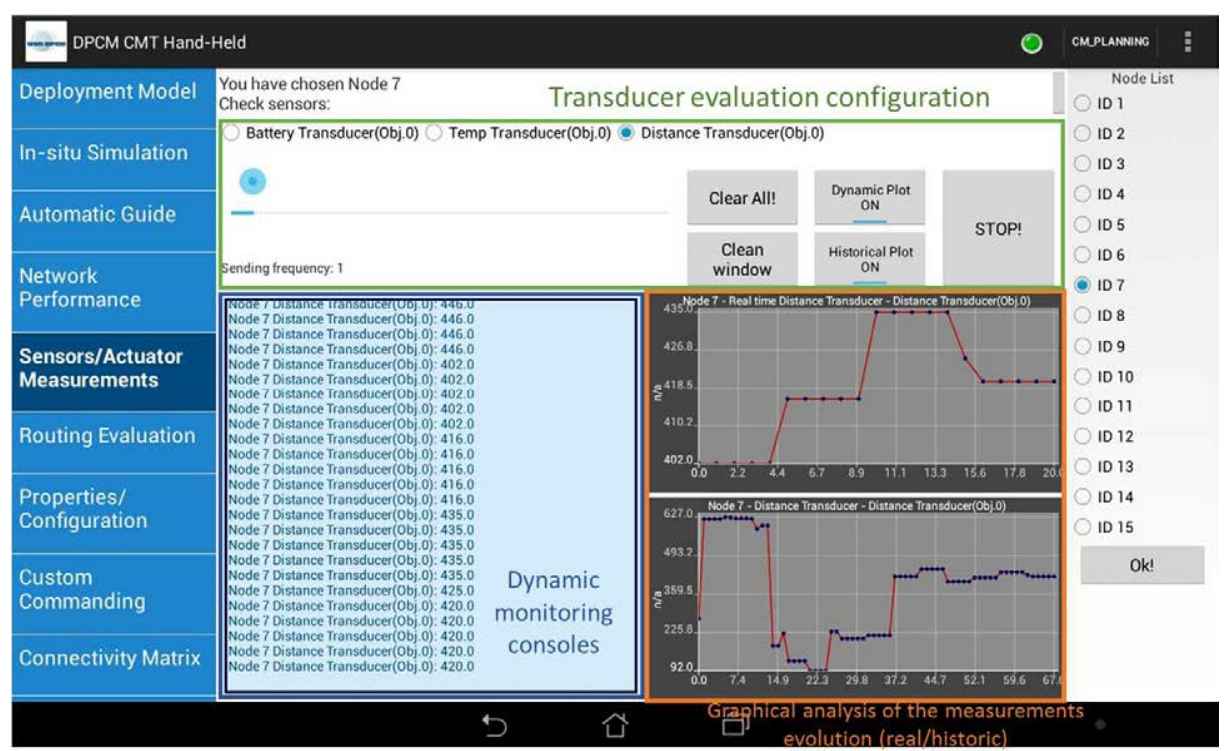

Fig. 4.13 Adaptable transducer management configuration and monitoring options based on dynamic interfaces.

These real-time plots can also be observed in combination with historic values already registered in the HHD (by activating both plot toggles), so that data evolution patterns could lead to performing correction actions either at software/processing level or data acquisition level. Moreover, users have the possibility of changing the graphical distribution of the fragment for a better/more-detailed view of the data presentation windows. For this, plots can be expanded to display individual acquisitions, or reduced to gather several parallel monitoring views within the same presentation area.

The Routing Evaluation module is intended to aid users in the performance analysis and assessment of the multi-hop mechanisms to be used in the WSN, particularly considering routing metrics and data delivery transactions tests. Following the same philosophy of dynamic configuration and adaptation of the fragments for the target deployment, users shall select the property blocks to be used for the performance evaluation activities, and then the data representation offers graphical views of the test results as in previous described modules (see Fig. 4.14). 


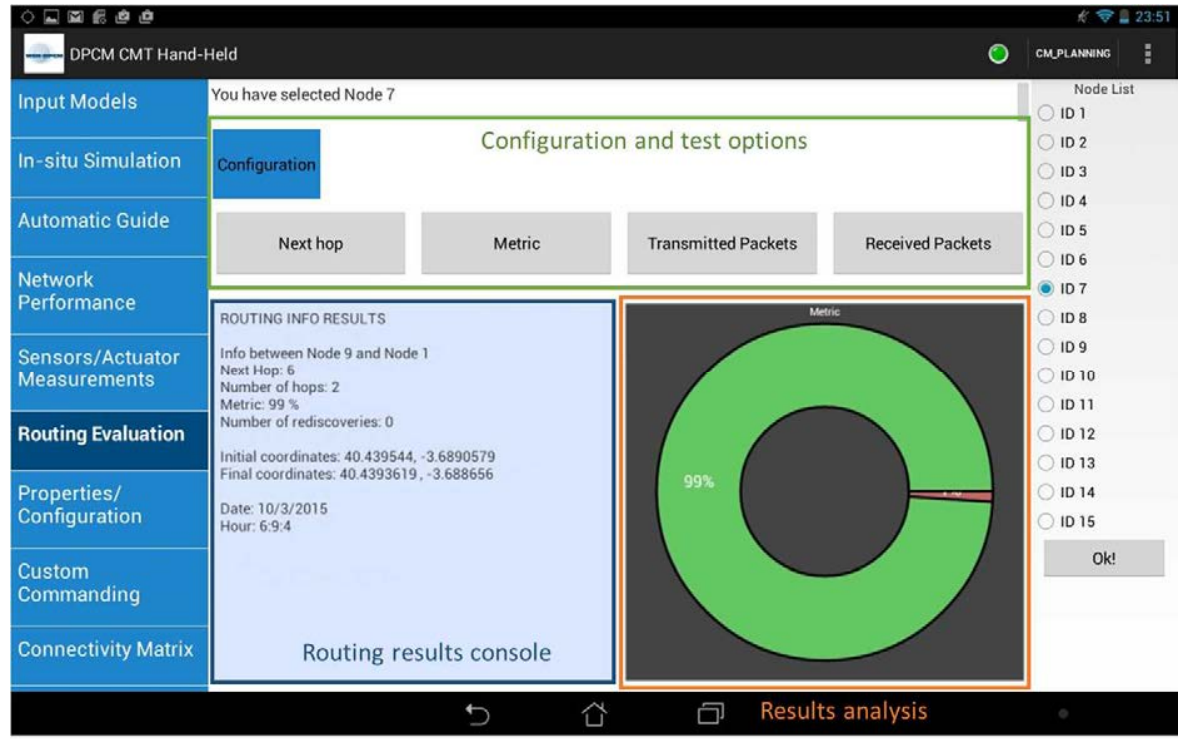

Fig. 4.14 Performance evaluation options and test results for WSN routing strategies.

The Properties/Configuration modules provides users with a dynamic graphical interface to interact with the functional blocks that are implemented in the sensor nodes, particularly serving as a high-level access to the middleware components of the HW-SW platform. Such a functionality allows on one hand verifying the status of any node and related component of the network at runtime, and on the other hand reconfigure parameters to change the operation of specific elements of the platform, so that runtime updates during the deployment/maintenance tasks can be supported. By selecting one of the sensor nodes at the identification panel, a repository of block types is expanded which can be pressed to automatically display the instances that are associated with such a block, as well as the properties and configuration parameters that define the implementation of them.

As shown in Fig. 4.15, users can trigger a background monitoring of various types of parameters that will be prompted at the properties console in real-time, in addiction to sending configuration messages to the sensor nodes under evaluation. Blocks are also classified in relation with their read-write nature, whose condition is highlighted with active icons that change according to the status of the block parameter (sending data to and receiving from the embedded middleware component). This module represents a fundamental capability of 
the HHD to assess and validate the real implementation of the embedded software blocks of the wireless sensor nodes under deployment, in accordance with the network model definition created at the planning/development stage of the WSN.

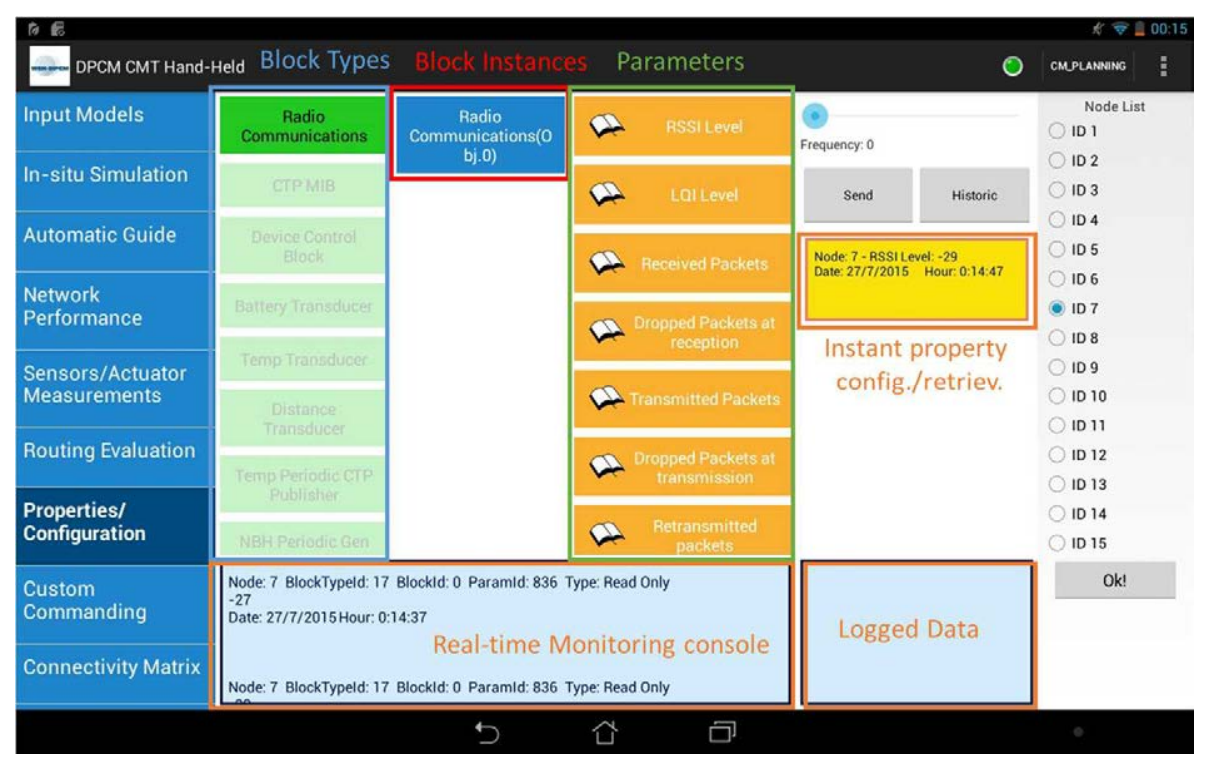

Fig. 4.15 Interface to the functional blocks of the WSN node/middleware implementation.

The Connectivity Map Module offers an overall view of the WSN connectivity both at link level as well as remote/multi-hop level, considering the estimation values from the simulation models as well as the real data obtained during the deployment/maintenance activities in-situ. The interactive comparison is provided in the form of a filtering matrix upon which users can select specific 2Ns to display further information related to connectivity metrics, routing performance, and registered status of the communication between the involved nodes. As shown in Fig. 4.16, tags at the upper-side of the fragment allows specifying the type of map to be used: simulated, real, simulated + real.

The Custom Commanding module allows performing low-level tests on functional components of the sensor nodes based on specifying the block and parameters to be acted on/read from. Thus, it is a more advanced fragment intended for experimented users to carry out verifications and specific readjustments on the component implementations. In this direction, the Application Data module also refers to a detailed view of the packet traffic handled by the toolset during the 
deployment activities, so that particular frames and underlying messages can be observed at runtime. Fig. 4.17 shows a general view of these fragments following a console-based structure for prompt capturing and packet string representation. These fragments also allow providing runtime view and registry of low-level frames from the WSN by configuring the HHNode component to act as a packet analyser interface, so that the underlying data exchange among the sensor nodes in the target area can be observed and subsequently decomposed.

Furthermore, as also shown in Fig. 4.17, a Radio Modelling module is also integrated into the deployment toolset to provide advanced users with a support fragment for creating and customizing connectivity estimation models, based on the in-field analysis of the wireless communication HW behaviour at the target environment.

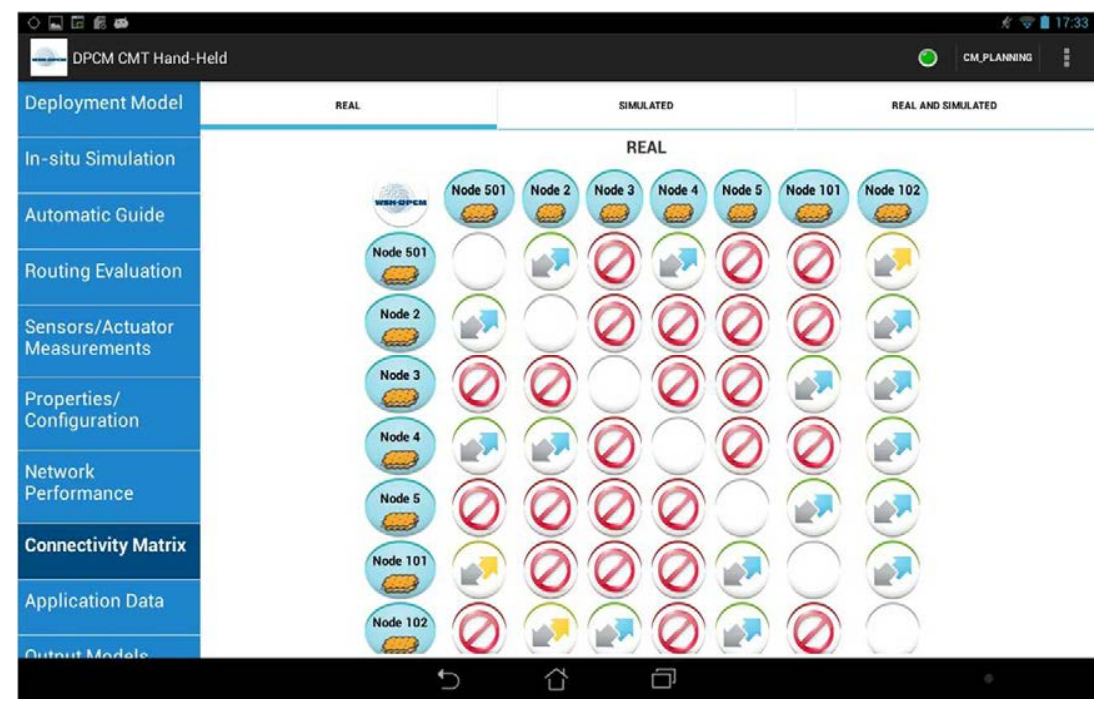

Fig. 4.16 Interactive map for the analysis of the network connectivity and real test results.

The Output Models module allows generating and uploading the results of the deployment, commissioning and maintenance activities to the project repository in the form of output reports. Users can select one of the available models to generate a new report from the basis of data automatically registered and processed during the evaluation procedure, and then visualize the results related to the nature of such a report at the preview panel as shown in Fig. 4.18, before sending it up to the remote repository. Apart from self-included 
assessment indicators provided by the deployment tool, users have also the opportunity to incorporate additional details along the generated reports based on their experience and evaluation result analysis when carrying out the in-field deployment and validation process. For this, dialogues allow introducing such information for its subsequent integration into the output report.

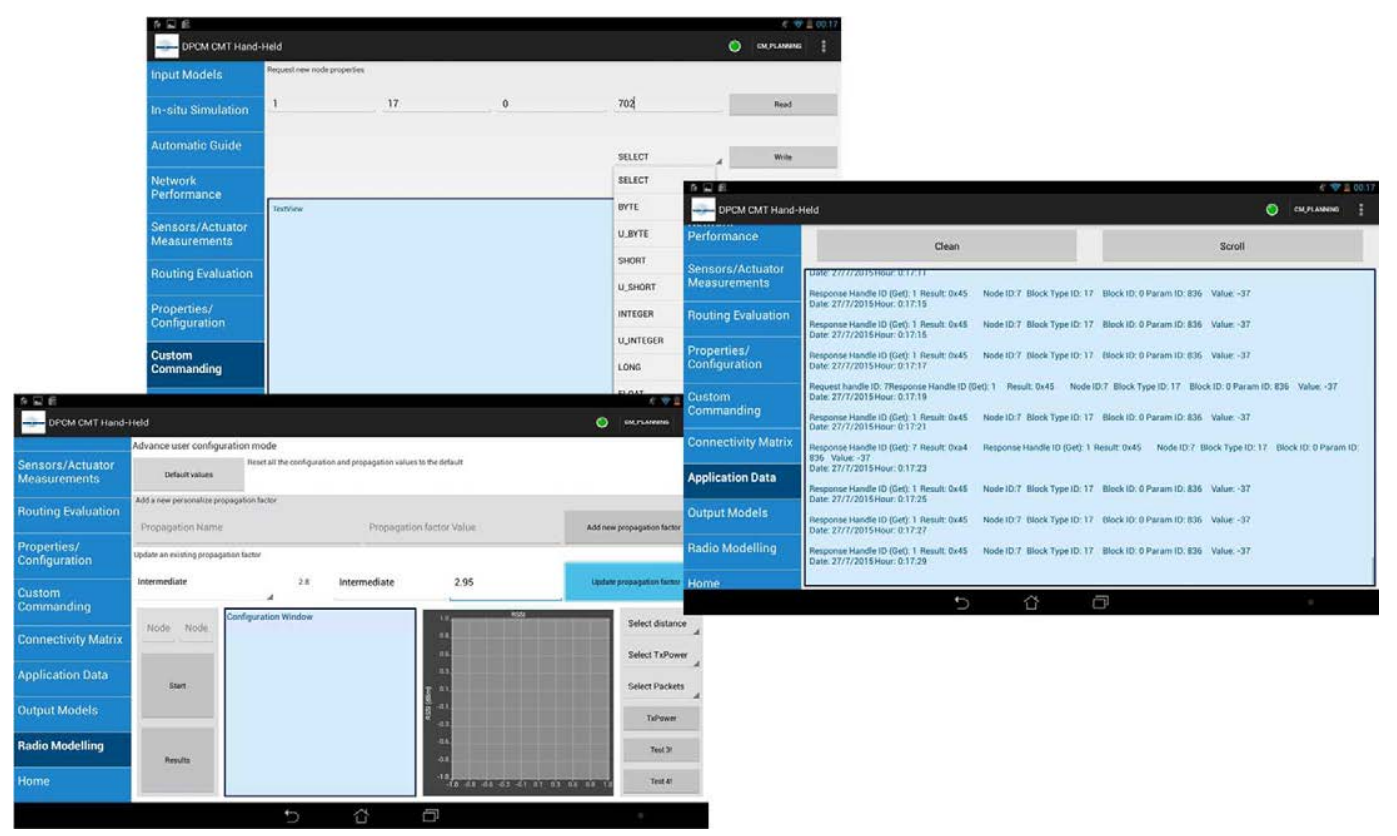

Fig. 4.17 Advanced-user configuration and monitoring modules.

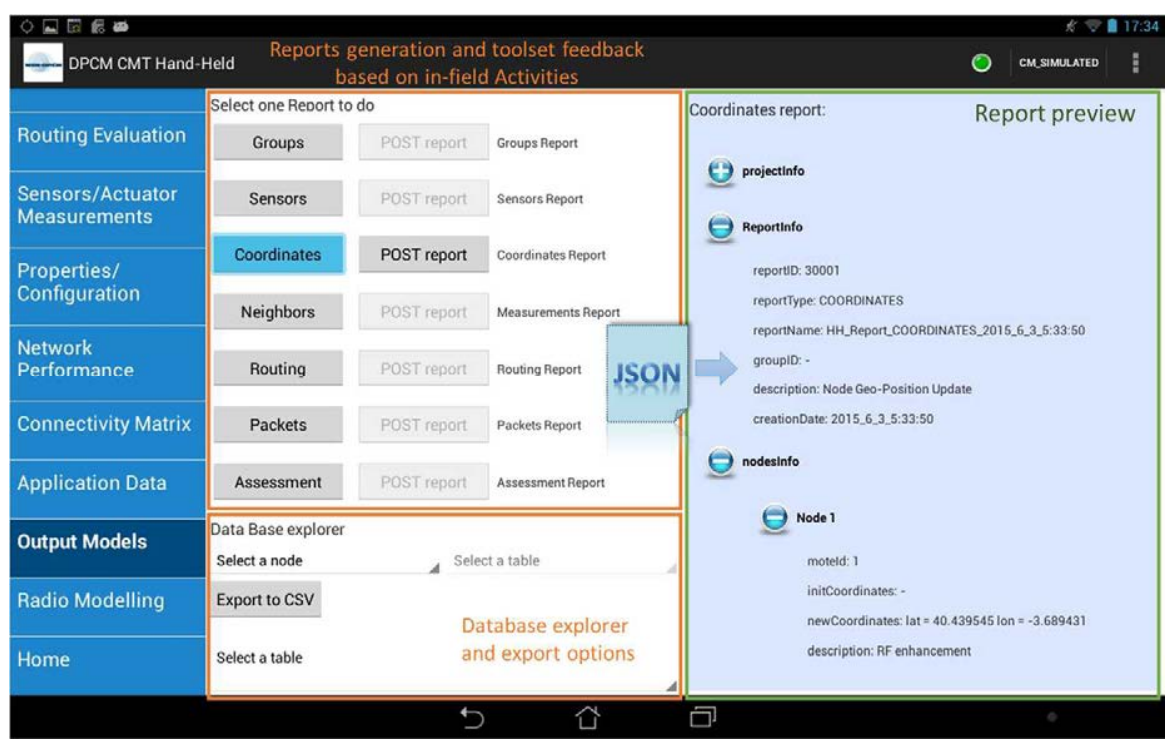

Fig. 4.18 Output reports generation. WSN deployment assessment and feedback. 
Fig. 4.19 shows the HHD, which is composed of the tablet device with a WSN platform, in this case a Cookie node, connected through the USB socket.

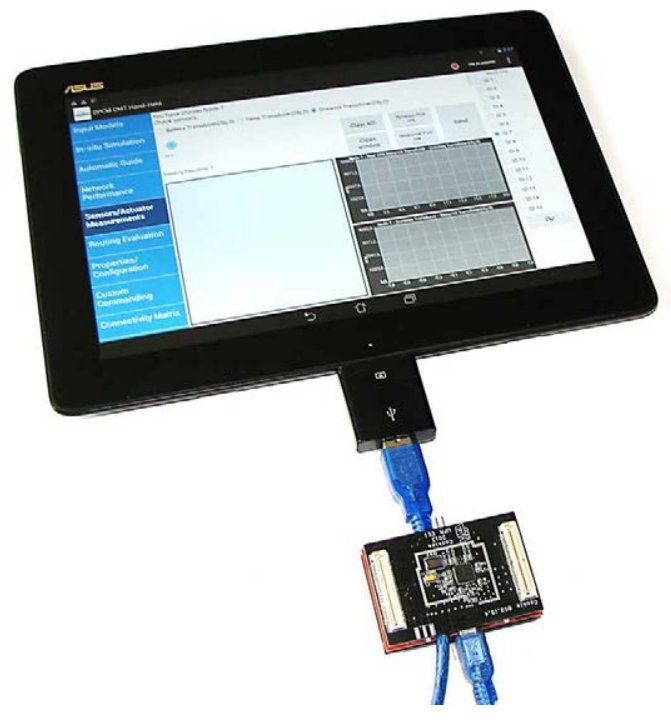

Fig. 4.19 General view of the HHD with the connection to HHNode.

\subsection{Conclusions}

The implementation of the HHD was conceived taking into account not only the effective integration of the proposed deployment optimization strategies within a unique in-field support toolset, but also an easy-to-use interactive interface for deployers to be guided throughout the commissioning, configuration and on-site evaluation of the wireless sensor network under deployment. The fragmentbased design approach allows the composition of a set of dynamic modules that represents the various subsystems that compose the overall deployment tool, whose underlying structure has been thought to easily accommodate additional components towards extending the functionalities of the platform. The Javabased component implementation indeed enhances this integration suitability thanks to its modular and object-oriented nature.

Furthermore, the synergy between the evaluation components and the real interaction of the HHD with the sensor network by using the HHNode provides the on-site access to and the comparison with the real capabilities of the nodes, so that a real-time performance analysis of the WSN deployment with the 
expected behaviour from the estimation outcomes can be carried out through the proposed deployment guidance.

\subsection{Bibliography of the chapter}

$\begin{array}{lllll}\text { [AndroidDev] } & \begin{array}{l}\text { Android Developers environment and API, available on: } \\ \text { https://developer.android.com/ }\end{array} \\ \text { [Maps] } & \begin{array}{l}\text { Google } \\ \text { https://developers.google.com/maps/documentation/android-api/ }\end{array}\end{array}$





\section{Chapter 5}

\section{EXPERIMENTAL TESTS AND USE}

CASES

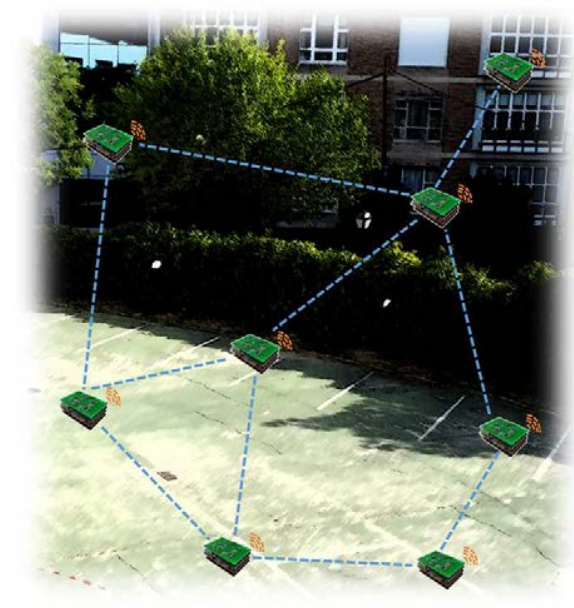
validated through several real deployments presented in this chapter, in which the analysis of the main results and test cases is detailed.

The practical focus of the Hand-Held Device and the hardware-software modular platform has leveraged their effective use within different application contexts not only to take advantage of the proposed toolset in the analysis and evaluation of real WSNs, but also to verify their applicability in various experimental scenarios.

The integration of the functional components that compose the overall deployment platform as well as a proper synergy with the development, planning and simulation stage of sensor networks have been 



\section{Experimental tests and use cases}

\subsection{Introduction}

The system implementation and integration of the proposed deployment capabilities in the Hand-Held Device has been conceived to provide deployers/maintainers with a real in-field perspective of the wireless sensor network under configuration and evaluation, so that a more comprehensive analysis of the actual behaviour of the involved components as well as the applicability of optimization strategies can be dynamically performed in-situ. In this way, the HHD and the associated functionalities have been used within different application contexts not only to take advantage of the designed and integrated techniques for the analysis of WSNs, but also to verify its applicability into real experimental scenarios.

On one hand, the validation of the CB-AODV implementation into the Cookies HW-SW platform has been carried out in several WSN deployments which also allowed for putting into context the modelling, optimization and testing resources of the deployment toolset, as well as producing an overall runtime configuration and assessment scheme for the mesh sensor network objective. On the other hand, the interoperability and adaptability of the HHD has been verified within the context of a WSN-based smart demonstration as part of the final results of an Artemis European project [DPCM], in which the commissioning, deployment and maintenance capabilities have been integrated into the design and implementation of a multi-domain platform for the development of WSN-based applications.

The experimental use cases shown in the following sections also served as an evaluation scenario to verify not only the various functional elements that compose the overall proposed framework, but also the interactions and data model generation among both the internal subsystems and the external platform interfaces, particularly considering the heterogeneous nature of the WSN development, planning, commissioning and maintenance toolset. Moreover, the 
HW-SW integration platform also proposed in this work including the designed processing and communication layers (particularly considering the IEEE 802.15.4-based layer and related controllers) has been employed in order to support the deployment and management of the sensor networks under commissioning and evaluation, so that a complete synergy between the implemented components of the HHD and the embedded WSN platform is indeed obtained. This leads to providing users with a comprehensive development and deployment support framework based on the modular platform, the network model definition and the commissioning toolset, so as to enhance how the in-field configuration, network assessment and system release is carried out.

\subsection{Test case 1: $C B-A O D V$ implementation and routing protocol verification}

The proposed CB-AODV routing protocol has been tested and analysed in real WSN deployments at the Centre of Industrial Electronics by means of using some of the capabilities of the HHD in order to verify and validate the designed functionalities as well as the components under the Cookie HW-SW architecture. The implementation of CB-AODV has been carried out based on the Cookie support platform proposed in this work, and by integrating the software components as depicted in Fig. 5.1. In terms of memory usage, the CB-AODV was included in the Cookie processing layers that incorporate the 8051-based microcontrollers, which provide up to $64 \mathrm{kB}$ of flash/program memory as well as $4 \mathrm{kB}$ of data storage, as described earlier in chapter 2. The final implementation fits in $8 \mathrm{kB}$ of program memory and less than $2 \mathrm{kB}$ of data memory (including the support software components for the diagnosis capabilities), which gives enough free memory space for Cookie-based application proposals. Moreover, this set of experimental tests allows the verification of the interactions between the HW controllers for the IEEE 802.15.4based Cookie communication layer and the routing protocol software implementation, so that the control packets and protocol management tasks are properly abstracted to the application level. 


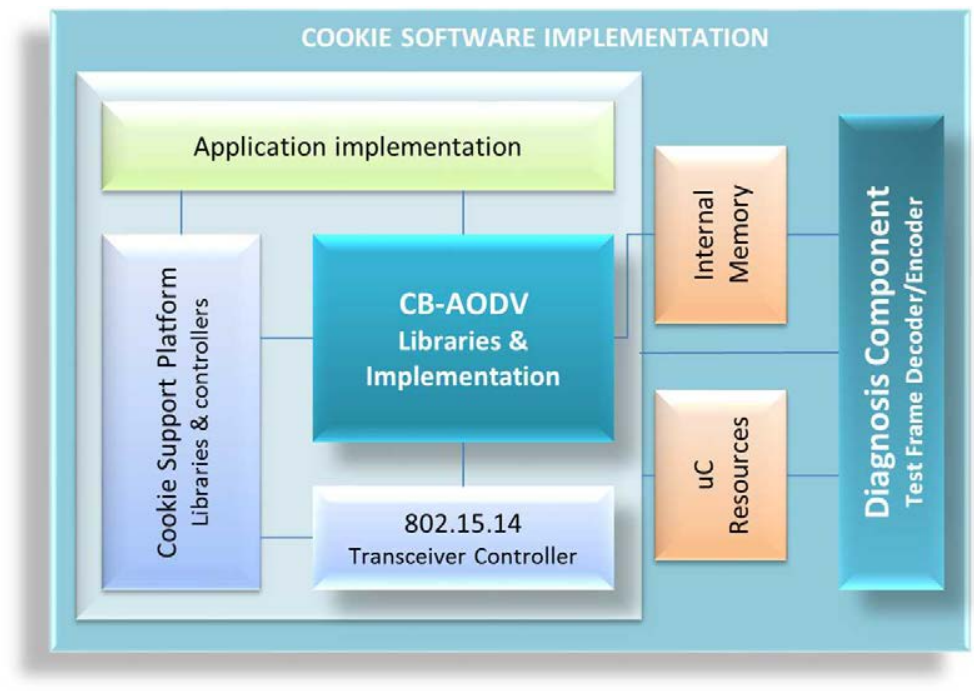

Fig. 5.1 General view of the CB-AODV integration in the Cookie platform.

Table 5-1 summarizes the main functionalities included in the configuration and diagnosis components in order to analyse the behaviour of the routing strategy, as well as the dynamics of the functionalities that compose the CB-AODV and its related libraries that generate the different stages of the routing mechanism. The debugging version of the implementation allows an easy tracking of the control packets that are generated when performing discovery, routing and maintenance tasks during the operation of the WSN, so that a more comprehensive view of the routing mechanisms can be produced.

Several controlled deployments and topology arrays were carried out in order to firstly verify that the different actions and control packets of the discovery mechanism performed as expected for a type of configuration, especially validating that CB-AODV properly generates and manages RREQ, RREP, RERR, DATA_ERR, and DATA in accordance with the routing creation/updating. Fig. 5.2 shows the deployment of 4 nodes that are used to preliminarily analyse the behaviour of the routing mechanisms and the PHY-MAC module controller. A burst of 1000 packets (100 ms period) was sent from N3 to node $\mathrm{N} 1$, obtaining $100 \%$ of packet delivery (the deployment and the transmission power were set up such that no direct/layer-2 connections between the 
origin and the destination points are achieved, i.e., obtaining a route N3-N2N1).

\begin{tabular}{|c|c|}
\hline Functionality & Description \\
\hline Module configuration & $\begin{array}{l}\text { Configuration of the radio transceiver, including the } \\
\text { parametrization of the transmission power, } \\
\text { operational modes and networkidentifiers. }\end{array}$ \\
\hline TX Power & $\begin{array}{l}\text { Runtime reconfiguration of the transmission power of } \\
\text { the communication module, in accordance with the } \\
\text { multi-hop objective under evaluation. }\end{array}$ \\
\hline Protocol initialization & $\begin{array}{l}\text { Initialization of the information management } \\
\text { components of the protocol, which refers to the } \\
\text { routing tables and buffer handling. This also allows a } \\
\text { regeneration of the routing information so that } \\
\text { selected network areas can execute clean discovery } \\
\text { tasks. }\end{array}$ \\
\hline Operational mode & $\begin{array}{l}\text { Activation of the different operational and power } \\
\text { down modes of the communication module, } \\
\text { particularly intended to analyse the presence of } \\
\text { dynamic changes in the connectivity behaviour of the } \\
\text { network and node's correlation evolution. }\end{array}$ \\
\hline $\begin{array}{l}\text { Routing entry } \\
\text { management }\end{array}$ & $\begin{array}{l}\text { Visualization of the current status of the routing } \\
\text { information manager, which includes the provision of } \\
\text { the routing entries that have been generated and the } \\
\text { associated information to reach a remote destination } \\
\text { point. }\end{array}$ \\
\hline Route indicators & $\begin{array}{l}\text { Provision of parameters related to the number of } \\
\text { transmitted and received packets, } \\
\text { discovery/rediscovery actions and link failures of the } \\
\text { target node during the network operation. }\end{array}$ \\
\hline Packet generator & $\begin{array}{l}\text { Transmission of data packets to random destination } \\
\text { points in accordance with a configurable period of } \\
\text { time, so that a traffic pattern is created in part of or } \\
\text { the overall network. }\end{array}$ \\
\hline Path assessment & $\begin{array}{l}\text { Configuration of data packet transmission between } \\
\text { two remote points with a configurable frequency, to } \\
\text { analyse route discovery/maintenance, packet drop } \\
\text { rate, path quality metrics, and rediscoveryactions. }\end{array}$ \\
\hline $\begin{array}{l}\text { Protocol behaviour } \\
\text { analysis }\end{array}$ & $\begin{array}{l}\text { Data flow debugging of the protocol implementation } \\
\text { by tracking the execution and state of the internal } \\
\text { routing functionalities as well as the generation of the } \\
\text { control packets in the nodes. }\end{array}$ \\
\hline
\end{tabular}

Table 5-1 Summary of configuration and diagnosis functionalities for the analysis of the CBAODV implementation. 


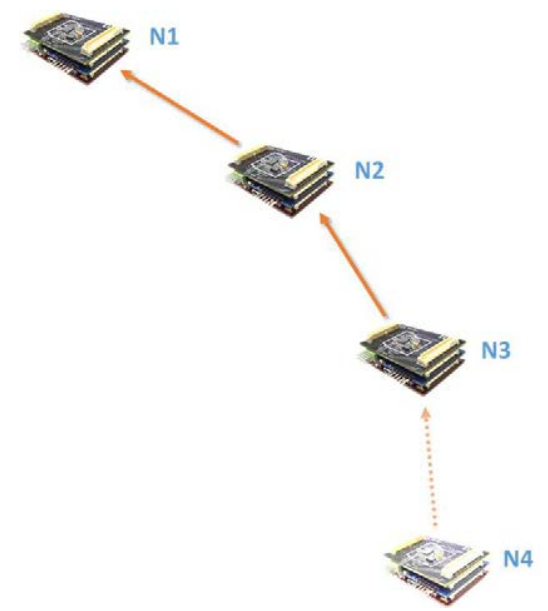

Fig. 5.2 Deployment of $3+1$ node to test the creation of the routing path.

Then, N4 was turned on and a route request to N1 was triggered. In Fig. 5.3, the route discovery process in which an intermediate node provides the origin point with an updated route to the destination point is highlighted, obtaining a route N4->N3->N2-N1. With this deployment array, combinations of traffic patterns were configured to evaluate the communications both at MAC and routing layers.

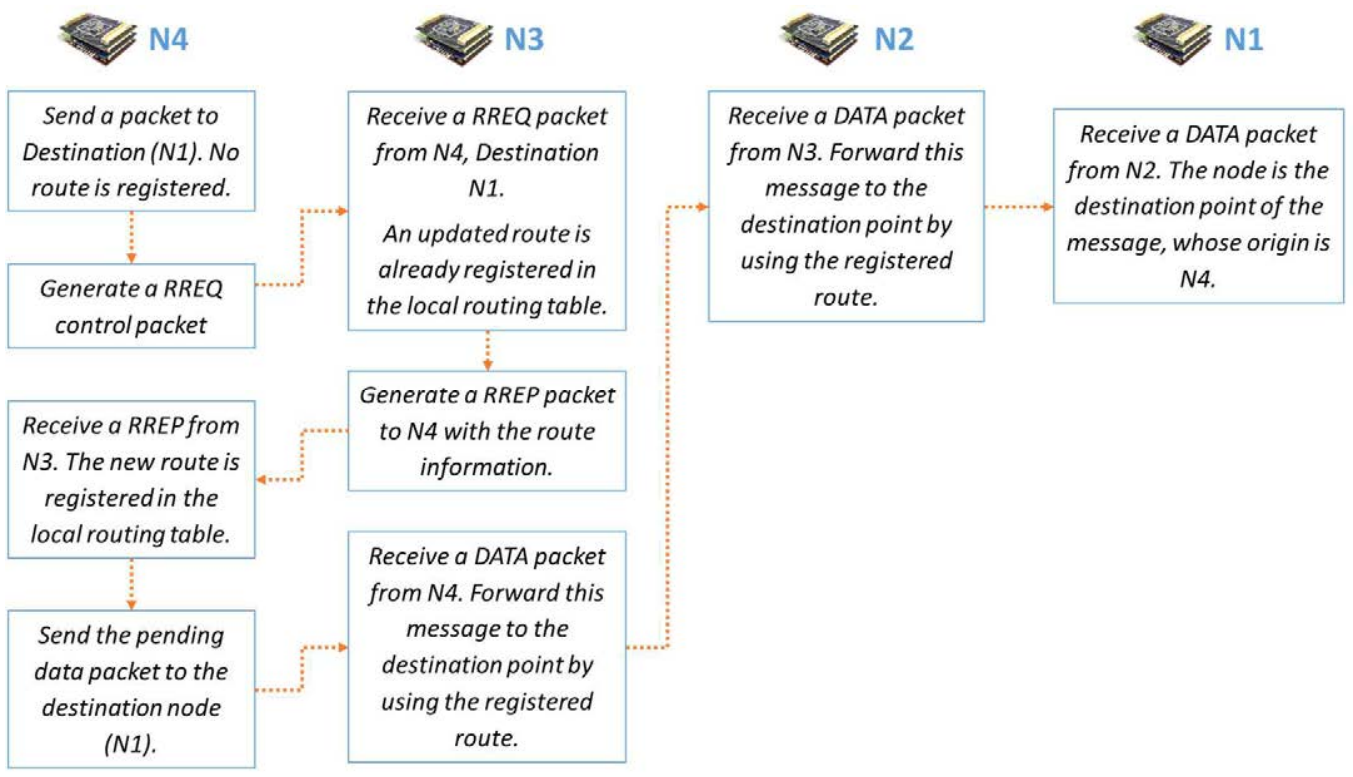

Fig. 5.3 Route discovery process based on the information already available in the routing tables of the nodes. 


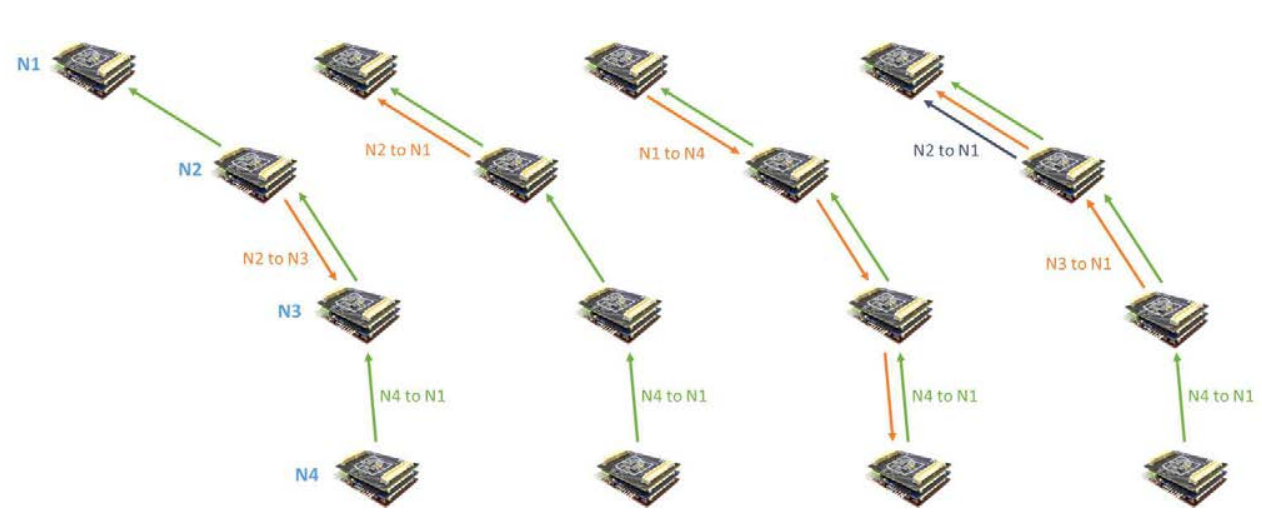

Fig. 5.4 Various data exchange configurations among the sensor nodes.

Fig. 5.4 shows different data exchange tests, whose results are summarized in Table 5-2, and in which the packet delivery in all cases is (or certainly closed to) $100 \%$, considering a high level of transaction frequency over the network. Regarding the connectivity of the nodes, the average link retry relies on the number of layer-2 retransmissions before getting the corresponding acknowledgement, which can be related both to the traffic congestion of the involved links, as well as the quality of such interconnections. In this case, MAC retries are higher in N4-N3 communications due to a worst connectivity between those nodes, as shown in Fig. 5.5, where the RSSI and LQI parameters for every link of the routing path were captured during the multi-hop processes.

\begin{tabular}{|c|c|c|}
\hline \multirow{2}{*}{ Test } & Data Transmissions & Packet Delivery (\%) \\
\hline \multirow{2}{*}{$\begin{array}{l}\text { N2->N3, } \\
\text { N4->N1 }\end{array}$} & 700 & 100 \\
\cline { 2 - 3 } & 500 & 100 \\
\hline \multirow{2}{*}{ N2->N1, } \\
N4->N1
\end{tabular}

Table 5-2 Summary of the packet delivery results for the various data exchange configurations. 
RSS

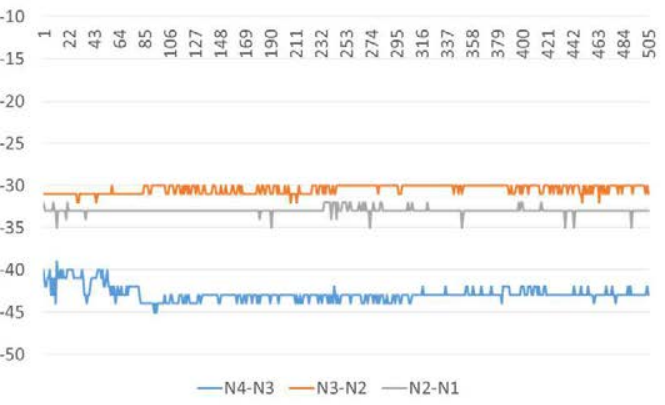

LQI

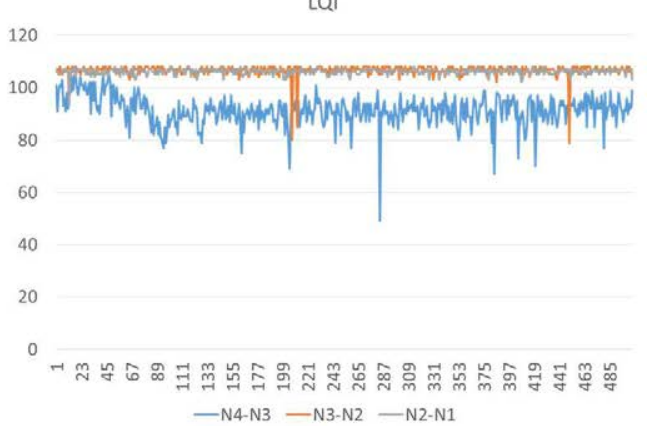

Fig. 5.5 Evolution of the RSSI and LQI values among the WSN links.

This topology has also been used for the evaluation of the routing performance in terms of packet transmission and routing delays. For this, both the Round-Trip delay Time (RTT) as well as the Route Discovery time (RD) are considered for the analysis of the multi-hop transactions among the sensor nodes. The first one lies on the time a packet takes to travel from the originator point to the destination node and then go back the source of such a packet (considering that the route is already created), whereas the second parameter refers to the latency associated with the creation of the multi-hop path between source and destination points.

Several packet delivery tests were performed to measure the RTT between $\mathrm{N} 1$ and N4 (3-hops), obtaining an average value of $41.7 \mathrm{~ms}$. When N1 discovers a route to $\mathrm{N} 4$ by using information already saved in $\mathrm{N} 2$, the RD delay is equal to $32 \mathrm{~ms}$. Moreover, when encountering the route through N3 (N1 and N2 generate the corresponding request messages, while N3 has a valid route to N1), the route discovery process produces an RD delay equal to $74 \mathrm{~ms}$. Fig. 5.6 shows some of the captures regarding the underlying measurements to obtain the round-trip latencies at the source node of the deployment.

In order to further explore these parameters within the performance analysis of CB-AODV, a topology composed of 4 nodes in a 2-hops, double-branch configuration has been used as represented in Fig. 5.7. Nodes are separated in a distance of $1 \mathrm{~m}$ between pairs of points roughly, with a transmission range 
such that the source node N1 shall use N2 or N3 to obtain a route towards N4.

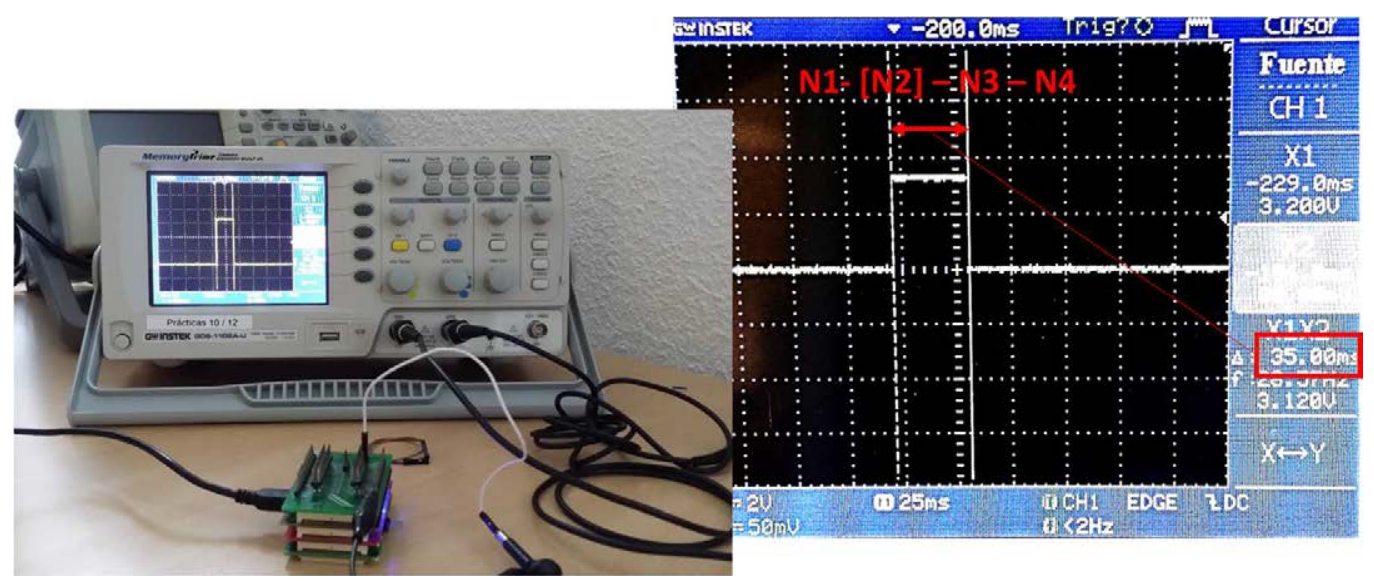

Fig. 5.6 RTT and RD delay measurement tests for the CB-AODV based multi-hop topology.

When triggering a route discovery between N1 and N4, a 2-hop RD delay equal to $74 \mathrm{~ms}$ is obtained for N1-N2-N4, while the average RTT in such scenario is $27.3 \mathrm{~ms}$, as represented in Fig. 5.8, where round-trip delay measurements are captured at the source node after performing a data transmission operation.

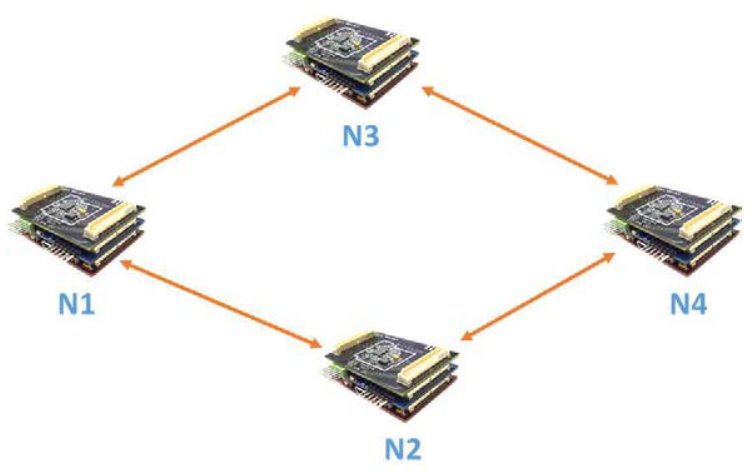

Fig. 5.7 2-Hop topology scenario for the routing delay analysis.

Based on these observations, it is important to highlight that CB-AODV outperforms NST-AODV considering around 30\% reduction of RTT for 2Hop and 3-Hop paths (e.g. $39.3 \mathrm{~ms}$ and $60.2 \mathrm{~ms}$ respectively in the second one), which represents another improvement of the proposed 
implementation, providing both robustness/reliability and efficiency on the multi-hop data packet delivery.

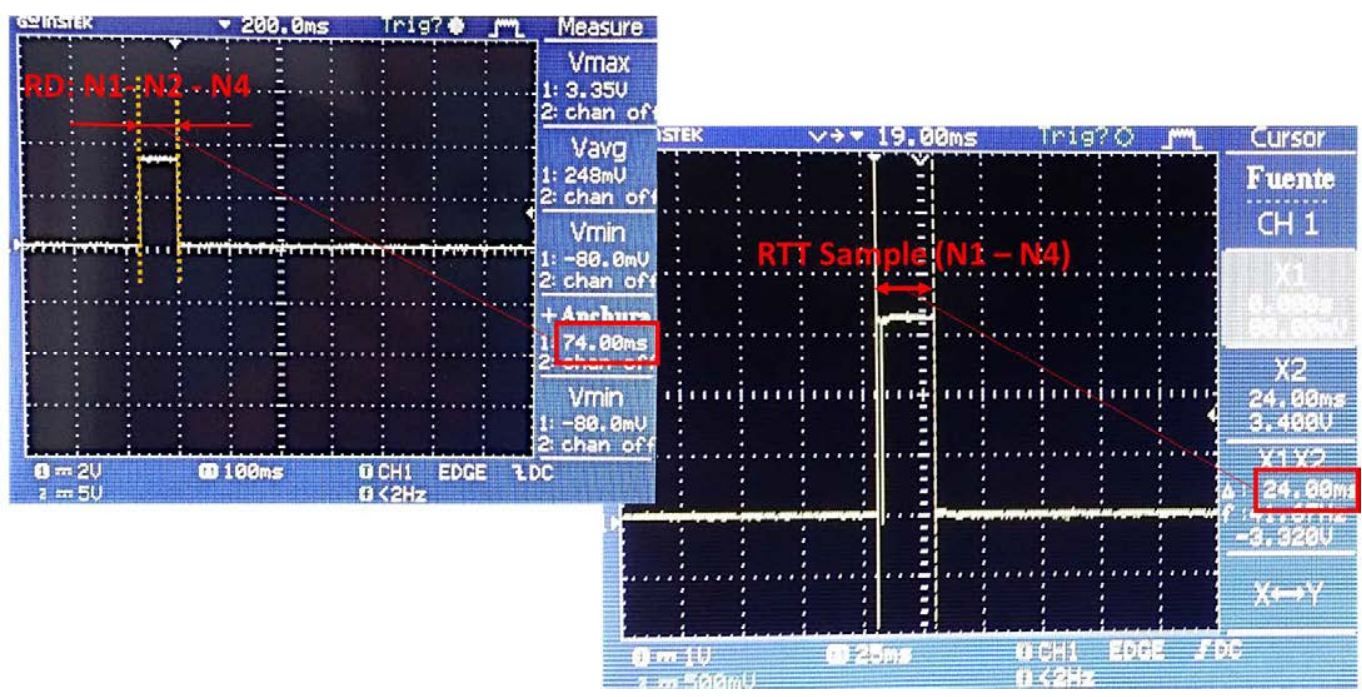

Fig. 5.8 Round-trip delay measurement samples for the 2-hop, double-branch topology scenario.

Going on with the preliminary evaluation tests, a deployment composed of 6 nodes has been realized to trigger the generation of multi-hop paths within another network array (see Fig. 5.9), by using the implemented routing mechanism. A summary of the main results for this case is also highlighted in Fig. 5.9, considering the data transmission from N6 to N1 both without additional data transactions in the network and with N5 and N3 also sending packets to N1, with a transmission time windows configuration equal to 250 ms.

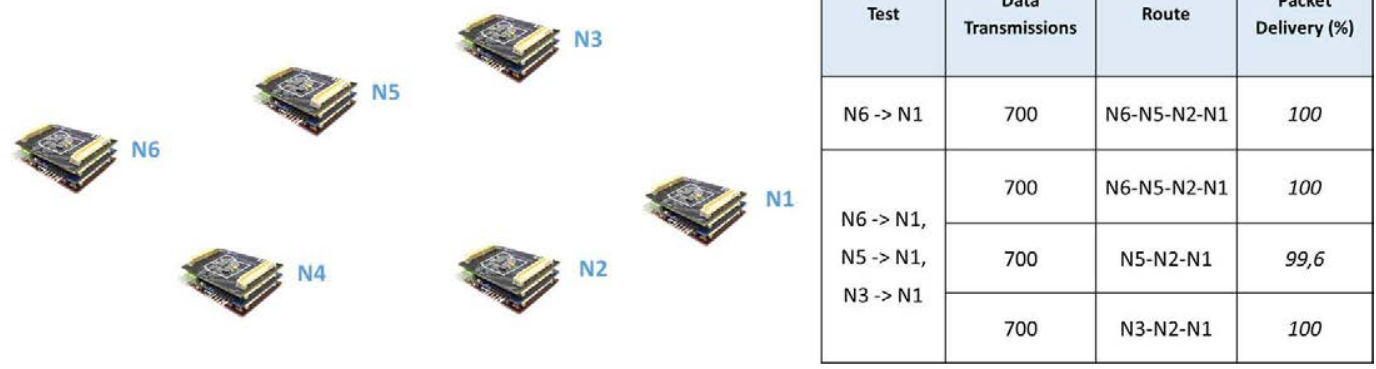

Fig. 5.9 Distribution of a 6-node sensor deployment to test the creation and data delivery by using the Cookies communication capabilities. 
As expected, in the former case the packet delivery is certainly $100 \%$ and the number of MAC retries as well as the medium assessment transactions are negligible, while in the latter case results show a good performance of the routing strategy in transferring data for such a traffic condition, obtaining an overall packet loss rate quite close to zero percent.

Thereafter, three different communication patterns were set up to further evaluate the behaviour and performance of CB-AODV. In this context, two of the topologies used to test CB-AODV are shown in Fig. 5.10 and Fig. 5.11 (left). First of all, in order to verify the expected operation of the routing protocol and its robustness throughout a period of time, nodes were configured to send packets every second to a random addresses. The packet loss rate and the network/routing map were then computed by the evaluation tool in order to obtain the degree of success during the transmission/reception process as well as the routing discovery procedure in such conditions. The TX power of the radio transceivers was also configured to modify the node coverage, so that the routing capabilities are evaluated by creating paths with the maximum amount of hops as possible (with minimum transmission power configuration), which helps developers to assess the performance of the integrated metrics. This random propagation was triggered during five consecutive hours without experimenting problems with particular nodes (considering these demanding conditions in terms of high transmission frequency and intensive traffic load pattern).

In this way, as a second test case, one of the nodes was configured to send 100 packets with a frequency of one message per second to its farthest node, i.e., a data exchange from the two most distant points of the network (nodes 1 and 9 in the figure). The main goal was to analyse the performance of both the PLR and the included metrics, especially the PATH-DR, E-aDM and the ETX, which depend on the link quality parameter. Furthermore, a third test case was also carried out in this scenario, in which a "crowded environment" was set up by configuring the rest of the nodes to send packets to random addresses, while the two distant nodes continued exchanging data between each other. 
In this traffic scenario, every node was defined to send packets to a different remote node every second, so that three main capabilities can be tested: a) the discovery process of the source node to the destination point in combination with the support of the discovery actions triggered by the rest of the nodes; $b$ ) the routing table information handled by the nodes according to the packet exchange to the request points (all the nodes are creating new routes, supporting the creation of them by other nodes and establishing the corresponding table correlations/updates based on the request-check-reply actions); c) the information processing by analysing how the management of the routing resources in the HW-SW platform can affect the normal operation of the application itself or even loose data during the transaction between two remote points in such situation.

Besides the MAC and routing frames of the protocol, the transmitted packets are composed of application information related to sensor measurements, battery level and energy consumption factors, data processing indicators and execution status. Metrics and the test configurations were launched by using the evaluation tool, and an iterative execution was done three times per configuration in order to obtain as much information of the network behaviour as possible.

In Fig. 5.10 and Fig. 5.11 (right), the main results related to the proposed scenario are classified according to the applied metric and the differences in traffic conditions, in addition to the computed routing paths, which can be analysed by means of gathering nodes information with the tool. The percentage of received packets is very close to $100 \%$, being slightly inferior in the case of traffic conditions, which thus allows verifying the reliability and operability of the protocol in such demanding situations (taking into account that the normal operation of a typical WSN produces a much lower traffic load). 


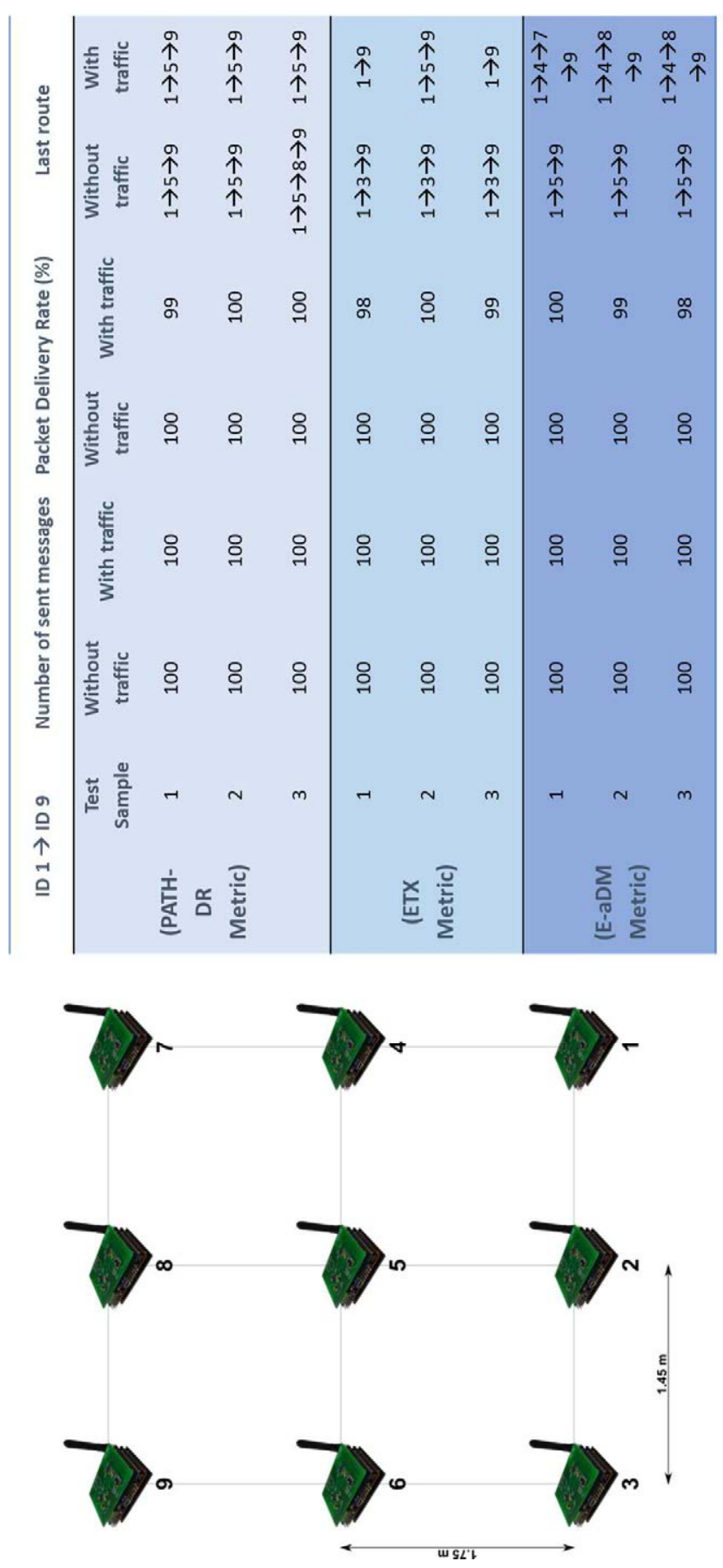

Fig. 5.10 Cookie-based mesh deployment array (left), and main results of the experimental scenario (right). 


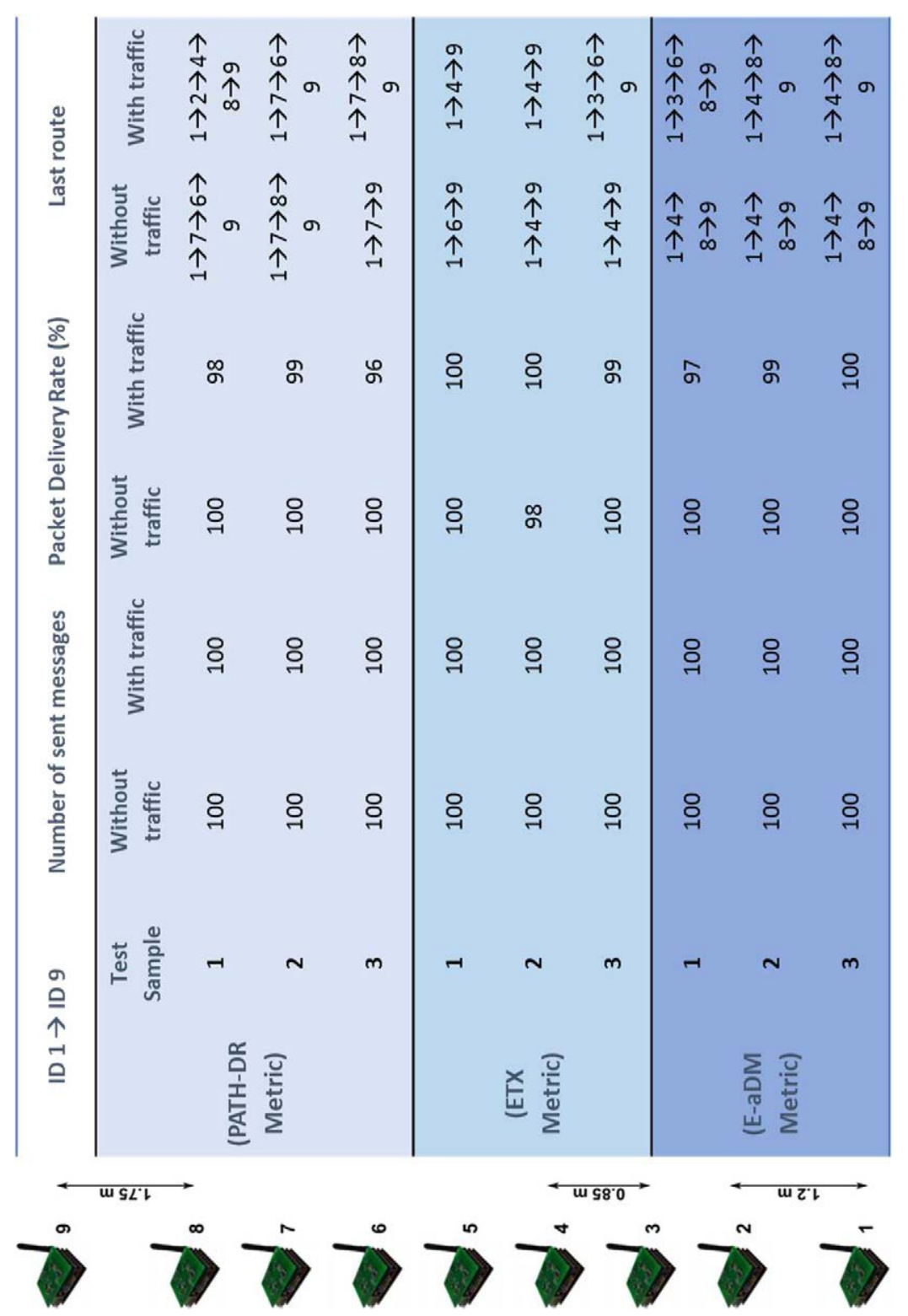

Fig. 5.11 Cookie-based aligned/string deployment array (left), and main results of the experimental scenario (right).

As a result of comparing the metric performances, the ETX has fewer hops than the PATH-DR, as expected (because ETX penalizes the number of hops). However, the percentage of messages that the final node receives is not higher in case of the PATH-DR than with the ETX, as it would be expected. Moreover, in 
case of the designed E-aDM metric, node 5 was configured to include a higher weight regarding the implemented functional blocks as well as the precursor list factor, so that a redistribution on the network routing rediscovery was obtained in the crowded environment, where packets from node 1 to node 9 follow a slightly different sequence compared to the PATH-DR, as shown in the results, thus verifying the influence of an energy conservation based approach and a balanced distribution of the packets.

\subsection{Metrics correlation and experimental characterization}

Experimental tests were carried out to establish additional correlations of the quality metrics to be considered in CB-AODV and the deployment tool, so that a better estimation and assessment of the correlation among nodes and routing discovery/maintenance mechanisms can be further approached. Apart from the experiments presented in chapter 3 related to the study of the connectivity of the modular platform, in this test case the relationship between node interconnection parameters are enhanced in order to provide additional estimation models based on the real sensor node behaviour. In this way, two Cookie nodes were configured and positioned in an outdoor environment at the same height ( $1 \mathrm{~m}$ from the ground) and distance samples starting from $1 \mathrm{~m}$ up to $35 \mathrm{~m}$. Moreover, the range of the TX power configuration of the radio modules is swept from $0 \mathrm{dBm}$ to $-25 \mathrm{dBm}$.

Besides the RSSI Threshold reference described in chapter 3, this model aims at establishing an experimental correlation between the RSSI and LQI parameters in order to include it in the on-site simulation engine of the HHD, so that a complete connectivity evaluation in terms of RSSI, LQI and LDR can be subsequently generated, analysed in-field and compared with the real behaviour of the deployment. Based on this, in Fig. 5.12 the main results of the tests are presented including the combination of values in terms of distance plus transmission power configuration, so that a defined correlation pattern between both parameters can be distinguished.

Considering this experimental correlation, a simple approximation model can be obtained based on the expression in Eq. 5.1: 


$$
l_{e}=\rho_{1} \cdot a^{3}+\rho_{2} \cdot a^{2}+\rho_{3} \cdot a+C
$$

where $\rho_{1}, \rho_{2}, \rho_{3}$ are the coefficients of the expression and respectively defined as $1.1856,-2.7009$ and $0.6305, C$ is the independent factor defined as 106.9160, a depends on the measured RSSI value (and considering a mean value $=-31.94$, a standard deviation $=14.85$, with an offset of $40 \mathrm{dBm}$ ), and finally $l_{e}$ is the resulting LQI.

\section{RSSI vs LQI}

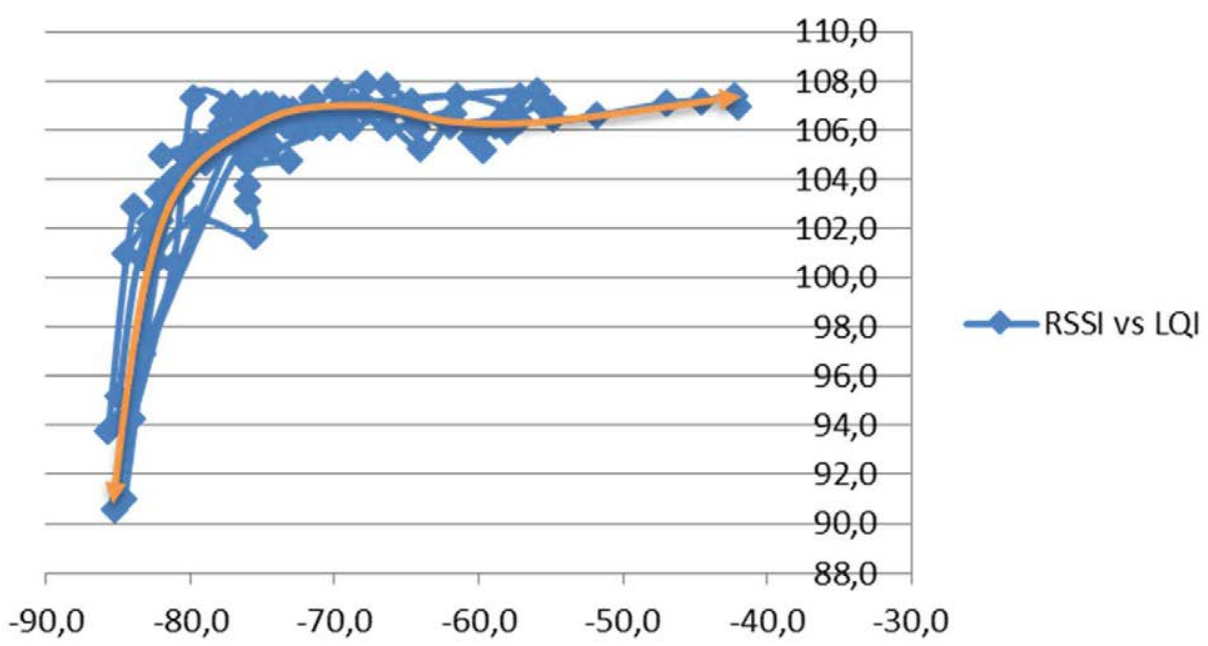

Fig. 5.12 Experimental correlation of RSSI and LQI parameters obtained from real tests and to be included within the estimation of quality metrics.

\subsection{Test case 2: Outdoor Scenario A}

In this experimental test case de main target was to analyse and evaluate the deployment cohesion of a WSN in an outdoor environment with the support of the HHD, particularly considering the generation of the real network connectivity correlation scheme from the basis of the implemented estimation and optimization strategies. The WSN is composed of 16 Cookie nodes deployed at ETSII-UPM, by combining in the same deployment a heterogeneous distribution of the nodes in terms of connectivity schema, as shown in Fig. 5.13. Considering the experimental results regarding the reliability of the links by defining a minimum RSSI to assure a stable connection between two points (-85 
$\mathrm{dBm})$, the TX power optimization mechanism provides a homogeneous configuration of the transmission parameter to be set to $-5 \mathrm{dBm}$, from which the connectivity map for the target deployment has been built as shown in Fig. 5.15 in accordance with the estimation models (bottom-left side).

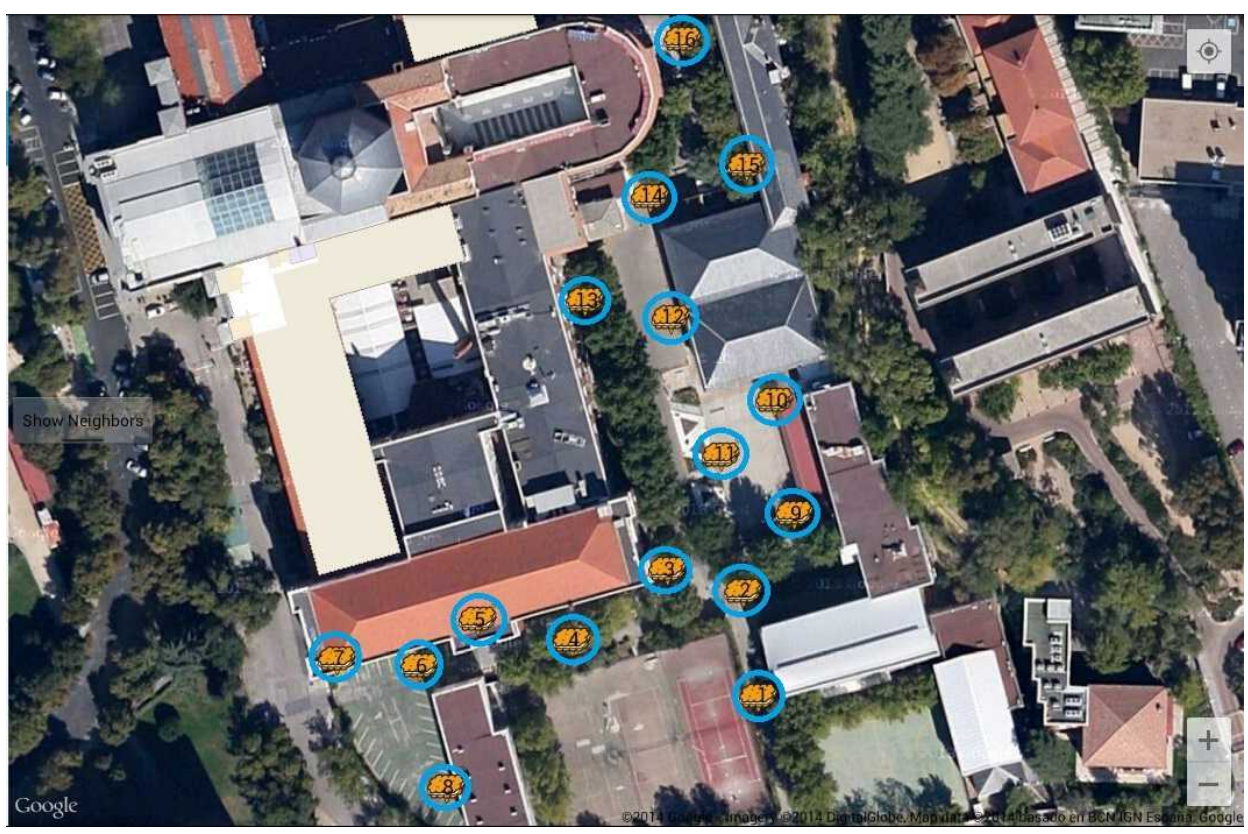

Fig. 5.13 WSN deployment scenario composed of 16 Cookie-based sensor nodes at ETSII-UPM.

The HHD establishes the correlation points where a connection shall be obtained (in white colour). The purple connections directly affected by obstacles are highlighted, so that no links shall be expected in those pairs of points. In red are the values above the defined RSSI threshold, which defines the limit below which the connectivity is not assured. Such a connectivity array serves as the input for the optimization strategies to produce the deployment points to be used in the generation of the real connectivity correlation scheme. Table 5-3 summarizes the main configurations that are considered for the execution of the proposed algorithms, from which the different combinations taking into account the deployment methods have been analysed. The outcomes of the optimization execution process are shown in Fig. 5.14, where a comparison of the GA-based and CA-based mechanisms for methods 1 and 2 is provided. 


\begin{tabular}{|c|c|}
\hline Parameter/Attribute & Configuration \\
\hline TX Power & $\begin{array}{r}\text { Type of strategy }=\text { Homogeneous } \\
P t=-5 \mathrm{dBm}\end{array}$ \\
\hline RSSI Threshold & RSSIlim $=-85 \mathrm{dBm}$ \\
\hline GA-Based & Population $=100$ \\
Optimization & $\begin{array}{c}\text { Generation limit }=300 \\
\text { Convergence rate }=90 \%\end{array}$ \\
\hline $\begin{array}{c}\text { Optimization } \\
\text { OptBased }\end{array}$ & Maximum Size $=30 \%$ \\
\hline
\end{tabular}

Table 5-3 Configuration parameters of the deployment optimization strategies for the target application scenario.

Optimization algorithms - Fitness results

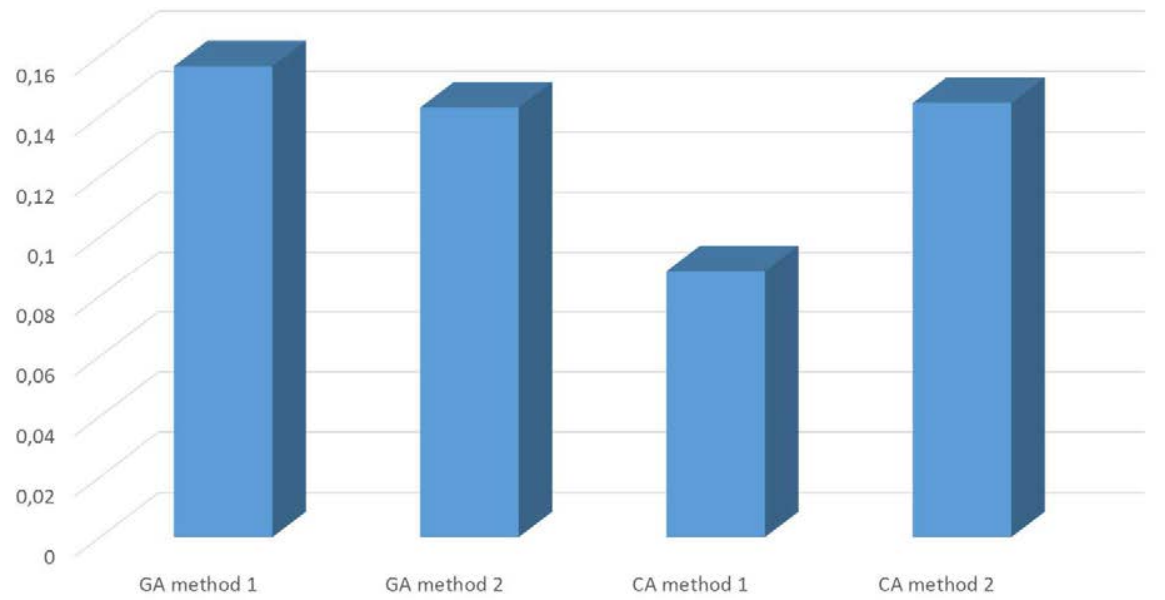

Fig. 5.14 result comparison of the GA and CA methods for the WSN experimental scenario.

Taking into account that the Kps-based strategy produces proper results in both cases, which are certainly closed to the optimal value obtained for the point-topoint technique with GA, and considering the deployment evaluation and maintenance suitability of the WSN based on the key strategy-points, the NDoSq from the second combination of such results has been used to carry out the real 
generation and analysis of the in-field network correlation scheme and connectivity evaluation. This means that several sensor nodes are automatically correlated through the evaluation of Kps points of $\operatorname{NDoSq}(1,4,8,9,13$ y 16).

Based on this the real connectivity map is built in-field to be compared with the simulated one, by performing the WSN deployment with the support of the HHD. According to the previous results, nodes have been configured with the homogeneous transmission power of $-5 \mathrm{dBm}$. In Fig. 5.15 (upper-right side), the main average experimental results are reflected by computing 4 consecutive tests per link (bidirectional connections) and the comparison with the theoretical calculation are generated and analysed. Highlighted in white are the real and estimated values between pairs of nodes, whereas in blue are the connections that were not expected from the simulation though in the real implementation such pairs of nodes did connect. In orange those connections that were not established are represented.

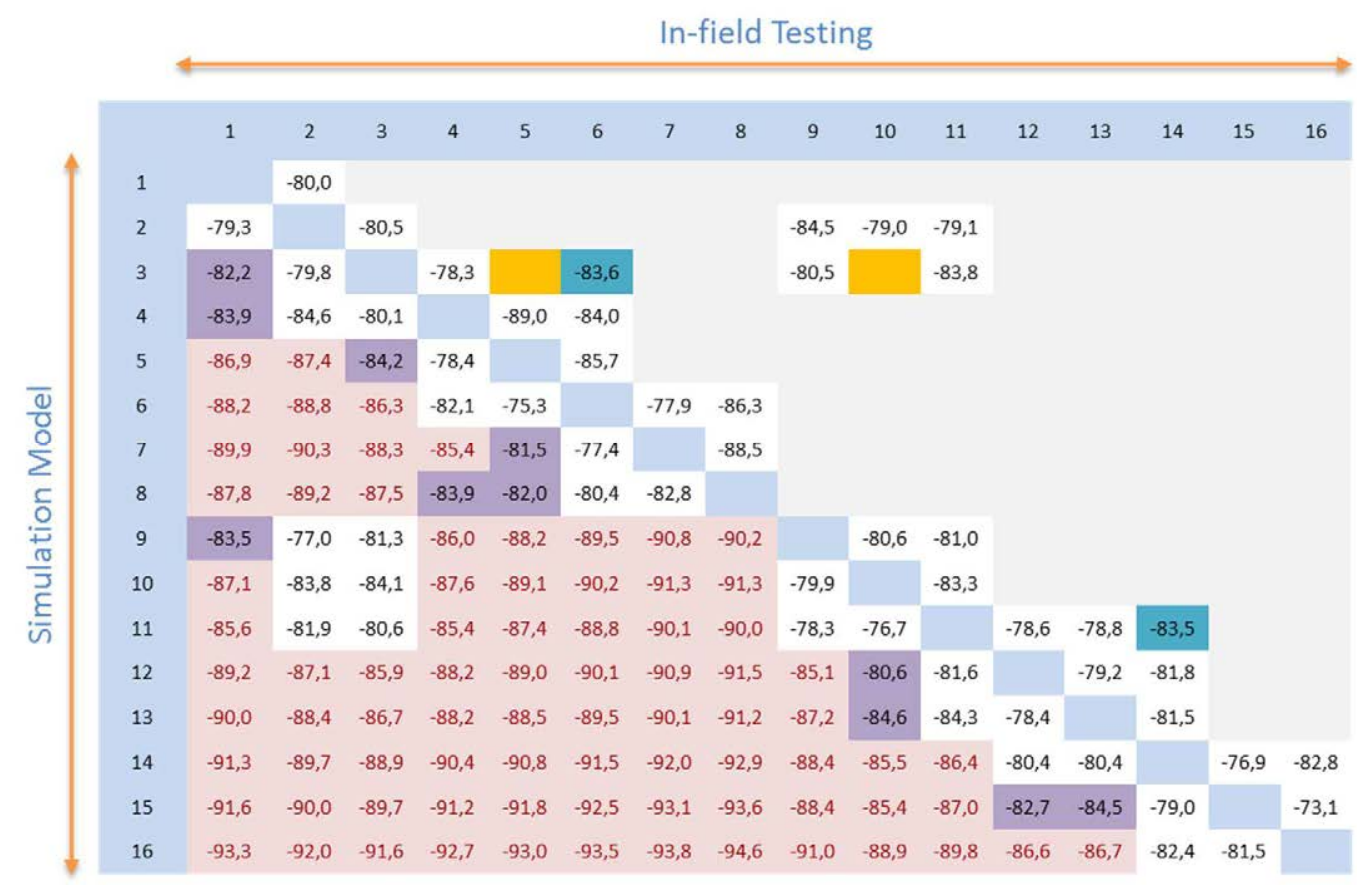

Fig. 5.15 Comparative analysis of the in-field connectivity results with the simulation model outcome. 
In such cases (connection mismatches), the real values are very close to the defined quality limit, so the estimation is pretty much accurate to predict those links that are prone to suffer instability and, therefore, to be marked as unconnected points. The rest of the simulated 2-Ns that produce stable connections were indeed validated in the real implementation when establishing the comparison with the HHD in-situ. As a result of the deployment and evaluation tool, the average error of the comparative analysis in terms of the simulated and real metrics is $4 \%$, having maximum deviations $(12 \%)$ in those communications where unexpected/not-considered obstacles have influenced the performance of the connections. That thus provides a proper practical estimation level of the network connectivity scheme, so that on-site real time configurations and readjustments on the functional components of the sensor platforms can be performed in order to meet the expected requirements in terms of overall network cohesion and nodes correlation establishment.

These types of on-site study can also be carried out by graphically comparing the connectivity performance of every pair of points as represented in Fig. 5.16 for this particular outdoor scenario, in which the orange lines indicate the simulation results while the green ones correspond to the real gathered data. The thickness of the lines matches with the quality and stability of the interconnections between pairs of nodes. This provides users with the possibility of directly analysing in real time how reconfigurations in terms of communication capabilities (consumption modes and transmission power) and location changes might influence the surrounding neighbourhood or even the whole deployment. When changing the properties of a node at runtime, the connectivity correlation is dynamically recalculated, so that deployers can observe how such modifications affect the overall performance and cohesion of the surrounding area, as well as the overall sensor network.

This leads user to having a more comprehensive understanding about the effects of reconfiguring parameters of the sensor platform and its physical installation in the target area, whose outcome may change the behaviour of the local node but also the ones that are correlated to it. 


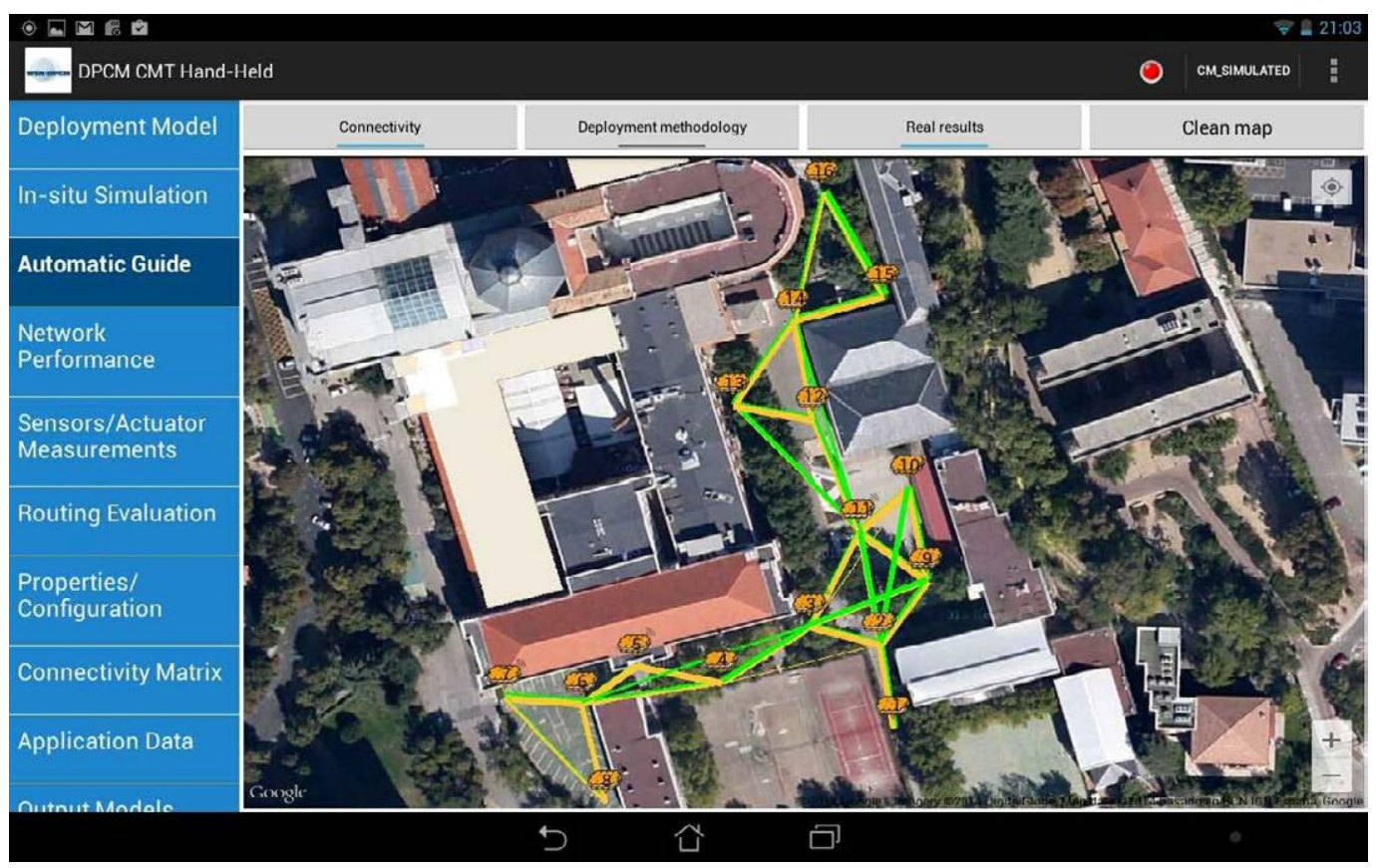

Fig. 5.16 Graphical representation of the comparative analysis between the estimation and real on-site experimental data by using the HH interface.

Regarding the performance of the routing capabilities based on this real connectivity map, packet delivery tests were triggered to mainly compare the behaviour of CB-AODV by including ETX and E-aDM in the study, both targeting quality of the interconnections but the former one penalizing the number of hops whereas the latest one balancing the distribution of the paths. Under these outdoor conditions, tests were launched from different deployment areas, and considering nodes 8 and 13 as remote pairs of points to show the result analysis, in Table 5-4 the comparison of the routing metrics for a total of 100 packets per test is represented. It can be seen the effect of hop penalization in ETX, whereas with E-aDM the routing load balance has been distributed among nodes 2, 9 and 11 to guarantee that the creation of critical points is avoided as much as the deployment topology allows such optimization.

The use of the ETX metric may be suitable for those applications in which transmission and processing delays (based on hop reduction) are a key factor for the performance of the required service, whereas E-aDM provides a more balanced link correlation among remote points in terms of energy conservation 
in addition to resource/processing constraint awareness in specific nodes of the deployment. E-aDM allows a more precise tuning of the path selection targeting energy and processing equilibrium among the deployed nodes, although for more directional topologies in with the routing capabilities are more concentrated in specific rely points of the network, ETX can provide good results without specific parametrization. One of the advantages of the proposed evaluation tool is that by using the HHD deployers can analyse in real time what metrics can be more adapted and suitable depending of the specific requirements and behaviour of the in-field target application, so that a runtime reconfiguration process can be performed in-situ accordingly.

\begin{tabular}{|c|c|c|c|c|}
\hline ID $8 \rightarrow$ ID 13 & Test Sample & $\begin{array}{c}\text { Number of sent } \\
\text { messages }\end{array}$ & $\begin{array}{l}\text { Packet Delivery Rate } \\
\text { (\%) }\end{array}$ & Last route \\
\hline \multirow{3}{*}{$\begin{array}{c}\text { ETX } \\
\text { Metric }\end{array}$} & 1 & 100 & 100 & $8 \rightarrow 6 \rightarrow 5 \rightarrow 3 \rightarrow 11 \rightarrow 13$ \\
\hline & 2 & 100 & 98 & $\begin{aligned} 8 \rightarrow 6 \rightarrow 5 & \rightarrow 4 \rightarrow 3 \rightarrow 11 \\
\rightarrow & 13\end{aligned}$ \\
\hline & 3 & 100 & 100 & $8 \rightarrow 6 \rightarrow 5 \rightarrow 3 \rightarrow 11 \rightarrow 13$ \\
\hline \multirow{3}{*}{$\begin{array}{l}\text { E-aDM } \\
\text { Metric }\end{array}$} & 1 & 100 & 100 & $\begin{aligned} 8 \rightarrow 6 & \rightarrow 5 \rightarrow 3 \rightarrow 2 \rightarrow 11 \\
& \rightarrow 12 \rightarrow 13\end{aligned}$ \\
\hline & 2 & 100 & 100 & $\begin{array}{c}8 \rightarrow 6 \rightarrow 5 \rightarrow 4 \rightarrow 3 \rightarrow 2 \rightarrow \\
11 \rightarrow 12 \rightarrow 13\end{array}$ \\
\hline & 3 & 100 & 99 & $\begin{aligned} 8 \rightarrow 6 & \rightarrow 5 \rightarrow 3 \rightarrow 2 \rightarrow 11 \\
& \rightarrow 12 \rightarrow 13\end{aligned}$ \\
\hline
\end{tabular}

Table 5-4 Experimental results of the routing strategies by using ETX and E-aDM in the comparative analysis.

\subsection{Test case 2: Outdoor Scenario B}

This experimental case was focused on the use of the HHD to properly produce the desired network connectivity and real nodes correlation scheme from the basis of the topology and deployment optimization strategies, so as to install the sensor nodes with a balanced configuration regarding the transmission parameters, in addition to validating the functional component implementation of the embedded application level in terms of sensor processing and in-field environmental measurements. 
The target distribution is based on an outdoor scenario comprising 9 nodes with a mesh topology, as shown in Fig. 5.17, and with the inclusion of environmental sensors in the platform implementation: temperature, humidity and light intensity. As shown in Fig. 5.17 the TX power optimization mechanism provided the generation of a balanced configuration of the transmission parameter to be set to $-10 \mathrm{dBm}$. Based on that, the connectivity estimation for the sensor network is generated in order to provide the deployment methodology with the overall correlation scheme of the nodes, so that and optimal NDoSq can be created for the in-filed configuration and evaluation of the sensor platforms. The GA-based strategy for the point-to-point method provides an optimal $N D o S q=\{5,6,3,2,1$, $4,7,8,9\}$ upon which the runtime evaluation tests were triggered.

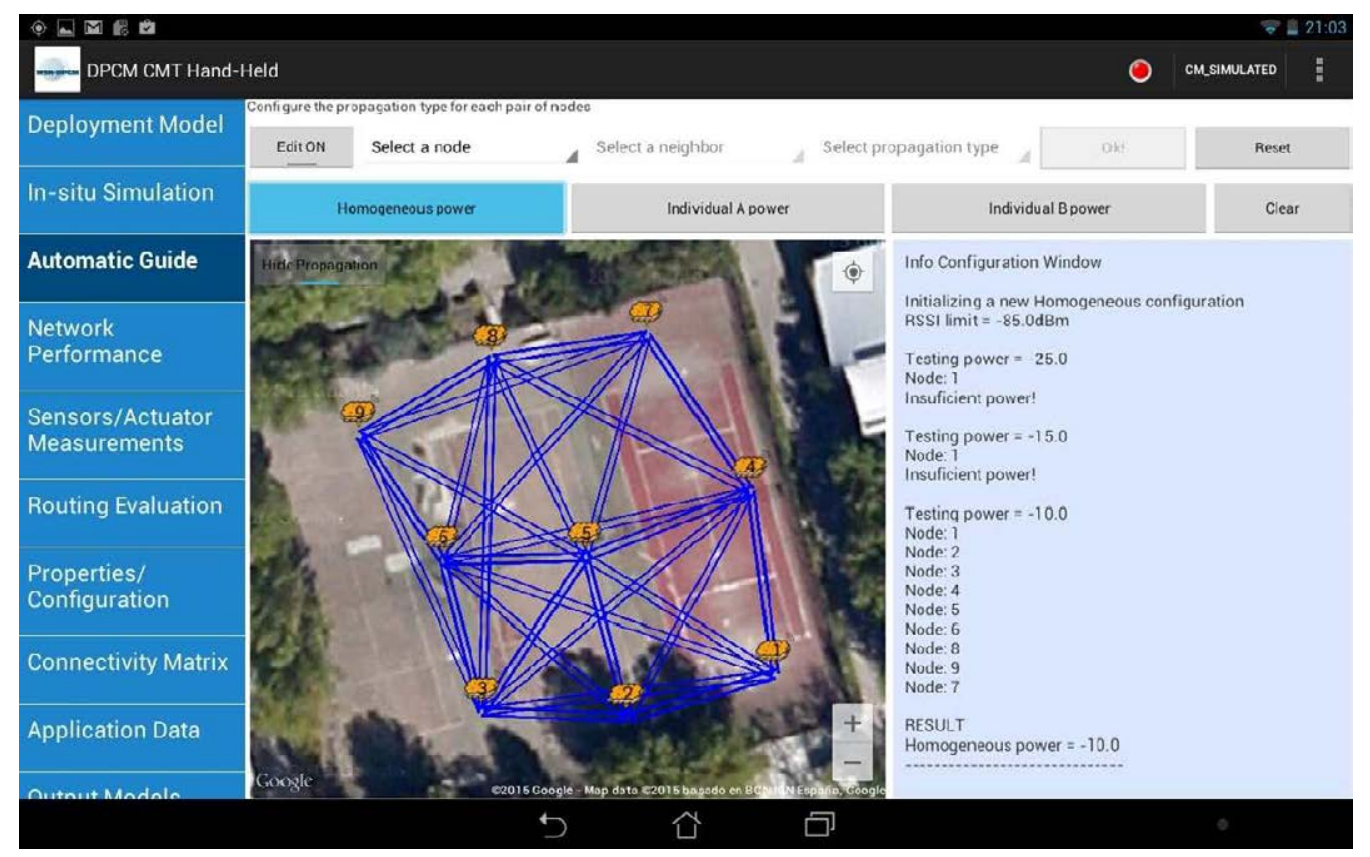

Fig. 5.17 TX power optimization and configuration for the proposed experimental scenario.

Table 5-5 summarizes the main results regarding the in-field connectivity scheme compared to the estimation values for the network objective, whose graphical representation is provided in Fig. 5.18 (orange lines represent estimated connections whereas green ones correspond to the real gathered data, and considering the thickness as the quality of the connection). On one hand, 3 nonexpected points where encountered as connected links though 2 of them are 
certainly close to the quality boundaries, and on the other hand two expected connections where not obtained as a result of the evaluation process. In both cases the ratio of the value deviation corresponds to less than $2 \mathrm{dBm}$ around the limit.

\begin{tabular}{|c|c|}
\hline Parameters & Outcome \\
\hline Number of pair-point evaluations & 36 \\
\hline Average Error & $4.1 \%$ \\
\hline Non-expected connected pair-points & $3(1)$ \\
\hline Unexpected Unconnected pair-points & $2(0)$ \\
\hline
\end{tabular}

Table 5-5 General results of the comparative analysis of the estimation + real nodes correlation.

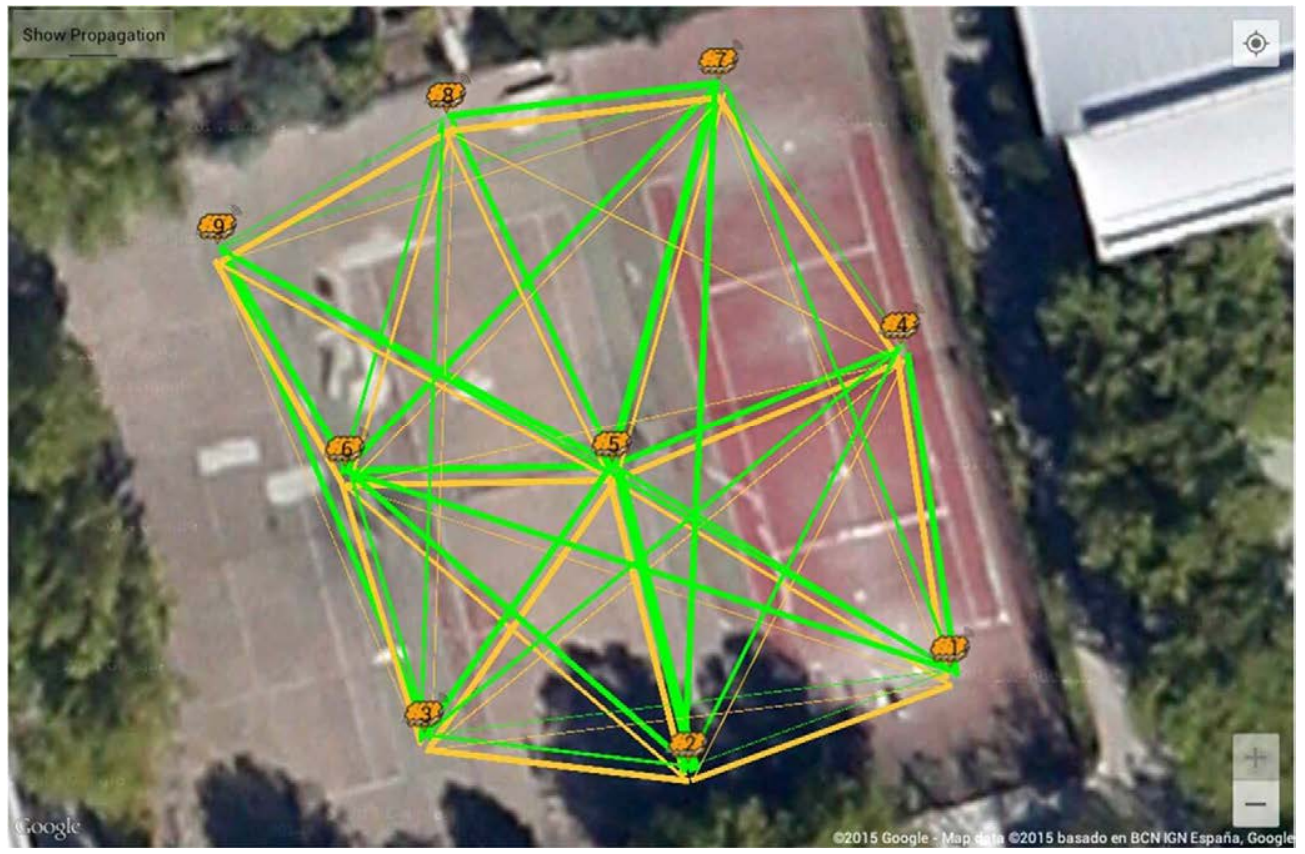

Fig. 5.18 Graphical representation of the comparative analysis between the estimation and real on-site experimental data of scenario B, by using the HH interface.

Apart from this comparative analysis, Fig. 5.19a) shows how runtime verification of the functional components related to implemented sensors is performed and studied, in addition to the bidirectional evaluation of correlated points under test (Fig. 5.19b)). 
You've chosen Node

Check sensors:

$\checkmark$ Temperature $\checkmark$ Humidity $\checkmark$ LOR $\square$ Battery Level

$\odot$
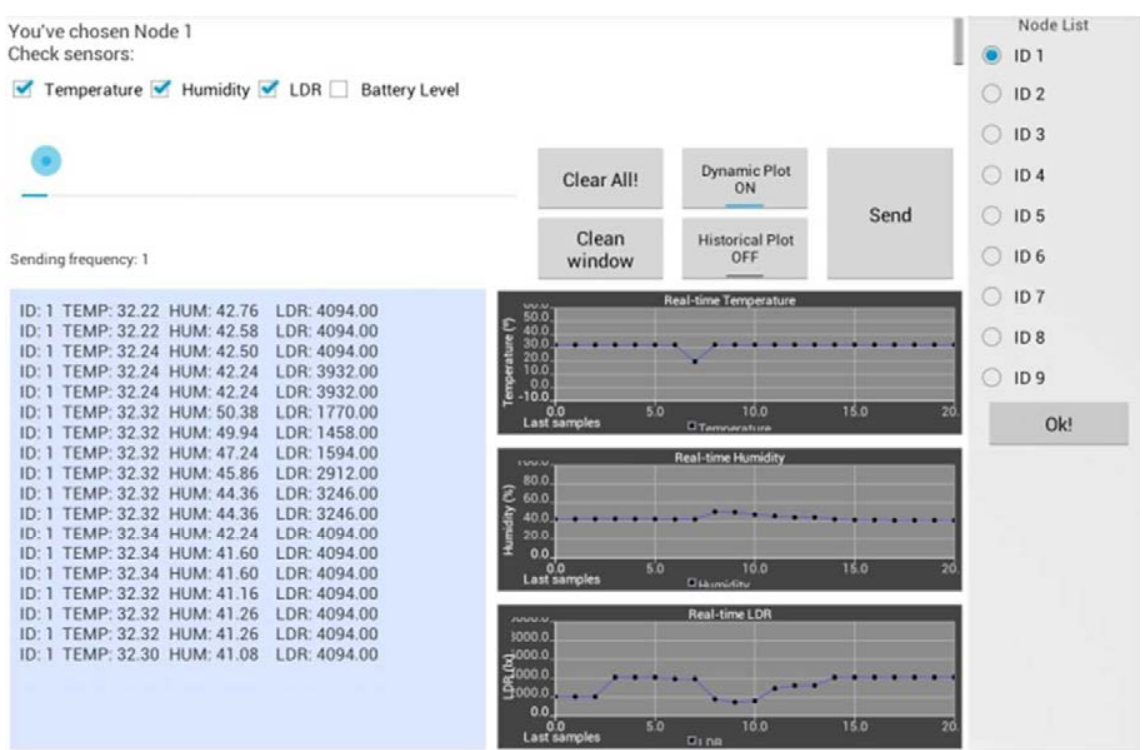

ID: 1 TEMP: 32.22 HUM: 42.76 LDR: 4094.00 ID: 1 TEMP: 32.22 HUM: 42.58 LDR: 4094.00 ID: 1 TEMP: 32.24 HUM 4250 LDR: 4094.00 ID: 1 TEMP: 3224 HUM: 4224 LDP: 393200 ID: 1 TEMP: 32.32 HUM: 50.38 LDR: 1770.00 ID: 1 TEMP: 32.32 HUM: 49.94 LDR: 1458.00 ID: 1 TEMP: 32.32 HUM: 47.24 LDR: 1594.00 ID: I TEMP: 32.32 HUM: 45.86 LDR: 2912.00 D. T TEMP. 32.32 HUM: 44.36 LDR: 3246.00 D. TEMP: 32.32 HUM: 44.36 LDR: 3246.00 D: 1 TEMP: 3234 HUM: 41.60 LDP: 409400 ID: 1 TEMP: 32.34 HUM: 41.60 LDR: 4094.00 ID: 1 TEMP: 32.32 HUM: 41.16 LDR: 4094.00 ID: 1 TEMP: 32.32 HUM: 41.26 LDR: 4094.00 D.1 TEMPP 32.32 HUM: 41.26 LDR: 4094.00 D: 1 TEMP: 32.30 HUM: 41.08 LDR: 4094.00

a)

You've chosen Node 2

\begin{tabular}{|c|c|}
\hline Test 1 & Hand-Held neighbou \\
\hline Test 2 & Node neighbours \\
\hline Test 3 & Neighbours connecti \\
\hline Test 4 & Packets delivery \\
\hline \multicolumn{2}{|c|}{ Select a destination node 1D: Neighbor 3} \\
\hline \multicolumn{2}{|c|}{ Info from Node 2 to Node 3} \\
\hline \\
\hline \multicolumn{2}{|l|}{$\begin{array}{l}\text { RSSI to: }-42 \\
\text { Lal to: } 89\end{array}$} \\
\hline \multicolumn{2}{|c|}{ Metric LDR to: $94 \%$} \\
\hline \multicolumn{2}{|c|}{ RSSI from: -44} \\
\hline \multicolumn{2}{|c|}{ LQI from: 87} \\
\hline \multicolumn{2}{|c|}{ Metric LDR from: $91 \%$} \\
\hline \\
\hline \multicolumn{2}{|c|}{$\begin{array}{l}\text { Initial coordinates: } \\
40.4393309\end{array}$} \\
\hline \multicolumn{2}{|c|}{-3.68879399} \\
\hline \multicolumn{2}{|c|}{$\begin{array}{l}\text { Final coordinates: } \\
40.43934\end{array}$} \\
\hline \multicolumn{2}{|c|}{$\begin{array}{l}40.43934 \\
-3688942\end{array}$} \\
\hline \multirow{2}{*}{\multicolumn{2}{|c|}{ Date: $10 / 3 / 2015$}} \\
\hline Hour: 5:36:2 & \\
\hline
\end{tabular}
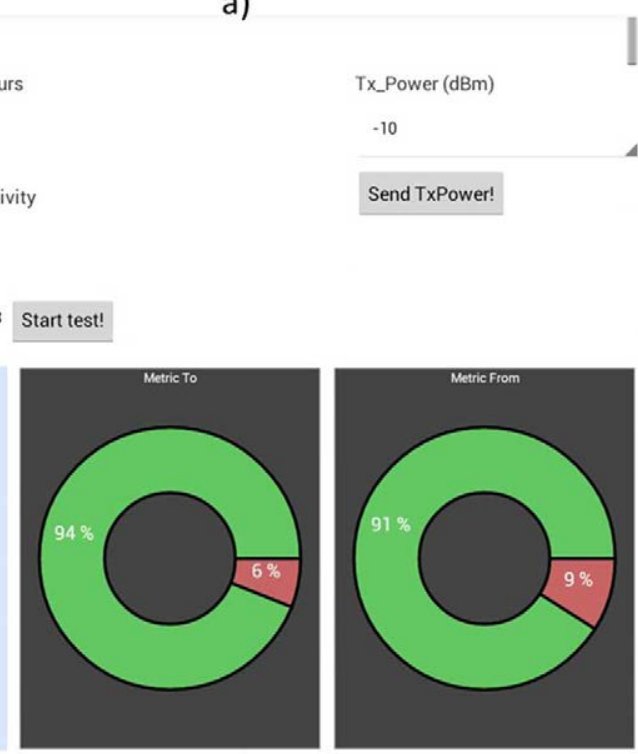

b)

Fig. 5.19 in-field verification of node functionalities, a) sensor retrieving configuration and runtime measurement behaviour b) medium access assessment and analysis of connectivity metrics.

Regarding the performance of CB-AODV, computations of packets delivery tests were performed between nodes 1 and 9 with the configured intermediate TX power and applying E-aDM by assigning node 5 a weight in terms of blocks processing equal to 4 , and the results generated the creation of a route passing 
through nodes $1 \rightarrow 6 \rightarrow 9$ with a metric $=99 \%$ (see Fig. 5.20a)). Fig. 5.20b) shows the result of configuring a packets delivery test between these points to analyse remote data exchange by using CB-AODV and the HHD.

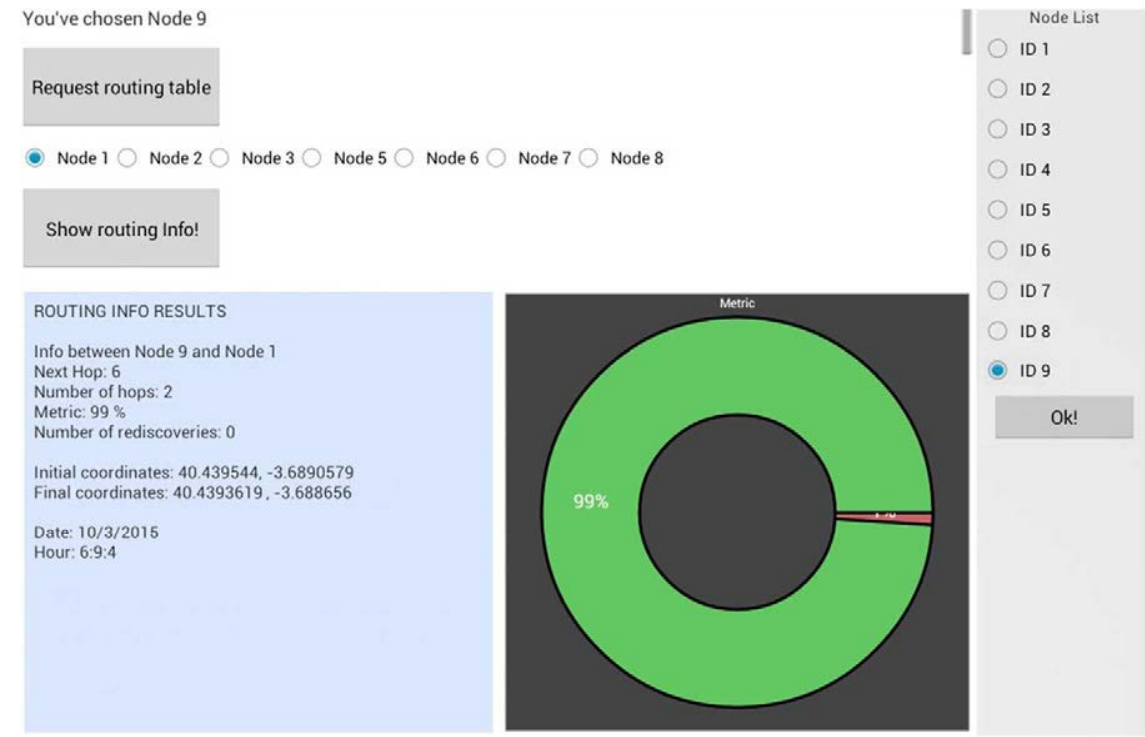

a)

You've chosen Node 9

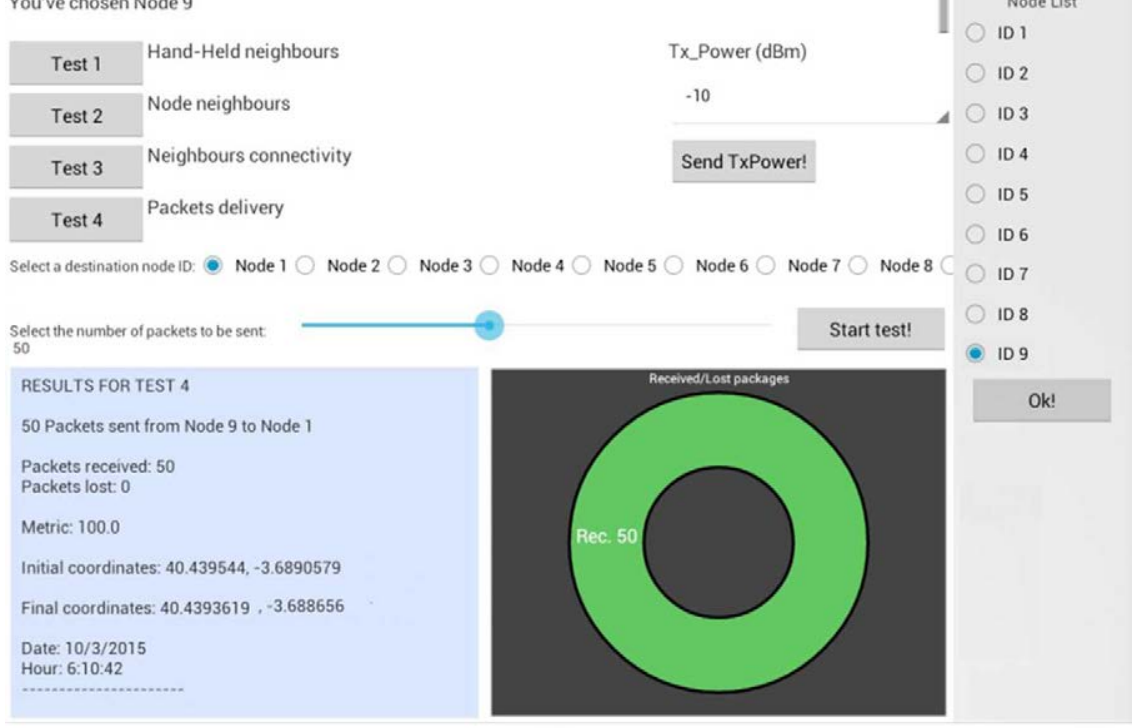

b)

Fig. 5.20 analysis of the routing performance, a) creation of a route from node 9 to $1, b$ ) packet delivery configuration and test results presentation. 
Moreover, subsequent delivery tests have been performed to analyse the impact of insolating part of the nodes along the paths, so as to verify the dynamic reconfigurability of $\mathrm{CB}-\mathrm{AODV}$ in such conditions, as the one depicted in Fig. 5.21 , where the rediscovery mechanism produces a new route (from node 1 to node 3 ) of $1 \rightarrow 4 \rightarrow 5 \rightarrow 3$ and with an average delivery ratio of $98 \%$ for the total amount of tests (more than 900 in-field test verifications automatically handled by the evaluation tool). Fig. 5.22 provides a screenshot of the routing information of the involved sensor nodes in order to verify how multi-hop communication is produced by means of using the routing strategy under evaluation.

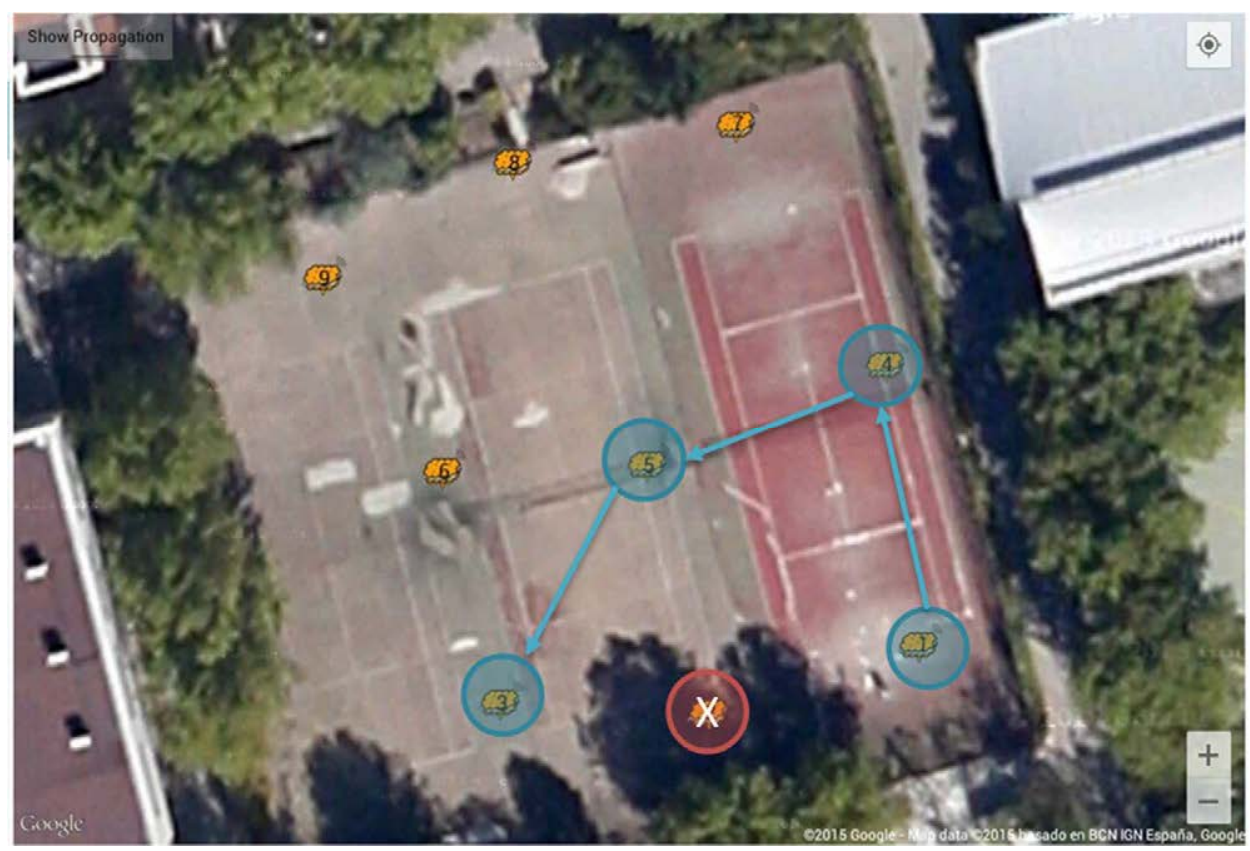

Fig. 5.21 Evaluation of the routing rediscovery when detecting errors in route entries. 


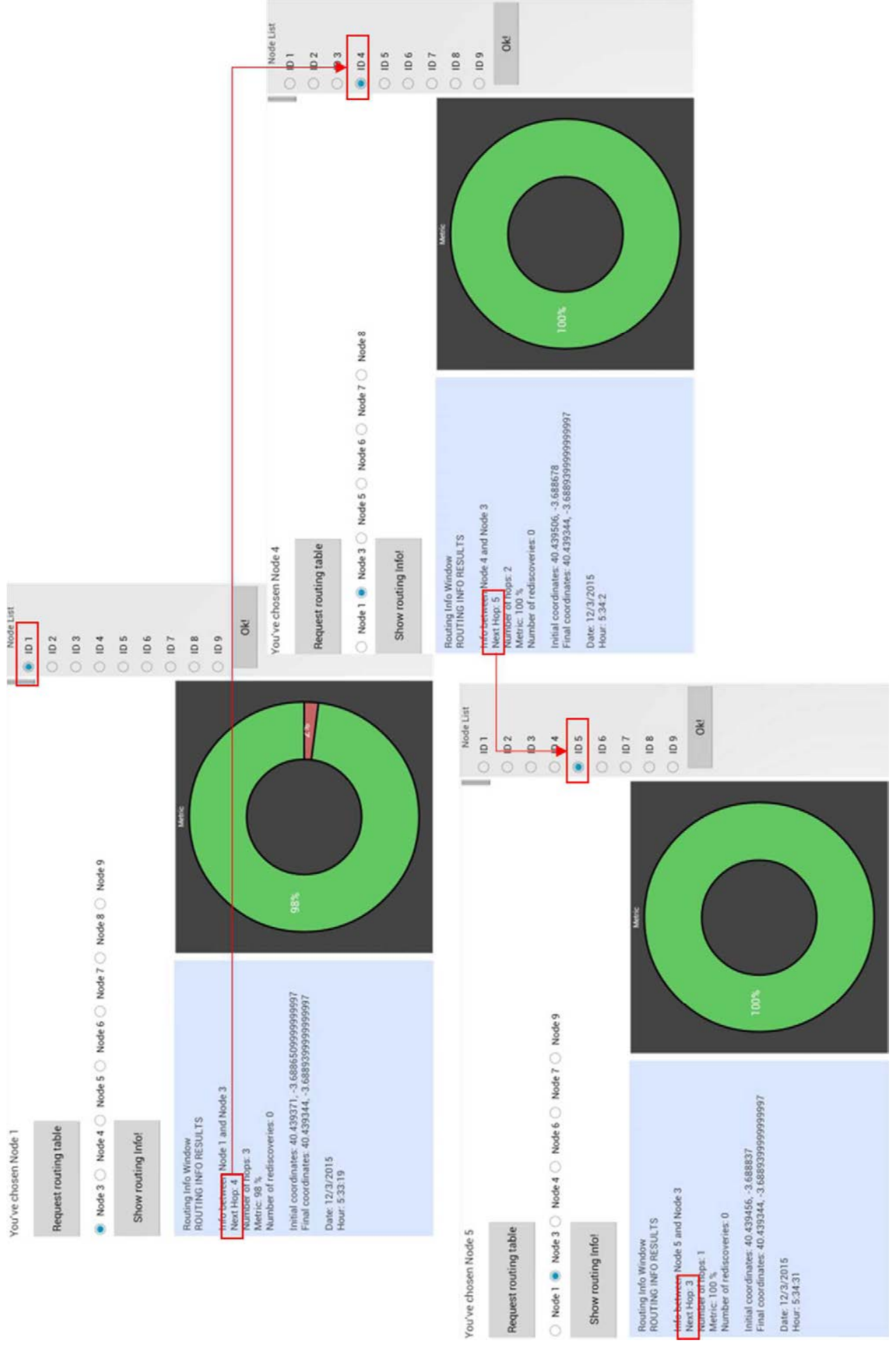

Fig. 5.22 Creation of an alternative route and results representation of the rediscovery mechanism by using the HH diagnosis tests visualization. 


\subsection{Smart parking demonstrator: WSN-DPCM European project validation}

The design and implementation of the HHD has also been part of a very challenging framework for the development of WSN-based applications, within the scope of the ARTEMIS/ECSEL-JU WSN-DPCM European funded project [DPCM]. The main target of the DPCM project was to build a multi-domain platform in which the various stages that involve the planning, development, commissioning and maintenance of wireless sensor systems are integrated into a hardware-software tool-chain. The architectural definition of this support platform relies on the reusability and enhancement of existing technologies that are integrated by means of using common interfaces and data model exchange among the participant tools and functional components, so that the WSN design, implementation, testing, and deployment efforts from the developer perspective are simplified, who can then focus on application requirements and its implementation based on the integrated components.

The WSN-DPCM project is aligned with and provides inputs to several ARTEMIS subprograms, highlighting ASP 3 Smart Environments, which aims at providing methods, tools, and innovative technologies to leverage the building of smart systems composed of heterogeneous devices that cooperatively interact with each other and the target environment to produce application services. Partners' main focus and efforts are concentrated on spreading the use of WSN technologies not only from the research and academy perspective but also enclosing industrial contexts, by means of offering an overall support sensor-touser integrated framework that fosters the development of novel smart solutions for the community, corporations, and institutions.

In order to address such challenging objectives, the DPCM toolset considers on one hand three different systems within the integration process, that is, development, planning, and commissioning and maintenance tools and related components; and on the other hand the definition of a middleware that provides cross-connectivity, interoperability, and technology encapsulation among different WSN hardware-software platforms and vendor providers. In this 
direction, three main capabilities can be distinguished to establish the integration of the heterogeneous components as well as the top-level system interface approach, as follows.

- WebTop Environment: provides the top level interface that serves as the main entry point for users to access the rest of the tools and functionalities of the integrated platform. It allows triggering and checking the different status of the development flow in accordance with the execution and outcomes of the involved tools and components.

- Common Data Repository: contains the information generated by the different tools which serves as the input/output data for the various components and subsystems that participate in the development flow, so as to execute the corresponding implementation, testing, and/or monitoring tasks.

- Service Interfaces: provide the entry point for accessing the core functionalities of the various subsystems as well as the data models reported by the tools, in order to feed the overall platform with the capabilities of the integrated hardware-software components.

While the planning tool (PT) provides users with a graphical interface for the definition of the WSN to be developed based on input requirements (types of sensor nodes, location details and positioning constraints, sensing objectives), as well as pre-deployment analysis and simulation capabilities; and the development tool (DT) allows the production of application-specific software to be included in the embedded devices from the basis of programming and automatic synthesis capabilities considering the use of libraries of hardware and software components, and behavioural simulation engines (thus hiding lowlevel implementation details, so that users can be certainly focused on the required application details by employing a top-level abstraction interface); the commissioning and maintenance tool (CMT) is the main responsible element for assisting developers in the configuration, on-site installation and evaluation, runtime optimization based on specific details encountered when carrying out the deployment tasks, and in-field validation/operational release of the overall platform, which also supports the monitoring, debugging, and maintenance 
stages once the WSN application is running. The middleware technology (MW) implemented in the DPCM toolset supports not only software reusability and development productivity but also the runtime operation with the commissioning and maintenance tool in order to establish a proper interaction with the hardware and software components that encompass the smart embedded system under deployment/monitoring.

In this way, the Hand-Held device designed and implemented in this thesis represents an integral part of the DPCM platform and contributes especially to the commissioning and maintenance tool as the reference on-site technology to perform the described deployment capabilities. The CMT-HHD is fully integrated into the overall toolset with a particular focus on the middleware interaction so as to assure a high degree of interoperability, portability, heterogeneity, and ease of integration with different embedded platforms. Furthermore, the CMT-HHD establishes a tied cooperation with the CMT-Server in order to properly realize the WSN application monitoring and debugging by interactively exchanging information of both the gathered data from the sensor platforms and runtime reconfiguration tasks, so that further optimization and refinement actions can be performed accurately.

The effectiveness of the WSN-DPCM toolset has been evaluated through the design and implementation of a smart-application demonstrator environment, where the development flow of the WSN-based system considering the various tools involved in the overall platform and their resulting integration is validated in the context of a real scenario. The aim of this experimental test case was to build and assist the deployment of a smart parking in which the top-level application consisted of managing and monitoring the occupancy of the parking slots in an objective area by using a wireless sensor network, whose prototype has been tested within the facilities of the ETSII-UPM.

The main goal of this scenario included in the context of smart and sustainable city applications is the detection of free parking places so that motorists can be automatically guided to those parking spaces where there is availability for parking their cars, providing relevant and up-to-date information of the state of the car park as well as the associated booking system through the use of smart 
devices. To accomplish that, the WSN system shall be built from the basis of the middleware architecture and the development, testing and commissioning components of the DPCM toolset, so that the benefits and usability of the proposed framework can be clearly shown under a smart environment that may be scaled or extrapolated to other application scenarios as well.

\subsubsection{Demonstrator requirements and system architecture}

The smart parking management relies on the combination of a wireless sensor network that allows the effective monitoring of the state of the target car park area with the interaction of the users by means of their mobile devices (smartphones and/or tablets), from which they can, on one hand, be guided to the location of the parking space that has been booked, and on the other hand manage their reservation account in order to perform booking actions in specific time slots. This means that a real time monitoring system has to be built in order to cope with the online and remote data exchange nature of the proposed smart environment. This system specification leads to defining key input requirements to approach the design and implementation of the smart application, summarized as follows.

User/System interaction: users are to be provided with a set of capabilities to interact with the smart parking application by using their smart devices, considering the following functions:

- App account: allows the creation and edition of personal accounts in order to access the smart parking, and create a user profile to start using the capabilities of the booking and monitoring system.

- Parking areas: refers to an overall view of the different available parking areas where users can book a parking slot.

- Slot availability and booking: shall include the monitoring of the status of parking space as well as their time remaining in case they are already booked/occupied. Alarm configurations as well as special messages to indicate status changes are to be provided. Based on this information, users shall be capable of reserving a specific place for a configurable time slot, in addition to being notified about the status of its reservation. 
- Parking guidance: this function shall provide users with an automatic indication to the corresponding parking space location where they made a reservation.

Deployment structure: the WSN that supports the smart environment shall be primarily composed of at least one sensor node per parking space in order to detect the presence of cars in the target area. The sensor platforms are to be equipped with batteries and shall encompass low-power-consumption components that allow the management of several operational modes of the devices. Furthermore, environmental sensors shall also be included in order to provide the top-level application with additional information of the scenario, particularly related to temperature and humidity measurements. The runtime information provided by the deployed sensor network is to be collected by gateway elements (with the support of router points if necessary) that establish the communication between the WSN and remote servers, so that updated data can be supplied to the different components that take part in the remote management and monitoring of the smart environment. In this direction, the server side will provide the rest of the top-level platform with the state of the smart parking, not only from the point of view of the detector sensors themselves, but also relevant information related to the sensor network behaviour, such as alive nodes, connectivity status, packet delivery rates, environmental measurements, battery level, etc., which is to be supported by the on-site inspection and assessment of the deployed system.

Communication capabilities: the communication technology that shall take part in the data exchange among the sensor nodes of the smart environment is to be enclosed by low-power protocols from the basis of the IEEE 802.15.4 standard, upon which routing and dissemination techniques are to be included in order to guarantee a proper data distribution throughout the overall system. Moreover, high-level communication protocols shall also be considered in order to establish a proper interaction between the gateway elements, the server side and the mobile agents that contribute to the dynamics of the smart environment, taking into account IEEE 802.11/802.3 among the deployment structure, and 3G/4G connections among the participant elements of the smart parking application. 
Software components: various software levels shall build the overall architecture of the smart environment, considering the support of the middleware not only to manage the embedded sensor nodes, but also to establish a proper interaction with the CMT and thus the top-level application. In this way, three different profiles are to be considered within the interface definition for the smart environment: a client profile that allows monitoring and managing the parking slots from the user perspective in order to be capable of booking and following the state of the target area; a user profile that is intended to observe the behaviour of the sensor network, which is certainly associated with a first/basic developer level; and an expert profile that provides more advanced capabilities related to the control and monitoring of the WSN considering runtime configuration and evaluation actions, node diagnosis tasks, monitoring of performance parameters, and statistical analysis of the platform operation.
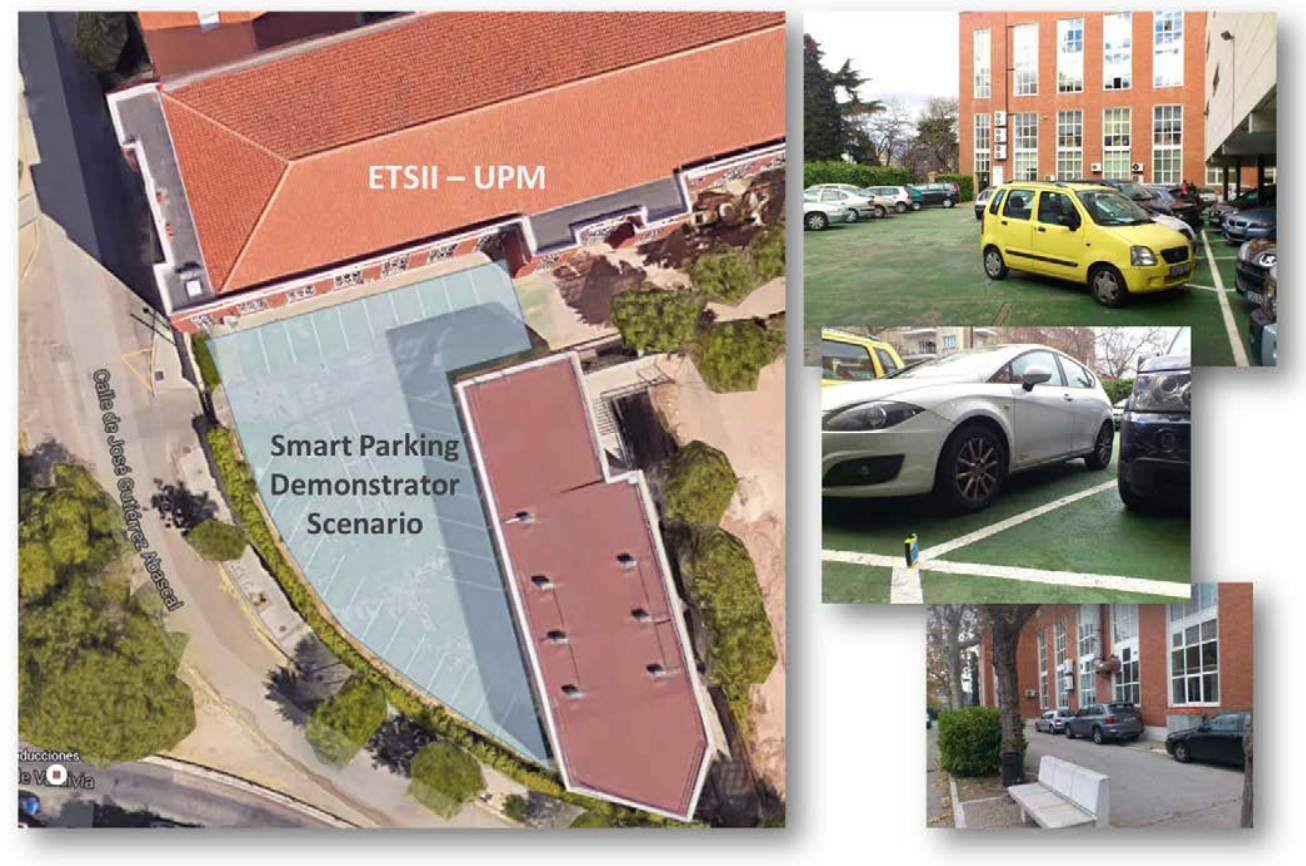

Fig. 5.23 Smart parking demonstrator scenario for the DPCM toolset validation.

Based on these input conditions, a system architecture for the proposed smart application scenario has been defined in order tackle the aforementioned requirements with the support of the deployment, commissioning, and 
maintenance tools, so that the validation of the sensor network developed through the use of WSN-DPCM capabilities can be carried out accordingly. As mentioned before, the application scenario for the smart parking was a car park of the ETSII-UPM as shown in Fig. 5.23, in which the area of interest is delimited to carry out the deployment of the WSN. On the other hand, the overall architecture that encompasses the hardware and software components to build the WSN-based smart parking is depicted in Fig. 5.24.

In terms of the hardware elements that compose the smart parking demonstrator, four main components can be distinguished in line with the described requirements: Sensor nodes, Gateway platform, Server infrastructure and the Hand-Held Device. The system prototype comprised a WSN with 14 sensor nodes and a root node connected to the gateway device through a USB connection, from which the runtime network and measurement data are gathered, considering the following specifications.

Sensor nodes: A TelosB-based platform [Polastre'05] has been used as the sensor node for measuring and processing the state of the parking spaces as well as the environment parameters of the deployment area, whose design includes an IEEE 802.15.4 transceiver as well as a low-power MCU for local processing of the sensing and communication capabilities. Fig. 5.25 highlights the external design of the sensor nodes (right-side) and the block diagram of the hardware components included in the platform structure (left-side). These sensor nodes are equipped with temperature, humidity and light sensors in order to monitor the environmental conditions of the application area, though dedicated interfaces are provided through expansion connections for attaching external components such as analogue and digital sensors or peripherals. 


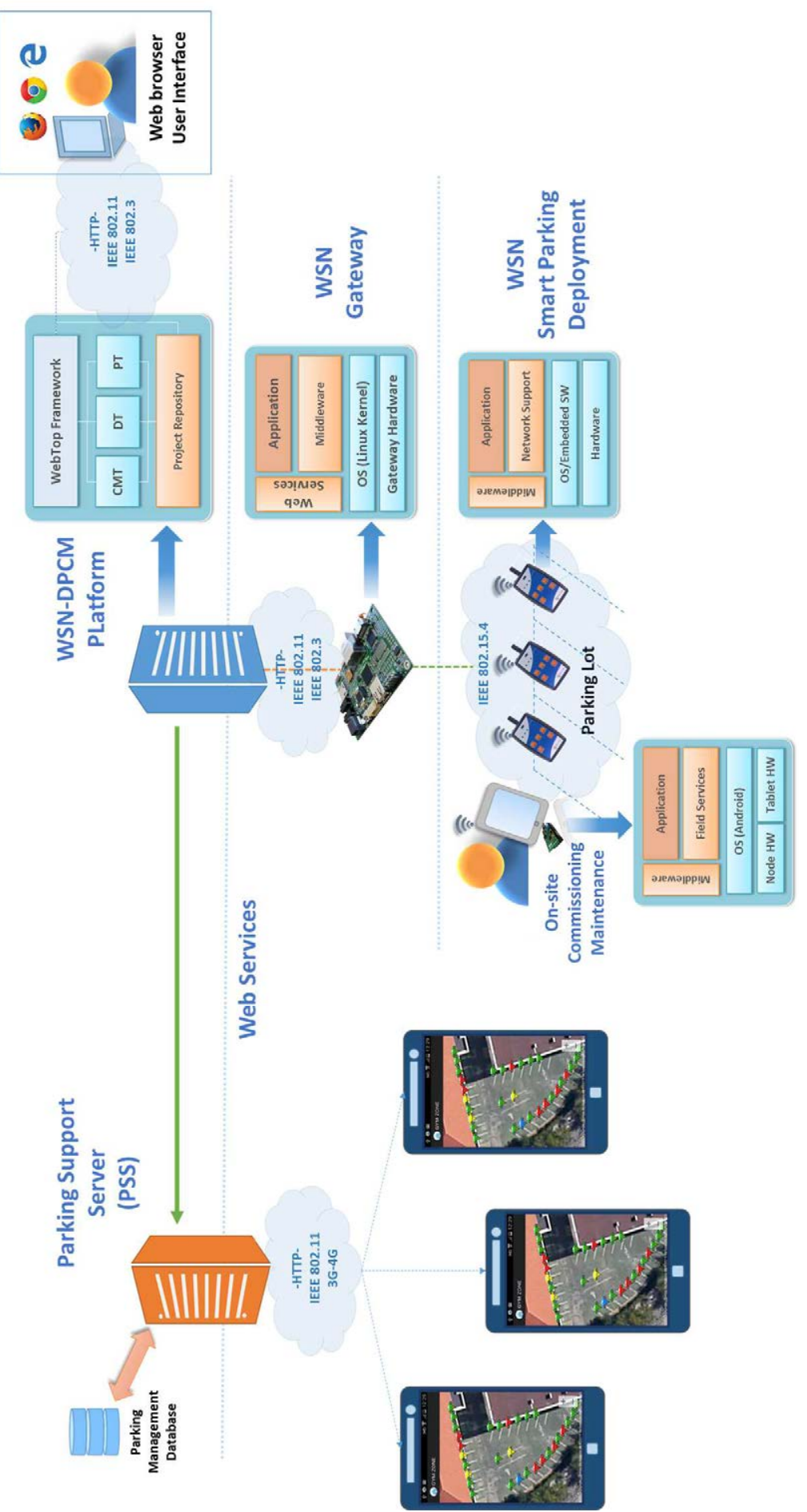

Fig. 5.24 System architecture of the proposed smart parking demonstrator with the support of the WSN-DPCM platform. 

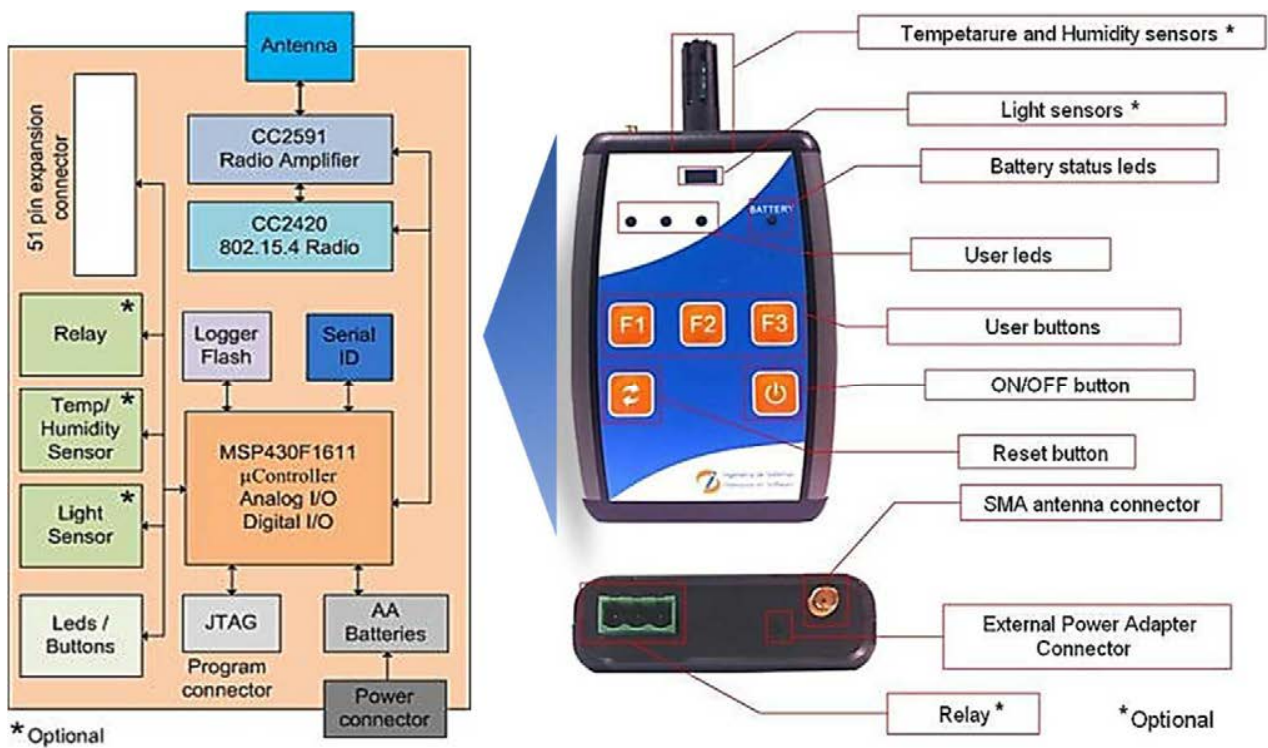

Fig. 5.25 Internal block diagram of the TelosB-based sensor node and external structure (Inetsis platform [DPCM-SD]).

Regarding the detection of the car occupancy in the parking spaces, proximity sensors are included in the implementation of the WSN prototype. The vehicle presence is monitored by combining light dependent and an infrared sensors, considering for such selection a trade-off between fast integration, low-cost, and reliability of the measurements. Fig. 5.26 shows how infrared sensors work, in which two elements are used for the proximity detection, i.e., and infrared led that beams an infrared signal towards the target object, and an optical sensor that received a reflected signal in case the object is under the distance boundaries, taking into account that the obtained measurement is proportional to the intensity of the captured infrared-light signal and inversely proportional to the square of the distance.

A distance sensor from Sharp [GP2Y0A60S] has been selected to be integrated into the hardware platform in combination with the available sensors. This device is composed of a position sensitive detector (PSD) and the infrared emitting diode (IR-LED) supported by a conditioning and signal processing circuit, which provides an analogue output (whose voltage is proportional to the detection distance), as shown in Fig. 5.26. 
Gateway platform: it was composed of an embedded PC to which the root node of the sensor network is attached (via USB port), so that the low-rate communication protocol provided by the TelosB-based node allows setting up a WSN data interface to the server side of the overall system, whose access is performed through internet connection. Apart from establishing the communication with the deployed sensor nodes, the Gateway plays a fundamental role in terms of implementing the middleware services and WSN high-level interfaces in order for the CMT-server to access, update, and retrieve parameters from/to the functional blocks implemented into the sensor platforms, so that an end-to-end seamless integration with the remote monitoring and management system is properly achieved.
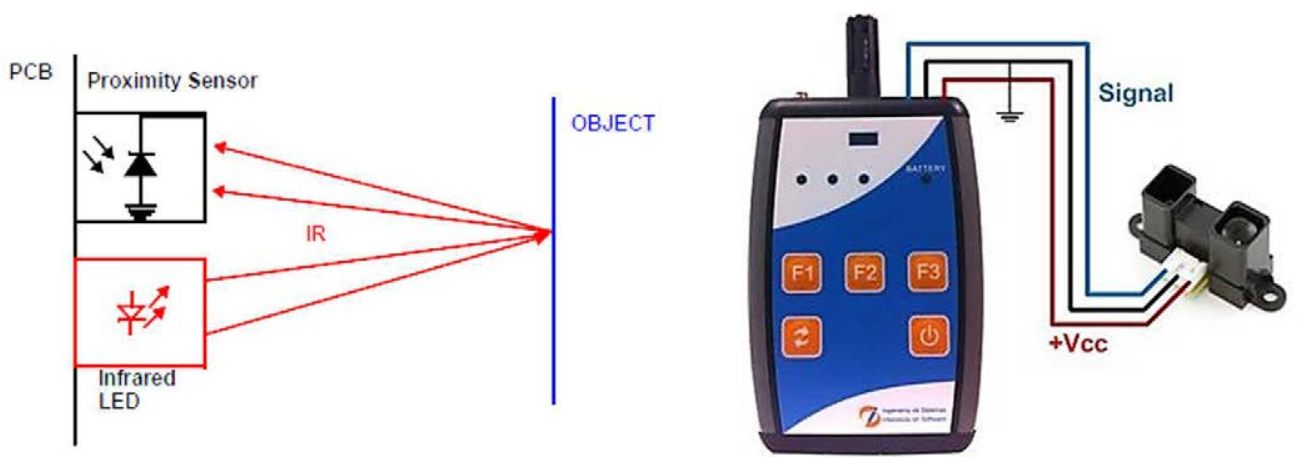

Fig. 5.26 graphical representation of the infrared sensor and integration into the sensor node [DPCM-SD].

Server infrastructure: the server side of the system architecture relies on a distributed array of remote machines which provides the functional components of the DPCM toolset as well as the runtime monitoring and control of the deployed WSN. As shown in subsequent paragraphs, the CMT-Server also provides additional services for feeding the smart parking application structure with data related to the detection sensors, so that a proper management of the parking occupancy can be realized.

Hand-Held Device: the proposed deployment tool constitutes a fundamental component to carry out the on-site configuration, performance evaluation and verification of the WSN system at the target area, so that the expected operation of the smart demonstrator can be validated in addition to providing the 
monitoring system with on-site support for the diagnosis of possible mismatches between the top-level parking application states and the real behaviour of the sensor network in-field. The CMT-HHD is composed of a tablet Asus Transformer (which includes a NVIDIA Tegra3 Quad Core processor at $1 \mathrm{GHz}$, and 1GB of RAM memory) running an Android Operating System Ice Scream Sandwich and with the IEEE 802.15.4-based HHNode connected through the USB host port. The support to the middleware integration within the deployment tool is also a key aspect for the proper in-field evaluation of the sensor network, as shown in next paragraphs.

On the other hand, the middleware provides common abstraction for interfacing the different components and tools that take part in the hardware-software architecture of the smart application, particularly considering the end-to-end integration of the wireless sensor nodes, gateway and network interfaces, and the CMT. The MW functional block implementation is in line with those included in the network model during the development process, whose correlation is performed by using SysML block description and the MW buildenvironment that allows binding various block interfaces in accordance with the information from the data repository. As a result, the CMT-Server, the CMTHHD, the WSN nodes and the WSN Gateway elements are interfaced by means of the network model that contains information and identification of the blocks implemented by the MW, so that a proper access, configuration, and monitoring of the running components can be seamless performed. As shown in Fig. 5.24, while the CMT-Server realizes the remote interaction with the WSN-MW through the Gateway/network-interfaces by using WS, the CMT-HHD, the sensor nodes and the Gateway run the MW implementation for direct in-field interfacing, based on the HH-MW integration and high-level interface presented in chapter 3.

In this way, MW abstracts the underlying library details of the software implementation during the development and synthesis time, so the core functional components are described as MW Transducer/Sensor Channel blocks for the parking sensors, Communication blocks for device port abstractions, and 
Parameter blocks for monitoring and configuration of the MW block implementations.

As represented in Fig. 5.27, the network model is composed of three main device definition entities in accordance with the development of the aforementioned architecture, as follows:

- The network interface definition (TypeID $=0$ ) describes the interfaces of the external components, i.e., the CMT-server, to the Gateway device and thus to the MW services, in order to remotely access the WSN capabilities, including on one hand connection parameters (host and port definitions) and on the other hand the specification of configuration and monitoring parameters of the high-level services.

- Sensor \& route node definition (TypeID = 1) depicts the functional components implemented in the deployable nodes, in accordance with the software development and the MW embedded interface. This includes the transducer blocks that interface the detector and environment sensors; the communication blocks that allow accessing the transceiver and protocol properties; and configuration blocks related to the behaviour of the wireless node.

- The root node definition (TypeID = 2) contains the functional blocks that describe the implementation of the WSN sink node, which is physically connected to the Gateway device in order to be able to gather the wireless network data through the low-rate communication protocol, i.e., IEEE 802.15.4 in this case.

As shown in the following section, the CMT is fed with the produced network model in order to properly carry out the installation, configuration and verification of the sensor nodes in-situ by using the HHD, as well as the WSN remote monitoring from the server side once the network structure is deployed and released, in accordance with the system planned and developed through the DPCM toolset. 


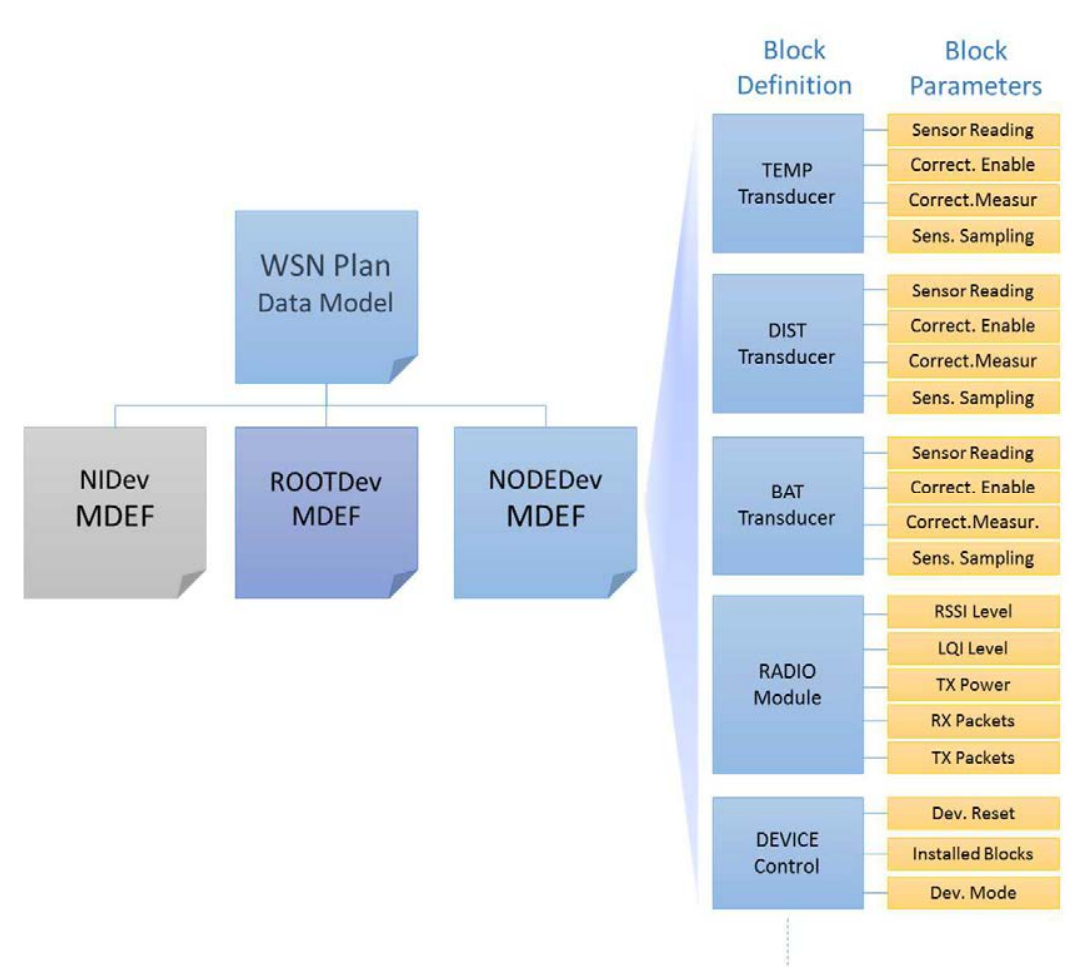

Fig. 5.27 Deployment model representation for the planned WSN demonstrator.

\subsubsection{Demonstrator implementation and on-site WSN deployment}

Fig. 5.28 depicts a typical session for the development of the WSN-based system by using the toolset, which has been followed to build the proposed smart parking demonstrator and validate the overall implementation and integration of the various elements that compose the DPCM platform. By using the Web-top interface, users can start the design of the WSN by specifying input parameters related to the types and minimum number of sensors, area of interest, and node positioning, whose information is used to trigger simulations in order to estimate how the communication among the wireless devices would be under the defined constraints.

Based on the input definitions and node requirements, the development services allow the completion of the embedded software by creating the top-level application with the support of platform library selection and synthesis triggering, whose results can be used to generate both executable code for the 
corresponding node type and platform/network simulation for behavioural analysis of the target WSN.

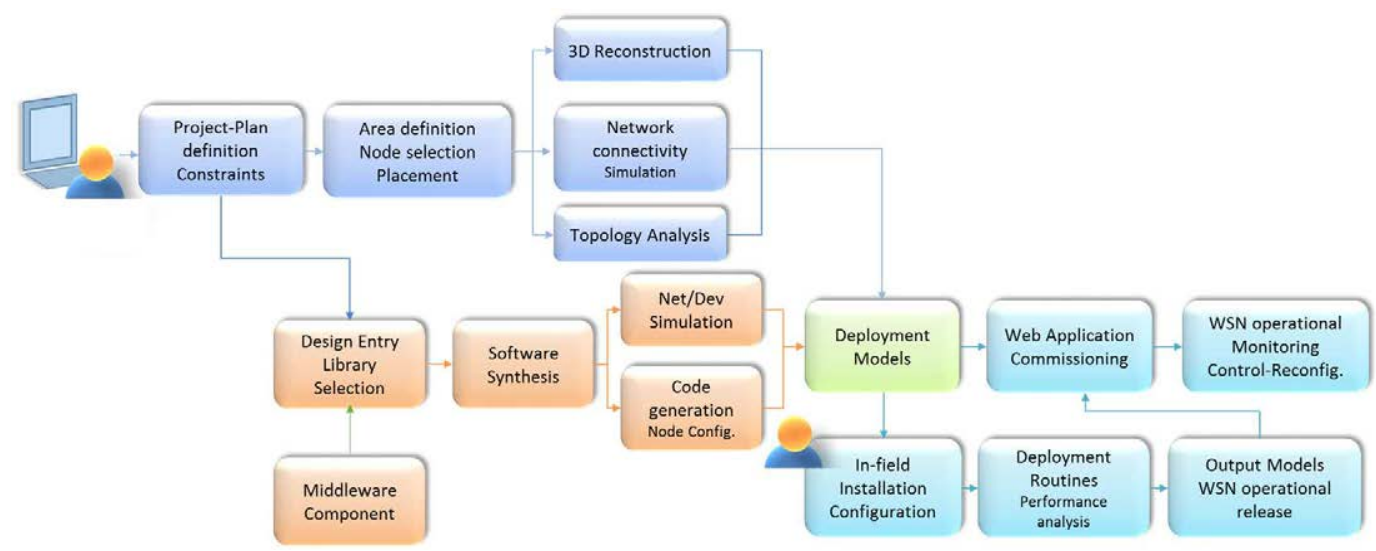

Fig. 5.28 DPCM toolset workflow for the design and implementation of the WSN.

As a result of this process, the common repository is automatically updated with the network models that will be used by the CMT to carry out the WSN installation, on-site configuration and network evaluation. The HHD is then used to perform the WSN deployment at the target area based on the proposed optimization and on-site analysis capabilities, so the node placement, sensor relocation and functional block reconfiguration based on in-situ performance assessment can be realized with the assistance of the commissioning and deployment tool, which also allows automatic generation of output reports that will update the common repository with actual data from the terrain. Such information is used by developers to interactively readjust the definition of design and development parameters, seeking a proper accomplishment of the application requirements.

Fig. 5.29 shows the distribution of the parking area to be monitored, in which $A x$ nodes represent car spaces under control and $R-x$ locations where additional nodes are deployed for environmental sensing and routing purposes. The sink node (base station) is attached to the embedded PC (located in one of the rooms in the ETSII building) that runs the MW Gateway implementation to interface the remote CMT Server and provides it with the middleware core services (based on an apache tomcat server over the eclipse integrated environment, and including the Java-based library of components), whose access information 
(host, port, and resource interfaces) is included in the network interface description at the common repository.

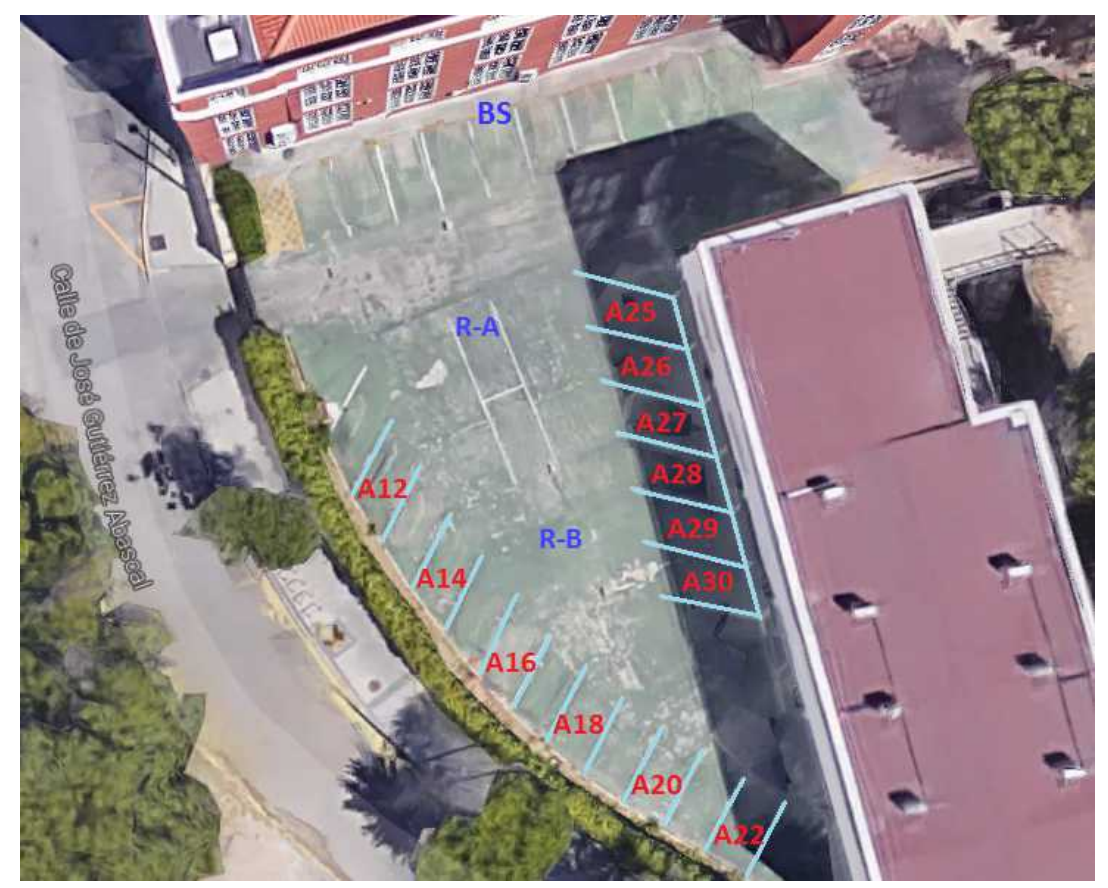

Fig. 5.29 Distribution of the car spaces in the target parking area at ETSII - UPM.

The wireless nodes were pre-programmed with the embedded code built during the software development, in which the underlying MW functional blocks are instantiated according to the network model description for the target environment, and the node types to be part of the WSN. Once the HHD downloads the corresponding network plan, the deployment tool is automatically configured to adapt the interface to the target environment, and the functional block setting panel is loaded with the current device models, so that deployers can carry out the configuration and evaluation of the sensor nodes in accordance with the running embedded system, as shown in Fig. 5.30, where several captures of the tool interface for the WSN under deployment are highlighted, particularly allowing for the consistency of the component definitions and block instances in relation with the designed smart parking demonstrator.

The transmission optimization method provides a homogeneous TX power of $10 \mathrm{dBm}$ to be configured through the Radio Block instance, and the result of the 
connectivity correlation scheme is shown in Fig. 5.31. A point-to-point/GAbased methodology was followed to carry out the in-field installation of the sensor node in the car park area (Fig. 5.32, representing a quite straight forward configuration due to the string distributions of the deployment), from which parametrization actions were performed mainly considering the evaluation of the detection sensor block, the effective communication of the wireless nodes to the base station (for which the HHD connectivity tests were triggered according to the NDoSq), and the configuration of the sensor publication for runtime data observation and calibration.

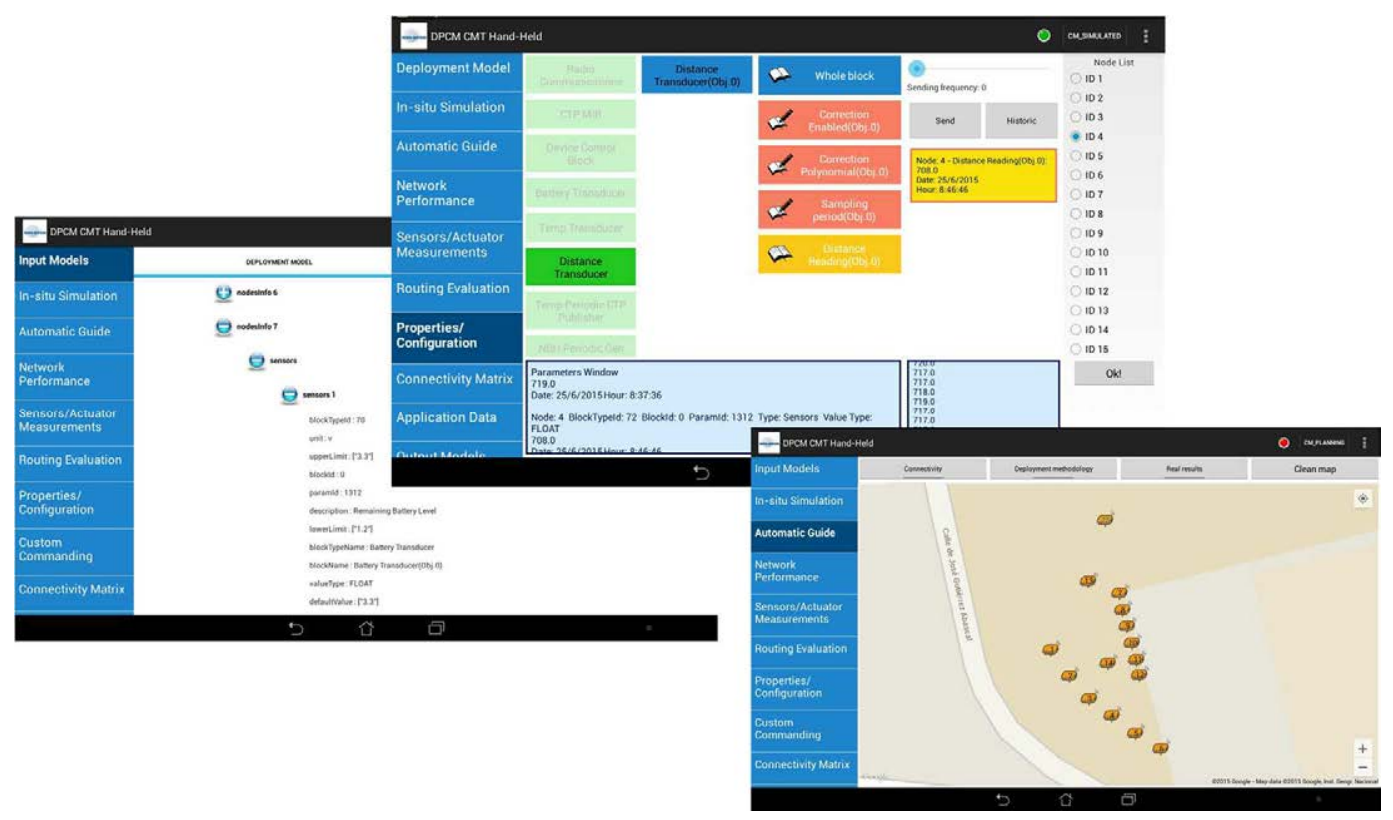

Fig. 5.30 HHD interface visualization in accordance with the defined deployment model for the ETSII smart parking demonstrator. 


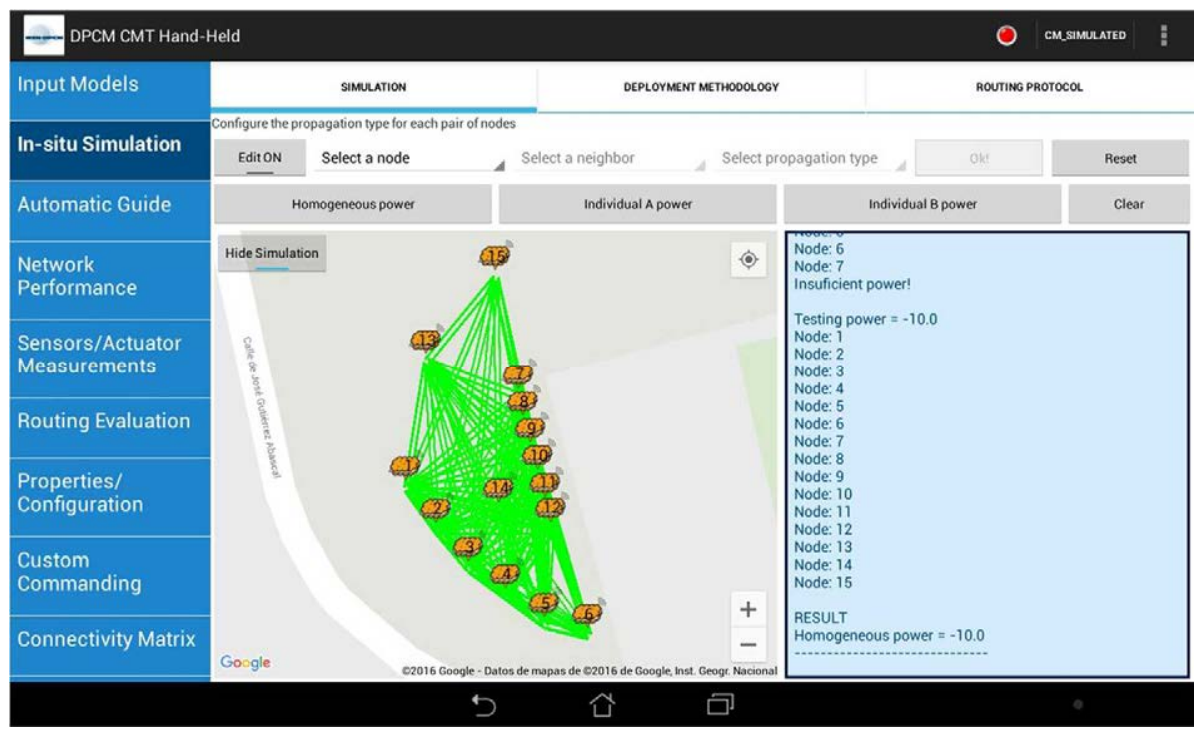

Fig. 5.31 nodes connectivity scheme results for the target WSN deployment.

Fig. 5.33 provides a series of measurements to show the car occupancy detection evaluation and calibration in some of the deployed sensor nodes (NodeID $=7$ in this case, Distance Transducer block), in which the HHD allows analysing the information gathered at runtime from the target node in combination with the data retrieved and handled previously by the internal management system, so that sensor corrections and/or block reconfigurations are performed in-situ.

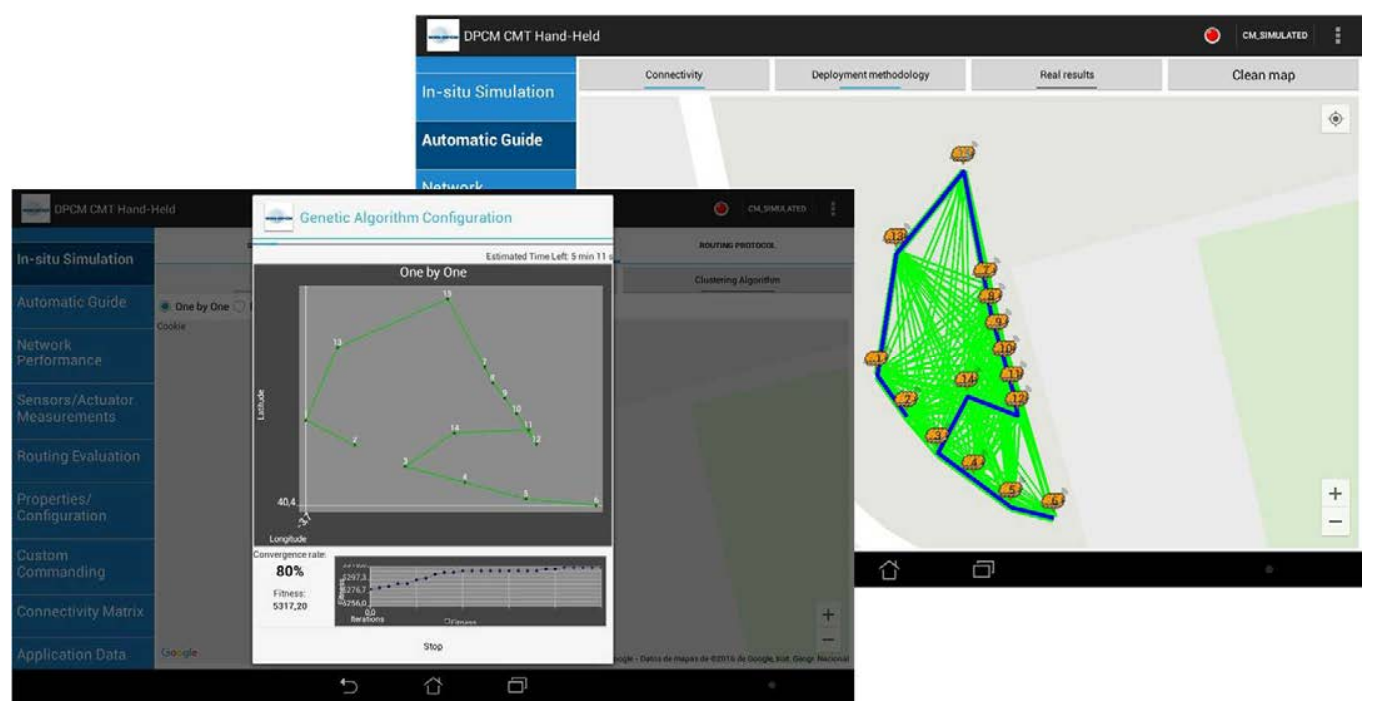

Fig. 5.32 HHD interface for the deployment methodology and WSN device connections. 
Then, output reports were generated and successfully uploaded to the project common repository, in accordance with the on-site evaluation and testing of the network functionality, as well as the behaviour of the sensor nodes after the configuration process was performed.

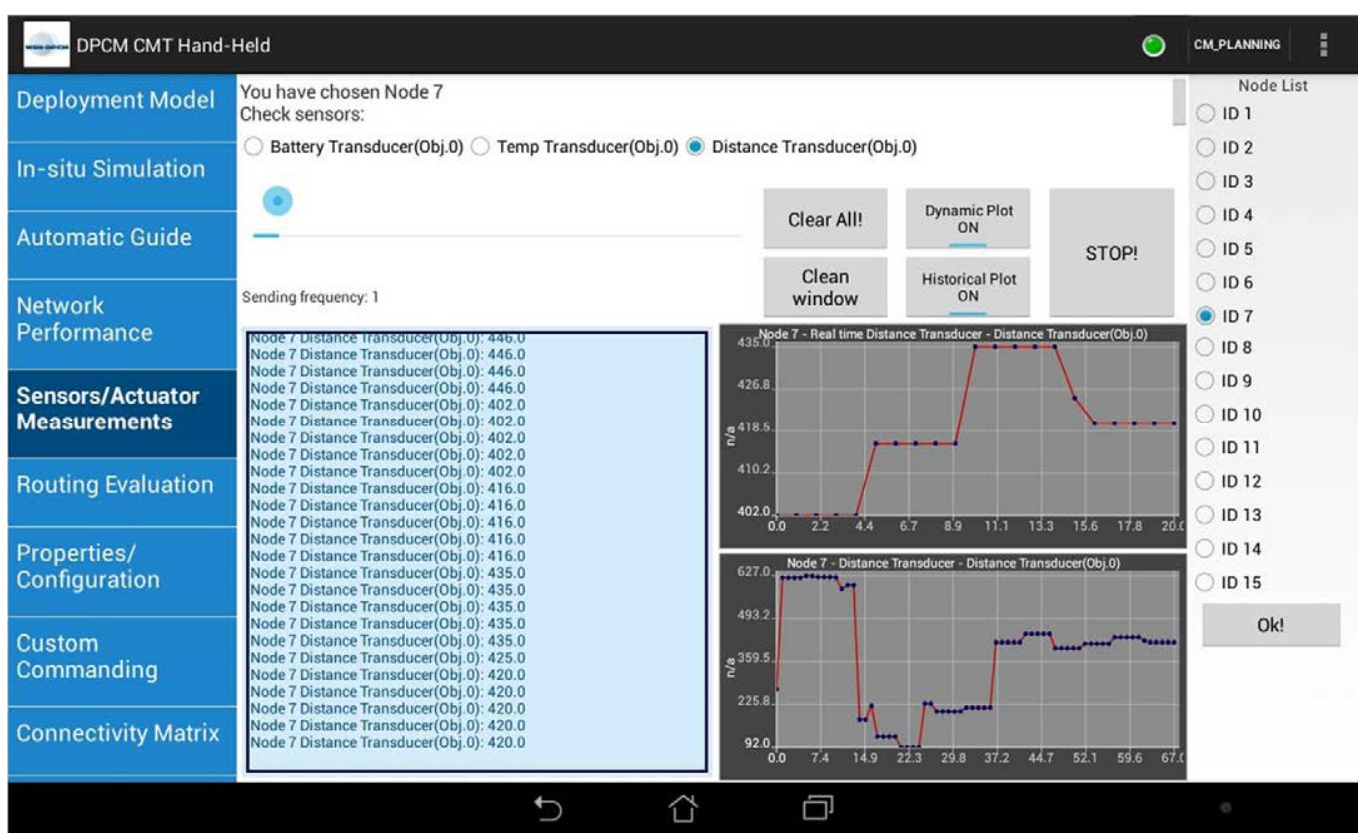

Fig. 5.33 HHD interface capture related to the evaluation of the occupancy detection sensor.

Once the sensor network is verified and released, users can remotely carry out the control and data gathering activities by triggering the CMT Web application, which is in charge of monitoring the operation of the WSN from the basis of the MW interface definitions. The CMT-HHD can be used to assist the execution of in-field maintenance and debugging actions that could result in new reconfiguration and implementation modifications of the WSN, particularly when changes in the conditions of the network or the application environment are detected.

\subsubsection{Domain-specific end-user application}

In addition to the support of the CMT to the WSN, both from the HHD and the Server sides, further domain-specific functionalities were implemented within the scope of the smart parking demonstrator in order to highlight the usability of the deployed system from the user point of view. The main focus was the 
creation of a top-level application that benefits from the sensor network developed through the DPCM toolset in order to provide application-context features related to the monitoring and management of the parking occupancy and booking system.

In this way, users can manage a personal account by using their smart devices from which booking and monitoring actions shall be performed, in accordance with the state of the parking space provided by the WSN. For that, the DPCM deployment structure is complemented with the architecture represented in Fig. 5.24 (left-side of the overall structure). While the commissioning server is responsible for accessing and monitoring intrinsic parameters of the WSN related to the sensor measurements, network connectivity, and functional components of the nodes, the Parking Support Server (PSS) is mainly in charge of providing the client applications with information regarding the state of parking occupancy (by transforming the detection values into slot status) as well as handling data regarding booking actions and users notifications.

From the point of view of the communication among the involved elements of the demonstrator, the data exchange and service provision both between the CMT and the PSS, and between PSS and the smart App, is performed via the implementation of WS (considering the Jersey Framework, an Apache Tomcat server for deploying the services, and JSON representation for data provision). On the other hand, a MySQL-based data-base is used to handle the information related to the parking management system, which mainly provides the following properties (using GET operations from the application side via RESTful):

- User credentials for log-in/registering verification, as well as account information retrieving.

- State of the parking spaces, from the basis of the detection sensors.

- List of reservations for a selected parking space, and information related to time-slots occupancy/availability.

- Registered information associated with a reservation (booking code as request parameter). 
- Registered information associated with the user account.

- Cancel a reservation (username as request parameter).

On the other hand, user application sends information to the parking management system related to the following actions (using POST operations from the application side via RESTful):

- Register a new user account.

- Book a new parking space for a particular time slot.

- Report an incidence related to a reservation or the state of the parking area.

It can also be realized that the PSS acts as the server for the user App while performing request actions to the CMT server in order to obtain data related to the sensor measurements (JSON file containing functional block and parameter associated with the implementation of the detector sensor), whose information is used to update the state of the parking occupancy in the data-base, as mentioned before. Therefore, the PSS serves as an intermediate element for the end user devices to abstract the underlying WSN technology, so that a transparent use of the smart parking environment is carried out. The PSS automatically checks and manages the registered reservations in order to update the expired and remaining time-slots, so that the state of the booking information is also refreshed in the user Apps.

In this context, a mobile smart-parking application has been designed and implemented in order for users to benefit from the monitoring and booking system of the developed demonstrator. As mentioned before, the parking App makes use of the PSS as a web-service client, and the graphical interface allows accessing the car-park display and reservation engine. The Android-based App (version 4.0.3 or later) relies on the creation of a user account in order to be able to use the smart parking system. Once a valid user is entered/registered, the parking area is presented to the user as shown in Fig. 5.34. The Google Map service is employed to visualize the target area, so typical map features such as zooming, rotating, etc. are integrated. By selecting the parking zone to be displayed, a detailed view of the status of the car park will be shown (see Fig. 
5.35, left), where markers and colours represent the current state of the available spaces, considering the nomenclature detailed in Fig. 5.35 (right). This information is updated periodically in accordance with the information provided by the PSS (refresh period can be configured by users, considering 1 minute as the minimum value).
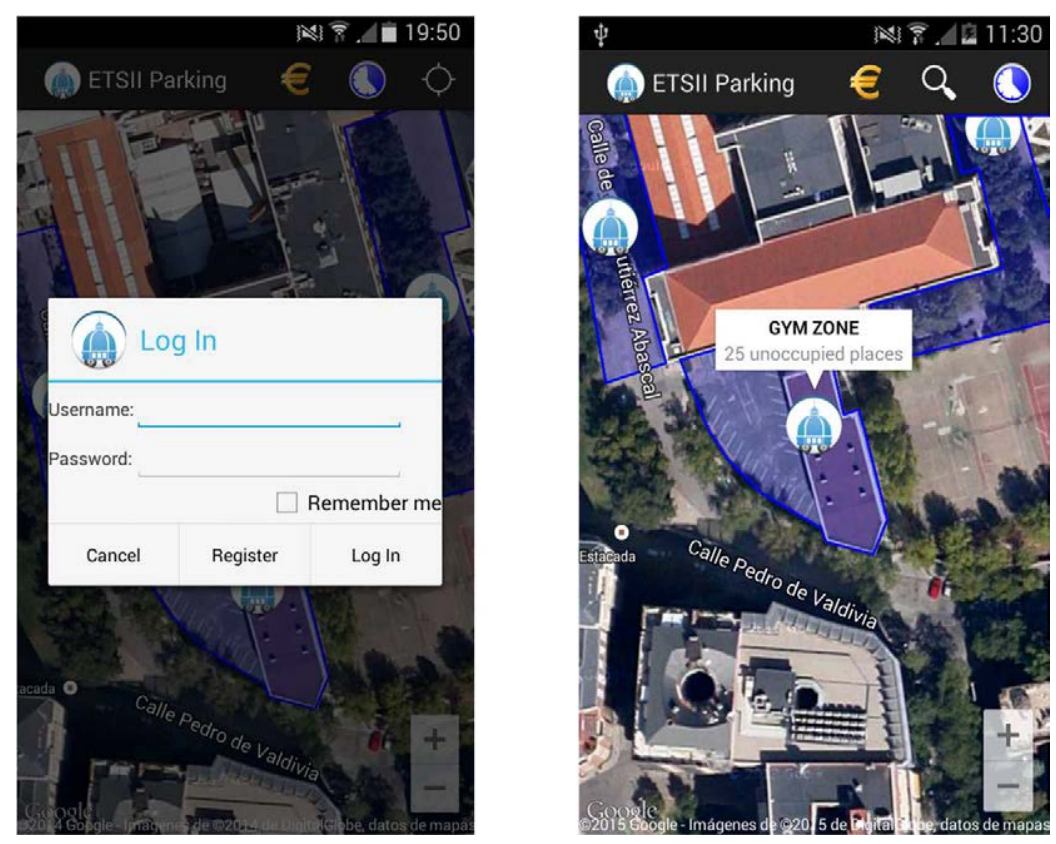

Fig. 5.34 App interface, log-in menu (left), and parking areas (right).

By selecting any of the parking spaces, a list of reservation time-slots and occupancy periods is visualized, so users can book one of the available slots or specify a reservation period as shown in Fig. 5.36 (left), which is then filtered to assure no overlapping among reservations. If the booking information entered by the user is valid, the smart parking management system registers it and provides a unique reservation code for tracking and reference purposes. However, since usually the main objective from the user perspective is to book any free space for a particular period of time, the App also offers an automatic searching option in which, after entering a target time-slot, the application displays the available parking spaces that can be reserved (no reservations registered for that time-slot), as shown in Fig. 5.36 (right). 


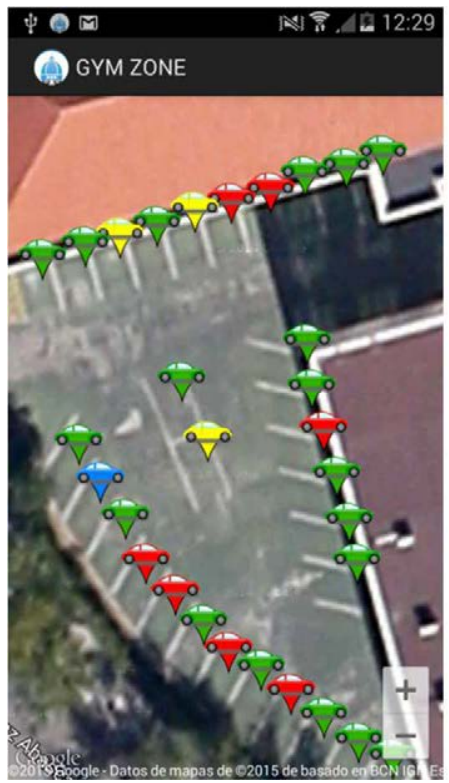

\begin{tabular}{|c|c|}
\hline Icon Colour & Symbol meaning \\
\hline Green & $\begin{array}{c}\text { Unoccupied parking space, with } \\
\text { no reservations registered } \\
\text { (current and next day) }\end{array}$ \\
\hline Yellow & $\begin{array}{c}\text { Unoccupied parking space, with } \\
\text { reservations for other time slots }\end{array}$ \\
\hline Red & Parking space currently \\
\hline Blue & $\begin{array}{c}\text { Parking space reserved by the } \\
\text { user }\end{array}$ \\
\hline Green & $\begin{array}{c}\text { No information of the current } \\
\text { status of the parking space }\end{array}$ \\
\hline
\end{tabular}

Fig. 5.35 App parking map and booking interface (left), slot status information (right).

Once a booking process is realized, users can access the information and status of the reservation, including the specification of the parking area where the space is located, slot identifier, reservation code, starting and ending time (also showing a status bar regarding the remaining time before starting the reservation time-slot), as shown in Fig. 5.37. The option "Drive me here" allows user to launch the predefined navigator (configured as predefined in Android) in order to guide drivers from their current position to the reserved parking space.

The designed App also makes use of Android notifications in order to alert users whenever a reservation is about to start/expired (10 minutes before the event), as presented in Fig. 5.38. Users can enable/disable this option from the App configuration panel any time. Moreover, users can report possible irregularities to the management system, so as to notify problems or incidences when reserving/using the smart parking, which is particularly intended to be used when not-registered, expired, or irregular car occupancy is encountered. In this way, users can specify: a) reason of the irregularity to be reported; b) licence plate of the involved car, if applicable; c) slot identifier, either entered manually by the user or selected from the parking map. This form is automatically filled 
when the irregularity report is uploaded from the reservation status panel (i.e. another car is occupying the reserved parking space).
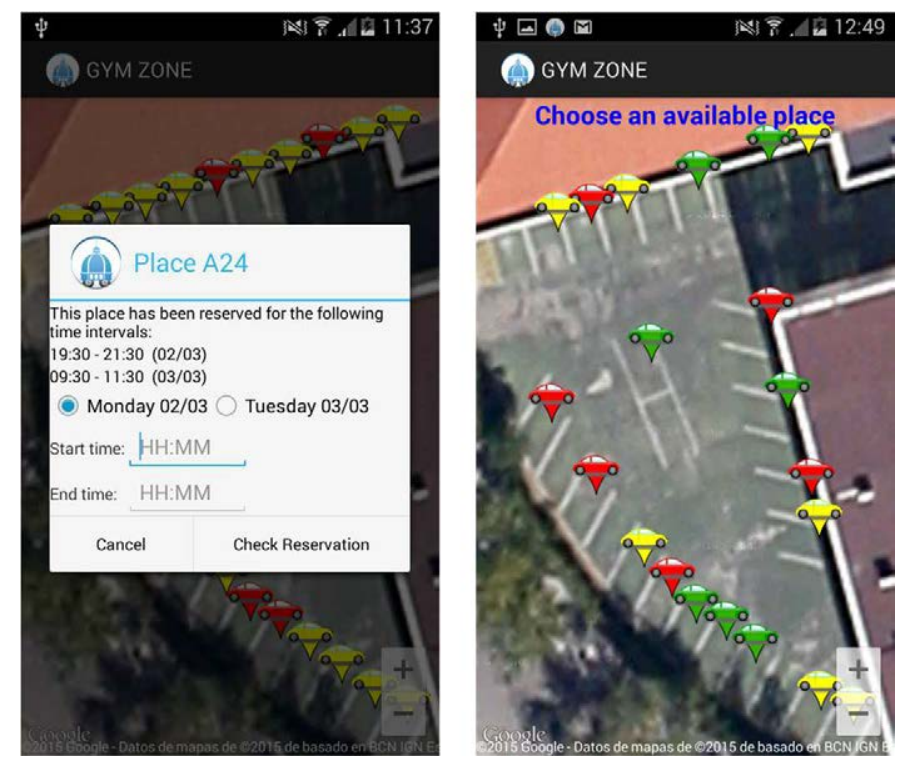

Fig. 5.36 Booking reservation panel (left), parking place filtering for a specified time-slot (right).

There are some similarities of the proposed environment in terms of system functionalities with respect to state-of-the-art solutions for parking monitoring, such as the Smart Santander [Santander], in which the IEEE 802.15.4 protocol is used in combination with Gateway elements to gather the detection measurements from the sensor nodes, which then provide information regarding available parking spaces of the target area, both in installed panels as well as in online maps that can be accessed by citizens. The DPCM smart parking demonstrator is more focused on wireless multi-hop networking and middleware-based support in order to provide a high degree of flexibility, reusability and scalability of the sensor deployment with the interactive assistance of the development, planning and CMT platform. Moreover, in addition to a status map view, the proposed smart parking provides a top-level mobile application that allows end-users to access a parking management system for monitoring and booking free spaces in an online fashion, so that the dynamic interaction user-system is totally covered by the overall platform. 


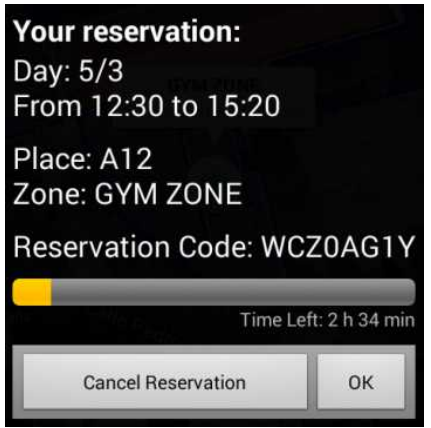

Fig. 5.37 User reservation details and current status.

\subsubsection{Platform validation through the smart demonstrator operation}

The described smart parking demonstrator structure supports the end-to-end verification of the developed WSN-based system using the DPCM toolset, taking into account the CMT assistance, the MW support for managing the sensor network implementation, and the top-level/end-user application.

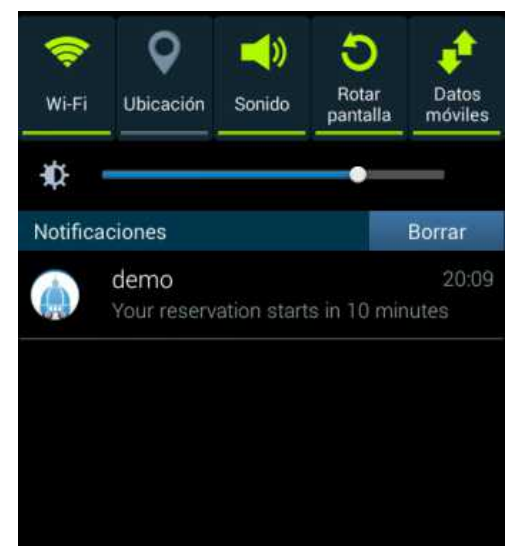

Fig. 5.38 Android notifications related to the user reservation status.

In this context, cars were used to test the smart parking monitoring system both through the direct analysis of the HHD in field as well as by using the end-user App, which implies the verification of the whole operability of the underlying system architecture, from the sensing measurement acquisition of the wireless nodes and their communication to the WSN up until the event notification to the user smartphone, with the support of the MW, CMT server and PSS, and the smart parking management system in the middle of such a process. 
The validation of the overall toolset through the implemented demonstrator was mainly approached from an integration perspective, considering the suitability of using heterogeneous technologies for the development of a WSN-based application in a unified platform. This was realized with the support of the DPCM toolset-based development flow, which allows the validation of the various functional components, services, and sub-systems that take part in each step of such an operational chain, along with their usability throughout the WSN design, development and implementation process. A particular focus on the interaction among the involved tools was highlighted during the toolset session, where the integration of the CMT-HHD with the rest of the system was indeed encompassed, especially taking into account that related to the MW and the CMT workability.

\subsection{Conclusions}

Several real test cases have served as the main scenarios for the evaluation and validation of the functional components of the deployment toolset, in addition to the multi-hop mesh networking optimization mechanisms proposed in this work, particularly considering the embedded implementation of CB-AODV as well as configuration capabilities for the sensor nodes. The performance assessment of the routing strategies has been carried out following a variety of network topologies and communication patterns, including protocol metric analysis under intensive traffic conditions. This led to test how transitions among the different states of the discovery and maintenance mechanisms can properly evolve in accordance with events on the data communication exchange between remote 2 -Ns. The comparative analysis of the integrated metrics allows evaluating their suitability to obtain a proper trade-off between reliability, energy awareness and end-to-end effective communication, based on deployment requirements (including network distribution and topology constraints) as well as the dynamics of the mesh network.

The HHD was indeed used not only within the deployment of Cookie-based sensor networks, but also during the validation of the DPCM multi-domain toolset through the implementation and evaluation of the WSN smart parking 
demonstrator presented in this chapter. The integration of the deployment tool with the rest of the planning and development chain was verified considering the effective production and management of the deployment models, the in-field optimization and automatic guidance, and the middleware support for the onsite access to the embedded functional components of the sensor nodes under deployment. This also allowed the validation of the heterogeneous support of the HHD to different sensor node platforms.

\subsection{Bibliography of the chapter}

[DPCM] Development, Planning, Commissioning and Maintenance Toolset for Wireless Sensor Networks (WSN-DPCM), ARTEMIS-JU European project, http://www.wsn-dpcm.eu/

[DPCM-SD] WSN-DPCM European Project, Demonstrators Architecture, Smart Parking Demonstrator, http://www.wsn-dpcm.eu/

[GP2Y0A60S] Distance Measuring Sensor Unit GP2Y0A60SZ0F, Sharp Corporation http://www.sharp-world.com/products/

[Maps] Google Maps Android API, available on: https://developers.google.com/maps/documentation/android-api/

[Polastre'05] J. Polastre, R. Szewczyk, D. Culler, “Telos: enabling ultra-low power wireless research", in International Symposium on Information Processing in Sensor Networks (IPSN'05), pp. 364-369, April 2005.

[Santander] SmartSantander Research Facility, Outdoor parking management area, Santander Smart City, http://www.smartsantander.eu/ 



\section{Chapter 6}

\section{CONCLUSIONS AND FUTURE WORK}

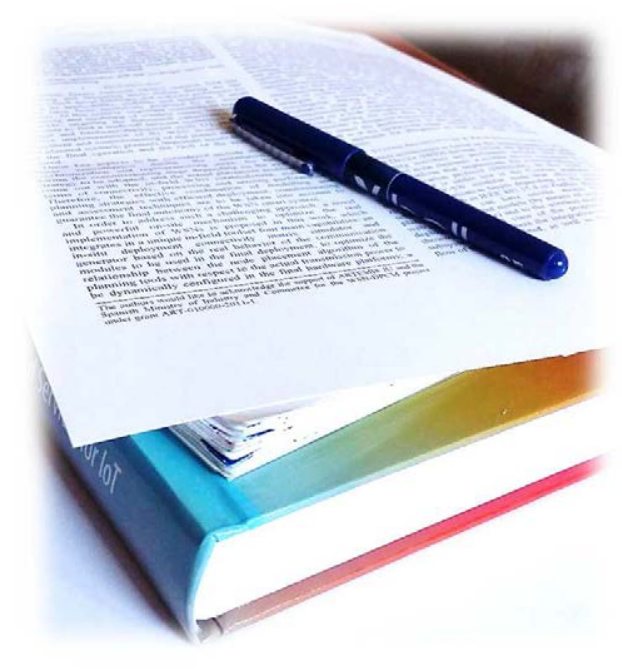

The pre-deployment and deployment strategies proposed in this thesis have been deeply discussed in previous chapters in order to provide a detailed overview of the main capabilities for supporting the experimental prototyping, on-site performance evaluation and optimization of sensor networks.

In this way, this chapter is devoted to highlight the main contributions that allowed obtaining a new integrated HW-SW toolset to assist developers and deployers in the achievement of real WSN-based systems. Indeed, this work also allows opening new research lines in order to broaden the capabilities associated with the modular sensor platform as well as the Hand-Held Device, hence a discussion related to future perspectives for this promising technology is subsequently presented. 



\section{Conclusions and future work}

\subsection{Conclusions}

In this work a set of deployment tools for the evaluation and optimization of real Wireless Sensor Networks has been proposed, including the analysis of the experimental stages that take place during the development cycle of WSN-based systems. This encompasses pre-deployment and in-field deployment techniques to leverage the validation, operational release and subsequent maintenance of WSNs. On one hand, the implementation of sensor networks is to be supported by flexible prototyping and testing mechanisms in order for developers to be capable of optimizing the designed platform based on real data, beyond the expected results from the simulation and code generation process. To this extent, the pre-deployment stage shall include the integration of hardware-software components for the composition of an experimental framework under which prototypes can be studied and properly evaluated. On the other hand, the infield deployment represents a fundamental stage within the overall system implementation towards the optimization, refinement and verification of the WSN at the target application scenario.

In this thesis the former phase has been approached by proposing a hybrid multi-purpose testbed support platform based on the modularity and flexibility of the Cookie architecture, in which a testing and debugging infrastructure is combined with a remote partial reprogramming mechanism in order to enhance how the embedded software of the sensor nodes under test can be remotely included, updated and replaced at runtime, highlighting the following features:

- The backchannel-based approach allows creating a seamless experimentation without invading the main communication link of the network, and its flexibility is increased in this work by integrating both a PoE-based wired and a wireless support layer in conjunction with innode debugging blocks. They allow changing dynamically the experimentation scheme of the node in order to evaluate particular 
functionalities at various abstraction levels, such as module-host interaction, platform configuration/parametrization and software execution process, node interface handling, and protocols implementation.

- The in-node debugging and testing capabilities contribute to achieve the flexibility, adaptability and nomadic-based approach of the testbed, unlike state-of-the-art works where more rigid solutions for creating the experimental scenario are used.

- The partial reprogramming approach proposed in this work aims at addressing a modular composition of the software components in the sensor node in order to optimize not only the way developers make use of the HW-SW support platform from higher abstraction levels, but also how software prototypes are included or replaced in an efficient/costeffective fashion, considering the resource constraints of the WSN platforms. For this, the MS-PRS has been created to allow independent reprogramming of software components as well as a seamless interaction among application-level and platform support components. This means that, in case a user's software prototype that makes use of the software support platform is to be reprogrammed, the rest of the structure remains unaltered, thus enhancing both the reprogramming process itself and the modularity of the overall software implementation.

- The benefits of this technique is indeed intended to be extrapolated from the pre-deployment/testbed stage to the site operation of the sensor network, where runtime reprogramming tasks must be enclosed by efficient mechanisms so as to guarantee a trade-off between reliability of the transaction and its power/resource consumption efficiency.

The latter phase related to the in-field deployment of WSNs has been addressed in this thesis by proposing a novel toolset for assisting deployers in performing the on-site configuration, evaluation and optimization of the real sensor network prior to its final operational release. The basis of this set of capabilities relied on the implementation of an intelligent mobile device, referred to as a Hand-Held Device, with which the integration of optimization strategies for an efficient 
deployment of planned and developed sensor systems take place. The HHD allows achieving a proper cohesion of the wireless network by taking into account both the simulation outcomes of the WSN and the real behaviour of the nodes under installation, so that a more accurate refinement of the overall system can be properly performed, and considering the following contributions:

- The integration in a unique deployment platform a set of in-field simulation and optimization engines based on experimental data, from which a comprehensive deployment methodology and guidance is provided to support users during those tasks associated with the on-site evaluation and validation of WSNs at the target scenario. This toolset has been successfully implemented considering a two-side integration approach. On one hand from the perspective of the development-cycle, by proposing a network definition where the results of the planning, simulation and code generation process are expressed in the form of well-defined models that encompass key development information to be used at the deployment phase. On the other hand from the real sensor network itself, by means of integrating a WSN interface in the HHD that allows communicating with the nodes through the proper low-rate wireless protocol.

- Such interface is supported by the implementation of a middlewarebased abstraction layer for accessing the embedded HW-SW platform, so that reconfiguration and parametrization actions can be directly done insitu from the mobile device.

- The design and development of the proposed deployment toolset contributed to tackle the lack of well-defined platforms, tools and frameworks in the state of the art to leverage an efficient accomplishment of the configuration, in-field operation assessment and validation of WSNs.

- A particular focus on the analysis and optimization of platform connectivity is taken into account at different levels of implementation, especially regarding link layer performance evaluation and routing techniques. The latter has been comprehensively approached in this 
work through the implementation of multi-hop communication strategies targeting the adaptability of Cookie-based WSNs to different application scenarios, where the flexibility of mesh networking techniques is a must.

In this way, a real perspective for the integration and testing of routing protocols in WSN nodes has been discussed in this thesis by means of implementing an optimized distance-vector algorithm into the Cookie HW-SW platform (CBAODV). This has been created from the basis of the design of a new Cookie communication layer that incorporates the IEEE 802.15.4 standard. The actual implementation of this routing protocol into the modular platform contributed to provide an adaptable multi-hop communication mechanism that can be reconfigured depending on the power and resource utilization requirements, allowing for the parametrization of connectivity metrics, discovery and maintenance functionalities.

The HHD fully supports users to evaluate the implementation of communication and routing techniques in real sensor nodes and within application environments, particularly in case of CB-AODV. Real test cases served as the main experimental scenarios to put the deployment capabilities proposed in this thesis into various application contexts. These results allowed verifying the benefits of the implemented strategies and tools in pursuing a proper deployment and in-field operation of wireless sensor networks. Besides the experimental cases presented in this work, part of the proposed capabilities and particularly the HW-SW platform have successfully contributed to support the implementation of Cookie-based WSN applications in the context of industrial and research projects.

\subsection{Research results and scientific references of the work}

\subsubsection{Related projects}

Part of this work was implemented within the scope the European project WSNDPCM, funded by ARTEMIS Joint Undertaking and national authorities, under grant ART-010000-2011-1. The consortium that participated in this 
challenging project was composed of European companies, universities and research centres targeting the design and implementation of a multi-domain planning, development, commissioning and maintenance toolset based on the integration of heterogeneous hardware and software technologies. The WSNDPCM project started in 2011 and ended with successful results in September 2015, and with the accomplishment of the smart parking demonstrator where the main capabilities of the overall DPCM tool-chain, including the integration of the HHD, were properly validated.

Moreover, several undergraduate projects have been accomplished within the scope of this research work, where the author has been involved as a cosupervisor of the students. They are listed as follows:

i. Gordillo, Javier. "Algoritmos de Optimización de Despliegue en Redes de Sensores Inalámbricas y su Aplicabilidad en Smart Cities". Undergraduate thesis. Co-supervised with Jorge Portilla. Universidad Politécnica de Madrid, April 2015.

ii. García, Alejandro. “Diseño de una herramienta de soporte inteligente para el despliegue y configuración en campo de redes de sensores inalámbricas". Undergraduate thesis. Co-supervised with Jorge Portilla. Universidad Politécnica de Madrid, April 2015.

iii. Zamacola, Rafael. "Diseño de una capa de comunicaciones basada en el estándar IEEE 802.15.4 e implementación del protocolo de rutado AODV en redes de sensores inalámbricas". Undergraduate thesis. Co-supervised with Jorge Portilla. Universidad Politécnica de Madrid, April 2014.

iv. Hernando, Álvaro. “Diseño de una capa de comunicaciones basada en Low-Power Wi-Fi e implementación de estrategias de reprogramación remota orientada a testbeds en redes de sensores inalámbricas". Undergraduate thesis. Co-supervised with Jorge Portilla. Universidad Politécnica de Madrid, November 2013.

v. Guijarro, Mario. “Diseño e implementación de capa de alimentación y comunicaciones de segundo nivel basado en Power over Ethernet 
para banco de pruebas de plataforma modular de redes de sensores inalámbricas". Undergraduate thesis. Co-supervised with Jorge Portilla. Universidad Politécnica de Madrid, November 2012.

vi. Antón, David. "Diseño de capa de procesamiento altamente configurable y de ultra bajo consumo de plataforma modular para redes de sensores inalámbricas y reprogramación remota". Undergraduate thesis. Co-supervised with Jorge Portilla. Universidad Politécnica de Madrid, November 2012.

\subsubsection{Publications}

The list of scientific publications derived from this research work is classified as follows.

\subsubsection{Book Chapters}

i. G. Mujica, J. Portilla, T. Riesgo, "Deployment Strategies of Wireless Sensor Networks for IoT: Challenges, Trends, and Solutions Based on Novel Tools and HW/SW Platforms", in book Components and Services for IoT Platforms: Paving the Way for IoT Standards. Springer International Publishing, pp. 133-154, 2016. DOI: 10.1007/978-3-31 9-423 04-3.

\subsubsection{Journals}

i. G. Mujica, J. Portilla, T. Riesgo, et al., "Integrated Toolset for WSN Application Planning, Development, Commissioning and Maintenance: The WSN-DPCM ARTEMIS-JU Project", in Sensors Journal, vol. 16, n. 6: 804; 2016. DOI: 10.3390/s16060804.

ii. G. Mujica, J. Portilla, T. Riesgo, "Performance evaluation of an AODV-based routing protocol implementation by using a novel infield WSN diagnosis tool", in Microprocessors and Microsystems Journal, vol. 39, n. 8, 2015. DOI: 10.1016/j.micpro.2015.10.007.

iii. D. He, G. Mujica, J. Portilla, T. Riesgo, “Modelling and planning reliable wireless sensor networks based on multi-objective 
optimization genetic algorithm with changeable length", in Journal of Heuristics, 21: 257, 2014. DOI: 10.1007/s10732-014-9261-2.

iv. D. He, G. Mujica, G. Liang, J. Portilla, T. Riesgo, “Radio propagation modeling and real test of ZigBee based indoor wireless sensor networks", in Journal of Systems Architecture, vol. 60, no. 9, 2014. DOI: 10.1016/j.sysarc.2014.08.002.

v. J. Valverde, V. Rosello, G. Mujica, J. Portilla, A. Uriarte, T. Riesgo, "Wireless Sensor Network for Environmental Monitoring: Application in a Coffee Factory", in International Journal of Distributed Sensor Networks, vol. 8, n. 1, 2012. DOI: $10.1155 / 2012 / 638067$.

\subsubsection{Peer-review conferences}

i. G. Mujica, V. Rosello, J. Portilla, T. Riesgo, "CookieLibs: An Intuitive Software Platform For Controlling Cookies Wireless Sensor Nodes", in proceedings of International Conference on Design of Circuits and Integrated Systems (DCIS'12), Avignon, France, November 2012.

ii. G. Mujica, V. Rosello, J. Portilla, T. Riesgo, "Hardware-Software Integration Platform for a WSN Testbed Based on Cookies Nodes", in proceedings of IEEE Industrial Electronics Society (IECON'12), Montreal, Canada, November 2012.

iii. D. He, G. Mujica, J. Portilla, T. Riesgo, "Simulation Tool and Case Study for Planning Wireless Sensor Network", in proceedings of IEEE Industrial Electronics Society (IECON'12), Montreal, Canada, November 2012.

iv. G. Mujica, J. Portilla, T. Riesgo, "A Reliable Support Tool for Monitoring, Testing and Debugging Wireless Sensor Cookie Nodes", in European Conference on Wireless Sensor Networks (EWSN'13), Ghent, Belgium, February 2013.

v. D. He, G. Mujica, G. Liang, J. Portilla, T. Riesgo, "Radio Propagation Modeling and Measurements for ZigBee Based Indoor Wireless 
Sensor Networks", in proceedings of IV Jornadas de Computación Empotrada (JCE'13) - SARTECO. Madrid, Spain, September 2013. Best Paper Award of the Conference.

vi. G. Mujica, V. Rosello, A. Hernando, J. Portilla, T. Riesgo, “New Strategies on Remote Reprogramming for Supporting MultiApplication Experiments in Wireless Sensor Nodes", in proceedings of International Conference on Design of Circuits and Integrated Systems (DCIS'13), San Sebastian, Spain, November 2013.

vii. G. Mujica, V. Rosello, J. Portilla, T. Riesgo, “On-the-fly Dynamic Reprogramming Mechanism for Increasing the Energy Efficiency and Supporting Multi-Experimental Capabilities in WSNs", in proceedings of IEEE Industrial Electronics Society (IECON'13), Vienna, Austria, November 2013.

viii. G. Mujica, J. Portilla, T. Riesgo, “Testbed Infrastructure for Debugging, Analyzing and Optimizing WSN Nodes Based on a Modular HW-SW Architecture", in proceedings of V Jornadas de Computación Empotrada (JCE'14) - SARTECO, Valladolid, Spain, September 2014.

ix. G. Mujica, R. Zamacola, J. Portilla, T. Riesgo, “Performance Evaluation of an AODV-Based Routing Protocol Implementation by Using a Novel In-Field WSN Diagnosis Tool", in proceedings of IEEE International Conference on Design of Circuits and Integrated Systems (DCIS'14), Madrid, Spain, November 2014.

x. G. Mujica, A. Garcia, J. Gordillo, J. Portilla, T. Riesgo, “A Novel Onsite Deployment, Commissioning and Debugging Technique to Assess and Validate WSN Based Smart Systems", in proceedings of IEEE International Conference on Circuits and Systems (ISCAS'15), Lisbon, Portugal, May 2015.

xi. J. Gordillo, G. Mujica, J. Portilla, T. Riesgo, “A Novel Cluster-based Routing Protocol Optimization Approach for Wireless Sensor Mesh 
Networking", in proceedings of VI Jornadas de Computación Empotrada (JCE'15) - SARTECO. Cordoba, Spain, September 2015.

xii. G. Mujica, J. Portilla, T. Riesgo, “Testbed Architecture and Framework for Debugging Wireless Sensor Networks", in proceedings of IEEE International Conference on Design of Circuits and Integrated Systems (DCIS'15), Estoril, Portugal, November 2015.

xiii. G. Mujica, J. Portilla, T. Riesgo, "Merging WSN and IoT Technologies towards Smart Urban Networking", in proceedings of Jornadas de Computación Empotrada y Reconfigurable (JCER'16) - CEDI 2016, Salamanca, Spain, September 2016.

xiv. G. Mujica, J. Portilla, T. Riesgo, “Teaching Wireless Sensor Networks to Master and PhD Students Based on the Cookie Node", in proceedings of IEEE International Conference on Design of Circuits and Integrated Systems (DCIS'16), Granada, Spain, November 2016.

\subsection{Future work}

This $\mathrm{PhD}$ thesis has opened several future research lines to broaden the capabilities associated with the Cookie HW-SW support platform and the deployment toolset. From the point of view of the WSN testbed, key features are to be further explored to enhance the support of the proposed platform, as follows.

- The integration of server-side technologies will contribute to provide developers with a session-driven experimentation where the top-level software layer allows managing the testing and debugging schemes in accordance with users' requirements, such as the automatic generation of actions and events in particular components of the network.

- The console-based options of the reprogramming strategies can be extended by a graphical model of the status of the memory-segmentsbased architecture, so that developers can easily track the functionalities to be replaced or updated based on the definition of an experiment schedule. This shall be directly linked to the code generation and 
reprogramming process when a new application slot is to be tested, so that the overall programming chain from the user side can be further automated.

- The partial reprogramming can also be combined with efficient code distribution techniques when applying it to deployed sensor nodes. Efforts have been put into designing packet partitioning and dissemination control strategies to produce a trade-off between transmission efficiency and code decomposition at the destination side. The integration of these code distribution algorithms will contribute to increase the adaptability of the operation of Cookie WSNs when specific conditions or requirements are to be met on-demand, with the support of the partial reprogramming strategies.

- Moreover, the synergy of the Cookie HW-SW platform with IoT technologies such as OpenHAB or middleware-based integration techniques will leverage its applicability to a wider range of today's smart city contexts. In this way, the potential of the proposed framework is also being extended with the inclusion of additional protocols such as 6LowPAN, so that users can have a broader spectrum of possibilities when experimenting with the modular platform.

On the other hand, the deployment toolset has been conceived to allow further analysis and optimization strategies to be included, particularly within the connectivity and routing evaluation engines. In this way, the following functionalities can extend the potential of the platform, as commented below.

- The integration of additional multi-hop mechanisms, such as tree-based routing or spatial/zone algorithms, will contribute to provide users with an enhanced comparative outline on how WSNs can be configured to properly adapt to the specifics of the target application scenario and constraints.

- Likewise, even though the tool is ready to handle modelling results that come from the input deployment definition, the study of additional infield simulation models based on experimental data (and considering 
additional correction factors within the path loss estimation), besides the ones integrated in this work, will enrich the capability of dynamically generating an accurate connectivity correlation scheme in accordance with the installation tasks that the deployer performs, such as node positioning readjustment, additional relay node placement, or runtime reconfiguration of communication parameters in-situ.

- The aforementioned objective also applies to the improvement of the transmission optimization algorithms, where the uniform and cohesionbased techniques can be combined with the adapted GA-based method in order to produce additional solutions for the transmission reconfiguration of the communication modules in-field.

- The applicability of the HHD within different WSN deployment contexts will also reinforce not only the usability of the implemented techniques for assisting users during in-field commissioning and maintenance tasks, but also the creation of new methods within the deployment methodology and automatic guidance proposed in this work. Therefore, the operation of the toolset in further smart environments shall contribute to the refinement of the HHD capabilities, based on the experimental experience provided by users.

- Based on the integration of additional analysis and optimization methods into the deployment toolset, the output models definition shall be extended following the modular structure proposed in this work (which allows a proper scalability of the models), so that the implementation refinement is enriched with the results obtained from applying such new functionalities at the target scenario. 



\section{Bibliography of the Thesis}

[802.15.4] IEEE Standard Part 15.4: Wireless Medium Access Control (MAC) and Physical Layer (PHY) Specifications for Low-Rate Wireless Personal Area Networks (LRWPANs) 1 October 2003.

[802.3] IEEE 802.3 Working Group, standards for Ethernet network, http://www.ieee802.org/3/

[ADuC841] ADuC841 Microcontroller, Analog Devices, http://www.analog.com/

[ADXL210] ADXL210 Low-Cost $\pm 10 \mathrm{~g} \quad$ Dual-Axis Accelerometer http://www.analog.com/en/products/obsolete/adxl210.html

[Ag9400] IEEE 802.3af PoE PD, Ag9400 Series Sivertel, http://www.silvertel.com/products/poe-power-over-ethernet/26-poemodules/165-ag9400.html

[Ammari'12] H. M. Ammari, N. Gomes, W. I. Grosky, et al., "Review of Applications of Wireless Sensor Networks", in Wireless Sensor Networks: Current Status and Future Trends, CRC Press, 1srt. Edition, 2012, DOI: 10.1201/b13092-3.

[AndroidDev] Android Developers environment and API, available on: https://developer.android.com/

[Apache] Apache HTTP server, an open-source web server, Apache Software Foundation, https://httpd.apache.org/

[Aslam'12] M. Aslam, N. Javaid, A. Rahim, U. Nazir, A. Bibi and Z.A. Khan, “Survey of extended LEACH-based clustering routing protocols for wireless sensor networks", in proceedings of IEEE 9th International Conference on High Performance Computing and Communication and IEEE 14th International Conference on Embedded Software and Systems (HPCC-ICESS), pp. 1232-1238, June 2012.

[AT91SAM7X] 32-bit ARM-based microcontroller, Atmel SAM7X series http://www.atmel.com/devices/SAM7X256.aspx

[Atzori'10] L. Atzori, A. Iera, Giacomo Morabito, "The Internet of Things: A survey", in Computer Networks, Vol. 54, Issue 15, October 2010, pp. 2787-2805, ISSN 13891286, http:/ / dx.doi.org/10.1016/j.comnet.2010.05.010. 
[Avilés'09] E. Avilés, J.A. García, “TinySOA: a service-oriented architecture for wireless sensor networks", in Service Oriented Computing and Applications, Vol. 3, Issue 2, pp. 99-108, June 2009.

[Bal'09] M. Bal, H. Xue, W. Shen, and H. Ghenniwa, "A testbed for localization and tracking in wireless sensor networks" in IEEE Systems, Man and Cybernetics, SMC 2009. pp. $3581-3586$.

[Belqasmi'11] F. Belqasmi, R. Glitho and C. Fu, "RESTful web services for service provisioning in next-generation networks: a survey," in IEEE Communications Magazine, vol. 49, no. 12, pp. 66-73, December 2011.

[Botta'13] M. Botta, M. Simek, "Adaptive Distance Estimation Based on RSSI in 802.15.4 Network", in Journal of Radioingenieering, vol.2, no.4, 2013.

[Brouwers'09] N. Brouwers, K. Langendoen, P. Corke, "Darjeeling, a feature-rich VM for the resource poor", In 7th ACM Conference on Embedded Networked Sensor Systems (SenSys '09), pp. 169-182, New York, NY, USA, 2009.

[Buckl'08] C. Buckl, S. Sommer, A. Scholz, et al., "Generating a Tailored Middleware for Wireless Sensor Network Applications," in IEEE International Conference on Sensor Networks, Ubiquitous and Trustworthy Computing (SUTC'08), pp. 162169, June 2008.

[C8051F930] C8051F92x-F93x Ultra Low-Power MCUs, Silicon Labs, http://www.silabs.com/

[Cao'08] Q. Cao and J. A. Stankovic, "An In-Field-Maintenance Framework for Wireless Sensor Networks", in Proceedings of the 4th IEEE international conference on Distributed Computing in Sensor Systems (DCOSS '08), Springer-Verlag, Berlin, Heidelberg, 457-468, 2008.

[Castalia] Castalia, a wireless sensor network simulator for low-power pervasive computing. https://castalia.forge.nicta.com.au.

[CC2420] Single-Chip 2.4 GHz IEEE 802.15.4 Compliant and ZigBee Ready RF Transceiver, http://www.ti.com/product/CC2420

[Chatterjee'2014] P. Chatterjee and N. Das, "Coverage constrained non-uniform node deployment in wireless sensor networks for load balancing," in Applications and Innovations in Mobile Computing (AIMoC'14), pp.126-132, Kolkata, 2014.

[Chaudhary'11] D.D. Chaudhary, S.P. Nayse, L.M. Waghmare, “Application of Wireless Sensor Networks for Greenhouse Parameter Control in Precision Agriculture", in International Journal of Wireless \& Mobile Networks (IJWMN'11) Vol. 3, No. 1, February 2011.

[Chen'06] B. Chen, K. Muniswamy-Reddy, and M. Welsh, "Ad-hoc multicast routing on resource-limited sensor nodes", in Proceedings of the 2nd international 
workshop on Multi-hop ad hoc networks: from theory to reality (REALMAN'06), New York, NY, USA, pp. 87-94, 2006.

[Chen'09] M. Chen, V. Leung, S. Mao, Y. Xiao and I. Chlamtac, “Hybrid geographical routing for flexible energy-delay trade-offs", in IEEE Transactions on Vehicular Technology, vol. 58, no. 9, pp. 4976-4988, Nov. 2009.

[Couto'03] D. De Couto, D. Aguayo, J. Bicket, and R. Morris, "A high-throughput path metric for multi-hop wireless routing", in Proceedings of the 9th annual international conference on Mobile computing and networking (MobiCom'03), New York, NY, USA, pp. 134-146, 2003.

[Cyclone] FPGA Cyclone II $\quad$ Family, Altera http://wl.altera.com/devices/fpga/cyclone2/overview/cy2-overview.html

[Dimitriou'07] T. Dimitriou, J. Kolokouris, and N. Zarokostas, “Sensenet: a wireless sensor network testbed", In Symposium on Modeling, analysis, and simulation of wireless and mobile systems (MSWiM '07). ACM, pp. 143-150, New York, USA, 2007.

[Dongliang'11] X. Dongliang and W. Guoying, "SPAE: a dynamic wireless sensor network performance analysis and evaluation platform", in Proceedings of 4th IEEE International Conference on Broadband Network and Multimedia Technology (IC-BNMT'11), October 2011, pp. 545-548.

[DPCM] Development, Planning, Commissioning and Maintenance Toolset for Wireless Sensor Networks (WSN-DPCM), ARTEMIS-JU European project, http://www.wsn-dpcm.eu/

[DPCM-MW] WSN-DPCM European Project, Platform heterogeneity \& Middleware, http://www.wsn-dpcm.eu/

[DPCM-SD] WSN-DPCM European Project, Demonstrators Architecture, Smart Parking Demonstrator, http://www.wsn-dpcm.eu/

[Du'14] W. Du, F. Mieyeville, D. Navarro , I. O'Connor, L. Carrel, "Modeling and simulation of networked low-power embedded systems: a taxonomy", in EURASIP Journal on Wireless Communications and Networking, Vol. 2014:106, doi:10.1186/1687-1499-2014-106.

[Dunkels'05] A. Dunkels, B. Grönvall, T. Voigt, “Contiki - a lightweight and flexible operating system for tiny networked sensors", in 29th IEEE International Conference on Local Computer Networks, pp. 455-462, 2004.

[Dunkels'06] A. Dunkels, N. Finne, J. Eriksson, T. Voigt, "Run-time dynamic linking for reprogramming wireless sensor networks" In 4th international conference on 
Embedded networked sensor systems (SenSys'06), pp. 15-28, New York, NY, USA, 2006.

[Erdelj'13] M. Erdelj, N. Mitton and E. Natalizio, "Applications of industrial wireless sensor networks", in Industrial Wireless Sensor Networks: Applications, Protocols, and Standards, CRC Press 2013, DOI: 10.1201/b14072-2.

[ETRX2] ETRX2 2.4GHz ISM band transceiver based on the Ember EM250 single chip ZigBee/IEEE802.15.4 solution, Telegesis http://www.telegesis.com/support/legacy-products/etrx2-zigbee-module/

[Fischer'09] I. Chatzigiannakis, S. Fischer, C. Koninis, et al., “WISEBED: An Open Large-Scale Wireless Sensor Network Testbed", In International conference on Sensor Applications, Experimentation, and Logistics (SENSAPPEAL'09), Athens, 2009.

[Flukso] Flukso, an open-source web-based community metering application, https://www.flukso.net/

[Förster'11] A. Förster et al., "Poster abstract: MOTEL: Towards flexible mobile wireless sensor network testbeds," in European Conference on Wireless Sensor Networks (EWSN'11), Poster Session, pp. 1-2, Germany, Feb. 2011.

[Gauger'09] M. Gauger, P. Marrón, C. Niedermeier, "TinyModules: code module exchange in TinyOS", in 6th international conference on Networked sensing systems (INSS'09), pp. 175-182, Piscataway, NJ, USA, 2009.

[Gavalas'06] D. Gavalas, G. Pantziou, C. Konstantopoulos, B. Mamalis, "Lowest-ID with Adaptive ID Reassignment: A Novel Mobile Ad-Hoc Networks Clustering Algorithm", in Proceedings the International Symposium of Wireless Pervasive Computing, January 2006.

[Gnawali'13] O. Gnawali, R. Fonseca, K. Jamieson, M. Kazandjieva, D. Moss and P. Levis, "CTP: an efficient, robust, and reliable collection tree protocol for wireless sensor networks", in ACM Transaction on Sensor Networks (TOSN), vol. 10, no. 3, 2013.

[Gomez'06] C. Gomez, P. Salvatella, O. Alonso and J. Paradells, “Adapting AODV for IEEE 802.15.4 Mesh Sensor Networks: theoretical discussion and performance evaluation in a real environment", in proceedings of the International Symposium on a World of Wireless, Mobile and Multimedia Networks (WoWMoM'06), Niagara-Falls, Buffalo, NY, June 2006.

[Gomez'10] C. Gomez, A. Boix and J. Paradells, "Impact of LQI-based routing metrics on the performance of a one-to-one routing protocol for IEEE 802.15.4 multihop networks", in EURASIP Journal of Wireless Communication and Networking. 2010, pp 1-20. 
[Goyal'12] D. Goyal and M. Tripathy, "Routing protocols in wireless sensor networks: a survey", in Proceedings of Second International Conference on Advanced Computing Communication Technologies (ACCT'12), pp. 474-480, January 2012.

[GP2Y0A60S] Distance Measuring Sensor Unit GP2Y0A60SZ0F, Sharp Corporation http://www.sharp-world.com/products/

[GS110TP] Netgear GS110TP, 8-port Gigabit PoE Smart Managed Switch http://support.netgear.com/product/GS110TP

[Gubbi'12] J. Gubbi, R. Buyya, S. Marusic, M. Palaniswami, “Internet of Things (IoT): A vision, architectural elements, and future directions", in Future Generation Computer Systems, Vol. 29, Issue 7, Sept. 2013, pp. 1645-1660, http://dx.doi.org/10.1016/j.future.2013.01.010.

[Haas'02] Z.J. Haas, M.R. Pearlman, P. Samar, The Zone Routing Protocol (ZRP) for Ad Hoc Networks, draft-ietf, July 2002.

[Han'05] C. Han, R. Kumar, R. Shea, E. Kohler, M. Srivastava, “A dynamic operating system for sensor nodes", In 3rd international conference on Mobile systems, applications, and services (MobiSys'05), pp. 163-176, New York, USA, 2005.

[Handziski'06] V. Handziski, A. Köpke, A. Willig, and Adam Wolisz, “TWIST: a scalable and reconfigurable testbed for wireless indoor experiments with sensor networks" In international workshop on Multi-hop ad hoc networks: from theory to reality (REALMAN '06). ACM, pp. 63-70, New York, USA, 2006.

[Hanson'09] M.A. Hanson, H.C. Powell, A.T. Barth, et Al., "Body Area Sensor Networks: Challenges and Opportunities," in IEEE Computer, Vol. 42, No. 1, pp. 58-65, Jan. 2009, doi: 10.1109/MC.2009.5.

[He'12] D. He, G. Mujica, J. Portilla, T. Riesgo, "Simulation tool and case study for planning wireless sensor network," in 38th Annual Conference on IEEE Industrial Electronics Society (IECON 2012), pp. 6024-6028, Oct. 2012.

[Heinzelman'00] W. R. Heinzelman, A. Chandrakasan, H. Balakrishnan, “Energy efficient communication protocol or wireless microsensor networks", in Annual Hawaii International Conference on System Sciences, Maui, 2000.

[Hermans'10] F. Hermans, O. Rensfelt, P. Gunningberg, L.A. Larzon, E. Ngai, “Sensei-UU - A Relocatable WSN Testbed Supporting Repeatable Node Mobility", in international workshop on Wireless network testbeds, experimental evaluation and characterization (WiNTECH '10), pp. 63-70, New York, USA, 2010.

[HEX] ARM Technical Support, "Intel Hex File Format". [Online]. Available: http://www.keil.com/support/docs/1584. 
[Horneber'14] J. Horneber, A. Hergenroder, "A Survey on Testbeds and Experimentation Environments for Wireless Sensor Networks," in IEEE Communications Surveys \& Tutorials, Vol. 16, no. 4, pp. 1820-1838, Fourth quarter 2014, doi: 10.1109/COMST.2014.2320051.

[Huangfu'10] W. Huangfu, L. Sun, and J. Liu, “A High-Accuracy Nonintrusive Networking Testbed for Wireless Sensor Networks", in EURASIP Journal on Wireless Communications and Networking, Vol. 2010, 15 pages, doi:10.1155/2010/642531.

[Hui'04] J. W. Hui and D. Culler, "The Dynamic Behavior of a Data Dissemination Protocol for Network Programming at Scale Categories and Subject Descriptors", In International Conference on Embedded Networked Sensor Systems (SenSys '04), pages 81-94, Baltimore, USA, 2004.

[Hunkeler'08] U. Hunkeler, H. L. Truong and A. Stanford-Clark, "MQTT-S - A publish/subscribe protocol for Wireless Sensor Networks," in Conference on Communication Systems Software and Middleware and Workshops, pp. 791798, Bangalore, 2008.

[IGLOO] IGLOO Low-Power Flash FPGAs family, Microsemi, http://www.microsemi.com/products/fpga-soc/fpga/igloo-overview

[Imran'10] M. Imran, A.M. Said, H. Hasbullah, "A survey of simulators, emulators and testbeds for wireless sensor networks," in International Symposium in Information Technology (ITSim 2010), vol.2, pp.897-902, June 2010, doi: 10.1109/ITSIM.2010.5561571.

[Indriya] M. Doddavenkatappa, M. C. Chan, and A. L. Ananda, “Indriya: A lowcost, 3D wireless sensor network testbed," in TridentCom, pp. 302-316, Apr. 2011.

[ISD51] In-System Debugger, ARMKEIL microcontroller tools, http://www.keil.com/c51/isd51.asp

[Jacquet'03] P. Jacquet and T. Clausen, Optimized Link State Routing Protocol (OLSR) 2003, RFC 3626.

[JavaEE] Java Enterprise Edition and Web application development, Oracle Technology Network, http://www.oracle.com/technetwork/java/javaee

[Jeong'09] J. Jeong, D. Culler, "Incremental Network Programming for Wireless Sensors", in International Journal of Communications, Network and System Sciences (IJCNS), Vol. 2 No. 5, pp. 433-452, 2009.

[Jersey] Jersey RESTful Web Services framework, open-source support for developing RESTful in Java, https://jersey.java.net/ 
[Johnson'09] D. Johnson, Y. Hu and D. Maltz, The Dynamic Source Routing Protocol (DSR) for Mobile Ad Hoc Networks for IPv4 2009, IETF RFC 4728.

[Kang'07] J. Kang, D. Kim and Y. Kim, "RSS Self-calibration Protocol for WSN Localization", in 2nd International Symposium on Wireless Pervasive Computing, San Juan, 2007.

[Keil] Keil development tools for the 8051 Microcontroller Architecture, http://www.keil.com/c51/

[Kenan'10] Xu Kenan, H. Hassanein, G. Takahara, W. Quanhong, "Relay Node Deployment Strategies in Heterogeneous Wireless Sensor Networks," in IEEE Transactions on in Mobile Computing, vol.9, no.2, pp.145-159, Feb. 2010.

[Khemapech'07] I. Khemapech, A. Miller, I. Duncan, “A survey of transmission power control in wireless sensor networks", in Proceedings of the 8th Annual Postgraduate Symposium on the Convergence of Telecommunications, Networking and Broadcasting, pp. 15-20, June 2007.

[Koshy'05] J. Koshy and R. Pandey, "Remote incremental linking for energy-efficient reprogramming of sensor networks," in Second European Workshop on Wireless Sensor Networks (EWSN’05), pp. 354-365, Istanbul, Turkey, 2005.

[Levis'02] P. Levis, D. Culler, "Maté: a tiny virtual machine for sensor networks", in 10th international conference on Architectural support for programming languages and operating systems (ASPLOS'02), pp. 85-95, New York, NY, USA, 2002.

[Levis'04] P. Levis, N. Patel, S. Shenker, D. Culler, “Trickle: A self-regulating algorithm for code propagation and maintenance in wireless sensor networks", Technical report, University of California at Berkeley, 2004.

[Levis'05] P. Levis, S. Madden, J. Polastre, R. Szewczyk et. al., “TinyOS: An operating system for sensor networks", In Ambient intelligence, pp. 115-148, Springer Berlin Heidelberg, 2005.

[Levis'09] P. Levis, D. Gay, "TinyOS Programming", Cambridge University Press: Cambridge, 2009.

[Lin'06] S. Lin, J. Zhang, G. Zhou, L. Gu, J. A. Stankovic, and T. He, “ATPC: adaptive transmission power control for wireless sensor networks", in Proceedings of the 4th international conference on Embedded networked sensor systems (SenSys'06). ACM, pp.223-236, New York, NY, USA, 2006.

[Liu'03] J. Liu and B. Li, "Distributed topology control in wireless sensor networks with asymmetric links," in IEEE Global Telecommunications Conference (GLOBECOM '03), 2003, pp. 1257-1262 vol.3. 
[Liu'11] X. Liu, K. M. Hou, H. Shi, C. Guo, H. Zhou, "Efficient and portable reprogramming method for high resource-constraint wireless sensor nodes," in IEEE 7th International Conference on Wireless and Mobile Computing, Networking and Communications (WiMob'11), Wuhan, pp. 455-460, 2011.

[Maps] Google Maps Android API, available on: https://developers.google.com/maps/documentation/android-api/

[Meier'09] A. Meier, M. Woehrle, M. Weise, J. Beutel and L. Thiele, "NoSE: Efficient Maintenance and Initialization of Wireless Sensor Networks," in 6th Annual IEEE Communications Society Conference on Sensor, Mesh and Ad Hoc Communications and Networks, pp. 1-9, Rome, Italy, 2009.

[MIB600] Memsic MIB600 Gateway for MICA motes http://www.memsic.com/wirelesssensor-networks/MIB600.

[Misra'10] S. Misra, D. H. Seung, X. Guoliang, T. Jian, "Constrained Relay Node Place-ment in Wireless Sensor Networks: Formulation and Approximations," in IEEE/ACM Transactions on Networking, vol.18, no.2, pp.434-447, April 2010.

[Mohseni'10] S. Mohseni, R. Hassan, A. Patel and R. Razali, "Comparative review study of reactive and proactive routing protocols in MANETs", in proceedings of 4th IEEE International Conference on Digital Ecosystems and Technologies (DEST'10), pp. 304-309, Dubai, April 2010.

[MSP430] 16-bits Ultra-low-power microcontroller produced by Texas Instruments, http://www.ti.com/product/MSP430F5438A

[Munawar'10] W. Munawar, M. H. Alizai, O. Landsiedel, K. Wehrle, "Dynamic TinyOS: Modular and Transparent Incremental Code-Updates for Sensor Networks", in IEEE International Conference on Communications (ICC'10), pp. 1-6, Cape Town, South Africa, 2010.

[NetBridge] Stargate NetBridge embedded sensor network gateway, Crossbow Technology, http://www-xbow-com/

[NGW100] NGW100 Network Gateway Kit, Atmel Corporation http://www.atmel.com/tools/NGW100MKIINETWORKGATEWAYKIT.aspx

[NSLU2] Network Storage Link for USB 2.0 Disk Drives from Linksys, http://www.linksys.com

[Nurseitov'09] N. Nurseitov, M. Paulson, R. Reynolds, C. Izurieta, “Comparison of JSON and XML Data Interchange Formats: A Case Study", Caine, 2009, 157-162.

[OpenHAB] OpenHAB, a framework for home automation systems and IoT technology integration, available online: http:/ /www.openhab.org/ 
[OSGi] OSGi, Dynamic module framework and specifications for Java programming, OSGi Alliance, https://www.osgi.org/

[Othman'12] M. F. Othman, K. Shazali, “Wireless Sensor Network Applications: A Study in Environment Monitoring System", in Procedia Engineering, Vol. 41, 2012, pp. 1204-1210, ISSN 1877-7058, http:/ / dx.doi.org/10.1016/j.proeng.2012.07.302.

[Pang'06] K.L. Pang and Y. Qin, "The comparison study of flat routing and hierarchical routing in ad hoc wireless networks", in proceedings of 14th IEEE International Conference on Networks (ICON'06), 1, pp. 1-6, Singapore, September 2006.

[Panta'07] R. K. Panta, I. Khalil, S. Bagchi, "Stream: Low Overhead Wireless Reprogramming for Sensor Networks", in 26th IEEE International Conference on Computer Communications (INFOCOM’07), pp. 928-936, Anchorage, AK, 2007.

[Panta'11] R. K. Panta, S. Bagchi, S. P. Midkiff, "Efficient incremental code update for sensor networks," ACM Transactions on Sensor Networks, vol. 7, no. 4, pp. 1-32, Feb. 2011.

[Pantazis'13] N.A. Pantazis, S.A. Nikolidakis and D.D. Vergados, "Energy-efficient routing protocols in wireless sensor networks: a survey", in IEEE Communications. Survey and Tutorials, vol. 15, no. 2, 2013, pp. 551-591.

[Perkins'03] C. Perkins, E. Belding-Royer and S. Das, Ad hoc On-Demand Distance Vector (AODV) Routing, 2003, RFC 3561.

[Polastre'05] J. Polastre, R. Szewczyk, D. Culler, “Telos: enabling ultra-low power wireless research", in International Symposium on Information Processing in Sensor Networks (IPSN’05), pp. 364-369, April 2005.

[Portilla'07] J. Portilla, T. Riesgo, A. Abril, A. de Castro, “Rapid prototyping for multiapplication sensor networking", 12 November 2007, SPIE Newsroom. DOI: $10.1117 / 2.1200711 .0851$.

[Portilla'10] J. Portilla, "Plataforma Modular e Interfaces Genericas de Transductores para Redes de Sensores Inalambricas", PhD Thesis, Universidad Politécnica de Madrid, 2010.

[Raspberry] Raspberry Pi 2 Model B, the second generation Raspberry Pi, and Raspbian Debian-based Operating System, https://www.raspberrypi.org

[Rezaee'09] M. Rezaee, M. Yaghmaee, “Cluster based Routing Protocol for Mobile Ad Hoc Networks", INFOCOMP, Journal of Computer Science, Vol.8, no. 1, pp.30-36, Feb.2009.

[Ringwald'07] M. Ringwald, K. Römer, and A. Vitaletti, "Passive inspection of sensor networks", in Proceedings of the 3rd IEEE international conference on 
Distributed computing in sensor systems (DCOSS'07), Springer-Verlag, Berlin, Heidelberg, 205-222, 2007.

[RN131] RN131 ultra-low power embedded 802.11 b/g Wi-Fi module http://www.microchip.com/wwwproducts/Devices.aspx?product=RN131

[Romer'04] K. Romer, F. Mattern, "The design space of wireless sensor networks," in IEEE Wireless Communications, Vol. 11, no.6, pp. 54-61, Dec. 2004, doi: 10.1109/MWC.2004.1368897.

[Sakamuri'08] D. Sakamuri, "NetEye: A wireless sensor network testbed", Master's thesis, School of Wayne State University, Detroit, MI, USA, 2008.

[Santander] SmartSantander Research Facility, Outdoor parking management area, Santander Smart City, http:/ / www.smartsantander.eu/

[Selavo'06] L. Selavo, G. Zhou and J. A. Stankovic, "SeeMote: In-Situ Visualization and Logging Device for Wireless Sensor Networks," in 3rd International Conference on Broadband Communications, Networks and Systems, San Jose, CA, 2006, pp. $1-9$.

[Sharma'11] S. Sharma and S. K. Jena, "A survey on secure hierarchical routing protocols in wireless sensor networks", in Proceedings of the International Conference on Communication, Computing \& Security (ICCCS '11), ACM, New York, NY, USA, 146-151, 2011.

[Sheu'08] J. Sheu, C. Chang, C. Sun, W. Hu, “WSNTB: A Testbed for Heterogeneous Wireless Sensor Networks", in International Conference on Ubi-Media Computing (U-Media'12), 338 - 343, China, 2008.

[SHT11] SHT1x Digital Humidity \& Temperature Sensor, Sensirion https://www.sensirion.com/products/digital-humidity-sensors-for-reliablemeasurements/digital-humidity-sensors-for-accurate-measurements/

[SI-52008-F] Bel Fuse components, http://www.belfuse.com/

[Slipp'08] J. Slipp, C. Ma, N. Polu, J. Nicholson, M. Murillo, S. Hussain, “Winter: Architecture and applications of a wireless industrial sensor network testbed for radio-harsh environments", in IEEE conference of Communication Networks and Services Research Conference (CNSR'08), pp. 422 -431, 2008.

[SoAP] Simple Object Access Protocol, specifications and technical recommendations, https://www.w3.org/TR/soap/

[Sobeih'06] A. Sobeih, J.C. Hou, L.-C. Kung, N. Li, H. Zhang, W.-P. Chen, H.-Y. Tyan and H. Lim, "J-Sim: a simulation and emulation environment for wireless sensor networks", in IEEE Wireless Communications, vol. 13, no. 4, pp. 104-119, Aug. 2006. 
[Son'04] D. Son, B. Krishnamachari and J. Heidemann, "Experimental study of the effects of transmission power control and blacklisting in wireless sensor networks", in First Annual IEEE Communications Society Conference on Sensor and Ad Hoc Communications and Networks (SECON'04), pp. 289-298, 2004.

[SWE] Sensor Web Enablement (SWE) framework and standards, Open Geospatial Consortium (OGC).

[SysML] UML-based System Modelling Language, Object Management Group (OMG), http://www.omgsysml.org/

[TinyAODV] TinyAODV, AODV implementation for TinyOS, http:// tinyos.stanford.edu/tinyos-wiki

[Tomcat] Apache Tomcat Server, open-source webserver implementation of JavaEE specifications, http://tomcat.apache.org/

[Tozlu'12] S. Tozlu, M. Senel, M. Wei, A. Keshavarzian, “Wi-Fi Enabled Sensors for Internet of Things: A Practical Approach", in IEEE Communications Magazine, Vol. 50, No. 6, pp. $134-143,2012$.

[uVision] ARMKeil uVision IDE and uVision Debugger, http://www2.keil.com/mdk5/uvision/

[Varga'08] A. Varga and R. Hornig. "An overview of the OMNeT++ simulation environment", in Proceedings of the 1st international conference on Simulation tools and techniques for communications, networks and systems \& workshops (Simutools '08). ICST, Brussels, Belgium, Article 60, 10 pages.

[W5200] W5200 Hardwired TCP/IP embedded Ethernet controller, WIZnet http://www.wiznet.co.kr/product-item/w5200/

[Wang'13] Y. Wang, B. M. Kelly and X. Li, "On the network connectivity of wireless sensor net-works following a random and non-uniform distribution," in IEEE 9th Conference on Wireless and Mobile Computing, Networking and Communications (WiMob'13), pp.69-74, 7-9 Oct. 2013.

[Wei'13] S. Wei, M. Hashemi, I. Warsawski, M. Laifenfeld, D. Starobinski and A. Trachtenberg, "TeaCP: a toolkit for evaluation and analysis of collection protocols in wireless sensor networks", in Proceedings of IEEE International Conference on Microwaves, Communications, Antennas and Electronics Systems (COMCAS 2013), pp. 1-5, Tel Aviv, October 2013.

[Werner'05] G. Werner-Allen, P. Swieskowski, M. Welsh, "Motelab: a wireless sensor network testbed" in International Symposium on Information Processing in Sensor Networks (IPSN’05), pp. 483 - 488, USA, April 2005. 
[Weyer'09] C. Weyer, C. Renner, V. Turau, and H. Frey, "A roadmap for hardware and software support for developing energy-efficient sensor networks", in Proc. 8th GI/ITG KuVS FGSN, pp. 67-70, Aug. 2009.

[WifiPlug] Wi-Fi Plug for remote wireless control of powered devices, http://www.wifiplug.co.uk/

[Winter'12] T. Winter, P. Thubert, A. Brandt, J. Hui, R. Kelsey, P. Levis, K. Pister, R. Struik and J. Vasseur, RPL: IPv6 Routing Protocol for Low Power and Lossy Networks March 2012, IETF RFC 6550.

[WL-500G] Asus WL-500G, OpenWrt distribution https://wiki.openwrt.org/toh/asus/wl500gp

[Xu'01] Y. Xu, J. Heidemann and D. Estrin, "Geography-informed energy conservation for ad-hoc routing", in proceedings of ACM/IEEE International Conference on Mobile Computing and Networking (MobiCom'01), pp. 70-84, Rome, Italy, July 2001.

[YI'13] K. Yi, R. Feng, N. Yu, P. Chen, "PARED: A testbed with parallel reprogramming and multi-channel debugging for WSNs," in IEEE Wireless Communications and Networking Conference (WCNC'13), pp.4630-4635, China, April 2013.

[Yu'08] J. Y. Yu, P. H. J. Chong and M. Zhang, "Performance of Efficient CBRP in Mobile Ad Hoc Networks (MANETS)", in IEEE 68th Vehicular Technology Conference (VTC'08), pp. 1-7, Calgary, BC, 2008.

[Yu'10] D. Yu, "DiF: A Diagnosis Framework for Wireless Sensor Networks," in INFOCOM IEEE Conference on Computer Communications Workshops, San Diego, CA, 2010, pp. 1-5.

[ZigBee] IEEE 802.5.4-based specification for low-rate low-power wireless personal area networks, ZigBee Alliance.

[ZigBit] ZigBit $900 \mathrm{MHz}$ Module with Balanced RF Output, 784/868/915MHz 802.15.4/ZigBee OEM, Atmel http://www.atmel.com/tools/ZIGBIT900MHZMODULEWITHBALANCEDRF OUTPUT.aspx 


\section{Appendix A}

This appendix contains a representation of how to access and control the memory map of the Ethernet module integrated into the Cookie platform.

Chip Base Address $=0 \times 0000,512(0 \times 0200)$ bytes send
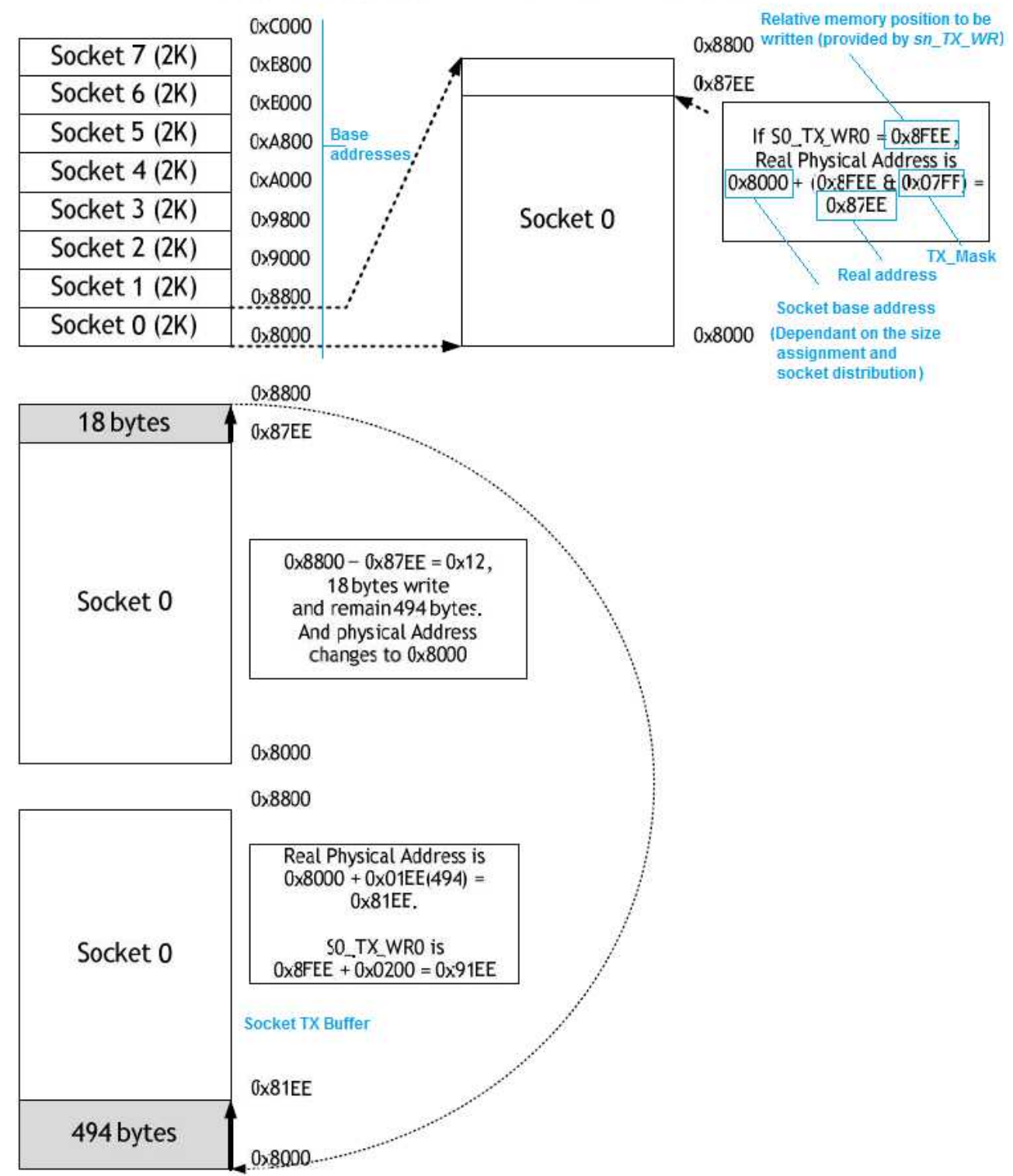

Fig. A-1 Memory scheme for TX/RX buffer handling of sockets [W5200]. 



\section{Appendix B}

Fig. B-I shows the node object representation (node ID 1 of a network in this case) within an input deployment model where four instances are created from various types of functional blocks.

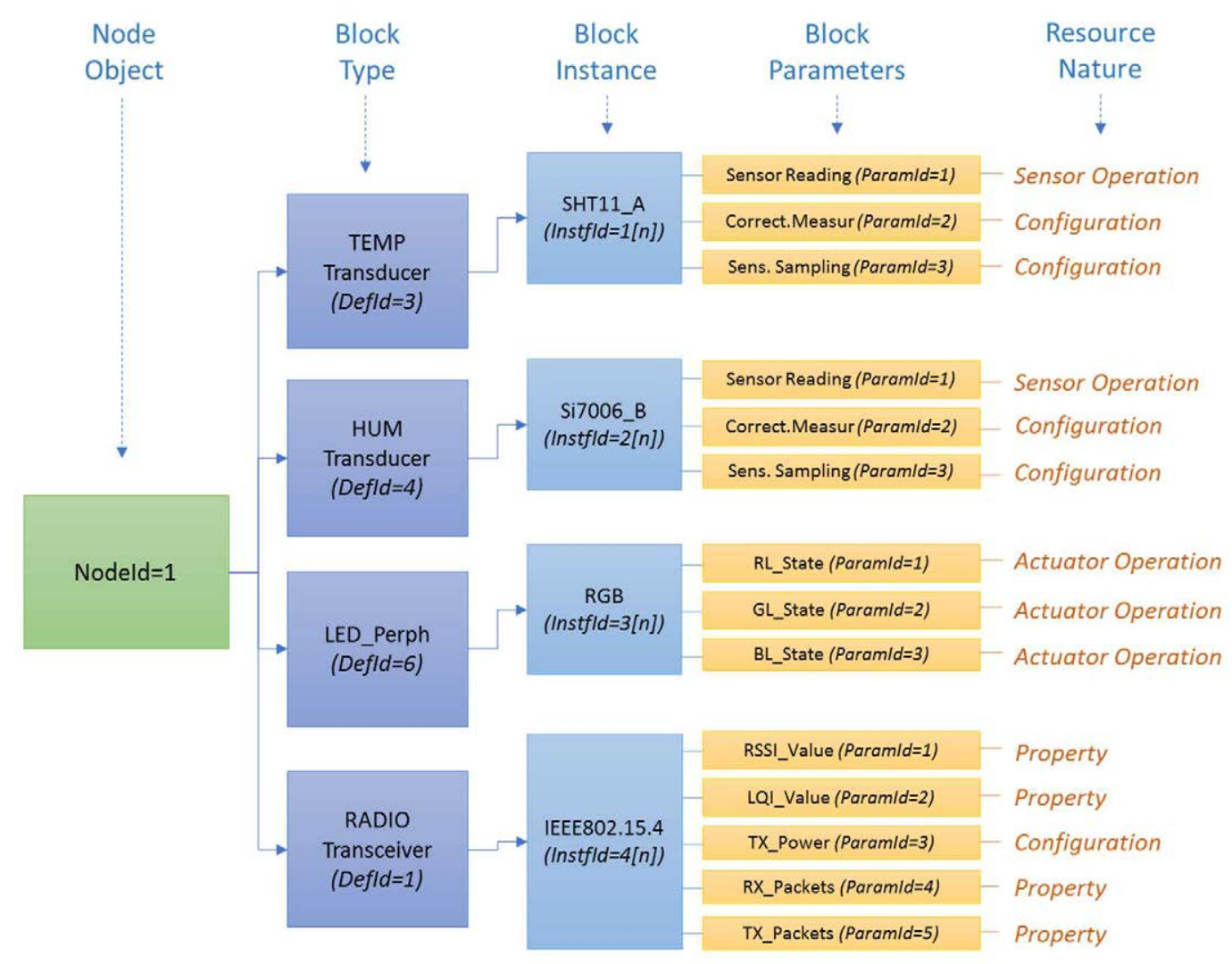

Fig. B-I Node object and functional block instance representation based on deployment model definition. 